\title{
Design Study for a Staged Very Large Hadron Collider
}

Report by the collaborators of The VLHC Design Study Group:

Brookhaven National Laboratory

Fermi National Accelerator Laboratory

Laboratory of Nuclear Studies, Cornell University

Lawrence Berkeley National Laboratory

Stanford Linear Accelerator Center

Stanford University, Stanford, CA, 94309

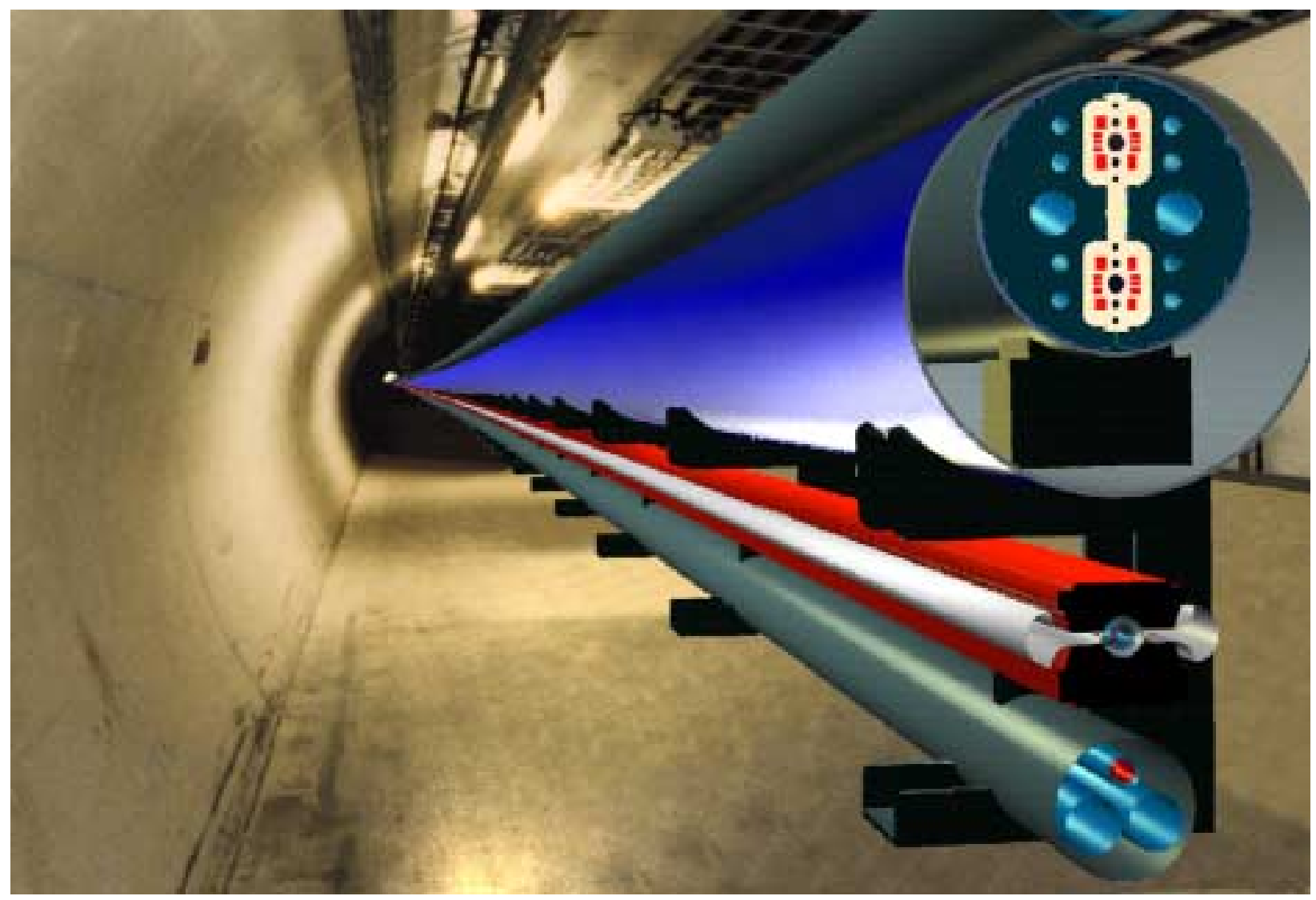




\section{Design Study for a Staged Very Large Hadron Collider}

\section{The VLHC Design Study Group:}

Giorgio Ambrosio ${ }^{1}$, Terry G. Anderson ${ }^{1}$, Nikolai Andreev ${ }^{1}$, Emanuela Z. Barzi ${ }^{1}$, Pierre C. Bauer ${ }^{1}$, Robert A. Bauer ${ }^{2}$, Sergey A. Belomestnykh ${ }^{3}$, Robert H. Bernstein ${ }^{1}$, Michael M. Blaskiewicz ${ }^{4}$, Rodger C. Bossert ${ }^{1}$, John A. Carson III ${ }^{1}$, Alexander W. Chao ${ }^{5}$, Deepak R. Chichili ${ }^{1}$, Peter Conroy ${ }^{6}$, Donald J. Cossairt ${ }^{1}$, Christine Darve ${ }^{1}$, Dmitri S. Denisov ${ }^{1}$, Angelika Drees ${ }^{4}$, Alexandr Drozhdin ${ }^{1}$, Luciano Elementi ${ }^{1}$, Kerry D. Ewald ${ }^{1}$, David A. Finley ${ }^{1}$,

Wolfram Fischer ${ }^{4}$, G. William Foster ${ }^{1}$, Peter H. Garbincius ${ }^{1}$, Norman M. Gelfand ${ }^{1}$,

Henry D. Glass ${ }^{1}$, Stephen A. Gourlay ${ }^{7}$, Ramesh C. Gupta ${ }^{4}$, Michael A. Harrison ${ }^{4}$, Steven L. Hays ${ }^{1}$, Yuenian Huang ${ }^{1}$, Linda Imbasciati ${ }^{1}$, Alan Jackson ${ }^{7}$, John A. Johnstone ${ }^{1}$,

Vadim V. Kashikhin ${ }^{1}$, Vladimir Kashikhin ${ }^{1}$, Kurt D. Kennedy ${ }^{7}$, James S. Kerby ${ }^{1}$,

Arkadiy L. Klebaner ${ }^{1}$, Glen R. Lambertson ${ }^{7}$, Michael J. Lamm ${ }^{1}$, Chris Laughton ${ }^{1}$,

Valery A. Lebedev ${ }^{1}$, Peter J. Limon ${ }^{1}$, Alexander Makarov ${ }^{1}$, Ernest I. Malamud ${ }^{1}$, John P. Marriner ${ }^{1}$, Philip S. Martin ${ }^{1}$, Michael P. May ${ }^{1}$, Nikolai Mokhov ${ }^{1}$, Charles Nelson ${ }^{8}$, King Yuen Ng${ }^{1}$, Thomas H. Nicol ${ }^{1}$, Barry L. Norris ${ }^{1}$, Igor V. Novitski ${ }^{1}$, Andrew R. Oleck ${ }^{1}$, Thomas M. Page ${ }^{1}$, Stephen G. Peggs ${ }^{4}$, Lee Petersen ${ }^{8}$, Thomas J. Peterson ${ }^{1}$, Henryk Piekarz ${ }^{1}$,

Fulvia C. Pilat ${ }^{4}$, Mauro Pivi ${ }^{7}$, Duane A. Plant ${ }^{1}$, Vadim I. Ptitsyn ${ }^{4}$, Roger J. Rabehl ${ }^{1}$,

Gianluca Sabbi ${ }^{7}$, Philip Schlabach ${ }^{1}$, Tanaji Sen ${ }^{1}$, Vladimir Shiltsev ${ }^{1}$, Jeffrey A. Sims ${ }^{1}$, Nikolai A. Solyak ${ }^{1}$, James B. Strait ${ }^{1}$, Michael Syphers ${ }^{1}$, Gianni R. Tassotto ${ }^{1}$, Steven Tepikian ${ }^{4}$, Iouri Terechkine ${ }^{1}$, Jay C. Theilacker ${ }^{1}$, John C. Tompkins ${ }^{1}$, Dejan Trbojevic ${ }^{4}$,

Vladimir Tsvetkov ${ }^{1}$, William C. Turner ${ }^{7}$, James T. Volk ${ }^{1}$, Bruce Wagener ${ }^{8}$, Masayoshi Wake ${ }^{9}$,

Ronald J. Walker ${ }^{1}$, George J. Wojcik ${ }^{1}$, Meiqin Xiao ${ }^{1}$, Ryuji Yamada ${ }^{1}$, Victor A. Yarba ${ }^{1}$, Shou-Yuan Zhang ${ }^{4}$, Alexander Zlobin ${ }^{1}$

\section{Editors:}

H.D. Glass, G.W. Foster, P.J. Limon, E.I. Malamud, P.H. Garbincius, S.G. Peggs, J.B. Strait, M. Syphers, J.C. Tompkins, A. Zlobin

\section{Acknowledgments}

The authors thank Michael Witherell, Director of Fermilab, and the U.S. Department of Energy for their support of this study. They also thank Ping Wang (Fermilab) for creating the web site for maintaining this document.

\footnotetext{
${ }^{1}$ Fermilab

${ }^{2}$ Illinois State Geological Survey, Champaign, IL

${ }^{3}$ Laboratory of Nuclear Studies, Cornell University

${ }^{4}$ Brookhaven National Laboratory

${ }^{5}$ Stanford Linear Accelerator Center

${ }^{6}$ consultant, Elmhurst, IL

${ }^{7}$ Lawrence Berkeley National Laboratory

${ }^{8}$ CNA Consulting Engineers, Minneapolis, MN

${ }^{9}$ KEK, Japan
} 
Fermilab-TM-2149 


\section{Table of Contents}

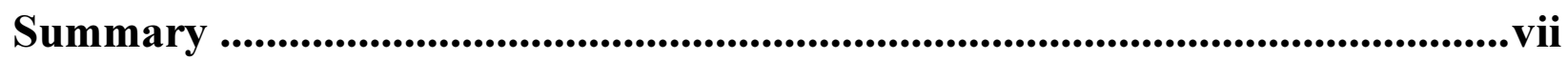

Chapter 1. Introduction.....................................................................1-1

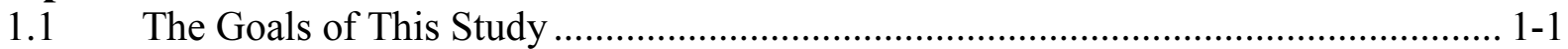

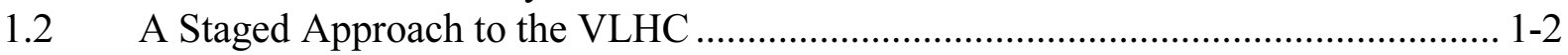

1.3 The Technical Description and Challenges ........................................................... 1-3

$1.4 \quad$ Gaining Public Support for a VLHC …………................................................... 1-5

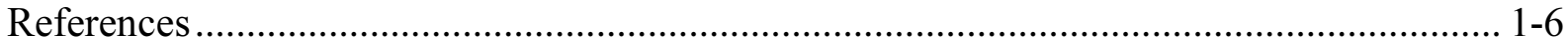

Chapter 2. Overview and Summary …...................................................2-1

$2.1 \quad$ General Description ................................................................................... 2-1

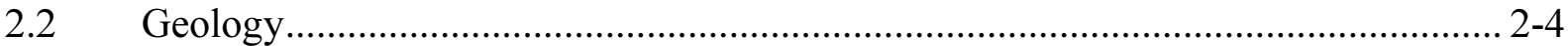

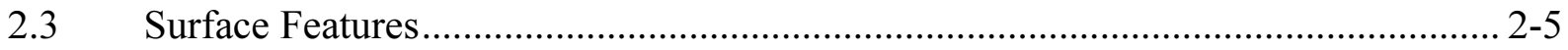

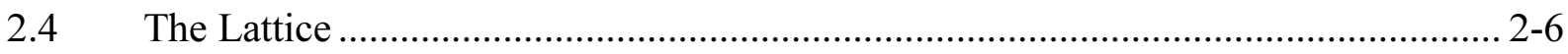

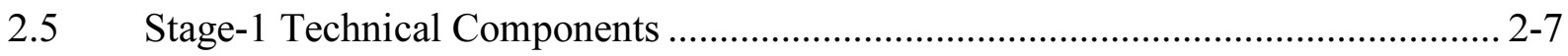

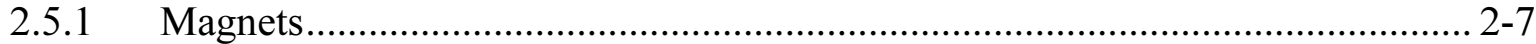

2.5.2 Other Stage-1 Technical Components ………................................................ 2-9

2.6 Stage-1 Construction and Installation Schedule .................................................. 2-9

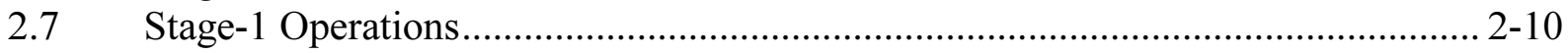

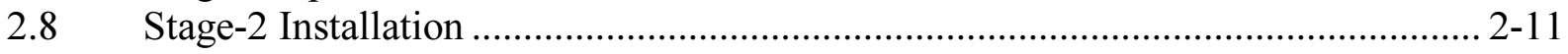

$2.9 \quad$ Stage-2 Operation ……………..................................................................... 2-12

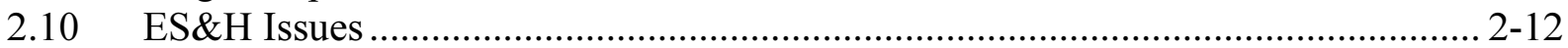

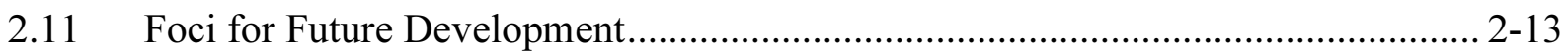

2.11.1 Performance Issues for Stage 1 …………................................................. 2-13

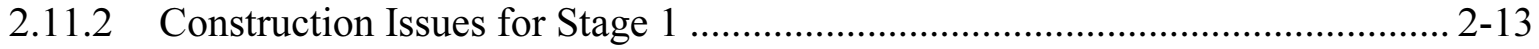

2.11.3 R\&D Aimed at Improvements and Cost Reduction for Stage 1 ....................... 2-14

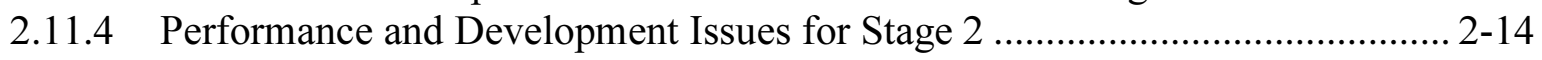

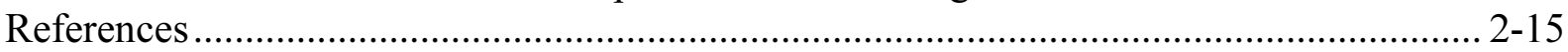

Chapter 3. Collider Accelerator Physics and Design .............................3-1

3.1 Two Colliders in One Tunnel ...................................................................... 3-1

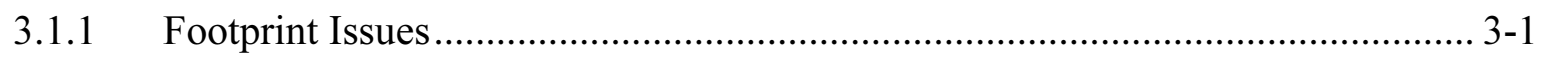

3.1.2 Optics Issues ..................................................................................... 3-1

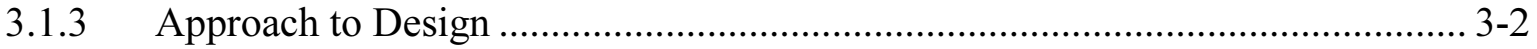

3.1.4 Footprint Parameters ............................................................................... 3-2

3.1.5 Half-Cell Length ..................................................................................... 3-3

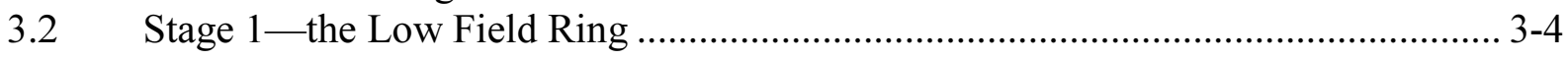

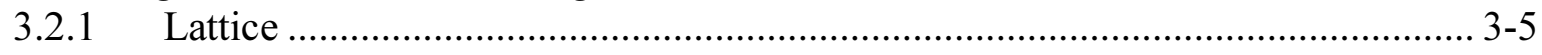

3.2.2 Magnet Apertures and Field Quality............................................................... 3-9

3.2.3 Tolerances and Corrections ........................................................................ 3-13

3.2.4 Ground Motion and Emittance Growth ………............................................. 3-18

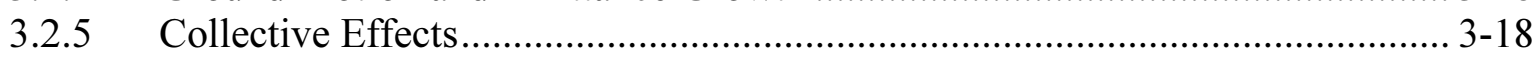

3.3 Stage 2 - the High Field Ring ....................................................................... 3-21

3.3.1 Luminosity versus Energy ………........................................................... 3-21

3.3.2 Operational Performance ........................................................................ 3-21 
3.3.3 Advantages and Disadvantages of Flat Beams ……....................................... 3-26

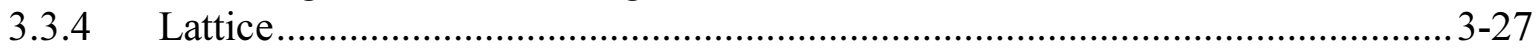

3.3.5 Magnet Apertures and Field Quality ................................................................ 3-29

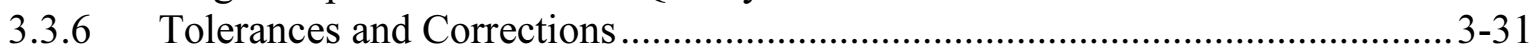

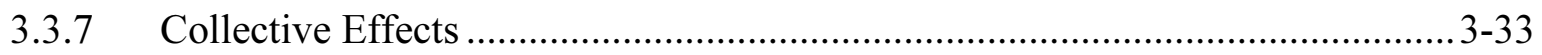

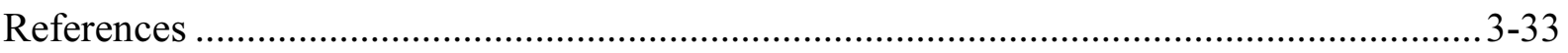

Chapter 4. The Fermilab Complex as Injector ........................................4-1

4.1 The Fermilab Complex and Beam Properties ........................................................... 4-1

$4.2 \quad$ Operational Scenarios ..................................................................................... $4-3$

$4.3 \quad$ Tevatron Extraction Lines................................................................................ $4-4$

4.3.1 Option A. Unipolar Tevatron, Single Extraction Region..................................... 4-4

4.3.2 Option B. Unipolar Tevatron, Two Transfer Lines.............................................. 4-5

4.3.3 Option C (Preferred): Bipolar Tevatron …………............................................ $4-6$

4.3.4 Extraction from the Tevatron ......................................................................... $4-6$

4.3.5 Installation and Beamline Construction Issues ................................................. 4-8

4.4 Transfer Line Magnets and Kickers .................................................................... 4-8

Chapter 5. Stage-1 Components ............................................................5-1

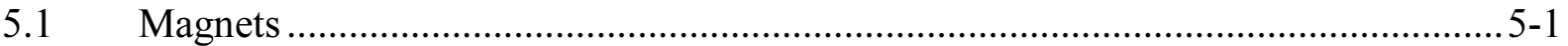

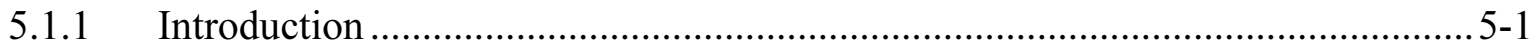

5.1.2 Combined Function Arc Magnets ……………..................................................5-4

5.1.3 Corrector Magnets...................................................................................... 5-18

5.1.4 Interaction Regions ………………………….....................................5-20

5.1.5 Special Collider Ring Magnets ……………............................................ 5-23

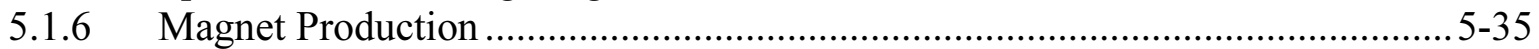

5.1.7 Magnetic Measurements and Testing.................................................... $5-42$

5.1.8 Magnet Installation................................................................................ 5-46

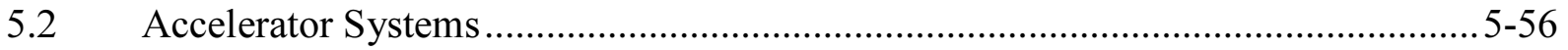

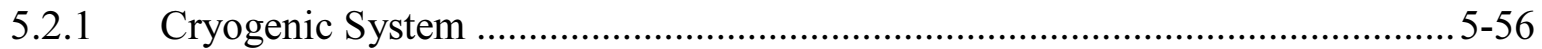

5.2.2 Magnet Power Supplies, Current Leads, and Quench Protection ......................5-66

5.2.3 Arc Instrumentation and Power Distribution .................................................5-72

5.2.4 Beam Vacuum System .......................................................................... 5-79

5.2.5 Once-per-Turn Instrumentation................................................................ $5-82$

5.2.6 Radio Frequency Systems …………………....................................... 5-82

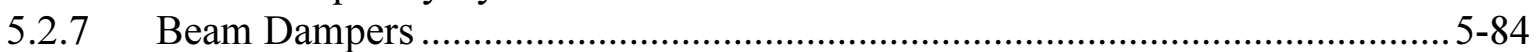

5.3 Radiation, Machine Protection, and Beam Abort ……….........................................5-87

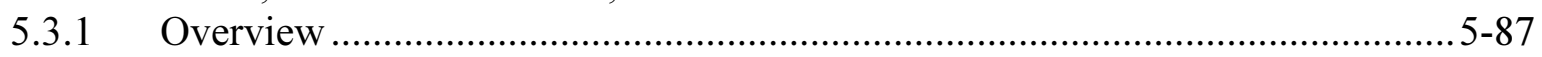

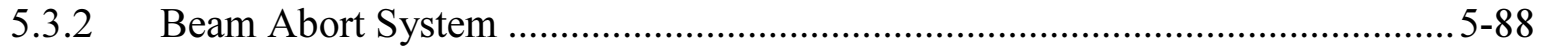

5.3.3 Radiation and Beam Loss...................................................................... 5-91

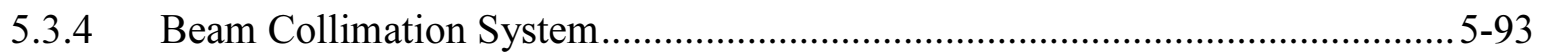

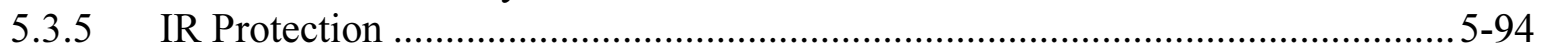

5.3.6 Worst-Case Beam Accidents.......................................................................... 5-94

5.3.7 General ES\&H Considerations................................................................. 5-96

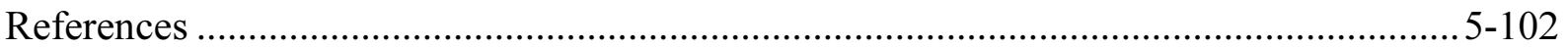


Chapter 6. Stage-2 Components ........................................................6-1

6.1 Superconducting Magnet Systems ..................................................................... 6-1

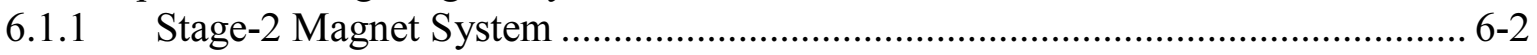

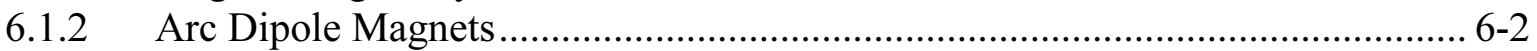

6.1.3 Arc Quadrupole Magnets.............................................................................. 6-5

6.1.4 Arc Corrector Magnets ………………………............................................. 6-7

6.1.5 IR Magnets............................................................................................. 6-9

6.1.6 Cryostat and Spool Pieces.............................................................................. 6-10

6.1.7 Magnet Quench Protection .......................................................................... 6-14

6.1.8 Magnet Production and Testing ...................................................................... 6-15

6.1.9 Magnet Installation, Including Survey and Alignment...................................... 6-16

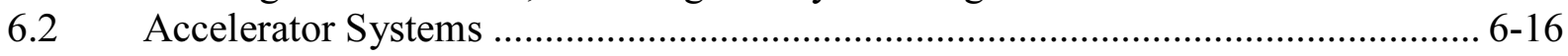

6.2.1 Stage-2 Cryogenic System Concept ………………..................................... 6-16

6.2.2 Vacuum System ....................................................................................... 6-21

6.2.3 Magnet Power Supply and Quench Protection .................................................. 6-28

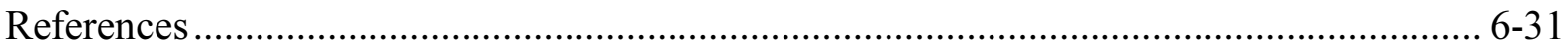

Chapter 7. Conventional Construction and Facilities .............................7-1

7.1 Geology of the Fermilab Region .................................................................... 7-1

7.2 Collider Tunnel and Enclosures......................................................................... 7-6

7.3 Various Tunnels and Ramps ……………...................................................... 7-11

7.4 Accelerator Utility Caverns …………………............................................... 7-13

7.4.1 Beam Stop Enclosure............................................................................... 7-13

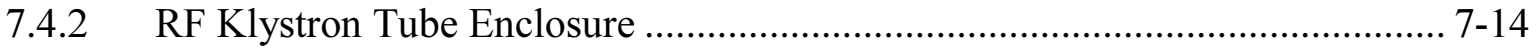

7.4.3 Kicker Magnet Power Supply Enclosures ……………………….................... 7-14

7.4.4 A-Site and B-Site Cryo Systems Caverns................................................... 7-14

7.4.5 Groundwater Collection Caverns and Pumping Stations ……………………... 7-15

7.4.6 AC Power Distribution Alcoves .................................................................. 7-16

7.4.7 Cryo Valve Alcoves................................................................................. 7-16

7.4.8 Quench Resistor Caverns ....................................................................... 7-16

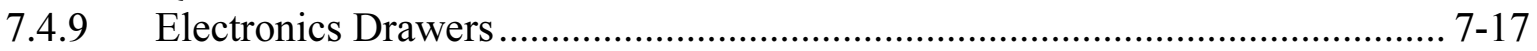

7.5 Experiment Caverns and Bypasses …………….............................................. 7-17

7.6 Surface Buildings, Utilities, Factories, and Footprints ............................................. 7-19

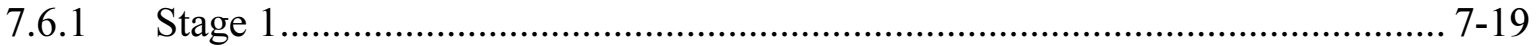

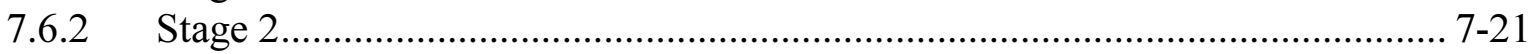

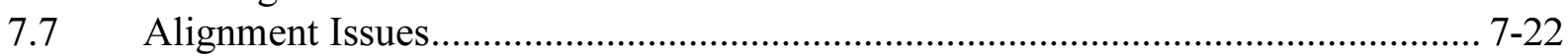

7.7.1 Tunnel Alignment During Construction ............................................................ 7-22

7.7.2 Reference Network and Component Alignment................................................ 7-22

7.8 ES\&H Issues During Construction, Installation, and Operations............................ 7-27

7.9 Model of Construction Schedule .................................................................... 7-30

7.10 Construction Engineering and Design Challenges ............................................... 7-32

7.11 Cost and Risk Reduction................................................................................ 7-35

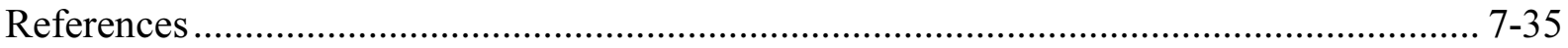

Chapter 8. VLHC Experiments and Detector Issues .............................8-1

8.1 Description of Experiment Parameters for Stage 1 ............................................... 8-1

8.2 Description of Major Experimental Challenges for Stage 2 …................................ 8-4 
8.3 Machine-Detector Interface Requirements for Stage 1 .......................................... 8-6

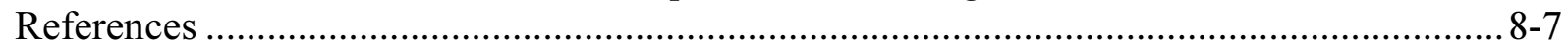

Chapter 9. Cost Analysis of the Stage-1 VLHC .....................................9-1

9.1 Uses and Limitations of This Cost Analysis ........................................................ 9-1

9.2 Identification of the Cost Drivers........................................................................

9.3 Models for Estimating the Cost Drivers.............................................................. 9-2

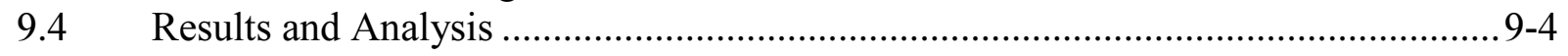

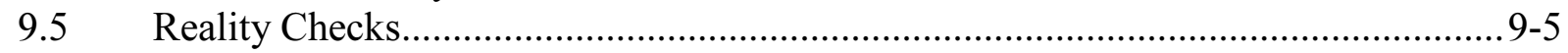

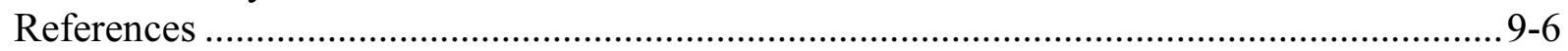

Chapter 10. $\quad$ R\&D Programs and Related Studies .................................10-1

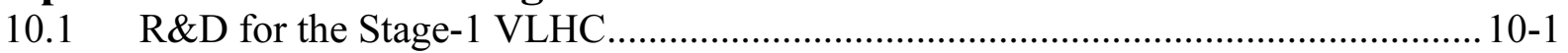

10.1.1 Tunneling R\&D and Engineering ……................................................... 10-1

10.1.2 Vacuum System....................................................................................

10.1.3 Beam Stability ................................................................................. 10-2

10.1.4 Magnetic Field Quality......................................................................... 10-3

10.1.5 High-Gradient IR Quadrupoles ................................................................ 10-4

10.1.6 Magnet Production, Handling and Operation ……........................................ 10-5

10.1.7 Cryogenic System .............................................................................. 10-5

10.1.8 Public Acceptance and Outreach................................................................. 10-6

10.1.9 Other Engineering Studies ' ........................................................................ 10-6

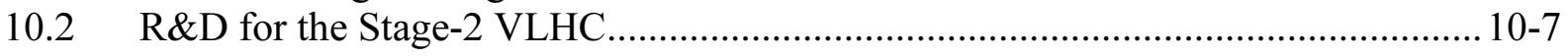

10.2.1 High Field Magnet R\&D...................................................................... 10-7

10.2.2 Strand and Cable R\&D......................................................................... 10-8

10.2.3 IR Magnets for Flat Beam Optics .......................................................10-10

10.2.4 Cryogenic-Related R\&D for a High Field VLHC ........................................ 10-12

10.2.5 Synchrotron Radiation and Vacuum ............................................................ 10-13

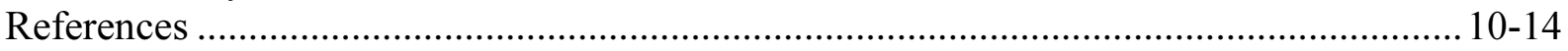




\section{Design Study for a Staged Very Large Hadron Collider}

\section{Summary}

Advancing accelerator designs and technology to achieve the highest energies has enabled remarkable discoveries in particle physics. This report presents the results of a design study for a new collider at Fermilab that will create exceptional opportunities for particle physics-a two-stage very large hadron collider. In its first stage, the machine provides a facility for energy-frontier particle physics research, at an affordable cost and on a reasonable time scale. In a second-stage upgrade in the same tunnel, the VLHC offers the possibility of reaching 100 times the collision energy of the Tevatron. The existing Fermilab accelerator complex serves as the injector, and the collision halls are on the Fermilab site.

The Stage-1 VLHC reaches a collision energy of $40 \mathrm{TeV}$ and a luminosity comparable to that of the LHC, using robust superferric magnets of elegant simplicity housed in a largecircumference tunnel. The Stage- 2 VLHC, constructed after the scientific potential of the first stage has been fully realized, reaches a collision energy of at least $175 \mathrm{TeV}$ with the installation of high-field magnets in the same tunnel. It makes optimal use of the infrastructure developed for the Stage-1 machine, using the Stage-1 accelerator itself as the injector.

The goals of this study, commissioned by the Fermilab Director in November 2000, are:

- To create reasonable designs for the Stage-1 and Stage-2 VLHC in the same tunnel

- To discover the technical challenges and potential impediments to building such a facility at Fermilab

- To determine the approximate costs of the major elements of the Stage-1 VLHC

- To identify areas requiring significant R\&D to establish the basis for the design

The answers to these questions, addressed in detail throughout the report, are encouraging. The low-field magnets used in the Stage-1 VLHC require a tunnel of $233 \mathrm{~km}$ to reach the desired energy. Although such a large tunnel represents significant construction, management and public outreach challenges, there appears to be no technical reason why it could not be built in about six years, permitting machine commissioning to begin 10 years after the start of construction. The intrinsic properties of the simple magnet design significantly reduce the extent and complexity of the supporting subsystems: the cryogenic load is comparable to the present-day Tevatron; excellent injection field quality results in good dynamic aperture; and low inductance and low stored energy in the magnetic field greatly simplify the power supply system. All of these factors combine to reduce the cost and complexity of the technical components to compensate for the cost of the large tunnel. The total cost of the collider facility is estimated to be comparable to the cost of a linear electron collider of $500 \mathrm{GeV}$, as recently estimated for the TESLA design.

The technical risks for the Stage-1 VLHC are few, suggesting a short and relatively inexpensive $R \& D$ program. The small beam tube and low injection energy from the Tevatron required an examination of the risk of beam instabilities at injection. This study concluded that these instabilities would yield to a combination of straightforward RF manipulations, injecting 
beam into the ring in a well-distributed sequence, and modest feedback systems. Nevertheless, risk-reducing $\mathrm{R} \& \mathrm{D}$ is indicated. Other technical uncertainties involve the unusual length of the magnet and the long distances between refrigerators. The fabrication of long prototype magnets and the commissioning of a realistic string test will answer many of the questions related to production, handling, transportation and installation of $65 \mathrm{~m}$ magnets. This test, plus calculations and laboratory measurements, will establish the cryogenic heat load and flow stability. The high-gradient quadrupoles needed for the collision optics are a natural result of high-field magnet programs at Fermilab and elsewhere. Most other magnet and accelerator systems are of conventional design, requiring little or no R\&D.

The underground construction represents about 50 percent of the cost of the Stage-1 VLHC, and is an obvious place to concentrate cost-reducing R\&D. Typically, more than 40 percent of underground construction costs are in labor; modest use of simple automation techniques might reduce the cost of the VLHC significantly and at the same time improve construction safety. Improvements in tunnel-boring machine reliability, utilization and logistical support could yield greatly reduced cost in return for $R \& D$ investments made feasible by the large scale of the project.

The very-large-circumference tunnel is an advantage to the Stage-2 VLHC, where the high beam energy makes synchrotron radiation significant. The design energy is $175 \mathrm{TeV}$, but the study concludes that reaching $200 \mathrm{TeV}$ with a luminosity greater than $2 \times 10^{34} \mathrm{~cm}^{-2} \mathrm{~s}^{-1}$ will not be difficult, particularly if $R \& D$ can show that synchrotron radiation masks are effective in intercepting the radiation at ambient temperature. The power required for cryogenic refrigeration for the Stage-2 VLHC operating at $200 \mathrm{TeV}$ is about $100 \mathrm{MW}$, which would decrease by 20 percent with the use of the masks. The major R\&D for the Stage- 2 machine is the development and commercialization of cost-effective high-field magnets. The large tunnel permits $200 \mathrm{TeV}$ operation with $12 \mathrm{~T}$ magnets, well within reach of present-day materials.

Overall, the results of this study show that a VLHC can be built at Fermilab during a construction period of about 10 years for a cost comparable to the cost of a linear collider. The energy-frontier Stage-1 VLHC is not only technically feasible, but contains no unusual difficulties that cannot be solved using technology available today. One must be willing to pursue a decades-long program to reach the ultimate energy, but energy-frontier physics could be available in a relatively short time. The study shows it is feasible to design efficient lattices for collider rings of two very different energies in the same tunnel. Continuation of the design and engineering study, with the addition of experts in underground construction and management, would result in a more complete parametric study of design options, with more focused details and a narrowed uncertainty in the cost within a few years.

The VLHC should certainly be built at an existing large accelerator laboratory to reduce the initial investment in injectors and to take advantage of the existing intellectual and management infrastructure. It will certainly be built by an international collaboration, to share the cost. This study assumes construction at Fermilab, and shows that Fermilab would be an excellent site. In reality, the VLHC could be built at any large laboratory with extensive hadron accelerator infrastructure. This facility would fit well within a worldwide plan that includes the Tevatron, followed by the LHC and a linear electron collider, and then a VLHC. The staged VLHC will take us quickly to the energy frontier; an upgrade in the same tunnel offers a straightforward path to the high-energy future. It should be strongly considered as a viable option for the next large high-energy physics initiative in the U.S. 


\section{Chapter 1. Introduction}

Particle physics makes its greatest advances with experiments at the highest energy. The only sure way to advance to a higher-energy regime is through hadron colliders - the Tevatron, the LHC, and then, beyond that, a Very Large Hadron Collider. At Snowmass-1996 [1], investigators explored the best way to build a VLHC, which they defined as a $100 \mathrm{TeV}$ collider. The goals in this study are different. The current study seeks to identify the best and cheapest way to arrive at frontier-energy physics, while simultaneously starting down a path that will eventually lead to the highest-energy collisions technologically possible in any accelerator using presently conceivable technology. This study takes the first steps toward understanding the accelerator physics issues, the technological possibilities and the approximate cost of a particular model of the VLHC. It describes a staged approach that offers exciting physics at each stage for the least cost, and finally reaches an energy one-hundred times the highest energy currently achievable.

\subsection{The Goals of this Study}

In November, 2000, the Fermilab director commissioned a study for the purpose of beginning to understand the consequences of a staged approach to the VLHC [2]. The major goals of the study are:

- To determine the basic parameters of a proton-proton collider of $\mathrm{E}_{\mathrm{cm}}$ greater than $30 \mathrm{TeV}$ and luminosity of at least $10^{34} \mathrm{~cm}^{-2} \mathrm{~s}^{-1}$, while preserving the option of eventual operation of a collider with $\mathrm{E}_{\mathrm{cm}}$ greater than $150 \mathrm{TeV}$ in the same tunnel

- To identify the major technology and construction challenges, the important accelerator physics issues, and any unusual operational, environmental, safety and health requirements

- To estimate the current-day construction costs of the major cost drivers for the initial collider configuration, assuming it is built using Fermilab as the injector

- To identify areas requiring significant R\&D to establish the technical basis for the facility.

This study is not a conceptual design report, nor is it a complete cost estimate. To accomplish either of those goals would have taken more than the available time and resources. Instead, it is a broad-brush study, intended to describe the major issues. It provides information about the resources needed to construct such a facility and highlights any serious technical problems, allowing concentration of future effort. Since strategic planning requires accurate conclusions about feasibility and costs of facilities, this study is likely to be the first of a series of increasingly focused studies of the VLHC.

\subsection{A Staged Approach to the VLHC}

The staged approach to the VLHC starts with the construction and operation of a collider made from simple and inexpensive components, followed at a later time by a higher-energy collider in the same tunnel. The plan has the following guidelines: 
- Each stage must hold the promise of new and exciting particle physics.

- The first stage should lead to and assist in the realization of the next stage.

- Each stage should be a reasonably low-cost step into the energy frontier.

The VLHC satisfies all of these guidelines. The cost of tunneling is in general significantly less than the cost of a collider's technical components. Thus, it is cost-effective to increase tunnel circumference if doing so lowers the cost of the expensive technical components enough to reduce the overall cost of the collider. Hence, Stage 1 of this design uses low-field superferric magnets that are themselves inexpensive, and also require simple and less costly support systems, such as cryogenics and power supplies. However, the use of a low-field magnet requires a large tunnel to reach the energy frontier. In this design, we have settled on $40 \mathrm{TeV}$ collision energy with two detectors, requiring a ring circumference of $233 \mathrm{~km}$. In a further attempt to reduce costs, we have sited the collider at Fermilab, permitting the use of the existing Fermilab injector chain and physical plant, valued at well over $\$ 1$ billion. It also takes advantage of Fermilab's irreplaceable organizational infrastructure and expertise, further reducing design and startup costs.

The large circumference of the collider ring also has advantages for Stage 2. Above $30 \mathrm{TeV}$ beam energy, synchrotron radiation becomes an important factor in high-energy proton colliders. In a cryogenic environment, it is one of the properties that limits the ultimate energy and luminosity of such machines. The design operating energy of the high-energy collider is 175 $\mathrm{TeV}$, but the $35-\mathrm{km}$ radius of curvature of the VLHC would permit it to reach $200 \mathrm{TeV}$ collision energy with reasonable luminosity and power consumption. Since the first collider serves as the injector into the second collider, the common circumference permits a straightforward and fast filling scheme for the second machine, eliminating potentially troublesome issues connected with field quality in high-field magnets.

There are disadvantages to a staging scenario. It requires patience and the willingness to start down a multi-decade path toward the highest collision energy. The need to anticipate the approximate design of both stages at the time civil construction begins, may dictate certain conservative allowances in the design that a single-step plan would not require. The inside diameter of the tunnel or additional surface service areas are obvious examples. Both colliders are in the same tunnel, requiring a period of six years or more for the conversion from the initial configuration to the higher-energy one. During this time there would be no physics program. A staging scenario using a very large tunnel suffers from potential additional cost, not only because the tunnel is longer, but also because it traverses more disparate geology, potentially incurring higher costs per unit length. Whether this is true depends on the geology of the various possible Fermilab sites. This study addresses the topic. Finally, although staging the colliders may be a low-cost approach, a non-staged approach might be an even lower-cost way to build a collider of a specific energy.

Other concepts for a VLHC, such as a big tunnel and moderate-field magnets, or a much smaller tunnel with much stronger magnets and a new purpose-built injector, might reach higher energy sooner but would cost more than Stage 1 of this design. Each of these concepts deserves exploration. This study will offer a baseline for comparison. The staged approach has the singular merit that the relatively inexpensive Stage 1 would address the issues of siting, 
tunneling, injector performance and survival of a frontier U.S. physics program, allowing the field to address the technical and fiscal challenges of Stage 2 with a healthy program in place.

\subsection{The Technical Description and Challenges}

Table 1.1 shows the high-level parameters of both stages of the VLHC. To arrive at these parameters required addressing a number of challenging accelerator physics issues. At this stage there appear to be few technical problems in reaching the listed performance of Stage 1. Making the arc magnets inexpensively and very long, as well as learning how to transport and install them in a tunnel, will take R\&D investment over the next few years. The small beam pipe and large circumference dictate the need to study and understand beam instabilities at injection. Preliminary evidence indicates that feedback and RF manipulations within the current state-of-the-art will control these instabilities. If further study points to a problem, the beam pipe size could be increased with tolerable effects on the total project cost. The dynamic aperture is more than adequate and closed orbit distortions are benign and easily corrected when simulated using expected magnet and alignment errors. Strong, large-aperture quadrupoles for the interaction insertions will require considerable $R \& D$ in the next few years. It is particularly interesting to note the low average power consumption, comparable to that of Fermilab's $800 \mathrm{GeV}$ fixed-target program. Power is mostly concentrated at the cryogenic service buildings, of which there are five off the existing Fermilab site. These double in number and grow larger for Stage 2.

Table 1.1. The high-level parameters of both stages of the VLHC.

\begin{tabular}{lcc}
\hline & Stage 1 & Stage 2 \\
\hline Total Circumference $(\mathrm{km})$ & 233 & 233 \\
Center-of-Mass Energy (TeV) & 40 & 175 \\
Number of interaction regions & 2 & 2 \\
Peak luminosity $\left(\mathrm{cm}^{-2} \mathrm{~s}^{-1}\right)$ & $1 \times 10^{34}$ & $2.0 \times 10^{34}$ \\
Luminosity lifetime $(\mathrm{hrs})$ & 24 & 8 \\
Injection energy (TeV) & 0.9 & 10.0 \\
Dipole field at collision energy (T) & 2 & 9.8 \\
Average arc bend radius (km) & 35.0 & 35.0 \\
Initial number of protons per bunch & $2.6 \times 10^{10}$ & $7.5 \times 10^{9}$ \\
Bunch spacing (ns) & 18.8 & 18.8 \\
$\beta^{*}$ at collision (m) & 0.3 & 0.71 \\
Free space in the interaction region $(\mathrm{m})$ & \pm 20 & \pm 30 \\
Inelastic cross section $(\mathrm{mb})$ & 100 & 130 \\
Interactions per bunch crossing at $\mathrm{L}_{\text {peak }}$ & 21 & 54 \\
Synchrotron radiation power per meter $(\mathrm{W} / \mathrm{m} /$ beam $)$ & 0.03 & 4.7 \\
Average power use $(\mathrm{MW})$ for collider ring & 25 & 100 \\
Total installed power $(\mathrm{MW})$ for collider ring & 35 & 250 \\
\hline
\end{tabular}

Stage 2 presents more technical challenges. First, discovering how to build cost-effective high-field superconducting magnets will require a significant investment over the next 10 years or more, although with a large-circumference ring the magnets are not extraordinarily strong. 
Perhaps the most difficult problem is one that this report barely addresses: how to deal with the large number of interactions at each bunch crossing. The energy carried by collision debris equals about $50 \mathrm{~kW}$ per beam, most of which goes forward into the insertion region collimators and magnets. It will require a major $\mathrm{R} \& \mathrm{D}$ effort for the detector and magnet designers to deal with this issue. The next most important issue for Stage 2 is synchrotron radiation power. It appears that 5 watts per meter, or even 10, can be removed from the magnets, and that synchrotron radiation will not cause a vacuum problem at those power levels. The power does show up in the cryogenic system, however, and must be dealt with.

Figure 1.1 shows an artist's conception of the physical layout of the injectors and the collision halls. The VLHC ring is tangent to the Tevatron, but much deeper. The injection lines bend very gradually, because they also serve as ramps to install the very long (6-m) Stage-1 magnets. The collider is deep in order to permit tunneling mostly in the extensive layer of excellent Galena-Platteville Dolomite. The collision halls are large and typical of those at LHC.

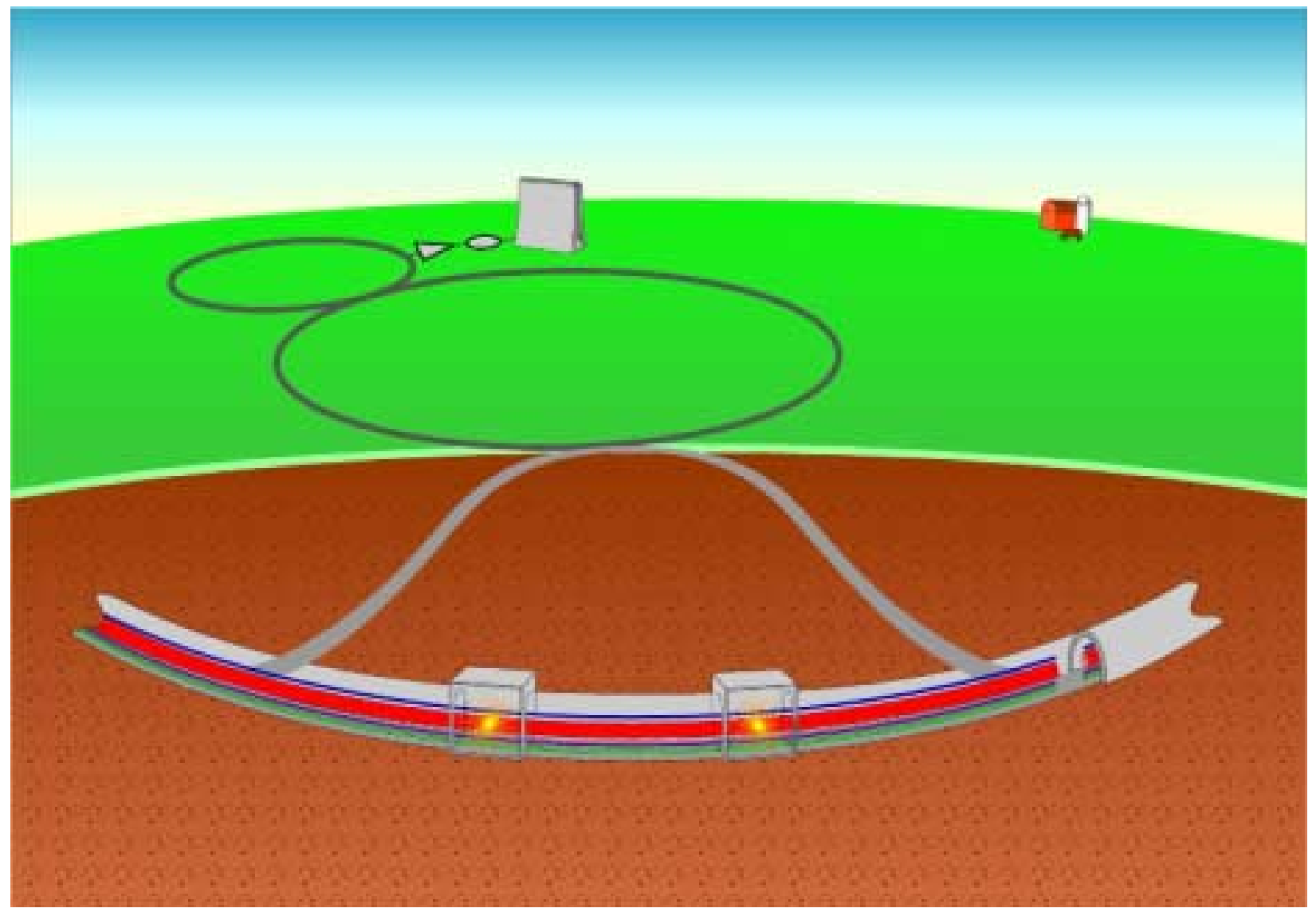

Figure 1.1. A cartoon diagram of the VLHC showing Fermilab's existing accelerator complex, the new injection line tunnels and the approximate locations of the detector halls.

The significant synchrotron radiation power present in the Stage-2 VLHC allows a trade-off of energy for luminosity. This study chooses $175 \mathrm{TeV}$ as the design energy and $2 \times 10^{34}$ as the design peak luminosity for Stage 2. At slightly lower luminosity, and with higher but still achievable magnetic field strength, this design could reach $200 \mathrm{TeV}$ collision energy, as shown in Table 1.2. At lower energy, higher luminosity is possible. Even better luminosity perform- 
ance can be achieved by "leveling" the luminosity to limit the inelastic collision debris power at the interaction point.

Table 1.2. Properties of the Stage-2 VLHC at various energies. The luminosity is limited by synchrotron radiation power and damping time, power at the interaction point due to inelastic collisions, and the beam-beam tune shift.

\begin{tabular}{|lc|c|c|c|}
\hline Collision Energy $(\mathrm{TeV})$ & Magnetic Field (T) & $\begin{array}{c}\text { Leveled Luminosity } \\
\left(\mathrm{cm}^{-2} \mathrm{~s}^{-1}\right)\end{array}$ & $\begin{array}{c}\text { Optimum Storage } \\
\text { Time }(\mathrm{hrs})\end{array}$ \\
\hline Stage 1 & 40 & 2 & $1.0 \times 10^{34}$ & 20 \\
\hline Stage 2 & 125 & 7.1 & $5.1 \times 10^{34}$ & 13 \\
\hline Stage 2 & 150 & 8.6 & $3.6 \times 10^{34}$ & 11 \\
\hline Stage 2 & $\mathbf{1 7 5}$ & $\mathbf{1 0}$ & $\mathbf{2 . 7} \times \mathbf{1 0}^{\mathbf{3 4}}$ & $\mathbf{8}$ \\
\hline Stage 2 & 200 & 11.4 & $2.1 \times 10^{34}$ & 7 \\
\hline
\end{tabular}

\subsection{Gaining Public Support for a Very Large Hadron Collider}

Construction of a new frontier accelerator at Fermilab will require not only the support of the national and international scientific community and U.S. and foreign governments, but also the support of Fermilab's neighbors, the people who live in surrounding communities. Just as important as technology development, infrastructure and site geology in determining whether Fermilab will be able to build a new accelerator is sociology. It is all too easy to imagine a scenario in which local opposition to an offsite accelerator makes it impossible for Fermilab to build such a machine. While community support will be necessary to some degree no matter what future accelerator Fermilab ultimately builds, it is a particularly important issue in the consideration of accelerators that would extend beyond the Fermilab site. And since, of all the proposed future Fermilab accelerators, the Very Large Hadron Collider would extend the farthest beyond Fermilab's borders, issues of public support are likely to have the greatest impact.

Although we cannot predict exactly what will most concern community members, the proposal to construct an accelerator beyond the Fermilab site is likely to raise many issues including: risks to environment, safety and health; effects on property values; esthetics; perceptions of the degree of community control in the decision-making process; neighborhood disruption during construction; and appropriate use of government funds.

How can Fermilab address such issues and build local public support for future accelerators? Clearly, the traditional "decide, announce, defend" model is a formula for failure. Fermilab needs to build the level of community dialogue, trust, understanding and support that building a VLHC, or any future accelerator, will require.

Some steps that Fermilab is now taking or has planned include: a comprehensive community opinion survey that will provide invaluable baseline information on the current perception of Fermilab, including questions about attitudes toward possible laboratory expansion beyond the current site; creating a long-term community outreach plan that includes future accelerators at Fermilab; forming a laboratory-community organization to serve as a public advisory group; consulting with other laboratories that have successfully dealt with similar community outreach issues; and the use of various Fermilab resources, e.g., Science Education programs, Saturday 
Morning Physics, open houses, and the Office of Public Affairs, to build support for future facilities.

Building a new frontier accelerator at Fermilab will not only have a profound effect on the future of our own laboratory and of U.S. high-energy physics but on the future of our local community. We believe that most of its effects would be positive, in the form of the economic, cultural and environmental benefits that it will bring to the region. However, it will be up to Fermilab to communicate both the benefits AND the costs of such a project. Involving the community from the beginning in planning for a future accelerator will be challenging and time-consuming, but ultimately it is likely to be the only way to create the level of community trust and support that such a project will require.

\section{References}

[1] D.G. Cassel (ed.) et al., "New Directions for High-Energy Physics," Proceedings of the 1996 DPF/DPB Summer Study on High-Energy Physics, Vol. 1, p. 251.

[2] Charge to the VLHC Study from the Fermilab Director, Nov 2000. 


\section{Chapter 2. Overview and Summary}

\subsection{General Description}

The staged approach to the VLHC described in this feasibility study requires placing two collider rings in a common tunnel. This requirement imposes constraints on the lattices of the rings as well as on the civil construction parameters. The injector to the collider is the existing Fermilab accelerator complex, hence the collider must pass through or close to Fermilab.

The plan view of the 233-km VLHC collider is shown in Figure 2.1, which shows the service buildings that contain cryogenic refrigerators and other utilities. Stage 1 has six service areas (one on the Fermilab site) for cryogenics and other operational requirements. Stage 2 requires six additional service sites.

The ring is almost circular, comprising two great arcs of $35-\mathrm{km}$ average radius connected by two clusters, one at Fermilab, one exactly opposite, each six kilometers long and each containing straight sections and dispersion suppressor arcs. The straight sections are needed for various functions necessary for operation, such as injection, extraction, beam cleaning, and the physics detectors.

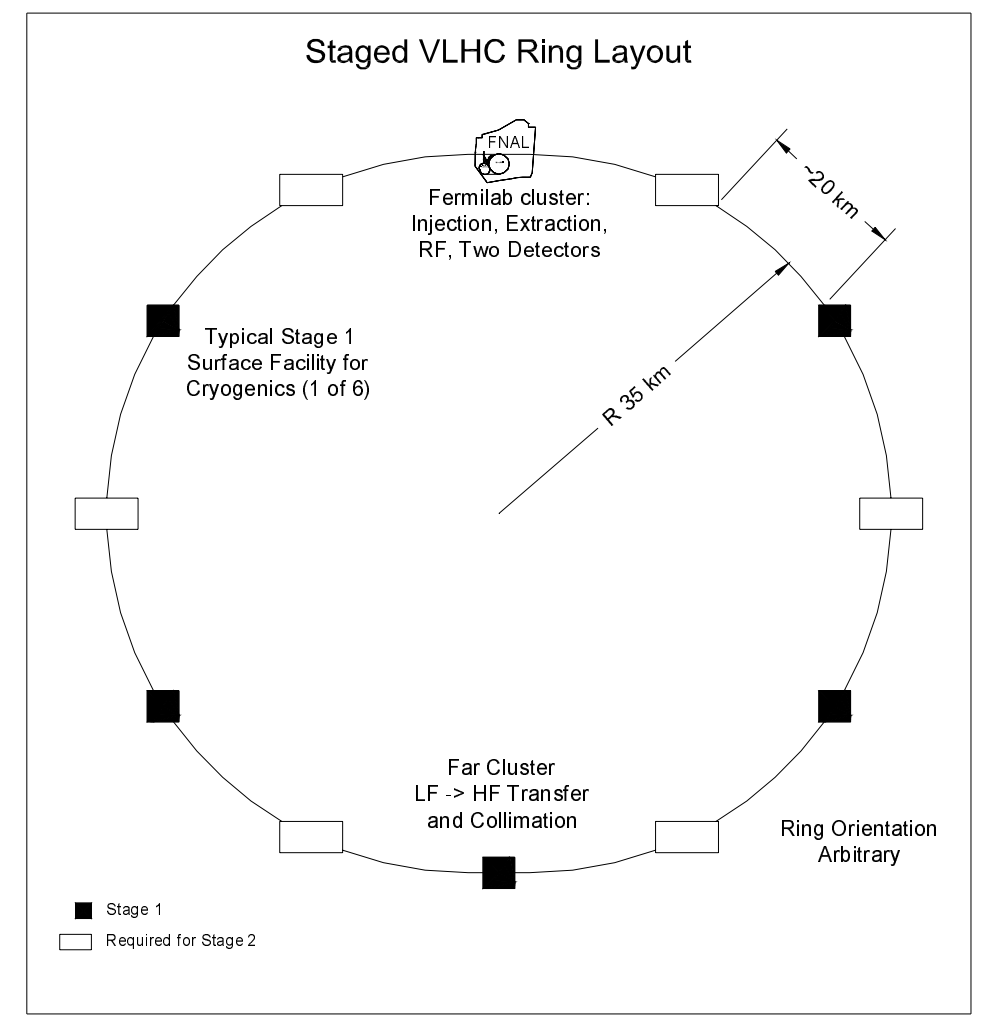

Figure 2.1. The 233-km VLHC ring.

A magnified view of the VLHC Fermilab cluster is shown in Figure 2.2 with one scale extremely expanded relative to the other. Grouped below or nearly below the existing Fermilab 
site are the underground injection and extraction beam lines, the beam absorber, and the RF acceleration stations. In the on-site straight sections are two detectors, separated sufficiently

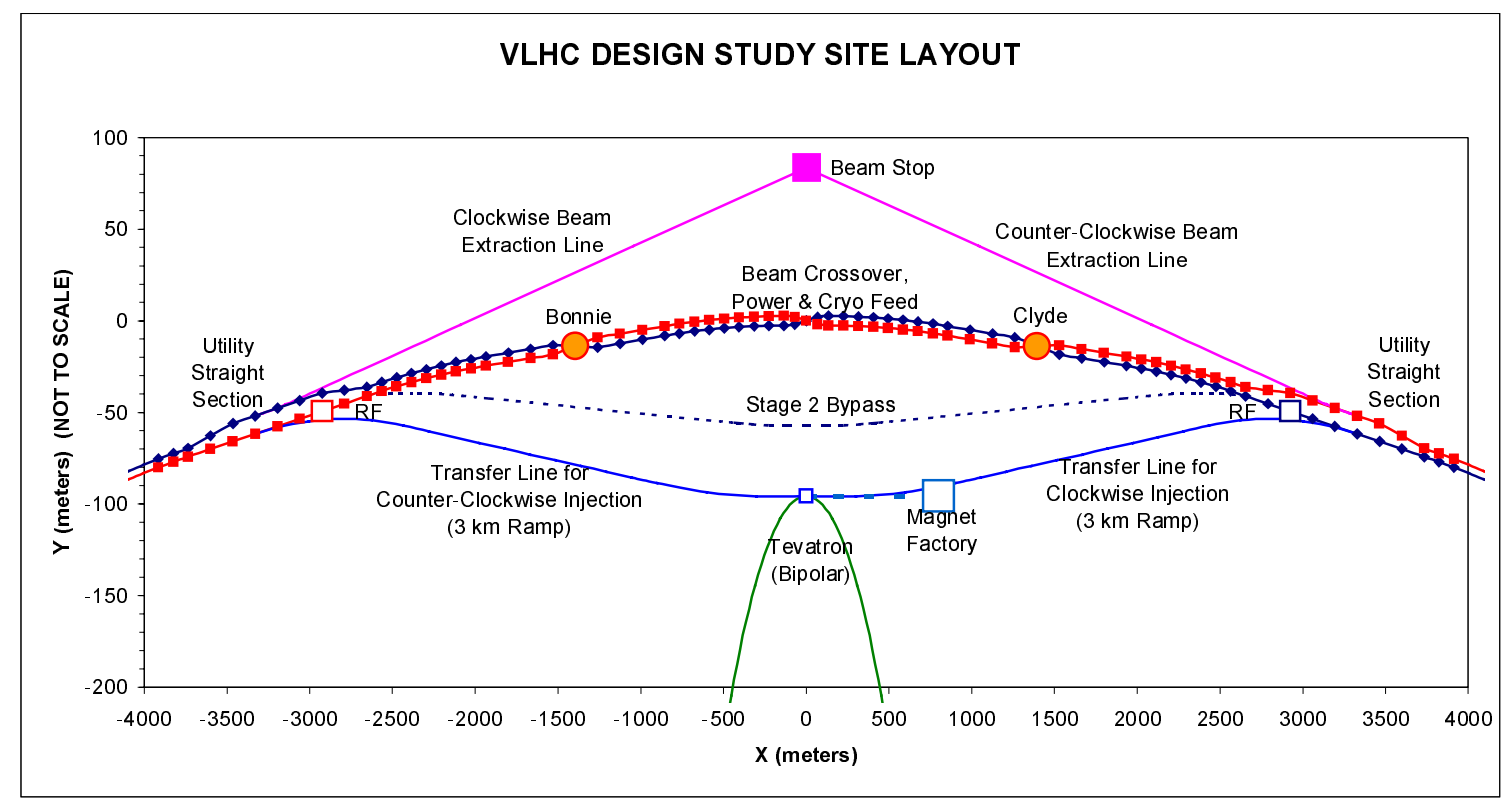

Figure 2.2. VLHC cluster at Fermilab, showing the various functions. Note the differences in $E-W$ and $N-S$ scales.

in distance and angle to avoid muon background from one detector hitting the other. The cluster opposite the Fermilab site is mostly passive, although a second campus could be developed there. The machine functions at the far cluster are beam cleaning and, eventually, the systems for beam transfer from Stage 1 to Stage 2 .

Several different injection layouts from the Tevatron are possible and are discussed in Chapter 4. To take advantage of the best geology in the area, the VLHC tunnel is approximately $120 \mathrm{~m}$ below the surface at Fermilab (Figure 2.5), much deeper than the Tevatron, but about the same as the LHC.

The lengths of the injection lines are determined by the desire to descend gradually to that depth so that these ramps can be used during the construction phase for the installation of magnets and other technical components. A similar ramp is used for installation on the far side of the VLHC ring.

The tunnel for this Design Study is a standard tunnel, similar in size to the SSC and LHC tunnels, and made by standard construction techniques. A cross section view of the tunnel at its minimum finished diameter of 12 feet and minimum floor width of 10 feet is shown in Figure 2.3 with the Stage-1 collider installed. Notable are the small Stage- 1 combined-function magnet installed on stands on the floor of the tunnel, and the small amount of necessary infrastructure. For example, there is only one small cable tray, because all of the correction elements are powered from local supplies installed in wall penetrations, and all of the instrumentation and controls are local, with only fast communication to the rest of the world. An electric trolley line provides power for tunnel transportation and local work power. This design eliminates almost all long cables except for ring-wide power cables and some bundles of optical fiber. 
The Stage-2 collider is shown in Figure 2.4. The Stage-2 magnets are installed vertically above the Stage-1 magnets to preserve the ring circumference. Additional cryogenic service piping, power and cable trays are required.

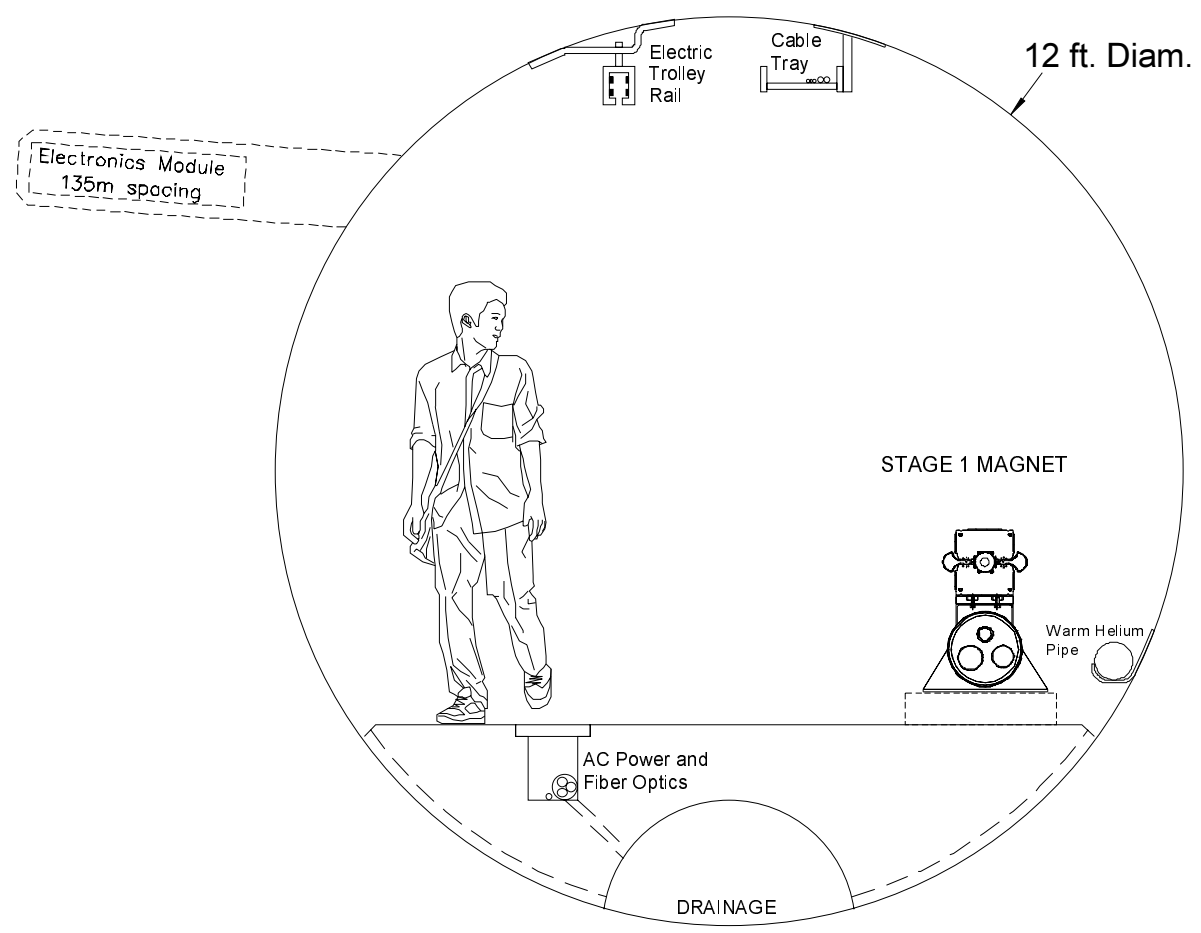

Figure 2.3. A cross section of the VLHC tunnel with the Stage-1 magnets in place.

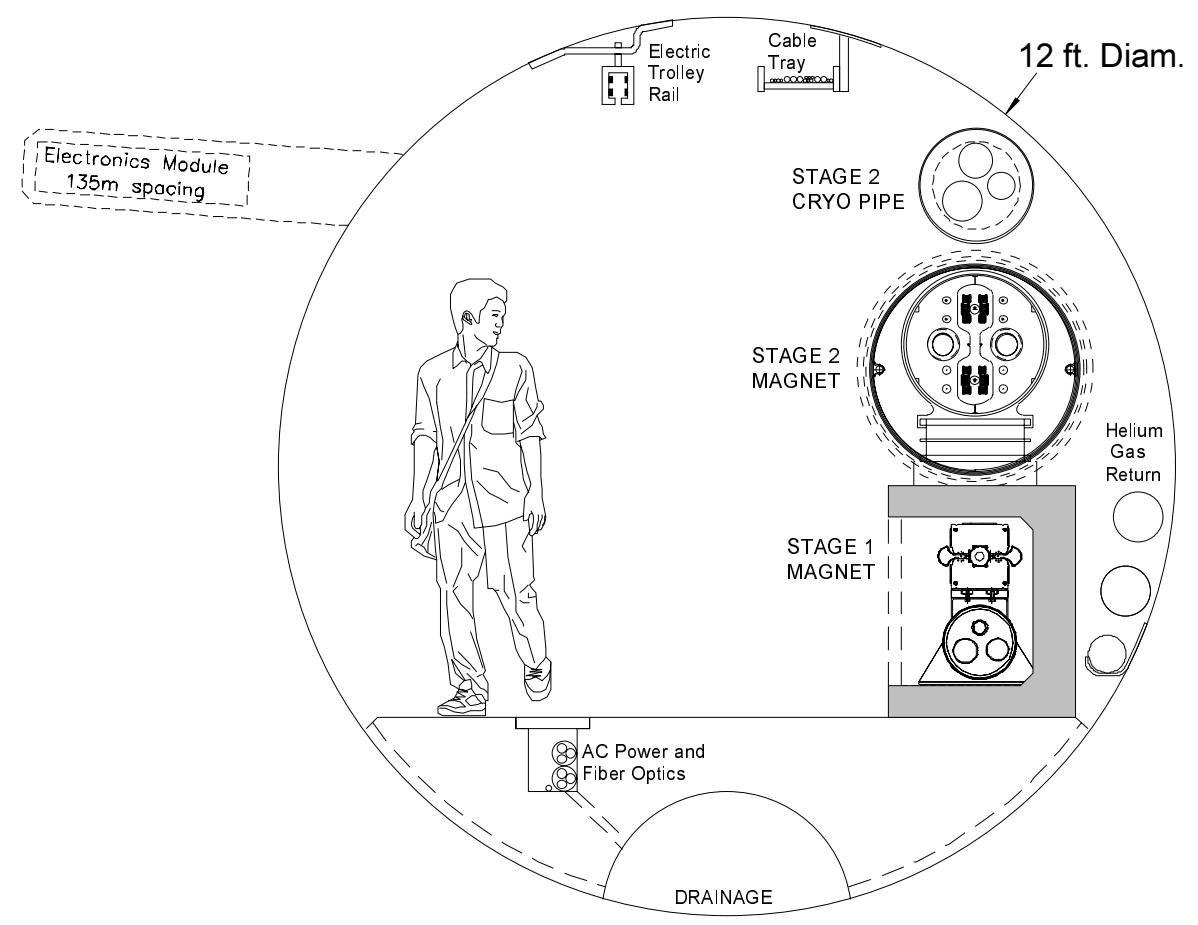

Figure 2.4. A cross section of the VLHC tunnel after the Stage-2 magnets have been installed. 
Sections of the tunnel used for services will have to be larger than the nominal minimum of 12 feet. The long straight sections for injection and extraction and experimental areas, the tunnel below the major service buildings, and anywhere where two tunnels join will be among them. In addition, there is an adit to the tunnel every $10 \mathrm{~km}$ that contains cryogenic valve boxes and transformers and bulk power supplies for DC power distribution in the tunnel. There are additional personnel exits every $5 \mathrm{~km}$ between the $10 \mathrm{~km}$ points. Tunnel locations under the service areas and the future locations of the Stage-2 service areas will have adits sized for Stage 2 during the initial construction.

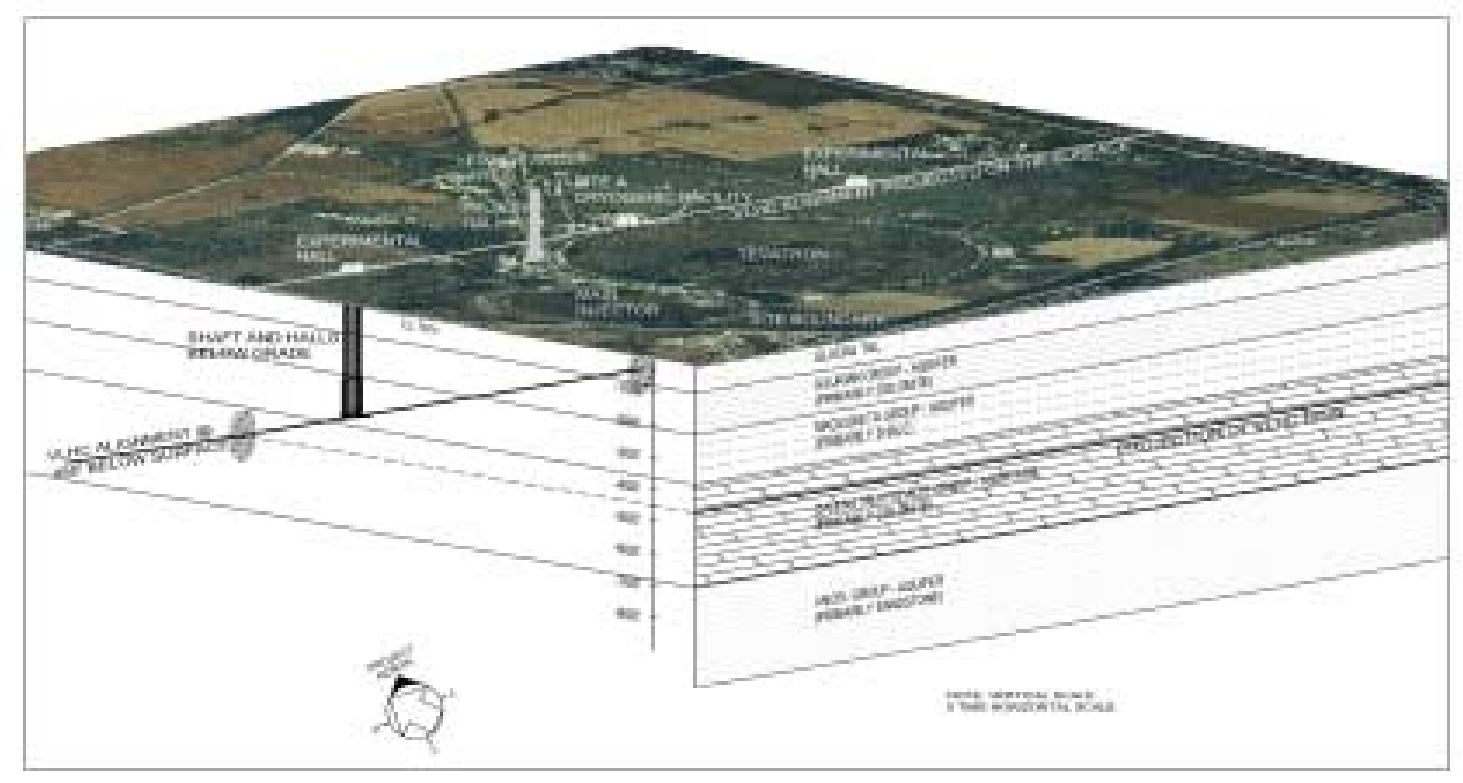

Figure 2.5. A cutaway view of the underground geology at Fermilab with the VLHC tunnel in its approximate location. The vertical scale is expanded relative to the horizontal scales.

\subsection{Geology}

The terrain in northern Illinois is excellent for building large accelerators. The surface is relatively flat, and there are thick layers of self-supporting and competent rock underground, as can be seen in Figure 2.5. The geology is not perfect, however. The best rock for tunneling, the Galena-Platteville dolomite, is deep underground and is tilted down to the east. There is an inactive fault west and south of Fermilab with a large mismatch of geological properties on either side, and a disturbance from a large meteor strike near Des Plaines. In order to sample all of the features that are in the area, and to get an idea of the technical, environmental and financial issues involved in tunneling through various media, we have included three tunnel orientations discussed in detail in Chapter 7:

- A south ring with a $0.08 \%$ incline and a depth at Fermilab of 235 feet. This orientation requires tunneling through the Sandwich fault and is relatively shallow.

- A north ring, with no incline and a depth at Fermilab of 330 feet. This orientation samples numerous types of underground media. 
- A north ring with a $0.20 \%$ incline and depth at Fermilab of 500 feet. This ring is inclined to stay exclusively in the Galena-Platteville dolomite. It is very deep in some locations.

The tunnel in the dolomite will not be lined but may have to be grouted and sealed in places. Some of the tunnel will have to be lined in order support poorer quality material and to avoid excessive inflow of water, which we have specified as no more than an average of 50 gallons per minute per mile of tunnel. Large underground cisterns are constructed as part of the tunnel near the six major service areas, and pumps with emergency backup empty them. This is a potential environmental issue and the possibility of lining the entire tunnel to reduce the water inflow is being studied.

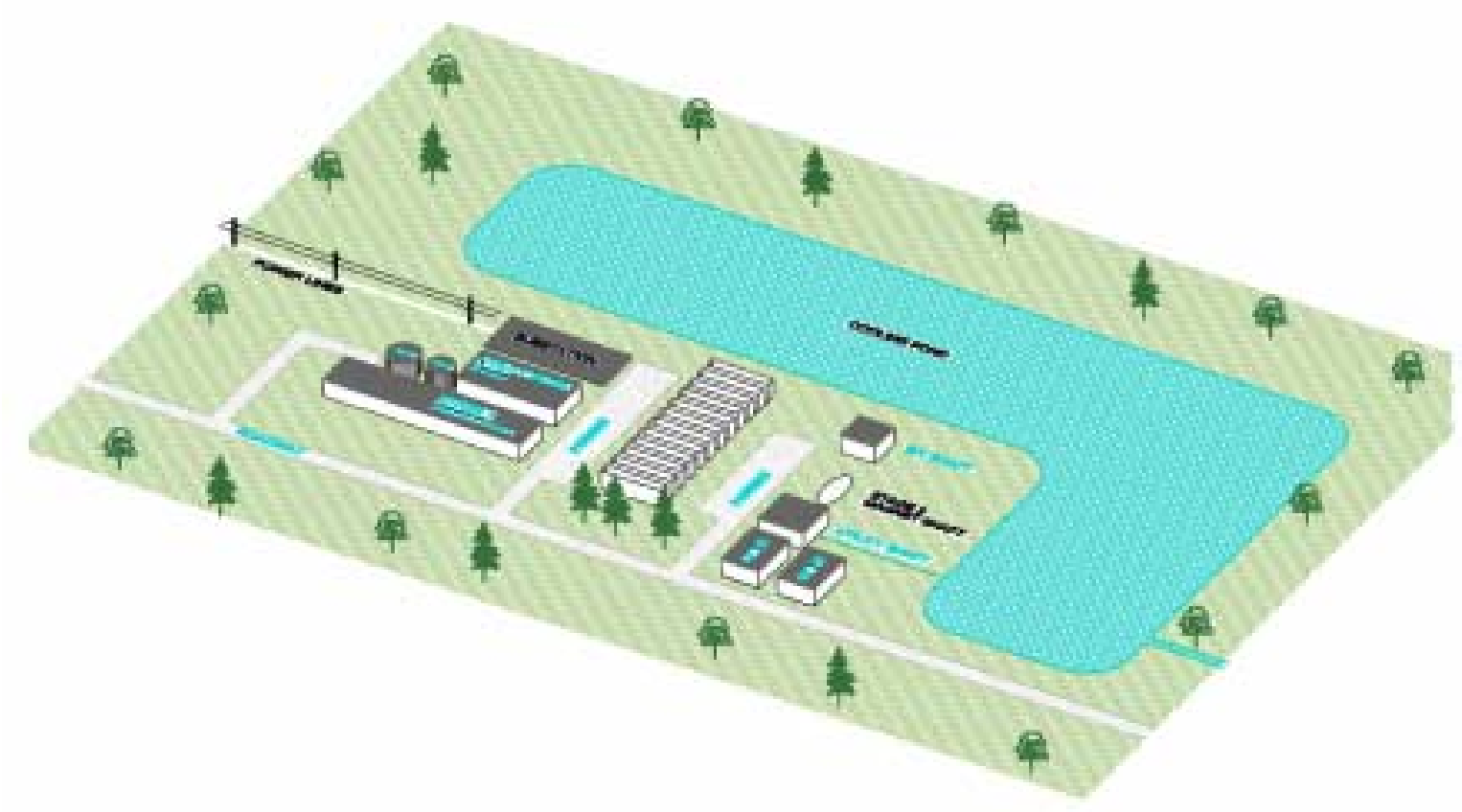

Figure 2.6. A typical service area for the Stage-1 VLHC. The layout of trees, low-profile buildings, and cooling pond provides an aesthetic 10-acre site appealing to the local community.

\subsection{Surface Features}

The most prominent off-site surface feature of the VLHC will be the service buildings required for refrigeration and other utilities for the collider. Stage 1 needs only six service buildings, roughly $40 \mathrm{~km}$ apart. A typical Stage-1 service area is shown in Figure 2.6. One of these is on the Fermilab site and is larger than the others to accommodate additional utilities and cryogenic power required by the experimental areas.

The six Stage-1 service areas are modest, each requiring up to $\sim 10$ acres of space, including cooling ponds for $4 \mathrm{MW}$ of installed power. Stage 2 will require six additional sites approximately halfway between the Stage- 1 service areas. In this upgrade, all 12 sites will require 40 acres each, with approximately $20 \mathrm{MW}$ of installed power per site. The land use requirements will depend on the mix of cooling ponds versus cooling towers chosen for each service area. Land availability and aesthetics are as important as the technical cost issues in these choices. 
The heat rejection capability for Stage- 2 compressors is about $8 \mathrm{MW}$ per service area. Minor areas are present at approximately five-kilometer intervals. They will have small buildings to cover and interlock the personnel exits and contain backup (generator) power for the elevators and lights. Every $10 \mathrm{~km}$ these buildings will be slightly larger because of additional underground utilities and power requirements.

The two detector caverns and associated service buildings are on the Fermilab site. These caverns are sized according to the SSC design and are $100 \mathrm{~m}$ by $30 \mathrm{~m}$ by $45 \mathrm{~m}$ high. It will be convenient but not necessarily possible to construct them sufficiently deep that their roofs have a thick enough dolomite cover that they will be self-supporting over that large span. The two areas have bending between them so that the background, particularly muons generated in one interaction region will not appear in the other. Experiments similar to those designed for SSC or being built for LHC are thought to be adequate for the $40 \mathrm{TeV}$ collisions of the Stage-1 VLHC.

\subsection{The Lattice}

While the Stage-2 collider is far in the future, one needs to anticipate its requirements as best one can before laying out the components of the first machine, since they ultimately must reside in same tunnel. This entails anticipating the geometric, space and beam optics requirements for future technical equipment - much of which may not yet be known. This creates the need for conservatism and some compromises. For example, while the size of the arcs is determined by the energy and bend fields of both colliders, the lengths of the straight sections and the interaction regions are determined by the high energy (magnetic rigidity) of the Stage- 2 beam. Another complication of the geometry comes about at the transition regions between bending and nonbending portions of the accelerators. Special optical modules - dispersion suppressors - are used to bring the orbits of off-momentum particles to coincide with each other in the long straight sections. Since the Stage-1 and Stage-2 designs have different focusing characteristics, the dispersion suppressors must be designed simultaneously to ensure that the Stage-1 and Stage-2 orbits line up appropriately when entering the long straight sections for the interaction regions and utility regions.

To allow for lattice and component designs to progress simultaneously for both the Stage-1 and Stage-2 colliders, a general layout composed of basic modules was developed. Each collider is made up of two major arcs that connect two clusters of straight sections. The clusters contain space for two nearby interaction regions and two utility regions that are used for injection and extraction, accelerating cavities, and other necessary functions. Short bending regions are located between each interaction region and utility region to allow collision debris and muon vectors to miss the downstream detector and utility enclosures. At the outset, only one cluster region - the one located at the Fermilab site - will be equipped with full interaction region and utility region optics. The other cluster region will have the same straight section lengths, but optically will consist of simple FODO-type modules, with the exception of one area with large dispersion used for beam collimation. Since the Stage-1 magnet has side-by-side apertures, each cluster contains one insertion in which the beams are crossed without colliding, to guarantee equal path lengths for the two beams. The exact length of the standard cell is determined by the chosen bunch spacing. To ensure that bunches will collide at any detector in the design, regardless of what modules exist between interaction points, the ring module lengths are designed in units of the bunch spacing. Details of both colliders are shown in Table 1.1 and 1.2 , and in Chapter 3. 


\subsection{Stage-1 Technical Components}

\subsubsection{Magnets}

The major technical component of the VLHC is, of course, the main arc magnet. The Stage-1 magnet, shown in perspective in Figure 2.7, is a superferric gradient magnet of simple and elegant design. It is not only less costly per Tesla-meter than the typical high-field superconducting magnet, but also requires much simpler and less costly services, such as cryogenics, power supplies, quench protection, and so forth. Since it is a gradient magnet, quadrupoles are not required in the arcs. Its long length, $65 \mathrm{~m}$, presents some challenges in handling and transportation, but reduces the number that need to be fabricated and tested and greatly decreases the overall cost of production and installation.

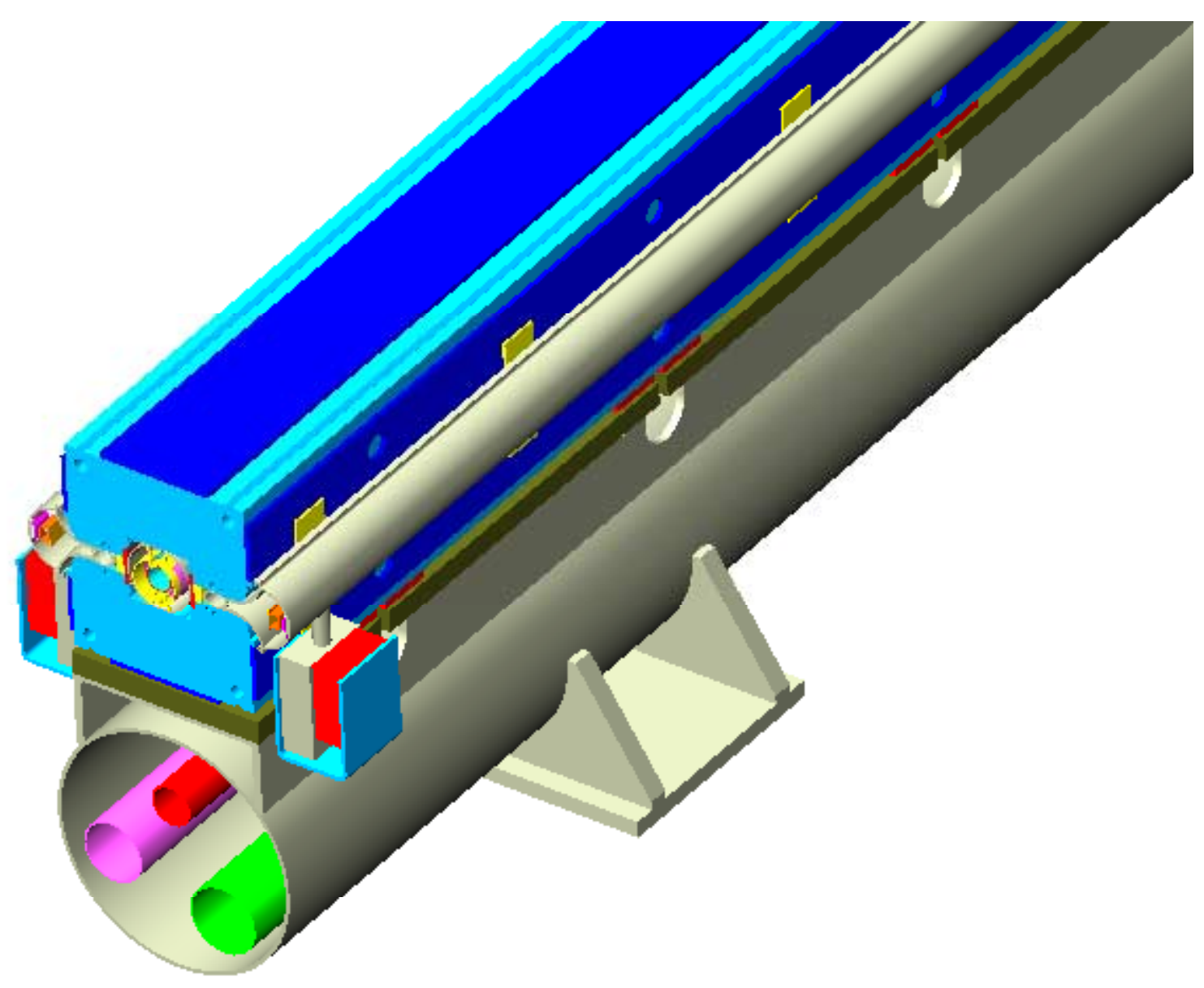

Figure 2.7. The superferric "Transmission-line" gradient magnet used in the arcs for the Stage-1 VLHC.

The magnet and its associated systems are discussed in detail in Chapter 5. The magnet is energized by passing $100 \mathrm{kA}$ through the superconducting cable in the center of the steel yoke, which induces a magnetic field that circulates in the yoke and through the gaps. The magnetic fields in the gaps on either side are in opposite directions for the oppositely directed beams of 
protons. The current returns in a similar cable in the helium supply/return line in the cryogenic service pipe below the magnet. The separation of the two cables generates a small dipole fringe field in the tunnel. The gaps are narrow to keep the magnet and the excitation current as small as possible. With a two-centimeter gap on either side the magnet will reach $2 \mathrm{~T}$ at $90 \mathrm{kA}$. The small beam pipe creates some stability issues for the beam, and although standard methods seem to be able to keep them within bounds, it is a subject for further study and R\&D. Since the steel yoke and the beam pipes of this magnet are warm, the cold mass of this design is very low, so cool-down times and inventory is impressively small. In addition, the electromagnetic force on the conductor is small, permitting a support structure with very low heat leak, hence, low operating power.

Every half-cell, $135 \mathrm{~m}$, the conductor in the center of the magnet is bent down to be close to the return conductor. This is a complication in the cryostat, but creates a field-free region at room-temperature that is used for the various correction magnets: a closed-orbit dipole, a tune quadrupole and a sextupole. Other correctors could be added as needed. All of the correctors are conventional air-cooled iron and copper magnets powered by local DC-to-DC converters. There are also the usual collection of special magnets in the utility straight sections. These comprise Lambertson-style septa for the beam-extraction system, which operate in series with the main excitation bus so they are always at the correct bend strength; injection and extraction kicker magnets, also in the utility straight sections; conventional quadrupoles that carry the FODO lattice across the utility straight sections; and strong, single aperture quadrupoles and bending magnets to focus the beams and bring them into collision at the two interaction regions.

The interaction region quadrupoles are typical of the magnets that will be developed for LHC second-generation IRs. They operate at about $300 \mathrm{~T} / \mathrm{m}$ gradient and have a larger aperture than the quadrupoles now being built by Fermilab for the LHC, but otherwise look very similar. The improved performance is gained by using $\mathrm{Nb}_{3} \mathrm{Sn}$ as the conductor.

Table 2.1. Main parameters and count of magnets in the Stage-1 VLHC.

\begin{tabular}{|l|c|c|c|c|l|}
\hline \multicolumn{1}{|c|}{ Magnet Type } & $\mathbf{B}_{\text {nom }} \mathbf{( T )}$ & $\begin{array}{c}\mathbf{G}_{\mathbf{n o m}} \\
\mathbf{( T / m )}\end{array}$ & $\mathbf{L}_{\mathbf{m a g}} \mathbf{( m )}$ & $\begin{array}{l}\text { Number of } \\
\text { elements }\end{array}$ & \multicolumn{1}{|c|}{ Notes } \\
\hline $\begin{array}{l}\text { Gradient dipole } \\
\text { (arc) }\end{array}$ & 1.97 & 9.73 & 65.75 & 3136 & $\begin{array}{l}\text { Superferric transmission } \\
\text { line }\end{array}$ \\
\hline $\begin{array}{l}\text { Gradient dipole } \\
\text { (Disp. Suppressor) }\end{array}$ & 1.80 & 16.88 & 48.81 & 160 & $\begin{array}{l}\text { Superferric transmission } \\
\text { line }\end{array}$ \\
\hline Special dipoles & 1.95 & & $25-35$ & 52 & $\begin{array}{l}\text { Separation, recombina- } \\
\text { tion, and cross-over }\end{array}$ \\
\hline Straight sect quads & & 70 & $4.8-6.8$ & 464 & $\begin{array}{l}\text { Water-cooled copper \& } \\
\text { steel }\end{array}$ \\
\hline Low $\beta$ quadrupoles & & 300 & $9.2-10.9$ & 16 & Nb ${ }_{3}$ Sn Conductor \\
\hline Correctors & & & & & $\begin{array}{l}\text { Air-cooled copper \& } \\
\text { teel. }\end{array}$ \\
\hline Dipole (horiz.) & 1.0 & & 0.50 & 1648 & \\
\hline Dipole (vert.) & 1.0 & & 0.50 & 1648 & \\
\hline Quadrupole & & 25 & 0.50 & 3296 & \\
\hline Sextupole & & $1750 \mathrm{~T} / \mathrm{m}^{2}$ & 0.80 & 3296 & \\
\hline
\end{tabular}




\subsubsection{Other Stage-1 Technical Components}

\subsubsection{Cryogenics}

The cryogenic system of the Stage- 1 collider is distributed over a wide area, but is about the same total refrigeration power as for the Tevatron - $20 \mathrm{MW}$ installed, including $50 \%$ overcapacity. It is a very simple system, with only piping and no pressure vessels in the tunnel. Each refrigeration plant services $38 \mathrm{~km}$ of arc magnets. The refrigerator cold box is split, so that the cold stages of the heat exchangers are at tunnel elevation. This reduces the helium gravity head pressure. There are two upstream and two downstream helium loops at each refrigerator. One loop in each direction passes through the transmission line and returns after $10 \mathrm{~km}$. The other loop is bypassed for the first $10 \mathrm{~km}$, and then is switched into the transmission line. This design reduces the size of the transmission line cryostat. There is no liquid nitrogen in the tunnel. The thermal shield for the transmission line and for the cryogenic service pipe is cooled with $40 \mathrm{~K}$ helium.

\subsubsection{Tunnel-Resident Systems}

Because of the size of the ring, we have taken special precautions to maximize reliability and minimize maintenance in the long arcs. There is no low-conductivity water system in the arcs, since the corrector magnets are air-cooled. Only repetitive instrumentation and corrector power supplies at each corrector location are required. These electronics are located in radiationshielded modules buried in cavities (holes in the wall) at each quadrupole location. Average power dissipation is approximately $15 \mathrm{~W} / \mathrm{m}$ of tunnel. All cables for instrumentation and corrector magnets are pre-assembled on the magnet and factory tested before installation. At alternate half-cells $(270 \mathrm{~m})$, cryogenic thermometry and valve controllers are used to regulate the shield flow. Every $10 \mathrm{~km}$ there is a walk-in alcove that contains conventional electronics racks and provides network connections, tunnel safety systems, bulk DC power for the instrumentation modules, and cryogenic instrumentation for the cool down valve box.

\subsection{Stage-1 Construction and Installation Schedule}

The construction of the tunnel is the major cost and schedule issue of the VLHC. It is our goal that the entire construction, installation and commissioning sequence be completed in 10 years. This will require a major logistical effort, since the installation of infrastructure and magnets must be done in one part of the ring while construction is proceeding in another part of the ring. Because the magnets are so long, they must be installed using ramps, not drops. It is presently planned to have installation ramps in two locations, enabling installation in four areas of the tunnel simultaneously. Although infrastructure can be installed in any area in which we have occupancy, magnets can only be installed in areas that are continuous with at least one ramp. To be able to commission the installed components immediately after they are installed, the surface facilities construction must also be coordinated with the tunnel construction.

Because the VLHC is being built at Fermilab, there is the organizational structure to move quickly to plan the construction and installation, and to establish construction activities. Even so, we believe that it will take about one year for the first tunneling to begin at the Fermilab cluster and that the first section in which we can sensibly install will be available at the start of 
year four. The tunneling and service contracts will follow in sequence, with the last sector completed for installation at the start of year eight. Hence, the complete tunnel construction period is thought to take about six years, and the installation period another six years, overlapped so that the total is ten years, as shown in Figure 2.8. It will take a significant effort to finish the collision halls by the end of year five so that detector installation can start. After magnet installation is completed in a sector, that sector will be commissioned, that is, cooled down and energized, even while installation and perhaps tunneling is continuing in other sectors of the ring.

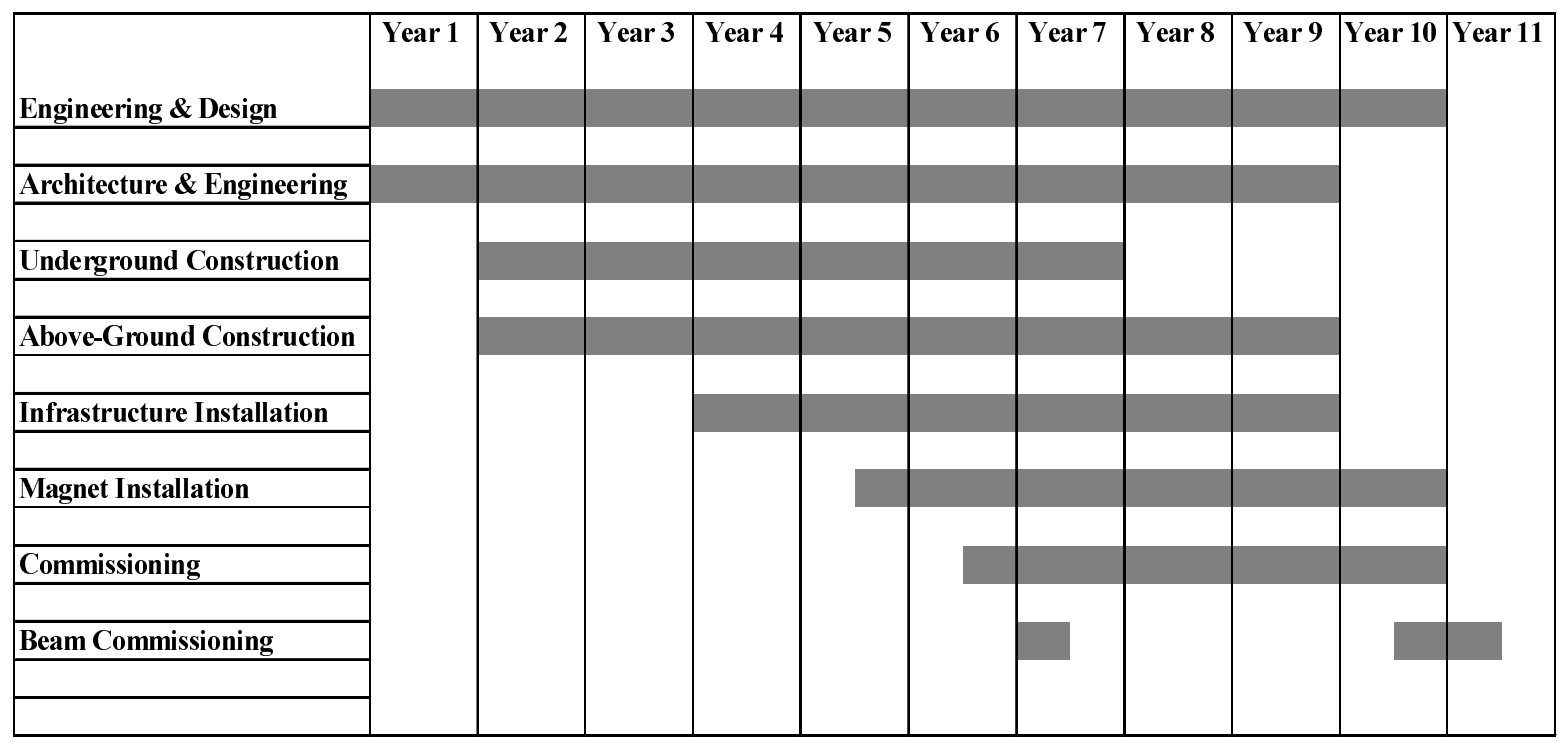

Figure 2.8. An overview of the schedule for construction, installation of infrastructure and magnets, and commissioning.

There are about 600 magnets per sector, so magnet installation will take about 2.5 years per sector at the rate of one magnet per day. Hence, the total installation rate in all sectors will be about four magnets per day. Since our plan has only two magnet-assembly factories, the magnet production will need to start earlier than installation, and a significant amount of magnet storage space will need to be constructed. Other possibilities are to have more magnet-assembly areas or to extend the construction period to 12 years. It is assumed that once a sector adjacent to Fermilab is commissioned, the magnets will be energized and beam may be injected. This will cause some delay in the installation process.

\subsection{Stage-1 Operations}

The Stage- 1 collider can be filled in about one hour from the Tevatron. Because of the cryogenic load of the rapid ramping cycle $(50 \mathrm{~s})$, the Tevatron can inject at no more than $900 \mathrm{GeV}$. The acceleration time to $20 \mathrm{TeV}$ per beam is about 20 minutes, which could be shortened at the expense of more RF. The long luminosity lifetime and long fill time indicate that it will not be a significant advantage to decrease the acceleration time.

Because of the size of the ring, emergency repairs will be time consuming. This will put a premium on reliability, redundancy and regular maintenance. Fully redundant capability is 
provided for all mission-critical subsystems in the arcs. Power distribution is provided to each module by two independent loop feeders with auto-resetting circuit breakers and remote disconnects. Two independent power supplies are provided for each corrector magnet and control function. Redundant network connections to each module are provided. The critical function of monitoring beam loss is provided by redundant sensors read out from alternate quadrupole modules, so that even the failure of a handful of control modules will not require immediate maintenance. In order to keep such a system reliable, maintenance periods every two weeks will be required to repair the "half-power supplies" that have failed during that period. Because the helium refrigerators have expanders in the tunnel, that part of the cold box is in an alcove far enough from the tunnel to allow access while beam is on, which, in conjunction with generous liquid helium storage, will lead to improved availability.

\subsection{Stage-2 Installation}

At some point in the operation of the Stage-1 VLHC it will be decided that an energy upgrade is necessary. Production will begin on the high-field dipoles, which although more difficult technically than the superferric magnets of Stage 1 are more conventional in size and appearance. Since Stage 2 will require more than 11,400 double-aperture dipoles, 1700 doubleaperture quadrupoles, and about 1700 spool pieces with more than 10,000 correctors of different types, production will require the participation of many industrial companies throughout the world. The magnets can be transported by conventional means and accumulated in industrial storage space so that a large inventory is available at the start of the shutdown. Since the tunnel has a complete infrastructure, magnets can be installed in many locations simultaneously. It is anticipated that the peak installation rate will be about 12 magnets per day, and that the conversion from Stage 1 to Stage 2 will take less than seven years. The major impediment to this fasttrack schedule appears to be the ability of what is now a small industrial base to make enough $\mathrm{Nb}_{3} \mathrm{Sn}$ wire and cable in the five-year magnet production period. If the magnet production begins earlier and more effort is put into installation, the conversion time could be made shorter.

At the same time as magnet installation is proceeding, the tunnels must be constructed for the Stage-1 bypass, the Stage-1 machine must be rerouted through the bypasses with a few higher-field (about $4 \mathrm{~T}$ ) magnets, and two new or upgraded detectors must be assembled in the existing collider halls. It seems reasonable that all of these operations could be completed in less than the seven-year conversion time.

\subsection{Stage-2 Operation}

Since the Stage- 2 collider is the same size as the Stage- 1 collider, it can be filled in about 30 seconds by transferring multiple Tevatron-length batches already stored in the Stage-1 ring, freight-car style, into the Stage-2 ring. The ramp up to full energy of $87.5 \mathrm{TeV}$ per beam will take about 35 minutes, limited first by the RF accelerating voltage and later by the amount of installed power for the magnet supplies. The energy stored in the magnetic field is returned to the power grid when the magnets are ramped down in preparation for the next cycle. Hence, the minimum time from physics collisions to physics collisions will be about 1.2 hours. We have chosen $10 \mathrm{TeV}$ as the injection energy since that seems completely adequate from the point of view of beam instabilities and dynamic aperture, and because the lower the injection energy, the less costly is the injection equipment, such as kickers and septa. 
From an accelerator physics viewpoint the most interesting feature of the Stage- 2 machine is that synchrotron radiation will lead to damping of the beam emittance, and hence, a noticeable improvement in luminosity during the store even as the beam is evaporating due to inelastic collisions at the interaction points. This enhancement allows excellent luminosity at reduced beam current, which has the effect of limiting the stored energy in the beam and the compressor power needed to remove the synchrotron radiation power in the cryogenic system. The reduced beam current also reduces the optimum storage time, but at eight hours, that is a small effect on the integrated luminosity. Another interesting consequence of synchrotron radiation is that one can trade off luminosity against operating energy: going up in energy, one does not lose as fast as one might imagine, because the damping becomes more effective. It appears that the Stage-2 VLHC could operate at $200 \mathrm{TeV}$ and $2 \times 10^{34}$, as shown in Table 1.2.

\subsection{ES\&H Issues}

During construction, the major environmental issues will be disposal of rock chips from the tunnel and the disturbance to neighborhoods during the construction period. These are nontrivial issues, but they can be handled with appropriate planning, communication and sensitivity. It is clear that a lot of thought will need to be put into organizing the construction effort, particularly since most of the construction will be staged off site in close proximity to private land.

During operation there are a few issues that are similar to those occurring now in all accelerators, but exacerbated by the higher particle energy and total stored beam energy of these machines, and the fact that the VLHC is so large in circumference. Most obvious among these is the so-called "worst-case accident," which we believe to be the loss of the entire beam at a single point in the arcs of the Stage- 2 collider. We have made a preliminary study of this accident, and it appears that it can be handled. It will scar a section of tunnel wall and create a local radiation cleanup problem in the tunnel. It will also irradiate some ground water with tritium and sodium 22, but not to a level that creates a severe environmental or health hazard. The total activation is equivalent to losing the Main Injector beams at a single point for an eight-hour shift. A more plausible accident involves beam damage from an accidental partial firing of the beam extraction kickers. The potential beam damage in this case is confined to several well-defined places which can be protected by special graphite collimators to intercept the beams [1].

One interesting equipment hazard that is unique to the Stage-1 machine is the fringe field caused by the large separation of the drive and return currents in the magnets. When the magnets are energized to full field, there is a region of about $30 \mathrm{~cm}$ radius around the magnets and centered approximately at the bottom of the steel yoke, where steel tools can be levitated and accelerated toward the magnets. If they hit the magnets in particular spots they could cause localized but significant damage. This is not a personnel safety issue because no one can be in the tunnel when the magnets are energized, but precautions need to be taken to avoid equipment damage. There will be no measurable magnetic field at the surface for either machine. 


\subsection{Foci for Future Development}

In order to focus the R\&D over the next few years, it is first necessary to understand the technical problems that might limit performance or even make the operation of the collider impossible or unsafe; second, to understand the issues connected with construction, fabrication and installation that could delay startup or create unwanted environmental concerns; and third, to enumerate those technical or scientific developments that could, if successful, make the VLHC less costly or lead to improved performance.

\subsubsection{Performance Issues for Stage 1}

The relatively low energy at injection, the small beam-tube aperture and the large-circumference ring have led to some concern about beam instabilities and tune shifts at injection. Calculations to date and experience on working machines show that a combination of active feedback systems and natural damping mechanisms can handle the instabilities.

During the course of this study we have identified and incorporated two design changes to minimize these issues. Firstly, a bunch coalescing scheme similar to the one used in Tevatron Collider operations has been incorporated to avoid potential single-bunch instability problems at injection. In this scheme, four low intensity $200 \mathrm{MHz}$ bunches are injected, accelerated, and then combined into single high-intensity $53 \mathrm{MHz}$ bunch at collision energy where the instabilities are not an issue.

A second change was made to minimize the tune spread due to time-dependent magnetic image currents in the steel pole tips. This effect is minimized by loading the VLHC ring uniformly around the circumference as described in Chapters 3 and 5. Residual tune spread can be eliminated, if necessary, with a pair of trim quadrupoles operating at audio frequencies.

At this time these appear to be solutions for the most serious potential accelerator physics issue for the Stage-1 VLHC. If further calculations show this to be inadequate, the magnets will have to be redesigned with larger gaps, requiring more steel and a higher drive current. Both of these are cost issues but neither affect the feasibility of the machine. Beam stability will be one of the major R\&D efforts for the VLHC.

Other magnet issues are verifying the magnetic field quality for significant quantities of the final magnet design, and systems issues such as thermal load in the transmission line and interconnect design and tests. The issues associated with producing and handling very long magnets need to be simulated and then tested. The insertion quadrupoles have challenges that appear able to be conquered, but the realization needs to be demonstrated. They will require $\mathrm{Nb}_{3} \mathrm{Sn}$ technology. A fallback using $\mathrm{NbTi}$ would entail approximately a $25 \%$ luminosity penalty.

Other issues that might seem problematic have been looked at and although they all need further study, they do not at this time appear to be performance limiting. Among these are ground motion degradation of beam emittance or alignment, the alignment process itself, and the study of potential supercritical flow instabilities in the cryogenic piping of Stage 1. 


\subsubsection{Construction Issues for Stage 1}

The most obvious construction issues for Stage 1 are the long tunnel running under land that is in private or public use, and the massive amount of fabrication and installation. The tunnel construction was mentioned above as an environmental issue, and in Chapter 1 as a public acceptance issue. The planning, tooling and equipment required for handling, assembling, testing, storing and installing and commissioning the long magnets and the subsystems and infrastructure will be a significant challenge. We have created a model of a ten-year construction period with two factories and two installation ramps. It appears that this schedule may be hard to keep, and we may need either more assembly factories and installation ramps, more installation equipment, or a few years longer to complete the job. It may be more cost-effective to have an additional factory, since it also opens up more of the ring to timely installation.

\subsubsection{R\&D Aimed at Improvements and Cost Reduction for Stage 1}

Considering the cost drivers, there are two obvious possibilities for cost savings in the construction of the Stage-1 VLHC: improved and less costly tunneling and underground construction techniques, and cheaper magnets. Investing in R\&D in these subjects holds promise for significant reductions in the cost of the VLHC. The major component of tunneling costs is labor, and automation of the process would not only reduce the cost, but make tunneling safer. This R\&D would be useful to society at large, in addition to its specific application to the VLHC. There are also many possible tunnel designs. Some may eliminate many of the special underground additions, such as sump pits, egress and access side-rooms, and so forth, all of which add greatly to the cost. These issues need serious engineering that they have not yet received.

Our cost-driver analysis indicates that the superferric magnet is between two and three times less costly per Tesla-meter than conventional superconducting magnets. Automation of the production might make them even more cost effective by reducing assembly and sub-assembly labor. The single most expensive component is the yoke steel, so being able to produce highquality magnet steel assemblies inexpensively would be a big money saver.

The vacuum system is surprisingly expensive, driven by the costs of the standard ion pumps needed to reduce the partial pressure of non-reactive gasses. Finding a simpler pump design or a getter that will pump methane would drastically reduce the cost of the vacuum system.

\subsubsection{Performance and Development Issues for Stage 2}

We chose $175 \mathrm{TeV}$ collision energy as the operating energy of the high-energy VLHC, but this study has shown that neither vacuum problems nor local heat-removal issues will arise from synchrotron radiation impinging on the beam-tube liner. The only limitations to operation up to $200 \mathrm{TeV}$ and $2 \times 10^{34} \mathrm{~cm}^{-2} \mathrm{~s}^{-1}$ luminosity appear to be the amount of beam debris power in the IRs and the total compressor power needed to remove the synchrotron radiation power. The first of these will need a dedicated team to study the flow of particles and energy into the detectors and into the magnets close by the interaction point. More sophisticated algorithms to match finer grained and faster detectors close to the IP may be developed and may make a big improvement. The development programs on more radiation-resistant electronics, such as deep sub-micron silicon or diamond-based detectors need to be continued and enhanced. Computer 
simulations of energy deposition and tests on radiation damage need to be started to understand design and materials issues that may influence the parameters of the interaction insertions and the detectors.

As to the removal of synchrotron radiation power, it appears entirely feasible to place a room-temperature "finger," or synchrotron radiation mask between magnets that are about 14 meters long that will intercept all or most of the synchrotron radiation, and that will not interfere with the beam or create impedance problems. This can greatly reduce the compressor power, and allow higher luminosity, higher energy, or both. With such a device, collision energies greater than $200 \mathrm{TeV}$ and luminosities greater than $2 \times 10^{34} \mathrm{~cm}^{-2} \mathrm{~s}^{-1}$ are certainly possible.

A possible enhancement of performance could come from using the synchrotron radiation damping to obtain flat beams in collision as is done in all electron colliders. The advantages appear to be in doublet optics in the interaction region, and smaller $\beta_{\max }$ in the quadrupoles. The disadvantages have to do with extremely challenging magnets that would have to operate in the environment of the interaction insertion. This issue and the necessary magnets deserve some study.

\section{References}

[1] J. Donald Cossairt et al., "Environment, Safety \& Health Considerations for a New Accelerator Facility," FERMILAB-Conf-01/051-E, April, 2001. 


\section{Chapter 3. Collider Accelerator Physics and Design}

\subsection{Two Colliders in One Tunnel}

\subsubsection{Footprint Issues}

For the design study it has been envisioned that the Low Field (LF) ring will be comprised of long, combined function magnets - the bending of the central trajectory and the focusing of the particle beam will be performed using gradient magnets with central field strength on the order of $2 \mathrm{~T}$. The High Field (HF) ring will use separated function magnets — dipole magnets for bending the central trajectory and quadrupole magnets for focusing. Since the LF and HF designs have different focusing characteristics, the dispersion suppressors must also be designed simultaneously to ensure that the LF and HF orbits line up appropriately when entering the long straight sections for the Interaction Regions and Utility Regions.

As stated earlier, the size of the arcs is determined primarily by the low magnetic field of the first stage collider, while the lengths of the major straight sections are determined by the high magnetic rigidity of the beam in the second stage collider. The interaction regions must be made long enough to accommodate trajectory and optics manipulations of high energy proton beams using reasonable guesses for sizes of future high energy, high luminosity detectors. The utility straight section regions must be made long enough to provide space for extracting a very high energy proton beam toward a beam dump and for beam scraping and beam instrumentation at high energy. All of these functions will be required for the LF collider as well, of course, but the technologies used for injection, extraction, acceleration, and so forth will be similar for the two rings. Thus, the requirements of the HF design will set the scale for the straight sections.

\subsubsection{Optics Issues}

In addition to the geometric layout of the two rings, many optical properties must be decided simultaneously as well. The Low Field ring uses gradient magnets in the arcs of the collider, while the High Field ring uses quadrupole FODO cells. For simplicity of instrumentation, power distribution, cable runs, etc., it is desirable for the maximum beam size locations in the HF arc to correspond with those in the LF arc. Dispersion suppressor regions typically are made by reducing the total bending in two consecutive cells by half. The proportion of bending to leave out in each of the cells depends upon the phase advance per cell. In combined function lattices, such as the LF design, the bending magnets also provide focusing, so this tactic must be modified. While combined function designs of dispersion suppressors have been performed in the past (e.g., Fermilab Recycler), the choice of cell phase advance and cell lengths must be settled upon for both rings to ensure a consistent dispersion suppressor design. (Obviously, this is an issue for both optics and geometry as well.) This is another reason that it was chosen to give the two colliders equal cell lengths and equal betatron phase advances per cell. Each collider, LF and HF, is made of two separate, but linked, accelerators. The choice must be made as to whether adjacent magnets have the same or opposite focusing characteristic with respect to each other for the beam traveling through them. This affects the matching of sections 
going into and out of straight sections, as well as the design of the beam transfer system joining the LF and HF rings. The decision must be made in coordination with the magnet design effort. Finally, the magnet choice for the LF collider is a horizontally separated two-in-one iron dominated magnet. To ensure that the two beam trajectories have the same path length, the beams will need to be crossed several times about the circumference. Space and optical designs must be incorporated into the layout to accommodate this feature. The two beams of the high field collider are naturally separated vertically in the common coil arc magnets.

\subsubsection{Approach to Design}

As indicated in Figure 3.1 each collider is made up of two major arcs that connect two clusters of straight sections. At the outset, only one cluster region-located at or near the Fermilab site - will be equipped with full interaction region and utility region optics. The opposite cluster will have the same straight section lengths, but optically will consist of simpler, FODOtype modules where applicable, with the exception of a special straight section designs to accommodate beam scraping and future beam transfers between the LF and HF accelerators.

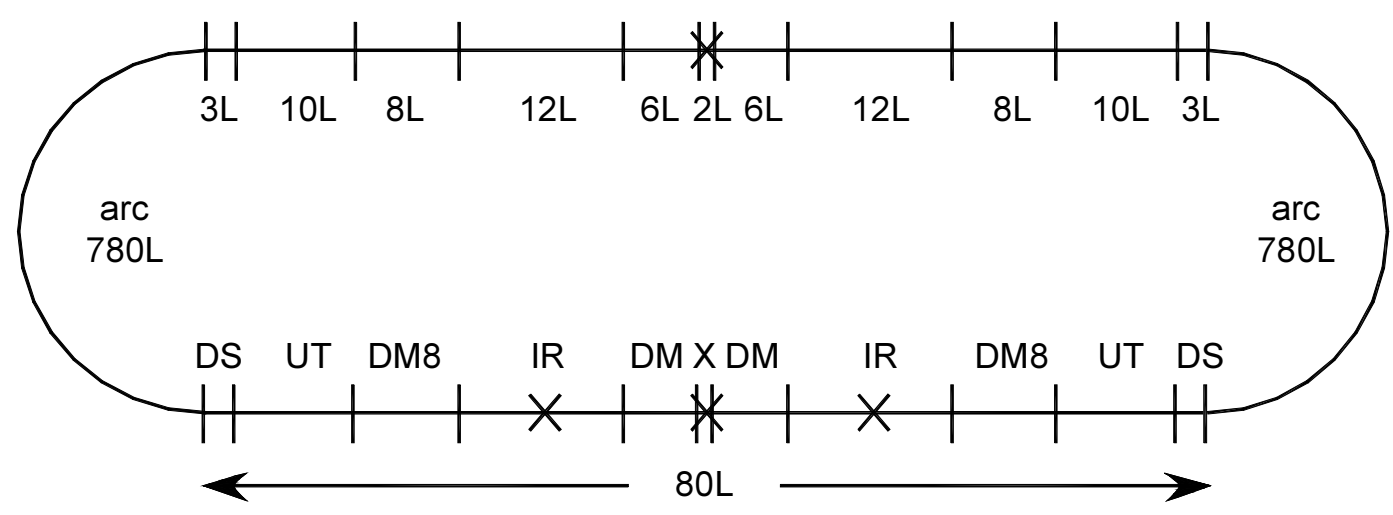

Figure 3.1. Schematic layout of collider modules. The figure is not to scale, emphasizing the straight section functions.

Dispersion suppressor modules are adopted following the SSC design, which was also later used in the Fermilab Main Injector. A cell phase advance of 90 degrees has been chosen for which a dispersion suppressor can be made using two special cells, each $3 / 4$ the length of a standard cell and each containing $2 / 3$ the bending of a standard cell. These modules reduce the periodic arc dispersion function to zero at the output of two such cells. The short bending regions between Interaction Regions and Utility Regions will be composed of back-to-back dispersion suppressor modules. Any additional bending required in these regions is generated by inserting standard half-cells between the DS modules.

\subsubsection{Footprint Parameters}

Table 3.1 lists the fundamental parameters of the footprint. In the transition from Stage- 1 to Stage- 2 operation, the experiments will remain centered on the same interaction points. At the same time it is envisaged that they will be upgraded to take up more space along the beam line, increasing the distance from the IP to the first magnet, $L^{*}$, from 20 to $30 \mathrm{~m}$. It is therefore 
necessary to allow for a bypass to keep the low field ring beams well clear of the experiments. This must be done without changing the total low field ring circumference.

One advantage of modular construction is that low and high field lattices are guaranteed to have almost identical footprints, and therefore to fit in the same tunnel, so long as corresponding low and high field modules are placed on top of each other. The maximum deviation is only a few millimeters, easily allowing one ring to be placed on top of the other at all locations in the VLHC tunnel.

Table 3.1. Fundamental lattice parameters common to both low and high field rings.

\begin{tabular}{|lrl|}
\hline Circumference, $C$ & 233.037 & $\mathrm{~km}$ \\
Average arc radius, $R$ & 35.0 & $\mathrm{~km}$ \\
Number of interaction points & 2 & \\
Half cell length, $L_{h c}$ & 135.486 & $\mathrm{~m}$ \\
Half cell bend angle, $\theta_{\mathrm{hc}}$ & 3.875 & $\mathrm{mrad}$ \\
Half cell count & 1720 & \\
Half cell harmonic, $n_{\lambda}$ & 24 & \\
Bunch spacing $(53.1 \mathrm{MHz}), S_{B}$ & 5.645 & $\mathrm{~m}$ \\
Time between bunches & 18.8 & $\mathrm{~ns}$ \\
Number of buckets & 41280 & \\
Number of bunches, $M$ & 37152 & \\
Phase advance per cell & 90.0 & $\mathrm{deg}$ \\
Revolution frequency & 1.286 & $\mathrm{kHz}$ \\
Revolution period, $T$ & 0.778 & $\mathrm{~ms}$ \\
Harmonic number, $h$ & 371520 & \\
RF frequency $(9 x 53.1)$ & 478.0 & $\mathrm{MHz}$ \\
\hline
\end{tabular}

\subsubsection{Half-cell Length}

The average bending radius of the arcs is made as close as possible to the goal value of $R=35$ $\mathrm{km}$ by adjusting the total equivalent number of (bending) arc cells in each ring. Each of the 4 short half-cells in each dispersion suppressor has $2 / 3$ the bending of an arc half-cell. Thus, the 20 dispersion suppressors (on either side of 10 straights) have a total bending equivalent to $160 / 3$ arc half-cells. If there are $N=784$ arc half cells in each half of the VLHC, then the bend per half cell is

$$
\theta_{h c}=\frac{2 \pi}{2 N+160 / 3}=3.875 \mathrm{mrad}
$$

and the average arc bend radius is

$$
R=\frac{L_{h c}}{\theta_{h c}}=34.961 \mathrm{~km}
$$

The (minimum) bunch spacing $S_{B}$ is taken to be one 53.1 MHz RF wavelength in the Tevatron

$$
S_{B}=\lambda_{53}=\frac{(2000 \mathrm{~m}) \pi}{1113}=5.645 \mathrm{~m}
$$

This guarantees that the filling schemes from the Tevatron will be relatively simple. The bunch time spacing of about $18.8 \mathrm{~ns}$ is not unreasonably challenging to the experiments. 
The half-cell length is

$$
L_{h c}=n_{\lambda} S_{B}
$$

The bunch spacing can trivially be increased (for example during commissioning) by any of the common factors of the "half cell harmonic number" $n_{\lambda}$. The value of $n_{\lambda}=24$ was chosen for both low and high field rings, corresponding to a half-cell length of $L_{h c}=135.4865$ meters.

This half cell length is short enough to keep the natural horizontal emittance due to synchrotron radiation in the high field ring, $\varepsilon_{\text {nat }}=0.04 \mu \mathrm{m}$, much smaller than the horizontal emittance $\varepsilon_{x} \approx 0.20 \mu \mathrm{m}$ which must be maintained (by heating) to avoid beam-beam limits in the store. A stronger limit to the maximum arc half-cell length is the need to limit the vulnerability of the low and high field rings to systematic field harmonic errors in the arc bending magnets. This is more critical for the low field ring, simply because the injection energy is an order of magnitude lower $(1 \mathrm{TeV})$ than for the high field ring $(10 \mathrm{TeV})$.

The current value of $L_{h c}=135.4865 \mathrm{~m}$ is by no means fully optimized, not least because we do not accurately understand what values of systematic magnet harmonics might be achieved in industrial production of the arc bending magnets.

\subsection{Stage 1 - the Low Field Ring}

The first stage low-field collider will have a top energy of $20 \mathrm{TeV}$ and peak (initial) luminosity of $10^{34} \mathrm{~cm}^{-2} \mathrm{sec}^{-1}$. The collider will use the Tevatron as its injector operating at a transfer energy of $900 \mathrm{GeV}$. For this study a transverse emittance of $1.5 \pi \mathrm{mm}-\mathrm{mrad}$ (rms, normalized) is used, which is typical for the Fermilab Booster, though about half the value at the Tevatron under recent normal operations. It is anticipated that with further Run II experience, the efficiency of emittance preservation will increase. Using the present acceleration systems of the Tevatron injector chain, the collider has a bunch spacing of $5.645 \mathrm{~m}(53.1 \mathrm{MHz})$, which sets the number of available RF buckets. With a $90 \%$ filling fraction to allow for gaps in the beam for various kicker rise times, the number of protons per bunch required in the Stage-1 collider is approximately $2.6 \times 10^{10}$, similar to Tevatron bunch intensities during previous Fixed Target operations. Using an interaction region design with a $\beta^{*}=0.3 \mathrm{~m}$, we arrive at the desired initial luminosity. Table 3.2 lists the general parameters of the Stage-1 collider.

The typical store parameter evolution is depicted in Figure 3.2. The figure shows the proton bunch intensity and luminosity decreasing due to collisions. The vertical emittance also decreases slightly due to synchrotron radiation damping which occurs with a damping time of about $100 \mathrm{hr}$. Due to the use of gradient magnets in the Stage-1 collider the horizontal emittance actually will be anti-damped, and will increase at approximately the same rate. This should not be a problem since store times will be much less than 100 hours. 
Table 3.2. Nominal store parameters for the low field collider.

\begin{tabular}{|c|c|c|}
\hline Storage energy & 20 & $\mathrm{TeV}$ \\
\hline Peak luminosity & $10^{34}$ & $\mathrm{~cm}^{-2} \mathrm{sec}^{-1}$ \\
\hline Packing fraction & 89 & $\%$ \\
\hline Injection energy & 0.9 & $\mathrm{TeV}$ \\
\hline Transverse normalized emittance, rms (H\&V, inject) & $1.5 \pi$ & $\mu \mathrm{m}$ \\
\hline Initial bunch intensity & $2.6 \times 10^{10}$ & \\
\hline Average beam current & 195 & $\mathrm{~mA}$ \\
\hline Stored energy per beam at collision & 3.0 & GJ \\
\hline Bend field at storage & 2.0 & $\mathrm{~T}$ \\
\hline Bend magnet gradient & 9.0 & $\mathrm{~T} / \mathrm{m}$ \\
\hline Phase advance per cell & 90.0 & $\operatorname{deg}$ \\
\hline Max rms arc beam size (inject) & 1.2 & $\mathrm{~mm}$ \\
\hline Bunch spacing (53.1 MHz) & 5.645 & $\mathrm{~m}$ \\
\hline Time between bunches & 18.8 & nsec \\
\hline Bunch length & 30 & $\mathrm{~mm}$ \\
\hline Longitudinal emittance, rms (inject) & 0.4 & eV-s \\
\hline $\mathrm{RF}$ voltage at storage & 50 & MV \\
\hline Fill time & 60 & $\min$ \\
\hline Acceleration time & 1000 & $\sec$ \\
\hline Beam size (rms) at IP (storage) & 4.6 & $\mu \mathrm{m}$ \\
\hline Total crossing angle ( $10 \sigma$ separation in drift space) & 153 & $\mu \mathrm{rad}$ \\
\hline Distance from IP to first magnet & 21 & $\mathrm{~m}$ \\
\hline$\beta^{*}$ at IP $(\mathrm{H} \& \mathrm{~V})$ & 0.3 & $\mathrm{~m}$ \\
\hline Maximum interactions per crossing & 20 & \\
\hline Debris power at IP (each direction) & 3 & $\mathrm{~kW}$ \\
\hline SYNCHROTRON RADIATION AT STORE & & \\
\hline Energy loss per turn per particle & 38 & $\mathrm{keV}$ \\
\hline $\begin{array}{l}\text { Radiation damping time, } \tau_{0} \\
\text { (anti-damping in H plane) }\end{array}$ & 100 & $\mathrm{hr}$ \\
\hline
\end{tabular}

\subsubsection{Lattice}

The bulk of the lattice is characterized by its construction from just three optical building blocks - arc, dispersion suppressing, and straight-section cells. The arc and suppressor cells consist of combined function gradient magnets and have space allocated at maximum- $\beta$ sites for 4 correction elements. Additional corrector space is available at the quarter-cell locations $\left(\beta_{\mathrm{x}}=\beta_{\mathrm{y}}\right)$ for lumped correction of systematic multipoles [1]. Two utility straights, each of which must accommodate $900 \mathrm{GeV}$ injection from the Tevatron plus abort of $20 \mathrm{TeV}$ beams are needed. The beams collide with a horizontal crossing angle at two interaction points where $\beta^{*}=30 \mathrm{~cm}$. In a special cell, midway between the IP's, the beams are made to cross over 
between the inner and outer horizontal apertures. Diametrically opposed in the ring, a mirror of this cell is installed to ensure identical path lengths for the two circulating beams.
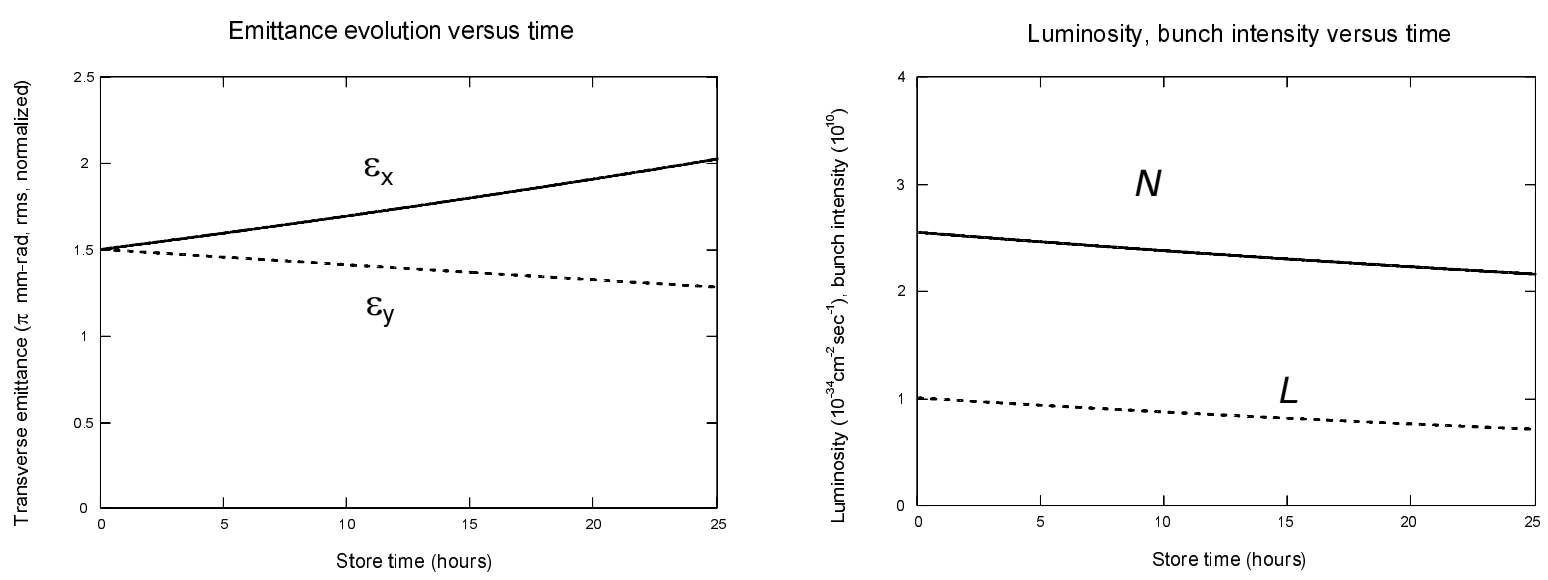

Figure 3.2. Evolution of collider parameters during a typical store.

\subsubsection{Interaction Regions}

The final foci in the IRs are anti-symmetric triplets, formed from single-bore, $300 \mathrm{~T} / \mathrm{m}$ magnets. Four additional quadrupole circuits, comprising double-bore, $70 \mathrm{~T} / \mathrm{m}$ magnets, are also used in optical matching. With a total of 6 independently-tunable quadrupole circuits available it is possible to match the four $\beta$ 's and $\alpha$ 's from the IP to regular FODO cells, and hold the phase advance $\Delta \mu$ constant across the IR through the squeeze from $\beta^{*}=6.00 \mathrm{~m} \rightarrow 0.30 \mathrm{~m}$. Fixing $\Delta \mu$ eliminates the necessity of a special phase trombone to maintain the nominal operating point. Figure 3.3 shows the lattice functions through the IR during collisions.

The circulating beams are separated horizontally by $15 \mathrm{~cm}$ throughout the arcs and $70 \mathrm{~T} / \mathrm{m}$ straight-section quads. Four dipoles, $13.6 \mathrm{~m}$ each at $1.97 \mathrm{~T}$, situated between the Q3 and Q4 quadrupoles bring the beams together at the entrance to the triplet for collisions at the IP. Dipoles downstream of the IP separate the beams again and channel them back into the inner and outer rings. A half-crossing angle of $77 \mu \mathrm{r}$ at the IP gives $10 \sigma$ separation between the beams at the first parasitic crossing.

\subsubsection{Injection and Abort Straight Sections}

To accommodate $900 \mathrm{GeV}$ injection from the Tevatron, as well as $20 \mathrm{TeV}$ proton abort, 5 straight-section cells are modified as shown in Figure 3.4. The long drift space $(377 \mathrm{~m}) \mathrm{created}$ by triplet focusing produces sufficient room for abort Lambertsons (see Section 5.1.5.4) and beam extraction.

Dipoles separate the circulating beams through the utility straight to make room for RF cavities downstream of the second triplet. Three $21.54 \mathrm{~m}$ dipoles at $2 \mathrm{~T}$ bend the beams apart by $40 \mathrm{~cm}$, followed immediately by 3 more dipoles that flatten out the trajectories. This pattern is reversed downstream of the RF to bring the beams back into standard double-bore magnets. 


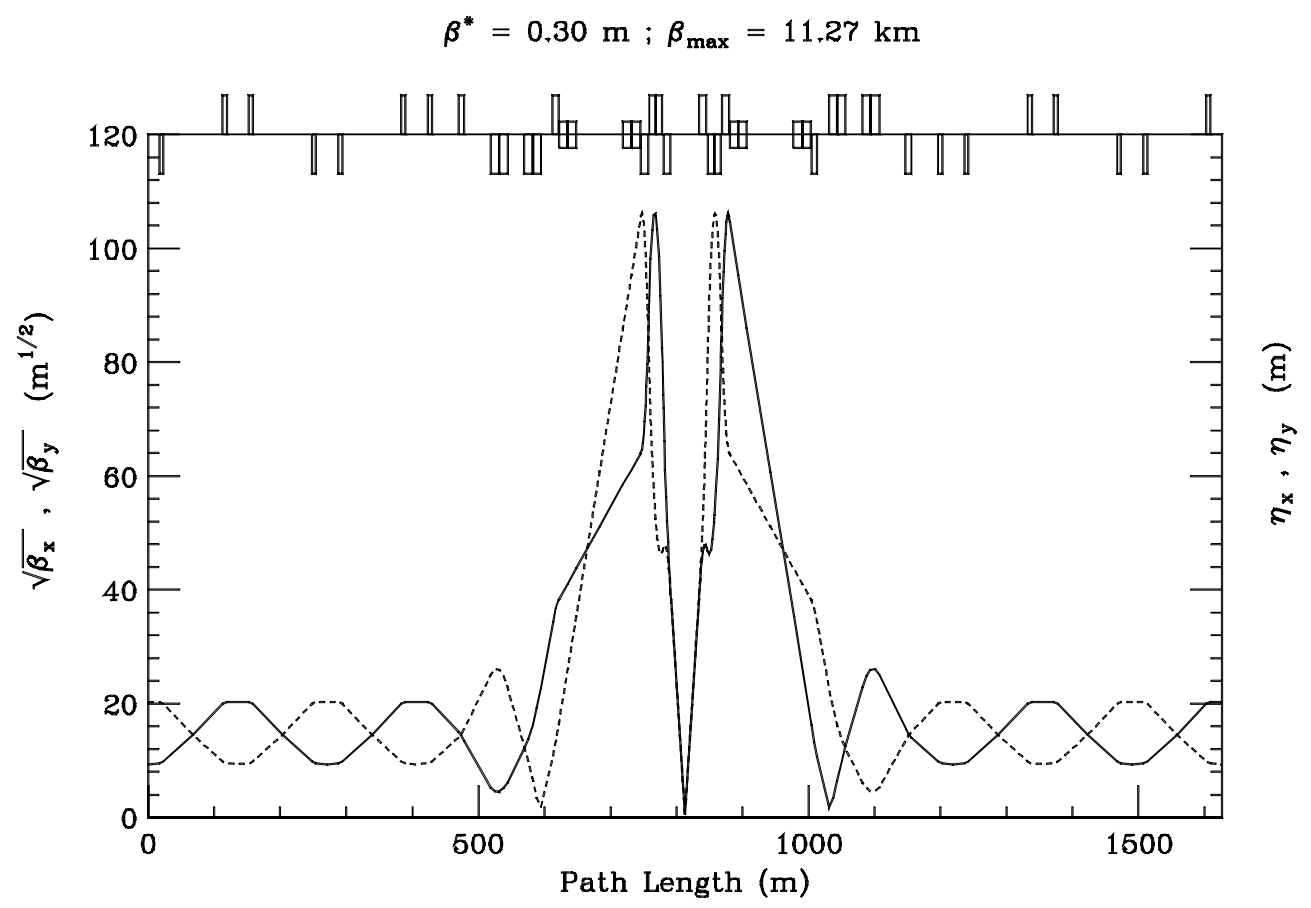

Figure 3.3. IR lattice functions in the collision configuration.

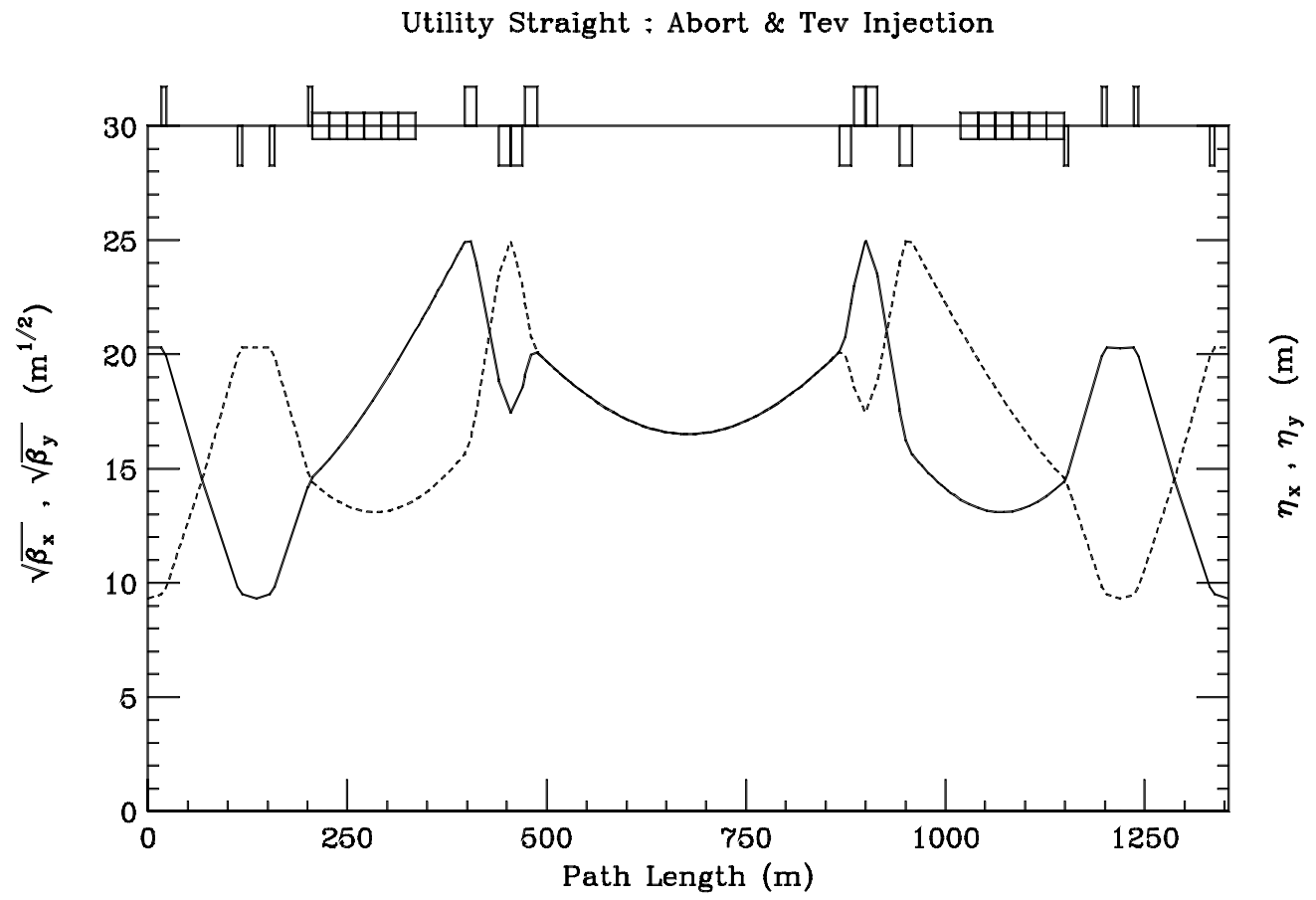

Figure 3.4. Layout of a utility straight supporting beam transfer from the Tevatron and 20 TeV abort. (See Section 5.2.5.4.) 


\subsubsection{Beam Crossovers}

With the beams separated horizontally in the arcs it is necessary to move the beams between the inner and outer rings to maintain identical path lengths. In the LF ring 2 crossover cells are inserted on opposite sides of the ring. Four rolled dipoles move the beams the $15 \mathrm{~cm}$ from one channel to the other. Each dipole is $36 \mathrm{~m}$ at $1.944 \mathrm{~T}$. The first dipole is rolled by $0.12 \mathrm{rad}$ to start separating the beams vertically, while bringing them closer together horizontally. The second bend is rolled by -0.12 rad to flatten the vertical trajectory. At the crossing point the beams are separated vertically by $9 \mathrm{~mm}$. This is $17.8 \sigma$ at the injection energy of $900 \mathrm{GeV}$. Rolled dipoles after the crossing point remove the vertical offsets and complete the $15 \mathrm{~cm}$ transverse transfer of beams to the opposite channel.

\subsubsection{Miscellaneous Insertions}

For momentum collimation a localized dispersion wave is generated in a 5-cell straight section (Figure 3.5). Four $45 \mathrm{~m}$ dipoles (2 T@20 TeV) at the upstream end of the straight move the beams transversely by $0.36 \mathrm{~m}$, creating $\pm 70 \mathrm{~cm}$ of dispersion. Four more dipoles downstream bring the beams back onto their nominal trajectory and cancel the dispersion.

Free space for damping systems, beam instrumentation, etc. (see, for example, Figure 5.53) can be generated by replacing standard arc gradient magnets with a shorter, modified version plus a small quadrupole. Through this insertion, the $\beta$ 's, $\alpha$ 's, and phase advance of an arc cell are exactly reproduced by a gradient magnet with $B^{\prime}=9.185 \mathrm{~T} / \mathrm{m}(1 \%$ weaker than the regular arc magnets) plus a $2.0 \mathrm{~m}$ quadrupole with $B^{\prime}=26.14 \mathrm{~T} / \mathrm{m}$. The low field of the quadrupole makes it just a longer version of the normal arc trim quads. The dispersion wave created by the holes is cancelled locally with $90^{\circ}$ of phase advance per cell.

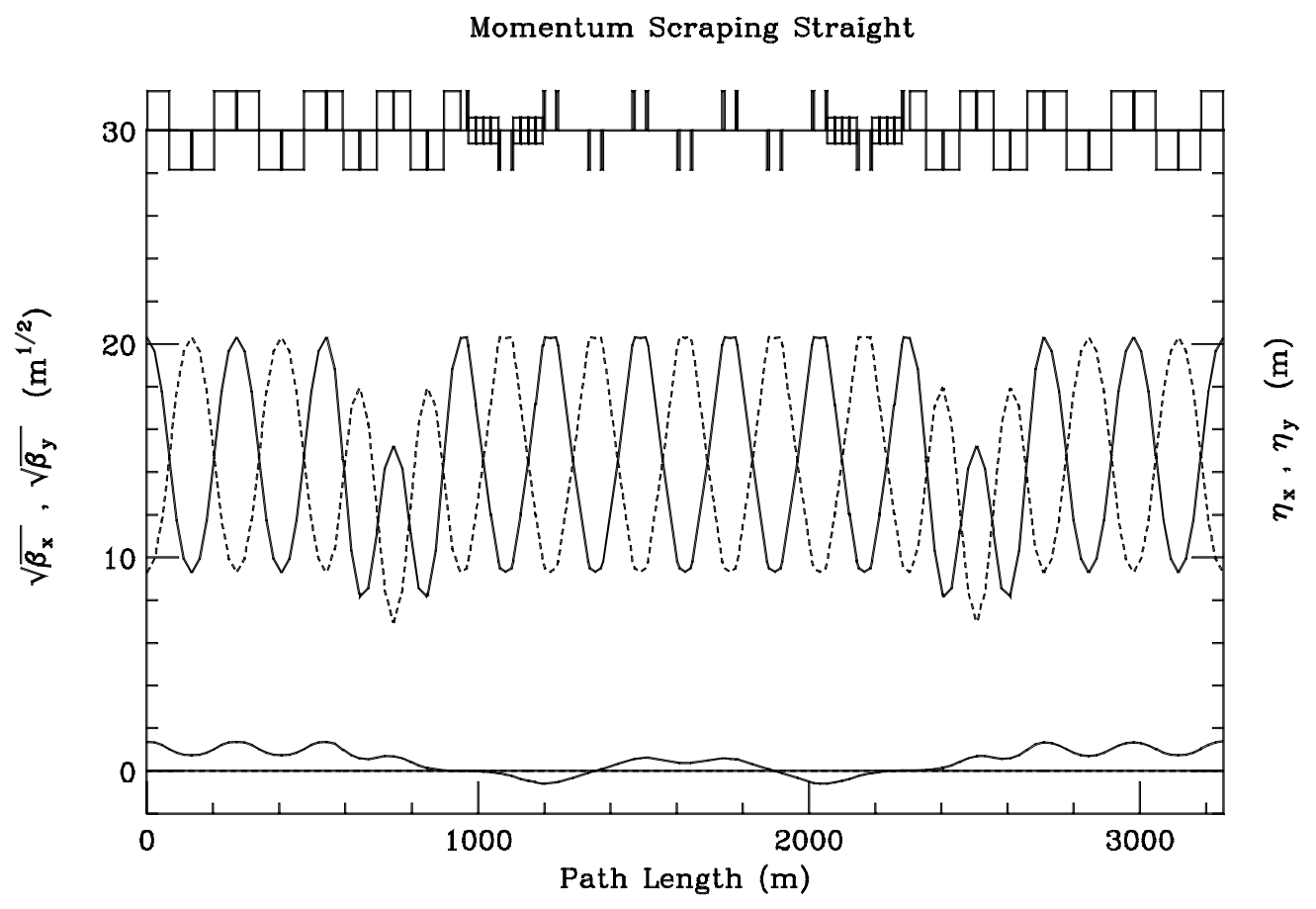

Figure 3.5. Dispersion creation in a 5-cell straight for collimation. 


\subsubsection{Magnet Apertures and Field Quality}

The available mechanical aperture determines the required dynamic aperture. Collimators define the limiting aperture in most cases and they are usually placed at around $6 \sigma$ from the center of the beam pipe. This allows $3 \sigma$ for the beam distribution, and another $3 \sigma$ for injection errors and orbit drifts over time. The real dynamic aperture therefore must be greater than $6 \sigma$ in order to avoid significant losses of particles at places in the ring outside the beam cleaning section.

Errors, time dependent fields and other imperfections in a real machine can never be modeled accurately enough in a simulation, so the dynamic aperture calculated by a simulation always exceeds the measured dynamic aperture. Observations at the Tevatron, HERA, and SPS have shown that the agreement between the measured and calculated dynamic aperture varies between $20-100 \%$. As a consequence the LHC, for example, requires that the calculated dynamic aperture after $10^{5}$ turns at both injection and collision (with only magnetic nonlinearities) equal $12 \sigma$. Since the errors listed above are just as likely for the VLHC, it seems reasonable to adopt a dynamic aperture requirement of $12 \sigma$ after $10^{5}$ turns.

\subsubsection{Scaling the Main Injector Errors}

As a starting hypothesis, the errors in the Main Injector are used as a scaling basis, assuming that the mechanical errors scale with the pole tip gap. Thus we assume that the field errors in the VLHC low field transmission line magnets at a pole tip radius of $10 \mathrm{~mm}$ are the same as the errors in the Main Injector magnets at a pole tip radius of 1 inch. The bend fields in the Main Injector at $8 \mathrm{GeV}$ and in the VLHC at an injection energy of $1 \mathrm{TeV}$ are nearly the same: $B_{0}{ }^{M I}=$ $0.101 \mathrm{~T}, B_{0}{ }^{V L H C}=0.098 \mathrm{~T}$.

We make the somewhat stronger assumption that the equality is satisfied for every harmonic $n$ so that we obtain the following scaling relation between the multipole coefficients in the Main Injector and those in the VLHC,

$$
\left[\frac{b_{n}}{R_{r e f}^{n}}, \frac{a_{n}}{R_{r e f}^{n}}\right]^{V L H C}=(2.54)^{n} \times\left[\frac{b_{n}}{R_{r e f}^{n}}, \frac{a_{n}}{R_{r e f}^{n}}\right]^{M I}
$$

Table 3.3. Systematic <> and random $\sigma$ of multipole coefficients used for the VLHC, scaled from Main Injector magnets using Equation 3.5. The coefficients are shown in units of $10^{-4}$ at a reference radius of $10 \mathrm{~mm}$.

\begin{tabular}{|ccccc|}
\hline Order, $\boldsymbol{n}$ & $<\mathbf{b}_{\mathbf{n}}>$ & $<\mathbf{a}_{\mathbf{n}}>$ & $\sigma\left(\mathbf{b}_{\mathbf{n}}\right)$ & $\sigma\left(\mathbf{a}_{\mathbf{n}}\right)$ \\
\hline 2 & -0.600 & 0.000 & 0.600 & 0.200 \\
3 & 0.000 & 0.000 & 0.149 & 0.300 \\
4 & 0.300 & 0.000 & 0.300 & 0.150 \\
5 & 0.000 & 0.000 & 0.100 & 0.500 \\
6 & 0.000 & 0.000 & 0.250 & 0.250 \\
\hline
\end{tabular}

Table 3.3 shows the derived harmonics for the low field magnets. We have assumed that the measured systematic skew harmonics in the Main Injector magnets are the result of the top 
down asymmetry in the magnet bus work [2]. This should not be an issue for the low-field magnets since the bus work is symmetric, and so the nonlinear skew harmonics have been set to zero.

\subsubsection{Dynamic Aperture at Injection}

The main issue is to determine whether the field quality is good enough to meet the target set for the acceptable dynamic aperture. If not, a nonlinear corrector system that increases the dynamic aperture to the target value must be developed. The results shown below will be refined at a later stage when more information on the field quality is available. For most calculations reported here, particles are tracked for 1024 turns without synchrotron oscillations in a perfectly aligned lattice. The only nonlinearities are the chromaticity sextupoles and the field errors in the arc magnets. Sources of coupling are not introduced so correction with skew quadrupoles is not necessary. The fractional tunes are set to the Tevatron values $v_{\mathrm{x}}=0.581, v_{\mathrm{y}}=$ 0.575 .

The impact of linear coupling is reduced when there is a difference in horizontal and vertical integer tunes. In the perfect aligned lattice there are no sources of linear coupling. Nevertheless the influence of tune splits on the dynamic aperture for the cases $v_{x}-v_{y}= \pm 2, \pm 4, \pm 6$ were studied. In no case did the dynamic aperture improve and in some cases the aperture was reduced significantly, presumably due to the beta beating.

Figure 3.6 shows the dynamic aperture with only chromaticity sextupoles, only random errors, only systematic errors and all the errors. The dynamic aperture with all errors is nearly the same as that with only the random errors. Tracking with all the field errors at injection shows that the dynamic aperture is about $20 \sigma$ as shown in Table 3.4. Increasing the number of turns to 100,000 typically results in a decrease of the dynamic aperture by about 2-3 $\sigma$. This suggests that the target dynamic aperture of $12 \sigma$ at 100,000 turns should be met with this set of assumed errors.

\subsubsection{Dynamic Aperture at Collision}

The dynamic aperture at collision optics will be dominated by the field errors of the IR quadrupoles due to the large beam sizes in these magnets. The IR quadrupoles with their high gradients will be built with "conventional" superconducting magnets. The nonlinear harmonics in these magnets are well understood following decades of development and also through the Fermilab program of building these quadrupoles for the LHC. The errors in the arc magnets built with conventional technology usually are not significant in determining the dynamic aperture at top energy. For example, the target dynamic aperture in the LHC at top energy is set at $12 \sigma$ with only the IR quadrupole field errors. We will adopt the same target dynamic aperture of $12 \sigma$ in the simulations. 


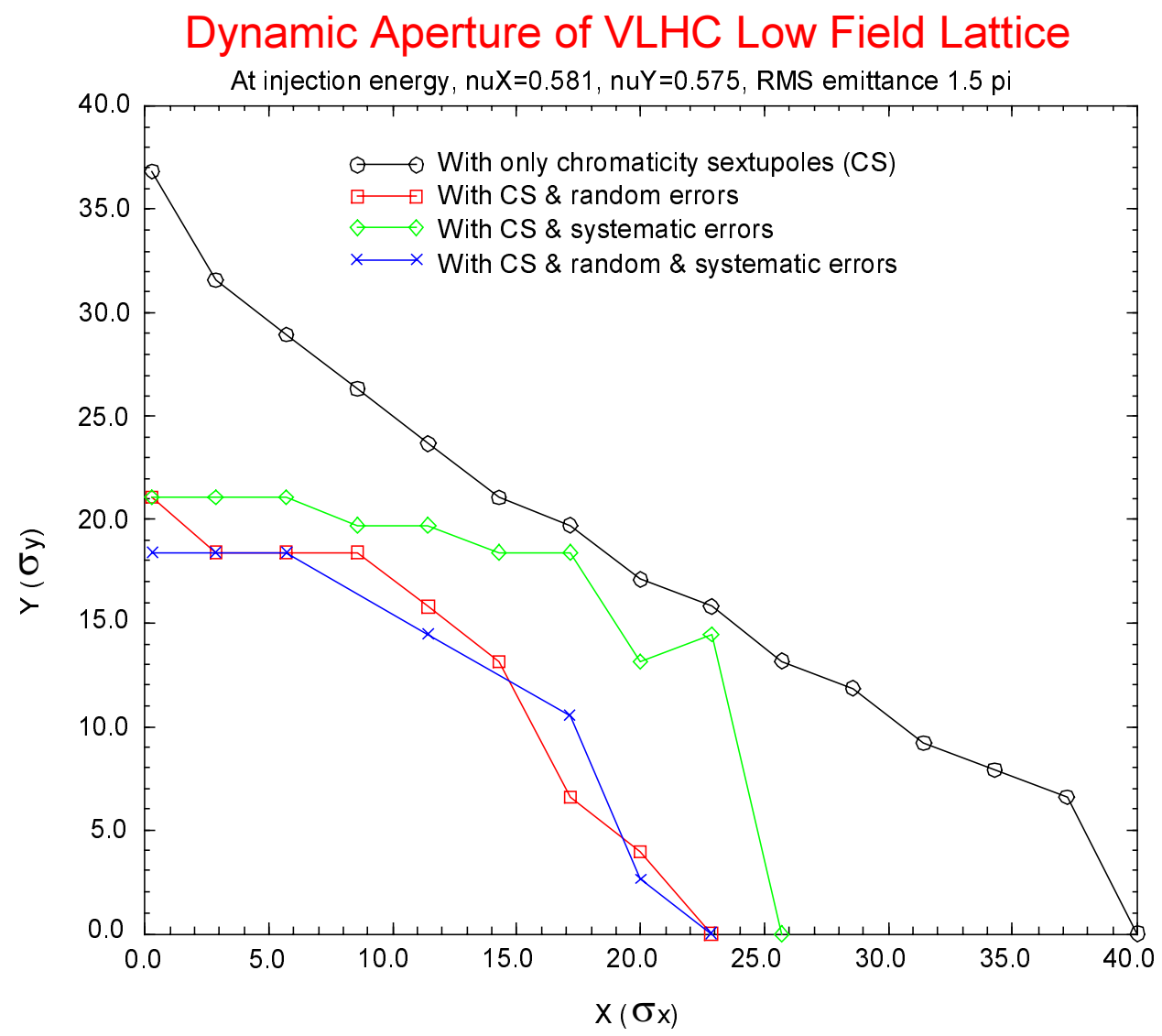

Figure 3.6. Dynamic aperture at injection with only chromaticity sextupoles(CS), CS and random errors only, CS and systematic errors only, and CS with all errors. The target dynamic aperture is $12 \sigma$.

Table 3.4. Dynamic aperture at injection energy with various sets of errors in the arc magnets. These results show that the random errors together have a somewhat larger impact on the dynamic aperture than the two systematic components $\left\langle b_{2}\right\rangle,\left\langle b_{4}\right\rangle$. The dynamic aperture with all the errors is about the same $(20 \sigma)$ as without the systematic errors.

\begin{tabular}{|lcc|}
\hline Errors & \multicolumn{2}{c|}{$\begin{array}{c}\text { Dynamic Aperture } \\
\text { Average }\end{array}$} \\
Only chromaticity sextupoles $(\mathrm{CS})$ & 30.9 & 25.4 \\
CS and only random errors & 20.0 & 18.4 \\
CS and only systematic errors & 23.4 & 21.1 \\
CS and all errors & 19.7 & 18.4 \\
CS and only random errors and $\sigma\left(b_{2}\right)=\sigma\left(a_{2}\right)=0$ & 20.4 & 18.6 \\
CS and only random errors and $\sigma\left(b_{3}\right)=\sigma\left(a_{3}\right)=0$ & 20.2 & 17.6 \\
CS and only $<b_{2}>\neq 0$ & 31.5 & 25.2 \\
CS and only $<b_{4}>\neq 0$ & 24.5 & 21.1 \\
\hline
\end{tabular}

The arc magnets in the Stage-1 collider, however, are built with transmission line magnets, which have different characteristics from conventional superconducting magnets. At high current, the field in the iron saturates leading to a drop in the gradient and a large negative sextupole component $\left(b_{2}\right)$. While quadrupole correctors in every cell to maintain the correct 
phase advance per cell will compensate the change in gradient, the compensation of the sextupole component is a more serious issue. If the nonlinear fields due to magnet saturation have a significant impact on the dynamic aperture, then this could potentially limit the maximum energy reach of the low field collider.

The straightforward approach to compensating the saturation $b_{2}$ component is to use the chromaticity sextupoles in the cells to correct the linear chromaticity. At large $\left|b_{2}\right|$ this leads to strong chromaticity sextupoles lumped at two locations in the cell, one SF next to the focusing magnet $(\mathrm{F})$, the other SD next to the defocusing magnet (D). A calculation of the dynamic aperture at $20 \mathrm{TeV}$ without the IR errors but with the $b_{2}$ component in the arc magnets due to saturation shows that the dynamic aperture is quite large even up to 10 units of $b_{2}$. At $b_{2}=0$, integrated strengths required for the F and D type sextupoles are $(946,1761) \mathrm{T} / \mathrm{m}$ respectively. At positive $b_{2} \leq 3$, the F sextupoles decrease and D sextupoles increase in magnitude and vice versa at negative $b_{2} \geq-3$. At larger absolute values of $b_{2}$, both sextupole strengths increase in magnitude. If required, additional sextupoles could be placed in the $1 \mathrm{~m}$ gap between the $\mathrm{F}$ and $\mathrm{D}$ and the $\mathrm{D}$ and $\mathrm{F}$ magnets to correct the chromaticity generated by the iron saturation. This would lower the integrated sextupole strengths and allow for larger values of $b_{2}$ to be compensated.

The impact of magnet saturation should, however, be determined in the presence of the dominant field errors of the IR quadrupoles. We have based our dynamic aperture calculations on the expected field errors calculated by G. Ambrosio et al. [3].

The dynamic aperture was calculated for three seeds for the random errors with the signs of the uncertainties in the systematics alternating between positive and negative. The random errors were truncated at $3 \sigma$. For each seed the dynamic aperture was calculated with $-10 \leq b_{2} \leq$ 10. At $b_{2}=0$, the dynamic aperture drops to around $20 \sigma$ in the presence of the IR errors, compared to a value of around $130 \sigma$ without these errors but only chromaticity sextupoles. The average dynamic aperture varies over a small range of 20-23 $\sigma$ when $b_{2} \leq 10$. Clearly the saturation sextupoles do not have a significant impact on the dynamic aperture at $20 \mathrm{TeV}$. The maximum tolerable value of $b_{2}$ may instead be limited by the sextupole strengths required to correct the chromaticity.

\subsubsection{Dynamic Aperture with Synchrotron Oscillations}

After correction of the orbit due to misaligned magnets, the rms closed orbit deviations were reduced to $\langle x\rangle_{\text {rms }}=0.22 \mathrm{~mm},\langle y\rangle_{\mathrm{rms}}=0.21 \mathrm{~mm}$. The fractional tunes were $v_{x}=0.585, v_{y}$ $=0.573$. Figure 3.7 shows the average and minimum dynamic aperture with synchrotron oscillations and closed orbit errors as a function of the momentum deviation amplitude. The average and minimum are taken over three random seeds. The average dynamic aperture at $\delta p / p=0$ is $19 \sigma$ while at $\delta p / p=3 \times 10^{-4} \approx 3 \sigma_{p}$, the average dynamic aperture drops to about $11 \sigma$ which is only slightly under the target value. 


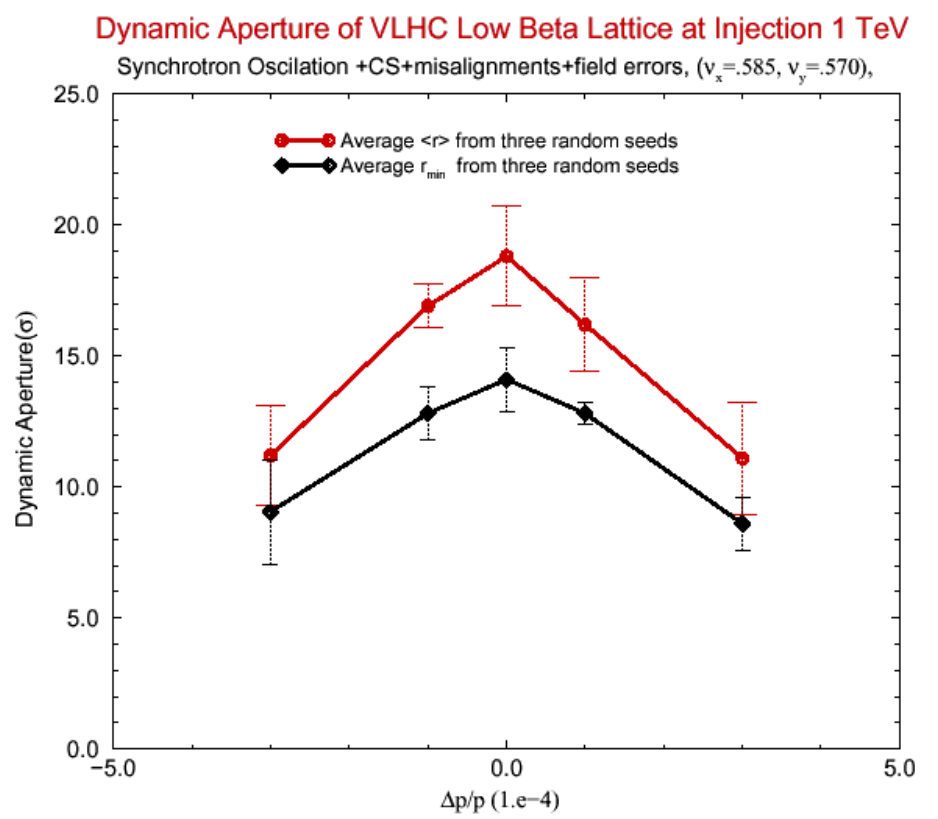

Figure 3.7. Dynamic aperture with synchrotron oscillations and closed orbit errors. Particles were tracked for 10,000 turns and with three random seeds.

\subsubsection{Tolerances and Corrections}

\subsubsection{Closed Orbit}

Distortions of the ideal closed trajectory in the collider will be primarily generated by transverse alignment errors. The trajectory in the horizontal plane will also be influenced by errors in the main bend field of the magnets, and the vertical trajectory will be further distorted due to roll errors in the main bending field.

For a bending magnet of length $L$ with gradient $B^{\prime}$ which is displaced transversely from its ideal position by an amount $d$, a particle with magnetic rigidity $B \rho=p / e$ will have its trajectory deflected through an angle $\theta=\left(B^{\prime} L / B \rho\right) d$. For the low field collider, the gradient magnets have lengths roughly equal to the half-cell length and will be supported along its length by several adjustable magnet stands. If the arc magnets are divided into $n$ equal-length sections for support and alignment, then the rms expected closed orbit deviations in the horizontal $(x)$ and vertical (y) degrees of freedom at maximum $\beta$ locations in the arcs are given by

$$
\Delta \widehat{x}_{c o}=\frac{\sqrt{\hat{\beta}\langle\beta\rangle}}{2|\sin \pi v|} \sqrt{\frac{N}{2}}\left(\frac{B_{0} L}{B \rho}\right) \sqrt{\frac{1}{n}\left(\frac{B^{\prime} d_{r m s}}{B_{0}}\right)^{2}+\left(\frac{\Delta B}{B_{0}}\right)_{r m s}^{2}}
$$

and

$$
\Delta \widehat{y}_{c o}=\frac{\sqrt{\hat{\beta}\langle\beta\rangle}}{2|\sin \pi v|} \sqrt{\frac{N}{2}}\left(\frac{B_{0} L}{B \rho}\right) \sqrt{\frac{1}{n}\left[\left(\frac{B^{\prime} d_{r m s}}{B_{0}}\right)^{2}+\phi_{r m s}^{2}\right.}
$$


where $\left(\Delta B / B_{0}\right)_{r m s}$ is the rms field strength deviation of the full magnet, $d_{r m s}$ is the rms displacement of the magnet sections, and $\phi_{r m s}$ is the rms roll angle (with respect to vertical) at the $n$ locations within a half-cell. $N$ is the number of half-cells in the collider.

For typical values of the above variables we use $n=5$ over a half cell length of $L=67 \mathrm{~m}$, $\beta_{\max }=411 \mathrm{~m},<\beta>=250 \mathrm{~m}, B_{0}=2 \mathrm{~T}, B^{\prime}=9 \mathrm{~T} / \mathrm{m}, d_{r m s}=250 \mu \mathrm{m}, \phi_{r m s}=0.5 \mathrm{mrad}$, and $\left(\Delta B / B_{0}\right)_{r m s}=10^{-4}$. From a distribution of errors over the 780 arc cells in the collider, we obtain expected rms orbit errors on the scale of

$$
\begin{aligned}
& \Delta \hat{x}_{c o} \approx 9.5 \mathrm{~mm} \\
& \Delta \widehat{y}_{c o} \approx 10.3 \mathrm{~mm}
\end{aligned}
$$

The maximum uncorrected closed orbit excursions clearly venture outside any reasonable beam pipe radius.

To correct the orbit distortions, we assume dipole correctors are placed at maximum $\beta$ locations in the arcs $(\mathrm{H} \& \mathrm{~V})$, and a standard 3-bump algorithm is performed. The corrector strength necessary to perform the correction using the parameters introduced above would be

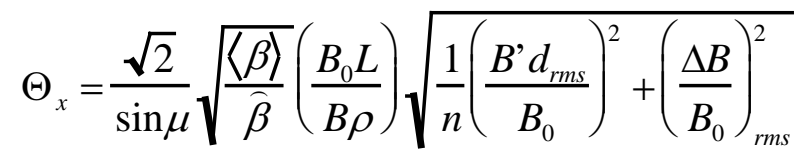

and

$$
\Theta_{y}=\frac{\sqrt{2}}{\sin \mu} \sqrt{\frac{\langle\beta\rangle}{\widehat{\beta}}}\left(\frac{B_{0} L}{B \rho}\right) \sqrt{\frac{1}{n}\left[\left(\frac{B^{\prime} d_{r m s}}{B_{0}}\right)^{2}+\phi_{r m s}^{2}\right]}
$$

where $\mu$ is the cell phase advance. For $\mu=90^{\circ}$, and the same parameter values as before, the rms corrector strengths are

$$
\begin{aligned}
& \Theta_{x} \approx 2.3 \mu \mathrm{rad} \\
& \Theta y \approx 3.2 \mu \mathrm{rad}
\end{aligned}
$$

At $20 \mathrm{TeV}$, a $3 \mu \mathrm{rad}$ bend angle requires an integrated field strength of $0.2 \mathrm{~T}-\mathrm{m}$. To be able to correct at the 2.5- $\sigma$ level, steering correctors should have strengths of approximately $0.5 \mathrm{~T}-\mathrm{m}$. The maximum corrector strengths will be dominated by displacement errors caused by diffusive ground movement and ground settlement over time.

\subsubsection{Quadrupole Effects}

The quadrupole gradient in the transmission line magnets drops steeply at high fields due to saturation of the iron. Quadrupole correctors, one in each plane, will be placed in every arc cell to maintain the required phase advance of $90^{\circ}$ per cell. At present these correctors are $0.5 \mathrm{~m}$ long and placed at the beginning of each half-cell. The correctors, independently powered, have been sized to correct the gradient shift due to magnet saturation at 2 Tesla.

Sources of errors in the tune include gradient errors and closed orbit distortions in the nonlinear magnets. Quadrupole correctors in the arcs will be used to control the global tunes and to split the integer tunes to reduce the sensitivity to systematic linear coupling sources. 
It will be important to restrict the beta beating to a low value everywhere, both for onmomentum and off-momentum particles. It will be especially important to isolate the interaction regions from beta-beating errors generated elsewhere in the ring. Quadrupoles at the entrances and exits of the arcs and at the entrances and exits of the straight sections can be used to control both the amplitude and phase of $\Delta \beta / \beta$.

Normal quadrupole gradient errors and skew quadrupole gradient errors will contribute to horizontal and vertical dispersion beating respectively. The errors generated in the arcs and utility straight sections will be corrected either by quadrupole correctors or by the closed orbit correctors. It will be important to keep the dispersion at the IPs very small. Local dispersion correction within the IRs will be necessary to correct the dispersion generated by the crossing angle.

\subsubsection{Coupling}

Sources of coupling include skew quadrupole errors in the main magnets, rolls of the gradient magnets and vertical orbit distortion through the nonlinear magnets. Requiring that the minimum tune split be less than 0.05 before correction may set the tolerance for the allowed coupling. Larger values make coupling correction with skew quadrupoles difficult.

Skew quadrupoles placed in regions of horizontal dispersion will generate vertical dispersion, which will need additional correction. It is therefore preferable to place these quadrupoles in straight sections. Two families of skew quadrupoles in the utility straight sections and the IRs will be sufficient to control the effects due to the difference resonance $v_{x}-v_{y}=p$. These families will be placed so that the difference in phases $\psi_{x}-\psi_{y}$ differs by $\pi / 2$ at the families. The coupling sum resonance $v_{x}+v_{y}=p$ may also need to be corrected (depending on the strength of the skew quadrupole errors). In this case an additional two families will be required, placed so that $\psi_{x}+\psi_{y}$ differs by $\pi / 2$ at these families.

\subsubsection{Chromaticity Effects}

The linear chromaticity is controlled by two families of sextupoles, one in each plane, placed in every arc cell. These families can be defined in software, since all correctors are independently powered, as described in Section 5.2.3. This will suffice provided the nonlinear change of tune with momentum is small over the required momentum aperture. If the global nonlinear chromaticity requires correction, then two families of sextupoles in each plane for the $90^{\circ}$ cells will be required. It would therefore be desirable to place sextupoles (of say the F type) in every other cell on one bus and the other family of sextupoles in the other cells on another bus.

In addition to the chromaticity due to the quadrupoles, there is a contribution due to sextupole fields caused by iron saturation at fields close to 2 Tesla. This chromaticity must also be compensated by the chromaticity sextupoles. This has been discussed in the section on the dynamic aperture at collision.

If the beta function is strongly dependent on the momentum, then it can lead to an increased spot size if not corrected at the IPs and to emittance growth due to injection errors if not corrected at the injection point. Correcting the momentum dependence of the beta functions will require additional sextupole families. 
For the IRs, a local chromaticity correction system was proposed for the SSC [4], which increased the momentum aperture from about $2.5 \sigma$ with linear chromaticity correction to about $8 \sigma$ with correction of the nonlinear chromaticity generated by the IRs. A similar system may be required for the IRs if the momentum aperture needs to be increased.

\subsubsection{Alignment Tolerances in the IRs}

These tolerances are more stringent for the IR quadrupoles than for any other magnets in the ring. Several criteria have to be met including:

- Dispersion at the IP should be small, both to attain maximum luminosity and to avoid synchro-betatron resonances.

- Beam excursions in the triplets should be limited to preserve the dynamic aperture.

- Separations between the beams at the parasitic collision points should not decrease.

In the IR quadrupoles the beta functions change rapidly and are not symmetric about the quadrupole center. As a consequence, pitch and yaw misalignments will introduce substantial beam separations at the IPs if not corrected. Due to the crossing angle a roll misalignment of an IR quadrupole will introduce orbit shifts in the plane orthogonal to the crossing plane. This is in addition to the extra coupling generated by the roll angle. Thus misalignments in all six degrees of freedom of these IR quadrupoles need to be well controlled and corrected.

\section{Orbit offsets at the IPs}

The orbit offset at the IP $z_{\text {offset }}(I P)$ due to a transverse displacement $\Delta z_{Q}$ of an IR quadrupole (assumed to be at 90 degrees from the IP) is

$$
\frac{z_{\text {offset }}(I P)}{\Delta z_{Q}} \propto \frac{\sqrt{\beta^{*} \beta_{Q}}}{F}
$$

Here $\beta_{\mathrm{Q}}$ is the beta function at the quadrupole and $F$ is the focal length of the displaced quadrupole. The maximum offset of the quadrupole that can be corrected with orbit correctors is proportional to this ratio $z_{\text {offset }}(I P) / \Delta z_{Q}$. In Table 3.5 we compare the parameters for the quadrupole where the beta function is the largest.

Tolerances for the LHC quadrupoles were derived in [5]. The rms tolerance for the offset was obtained to be $0.27 \mathrm{~mm}$. Scaling this number by the ratio 2.3/3.1, we obtain a tolerance of $0.2 \mathrm{~mm}$ for the VLHC IR quadrupoles. This assumes that corrector strengths similar to those in the LHC (in kick angle) will be used in the VLHC.

Table 3.5. Parameters of the strongest IR quadrupole in the LHC and the VLHC.

\begin{tabular}{|lcc|}
\hline Parameter & LHC IR & VLHC (LF) IR \\
$\beta^{*}[\mathrm{~m}]$ & 0.5 & 0.3 \\
$\beta_{\max }[\mathrm{m}]$ & 4567 & 11263 \\
Focal Length, $F[\mathrm{~m}]$ & 21.1 & 18.7 \\
$z_{\text {offset }}(\mathrm{IP}) / \Delta z_{\mathrm{Q}}$ & $\sim 2.3$ & $\sim 3.1$ \\
\hline
\end{tabular}


Correctors to correct the orbit at the IP are best placed at 90 degrees from the IP. As in the LHC, correction packages with dipole and skew dipole correction coils should be placed at several high beta locations next to the IR quadrupoles. Spaces between Q2 and Q3 and after Q3 would be ideal locations for these correctors.

\section{IR quadrupole roll angles}

Tolerances on the allowed roll angles of the IR quadrupoles may be set by specifying the minimum tune split tolerable before correction. We will assume that this tune split due to roll angles in all sixteen quadrupoles from the two IRs is less than 0.05 before correction. RMS roll angles of $0.5 \mathrm{mrad}$ can be tolerated for the IR quads, assuming that all the IR quadrupole assemblies include a skew quadrupole corrector.

Normally two families of skew quadrupoles are required to correct both the real and imaginary parts of the coupling resonance term. However the phase advance through the IRs is very small and so the imaginary part of the resonance term is negligibly small. Thus only a single skew quadrupole on each side of the IP will suffice to locally correct the coupling in the IR.

Table 3.6 summarizes the misalignment tolerances for the IR quadrupoles. These are based on scaling from tolerances derived for the LHC and should be replaced at a later stage with more exact calculations.

Table 3.6. Misalignment tolerances for the VLHC IR quadrupoles derived by scaling from the LHC IR quadrupole tolerances [5].

\begin{tabular}{|lc|}
\hline Misalignment & Tolerance \\
Offsets [mm] & 0.2 \\
Longitudinal placement [mm] & 1 \\
Roll [mrad] & 0.5 \\
\hline
\end{tabular}

\subsubsection{Crossing Angle}

A crossing angle is required to separate the beams at the parasitic collisions. As is well known, the crossing angle has several effects on the linear and nonlinear optics and beam dynamics, including generation of dispersion due to orbit offsets in the quadrupoles, coupling due to skew multipole errors, and feed down of higher order multipoles. Local dispersion control is required to prevent dispersion waves from propagating into the arcs. This can be performed adjusting pairs of quadrupoles in the cells adjacent to the IRs where there is natural dispersion. A scheme such as this was proposed for the SSC [6]. Correction of local coupling from this and other sources will require skew quadrupoles in the IR as already mentioned. The linear effects from feed-down of higher order multipoles will be corrected by orbit and tune correctors. Nonlinear effects will require nonlinear correction packages in the IRs similar to those in the LHC and RHIC.

\subsubsection{Ground Motion and Emittance Growth}

We have measured the slow ground motion in the 300-ft deep dolomite Conco mine in Aurora, IL, about 3 miles west of Fermilab. The goal of the studies is to record and analyze vertical 
ground motion in 8 points separated by 30 meters over a time interval of about 1 year. The experiment in the Aurora mine started in October 2000. The first data reveal that dolomite mine motion is rather small and can be described by the ATL law with coefficient of $A=(6.8 \pm 2.7)$ $\times 10^{-7} \mu \mathrm{m}^{2} / \mathrm{m} / \mathrm{s}$ [7]. The resulting maximum orbit distortion in the VLHC which will be caused by this coefficient is of the order of $9 \mathrm{~mm} /(\text { year })^{1 / 2}$.

The only drawback of the Aurora mine experiment is that there are too few sensors, which limits the statistics for studies of spatial correlations. Therefore, a system with about 20 sensors is needed, and long-term measurements should be repeated on a larger scale. Analytical/ computer studies of the slow ground motion effects and orbit correction system and procedures must be performed.

Turn-to-turn dipole magnetic field fluctuations and vibration of quadrupole magnets are of concern, because they can excite coherent beam motion. If the motion is not corrected over the decoherence time of about $1 / \xi_{\text {beam-beam }} \approx 1000$ turns, then the coherent motion will be converted into transverse emittance increase and can cause substantial emittance growth over about 10 hours of the store. So far, experimentally measured high-frequency ground or magnet vibrations do not pose a big problem for VLHC [8]. Nevertheless, more detailed analysis on how the lattice may affect the tolerances is needed. For example reference [9] claims that tolerances on vibration amplitude in a lattice with combined function magnets is about 3 times the tolerances in a separated function FODO lattices.

In 1999 experimental studies of high-frequency magnetic field fluctuations were carried out in a Tevatron superconducting dipole magnet. An effective $\mathrm{rms} \mathrm{dB} / \mathrm{B} \approx 10^{-10}$ which is about 10 times the tolerance for the VLHC, was measured. This amplitude could cause emittance doubling after about 6 minutes. The measurements have to be repeated in the VLHC dipole prototypes. Again, we need to analyze and perform computer simulations for lattices with separated and combined function magnets.

Suppression of the emittance growth can be provided by low noise feedback. The first analysis of the FB is presented in [10]. The system has to have sub-micron equivalent input noise. Design and test of such a system at the Tevatron is an important VLHC beam physics R\&D task.

\subsubsection{Collective Effects}

A VLHC Instability Workshop was held at SLAC, March 21-23, 2001 [11]. Most instability issues examined at this workshop are not expected to be serious concerns for the VLHC. Two exceptions are the transverse mode-coupling instability (TMCI) and the transverse resistive wall instability, which are both most pronounced for the Stage- 1 collider at injection. In particular, the design intensity of $2.5 \times 10^{10}$ particles per bunch is a factor of two higher than the expected TMCI threshold. The resistive wall instability will require an extension of the stateof-the-art feedback system. To compensate for the very large incoherent and coherent tune shifts, as well as tune variation along the bunch train due to resistive wall effects, the ring will need to be filled in an appropriate bunch pattern to mitigate this issue.

The rings of both stages have the same size, $2 \pi R=233.037 \mathrm{~km}$ in circumference, and the same RF frequency. In order to have shorter bunches, the RF frequencies in both phases are chosen 9 times higher than that of the Tevatron, so that the bunches will be separated by 9 RF 
buckets. The RF harmonic is $h=371520$, which is the Tevatron harmonic number scaled to the VLHC circumference.

In the low-field rings, the RF voltage has been chosen to be $V_{r f}=50 \mathrm{MV}$. The rms bunch area of bunches inside the buckets is chosen to be $0.4 \mathrm{eV}-\mathrm{sec}$, the rms bunch area [12] of the Tevatron bunches at $900 \mathrm{GeV}$. With $V_{r f}=50 \mathrm{MV}$, the maximum energy spread is $2 \times 10^{-3}$, and the bucket area is $5 \mathrm{eV}$-sec. Note that the present Tevatron RF system has a maximum voltage of $1 \mathrm{MV}$ only, thus the bunch length would be too long and the momentum spread too small to fit the higher harmonic buckets at injection. Thus, the Tevatron bunches must be shortened by either a bunch rotation or through the installation of a higher RF voltage before the transfer to the $20 \mathrm{TeV}$ ring is possible.

A bunch excited by forces in the vacuum chamber oscillates in the longitudinal phase space in modes describable by the radial mode parameter $n_{r}$ designating $n_{r}$ radial nodes and the azimuthal mode parameter $m$ designating $|m|$ azimuthal nodes. For a given $m$, the most easily excited radial mode is $n_{r}=|m|$. Almost distribution independent, the spectrum for the mode designated by $m \neq 0$ peaks at frequency $f(m) \approx(|m|+1) / 2 \tau_{L}$, where $\tau_{L}$ is the total length of the bunch in time. For a Gaussian distribution, we approximate it by the $95 \%$ length $\tau_{L}=2 \sqrt{6} 6 \sigma_{\tau}$, where $\sigma_{\tau}$ is the rms bunch length. We note that the frequencies for the various VLHC bunch modes of interest are in the GHz range.

\subsubsection{Transverse Mode Coupling Instability}

The TMCI threshold for the resistive wall has been calculated in various ways, including analytical formulas, a matrix approach for up to 25 modes, and through particle tracking simulations [11]. The analytic formulas estimate a threshold approximately $40 \%$ larger than the matrix approach. The difference between the matrix approach and particle tracking was about $30 \%$. These discrepancies were discussed at the workshop, and future calculations and checks have been planned.

The problem is very important for the low field VLHC. These calculations show that the threshold number for the bunch population at injection with the nominal set of parameters is equal to $1.4 \times 10^{10}$ protons, about half the nominal intensity. The above estimate assumes a round beam pipe of $9 \mathrm{~mm}$ radius. The threshold estimate needs to be redone for the nominal oval-shaped beam pipe.

Assuming that TMCI will exist in the VLHC, the following measures could be a cure:

- Inject low intensity bunches and coalesce them to make high intensity bunches at high field where the TMCI threshold is increased.

- Provide active feedback (resistive damping) at a few low order modes $l= \pm 1, \pm 2, \cdots$

- Introduce an RF quadrupole to provide a tune shift between the head and tail of the bunch.

For this reason, a bunch coalescing scheme has been adopted, which is described in a subsection below. The other two measures will continue to be studied.

The TMCI theory for proton beams is technically challenging because the bunches are longer and other forces may be present. Experimental data for comparison would be most 
useful. For instance, it may be possible to study the TMCI using the Tevatron Electron Lens (TEL).

\subsubsection{Resistive Wall Effects}

For the low field ring parameter range the various formulae for the resistive wall impedance agree within a factor of two. The e-folding time for the low frequency resistive wall instability is less than 1 turn. Additionally, the very low revolution frequency leads to a variation in the magnetic image Laslett tune shift when the ring is partially filled during the injection process. The variation is due to the fact that the revolution period is comparable to the magnetic diffusion time through the beam pipe. Initial estimates show a DC tune shift on the order of 0.5 units, with an $\mathrm{AC}$ component of about 0.1 units due to the time dependence of the magnetic diffusion into the beam pipe. Increasing the vacuum pipe thickness and/or radius could reduce both of these problems. Increasing the dipole magnet gap can reduce the DC Laslett tune shift, while the variation in tune along the bunch train could be reduced by quadrupoles running at multiples of the revolution frequency. It is envisioned, however, that injecting beam in an appropriately symmetric manner, with bunches entering the ring far apart from each other and gradually filling in between the already injected bunches, the tune variations can be reduced to a tolerable level.

The resistive wall instability needs to be damped. Realistic studies of the feedback system are needed. To fight the transverse resistive wall instability, a fancy feedback system is envisioned [11]. The system would have high-gain, low bandwidth (perhaps $100 \mathrm{kHz}$ ) and the pickup signal would arrive at the kicker slightly behind the beam bunch that produced the signal. This feedback system indeed is capable of suppressing the dipole multi-bunch resistive wall growth time of one turn. It is not $100 \%$ clear exactly how fast the growth rate is because the theory of resistive wall impedance in this complicated environment (cold, thin coating, magnets outside, etc.) is not easy to calculate. Whether it can suppress beyond one turn is yet to be demonstrated.

\subsubsection{Bunch Coalescing in the Stage-1 Collider}

To alleviate beam instabilities at its injection energy, the Stage-1 collider will have a second RF system for performing bunch coalescing. Five bunches each containing approximately $0.5 \times$ $10^{10}$ protons will be coalesced into one bunch of $2.5 \times 10^{10}$ using a $53.1 \mathrm{MHz}$ system in the Stage-1 collider [13]. If this is performed at $20 \mathrm{TeV}$, then the $478 \mathrm{MHz}$ system voltage must be reduced from $50 \mathrm{MV}$ to about $6.5 \mathrm{MV}$ and the bunches allowed to rotate $1 / 4$ of a synchrotron period. The main RF system is then turned off, and the second RF system, operating at 53.1 $\mathrm{MHz}$, is snapped on with voltage of 2-3 MV. After 1/4 period in this system, the $53.1 \mathrm{MHz} \mathrm{RF}$ is turned back off and the $478 \mathrm{MHz}$ turned on again at approximately $20 \mathrm{MV}$. The RF voltage is then raised adiabatically to $50 \mathrm{MV}$ completing the process.

This system naturally implies that a $478 \mathrm{MHz}$ RF system be employed at some early stage in the Tevatron injector system. The details of this injector upgrade are yet to be worked out. 


\subsection{Stage 2 - the High Field Ring}

\subsubsection{Luminosity versus Energy}

The High Field VLHC ring will be the first cryogenic collider to operate in the synchrotron radiation dominated regime, in which the radiation damping time is shorter than the storage time. In this regime there are practical and economic limits to the cryogenic system that can be installed. There is a maximum value $P_{\text {cryo }}$ for the synchrotron radiation, which can be absorbed in each ring. It is necessary to adjust the beam parameters to stay within the installed power limit, resulting in an average luminosity that depends on the beam energy $E$ according to a "maximum power law,"

$$
L_{\text {ave }}<\frac{P_{c r y o}}{E}\left(\frac{1}{N_{I P} \sigma_{\text {tot }}}\right)\left(\frac{T_{0}}{T_{\text {store }}}\right)
$$

Although there is a clear advantage in reducing the storage time, $T_{\text {store }}$ must remain significantly larger than the damping time $T_{0}$ in order to take advantage of radiatively damped beam sizes. Other factors (such as the refill time) will also play a role in determining the optimum value of $T_{\text {store }}$, but it is safe to estimate that

$$
\frac{T_{0}}{T_{\text {store }}} \approx 0.2
$$

Except for some uncertainty in this factor, the maximum power law clearly states that the installed capacity to absorb synchrotron radiation at cryogenic temperatures directly limits the attainable product of average luminosity and energy. This trade-off between higher luminosity and higher energy was emphasized in Table 1.2 in Chapter 1.

Similarly, if the beam stored energy per ring $U=N M E$ must be kept below a maximum value -- for example if the beam dump has a limited capacity -- then

$$
L_{\text {ave }}<\frac{U_{\max }}{E}\left(\frac{1}{N_{I P} \sigma_{\text {tot }}}\right)\left(\frac{1}{T_{\text {store }}}\right)
$$

Insofar as the stored energy is a practical limit to high field performance -- to the product $L_{\text {ave }} E$-- then there is pressure to reduce $T_{\text {store }}$, and hence to increase the dipole field, to reduce the circumference, and to reduce the refill time.

\subsubsection{Operational Performance}

For the Design Study, the most straightforward approach is to use as a baseline standard triplet optics and round beams, as in previous hadron colliders. It should be noted, however, that the High Field ring will be the first hadron collider with the potential to take advantage of naturally flat beams. While still under discussion, flat beams are sufficiently new and interesting for hadron colliders that most of the Stage-2 Design Study effort was devoted to analyzing this case up to now. The parameters described below have assumed that the beam emittances evolve into an asymmetric state characterized by $\kappa=\varepsilon_{y} / \varepsilon_{x}$. The high field ring should be able to achieve $\kappa<0.1$ during a store, consistent with conventional electron storage rings. 
Table 3.7. Nominal parameters for stores in the high field ring, using flat beams and doublet IR optics.

\begin{tabular}{|lrl|}
\hline Storage energy, $E$ & 87.5 & $\mathrm{TeV}$ \\
Peak luminosity, $L_{\max }$ & $2 x 10^{34}$ & $\mathrm{~cm}^{-2} \mathrm{sec}^{-1}$ \\
Collision debris power, per IP & 73 & $\mathrm{~kW}$ \\
Dipole field at storage & 9.765 & $\mathrm{~T}$ \\
Distance from IP to first magnet & 30 & $\mathrm{M}$ \\
Injection energy & 10 & $\mathrm{TeV}$ \\
Fill time & 30 & $\mathrm{sec}$ \\
Acceleration time & 2000 & $\mathrm{sec}$ \\
Synchrotron radiation damping time, $T_{0}$ & 2.48 & $\mathrm{hours}$ \\
Energy loss per turn, $U_{0}$ & 15.3 & $\mathrm{MeV}$ \\
Natural transverse emittance $(\mathrm{H})$ & 0397 & $\mu \mathrm{m}$ \\
Natural RMS momentum width & 5.5 & $10^{-6}$ \\
Collision beta horz, $\beta^{*}{ }_{x}$ & 3.7 & $\mathrm{~m}$ \\
Collision beta vert, $\beta^{*}{ }_{y}$ & 0.37 & $\mathrm{~m}$ \\
Equilibrium emittance ratio, $\kappa$ & 0.1 & \\
Initial bunch intensity, $N$ & 7.5 & $10^{9}$ \\
Beam current & 57.4 & $\mathrm{~mA}$ \\
Synch. rad. power, per beam, $P$ & .88 & $\mathrm{MW}$ \\
Dipole linear heat load & 4.7 & $\mathrm{~W} / \mathrm{m}$ \\
Stored energy, per beam, $U$ & 3.9 & $\mathrm{GJ}$ \\
\hline
\end{tabular}

The instantaneous luminosity is

$$
L=\frac{M}{4 \pi T} \frac{N^{2} \gamma}{\sqrt{\varepsilon_{x} \varepsilon_{y} \beta_{x}^{*} \beta_{y}^{*}}}
$$

where $T$ is the revolution period. The total number of protons in the ring, $M N$, is approximately set by the need to provide enough for "luminosity burn-off." Since the number of bunches $M$ is fixed, the need for a given peak luminosity then sets the single bunch population $N$. Nominal values for these and other parameters, including a conservative value of $\kappa=0.1$, are given in Table 3.7.

It is the head on beam-beam interaction that sets the minimum horizontal emittance, whether the beams are flat or round. Figure 3.8 shows the horizontal and vertical emittances decreasing to plateau values of $\varepsilon_{x}$ and $\varepsilon_{y}$ that are consistent with the beam-beam limit, and which are maintained by transverse beam heating. Figure 3.9 shows the corresponding evolution of instantaneous luminosity, and its average, during the store.

\subsubsection{The Head-on Beam-beam Interaction}

The horizontal and vertical tune shift parameters for bi-Gaussian round beams $\left(\beta^{*}{ }_{x}=\beta_{y}^{*}, \varepsilon_{x}=\right.$ $\left.\varepsilon_{y}\right)$ are

$$
\xi_{x}=\xi_{y}=\frac{r}{4 \pi} \frac{N}{\varepsilon_{x}}
$$




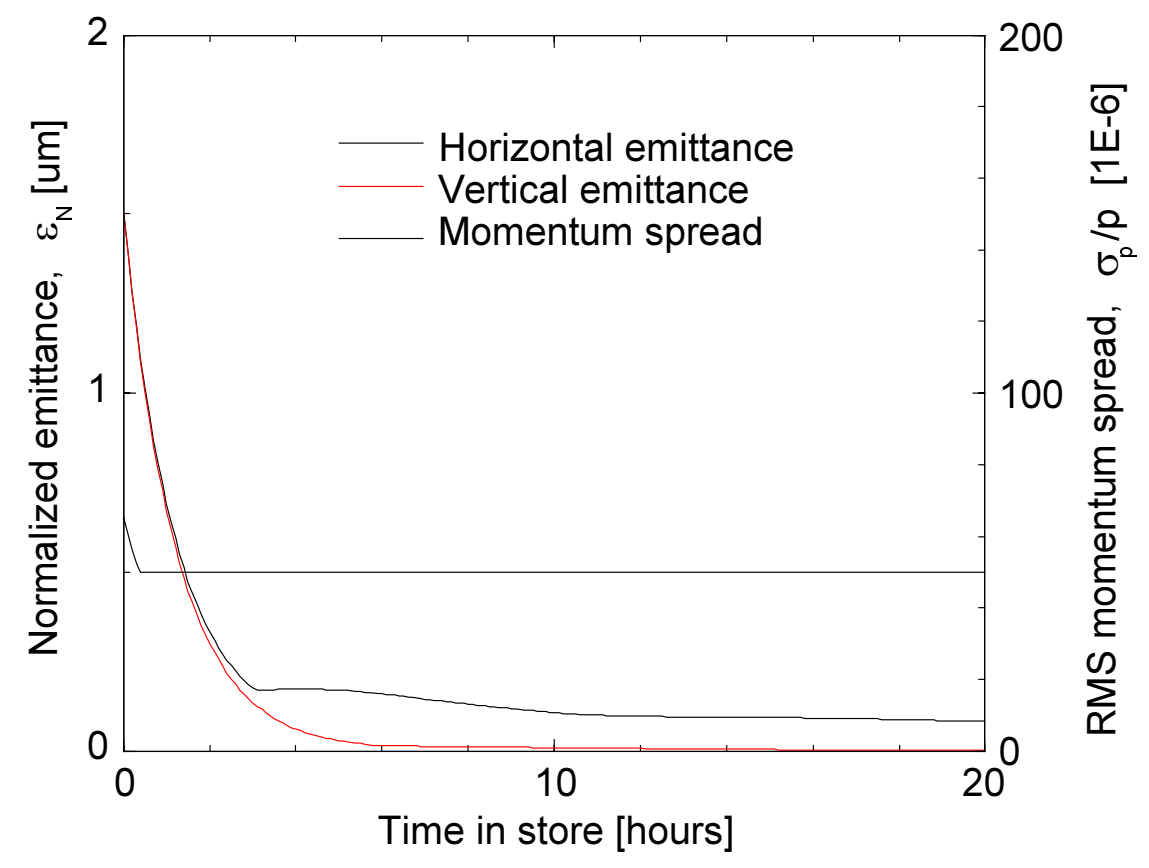

Figure 3.8. Evolution of the transverse emittances and the rms momentum spread during a store.

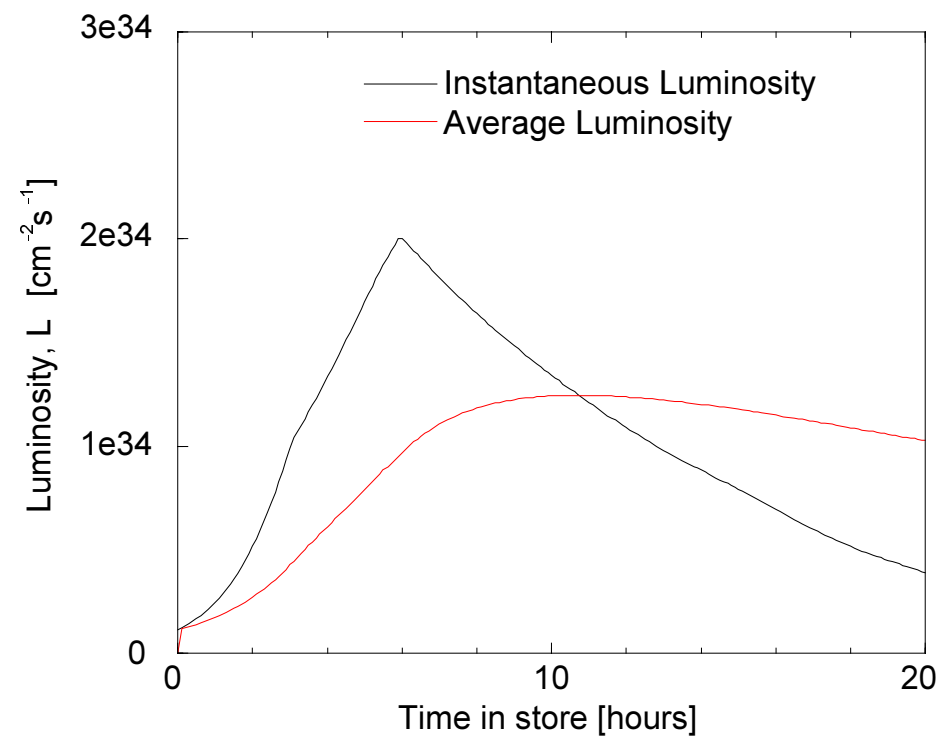

Figure 3.9. Evolution of the instantaneous and average luminosities, during a store.

The beam-beam limit is expected to be approximately the same for round or flat beams, given by

$$
\xi \leq 0.008 .
$$

This value is justified by practical experience at the SPS and at the Tevatron. The SPS operated at $\xi \approx 0.004$ (or slightly higher) with 6 collisions per turn. More recently, in Run Ib the Tevatron operated with $\xi \approx 0.0075$ with only 2 head on collisions per turn (as in the VLHC). Simulations predict that radiation damping might give the high field ring a slight additional advan- 
tage, which is by no means as strong as that commonly observed in electron storage rings. The numerical value of 0.008 is illustrated in Figure 3.10, which displays empirical data compiled by Keil and Talman for electron storage rings [14]. The "damping decrement" for the high field VLHC — the fraction of a damping period per head on collision-is approximately $10^{-7}$.

Then, re-writing Eqn. 3.18 as

$$
L=\frac{M}{4 \pi T} \frac{N^{2} \gamma}{\kappa \varepsilon_{x} \beta_{x}^{*}}
$$

it is explicitly clear that with flat beams the value of $\beta^{*}{ }_{\mathrm{x}}$ can be increased by a factor of about $1 / \kappa$, significantly simplifying the optics. Equation 3.21 then shows that the value of $\beta_{\mathrm{y}}{ }_{\mathrm{y}}$ is about the same for flat or round beams.

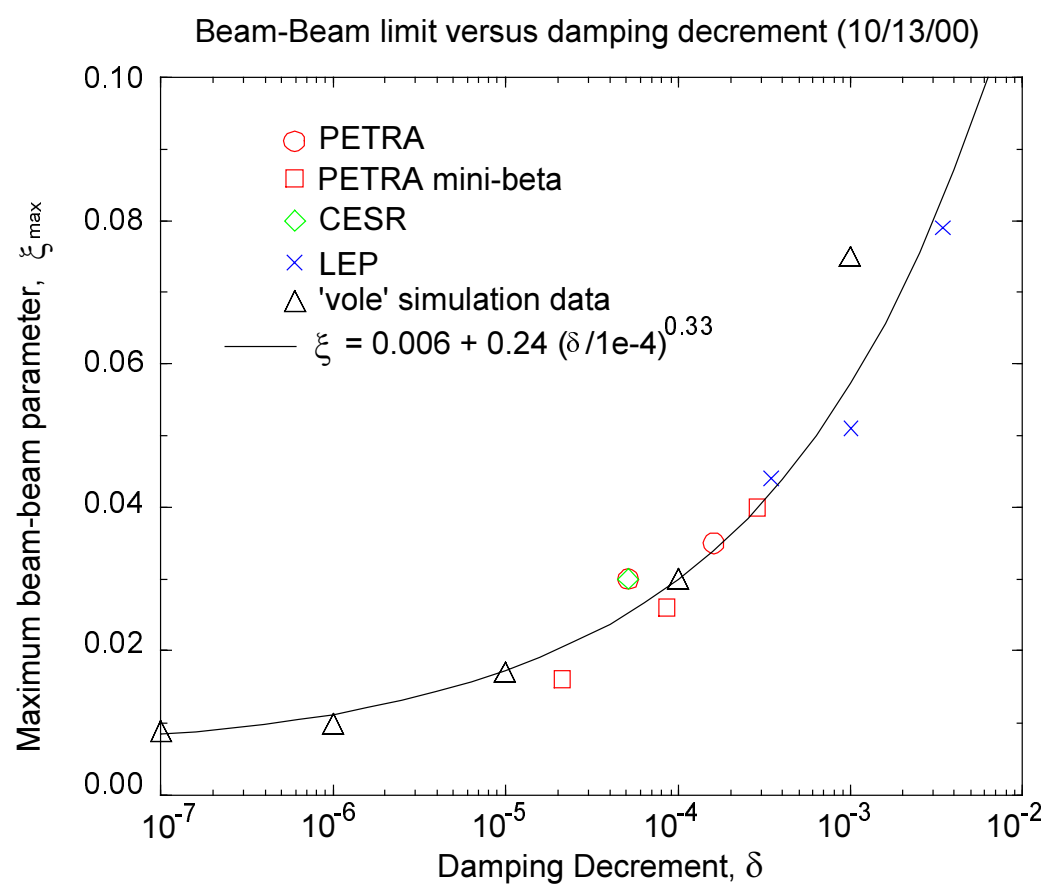

Figure 3.10. Maximum beam-beam parameter vs. damping decrement.

\subsubsection{Crossing Angles and Parasitic Beam-beam Collisions}

The horizontal and vertical tune shifts due to a single parasitic collision are

$$
\Delta q_{x, y} \approx \pm \frac{r N}{2 \pi \gamma} \frac{\beta_{x, y}}{\Delta^{2}}
$$

where the approximation is valid if the full beam separation $\Delta$ is much greater than both horizontal and vertical beam sizes, so that the beam acts like a moving line charge.

If the beams are thoroughly separated (into separate beam pipes, or with very large separations) at a distance $L_{\text {sep }}$ from the IP, then there are $4 L_{\text {sep }} / S_{B}$ parasitic collisions around each IP, where $S_{B}$ is the longitudinal bunch separation. The total tune shifts from all parasitic collisions with crossing angle $\alpha$ in one interaction region are 


$$
\Delta Q_{x, y} \approx \pm \frac{r N}{2 \pi \gamma} \frac{4}{S_{B}} \frac{L_{s e p}}{\beta_{x, y}^{*} \alpha^{2}}
$$

where the approximation is most valid if the beams are fully separated before the first IR quadrupole.

Again, assuming one exercises the flat beam option, the horizontal beta function at each collision is much less than the vertical

$$
\frac{\beta_{x}}{\beta_{y}} \approx \frac{\beta_{y}^{*}}{\beta_{x}^{*}}=\kappa
$$

so that, taking Eqns 3.24 and 3.25 together gives

$$
\Delta Q_{x} \approx-\kappa \Delta Q_{y}
$$

The horizontal tune shift is greatly suppressed with flat beams, to be much smaller than it is with round beams -- if the values of $L_{\text {sep }}$ and $\alpha$ compare favorably between the two cases.

\subsubsection{Advantages and Disadvantages of Flat Beams}

All electron colliders, whether circular or straight, take advantage of flat beams and use doublet optics. However, it has never before been possible to use flat beams in a hadron collider. Flat beams have the advantages and disadvantages discussed below, which continue to be investigated.

Flat beams require the first quadrupole on both sides of the IP to be vertically focusing to both counter-rotating beams (whether the crossing angle is vertical or horizontal). Thus, the optics must be symmetric across the IP, and the first quadrupole must be a 2-in-1 magnet. As seen in the next Section, both optical solutions have been developed.

Table 3.8 compares the performance of flat and round beam in the high field ring. In both cases the beam-beam limit of $\xi=0.008$ is reached 5 or 6 hours into the store, when the luminosity is at its peak. The horizontal and vertical emittances values recorded in Table 3.8 are those that are initially maintained (preventing further emittance shrinkage) when the horizontal and vertical beam-beam parameters first saturate. The same peak luminosity is achieved in both flat and round beam cases by adjusting the horizontal and vertical $\beta^{*}$ values.

The value of the total crossing angle $\alpha$ is set to be identical with flat or round beams-and is independent of the plane of the crossing angle. The major advantages and disadvantages of flat beams are outlined in the list below:

\section{Flat Beam Advantages}

1. Order of magnitude increase of $\beta_{x}^{*}$, and subsequent reduction of horizontal $\beta_{\max }$ in the doublet quadrupoles. While early separation pushes the first quadrupole further from the IP, the values of $\beta_{\max }$, both horizontal and vertical, are still reduced, as indicated in Table 3.8.

2. There are far fewer parasitic collisions per interaction region with flat beams than with round beams, greatly reducing the long-range (especially horizontal) tune shift. 


\section{Flat Beam Disadvantages}

1. The design of the first quadrupole and splitting dipole are difficult. A 2-in-1 quadrupole with relatively close separation will not have as good field quality a single bore element. But, with lower $\beta_{\max }$ in the doublet design, the tolerable field errors are likely to be larger.

2. Neutral particles generated at the IP will aim head-on for the conductor located at the center of the first 2-in-1 quadrupole.

3. Lack of energy flexibility may be a disadvantage to flat beams. At lower energies, damping times are longer and thus flat beams are no longer viable. (However, a lattice has been demonstrated in which 4 IR quadrupoles can act as a doublet or as a triplet [15].)

4. An operational disadvantage is the need for careful tuning to keep the vertical emittance small. Electron rings, however, routinely achieve beam flatness in the range $0.001<\kappa<$ 0.01 by controlling dispersion and global betatron coupling. Flatness values of $\kappa \approx 0.01$ should be achievable.

Table 3.8. Flat and round beam performance parameters after about 6 hours into the store, just after peak luminosity, when both horizontal and vertical beam-beam parameters are saturated.

\begin{tabular}{|lcc|}
\hline & FLAT & ROUND \\
Flatness parameter, $\kappa$ & 0.1 & 1 \\
Beam-beam parameter $\xi_{x}=\xi_{y}$ & 0.008 & 0.008 \\
Peak luminosity $L\left(10^{34} \mathrm{~cm}^{-2} \mathrm{sec}^{-1}\right)$ & 2.0 & 2.0 \\
& & \\
Initial bunch intensity $N\left(10^{9}\right)$ & 7.5 & 7.5 \\
Collision beta horz $\beta^{*}(\mathrm{~m})$ & 3.7 & 0.71 \\
Collision beta vert $\beta_{\mathrm{y}}^{*}(\mathrm{~m})$ & 0.37 & 0.71 \\
Maximum beta horz $\beta^{*}{ }_{\text {max }}(\mathrm{km})$ & 7.84 & 14.58 \\
Maximum beta vert $\beta_{\text {max }}(\mathrm{km})$ & 10.75 & 14.58 \\
Horizontal emittance $\varepsilon_{x}(\mu \mathrm{m})$ & 0.161 & 0.082 \\
Vertical emittance $\varepsilon_{y}(\mu \mathrm{m})$ & 0.016 & 0.082 \\
Collision beam size horz $\sigma_{x}^{*}(\mu \mathrm{m})$ & 2.53 & 0.79 \\
Collision beam size vert $\sigma_{y}^{*}(\mu \mathrm{m})$ & 0.25 & 0.79 \\
Maximum beam size horz $\sigma_{x-\max }^{*}(\mu \mathrm{m})$ & 116 & 113 \\
Maximum beam size vert $\sigma_{y-m a x}^{*}(\mu \mathrm{m})$ & 43 & 113 \\
& & \\
Total crossing angle $\alpha(\mu \mathrm{rad})$ & 10.0 & 10.0 \\
Separation distance, $L_{\text {sep }}(\mathrm{m})$ & 30 & 120 \\
Number of long range collisions per IR & 20 & 84 \\
Long range tune shift per IR, horz $\Delta Q_{x}$ & 0.0008 & 0.0166 \\
Long range tune shift per IR, vert $\Delta Q_{y}$ & 0.0081 & 0.0166 \\
\hline
\end{tabular}

A round beam solution for the VLHC is in hand, including IR magnet designs and optical layouts. Because of the difficult design of a 2-in-1 quadrupole magnet for the doublet optics, the flat beam scenario is at this time a promising upgrade path. The flat beam case with its possible advantages merits further work and discussion and will continue to be studied over the coming months. 


\subsubsection{Lattice}

The lattice geometrically matches the low field (LF) ring lattice so that they both fit in the same tunnel. See Table 3.9 for a selection of HF lattice parameters. The arcs are composed of 90 degree FODO cells using separated function magnets, as are the dispersion suppressors.

\subsubsection{Triplet IR Optics Approach}

Using interaction region optics standard in modern hadron colliders, collisions are created using anti-symmetric triplets for the final focusing. The "round beam" model discussed here is qualitatively similar to the low-field IR design. The triplet quadrupoles are $400 \mathrm{~T} / \mathrm{m}$ single-bore magnets. Four additional circuits, comprising double-bore $400 \mathrm{~T} / \mathrm{m}$ magnets are also used for optical matching. With a total of 6 independently-tunable quadrupole circuits available it is possible to match the four $\beta$ s and $\alpha$ 's from the IP into the regular FODO cells, plus hold the phase advance $\Delta \mu$ constant across the IR through the squeeze from $\beta^{*}=12.0 \rightarrow 0.50 \mathrm{~m}$. Fixing $\Delta \mu$ eliminates the need for a special phase trombone somewhere in the ring to maintain the nominal operating point. Figure 3.11 shows the lattice functions through the insertion region at collision.

Table 3.9. Short list of some high field lattice parameters.

\begin{tabular}{|lrl|}
\hline Horizontal tune & 218.19 & \\
Vertical tune & 212.18 & \\
Transition gamma & 194.13 & \\
Maximum arc beta & 459 & $\mathrm{~m}$ \\
Maximum arc dispersion & 1.42 & $\mathrm{~m}$ \\
Vertical $\beta$ (store) & 0.37 & $\mathrm{~m}$ \\
Horizontal $\beta$ (store) & 3.7 & $\mathrm{~m}$ \\
Maximum $\beta$, injection & 0.61 & $\mathrm{~km}$ \\
Maximum Horz $\beta$, store & 7.84 & $\mathrm{~km}$ \\
Maximum Vert $\beta$, store & 10.75 & $\mathrm{~km}$ \\
\hline
\end{tabular}

The circulating beams are separated vertically everywhere in the ring, except in the triplet quadrupoles. Four $10 \mathrm{~T}$ dipoles between the Q3 and Q4 quadrupoles bring the beams together at the entrance to the triplet for collisions at the IP. Dipoles downstream of the IP separate the beams again vertically and channel them back into the upper and lower rings. A half-crossing angle of $28.8 \mu \mathrm{r}$ gives $10 \sigma$ separation between the beams at the first parasitic crossing $2.823 \mathrm{~m}$ downstream of the IP $\left(\varepsilon_{N}=1.5 \pi \mu \mathrm{m}\right.$ at $\left.87.5 \mathrm{TeV} / \mathrm{c}\right)$.

\subsubsection{Doublet IR Optics Approach}

Doublet optics are more naturally suited to the flat beams of the high-field ring. The IR gives $30 \mathrm{~m}$ free space from the interaction point to the first magnetic element. For the doublet optics, the first magnet encountered is a high field small bore magnet of $16 \mathrm{~T}$ field. This can handle the beams until the total separation becomes $8 \mathrm{~mm}$. Then the beams enter a lower field, $12 \mathrm{~T}$, magnet with a larger bore. These beam separation dipoles bend the beam vertically. This crossing region requires that the beam separation at the first quadrupole be $30 \mathrm{~mm}$. With these 
dimensions the first quadrupole is limited to $400 \mathrm{~T} / \mathrm{m}$ gradient. As the beams separate, a higher quadrupole field can be attained with a limit of $600 \mathrm{~T} / \mathrm{m}$.

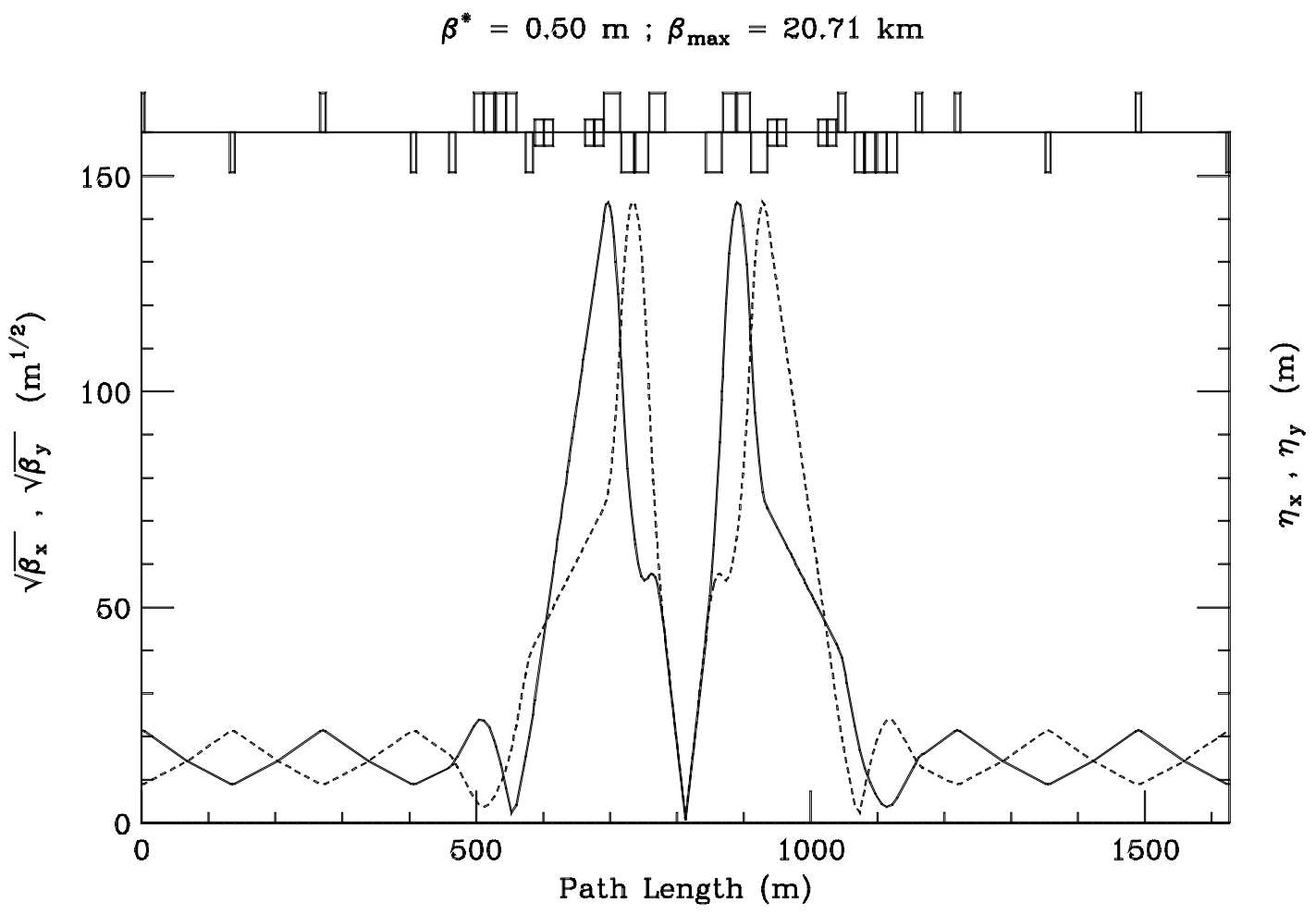

Figure 3.11 Lattice functions near the IP in collision optics, using triplet focusing.

With this design of the crossing region, two interaction regions can fit in the on-site cluster region. The $\beta^{*}$ can be varied from $0.37 \mathrm{~m}$ to $7.12 \mathrm{~m}$ (vertically), $\beta^{*}$ horizontally is 10 times larger). The maximum beta is $10.6 \mathrm{~km}$ with the collision optics and only about $760 \mathrm{~m}$ at injection. Figure 3.12 shows the collision optics of the interaction region.

\subsubsection{Magnet Apertures and Field Quality}

The effect of the magnetic field quality on the dynamic aperture was investigated at the injection energy of $10 \mathrm{TeV}$. At injection the beam size has its maximum and errors in the arc magnets dominate the dynamic aperture. With the current one-turn injection scenario, a storage time on the order of seconds is sufficient. To estimate the effect of the magnetic field errors on the single particle dynamic, the nonlinear chromaticity, the tune spread from nonlinear fields and the 1000-turn dynamic aperture were computed. 1000 turns is equivalent to 0.7 seconds real time in the VLHC. Experiments have shown that the dynamic aperture can be computed for storage rings with nonlinear field errors within a 30\% error when the field errors are well known $[16,17]$. For a future machine, however, a larger safety margin is required. 


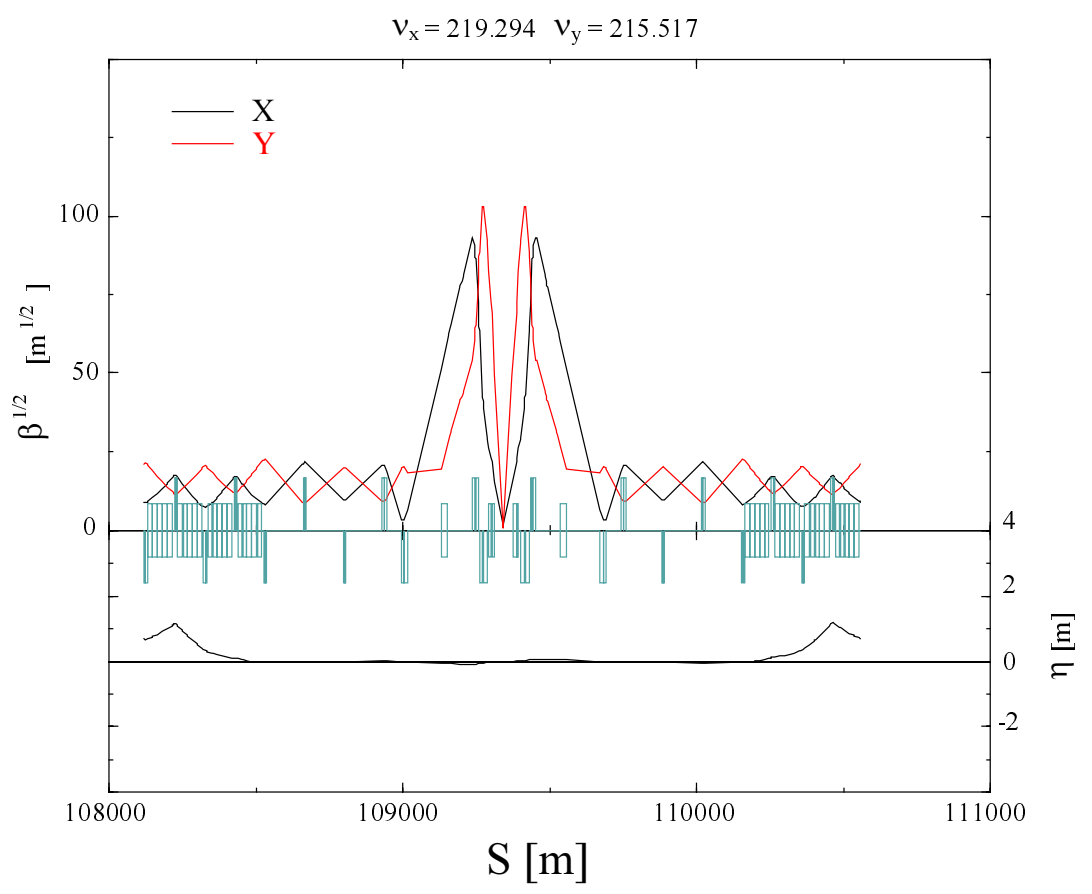

Figure 3.12. Lattice functions near the IP in collision optics, using doublet focusing.

In the lattice that is used for the evaluation, no interaction regions were inserted since the arcs dominate the dynamic aperture at injection. Arc magnet errors at injection were estimated for a 2.0 T field in the dipoles. The harmonic multipole content used in the simulation is given in Table 3.10. The systematic errors were computed as the maximum possible absolute value with given mean and uncertainties. Random errors were created from a Gaussian distribution, cut at two sigma.

Table 3.10. Systematic <> and random $\sigma$ of multipole coefficients used for Stage-2 tracking at injection. The coefficients are shown in units of $10^{-4}$ at a reference radius of $10 \mathrm{~mm}$.

\begin{tabular}{|ccccc|}
\hline Order, $\boldsymbol{n}$ & $<\mathbf{b}_{\mathbf{n}}>$ & $<\mathbf{a}_{\mathbf{n}}>$ & $\sigma\left(\mathbf{b}_{\mathbf{n}}\right)$ & $\sigma\left(\mathbf{a}_{\mathbf{n}}\right)$ \\
1 & 0.000 & 0.000 & 1.20 & 1.20 \\
2 & -2.000 & 0.000 & 0.85 & 0.85 \\
3 & 0.000 & 0.000 & 0.35 & 0.35 \\
4 & 0.500 & 0.000 & 0.12 & 0.12 \\
5 & -0.006 & 0.000 & 0.05 & 0.05 \\
6 & -0.004 & 0.000 & 0.02 & 0.02 \\
\hline
\end{tabular}

Quadrupoles were horizontally and vertically displaced randomly with a Gaussian distribution with a $0.3 \mathrm{~mm}$ rms value, cut at three sigma. In addition, beam position monitors were displaced with a $0.2 \mathrm{~mm}$ rms value. The resulting non-zero closed orbit was corrected to zero in the beam position monitors with horizontal orbit correctors at focusing quadrupoles and vertical orbit correctors at defocusing quadrupoles. Skew quadrupole errors in the arcs were disregarded and no coupling correction was necessary. The transverse tunes were set to (218.190, 212.180). Both horizontal and vertical chromaticities were set to 2. 
No synchrotron motion was included but particles had a relative momentum deviation of $\Delta p / p=7.2 \times 10^{-4}$, corresponding to three times the rms of the momentum distribution. The physical aperture of $10 \mathrm{~mm}$ in the arcs corresponds to $35 \sigma$ of the transverse beam size in the quadrupoles. Figure 3.13 shows dynamic aperture averaged over 10 seeds along with the minimum of the 10 seeds. The error bars of the average dynamic aperture are the rms value of 10 seeds.

Although linear coupling and synchrotron motion were disregarded in the determination of the dynamic aperture, the field quality with the magnet field errors used is sufficient for injection. At collision, we expect the dynamic aperture to be determined by the errors in the IR quadrupoles. Detailed modeling remains to be performed.

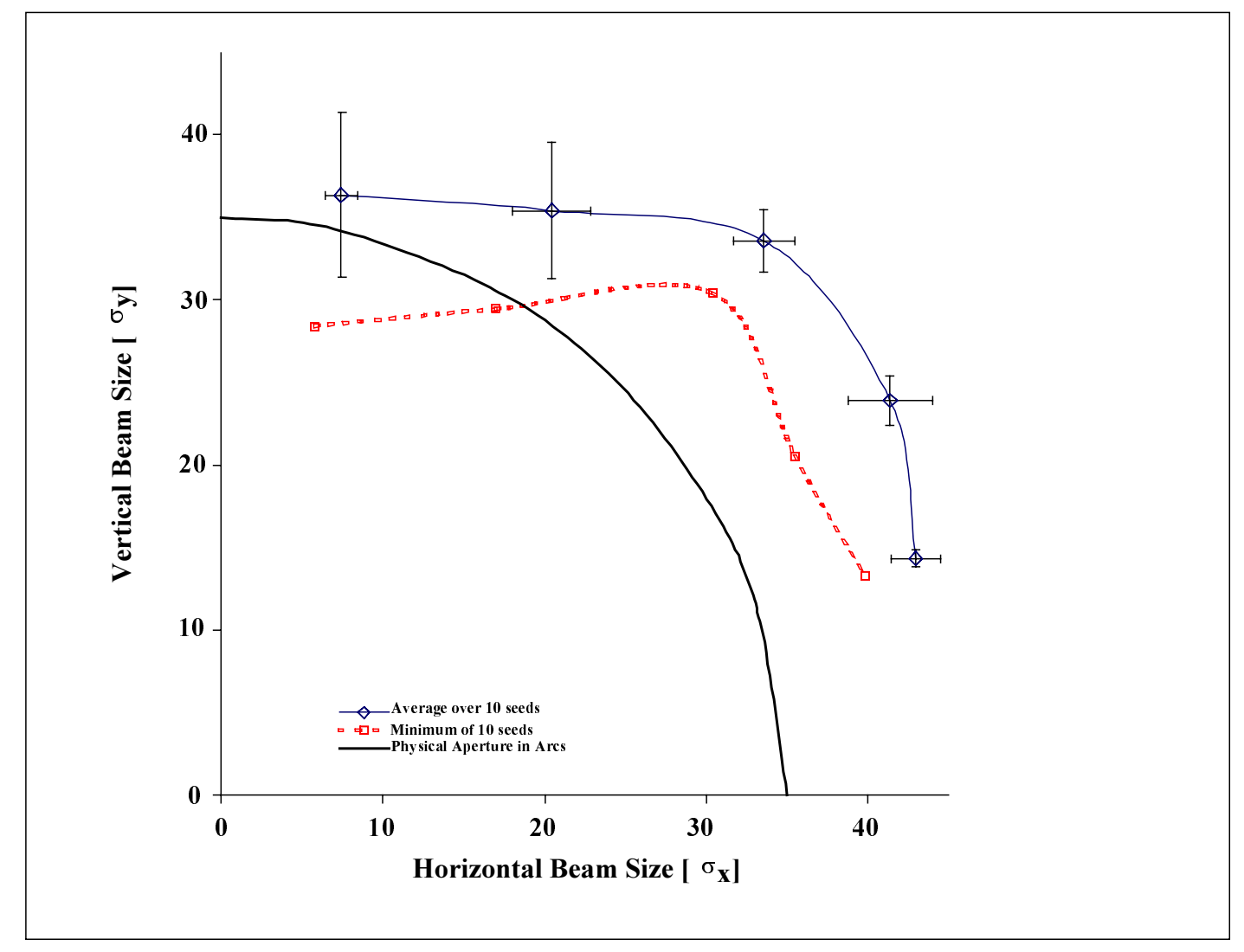

Figure 3.13. 1000-turn dynamic aperture at injection. Shown are the average and minimum over 10 seeds, and the physical aperture, all in units of the transverse rms beam size.

\subsubsection{Tolerances and Corrections}

In this section we consider the effect of magnet misalignments as well as the effect of main dipole and quadrupole field errors on the beam orbit and betatron tunes. We discuss the tolerances for misalignment and field errors listed in Table 3.11. To this purpose we use the highfield storage optics with $\beta_{x}^{*}=3.7 \mathrm{~m}$ and $\beta_{y}^{*}=0.37 \mathrm{~m}$. 
Table 3.11. Tolerance summary for magnet alignment.

\begin{tabular}{|lrll|}
\hline RMS ERROR & ARC & IR & \\
QUADRUPOLES & & & \\
Transverse offset & 0.25 & 0.2 & $\mathrm{~mm}$ \\
BPM to quad offset & 0.15 & 0.1 & $\mathrm{~mm}$ \\
Roll & 0.5 & 0.5 & $\mathrm{mrad}$ \\
Field Error $\Delta K / K$ & 0.5 & 0.5 & $10^{-3}$ \\
DIPOLES & & & \\
$\quad$ Roll & 0.5 & 0.5 & $\mathrm{mrad}$ \\
Field Error $\Delta B / B$ & 0.5 & 0.5 & $10^{-3}$ \\
\hline
\end{tabular}

\subsubsection{Orbit Errors}

Possible sources of orbit error include transverse quadrupole misalignments (in both the horizontal and vertical directions), main dipole field integral errors (for the horizontal orbit), and roll of the main dipole magnets (for the vertical orbit). In order to estimate and compare the value of the orbit distortion coming from these different types of error sources, the rms closed orbit has been calculated. The calculations assumed the rms magnet misalignments listed in Table 3.11. Each of five different individual perturbation sources were considered in turn. In some cases the errors are introduced in all magnets in the accelerator arcs, while in others the errors are applied to the quadrupoles of one of the interaction region doublets. In the interaction region doublets there is one corrector per plane. The biggest effect comes from quadrupole offsets. The dipole integral field error and dipole roll errors quoted in Table 3.11 have little influence on the required corrector strength.

\subsubsection{Tune Error, Coupling and IR Chromatic Effects}

The betatron tune shift caused by an rms quadrupole field error of $10^{-4}$ and the betatron coupling effect produced by rms quadrupole rolls of $1 \mathrm{mrad}$ have been calculated. The tolerance for arc quadrupole error can be put at $5 \times 10^{-4}$ providing a $2.5 \mathrm{rms}$ beta-beating value of about $9 \%$ is acceptable. A small number of quadrupole correctors placed in the arcs will easily compensate for this beta-beating. Field errors in interaction region quadrupoles can be corrected by adjusting the currents of power supply for these quads. After the current adjustment is done the current fluctuations should be kept well below the $10^{-4}$ level. Both global correction in the arcs and local skew quadrupole correction in the interaction regions are required for the compensation of betatron coupling effects. With these correction systems in place it is possible to relax the quad roll tolerances to about $0.5 \mathrm{mrad}$.

Because of the very large maximum $\beta$ values in the interaction region quadrupoles, the chromatic effects produced by these quadrupoles have been evaluated. For the design value of rms momentum spread of $5 \times 10^{-5}$ the amplitude of the chromatic vertical beta-wave was found to be just a few percent. Thus the effect is not large, mainly due to the small rms momentum spread. The vertical chromaticity produced by the quadrupoles from one interaction region has been found to be about -42 . The horizontal chromatic effects are smaller than the vertical. 


\subsubsection{Corrector Strengths}

The maximum dipole corrector strengths displayed in Table 3.12 are 2.5 times the rms value when all error sources are combined in quadrature. Assuming a maximum achievable field of 3.5 $\mathrm{T}$ for the IR dipole correctors, and 2.2 $\mathrm{T}$ for the arc dipole correctors, magnetic lengths of $1.05 \mathrm{~m}$ in the arcs and $2.5 \mathrm{~m}$ in the IRs are required. If the maximum corrector strengths are increased to higher values than those cited in Table 3.12 then the corresponding tolerances in Table 3.11 can be relaxed accordingly.

Even the maximum dipole corrector strengths listed in Table 3.12 cannot make up for the magnet alignment diffusion caused by ground motion. Ground motion might put magnet offsets well beyond the defined tolerances after a few years of movement. Thus, periodic magnet realignment will be necessary. Some means to simplify the realignment procedure, such as using stepping motors, should be evaluated.

Table 3.12. Corrector magnetic lengths and strengths. The maximum strengths are 2.5 times the rms value required when all contributions are added in quadrature.

\begin{tabular}{|lrl|}
\hline \multicolumn{1}{|c|}{ ARC CORRECTORS } & & \\
Dipole magnetic length & 1.05 & $\mathrm{~m}$ \\
Dipole max strength & 2.3 & $\mathrm{~T}-\mathrm{m}$ \\
Skew quad length & 0.95 & $\mathrm{~m}$ \\
Skew quad max strength & 95 & $\mathrm{~T}$ \\
Sextupole magnetic length & 0.95 & $\mathrm{~m}$ \\
Sextupole max strength, $L\left(d^{2} B / d x^{2}\right)$ & 7400 & $\mathrm{~T} / \mathrm{m}$ \\
$\quad$ IR CORRECTORS & & \\
Magnetic length & 2.5 & $\mathrm{~m}$ \\
Dipole max strength & 8.6 & $\mathrm{~T}-\mathrm{m}$ \\
Skew quad max strength & 95 & $\mathrm{~T}$ \\
\hline
\end{tabular}

\subsubsection{Collective Effects}

The reduced bunch population and higher energy of the Stage-2 collider greatly diminish the instability issues found in the Stage- 1 collider. While uncertainties of the transverse impedance are larger for the high field ring, worst case estimates lead to resistive wall e-folding times of order 3 turns, significantly slower than in the low field case. Any damper technology required should easily follow from that developed for the low field case. Future studies may indicate that increasing the thickness of the copper layer is beneficial for reducing any resistive wall effects. The effect of dipole magnetic fields on the resistivity of the various materials used in the high field design needs to be well understood.

\section{References}

[1] David Neuffer, Particle Accelerators 23 (1988), 21.

[2] F. Ostiguy, personal communication, March 19, 2001.

[3] G. Ambrosio, personal communication, March 22, 2001. 
[4] T. Sen et al, "Chromaticity Correction for the SSC Collider Rings," Proc. 1993 IEEE Part. Accel. Conf, 143 (1993).

[5] T. Sen, "Alignment tolerances of IR quadrupoles in the LHC," FNAL-Conf-99-304 (October 1999).

[6] Y. Nosochkov and D. Ritson, "Provision of IP crossing angles for the SSC," SSCL-Preprint-367 (1993).

[7] V. Shiltsev, Proc. ICFA Workshop on Ground Motion, Nov. 2000, SLAC; http://www-project.slac.stanford.edu/ls/wkshp/GM2000.

[8] B. Baklakov et al., PRST Accel. Beams, 031001, 1998.

[9] V. Shiltsev, FNAL TM-1987, 1996.

[10] A. Burov et al., "Beam Stability Issues in a Very Large Hadron Collider," NIMA 450, p. 194, 2000.

[11] Workshop information and presentations can be found at http://www.slac.stanford.edu/ achao/VLHCworkshop.html.

[12] See for example, K.Y. Ng, "Impedances and Collective instabilities of the Tevatron at Run II," Fermilab Report TM-2055, 1998.

[13] K.Y. Ng, "Bunch Coalescence in Low-Field VLHC Rings," report in progress (April 2001).

[14] E. Keil, R. Talman, "Scaling of Luminosity Data Between e $\mathrm{e}^{+}-$Storage Rings," Particle Accelerators, Vol. 14 pp. 109-118, 1983.

[15] S. Peggs, M. Harrison, F. Pilat, M. Syphers, "Flat beams in a $50 \mathrm{TeV}$ hadron collider," Vancouver PAC 97, 1997.

[16] W. Fischer, An Experimental Study on the Long-term Stability of Particle Motion in Hadron Storage Rings, Ph.D. thesis, Hamburg University, DESY 95-235 (1995) and CERN SL/96-10 (AP), 1996.

[17] W. Fischer, M. Giovannozzi and F. Schmidt, "Dynamic Aperture Experiment at a Synchrotron," Phys. Rev. E, Vol. 55, Number 3, p. 3507, 1997. 


\section{Chapter 4. The Fermilab Complex as Injector}

\subsection{The Fermilab Complex and Beam Properties}

The present Fermilab accelerator complex consists of a $750 \mathrm{keV}$ ion source, a $400 \mathrm{MeV}$ linac, the $8 \mathrm{GeV}$ Booster, the $150 \mathrm{GeV}$ Main Injector, and the $1 \mathrm{TeV}$ Tevatron. The first three machines operate at $15 \mathrm{~Hz}$; the Main Injector acceleration cycle to $150 \mathrm{GeV}$ is approximately 2.4 seconds, and the superconducting Tevatron acceleration cycle is approximately 40 seconds. The Tevatron has a circumference of $6283 \mathrm{~m}$, and has six long straight sections for injection and extraction. The F-0 straight section is used for the Tevatron RF and for injection from the Main Injector (for clockwise protons in the Tevatron). Figure 4.1 shows a schematic of the various machines.

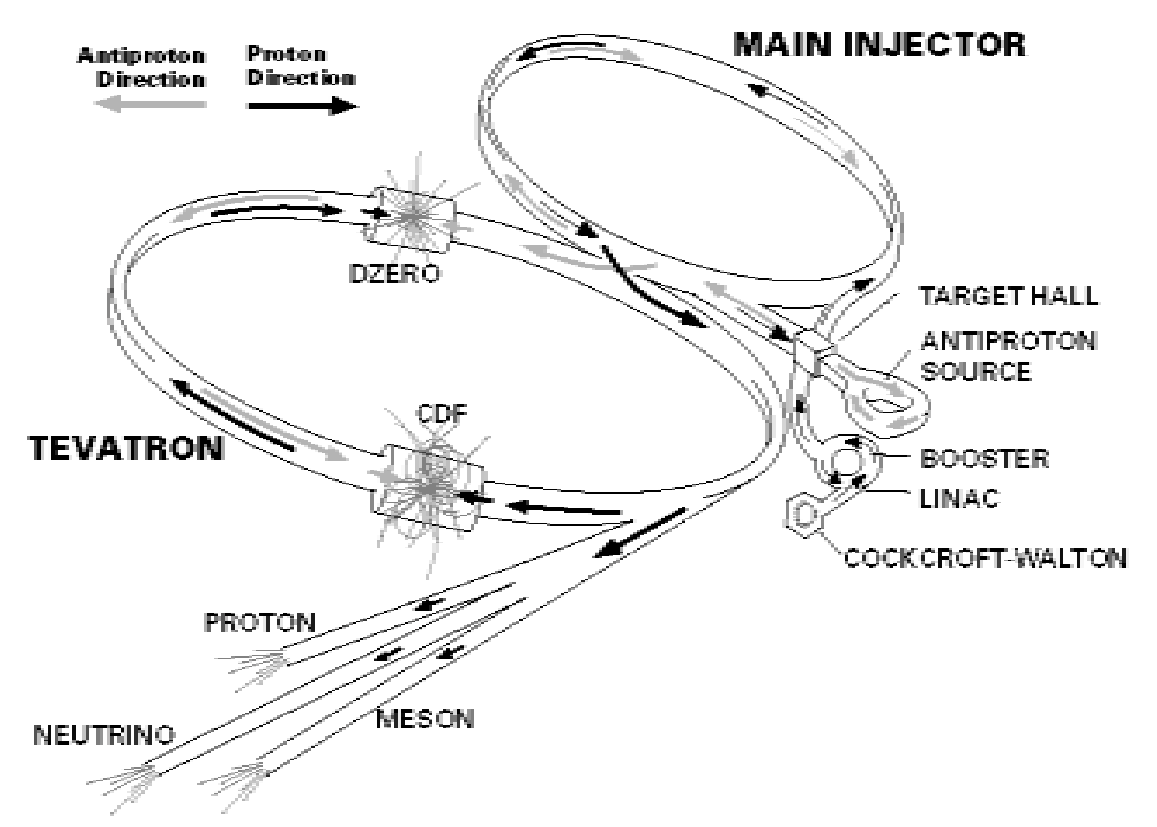

Figure 4.1. Schematic of the existing Fermilab accelerator complex..

The Fermilab accelerators produce beam of the desired emittances for use in the VLHC. In particular, the desired intensity per bunch is at the low end of what is demanded of the Booster by present operations, as shown in Figures 4.2 and 4.3. The questions related to preservation of these emittances through the Main Injector and the Tevatron will be addressed during the coming Collider run. Previous experience with the Main Ring as the injector to the Tevatron yielded transverse emittance dilutions of about $50-100 \%$, but that also involved bunch coalescing to produce bunches of greater than $2 \times 10^{11}$ protons. Careful attention and some possible upgrades will be required, but the emittances assumed in the Design Study represent reasonable projections from the current performance of the Fermilab injector. 


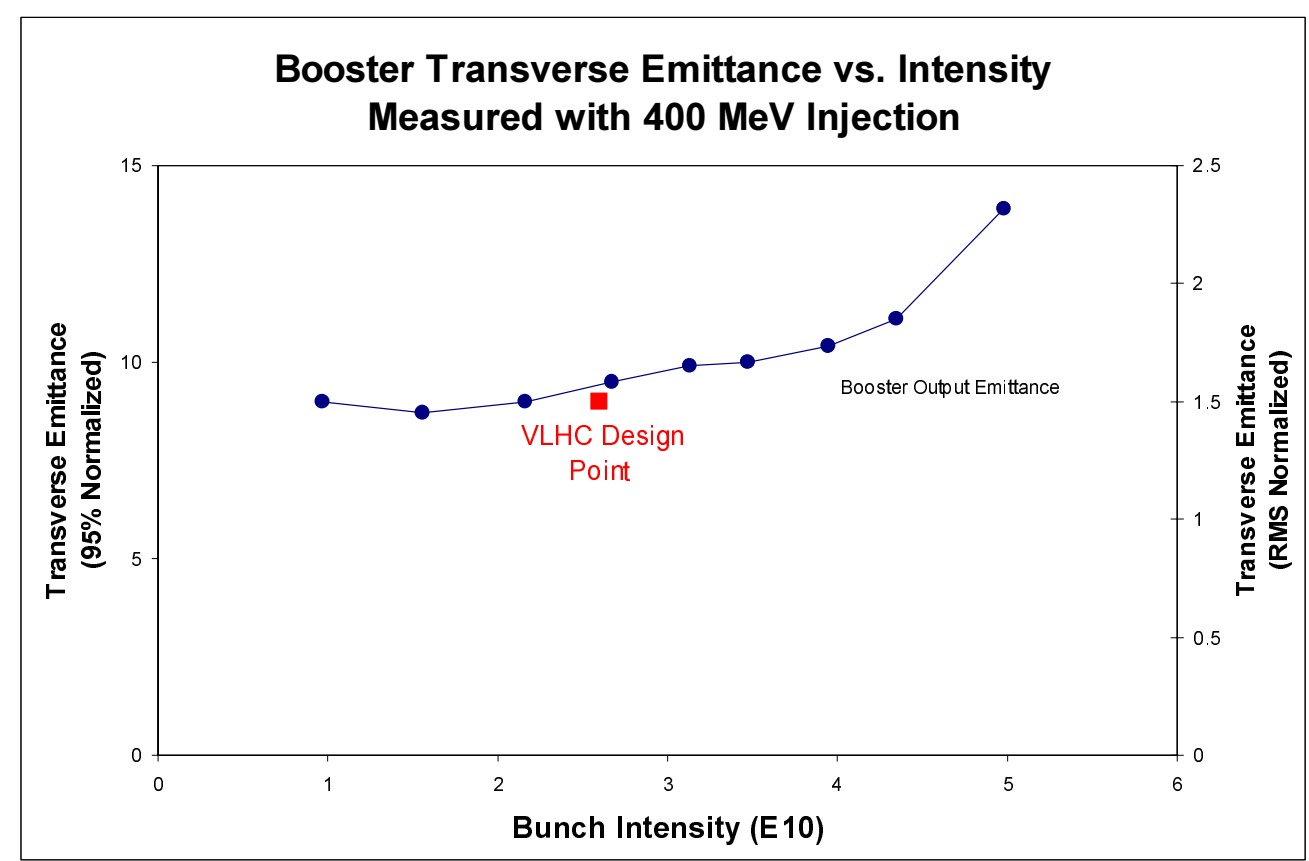

Figure 4.2. Measured transverse beam emittance (95\%, normalized) delivered from the $8 \mathrm{GeV}$ Booster as a function of beam intensity.

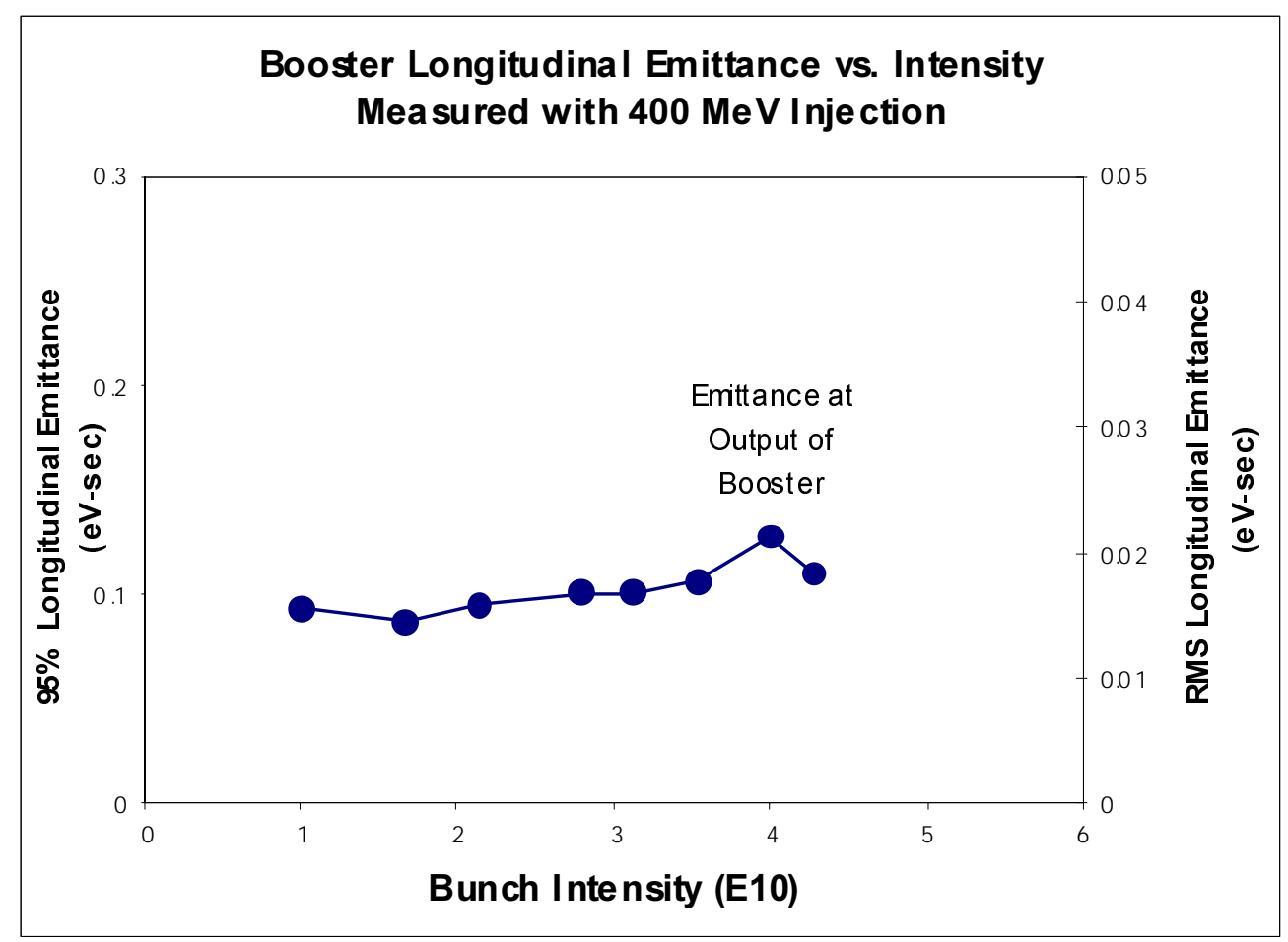

Figure 4.3. Measured longitudinal beam emittance delivered from the $8 \mathrm{GeV}$ Booster as a function of beam intensity. The longitudinal emittance at collision of $0.4 \mathrm{eV}$-sec rms assumed in the VLHC Design Study allows for significant emittance dilution during acceleration and possible coalescing operations. 


\subsection{Operational Scenarios}

The Booster, Main Injector and Tevatron all operate with 53-MHz RF systems, providing bunch spacings of $18.83 \mathrm{~ns}$; the harmonic numbers are 84, 588 and 1113 . These correspond to circumference ratios of 1:7:13.25. Due to the extraction kickers in the Booster, one to two buckets are lost at transfer time. Further, the requirement for kicker rise times in the Main Injector (for extraction and abort) implies that only about $6 / 7$ of the circumference is filled, and within the section that is mainly filled, there are the small (two to three bucket) gaps resulting from the rise time for the injection kickers. Since the Main Injector is only about one-half the size of the Tevatron, two Main Injector cycles are needed to load the Tevatron. Here again, the rise time of the Tevatron injection kickers requires a gap between the two beam-fills.

The scenario used during the 1999 fixed target run involved one Main Injector cycle with five Booster batches, followed by a second cycle with six Booster batches. Assuming 82 bunches per batch, each Tevatron cycle will accelerate $82 \times 11$ bunches $=902$ bunches. Interspersed between strings of filled buckets, there are nine gaps of $\sim$ two empty buckets each, and one large gap of about 50 buckets. Thus, the bunches span approximately 970 buckets, or 5.476 $\mathrm{km}$. To fill both rings of a 233-km circumference machine requires 84 Tevatron acceleration cycles, or one hour. The actual number may be slightly smaller due to the injection kicker rise times in the VLHC, or one may choose to change the filling structure of the Tevatron from that described above, e.g. inject five batches each time, for a total of ten batches, rather than eleven, so that a more uniform bunch pattern is created.

To use the Tevatron as the injector for a much larger machine, the geometric relationships between the two rings will determine the transfer line orientations. Several options were considered, as discussed below. In two of these options, the Main Injector and Tevatron continue to operate as they do now, with new extraction area(s) needing to be installed. At the present time, both the Main Injector and the Tevatron only accelerate protons in one direction, clockwise in the Tevatron and counterclockwise in the Main Injector. In the third option, the Tevatron is converted to bipolar operation, where protons are accelerated clockwise on some cycles, and counterclockwise on others, by reversing the magnetic fields of the magnets. To convert either machine to bipolar operation is relatively straightforward. Reversing switches need to be installed in each power supply circuit. In the Tevatron, a second set of quenchbypass SCRs is required to conduct the current around the quenching magnets.

The main issues with bipolar vs. unipolar operation have to do with injection and extraction. For a bipolar Main Injector, a new transfer line needs to be built to inject clockwise protons. The civil construction for such a line may be minimal, but the details of injection into the Main Injector need to be developed. Transfer to the Tevatron would be accomplished through the existing A-1 beamline, presently used for antiproton injection into the Tevatron. A new abort for clockwise Main Injector protons would need to be constructed. This could be an internal abort, i.e. one which does not require civil construction, if there is no other physics program associated with the clockwise protons.

For a unipolar Main Injector but a bipolar Tevatron, a new transfer line is required. The most likely route is from MI-40 to the Tevatron E-0 straight section. This distance is fairly straight, but will require an enclosure approximately $1300 \mathrm{~m}$ long, together with focussing magnets, utilities, etc. The MI-40 region also contains the Main Injector abort dump; a channel 
has been provided in the dump to allow a future beam extraction though this area. Therefore, no demolition of the MI enclosure would be required during the civil construction of the beamline.

For bipolar operation of the Tevatron, a new abort is required for the counterclockwise protons. This may be an internal abort as presently used in collider operation.

\subsection{Tevatron Extraction Lines}

The placement of the extraction lines from the Tevatron to the VLHC will be driven by the requirements of the new machine. The VLHC orientation and elevation are determined by the geology of northern Illinois. This in turn determines the geometric relationship between the VLHC and the Tevatron, with the remaining degrees of freedom being the choice of the extraction straight section(s), the amount of bending in the transfer lines, and shifting the VLHC ring by distances on the order of one to two times the Tevatron radius.

The distance between the injection regions in the VLHC is $10,839 \mathrm{~m}$ to the outside ends. With the length of the utility straight section of $1000 \mathrm{~m}$, then the length of each transfer line is approximately $4420 \mathrm{~m}$. At a depth of 100 to $150 \mathrm{~m}$ below the Tevatron, the line has an average slope of 23 to $34 \mathrm{mrad}$, or 2.3 to 3.4 percent slope. This is below the upper limit of 4 percent for using wheeled-vehicles for magnet installation.

The transfer line will necessarily change from cut-and-fill construction in the glacial till above the bedrock (elevation approx. 690 feet) to tunneling in the dolomite. Passing through this interface will present significant construction challenges with respect to water inflows over an excavation that is several hundred feet long. Based on the experience of the NuMI construction presently underway, the construction of the beamline enclosure will have to deal with hundreds of gallons per minute of water at a minimum. If blasting is used to begin the excavation into the bedrock, this could easily become thousands of gallons per minute.

In the following sections, some of the various options for beam transfers between the Tevatron and the VLHC are discussed in more detail. At any point in which a beamline connects to the Tevatron, it should be assumed that the building over that straight section must be demolished and rebuilt, and that a new building will be required for access to the beamlines. In this regard, F-0, which is used for Tevatron injection from the Main Injector, should not be used; to use A- 0 would be very expensive due to the need to demolish and reconstruct much of Transfer Hall and the Transfer Gallery. The presence of a large number of utilities in the vicinity of A-0 is a further complication. However, an extraction from A-0 only involving clockwise protons, in which the beam is first extracted in the direction of Switchyard, so that the actual civil construction does not disrupt the A-0 area, may be possible.

\subsubsection{Option A: Unipolar Tevatron, Single Extraction Region}

In this option the clockwise beam in the Tevatron is extracted at one straight section, and is then split into two lines, with a net bending of nearly $180^{\circ}$. See Figure 4.4 .

This option has the following advantages. It involves only one connection to the Tevatron, and no technical changes to the Main Injector or Tevatron are required. It could be configured to have only one section of the line penetrating the till/bedrock interface. The disadvantage is a 
potentially longer tunnel overall, and the amount of bending required (this option requires approximately half a Tevatron's worth of bend magnets). This scheme could be used at A-0 (as shown below), B-0, C-0, D-0 or E-0.

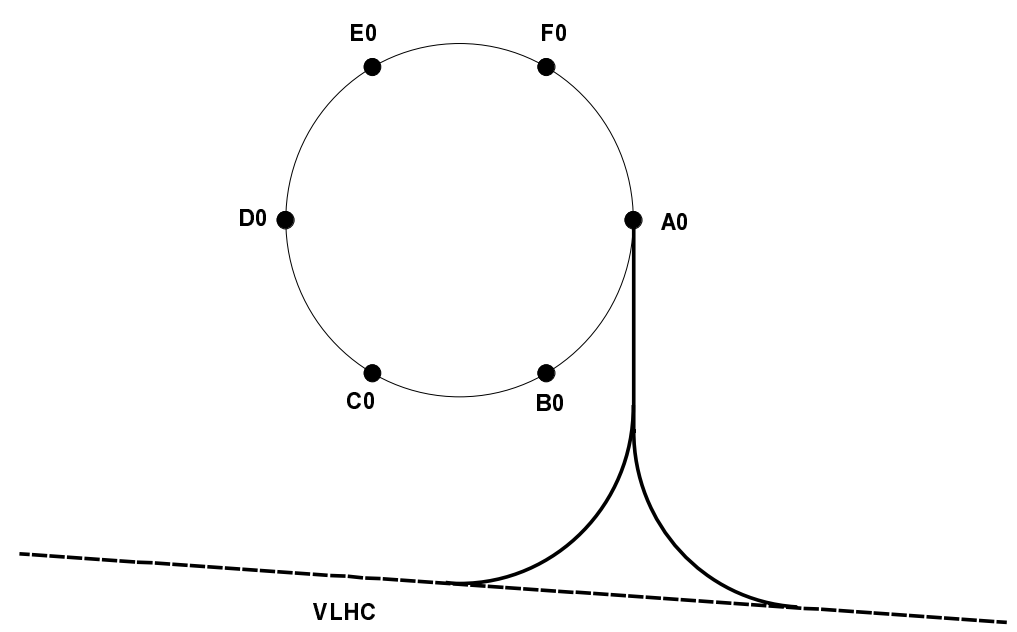

Figure 4.4. Unipolar Tevatron, single extraction line scheme.

\subsubsection{Option B: Unipolar Tevatron, Two Transfer Lines}

In this option, the clockwise beam in the Tevatron is extracted from two opposite straight sections, with a net bending of $20-30^{\circ}$ in each of the two beamlines. This is shown schematically in Figure 4.5.

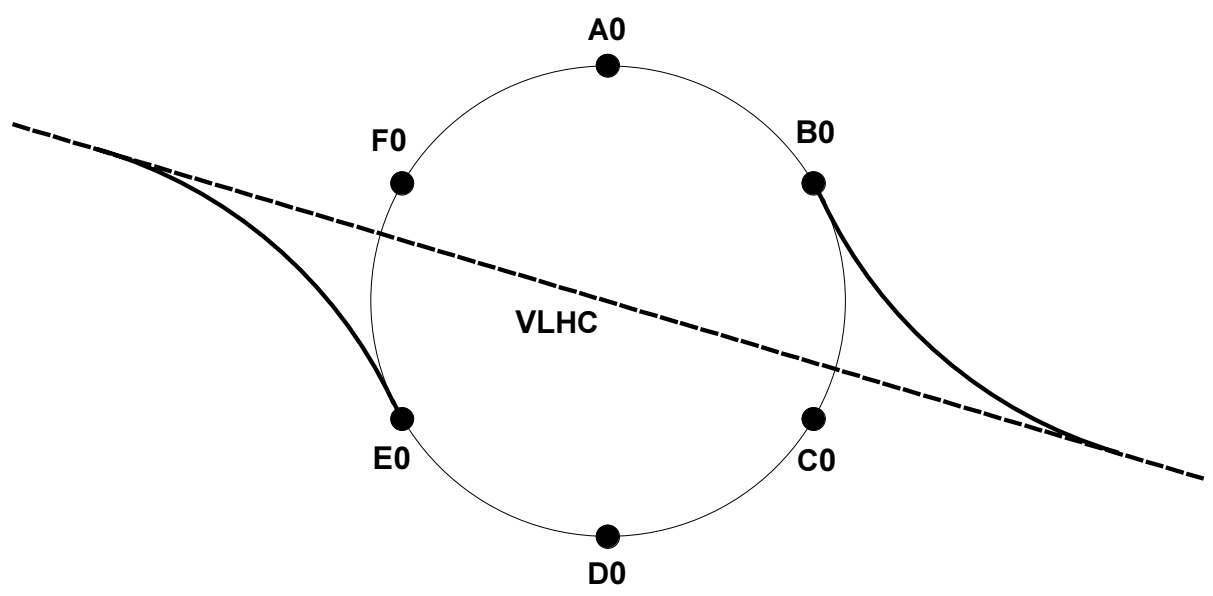

Figure 4.5. Unipolar Tevatron, two-extraction line scheme.

The advantage of this scheme is that no technical changes to the Main Injector or Tevatron are required. The disadvantages are that two separate beamlines must be constructed, each with a tie-in to the Tevatron, and a till/bedrock interface. Given the problems noted above regarding $\mathrm{F}-0$ and $\mathrm{A}-0$, this scheme best works for the combination of B- 0 plus E- 0 , although A- 0 plus D0 is also possible. With additional bending, B- 0 plus D- 0 , or C- 0 plus E- 0 could also be made to work. 


\subsubsection{Option C (Preferred): Bipolar Tevatron}

In this option, both clockwise and counterclockwise beams in the Tevatron are extracted from the same straight section, with almost no bending required in the beamlines, although the exact geometry of the VLHC vs. the Tevatron may require some bending. This is shown schematically in Figure 4.6.

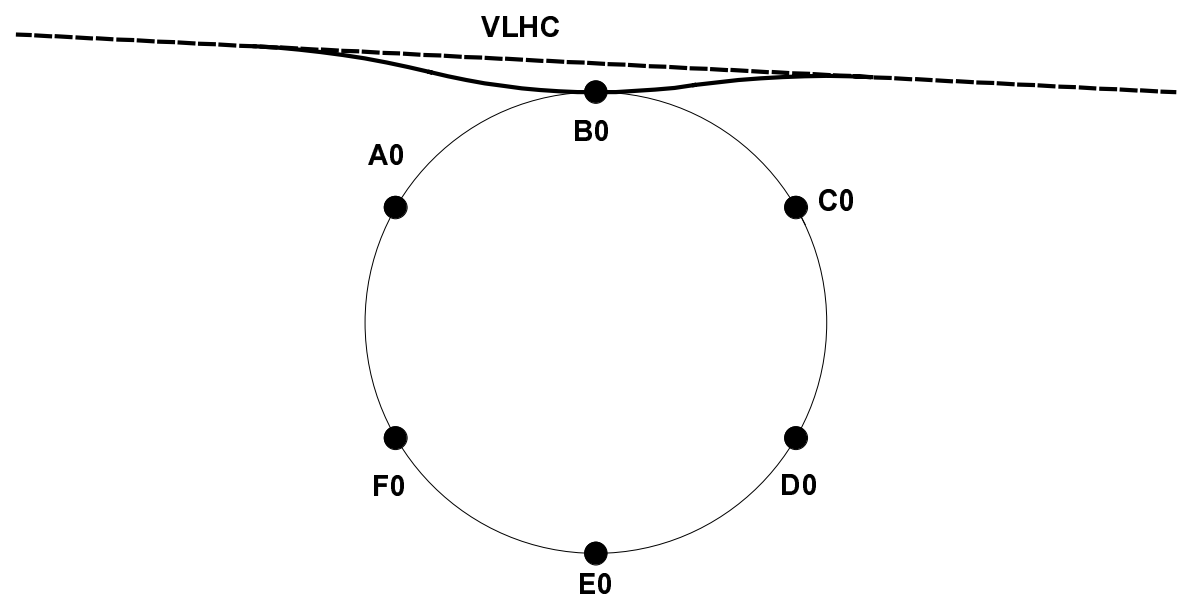

Figure 4.6. Bipolar Tevatron scheme. This option was chosen for the design study.

The advantages of the scheme are that a single tie-in to the Tevatron is required, and little bending is required in the beamlines. The disadvantages are that the Tevatron must be converted to bipolar operation, and the Main Injector either needs a new extraction line or must also be converted to bipolar operation with a new injection line; two till/bedrock interfaces are required for the Tevatron extraction lines. This scheme could be used at B- $0, \mathrm{C}-0, \mathrm{D}-0$ or E- 0 .

\subsubsection{Extraction From the Tevatron}

This section describes the requirements for Option A of the unipolar Tevatron scheme discussed above. For Option B, these requirements must be duplicated at two different areas. For Option C, the Lambertson magnets may be common, but all other aspects are duplicated. The discussion will be presented as occurring at the E-0 straight section, although it should be understood this can happen at B- 0, C- 0 or D- 0 as well. In the following, it is assumed that the extraction is performed with horizontal kickers and Lambertson magnets which provide a mainly vertical deflection. If the reverse is required, a slightly larger total deflection is required to clear the Tevatron quadrupole at the end of the straight section.

Extraction of the clockwise protons is initiated by firing kicker magnets located at D-48. The kicker magnet and power supply system should be similar to the system presently installed at B-48 for the Tevatron abort. This is discussed in more detail below. The kickers deflect the beam across the septum of a series of Lambertson magnets in the E-0 straight section. Following the Lambertson magnets, one or more $\mathrm{C}$-magnets provide additional downward bend. The various styles of existing Lambertson and C-magnets are detailed in the next section. As soon as sufficient vertical separation is achieved, it is extremely beneficial to add horizontal bending magnets to bend the extracted beam away from the Tevatron. The horizontal separation pro- 
vided by this additional bending minimizes the amount of Tevatron enclosure which must be demolished and reconstructed during the civil construction.

The layout of the E-0 straight section is shown in Figure 4.7. In this figure, the magnets that are shaded black are the Tevatron quads; the magnets shaded gray are the ones required for Option A or B; the kickers at D-48 are not shown.

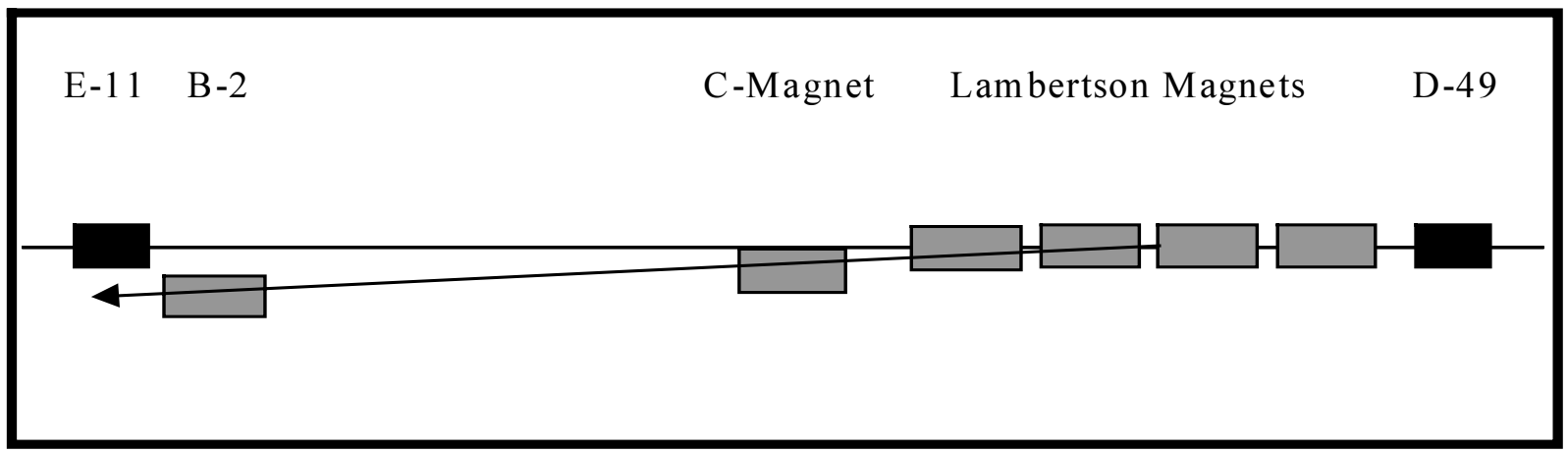

Figure 4.7. Tevatron extraction configuration for Options A or B viewed from radial outside.

Analysis of the required bending strength for the Lambertson magnets has been done. This is discussed further in Section 4.4. The two requirements are simply that the beam must be deflected enough to clear the quadrupole at the end of the straight section, but not so much as to run out of aperture within the Lambertson magnets themselves. The placement of the C-magnet has a similar constraint, in that it cannot be too close to the Tevatron beam pipe; something on the order of $75 \mathrm{~mm}$ of separation is needed between the circulating and the extracted beams.

For Option C, only the Main Injector Lambertsons have the required aperture in the bend plane to accommodate extraction in both directions. Since the 2-inch gap is not required, a new magnet with a 1-inch or smaller gap, but based on the MI design would probably be built. The magnet design must also be changed from the present symmetric design (with 7-in. above and below the field-free region) to an asymmetric design, with approximately 10-in. below. The extraction straight section for Option $\mathrm{C}$ is shown in Figure 4.8.

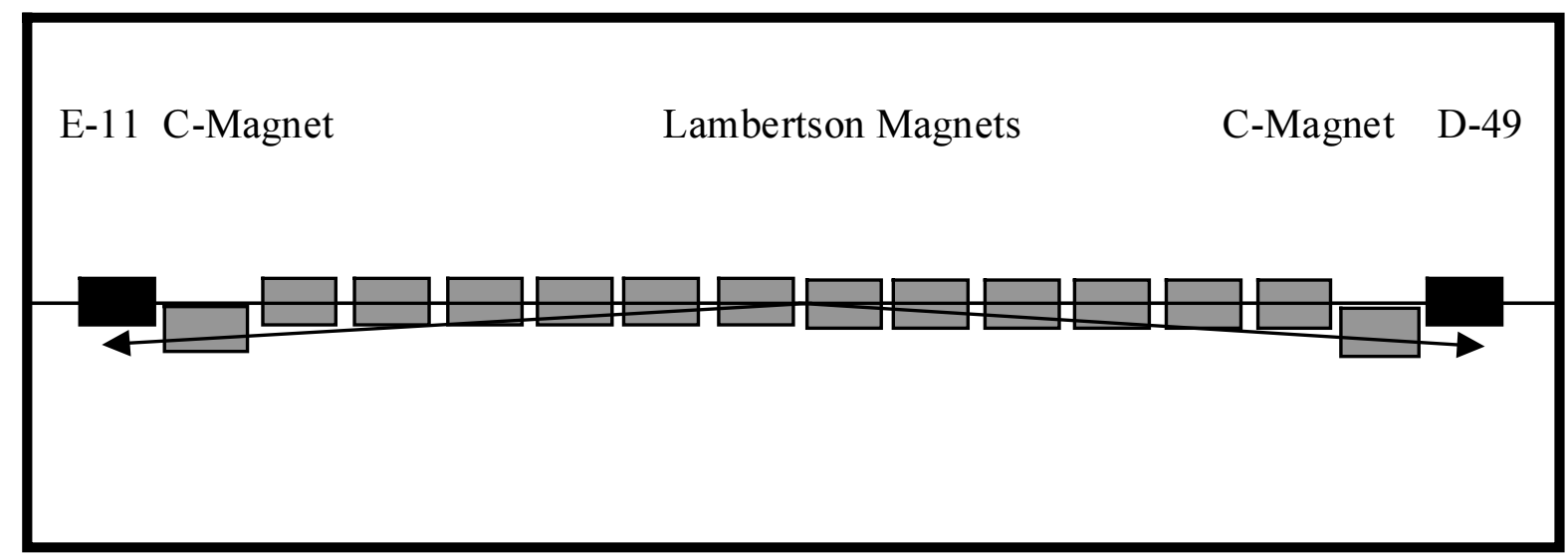

Figure 4.8. Tevatron extraction configuration for Option C. 


\subsubsection{Installation and Beamline Construction Issues Adjacent to the Tevatron}

As mentioned above, providing some small amount of horizontal bending early in the transfer line is useful. This avoids the situation of having one beamline sloping down close to another beamline that is flat, and the resulting civil construction and installation difficulties that arise as a result.

Another issue is radiation from the Tevatron if it is desired to have access to the beamlines while the Tevatron is operating. While 20 feet of shielding is adequate above the Tevatron, muon production produces a forward plume in the plane of the Tevatron. Analysis done as part of the Main Injector project indicated that having 60 feet of radial shielding is adequate.

To facilitate the installation of long magnets, the minimum radius of curvature of any portion of the beamline between the access point and the VLHC ring has been set at $1000 \mathrm{~m}$. This requirement does not pertain to the beamline enclosure in the immediate vicinity of the Tevatron in which shorter magnets will be placed. Thus, the outward horizontal bend mentioned above may wish to be cancelled by another bend to form a tight S-curve.

For magnet installation into the beamline or into the VLHC itself, an access ramp needs to be provided. Here it is assumed that this ramp begins at or near grade level and the installation ramp merges with the beamline enclosure above the dolomite. The portion of the beamline between the Tevatron and the installation ramp would need to be filled with shielding material if the Tevatron is operational during the installation phase. The portion between the surface and the beamline would need to be filled with some amount of shielding once the beamline becomes operational. For ease of reconfiguration, this material may be placed on rollers.

\subsection{Transfer Line Magnets and Kickers}

In Table 4.1, a combination of existing Fermilab magnet types is shown which meets the requirements for Options A or B; a similar table for Option C exists. Even with twelve MI-style Lambertsons, plus one $\mathrm{C}$-magnet, the beam just clears the quad at the end of the straight section. However, the MI Lambertsons could also be pushed slightly higher in strength.

Table 4.1. Possible set of Lambertson and C-magnets for Tevatron extraction for Option A or B.

\begin{tabular}{|l|c|c|c|c|c|c|}
\hline Magnet Type & Begin & $\underline{\text { Exit }}$ & $\underline{\underline{\text { Center }}}$ & $\begin{array}{c}\text { Angle from } \\
\text { Magnet }\end{array}$ & $\begin{array}{c}\text { Angle } \\
\text { at Exit }\end{array}$ & $\begin{array}{c}\text { Deflection } \\
\text { at Exit }\end{array}$ \\
\hline Sym. TeV Extraction & 0 & 5.68 & 2.8 & 0.00182 & 0.00182 & 0.005 \\
\hline Sym. TeV Extraction & 5.68 & 11.4 & 8.5 & 0.00182 & 0.00365 & 0.021 \\
\hline Sym. TeV Extraction & 11.4 & 17.0 & 14.2 & 0.00182 & 0.00547 & 0.047 \\
\hline Assym. TeV Extraction & 17.0 & 22.7 & 19.9 & 0.00246 & 0.00793 & 0.085 \\
\hline TeV Abort C-magnet & 22.7 & 26.6 & 24.6 & 0.00142 & 0.00935 & 0.118 \\
\hline End of Staight Section & & 51.3 & & & & 0.350 \\
\hline
\end{tabular}

The kicker magnets for Tevatron extraction should be fairly straightforward. The strength and rise time requirements should be the same as the existing B-48 abort kickers. The flatness over the extraction time of $20 \mu \mathrm{sec}$ may need to be improved, however. Also, the existing kickers are vertical, whereas horizontal kickers are required in the scenarios discussed above. 


\section{Chapter 5. Stage-1 Components}

\subsection{Magnets}

\subsubsection{Introduction}

The magnet system of the $20 \mathrm{TeV}$ Stage-1 VLHC is the main component of the collider, and has a strong influence on the total cost of machine. The general design goal is to reduce the complexity and cost per Tesla-meter of the collider. The arcs of the Stage-1 VLHC are based on a 2-in-1 warm-iron, combined function gradient dipole magnet $[1,2,3]$, excited by a single turn $100 \mathrm{kA}$ superconducting transmission line. A cross-section of the magnet, including the cryogenic service line and return bus, is shown in Figure 5.1. The flux lines surrounding the transmission line are shaped by an iron yoke, and two gaps in the yoke provide the apertures for the two beams. The $20 \mathrm{TeV}$ bending field is $1.966 \mathrm{~T}$ at the nominal current of $87.5 \mathrm{kA}$. The magnets have a field gradient of $\pm 4.73 \% / \mathrm{cm}$ which provides focusing and eliminates the need for separate quadrupoles. The $135.5-\mathrm{m}$ long half-cell is made of two $65.75-\mathrm{m}$ long gradient magnets.

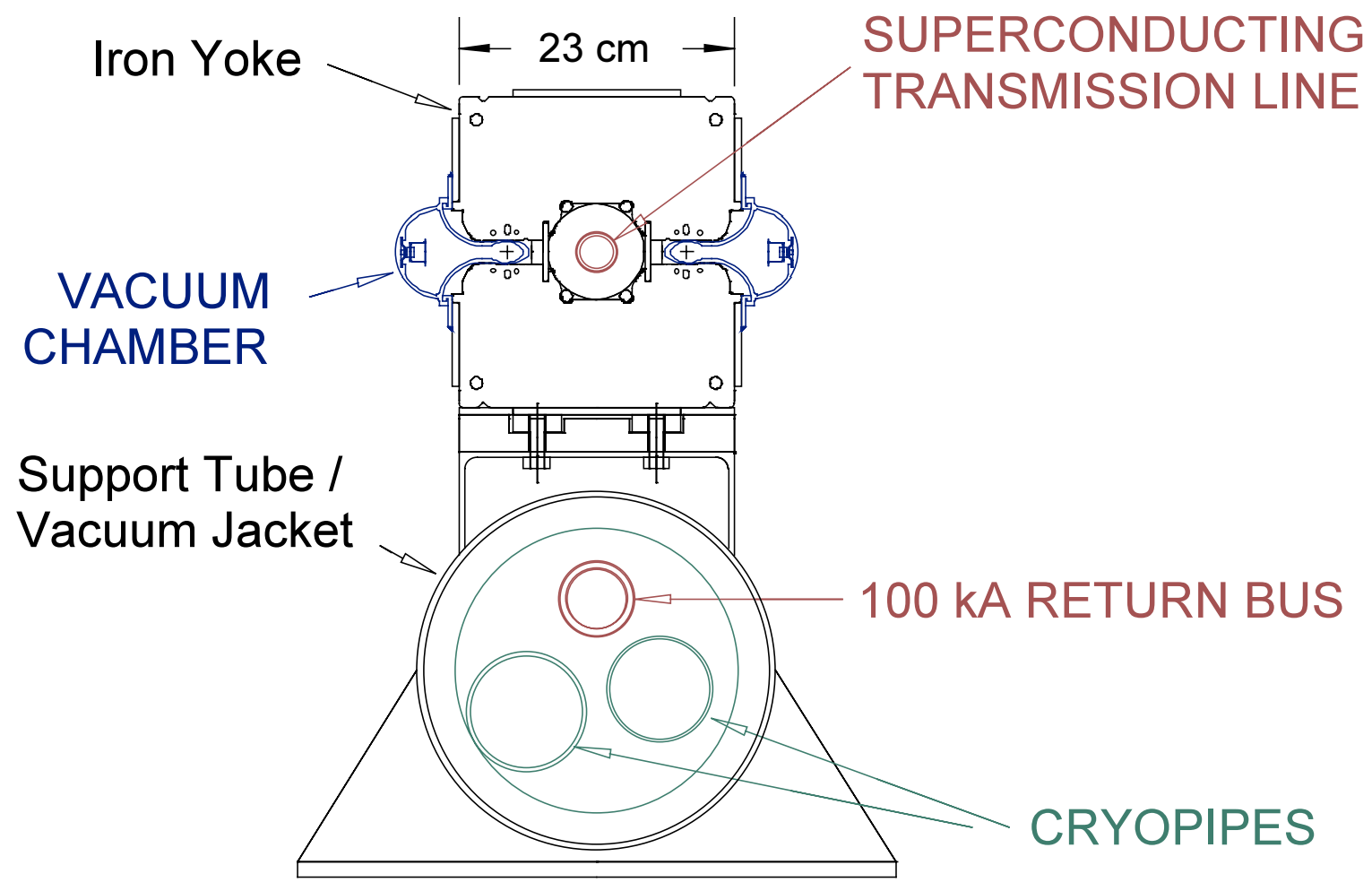

Figure 5.1. Cross-section of the gradient dipole magnet used in the arcs and dispersion suppressor of the Stage-1 VLHC. 
At the end of each half-cell, where the beta function is at a maximum in one plane and a minimum in the other, a $3.5-\mathrm{m}$ long space is left open for correction magnets. The transmission line is diverted into the cryogenic service pipe, near the return bus, allowing the use of conventional, room temperature, air cooled iron corrector magnets, as shown in Figure 5.2. Three correctors are installed at each location: a vertically or horizontally steering dipole, a quadrupole and a sextupole. The strength of these correctors is sufficient to accommodate alignment errors, ground motion, and iron saturation effects at full field (Section 3.2).

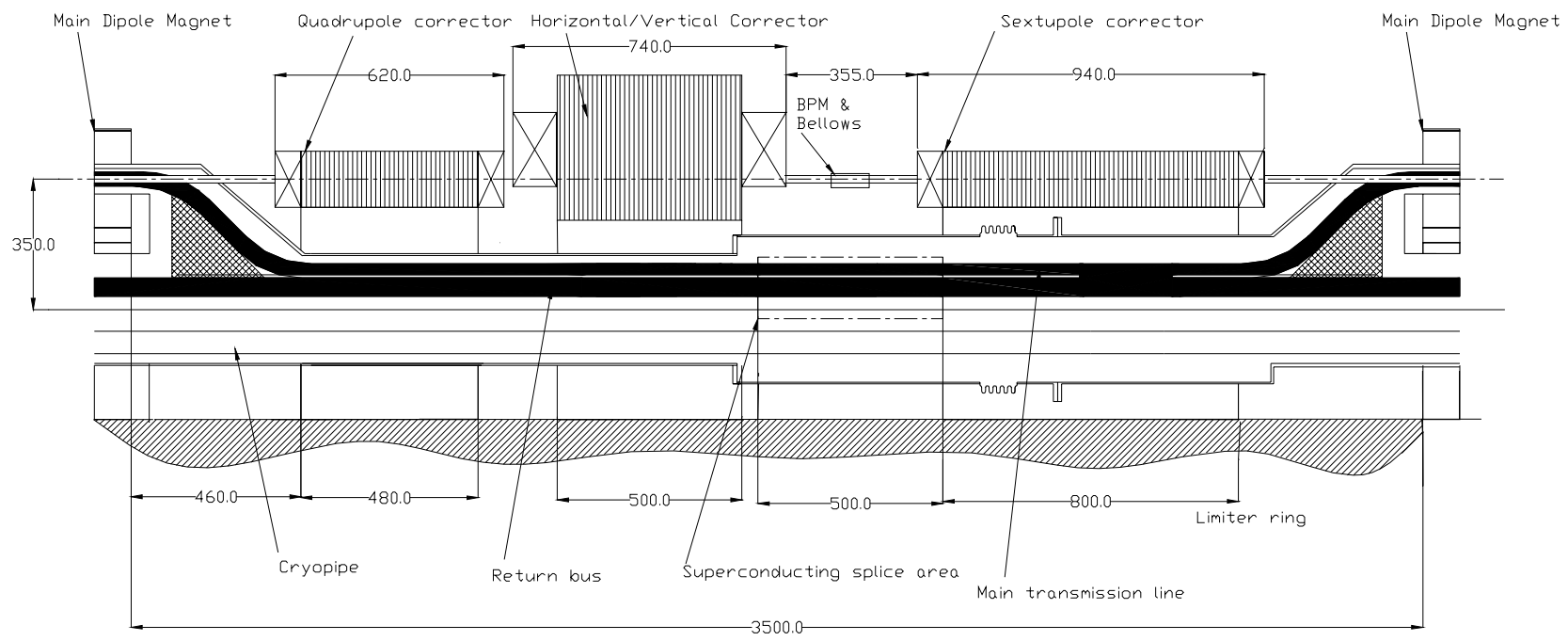

Figure 5.2. Correction magnets placed at each half-cell boundary (135-m spacing). The transmission line is diverted down near the current return bus to provide a field-free region for the correctors.

The dispersion suppressor sections are similar. Here the 101.6-m long half-cell is made of two 48.81-m long dipoles with correctors. The gradient is increased to $\pm 9.449 \% / \mathrm{cm}$ and the bend field reduced to $1.766 \mathrm{~T}$ to match the footprint of the Stage-2 machine (see Section 3.1.2).

Conventional water-cooled quadrupoles are used in the straight sections. Two 6.1-m long, $70-\mathrm{T} / \mathrm{m}$ quadrupoles are used in a typical $135-\mathrm{m}$ half-cell. Special optics for the machine utilities - injection, abort, the RF system, beam cleaning, and the matching sections of the interaction regions - are constructed using quadrupoles of the same design but with various lengths. The low-beta triplets utilize superconducting 300-T/m $\cos 2 \theta$ quadrupoles made from $\mathrm{Nb}_{3} \mathrm{Sn}$ cable. Special dipole magnets, similar in concept to the arc gradient magnets and excited by the transmission line, are used to bring the beams into collision at the interaction regions, to separate the beams to make room for RF cavities, and at the beam cross-over regions.

Table 5.1 summarizes the main parameters of the magnets used in the Stage-1 VLHC design. 
Table 5.1. Main parameters of magnets in the Stage-1 VLHC.

\begin{tabular}{|c|c|c|c|c|c|}
\hline Magnet Type & $\mathrm{B}_{\text {nom }}(\mathrm{T})$ & $\mathrm{G}_{\text {nom }}(\mathrm{T} / \mathrm{m})$ & $\mathrm{L}_{\text {mag }}(\mathrm{m})$ & $\begin{array}{c}\text { Number of } \\
\text { elements }\end{array}$ & Notes \\
\hline Gradient dipole (arc) & 1.97 & 9.73 & 67.75 & 3136 & Main Arc Magnets \\
\hline Gradient dipole (DS) & 1.80 & 16.88 & 48.81 & 160 & $\begin{array}{l}\text { Dispersion } \\
\text { Suppressors }\end{array}$ \\
\hline Straight sect quads & & 70 & $4.8-6.8$ & 464 & $\begin{array}{l}\text { Room temp. } \\
\text { conventional }\end{array}$ \\
\hline Low $\beta$ quadrupoles & & 300 & $9.2-10.9$ & 16 & Supercond. IR Quads \\
\hline Special dipoles & 1.95 & & $25-35$ & 52 & $\begin{array}{l}\text { Separation, recombi- } \\
\text { nation, and cross-over }\end{array}$ \\
\hline Correctors & & & & & $\begin{array}{l}\text { Air-cooled } \\
\text { Iron/Copper }\end{array}$ \\
\hline Dipole (horiz.) & 1.0 & & 0.50 & 1648 & Every "F" location \\
\hline Dipole (vert.) & 1.0 & & 0.50 & 1648 & Every "D" location \\
\hline Quadrupole & & 25 & 0.50 & 3296 & Every F\&D location \\
\hline Sextupole & & $1750 \mathrm{~T} / \mathrm{m}^{2}$ & 0.80 & 3296 & Every F\&D location \\
\hline
\end{tabular}

During the past three years, most of the R\&D effort has concentrated on the dipole design, with considerable attention focused on the 100-kA superconducting transmission line cable and cryogenic system. Conductors meeting all requirements have been developed and successfully tested at a 17-m length 100-kA superconducting test stand [4]. This design study is based on cables and splicing methods identical to those tested. A low heat-leak cold mass shielded support system was developed (Section 5.1.2.2). A 100-kA power supply and current leads (Figure 5.42) are being built for extended system tests. A 50-m Invar pipe was thermally cycled 1000 times to verify cryogenic weld reliability in the "zero-contraction" mode [5]. The parameters of the cryogenic system including pipe sizes, pressure drops, and refrigeration process flows have been calculated. Quench behavior has been calculated with a detailed magnetohydrodynamic simulation [6], and results were in adequate agreement with observations in the 17-m test loop. The magnetic and mechanical design was refined and several configurations tested using a room temperature magnetic test stand [7]. Two 6-m magnet prototypes with the parameters of this design study are now being manufactured to confirm the main technical decisions.

Section 5.1.2 describes the combined function arc and dispersion suppressor magnets in detail, including magnetic design, design of the 100-kA transmission line, and the mechanical and thermal designs. The correctors are described in Section 5.1.3 and the interaction region magnets in Section 5.1.4. Section 5.1.5 presents the various special magnets, including straight section quadrupoles; beam separation, recombination and cross-over dipoles; and injection and abort system magnets. Production of the 66-m long gradient dipoles and the testing and measuring of them are described in Sections 5.1.6 and 5.1.7 respectively. Transport, installation and alignment methods are summarized in Section 5.1.8. 


\subsubsection{Combined Function Arc Magnets}

The twin-aperture superconducting transmission line magnets [1-4,7-9] are shown in Figures 5.1 and 2.6. All bending magnets share a common single turn excitation transmission line winding. The return bus, located within the cryogenic service pipe, is $290 \mathrm{~mm}$ below the drive bus. There are several advantages of this single turn warm iron magnet: simple construction, low parts cost, low inductance, small cold mass, low field in the superconductor area, absence of turn to turn insulation, low magnetic forces and heat leak, high stability against beam losses and simple quench protection. Nevertheless there are some negative effects associated with the external return bus: a non-negligible fringe field, asymmetric saturation of the lower and upper iron half-cores, and partially uncompensated magnetic forces on the drive and return buses.

Table 5.2 shows the main parameters of the dipole magnets. Section 5.1.2.1 describes the magnetic design. The transmission line design is presented in Section 5.1.2.2. The mechanical and thermal designs are described in Section 5.1.2.3, including the magnet structure, the transmission line, the cryogenic service pipe and cryostat, and the beam pipe. The magnet ends, where space is provided for the correction magnets and interconnections from one magnet to the next are made, are described in Section 5.1.2.4.

Table 5.2. Main parameters of the dipole magnets.

\begin{tabular}{|c|c|c|c|}
\hline & Main Arc Dipole & Dispersion Suppressor \\
\hline \multicolumn{2}{|c|}{ Magnet air gap in the orbit center } & $20 \mathrm{~mm}$ & $22.26 \mathrm{~mm}$ \\
\hline \multicolumn{2}{|c|}{ Beam Pipe Inner Dimensions } & \multicolumn{2}{|c|}{$18 \mathrm{~mm} \times 28 \mathrm{~mm}$ (elliptical) } \\
\hline \multicolumn{2}{|c|}{ Separation Between Beams } & \multicolumn{2}{|c|}{$150 \mathrm{~mm}$} \\
\hline \multicolumn{2}{|c|}{ Magnet length } & $65.75 \mathrm{~m}$ & $48.81 \mathrm{~m}$ \\
\hline \multicolumn{2}{|l|}{ Half-cell length } & $135.5 \mathrm{~m}$ & $101.6 \mathrm{~m}$ \\
\hline \multicolumn{2}{|l|}{ Sagitta in Magnet } & $1.6 \mathrm{~cm}$ & $0.6 \mathrm{~cm}$ \\
\hline \multicolumn{2}{|l|}{ Gradient } & $\pm 4.73 \% / \mathrm{cm}$ & $\pm 9.449 \% / \mathrm{cm}$ \\
\hline \multirow[t]{2}{*}{ Magnetic field: } & injection & $0.1 \mathrm{~T}$ & $0.09 \mathrm{~T}$ \\
\hline & maximum & $1.966 \mathrm{~T}$ & $1.766 \mathrm{~T}$ \\
\hline \multirow{2}{*}{$\begin{array}{l}\text { Good field diameter } \\
(<0.02 \%) \text { : }\end{array}$} & injection & \multicolumn{2}{|c|}{$20 \mathrm{~mm}$} \\
\hline & maximum & \multicolumn{2}{|c|}{$10 \mathrm{~mm}$} \\
\hline \multicolumn{2}{|c|}{ Transmission Line Design Current } & \multirow{2}{*}{\multicolumn{2}{|c|}{$100 \mathrm{kA}$}} \\
\hline \multicolumn{2}{|c|}{ Current at $20 \mathrm{TeV}$} & & \\
\hline \multicolumn{2}{|c|}{ Magnetic field energy @100kA } & $790 \mathrm{~kJ} \quad(12 \mathrm{~kJ} / \mathrm{m})$ & $473 \mathrm{~kJ}(10 \mathrm{~kJ} / \mathrm{m})$ \\
\hline \multicolumn{2}{|c|}{ Superconducting cable } & \multicolumn{2}{|c|}{ braided NbTi with braided $\mathrm{Cu}$ stabilizer } \\
\hline \multicolumn{2}{|c|}{ Specified Max. Temp of Conductor } & \multicolumn{2}{|c|}{$6.5-6.7 \mathrm{~K}$} \\
\hline \multicolumn{2}{|c|}{ Nominal Max Temp of Cryo System } & \multicolumn{2}{|c|}{$6.0 \mathrm{~K}$} \\
\hline \multicolumn{2}{|c|}{ Iron Core } & \multicolumn{2}{|c|}{$\begin{array}{l}\text { 1-mm laminated low carbon steel } \\
\text { (AISI } 1008 \text { or better) }\end{array}$} \\
\hline
\end{tabular}

Figure 5.3 shows a detailed cross-section of the magnet, with an inset showing the dimensions and placement of the saturation control holes. The laminated core consists of upper and lower iron half cores, which are stamped from 1-mm thick low carbon steel. The upper and lower laminations are stacked and welded into 6-m long yoke blocks, which are then assembled into the complete yoke structure. The precise spacing between the two yoke halves is set by 
non-magnetic 316L stainless steel spacer bars, and the two halves are welded together with non-magnetic steel plates at the inner radius. The weld is done inside a press, and the weld shrinkage provides the preload necessary to assure precise and stable contact between all of the laminations and the spacer bar. The yoke blocks are mounted on the support tube, which also serves as the cryogenic service pipe for the magnet. The 12-m long yoke and support tube assemblies are shipped to Fermilab from outside vendors. Final assembly into 65-m magnets, including insertion of the transmission line cryostat, beam pipes, and cryo service pipes, is performed in final assembly buildings on site as described in Section 5.1.6.

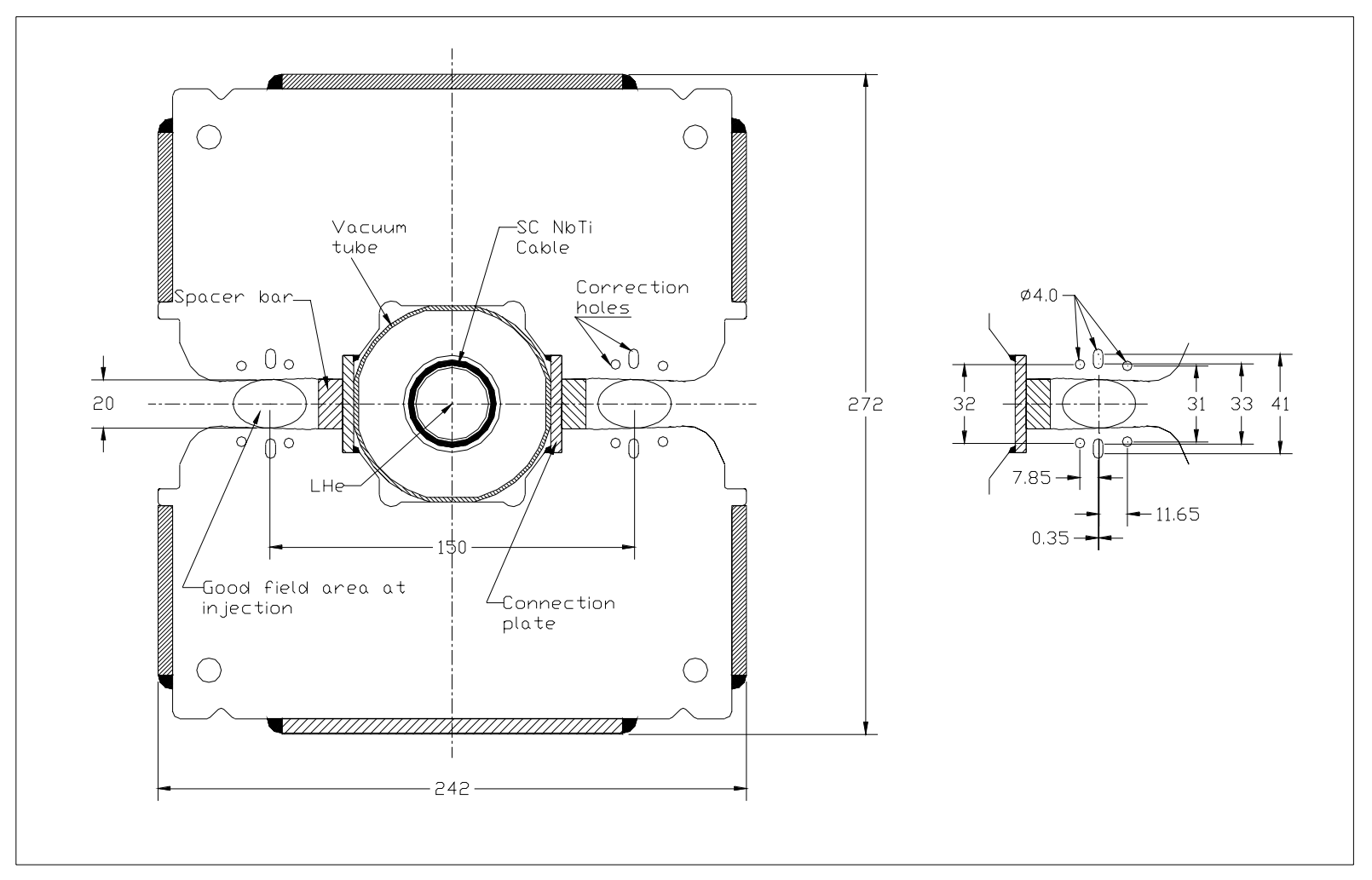

Figure 5.3. Cross-section of the transmission line magnet yoke. Inset shows holes used to control field defects from iron saturation. The vacuum system is installed after magnetic measurements are complete. Not shown are the vacuum antechambers shown in detail in Figure 5.52.

\subsubsection{Magnetic design}

The combined function gradient dipole is an iron-dominated magnet, in which the field quality is determined mainly by the shape of the pole tips and the properties of the iron from which they are made. The field quality is insensitive to conductor placement errors as large as $1 \mathrm{~mm}$. The magnet must maintain a high quality field over a 20:1 dynamic range.

\section{Field Quality at Injection}

Field quality requirements are most severe at injection where the beams are largest. The injection field of $0.1 \mathrm{~T}$ is above the range where hysteretic field defects cause difficulties. Recent experience building several hundred iron magnets for the Fermilab Main Injector and 
Recycler projects has shown that achieving the required 10-4 field quality in production is straightforward at this field level.

Mechanical tolerances on the pole tip shape will be tighter for the 2-cm magnet gap of the VLHC than for magnets with larger gaps. (Field error modeling is discussed in Section 3.2.2.) Relatively thin 1-mm steel laminations are chosen to improve this tolerance. If necessary, end shimming techniques similar to those used in Recycler production [10] can be used to reduce systematic and random multipoles at injection to a level limited by magnet measurement errors. Finally, a technique has been tested to put inexpensive multipole correctors in the body of the magnets by running wires down the saturation-control holes in the pole laminations [11]. It is unlikely that these measures will be needed.

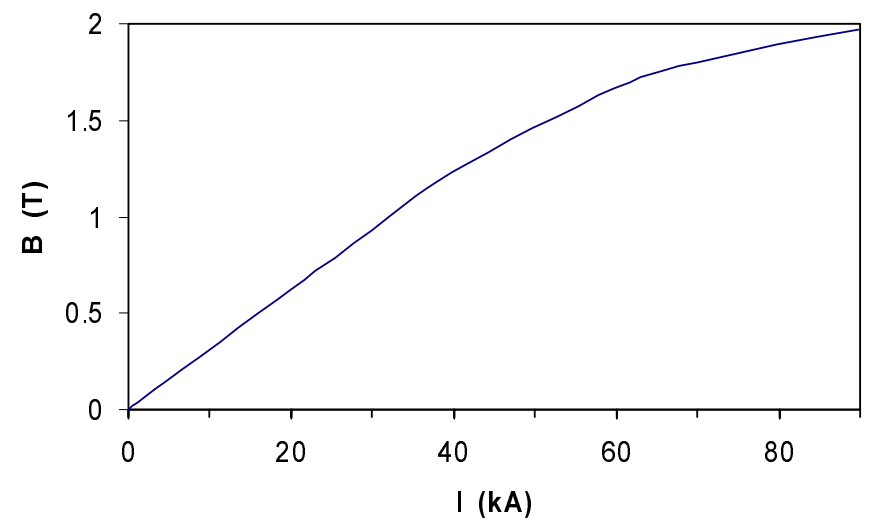

Figure 5.4. Magnetic field strength as a function of transmission line current. The $20 \mathrm{TeV}$ bend field of 1.966 Tesla occurs at $87.5 \mathrm{kA}$. The design current of the transmission line is $100 \mathrm{kA}$.

\section{Saturation Control and Maximum Dipole Field}

The main design challenge is dealing with field distortions induced by saturation of the iron at fields from 1.7 to $2 \mathrm{~T}$. Both sextupole and gradient shift are important. The sextupole defect is caused by early saturation of the corners of the pole tips, since they carry more flux than the centers of the pole. Gradient shifts are caused by asymmetric saturation on the high-field (narrow gap) side of the gradient magnet. Both of these saturation effects are controlled in the current design with a set of holes placed in the iron near the poles (see Figure 5.3 inset). At the highest fields, where the holes are insufficient, quadrupole and sextupole corrector magnets in each half-cell are needed to regulate tune and chromaticity. No significant loss of dynamic aperture results from this as discussed in Section 3.2.2.3.

There is a tradeoff between the maximum bend field (beam energy) and the strength of the corrector system required to compensate saturation effects. The present system allocates $1.2 \%$ of the ring circumference to sextupole and gradient corrector magnets. These allow a peak field $\sim 15 \%$ higher than would be possible without these strong correctors. This is worthwhile, even considering the larger cost-per-meter of the corrector magnets. On the other hand, doubling the current corrector strengths would increase the peak field by less than 5\% (see Figure 5.6). Thus the chosen corrector strengths seem to be roughly optimized.

Saturation control with "holes in the poles" proceeds as follows. Best results are obtained with a large number of holes, whereas field strength and mechanical considerations favor a 
smaller number of holes. The combined optimum was found to be three holes per pole, as shown in Figure 5.3. The field optimization objective function was the integral field homogeneity around a circular aperture. The optimization process was carried out simultaneously for three field levels (1 T, 1.7 T and 2.0 T). The optimization was done using an ANSYS Subproblem Optimizer, and a large number of local minima were investigated. The best solution was tested by OPERA $2 \mathrm{D}$ and had $\pm 0.02 \%$ field quality within a $20 \mathrm{~mm}$ diameter aperture for $0.1 \leq \mathrm{B} \leq 0.8 \mathrm{~T}, 15 \mathrm{~mm}$ for $0.8<\mathrm{B} \leq 1.5 \mathrm{~T}$, and $10 \mathrm{~mm}$ for $1.5 \mathrm{~T}<\mathrm{B}<2.0 \mathrm{~T}$. This shrinkage of good field region is acceptable, since the beam is $\sim 4 \mathrm{x}$ smaller $(\sigma=0.2 \mathrm{~mm})$ at full energy than at injection.

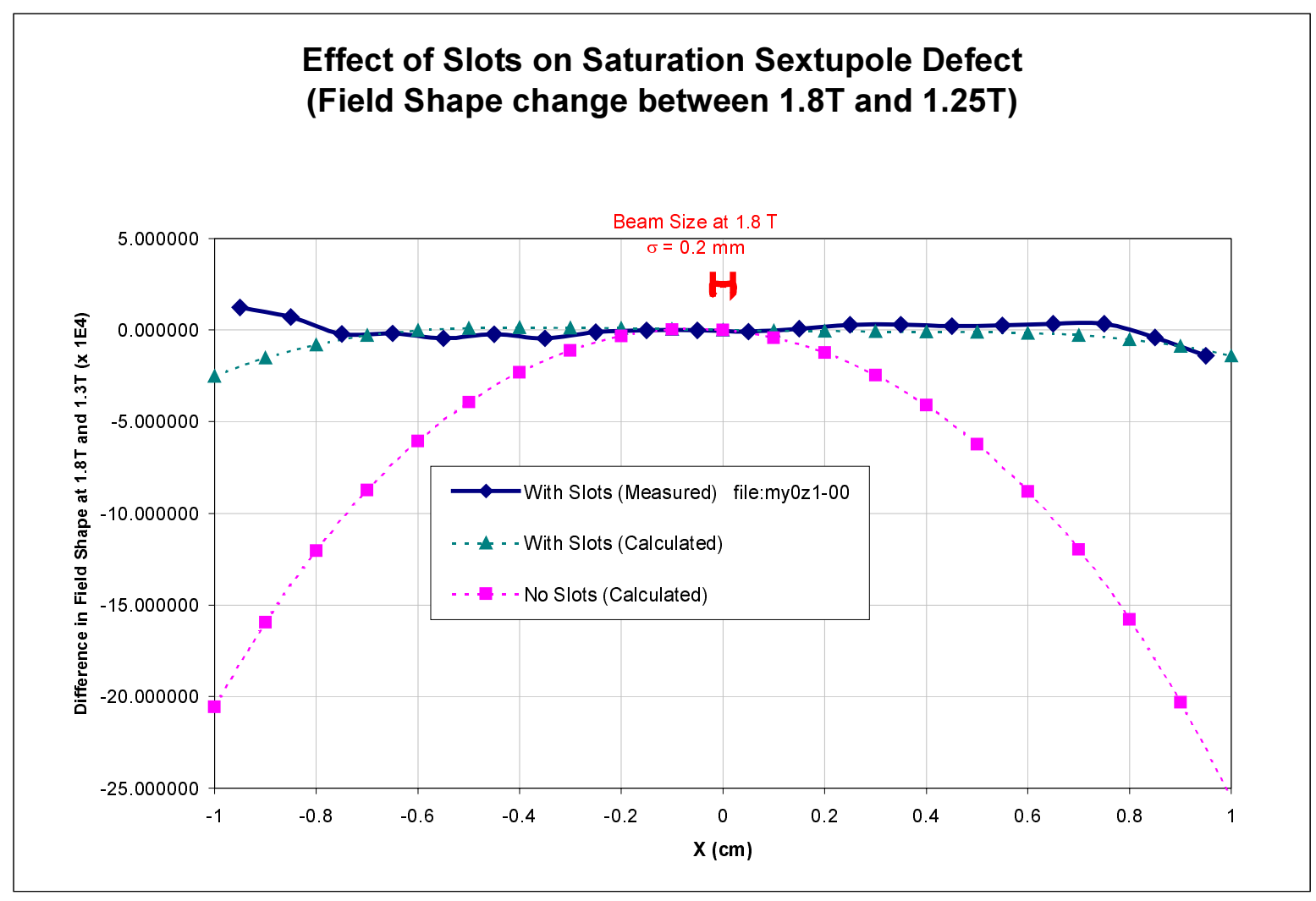

Figure 5.5. Measured effect of "Holes in the Poles" on the saturation sextupole defect as a function of transverse position in the transmission line magnet.

Both the Main Arc and Dispersion Suppressor magnets are excited by the common transmission line. The Dispersion Suppressor magnets operate at a higher gradient and reduced bend field to match the footprint of the Stage-2 dispersion suppressors. This results in similar pole tip fields and saturation characteristics for both magnet types.

We have not re-optimized the pole tip designs for the current design study parameters. The results shown below [6] correspond to an earlier parameter set with a smaller gradient of $\pm 3 \% / \mathrm{cm}$, corresponding to a lattice with $60 \%$ cell phase advance and $150-\mathrm{m}$ halfcell length, and a smaller spacing of $140 \mathrm{~mm}$ between beam apertures than the current value of $150 \mathrm{~mm}$. It is anticipated that performance similar to that described here will be achieved for the current designs when their optimization is complete. 
Figure 5.5 shows the measured effects of putting the correction holes in the pole laminations on the saturation sextupole defect at 1.8 T. The measured results agree well with the 2-D calculation, and demonstrate a large improvement with respect to the field shape without holes. Figure 5.6 compares the calculated and measured shift in the quadrupole moment as a function of excitation for a prototype yoke stack. The quadrupole and sextupole errors are \pm 2 units in the field range $0.1 \mathrm{~T}-1.85 \mathrm{~T}$ and both are about -8 units at $1.95 \mathrm{~T}$.

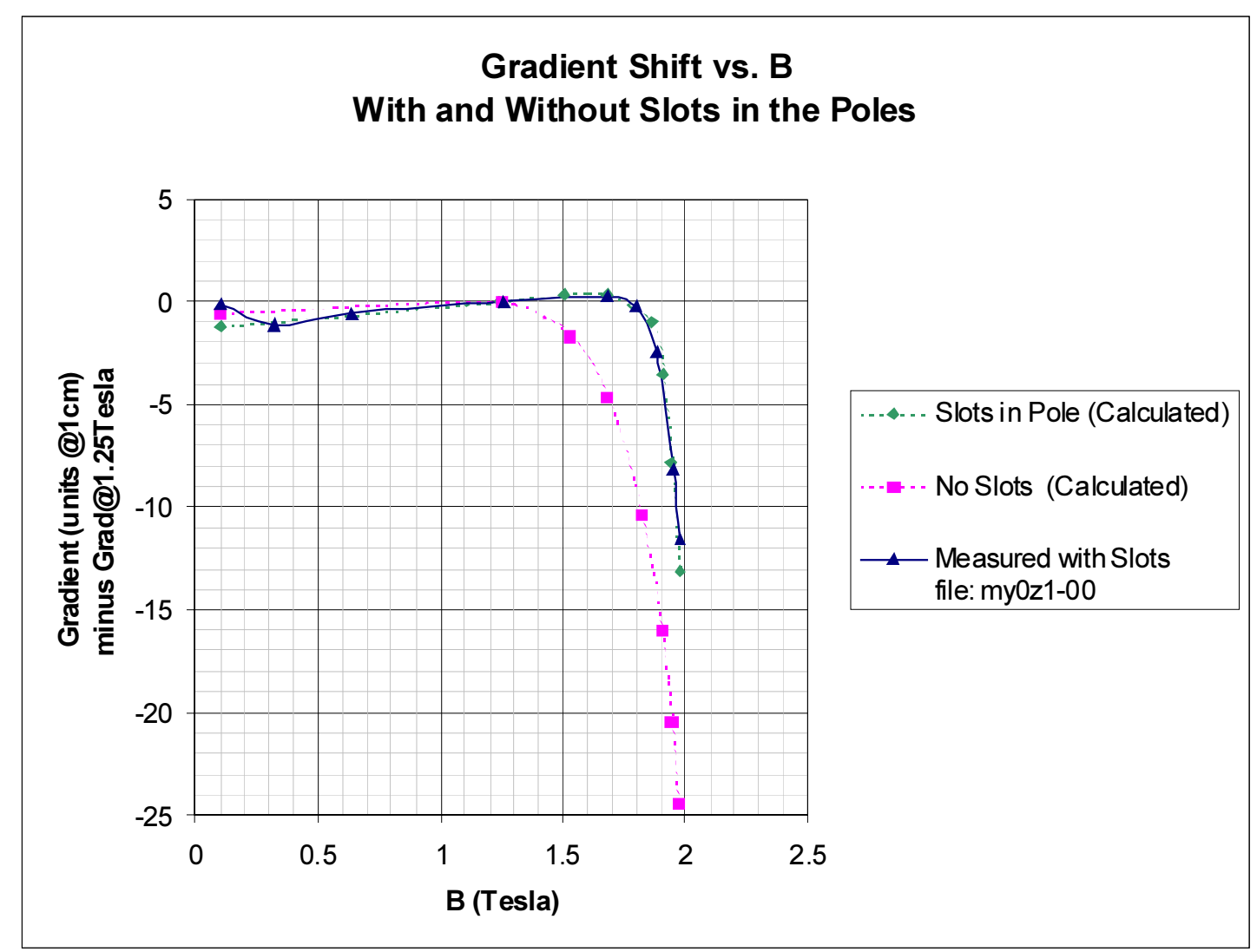

Figure 5.6. Effect of "Holes in the Poles" on the gradient shift in the transmission line magnet.

\section{Magnetic Forces and Asymmetric Saturation}

In order to suppress distant magnetic fringe fields and minimize the system inductance, a return conductor is placed outside the magnet laminations. At the midpoint of the accelerator, the drive conductor returns back through the first half of the magnets (but outside the lamination stack). At the points where the beams cross each other-at the interaction regions and the beam cross-over insertions - the drive and return buses trade places. See Figure 5.40.

The drive conductor is centered in the iron yoke and the return conductor is placed $290 \mathrm{~mm}$ below the drive conductor. The return conductor generates a fringing field that saturates the lower core more than the upper, which decreases the field quality in the gaps and increases the magnetic force on the drive conductor. This can be countered by increasing the thickness of the lower half core and adjusting the spacing of the return conductor. With these two adjustable parameters it is possible to adjust the magnetic force to zero on both the drive and return 
conductors when they are at their nominal position at full current. This same optimization largely eliminates the asymmetric (skew) multipoles in the magnet gap.

Although the force on the drive conductor in its nominal position is zero, it experiences a force gradient of about $200 \mathrm{~kg}$ per meter of magnet per $\mathrm{mm}$ of displacement. The force is horizontally stabilizing and vertically destabilizing (negative spring constant). The force gradient on the return conductor is 20 times smaller. The mechanical implications of this are discussed in Section 5.1.3.

\subsubsection{Superconducting Transmission Line}

The transmission line is a cylindrical superconducting braid inside a pipe cooled by supercritical 4.5-6.0 K helium, placed inside a co-axial cryostat, which in turn is placed at the center of the room temperature iron yoke. The transmission line (Figure 5.7) consists of (from inside out) a $2.5 \mathrm{~cm}$ helium flow space, a perforated Invar tube, a copper stabilizer braid, the braided superconductor cable, an Invar pipe to contain the helium, the cold pipe support, a cryoshield, the superinsulation blanket, and the vacuum jacket. The cold pipe support centers the conductor cable in the vacuum jacket, which in turn is placed centrally inside the magnet lamination. It also supports the cryoshield pipe, which in turn supports the superinsulation blanket.

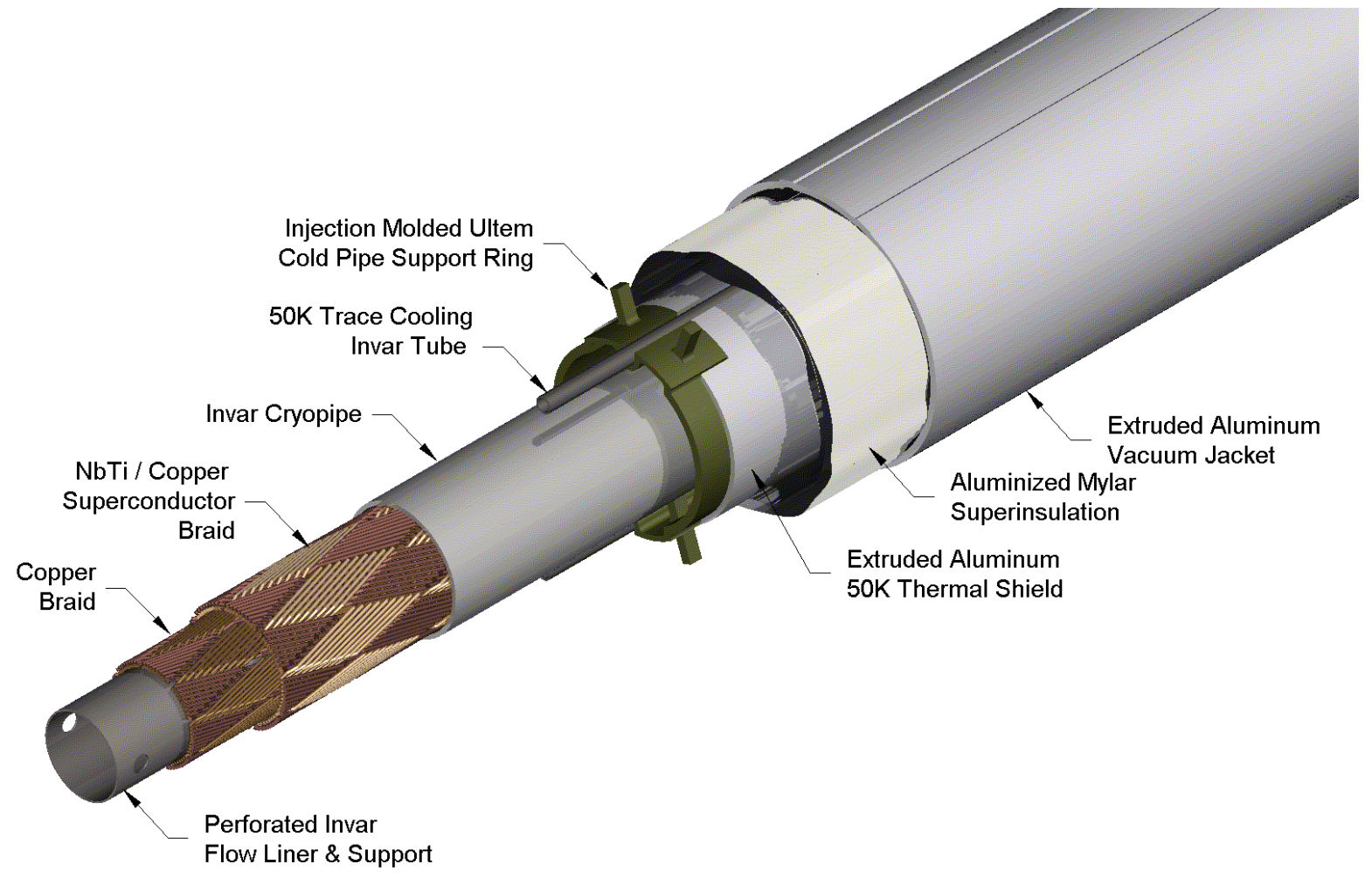

Figure 5.7. Transmission line drive conductor.

The main design requirements for the transmission line are as follows. First, it must contain enough superconductor to carry the specified current of $100 \mathrm{kA}$ at $6.5 \mathrm{~K}$. This includes a significant margin since the peak temperature of the cryogenic system is specified at $6.0 \mathrm{~K}$ (Section 5.2.1) and the required current for $20 \mathrm{TeV}$ is $87.5 \mathrm{kA}$. It must maintain a 2.5-cm clear bore for the helium flow in a 10-km loop. It must contain enough copper stabilizer and thermal 
mass to carry the full current in the non-superconducting state during a 1-second shutdown following a quench. The cold pipe must handle the maximum pressure transient of $\sim 35$ Bar which occurs during a quench. The cold mass supports must center the conductor with an accuracy of $0.5 \mathrm{~mm}$ and resist magnetic decentering forces while maintaining a low heat leak consistent with the cryogenic design. Finally, it must survive hundreds of temperature cycles with its ends constrained, since the transmission line forms a loop around the accelerator which cannot be allowed to shrink during cool down. This mandates the use of Invar $(36 \%$ Nickel steel) for the cold pipes.

\section{Conductor Design}

Three distinct conductor designs are utilized (see Table 5.3). The main drive bus that excites the dipole is optimized for minimum size consistent with operation at $100 \mathrm{kA}$ in a $\sim 1 \mathrm{~T}$ selffield. The current return bus in the cryogenic service pipe is identical to the drive bus but enlarged to increase flow area. A special type of conductor is required at the half-cell boundaries where the drive conductor is diverted downward to make space for the correction magnets (see Figure 5.2).

Table 5.3. Main parameters of the three types of transmission line conductors.

\begin{tabular}{|c|c|c|c|}
\hline & Drive Bus & Return Bus & Bus in Corr. Space \\
\hline $\mathrm{Cu} / \mathrm{SC}$ ratio in strand & 1.8 & 1.8 & 1.3 \\
\hline Diameter $(\mathrm{mm})$ & 0.648 & 0.648 & 0.808 \\
\hline Cond. Type & Braid & Braid & 9 Rutherford Cables \\
\hline Number of strands & 288 & 288 & 270 \\
\hline $\mathrm{Cu}$ Wire dia. $(\mathrm{mm})$ & 0.64 & 0.64 & 0.64 \\
\hline Number of wires & 240 & 288 & 288 \\
\hline Inner Pipe ID (mm) & 25.3 & 36.8 & 36.8 \\
\hline Outer Pipe OD (mm) & 38.1 & 47.1 & 50.1 \\
\hline Max working Pressure (bar) & 40 & 40 & 40 \\
\hline
\end{tabular}

A braided conductor is used for the main drive and return buses. The superconductor braids are sandwiched between an inner perforated Invar pipe, which serves as a liquid helium channel, and an outer Invar pressure pipe that closes the helium space. The braid consists of 288 NbTi strands arranged in a pattern of two sets of 24 crossing bundles with opposite pitch angle about the tube. It uses strands of the dimension of the SSC dipole outer layer design; current prototypes use actual SSC strand. For the Stage-1 VLHC it is planned to use a more cost effective wire optimized for the lower fields and without the constraint of small filament size. There is also a copper braid placed inside the superconductor to provide additional copper for carrying current during a quench. The conductor braid is secured in position by swaging the inner pipe against the outer one by drawing a series of carbide steel balls through the pipe. This provides adequate clamping and preload against the radial compressive forces of the magnetic self-fields, as evidenced by the fact that all of our test cables built this way achieved $\sim 100 \%$ of the theoretical current at quench. No training or degradation has been observed during the few quenches per sample tested.

The bus in the 3.5-m long corrector magnet region uses a spiral wrap of nine SSC inner dipole Rutherford cables in place of the braid. Here, due to the proximity of the two currents, the peak field rises to $1.5 \mathrm{~T}$, requiring more superconductor to carry the $100 \mathrm{kA}$ current. Cables are 
used here rather than braid to allow openings to be made in the superconductor assembly for pressure relief ports placed at every 4th half-cell $(542 \mathrm{~m})$. The Rutherford cables have less void space, which may help reduce conductor motion in the presence of azimuthal forces in this region.

Both braided and spiral-wrapped conductors (and the $10 \mathrm{~cm}$ long splice between them) have been successfully tested in the $100 \mathrm{kA}$ test facility. Power dissipation was $<0.2 \mathrm{~W}$ per splice at $100 \mathrm{kA}$.

The critical temperature vs. current for the braided conductor is shown in Figure 5.8. The data point is the measured performance of the conductor used in the prototype, and the diagonal line is the computed performance using the properties of NbTi and the self-field as a function of current. This slope has been measured on various transmission line samples and agrees with predictions derived from short sample parameters. At $90 \mathrm{kA}$ current the critical temperature is $6.8 \mathrm{~K}$. The temperature margin of $0.8 \mathrm{~K}$ with respect to the maximum helium temperature of $6.0 \mathrm{~K}$ (see Section 5.2.1) is sufficient both for stability of the superconductor and for operational "head room." The margin for the braided conductor, normalized to its higher self-field in the space between dipoles, is slightly larger than for the main conductor.

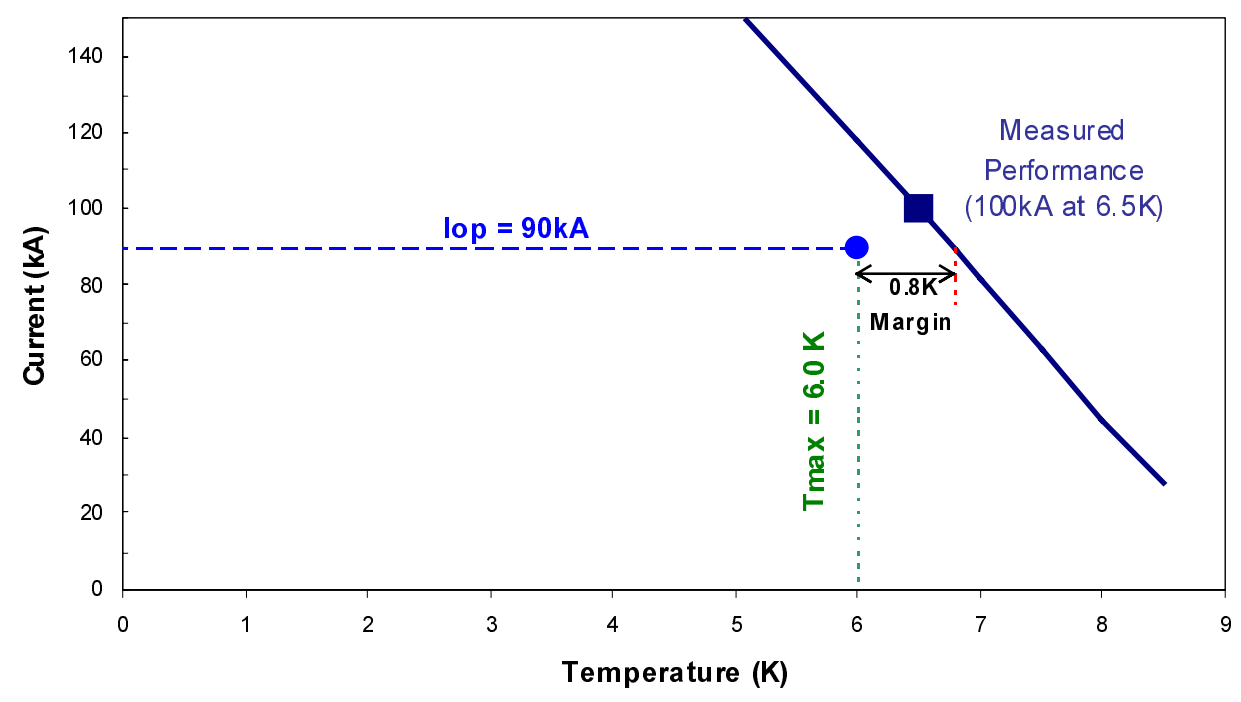

Figure 5.8. The critical temperature vs. current for main drive and return conductors. The design has a temperature margin of $0.8 \mathrm{~K}$ at $90 \mathrm{kA}$, or a current margin of $28 \mathrm{kA}$ at nominal temperature.

Detailed thermohydraulic simulations [8] have been performed on many quench scenarios with a number of different configurations of the transmission line. The simulations use a $1 \mathrm{~V}$ quench detection threshold and a 1 second decay time constant (see Section 5.2.2.3). The calculations demonstrate that, for conceivable worst-case scenarios, the peak temperature remains low $(<60 \mathrm{~K})$ and, with relief valves spaced every four half-cells, the peak pressure is below the 40 bar maximum for which the Invar pipe is rated without taking credit for its increased strength at low temperature. The simulations also show that the additional copper stabilizer braid is essential. Without it the peak temperature can reach $250 \mathrm{~K}$, and the higher temperature can result in peak pressure above 40 bar.

The quench propagation velocities were measured in a $17 \mathrm{~m}$ long conductor loop [4]. Although the properties of the conductor in the $17 \mathrm{~m}$ loop are not identical to the final design, the 
general behavior should be similar. It was found that in a forced quench by a local heating of $\sim 10 \mathrm{~K}$, quench propagation velocity rises linearly with current, and it reaches about (10-12) $\mathrm{m} / \mathrm{s}$ at $\sim 80 \mathrm{kA}$. Above this current, it rises exponentially reaching about (30-40) m/s at $100 \mathrm{kA}$. This indicates that by applying a current dump circuit of a time constant of 1 second, the quench zone would be at most half the length of a dipole magnet $(34 \mathrm{~m})$. Repeating these tests with long lengths of the final transmission line is an important goal of the R\&D program.

\section{Cryostat Design}

A cross-section of the drive conductor cryostat is shown in Figure 5.9. The support spider consists of an injection molded ULTEM ring with two pairs of pegs pointing up and down, the direction of the magnetic force. The inner pair of pegs support the cold pipe, while the outer pegs contact the vacuum shell. The conductor must be placed precisely on the central line of the magnet to minimize the de-centering force. Offsets of the order of $0.5 \mathrm{~mm}$, in excess of what can be expected due to part tolerances, lead to de-centering forces on the order of $100 \mathrm{~kg} / \mathrm{m}$. The supports are specified to withstand 100,000 mechanical cycles during the accelerator lifetime, or $10 \mathrm{ramps} /$ day for 30 years. Laboratory tests on these supports withstood 100,000 mechanical cycles of $300-\mathrm{kg} / \mathrm{m}$ force, three times that of the design requirement. Further design development, however, may be required to optimize the spider design with respect to axial motion, expected under thermal cycling. (See Section 5.1.2.3.)

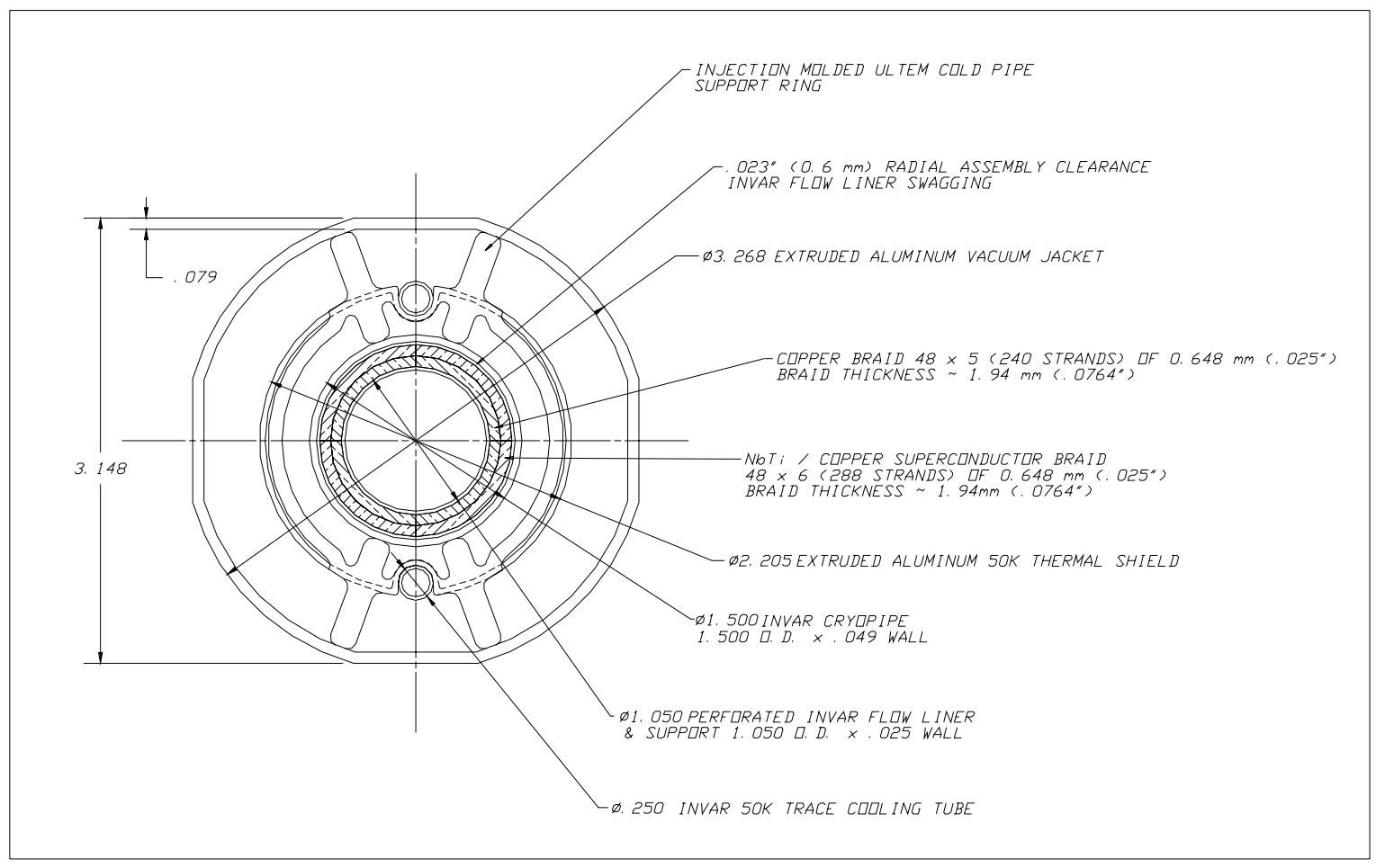

Figure 5.9. The drive conductor cryostat.

The cryo-shield is made of extruded aluminum pipe segments $\sim 50 \mathrm{~cm}$ long, which slide over opposite ends of each support spider. The 6.4-mm diameter Invar pipe that is used to provide the $50 \mathrm{~K}$ pressurized helium is "snapped in" to the cavities at the top and the bottom of 
both the shield and the supports once a magnet's length of shield has been assembled. The shield pipe is wrapped with 40 layers of a dimpled superinsulation blanket. The vacuum jacket is made of extruded aluminum, and it is of a "squared" shape to firmly fix its position inside the iron laminations. This also helps to prevent potential rotation (and/or twist) of the cold pipe supports.

\subsubsection{Mechanical and Thermal Design of Transmission Line Magnet}

\section{Magnet Length}

Many considerations entered into the $67.75 \mathrm{~m}$ magnet length chosen for this study. There appear to be no hard technological or financial limits to considering magnets either twice as long or half as long. The weight of 33 tonnes is slightly less that an LHC dipole so that considerations of floor loading, transportation power, etc. will be comparable. A magnet of this length can be picked up at two points and transported using a lifting fixture (load spreader bar) which weighs $1 / 3$ as much as the magnet and is small enough $(2 \mathrm{~m}$ height $)$ to fit into the tunnel. This greatly simplifies handling. With the electronics modules located at half-cell boundaries, all instrumentation and cables can be factory-installed and tested on our $1 / 4$ cell long magnets, but this is not true of shorter magnets. Cryogenic heat load from transmission line splices is about $0.4 \mathrm{~W}$ per magnet end or $<10 \%$ of the total $5 \mathrm{~K}$ heat load. Labor estimated for in-situ magnet splicing is $\sim 60$ person-hours per magnet, which corresponds to $<\$ 10 \mathrm{M}$ for 3000 magnets. This sets the scale for possible savings of in-tunnel labor from longer magnets. Doubling the magnet length would increase the floor space of the factory by about $50 \%$.

\section{Distance Between Support Posts and Gravitational Sag}

The main structural element of the magnet assembly is the $300 \mathrm{~mm}$ steel pipe which serves as the vacuum jacket for the cryogenic service lines and current return. The magnet cores act mainly as a dead weight on this pipe. The support pipe is made from A36 carbon steel $5 \mathrm{~mm}$ thick. The magnet core is welded to the tube every $0.5 \mathrm{~m}$ to increase the vertical stiffness of the assembly and to reduce system deflection.

The magnet is installed on vertical support/adjusters every $6 \mathrm{~m}$. This distance was chosen [12] to limit deflections due to gravity to $1-2 \mathrm{~mm}$ depending on assumptions about the connections between the cores and service pipe. As is common for long laminated magnets, this deflection will be precompensated by assembly tooling so the magnet sags into alignment. The present design also allows for a final shimming of the core position if needed. The maximum stress does not exceed $50-60 \mathrm{MPa}$.

\section{Force Needed to Introduce Alignment Bumps}

The force needed to introduce the 1.6- $\mathrm{cm}$ global sagitta into the $67-\mathrm{m}$ magnet assembly is very small $(<10 \mathrm{~kg})$. Large forces may be generated, however, on individual support/adjusters from gravity and friction, or if they are driven in opposing directions during magnet alignment in the tunnel. To estimate forces and deformations of the straight magnet structure during alignment, 3D numerical simulations were performed using a 12-m section model [13]. Forces were applied on a single support/adjuster connected to the vacuum tube at the center of the model, and the model ends were restricted from motion or rotation. The model also had a distributed 
weight load from the laminations in addition to the horizontal force applied by the adjuster. The force needed for $1-\mathrm{mm}$ motion is $450 \mathrm{~kg}$. The result shows the magnet twists slightly because the force is not applied along the neutral axis of the magnet assembly. The block moves about $0.9 \mathrm{~mm}$ for a $1-\mathrm{mm}$ movement of the vacuum tube. The difference in relative movement of the iron halves of the magnet can reach $30 \mu \mathrm{m}$.

\section{Yoke Deflection and Gap Stability under Magnetic Forces}

The magnetic force acting between the yokes rises quadratically from $6 \mathrm{~kg} / \mathrm{m}$ at injection to $2500 \mathrm{~kg} / \mathrm{m}$ at the maximum field of $2 \mathrm{~T}$. The outside of the gaps must remain open for magnetic measurements and vacuum chamber installation. Thus this force is taken across support spacers in the relatively small $\sim 1 \mathrm{~cm}$ distance between the transmission line vacuum jacket and the beam pipe. The yoke and gap spacer assembly must be rigid enough to keep the air gap stable within $\sim 20 \mu \mathrm{m}$. Several prototypes were built before identifying a viable and economical design.

The laminated magnet yoke (Figure 5.3) consists of upper and lower iron half cores. Core laminations are stamped from 1-mm thick low carbon steel. The stack of laminations is pressed and welded into a block. Two blocks, upper and lower, separated by two nonmagnetic 316L stainless steel spacer bars and are welded together by stainless steel strips from the inside of the central hole. The weld contraction provides a preload greater than 5 tons $/ \mathrm{m}$ which removes all clearances between the spacer bars and half-cores.

Tests of the final short mechanical model showed that under full load, the left gap shrank vertically by $12 \mu \mathrm{m}$ and the right gap by $6 \mu \mathrm{m}$. The horizontal change was less than $4 \mu \mathrm{m}$. These changes were reproducible indicating elastic deformation. In full magnetic testing a $1 \mathrm{~m}$ model with outer spacer bars was successfully tested and no gradient shift was observed (Figure 5.6) aside from that expected from iron saturation [14]. These measurements will be repeated with better statistics on longer magnets currently in production.

\section{Magnetic Forces on the Transmission Line and Cold-Mass Support Spider}

Loads from transmission line magnetic forces are transmitted from the drive conductor to the pipe, through the supports (spiders) to the vacuum jacket, and to the magnet core. In the ideal case, the magnetic forces are zero on both the drive and return conductors as described in Section 5.1.2.1. Nevertheless, iron saturation effects and assembly tolerances in the position of superconducting cable will cause uncompensated magnetic forces [15]. At a current of $100 \mathrm{kA}$, each meter of the transmission line experiences a decentering force gradient of $228 \mathrm{~kg}$ per mm of vertical displacement and a stabilizing force gradient of $100 \mathrm{~kg} / \mathrm{mm}$ in the horizontal direction. The vertical force gradient gives rise to an instability [16] in which the current-carrying pipe bends into a sinuous curve with a period of twice the support distance. This instability sets the maximum support interval of $0.5 \mathrm{~m}$.

Spiders made of ULTEM [17] centrally position the conductor. Pegs are nominally under pure compression inside the cryostat. The compression depends on the leg over-sizing and on the variation in pipe diameter. A $0.5 \mathrm{~mm}$ tolerance is required on the combined tolerance stackup of the cryopipe diameter, spider leg lengths, vacuum jacket wall thickness, and lamination geometry. See Section 5.1.6.3. The pressure range is $20-40 \mathrm{~kg}$. These numbers increase up to $70-100 \mathrm{~kg}$ in cold conditions and under the action of magnetic forces. 
Prototype injection-molded supports were exposed to 100,000 mechanical cycles with a load force of $300 \mathrm{~kg} / \mathrm{m}$ (three times that expected) [18]. This test indicates that in principle the transmission line supports would function with every second spider missing or destroyed.

The relative axial motion of the cold conductor line and the warm vacuum jacket tube requires the spiders to slide along the pipes to avoid a high stress in the spider material [19]. The sliding behavior needs to be carefully verified in system tests. If it is insufficient, other rolling or sliding support systems can be used [20,21,22].

\section{Magnetic Forces on the Current Return Conductor}

The return conductor is at a force null in the main body of the magnet, and experiences a force gradient 20 times smaller than the drive conductor. However in the corrector region where the buses are not shielded from each other, the magnetic forces are $700 \mathrm{~kg} / \mathrm{m}$ at the standard separation of $0.29 \mathrm{~m}$, increasing to $\sim 4000 \mathrm{~kg} / \mathrm{m}$ at the $10 \mathrm{~mm}$ separation in the corrector region. This force is handled by mechanically connecting the two buses together in this region (see Figure 5.11) with an insulating cold-to-cold support that does not generate a heat leak.

\section{Longitudinal Forces from Thermal Contraction of Invar Pipes}

The conductors in the transmission line magnet form a continuous loop around the ring circumference that can not be allowed to contract as the pipes cool down. Most materials will be damaged if they are cooled down to cryogenic temperatures but not allowed to contract (Table 5.4). Expansion bellows can be used for normal cryogenic piping but not with the transmission line conductor. The best solution for the conductor is to use materials such as Invar that will go into tension but will not be destroyed as they are cooled down with their ends constrained.

Table 5.4. Stress in pipe materials after cool down from $300 \mathrm{~K}$ to $5 \mathrm{~K}$ with ends constrained.

\begin{tabular}{|l|c|c|c|c|c|c|}
\hline Material & $\begin{array}{c}\int \alpha d t, \\
\mathrm{~mm} / \mathrm{m}\end{array}$ & $\begin{array}{c}E(5 \mathrm{~K}) \\
G P a\end{array}$ & $\begin{array}{c}\sigma=E^{*} \text { (adt } \\
M P a\end{array}$ & $\begin{array}{c}\text { oyield } \\
M P a\end{array}$ & oyield $/ \sigma$ & $\begin{array}{c}\Delta L(\mathrm{~mm}) \\
L=135 \mathrm{~m}\end{array}$ \\
\hline 304 Stainless Steel & 3 & 220 & 660 & $600-1200$ & $0.9-1.8$ & 405.0 \\
\hline 6061 Aluminum & 4.1 & 80 & 328 & 380 & 1.15 & 553.5 \\
\hline Invar (36\% Ni Steel) & 0.48 & 140 & 67 & $700-1000$ & $10.4-14.9$ & 64.8 \\
\hline
\end{tabular}

As the table above indicates, Invar will survive cooldown with $67 \mathrm{MPa}$ of thermal stress, corresponding to $\sim 1400 \mathrm{~kg}$ tension in our conductor (independent of length) at $5 \mathrm{~K}$. The hoop tension on the Invar pipe is provided by a radially outward force of $\sim 0.06 \mathrm{~kg}$ on each spider.

A test of the cryogenic reliability of Invar pipe welds was performed [5] as part of the VLHC R\&D program. A $50 \mathrm{~m}$ Invar tube containing 128 automatic girth welds was thermally cycled 1000 times from $300 \mathrm{~K}$ to $77 \mathrm{~K}$ with the ends constrained. No leaks were detected at a sensitivity of $10^{-10} \mathrm{std} \mathrm{cc} / \mathrm{sec}$.

\section{Longitudinal Anchoring for Magnet and Cryogenic Pipes}

The magnet anchor system consists of the vacuum barriers, the force-anchors, the transportation locks and the vacuum jacket anchors, as shown in Figure 5.10. 


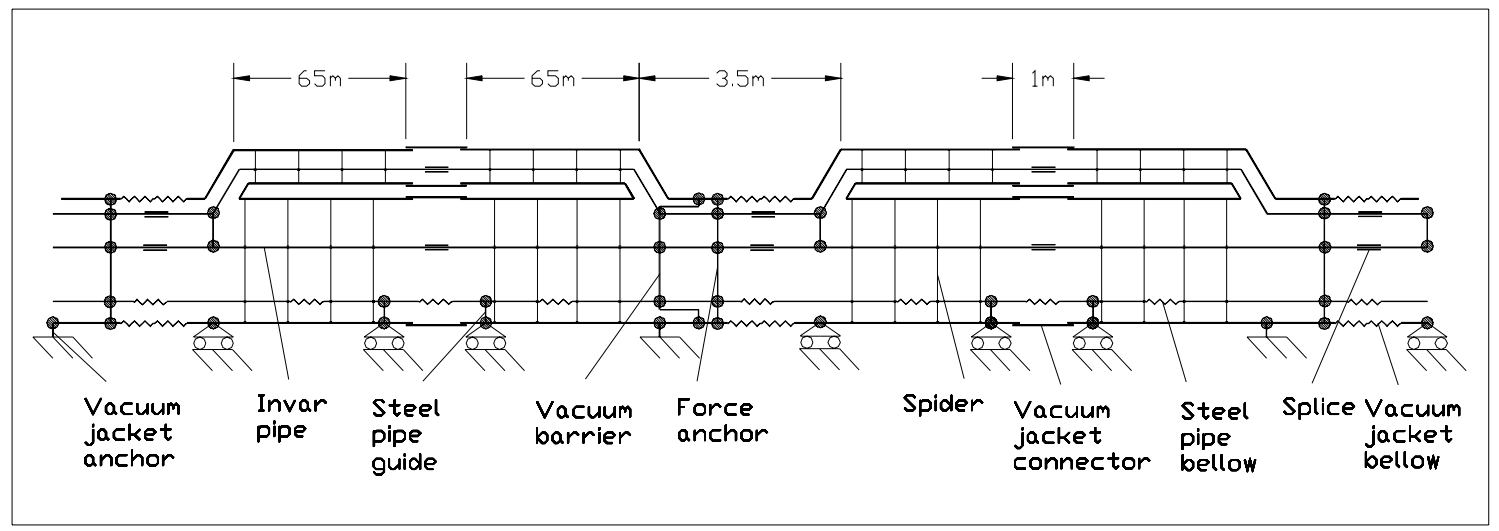

Figure 5.10. Longitudinal anchor schematic. The vacuum jacket and all pipes are anchored every $135 \mathrm{~m}$.

The basic philosophy is to longitudinally anchor the vacuum jacket and all piping at a single point near the corrector location at the half-cell boundary (every $135 \mathrm{~m}$ ). Two 67-m magnets will be hard-welded together into a single $135-\mathrm{m}$ assembly. The vacuum jacket is also connected to the ground every $135 \mathrm{~m}$ at the anchor point. These points define an immovable magnet cross-section in case of accidental vacuum loss and jacket thermal contraction from a cool down to about $250 \mathrm{~K}$ [23]. There will be expansion joints in the vacuum jacket and beam pipes on one side of this anchor. Magnet adjusters will permit 2-3 cm of longitudinal motion for normal tunnel temperature variations.

Cryogenic piping will be anchored at the $135-\mathrm{m}$ locations, and will have sliding supports in places where it is not anchored. Either Invar or stainless steel piping can be used for the normal cryogenic piping. Stainless pipes will need compensation bellows every $\sim 33 \mathrm{~m}$ to reduce shrinkage to the level of $\sim 100 \mathrm{~mm}$. The convoluted length of the bellow is $100 / 0.25=400 \mathrm{~mm}$. The force to expand the bellows by $50 \mathrm{~mm}$ is $300 \mathrm{~kg}$ for the bellows' spring constant $6 \mathrm{~kg} / \mathrm{mm}$. The bellows' stability problem is solved by using an internal tube liner and an outer protecting skin.

Vacuum barriers and quench pressure reliefs occur at the anchor location in every $8^{\text {th }}$ magnet $(540 \mathrm{~m})$. The vacuum breaks are used to isolate vacuum sectors in order to facilitate installation, troubleshooting, and repair. They provide a good vacuum in the transmission line ring section $[24,25]$. The vacuum barriers are designed to withstand a differential pressure of $0.15 \mathrm{MPa}$ and to minimize the heat in-leak [26]. They are made of an all-welded construction of thin-wall stainless steel tubes. The vacuum barrier is located close to the pipe longitudinal anchor to prevent damage to the ceramic insulators that electrically isolate the Invar conductors.

The longitudinal anchor restrains thermal forces from Invar pipe shrinkage and stainless steel bellows expansion ( $\sim 3000 \mathrm{~kg})$ during cool-down and warm-up. This anchor consists of graphite fiber composite tie rods and a G-10 spider, and connects all the pipes to the vacuum jacket.

The transportation lock is located on the opposite end of the magnet from the vacuum break. It is mounted in place during production and removed after magnet installation in the tunnel. 


\section{Cryostat Interconnection}

After magnet alignment, interconnection activities will take place. The magnet and the cryoservice line must be connected every $67.75 \mathrm{~m}$ at the $3.5-\mathrm{m}$ and $1-\mathrm{m}$ interconnection regions, as shown in Figure 5.11.

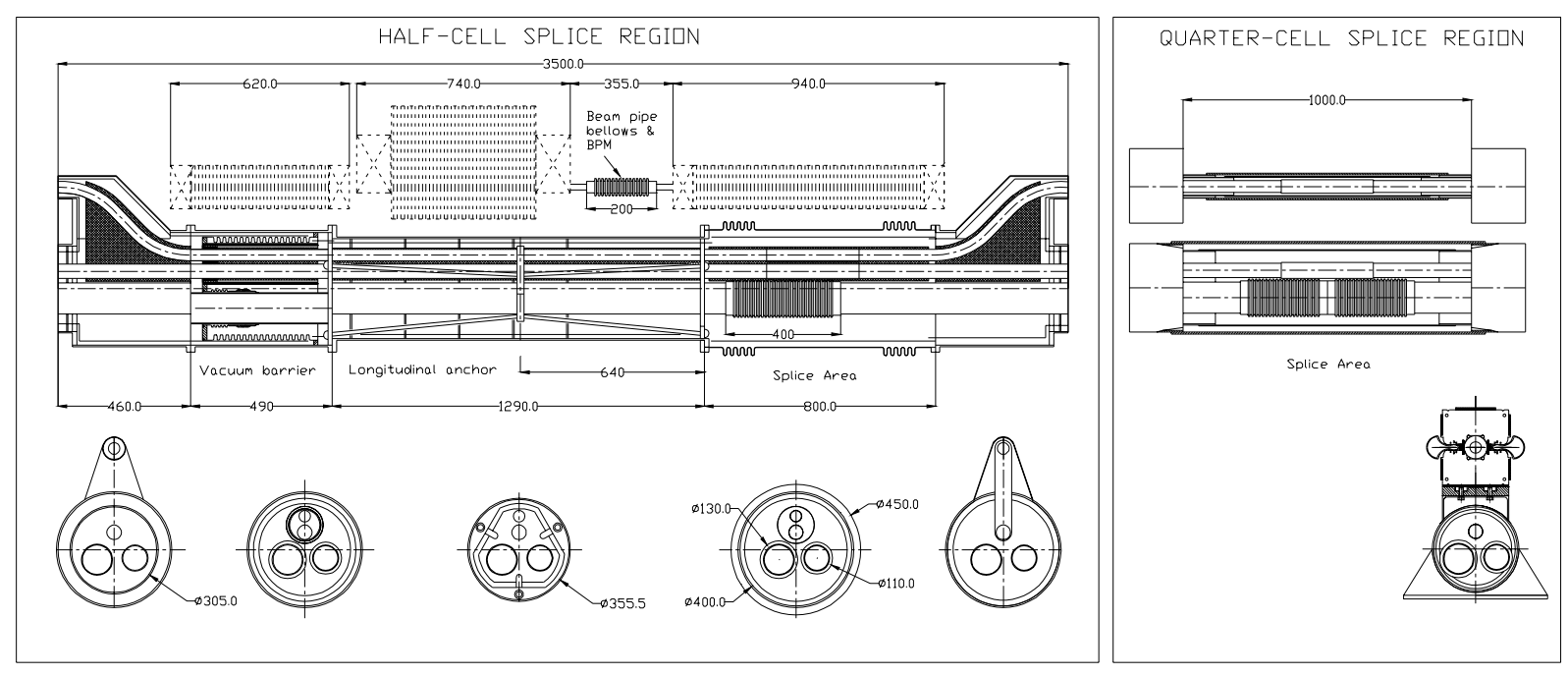

Figure 5.11. Magnet interconnections with bellows and correctors at the half-cell boundaries (left), and the hard-welded connection at the quarter-cell boundary (right).

Both conductors will be spliced at these areas. To compensate magnetic, thermal and hydraulic forces in the region where the transmission line conductors make a loop and are located close to each other, an additional set of special spacers and clamps will be used. The weld on the conductor line pipes can take place directly over the copper/superconductor structure without damaging it. A picture of the splicing operation is shown in Figure 5.33.

Three cryo-service pipes having bellows will be orbital welded automatically. An aluminum thermal shield made from two pieces will cover all the pipeline connections [27]. Multilayer insulation blankets are mounted around the shield to reduce radiation heat load. The vacuum jacket is connected by a bellows in the $3.5 \mathrm{~m}$ region and by a thin steel skin in the $1 \mathrm{~m}$ region.

Beam pipes are connected by bellows every $135 \mathrm{~m}$ in the space between corrector magnets to compensate $\sim 25 \mathrm{~mm}$ motion of the magnets from expected temperature excursions in the tunnel.

\subsubsection{Corrector Magnets}

In Stage-1 there are independently powered dipole, quadrupole and sextupole correctors in the $3.5 \mathrm{~m}$ free space at the boundary of each $135 \mathrm{~m}$ half-cell. See Figure 5.2. Horizontal or vertical dipole correctors occur at each horizontally focusing or defocusing location. The specified strength of $0.5 \mathrm{~T}-\mathrm{m}$ is sufficient to control closed orbit errors due to misalignments, dipole strength errors, and ground settling (Section 3.2.3). Dipole correctors can perform a full aperture scan at energies up to $7 \mathrm{TeV}$ and can produce a 3-mm "3-bump" in the closed orbit at full energy. Quadrupole correctors have sufficient strength to overcome the gradient shift from 
magnet saturation (Figure 5.6) and can be used to diagnose and correct $\beta$-waves in the lattice (Section 3.2.3.2). Sextupole correctors have the strength to overcome dipole saturation effects at full energy (Figure 5.5) as well as regulation of chromatic effects (Section 3.2.2.3).

Table 5.5. Corrector parameters.

\begin{tabular}{|l|c|c|c|c|}
\hline Corrector type & $\begin{array}{c}\text { Horizontal } \\
\text { Dipole }\end{array}$ & $\begin{array}{c}\text { Vertical } \\
\text { Dipole }\end{array}$ & $\begin{array}{c}\text { Quadru- } \\
\text { pole }\end{array}$ & Sextupole \\
\hline Quantity & 1720 & 1720 & 3136 & 3136 \\
\hline Maximum magnet strength & $1.0 \mathrm{~T}$ & $1.0 \mathrm{~T}$ & $25 \mathrm{~T} / \mathrm{m}$ & $1750 \mathrm{~T} / \mathrm{m}^{2}$ \\
\hline Field quality, 18 mm diameter area, \% & 1.0 & 1.0 & 1.0 & 1.0 \\
\hline Effective magnet field length, m & 0.5 & 0.5 & 0.5 & 0.8 \\
\hline Integrated field at r =1 cm, T-m & 0.5 & 0.5 & 0.125 & 0.14 \\
\hline Magnet core length, m & 0.48 & 0.48 & 0.5 & 0.8 \\
\hline Current, A & 25 & 25 & 1.0 & 1.0 \\
\hline Voltage, V & 23 & 23 & 140 & 175 \\
\hline Power, W & 600 & 600 & 150 & 200 \\
\hline Number of coils per magnet & 1 & 1 & 4 & 6 \\
\hline Number of turns per coil & 640 & 640 & 1240 & 633 \\
\hline Copper conductor dimensions, mm & $5 \times 5 \mathrm{sq}$. & $5 \times 5 \mathrm{sq}$. & $1 \mathrm{~mm} \varnothing$ & $1 \mathrm{~mm} \varnothing$ \\
\hline Copper weight, kg & 250 & 250 & 40 & 50 \\
\hline Core weight, kg & 300 & 220 & 30 & 50 \\
\hline
\end{tabular}

Higher multipole correctors have not yet been specified. In addition to the $3.5 \mathrm{~m}$ space at half-cell boundaries, the accelerator lattice design includes an unallocated $1 \mathrm{~m}$ space at the $1 / 4$ cell point that could be used for a Neuffer-Simpson type multipole corrector scheme.

The main design issue for the correction system is the fringing field generated by the two $100 \mathrm{kA}$ conductors in the drift space between dipole magnets. This field is $0.3 \mathrm{~T}$ in an unshielded beam pipe and causes magnetic forces of $700 \mathrm{~kg} / \mathrm{m}$ on the superconducting cable. The distance between the outer shell of the superconducting transmission line and the vacuum chamber center is only $30 \mathrm{~mm}$; it is a nontrivial task to form the needed correction field in this area. Several options were considered to solve this problem:

1. Compact superconducting correctors with cold ferromagnetic screen [28]

2. Room temperature correctors with iron core as a ferromagnetic screen

3. Room temperature correctors with the transmission line deflected downwards and out of the way in the corrector region

4. Pole tip windings on the main dipoles (effective at injection, less useful at high fields)

5. Iron-dominated mechanical correctors (stepping motor drive, low power)

6. Permanent magnet correctors (rotational multipoles, stepping motor drive)

Option 3 (warm correctors with out-of-the-way transmission line) was chosen for this Design Study on the basis of lowest overall design risk and predictable costs.

The six correctors in the $3.5 \mathrm{~m}$ space at each half-cell will be pre-aligned and transported on a common skid. See Section 5.1.8.6. All the correctors can be installed and removed without breaking the beam pipe vacuum. 


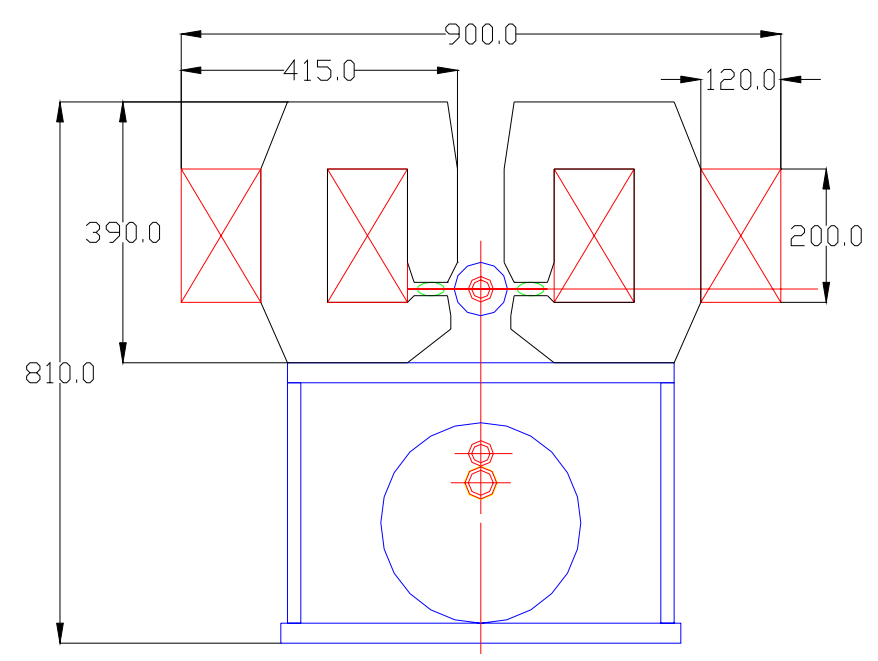

Figure 5.12. Horizontal dipole correctors.

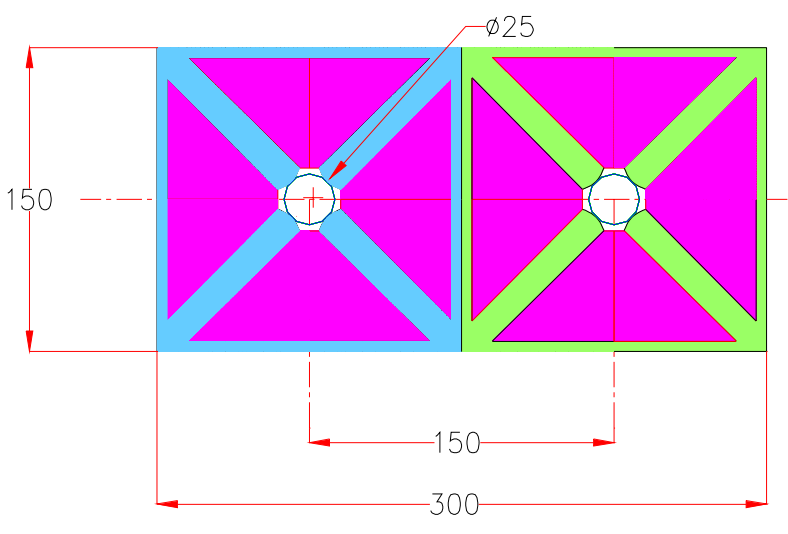

Figure 5.13. Quadrupole correctors.

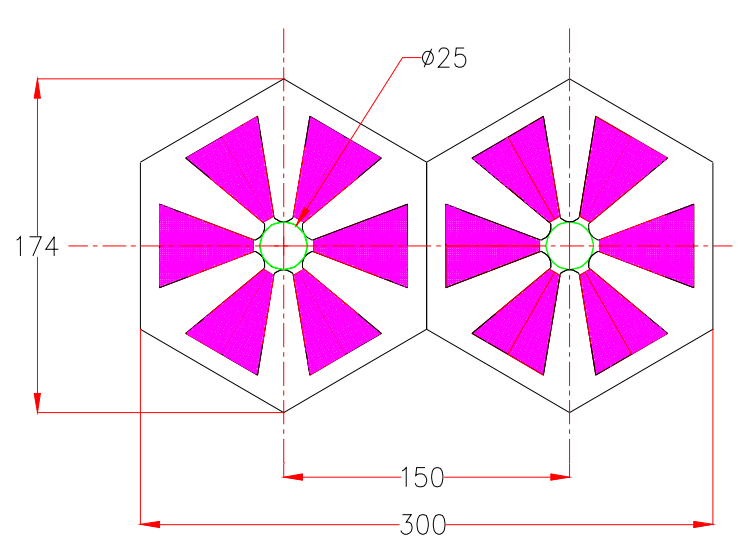

Figure 5.14. Sextupole correctors.

The powering scheme for the correctors is discussed in Section 5.2.3. All correctors are independently powered so that if necessary they can be programmed to overcome both random and systematic errors in the saturation characteristics of the magnets.

There is significant scope for reduction of corrector strengths. One rather promising approach is to optimize pole profiles for low field and saturation correction hole positions for maximum field. In this case, correctors will work at full power for only a short period of time during the acceleration cycle and will return to near zero at full field. This will reduce the operational cost of the corrector power supplies and simplify cooling.

\subsubsection{Interaction Regions}

The VLHC has provisions for two large colliding experiments located on the present Fermilab site. A system of magnets on each side of the interaction regions is required to bring the beams into collision. With these magnets come additional requirements for the accelerator including power supplies, beam absorbers to protect the magnets and prevent activation of the tunnel, and 
an alignment system for the magnetic elements. For the superconducting magnets there are quench protection systems, cryogenics, current leads, and warm-to-cold transitions for instrumentation and beam vacuum.

Figure 5.15 shows a schematic of the interaction region magnetic elements. Each interaction region spans a space of 6 half cells, from \pm 21 meters to approximately \pm 810 meters from the interaction points. The interaction region consists of eight high gradient "inner triplet" quadrupoles, four beam separation/recombination warm dipole elements, and an outer system of lower gradient quadrupoles. There will also be orbit and local field corrector magnets. The separation dipoles, "outer" quadrupoles and correctors are warm-iron magnets identical to those used elsewhere (Section 5.1.5).

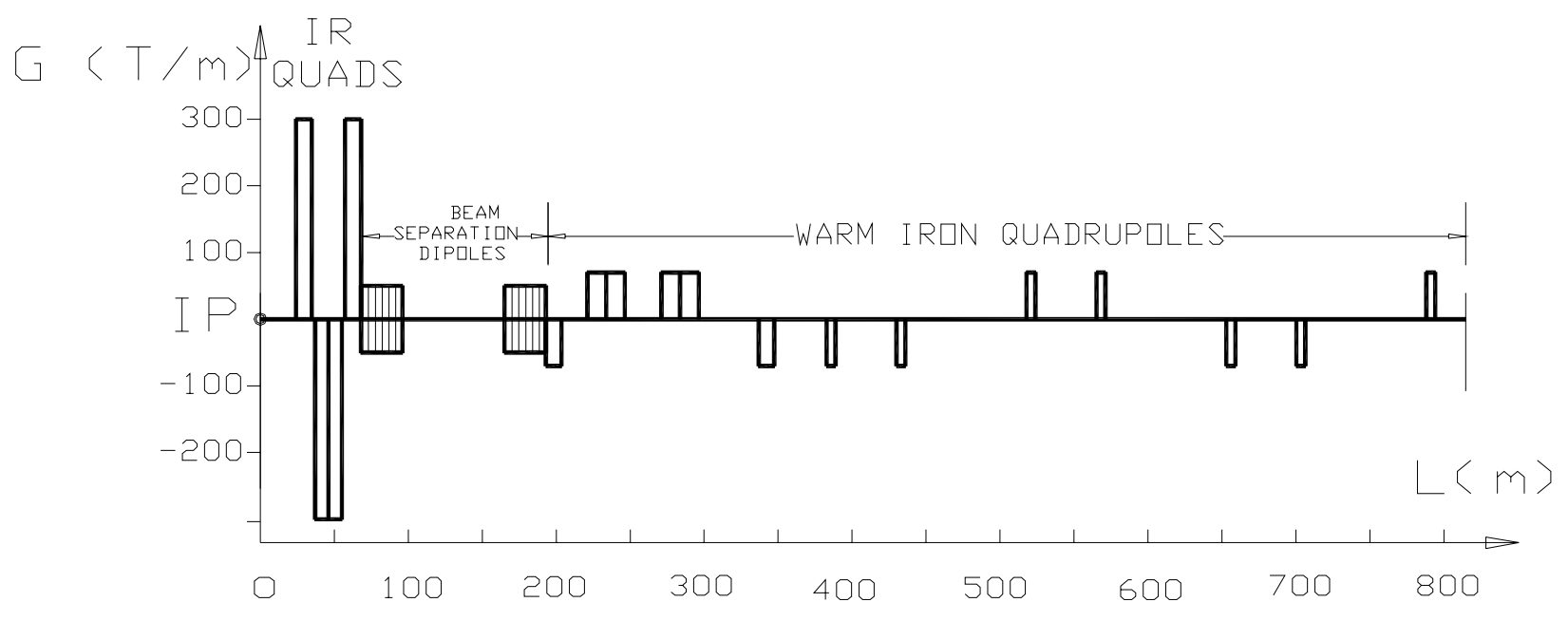

Figure 5.15. Principal magnetic elements for the VLHC-1 interaction region.

\subsubsection{Low-beta IR Quadrupoles}

These magnets form the final focus at the two collision points. The required gradient is $300 \mathrm{~T} / \mathrm{m}$ with a length of 10 meters and a single aperture of $\sim 80 \mathrm{~mm}$. The field quality must be very good because the beams could be separated by as much as $12.5 \mathrm{~mm}$ due to the crossing angle.

A design developed by INFN-LASA for a possible upgrade of the LHC low- $\beta$ quadrupoles, with an aperture of $85 \mathrm{~mm}$ [29], can be used as a starting point to meet these requirements. The basic design as shown in Figure 5.16 has two $\mathrm{Nb}_{3} \mathrm{Sn}$ layers in a $\cos (2 \theta)$ geometry, wound using the cable described in Table 5.6. The cable has a target critical current density of $3000 \mathrm{~A} / \mathrm{mm}^{2}$ at $4.2 \mathrm{~K}, 12 \mathrm{~T}$ [30]. Internal splices can be avoided using the double pancake technique. The main characteristics of the magnetic design are shown in Table 5.7. The field quality of the basic design (aperture $=85 \mathrm{~mm}$ ) gives acceptably low values for the allowed harmonics. The value of the b6, b10 and b14 multipoles, normalized to the gradient and using a $10 \mathrm{~mm}$ reference radius are respectively $1.5 \times 10^{-6}, 1.6 \times 10^{-8}$ and $<1.0 \times 10^{-9}$. The relative field error is lower than $1.1 \times 10^{-5}$, at $12.5 \mathrm{~mm}$ from the center on the midplane. Iron saturation effects remain to be studied. 
Table 5.6. Conductor characteristics for LHC upgrade low-beta quadrupoles.

\begin{tabular}{|l|c|}
\hline Superconductor & $\mathrm{Nb}_{3} \mathrm{Sn}$ \\
\hline Critical current non $\mathrm{Cu}(4.2 \mathrm{~K} 12 \mathrm{~T})$ & $3000 \mathrm{~A} / \mathrm{mm}^{2}$ \\
\hline Strand diameter & $0.825 \mathrm{~mm}$ \\
\hline$\alpha=\mathrm{Cu} /$ nonCu & 1.3 \\
\hline Number of strands & 36 \\
\hline Cable dimension & $(1.34 \leftrightarrow 1.60) \times 15.0 \mathrm{~mm}^{2}$ \\
\hline Effective filament diameter & $<40 \mu \mathrm{m}$ \\
\hline
\end{tabular}
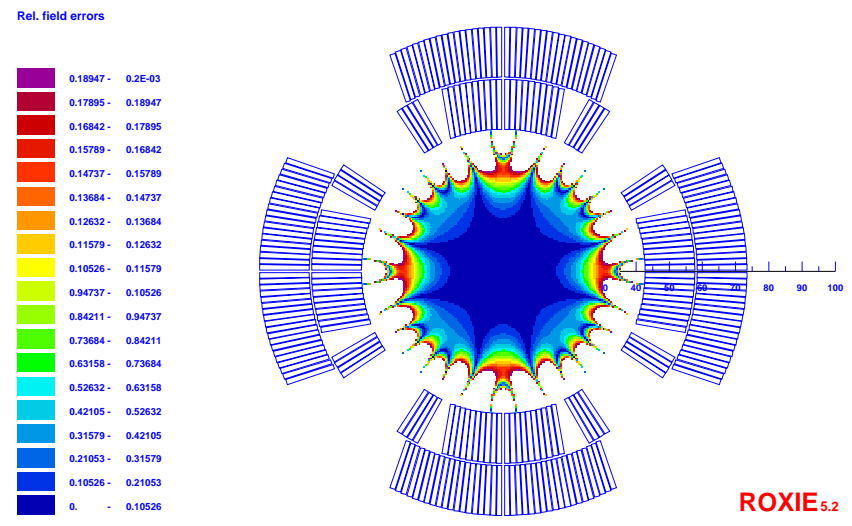

Table 5.7. Magnetic design characteristics at short sample limit.

Figure 5.16. Basic design cross section with field quality at maximum gradient.

Quench protection has been studied at an operating current corresponding to $93 \%$ of the short sample limit. Simulations were performed using a version of the QLASA code [31] modified at Fermilab [32] for the study of $\mathrm{FNAL} \mathrm{Nb}_{3} \mathrm{Sn}$ high field magnets. These studies show that full coil coverage by heaters on both layers is required, and the delay time between the spontaneous quench start and the beginning of the heater-induced quench must be very short. This solution has an impact on magnet technology, because if the insulation of the heaters between the inner and outer layers can not withstand the conductor heat treatment, the double pancake technique must be abandoned. Furthermore, protection redundancy will not be possible (or at least very complicated) and fault scenarios have to be addressed. The short time delay necessary to avoid excessive hot spot temperature and the lack of protection redundancy should be the object of an appropriate R\&D program.

A possible solution for the mechanical design is the one proposed for the upgraded version of the LHC IR low-beta quadrupoles presented in [33]. It uses $20 \mathrm{~mm}$ thick stainless steel collars, which can provide part of the required prestress and magnetic force containment. The rest of prestress and support are provided by a thick skin. The yoke is only in contact with the collars close to the midplanes, in order to transfer the stress from the skin to the coils where it is most necessary. The effectiveness of this design for the higher forces developed in the VLHC IR quadrupoles is still under investigation. A finite element analysis of coils and collars, simulating the rest of the mechanical structure by use of an external load, showed that the maximum stress in the coil can be kept below $160 \mathrm{MPa}$. The design of yoke, skin and contact regions must still be optimized to provide the required support on the coils. 
In summary, it can be concluded that a two layer design using $\mathrm{Nb}_{3} \mathrm{Sn}$ at $4.2 \mathrm{~K}$ is a good candidate to meet the requirements for these magnets, if the critical current target is reached. Optimization will be required for the quench protection and mechanical design.

\subsubsection{Interaction Region Subsystems}

Most of the 1600 meters of the interaction straight section will be in the standard VLHC tunnel. Since the IR magnetic elements need to be "tunable" they require a special gallery to house their power supply and electronics. There will likely be a global alignment system that connects the experiments to the final focussing elements. Finally, the tunnel will need to be wider near the IP to accommodate the extra shielding and vacuum connections and instrumentation associated with the detectors and IP interconnect.

Four independent power systems are required for each side of an interaction region: one for the inner triplet, one for each separation dipole and one for the outer quadrupoles. Additional quadruples in the IR region will likely be powered in series with other straight section quadrupoles in the accelerator and are not included here. The high gradient quadruples require a 25 $\mathrm{kA}, 10$ Volt supply, the conventional separation dipoles require $1 \mathrm{kA}, 100$ Volt supplies while the conventional outer quadrupoles need a $7 \mathrm{kA}, 10$ volt supply. Superconducting correctors will be powered independently with supplies comparable to the outer quadrupole conventional correctors except that quench and lead protection systems will be required.

Superconducting circuits will rely on quench heaters for protection. With the relatively small amount of stored energy (50 MJ) and small number of magnets/circuit (4 or less), an energy extraction circuit and bypass diodes are unnecessary. Instead, in the event of a detected quench, protection heaters on all the magnets in a circuit will be energized and the circuit power converter will be phased off.

As stated, inner triplet quadrupoles will likely need protection levels comparable to or greater than a similarly sized LHC IR quadrupole magnet. Thus 4-8 quench heater circuits plus the requisite electronics are required for each magnet. The total space for power supplies and electronics required for each quench heater circuit is approximately two 6U VME crates. Thus a space of approximately $706 \mathrm{U}$ crates are required for each side/IR.

The IR quadrupoles are cooled by $4.5 \mathrm{~K}$ helium. Since the operating temperature and pressure differs from the transmission lines, they will require separate transfer lines and cryogenic systems. The dynamic heat load is approximately $600 \mathrm{Watts} / \mathrm{side} / \mathrm{IP}$ or $2.4 \mathrm{~kW}$ for both interaction regions [34]. This heat load, if taken directly by the $4.5 \mathrm{~K}$ helium, will require approximately $1 / 2$ of a VLHC- 1 cryo plant. This heat load does not occur at the same time as ramping losses in the Tevatron injector, and thus might be provided for by load shifting from Fermilab's existing Central Helium Liquefier. Impact on the cryogenic system is discussed in 5.2.1. Local heating in the quadrupoles could be quite large due to the strong focusing elements (GL $\sim 3000$ $\mathrm{T}-\mathrm{m} / \mathrm{m}$ ) which sweep charged particles into the cold mass. The helium requirements might be reduced by carrying away some of the heat load by actively cooled absorbers in the $80 \mathrm{~mm}$ beam tubes.

A distribution box will be required in order to make a connection between the room temperature and cryogenic electrical elements, as well as to supply the cryogens to the magnetic elements. This will include voltage taps, strip heater leads, thermometry and other cryogenic instrumentation. 25-30 kA HTS leads would be used to supply the excitation currents. 
LCW and electrical power will be required for the power supplies and conventional magnets. The LCW system will be required to deliver several hundred liters/minute per IR and are included in the totals in Section 5.2.2.4.

A particle absorber system will be required to protect the IR magnets from the excessive radiation from particles produced from the interaction region. Using the LHC as a guide, the system will require a hadron and neutral particle absorber. The primary hadron absorber will be located on the IP side of the inner triplets. The size of the absorber will be comparable to the LHC absorber but will have significantly larger heat loads, estimated to be $2 \mathrm{~kW}$ per IR [35]. Thus an actively cooled absorber will likely be necessary. The neutral absorber, located between the separation dipoles would be necessary to protect the outer quadrupoles, possibly the transmission line magnets and the walls of the tunnel from activation. It, too, will likely need to be actively cooled. If water cooled the requirements would be modest and would be parasitic to the magnet and power supply LCW system.

Alignment tolerances are likely to be comparable to those of the LHC interaction region. Transverse alignment tolerances will thus need to be on the order of 100-300 microns over both sides of the IP. Thus an alignment system will be required to tie together the two sides of the IR and the detector.

\subsubsection{Special Collider Ring Magnets}

This section describes several different magnets installed in the straight sections of the ring. There are a total of 10 straight sections, 5 in each half of the ring. On the Fermilab site there are two Interaction Region straight sections, two Utility straight sections and one Beam Crossing straight section. The straight sections on the opposite side of the collider are basically the mirror reflection of what is on the Fermilab site, except that there are no IRs and minimal utility equipment installed in this area.

Beam focusing in the standard straight sections is provided using a classical FODO cell made of four quadrupoles. The cell half-length is $135 \mathrm{~m}$. The basic lattice is modified for special insertions as described in Section 3.2.1.

Both superconducting [36] and conventional quadrupoles were considered for the straight sections. Conventional water-cooled copper magnets are preferred because of their lower cost and ease of system integration. Warm magnets are more tolerant than superconducting magnets of the beam losses that occur in beam transfer and beam cleaning insertions. No warm-to-cold transitions, gate valves, etc. are required between the quadrupoles and the $\sim 100 \mathrm{~m}$ of warm beam pipe between quads. The compact quadrupoles in both rings can be installed side-by-side at the basic beam-beam spacing of $150 \mathrm{~mm}$ (Figure 5.17), or at non-standard beam spacings as required by special insertions. It easy for extracted beam lines to miss the warm quads due to their low profile and absence of a cryostat. The cooling water system needed for warm quadrupoles is absent in the arcs but is already present in the straight sections to service other systems.

\subsubsection{Straight Section Quadrupole}

This quadrupole uses water-cooled windings and has been designed so that a pair of them can be installed side-by-side in both rings. The $150 \mathrm{~mm}$ beam spacing and the required beam pipe 
size of $22 \mathrm{~mm}$ implies certain restrictions on the achievable quadrupole field gradient. The chosen gradient of $69.3 \mathrm{~T} / \mathrm{m}$ results in the basic quadrupole length of $6.1 \mathrm{~m}$. The preliminary magnetic design indicates that field quality is not going to be a problem since there is plenty of space to make the needed pole shaping. The magnetic field in the steel is acceptable, reaching $1.9 \mathrm{~T}$ at the pole base, but there are regions where the field exceeds $2.0 \mathrm{~T}$ at $70 \mathrm{~T} / \mathrm{m}$, so good quality low carbon steel must be used. Table 5.8 summarizes the quadrupole features.

Table 5.8. Straight section quadrupole parameter list.

\begin{tabular}{|l|r|}
\hline Number Required & 456 \\
\hline Gradient, T/m & 70 \\
\hline Pole diameter mm & 22 \\
\hline Length, m (typical) & 6.1 \\
\hline Width, mm & 148 \\
\hline Height, mm & 168 \\
\hline Number of coils per winding & 4 \\
\hline Number of turns per coil & 12 \\
\hline Conductor cross section area, $\mathrm{mm}^{2}$ & 61.5 \\
\hline Resistance, $\Omega$ & 0.18 \\
\hline Rated current, A & 285 \\
\hline Voltage drop, V & 53 \\
\hline Power consumption, $\mathrm{kW}$ & 15 \\
\hline Conductor hole diameter, $\mathrm{mm}$ & 5 \\
\hline Water temperature rise at $1 \mathrm{MPa}$ (deg. C) & 12 \\
\hline Water flow per quadrupole (l/min) & 17 \\
\hline
\end{tabular}

\subsubsection{Beam Cross-Over Section}

Two beam cross-over regions (Section 3.2.1.3) are needed to equalize the circumference of the two side-by-side rings. To accommodate the magnets in the cross-over region, a short straight section is used that consists of a focusing cell that differs from the standard focusing cell. At the entrance to the cross-over region, the two beams are in the horizontal plane, separated by $150 \mathrm{~mm}$. To make a cross-over, it is necessary to bring the beams together horizontally, separate them vertically before the crossing, and make the opposite transformation after the crossing. The vertical shift of each beam required to eliminate beam-beam interaction effects is $4.5 \mathrm{~mm}$, which results in a beam-beam separation of $9 \mathrm{~mm}$, more than $18 \sigma$. To reduce the number of magnets required for beam manipulation, tilted dipoles are used to bend beams both horizontally and vertically. The cell layout is shown schematically in Figure 5.18. 


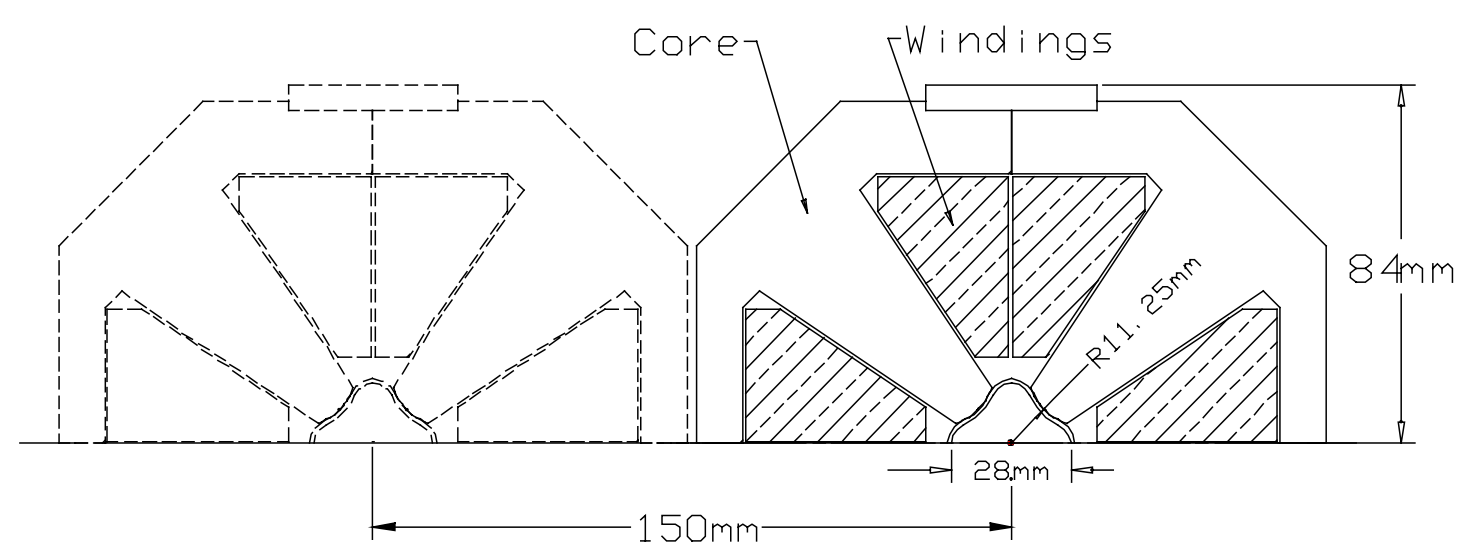

Figure 5.17. The straight section quadrupole is a conventional water-cooled copper design that allows two magnets to be mounted side-by-side with the standard beam separation of $150 \mathrm{~mm}$ or larger.

The magnet length $\mathrm{L}_{\mathrm{m}}$ and tilt angle $\theta$ can be found based on nominal magnetic field and length of gaps between the magnets in the string. As in the arc dipoles, the transmission line is used for magnet excitation. Moreover, because of the large required pole width of the magnet, the return line is also used as an active part of the magnet excitation winding. To minimize iron core saturation effects, a magnetic field of $\mathrm{B}_{\mathrm{m}}=1.95 \mathrm{~T}$ was chosen, as it was for the arc dipoles. With two-meter gaps introduced between the magnets to allow some space for repositioning of the transmission line, this results in a magnet length of $\sim 35 \mathrm{~m}$ and a tilt angle of $\theta \approx \pm 7^{\circ}$.

The total length of the beam cross-over magnetic system is about $150 \mathrm{~m}$. The magnet crosssection is shown in Figure 5.19. The dipole gap is $44 \mathrm{~mm}$ to accommodate (while tilted) the two beams with their vacuum pipes. The pole width provides a good field region for beams separated by $150 \mathrm{~mm}$. Because of the big difference in gap thickness for the pole and flux return regions, the force applied to the cable is significant, up to $2000 \mathrm{~kg} / \mathrm{m}$ for this magnet. Special measures must be taken to strengthen the cable "spider" to the needed level at this location.

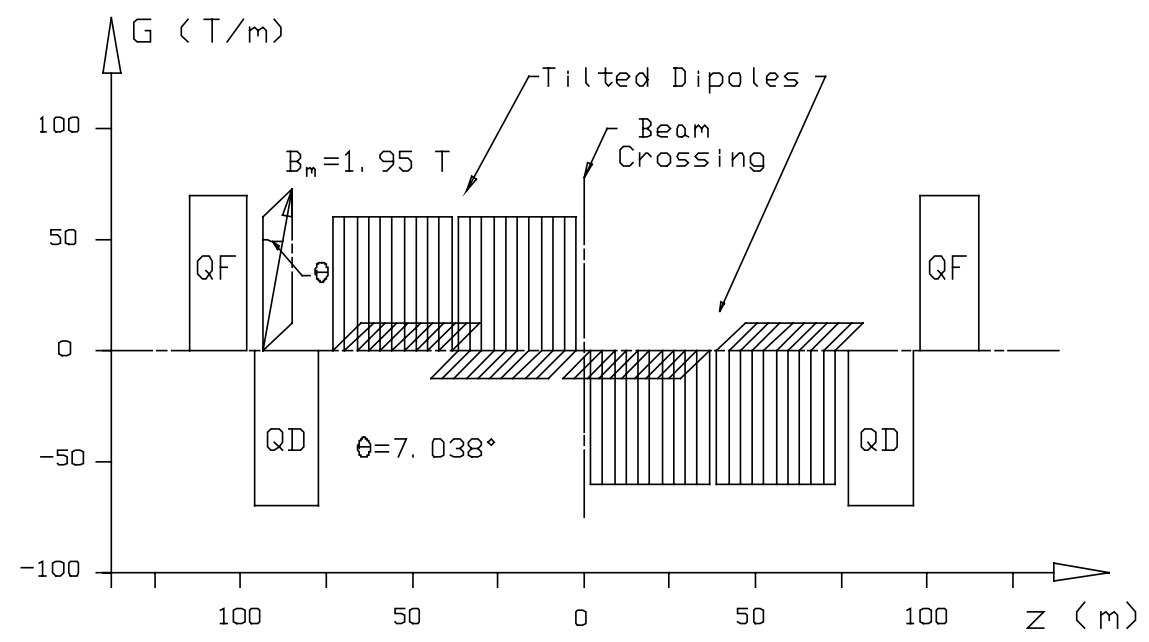

Figure 5.18. Beam cross-over region layout. See also Section 3.2.1.3. 


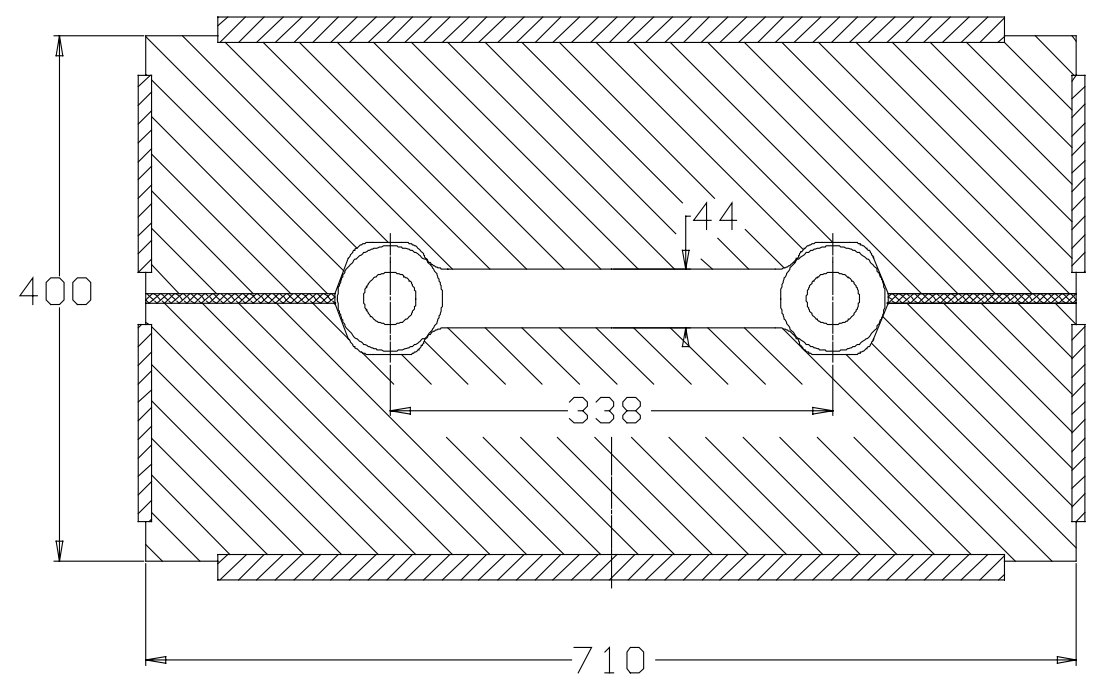

Figure 5.19. Beam crossing dipole. This is energized by the $100 \mathrm{kA}$ transmission line.

To allow a 150-m gap for the tilted magnet installation, the cell focusing was modified using different lengths of standard cell quadrupoles. Quadrupole lengths were limited to $6 \mathrm{~m}$ by water cooling considerations. The total length of quadrupoles installed in both rings for each of the two cross-over sections is $143.6 \mathrm{~m}$.

\subsubsection{Warm-Iron Magnets in the Interaction Region}

As described in Section 5.1.4, the IR straight section requires horizontal beam manipulations to collide the beams at the IP. For this purpose, so called "separation/ recombination" dipoles, as shown in Figure 5.15, are used that bring the two beams together within the low-beta lens string region and place the beams onto the nominal orbits before they go into the regular part of the straight section.

The main transmission line cable is used for excitation of these magnets, and a $1.95 \mathrm{~T}$ maximum magnetic field in the dipoles has been chosen. As it follows from Section 2.1.1, the gap available for installation of the bending magnets is about 125 meters. Leaving some space for the transfer line position manipulation, a magnet length of $28 \mathrm{~m}$ was chosen to insure that a $75-\mathrm{mm}$ beam shift within the 120-m gap in the lattice. Beam displacement inside this dipole is about $12 \mathrm{~mm}$. This displacement must be taken into account when the good field region of the dipoles is being specified.

The two beams, initially separated by $150 \mathrm{~mm}$, are simultaneously bent together by the recombination dipole, whose cross section is similar to the one shown in Figure 5.19. The separation dipole separates beams moving in the opposite directions. Final beam separation at the outer edge of the magnet is $24 \mathrm{~mm}$. To reduce the force applied to the transmission line and to avoid field asymmetry, a symmetrical iron core configuration was chosen. The magnet crosssection is shown in Figure 5.20. The good field region area for this dipole is $35 \times 20 \mathrm{~mm}$, which is enough to accommodate the two shifted beams.

The double-channel separation dipole makes use of both the arc dipole transmission line and current return. The gap height is $26 \mathrm{~mm}$. The maximum force applied to the superconducting cable is about $65 \mathrm{~kg} / \mathrm{m}$ at full field. The magnet cross-section is similar to shown in Figure 5.19 above, except the pole gap and pole width are smaller resulting in a more compact magnet 
with $650 \times 350 \mathrm{~mm}^{2}$ cross-section. The good field area of the dipole is $185 \times 20 \mathrm{~mm}^{2}$, which is slightly larger than necessary for this dipole location. This allows using the same dipole in the utility straight section where a larger beam separation is required.

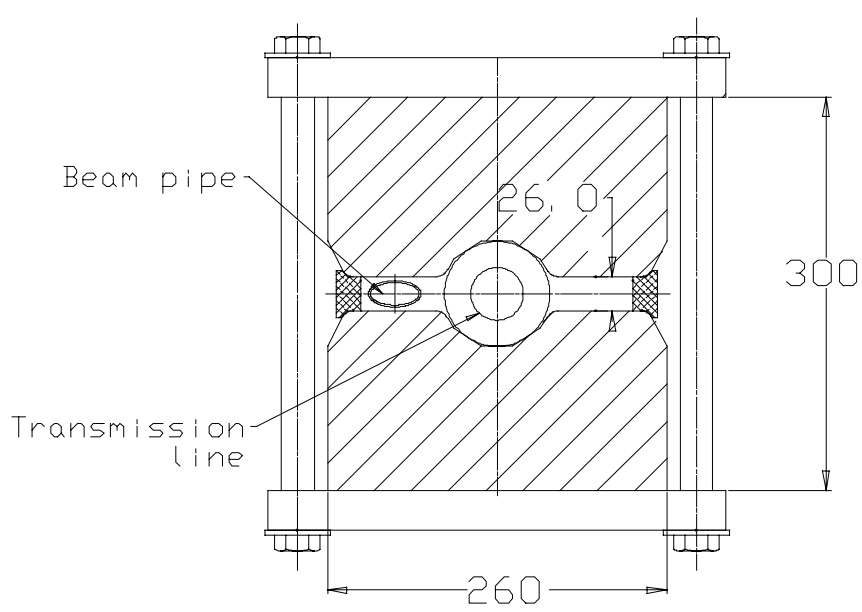

Figure 5.20. Separation/recombination dipole used for the utility straight sections and IRs.

Besides the separation/recombination dipoles, each of the two IR sections requires a set of focusing quadrupoles similar to that described in Section 5.1.5.1. The required strength of these quadrupoles can be found in Table 5.7. The total length of quadrupoles in each of the two FNAL-site IR sections is $445.1 \mathrm{~m}$. Quadrupoles with lengths longer than $6.1 \mathrm{~m}$ will be segmented into two magnets to limit water cooling requirements.

\subsubsection{Utility Section Conventional Magnets}

The utility straight section is introduced into the ring lattice to provide the space needed for various accelerator systems including RF cavities, instrumentation, injection, extraction and abort systems. At the RF cavities the required beam separation is $460 \mathrm{~mm}$. This is accomplished with separation dipoles similar to those used in the IR sections. At the input and the output of the section, these dipoles have an air gap width that accommodates both beams. In the middle of the section, individual separation dipoles are installed side-by-side in both rings. The cross-section of the dipole can be found above (Figure 5.20). The length of the dipoles in the utility section is $25 \mathrm{~m}$, which allows shifting each beam by $155 \mathrm{~mm}$ within the 242-m space as is shown in Figure 5.21 below.

Two types of quadrupoles are used in the Utility section. Most of the quadrupoles are identical to the $70 \mathrm{~T} / \mathrm{m}$ straight section quadrupoles described earlier. The total length of this type of quadrupole used in each of the two FNAL-site utility sections is $208 \mathrm{~m}$. The quadrupole triplet installed between the abort system kicker and Lambertson requires more radial space to accommodate the beam deflected by the kicker. In each of the two FNAL-site utility sections, 58.9 meters of these quadrupoles, described below, are needed. 

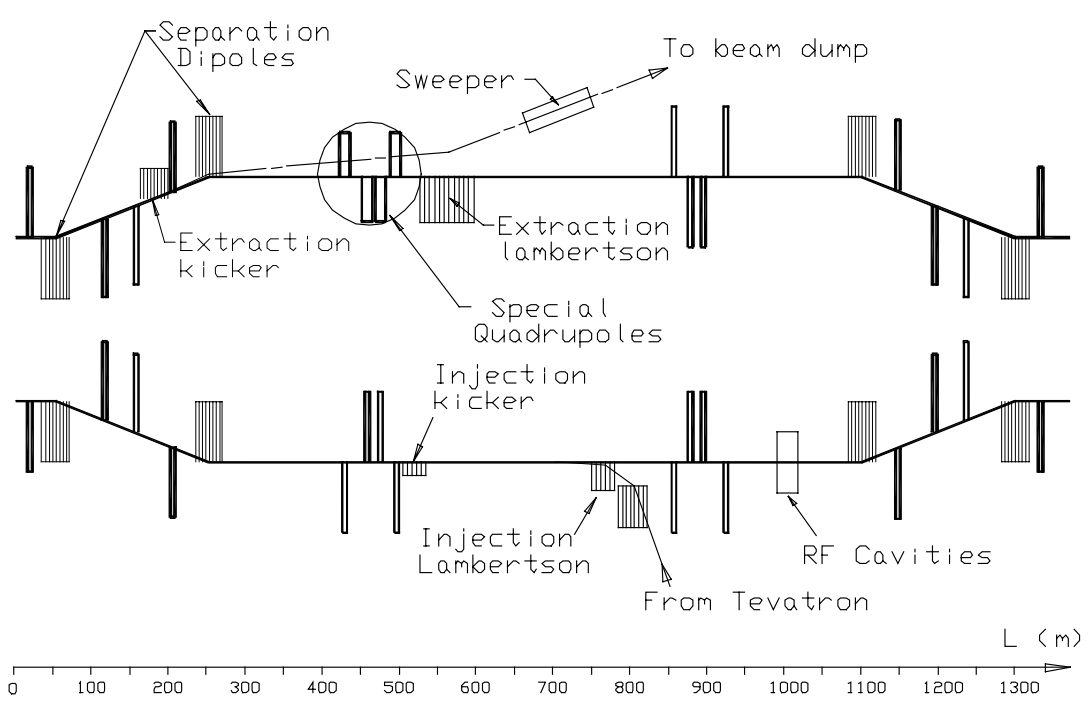

Figure 5.21. Utility straight section including injection, abort, and RF cavities.

\subsubsection{1}

Injection System

The overall injection/extraction scheme of the machine is shown in Figure 5.22. As described in Chapter 4, the Tevatron is run bipolar and is used to inject protons both directions into the collider rings. Beams for both collider rings are extracted using two extraction systems as described in Section 4.3.3. Two identical 3-km transfer lines slope downward at 3\% and end at the corresponding collider downstream utility section where Lambertsons and kickers inject the beam into the VLHC. In both utility sections the beam is injected into the radially inside ring, with the outboard ring reserved for extraction and abort.

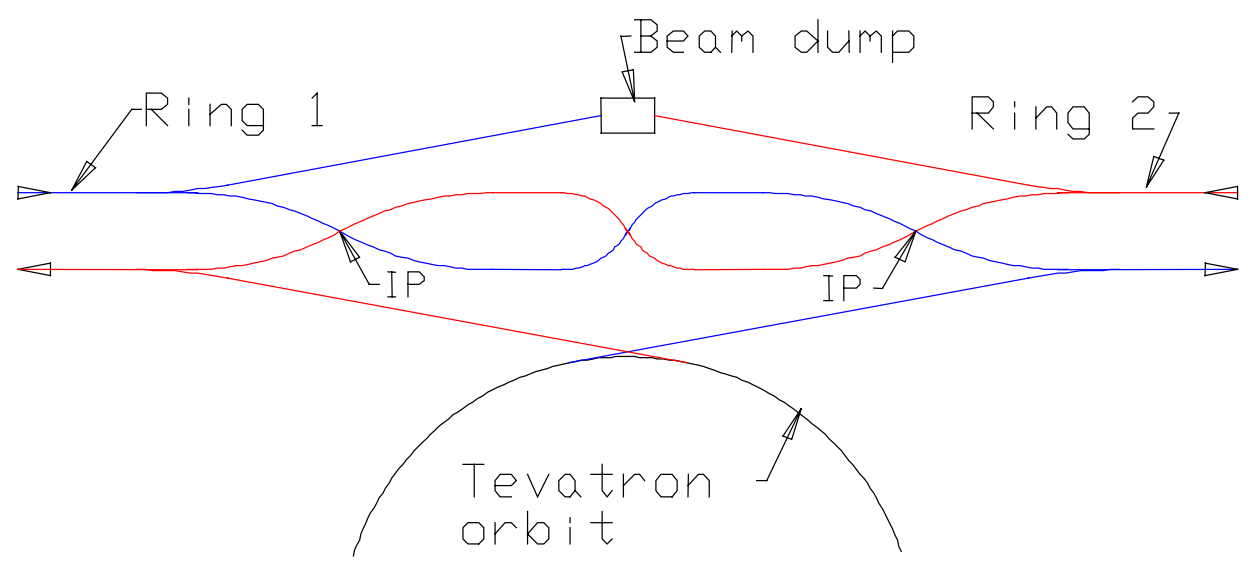

Figure 5.22. Injection and beam abort system scheme.

Downstream of the injection point it will be convenient to have an internal beam absorber for transfer line commissioning and tests which do not involve the arc magnets. The injected beam, after passing through two quad locations to measure position and steering errors, will be deflected $5-10 \mathrm{~cm}$ onto the absorber by a standard corrector magnet. The scope of absorber will be comparable to the Tevatron collider-mode abort. With reasonable intensity limits, air- 
cooling should be sufficient. A few bunches of $10^{9}$ protons are sufficient for a good BPM measurement.

The injection optics fit comfortably into the long 350-m drift in the utility region. The injection uses a full-aperture kick so that the Lambertsons do not restrict the circulating aperture. The layout, working backwards from the injection point, is as follows: six meters of pulsed kicker magnets with a magnetic field of $0.05 \mathrm{~T}$ provide the horizontal angular kick of $0.1 \mathrm{mrad}$ to the injected beam. The horizontal separation between the injected and circulating beams is $25 \mathrm{~mm}$ at the nearest Lambertson 250 meters away. A total of $6 \mathrm{~m}$ of Lambertsons operating at $0.8 \mathrm{~T}$ provide a vertical angular kick of $1.6 \mathrm{mrad}$. The Lambertsons are short enough that an asymmetric design is not needed. The clearance at the nearest quad (100 m away) is $160 \mathrm{~mm}$, large enough to allow unobstructed placement of standard quadrupoles on both the injected and circulating beam lines. The vertical clearance at the RF station (300 m from the Lambertsons) is $480 \mathrm{~mm}$. If desired this separation can be increased using normal dipoles to begin part of the 30-mrad up-bend needed for the slope of the transfer line ramp.

The injection kicker parameters are listed in Table 5.9. The system is composed of six separate modules, each 1-m long. A field strength of $0.05 \mathrm{~T}$ is used for this study, close to that used for SSC injection system [37] and similar kicker magnets at Fermilab. The duration of the kick provided to the injected beam is $\sim 20 \mu \mathrm{sec}$ and is defined by the length of the beam pulse extracted from the Tevatron. The injection kicker requires a magnetic field switching time of 150 ns. This determines the minimum time separation between successive injected batches.

Table 5.9. Injection kicker system parameters.

\begin{tabular}{|l|l|}
\hline Number of modules & 6 \\
\hline Length of Module $(\mathrm{m})$ & 1 \\
\hline Maximum field $(\mathrm{T})$ & 0.05 \\
\hline Rise time $(0$ to $100 \%)(\mathrm{nsec})$ & 150 \\
\hline Fall time $(100$ to $0 \%)(\mathrm{nsec})$ & 150 \\
\hline Impedance $(\Omega)$ & 16.6 \\
\hline Maximum current $(\mathrm{kA})$ & 2.1 \\
\hline Maximum PFN voltage $(\mathrm{kV})$ & 70 \\
\hline
\end{tabular}

Each module is fed by an individual pulse-forming network (PFN). A C-shaped magnet is made from ferrite blocks and with additional capacitors forms a twelve-cell lumped delay line with a characteristic impedance of $16.6 \mathrm{Ohm}$. The ceramic beam pipe has thin resistive coating on the inside surface, and conductive strips for image current on the outside.

Fast timing on both the rising and falling edges is required. This permits "box-car stacking" of a Tevatron batch in between two filled batches. This gives the freedom needed to produce a "smoothed" filling pattern (i.e. one without large low-frequency components that would produce a time-dependent Laslett tune shift). See Section 3.2.5.2. This will require a more complicated pulser than a Thyratron PFN, and will probably require a second Thyratron for the trailing edge. Ripple requirements after the trailing edge will probably require a cleanup kicker similar to one recently installed in the Tevatron. 
The injection Lambertson (Figure 5.23) steers the injected beam into the vicinity of the circulating beam without affecting the circulating orbit. Only a symmetric version is required due to the small length and sagitta. The magnet pole with the field free region is made of a solid piece of annealed, low carbon steel to reduce magnetic field in the field free region. To obtain good vacuum the pole is enclosed in a thin-wall stainless steel shell. The pole gap is fixed with the use of stainless steel spacers. For magnet excitation, conventional copper wire coils are used. Water-cooling can be omitted due to the low duty cycle (4-second flat top with a 40second cycle time). The main parameters of the injection Lambertson are summarized in Table 5.10 .

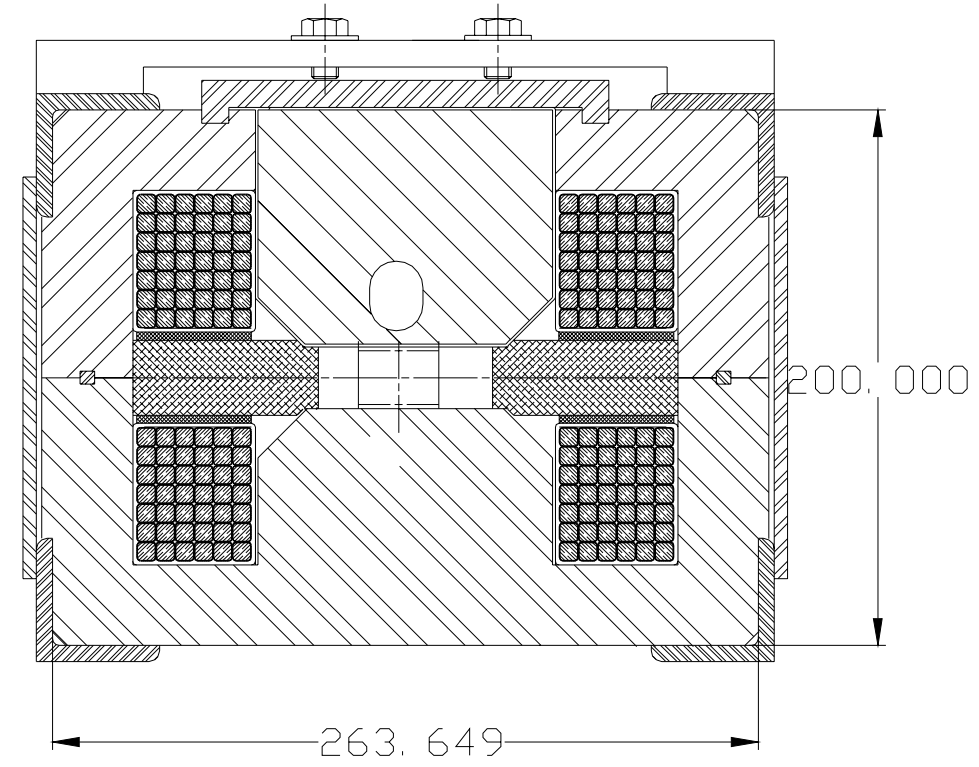

Figure 5.23. Injection Lambertson magnet.

Table 5.10. Parameter list for the injection Lambertson magnet.

\begin{tabular}{|l|r|}
\hline Magnetic field, $\mathrm{T}$ & 0.8 \\
\hline Pole spacing, $\mathrm{mm}$ & 25 \\
\hline Length, $\mathrm{m}$ & 4 \\
\hline Width, $\mathrm{mm}$ & 288 \\
\hline Height, $\mathrm{mm}$ & 260 \\
\hline Number of coils per winding & 2 \\
\hline Number of turns per coil & 42 \\
\hline Conductor cross section area, $\mathrm{mm}^{2}$ & 42 \\
\hline Resistance, $\Omega$ & 0.32 \\
\hline Rated current, $\mathrm{A}$ & 208 \\
\hline Voltage drop, $\mathrm{V}$ & 70 \\
\hline Peak power consumption, $\mathrm{kW}$ & 15 \\
\hline Average power consumption, $\mathrm{kW}$ & 1.25 \\
\hline Magnet surface temperature rise, ${ }^{\circ} \mathrm{C}$ & 20 \\
\hline
\end{tabular}

Transfer line dipole magnets, similar to the Lambertsons but without the field free region, have been designed. The coil cross-section is the same. Because the pole width can be made 
significantly smaller, and because no vacuum shell is required for this magnet, it is even more compact: $180 \times 180 \mathrm{~mm} 2$ in cross-section with pole gap of $25 \mathrm{~mm}$. The rest of the magnet parameters are very close to those shown in Table 5.10.

In addition to vertically bending magnets, the transfer line may require horizontal bending with an estimated total angle of $80 \mathrm{mrad}$ (depending on siting details). This bend can be made using the same type of magnets. The total dipole length required for this bend in both transfer lines is about $300 \mathrm{~m}$.

There are 86 quadrupoles installed in the transfer line. Most of them have a gradient of $40 \mathrm{~T} / \mathrm{m}$ and a length of $1 \mathrm{~m}$. Using a design similar to described in Section 5.1.5.1, and intermittent powering of about $0.8 \mathrm{~kW}$ peak power per lens, we can employ air cooling. This results in about $10{ }^{\circ} \mathrm{C}$ temperature rise at the surface of the magnet.

For this study, conventional magnets have been chosen to establish a reference point for future research work with the goal of building the transfer channel using only permanent magnet based equipment. Although this technique was well studied during fabrication of the Fermilab Recycler Ring, the requirement of having adjustable bending and focusing strength makes it difficult to scale the system cost without making first a thorough design study of this approach. An encouraging start to this study has been undertaken in [38].

\subsection{Beam Abort Magnets}

The abort magnet system (Figure 5.55) consists of a kicker magnet, a Lambertson, a beam sweeper magnet and defocusing quadrupoles. The beam absorber design and other system issues are discussed in Section 5.3.2. The circulating beam goes through the field free area of the Lambertson magnet. The beam is kicked radially outward into the bend channel of the Lambertson magnet, which deflects it vertically upward into the beam dump channel. The abort channel is equipped with a beam sweeper magnet to spread the energy on the graphite absorber.

As the machine ramps, the magnetic field of the abort kicker, Lambertson and sweeper magnet must track the beam energy. For the Lambertson this is accomplished automatically by energizing it with the transmission line current. The abort kicker and sweeper systems must rely on active systems to monitor the capacitor charging voltage and force an abort if it starts to go out of tolerance.

Abort Kicker Magnets switch the beam between the circulating orbit and the abort channel. Their strength is chosen to provide the needed beam separation of $17 \mathrm{~mm}$ at the entrance to the abort Lambertson located $300 \mathrm{~m}$ downstream. Assuming a kicker field strength of $0.2 \mathrm{~T}$, the required length is $30 \mathrm{~m}$. The cross-section of the kicker is shown in Figure 5.24.

The current rise time in the extraction kicker is about $3 \mu \mathrm{sec}$, so a beam abort gap of about $3 \mu \mathrm{sec}$ is needed. This requirement results in using a medium-high frequency magnetic material for magnet core fabrication. Mn-Zn MN8CX (Ceramic Magnetics) ferrite was chosen for this study. The core is made of ferrite blocks and assembled using epoxy bonding after the windings and ceramic vacuum pipe are installed inside. At nominal field of $0.2 \mathrm{~T}$ the current is $2410 \mathrm{~A}$. Maximum field in the core is about $0.45 \mathrm{~T}$, which is below the material saturation level. Pole width was optimized using pole profiling by attaching 1-mm ferrite stripes near the pole wedges. With this shaping, field uniformity in the entire vacuum pipe region is within $0.1 \%$, more than good enough for this device. In the configuration shown, the magnet inductance is 
$2.5 \mu \mathrm{H} / \mathrm{m}$. Ten $3-\mathrm{m}$ sections are used, installed one following another, with each section having $7.5 \mu \mathrm{H}$ inductance and $2.5 \mathrm{ohm}$ impedance. Each section is fed independently by its own pulser.

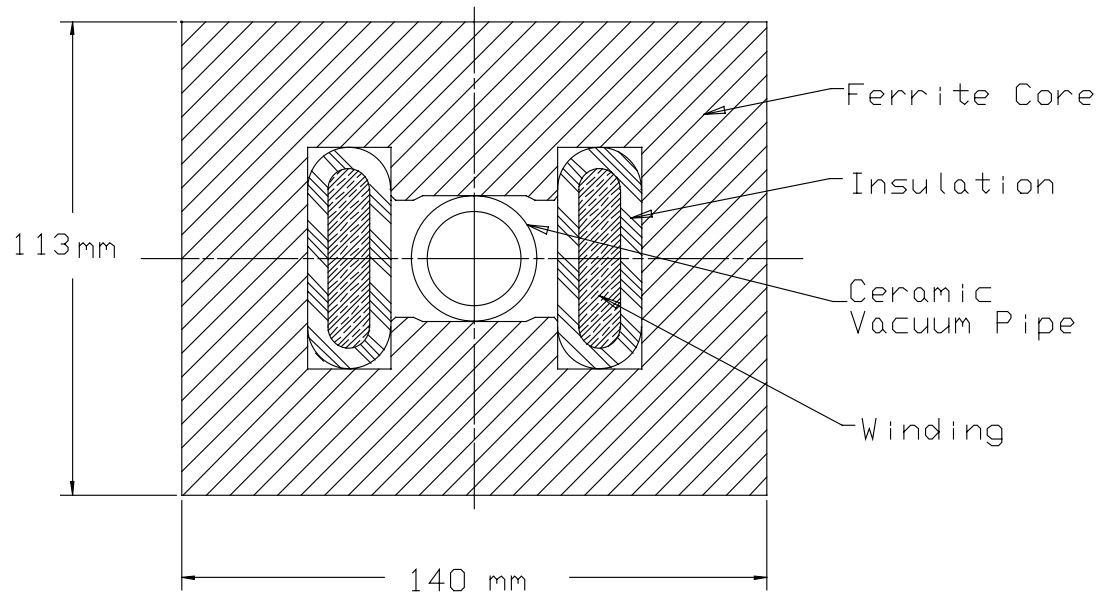

Figure 5.24. Abort system kicker magnet.

The abort kicker pulser uses a two-stage scheme. The first stage delivers high voltage for a fast current rise time. Capacitor discharge is used at this stage. Then, in the second stage, a long SCR-generated pulse with much lower voltage is used to support the flat top level. For the three-meter section, a discharge capacitor with $\mathrm{C}=0.5 \mu \mathrm{F}$ provides the needed current rise time. This results in maximum voltage at the first stage of $9600 \mathrm{~V}$. Energy stored in the capacitor bank or in the magnetic field is about $25 \mathrm{~J}$. The flat top length for the abort kicker magnet is defined by the ring circumference and is about $800 \mu \mathrm{sec}$. To have this long pulse shape, a separate power source must be switched on when current in the primary circuit starts to decay. This is a typical long-pulse pulser implementation (see, for example, [37]).

Large Aperture Quadrupoles are required between the Abort Kicker and the Lambertson to allow for the 17-mm radial displacement at the Lambertson (see Figure 5.21). This larger aperture means that a lower field gradient of about $45 \mathrm{~T} / \mathrm{m}$ can be achieved, and longer quadrupole lengths are required for focusing. A longer quadrupole results in higher power consumption and cooling water temperature rise. Using two 5-meter quadrupoles instead of one 10-meter, it is possible to have a lower water temperature rise at the expense of higher total water flux. Table 5.11 summarizes the large aperture quadrupole parameters.

Abort Lambertson Magnets use the superconducting transmission line as an excitation winding. This ensures that at each moment the magnetic field is proportional to the beam energy. The magnet cross-section is shown in Figure 5.25, and its main parameters are listed in Table 5.12. The magnet part that includes field free beam channels is placed inside a stainless steel vacuum box. Only one field free channel is used in this device; another channel has been added to obtain needed symmetry. The tolerable magnetic field in the field free area limits the maximum field in Lambertson magnet. The deflecting gap field value of $0.8 \mathrm{~T}$ was chosen to have field in the field free region on the level of 20 Gauss. The 67-m total length of the Lambertson gives the beam a kick of $0.8 \mathrm{mrad}$ that results in more than $200 \mathrm{~mm}$ of beam separation at the downstream quadrupole and horizontal dipole. Magnetic forces on the transmission line 
conductor are about $25 \mathrm{~kg} / \mathrm{m}$. This force can be handled by the standard transmission line support spider.

Table 5.11. Large aperture quadrupole parameters.

\begin{tabular}{|l|r|}
\hline Number Required (total both rings) & 8 \\
\hline Aperture radius, mm & 20 \\
\hline Gradient, T/m & 45 \\
\hline Length, $\mathrm{m}$ & 5 \\
\hline Quadrupole width, mm & 260 \\
\hline Quadrupole height, mm & 260 \\
\hline Number of coils per winding & 4 \\
\hline Number of turns per coil & 12 \\
\hline Conductor size, mm & 11.7 \\
\hline Conductor cross section area, $\mathrm{mm}^{2}$ & 104 \\
\hline Resistance, $\Omega$ & 0.091 \\
\hline Rated current, A & 625 \\
\hline Voltage drop, V & 61 \\
\hline Power consumption, $\mathrm{kW}$ & 38 \\
\hline Water Hole diameter, $\mathrm{mm}$ & 6.35 \\
\hline Water pressure drop, $\mathrm{MPa}$ & 1 \\
\hline Cooling circuits per winding & 8 \\
\hline Water temperature rise $\left({ }^{\circ} \mathrm{C}\right)$ & 16 \\
\hline Water flow per magnet $($ liter $/ \mathrm{min})$ & 35 \\
\hline
\end{tabular}

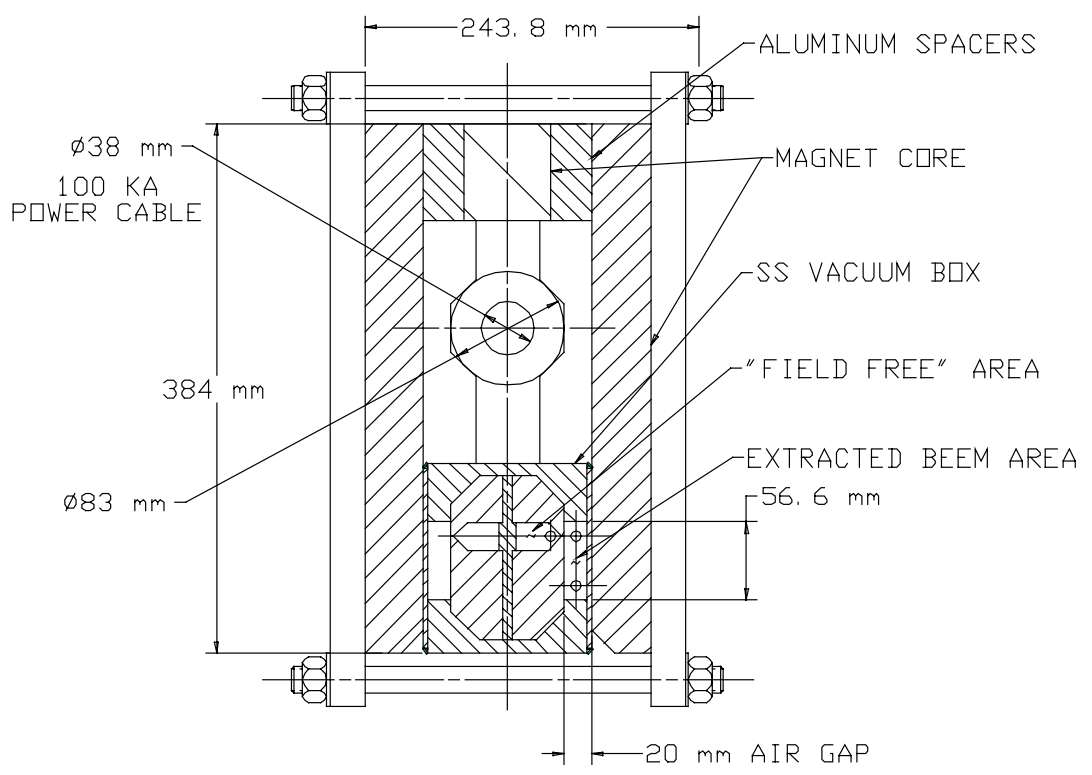

Figure 5.25. The abort Lambertson magnet. This is energized by the transmission line so that it automatically tracks the beam energy. 
Table 5.12. Main parameters of the abort system Lambertson magnet

\begin{tabular}{|l|r|}
\hline Maximum field, T & 0.8 \\
\hline Total length, m & 67 \\
\hline Air gap, mm & 20 \\
\hline Field homogeneity, \% & 0.1 \\
\hline Maximum field in the hole, Gauss & 20 \\
\hline Maximum current, kA & 100 \\
\hline Weight, kg/m & 330 \\
\hline
\end{tabular}

Abort Sweeping Magnets are introduced to reduce heating of the graphite absorber block by distributing the beam energy across a larger dump surface area. See Section 5.3.2. Although reliability of this sweeping system must be high, the field quality requirements for sweeping magnets can be quite modest, allowing about $10 \%$ field nonuniformity in the sweeping magnets. Sweeping magnets deflect the beam in the $\mathrm{X}$ and $\mathrm{Y}$ directions with amplitudes that correspond to the beam striking the dump block at a location close to its radial dimension. If the phase difference between $\mathrm{X}$ and $\mathrm{Y}$ oscillations is $90^{\circ}$, the beam projection on the dump surface will move along a circular path. The velocity of this movement must be high enough to allow beam circling around the block at least twice during the extraction time, $\sim 800 \mu \mathrm{sec}$. This requires a beam oscillation frequency of $\sim 2 \mathrm{kHz}$. The integrated strength of the sweeping magnets must be about $12 \mathrm{~T} \cdot \mathrm{m}$ in each coordinate. A field strength of $0.3 \mathrm{~T}$ was chosen for this device, resulting in a $40 \mathrm{~m}$ magnet. The required aperture is about $50 \mathrm{~mm}$ after taking into account possible steering mistakes due to kicker malfunction and unpredictable beam position in cases of emergency extraction. To make the system simple and reliable, the two steering magnets are combined in a single unit, as shown in Figure 5.26. Field uniformity in this magnet is at an acceptable level of about $5 \%$ at reference radius of $15 \mathrm{~mm}$. A positive sextupole component in the whole aperture results in additional beam defocusing on the target. The magnet yoke is made of standard toroidal iron powder cores (e.g. T520-40D by Micrometals). Core effective permeability is 60 to 150 in the frequency range up to $100 \mathrm{kHz}$ and in the flux density ranges up to $1 \mathrm{~T}$. The main parameters of the magnet are summarized in Table 5.13.

Table 5.13. Parameters of the sweeping magnet.

\begin{tabular}{|l|l|c|}
\hline Parameter & Unit & Value \\
\hline Magnetic field & $\mathrm{T}$ & 0.3 \\
\hline Magnet length (total) & $\mathrm{m}$ & 40 \\
\hline Maximum field in the core & $\mathrm{T}$ & 0.45 \\
\hline Stored energy / meter & $\mathrm{J} / \mathrm{m}$ & 160 \\
\hline Number of turns & & $2 \times 8$ \\
\hline Wire diameter & $\mathrm{mm}$ & 5 \\
\hline Maximum current & $\mathrm{A}$ & 2000 \\
\hline Inductance & $\mu \mathrm{H}$ & $2 \times 80$ \\
\hline
\end{tabular}




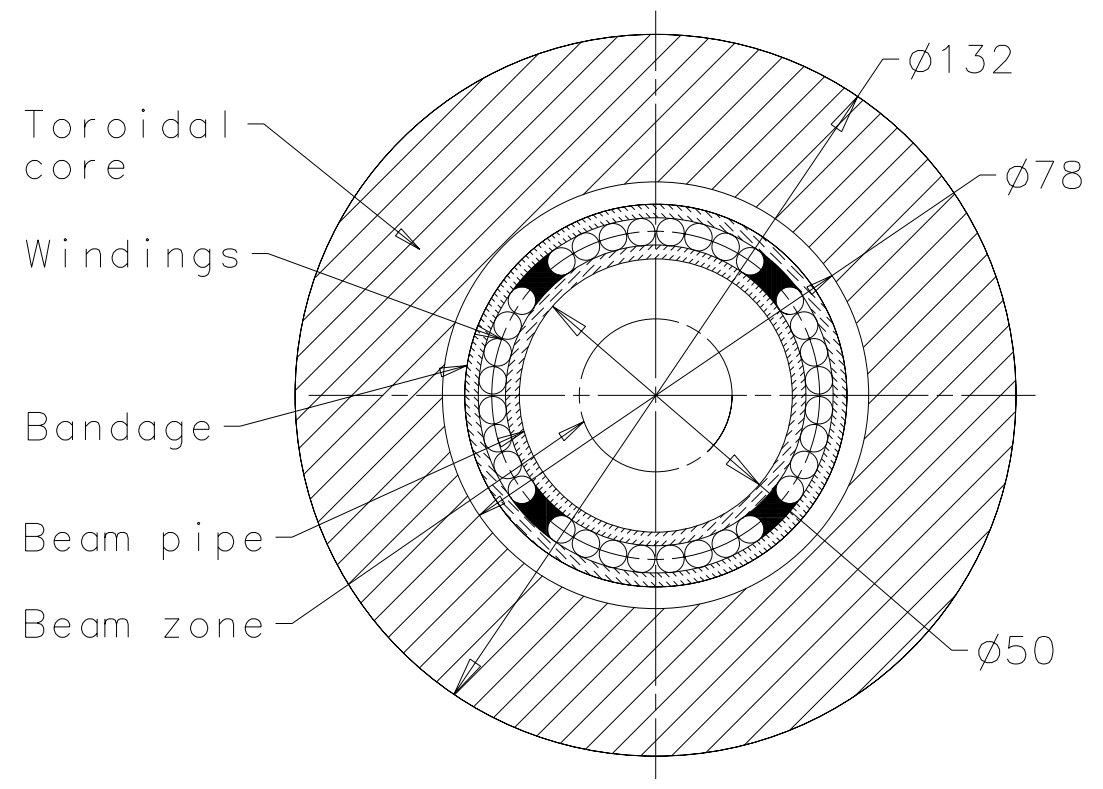

Figure 5.26. Sweeping magnet cross-section.

For equipment safety reasons the sweeping magnet is made of 10 separate 4-m sections powered by independent pulsers. The simultaneous failure of two sections, which is a very low probability event, will not compromize the safety of the absorber core. For each section, the current in the magnet windings is supplied by a simple pulser that discharges a capacitor bank into the windings. For each 4-meter section, the capacitance required to have an oscillation frequency of $2000 \mathrm{~Hz}$ is about $20 \mu \mathrm{F}$. With a circuit impedance of $4 \Omega$ and resistance of windings at $2 \mathrm{kHz}$ of about $0.1 \Omega$, one can expect periodic oscillations with a rather low rate of current decay. If desired, it is possible to increase the decay rate by adding some resistance to the circuit to ensure a better beam energy distribution across the dump block surface. Peak voltage is about $8 \mathrm{kV}$, requiring at least a $12 \mathrm{kV}$ rating on the windings and capacitor bank to ensure high reliability of the system. SCRs with conservative ratings can be used at these values of voltage, current, and repetition rate.

\subsubsection{Magnet Production}

Construction of the Stage-1 VLHC will require the fabrication of several thousand magnets. The largest and most numerous of these are the combined function arc magnets. They, along with the dispersion suppressor dipoles, represent the majority of magnet costs. They are also the most technically challenging as they are a significant departure from existing magnet technology, and because of their extreme length they require unique assembly procedures and facilities. All of the other required magnets are variations of magnets previously produced for other accelerators. They do not present any significant technical challenge, as fabrication methods are well understood and a broad experience base exists at various facilities within the labs and in private industry. A breakdown of magnet type and quantities is shown in Table 5.14 . 
Table 5.14. Magnets required for the Stage-1 VLHC.

\begin{tabular}{|l|c|c|}
\hline \multicolumn{1}{|c|}{ Magnet Type } & Physical Length, m & Quantity Required \\
\hline Main Arc Dipole & 67.75 & 3136 \\
\hline Dispersion Suppressor Dipole & 48.8 & 160 \\
\hline Straight Section Quadrupole & 6.0 typ. & 456 \\
\hline Quadrupole, Large Aperture & 10.0 & 8 \\
\hline Low Beta Quadrupole & 10.0 & $\sim 20$ \\
\hline Special Warm-Bore Magnets & Various & 3296 \\
\hline Dipole Corrector & 0.5 & 3296 \\
\hline Quadrupole Corrector & 0.5 & 3296 \\
\hline Sextupole Corrector & 0.8 & 16 \\
\hline
\end{tabular}

The extreme length of the combined function and dispersion suppressor dipole magnets makes the fabrication process unique. The obvious problems associated with the transport of these long devices make it necessary for the final assembly of the magnets to occur in a building strategically located to facilitate delivery of the completed and tested magnets directly to the accelerator tunnel. Two production facilities are envisioned. One facility will be located adjacent to the injection transfer line with the other remotely located on the opposite side of the ring. Magnet fabrication is projected to take place over approximately six years.

The facilities are considered assembly centers rather than manufacturing sites. The magnet components will be fabricated at subcontractor facilities off site to the highest level of completeness (value added) possible, considering that the components will need to be transported to the final assembly site. Constraints have been applied to the maximum length of component parts and subassemblies so as not to prohibit fabrication at remote locations around the country or overseas. Twelve meters was chosen, as it is the standard for shipboard containers and can easily be trucked over roadways without the need for special permits and vehicles. The on site assembly facilities will do only that work that cannot be done off site given the above constraints.

In any large-scale production program, a thorough quality assurance program must be carried out during all stages of manufacture. Inspection of parts upon receipt, testing of components where needed, and statistical sampling techniques will be employed to ensure that specifications are met. Warm magnetic measurements will be performed on all magnets as part of the QA program. Cold tests will be performed on all of the initial, low rate arc dipole production magnets and will continue during full production at rates determined by the test capacity. A discussion of magnet measurement and testing can be found in Section 5.1.7.

In this section we will concentrate on fabrication of the arc dipoles; they are a cost driver and present some unique requirements, including the superconducting transmission line. The facilities design, dominated by the $65-\mathrm{m}$ length of the final magnet assemblies, is discussed first. Factory throughput, labor required, and fabrication details associated with the arc dipole magnet are then proposed. Fabrication of the iron and structural assembly is discussed as well. Costs and schedules are covered elsewhere. 


\subsubsection{Production Facilities}

The fabrication of the transmission line magnets (combined function and dispersion suppressor dipole) takes place in two separate custom facilities each with an approximate size of 210 meters by 140 meters. A floor plan of the building is shown in Figure 5.27. Each facility has three distinct areas: 1) component storage, 2) assembly and test, 3) storage and staging into the tunnel.

Component storage. The facility will be used as the terminus for components fabricated off site. These components range from 12-m long iron core subassemblies to individual small parts. The space is sufficient to house stock for at least three months of production. The actual amount of storage needed will depend on vendor location and capabilities. In addition to bulk storage, space is allocated for staging (kitting) components at the various assembly area stations.

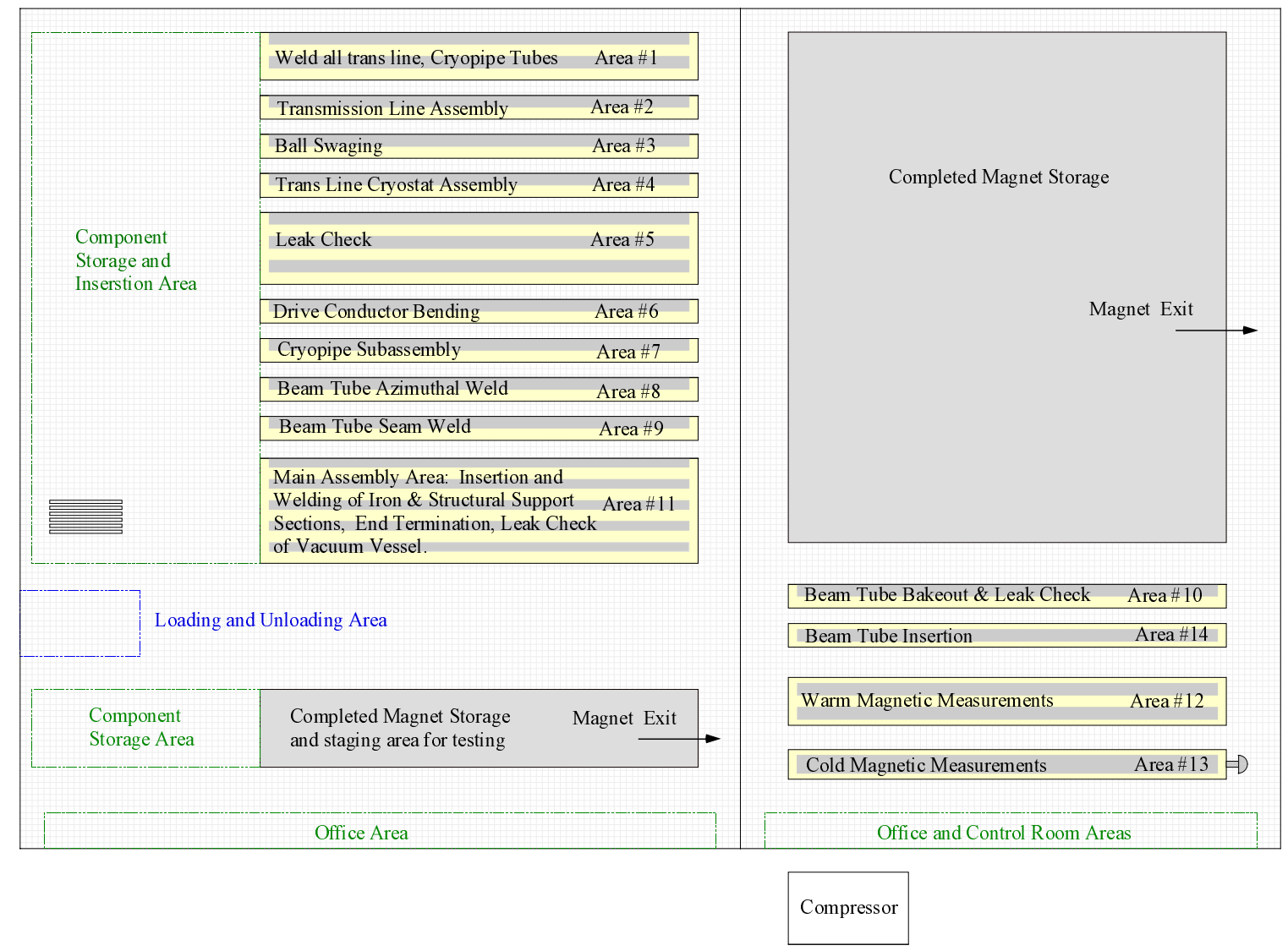

Figure 5.27. Transmission line magnet assembly factory floor plan.

Assembly and test area. The bulk of the facility is dedicated to magnet assembly. There are fourteen "areas," each devoted to a specific step in the fabrication process. Each fabrication area includes one or more stations needed to achieve the required throughput for a specific operation. Each area will work autonomously and have the capability of locally storing one week's production, so that glitches in one manufacturing step do not immediately halt the production pipeline. The facility will be manned 24 hours per day (three shifts) five days per week. 
Two shifts (shifts 2 and 3) will do production at the assembly stations. One shift (shift 1) will be used exclusively to move product between the various stations, stage components at the stations and provide routine maintenance. The output of each facility is one magnet per day. Table 5.15 lists each area, its function, number of stations required, and a list of the tooling necessary to accomplish the stated function. A more detailed description of the tooling necessary is included in reference [39].

Table 5.15. Assembly area and tooling summary.

\begin{tabular}{|c|c|c|c|}
\hline $\begin{array}{l}\text { Assy } \\
\text { Area }\end{array}$ & $\begin{array}{l}\text { Stations } \\
\text { Reqd }\end{array}$ & Function & Special Tooling Required \\
\hline Area 1 & 2 & $\begin{array}{l}\text { Tube subassembly: Weld } 12 \mathrm{~m} \text { tubes segments together to make a } \\
68 \mathrm{~m} \text { tube: eight varieties, trim ends six varieties to final length }\end{array}$ & $\begin{array}{l}\text { (1) storage rack, (2) welding machines, (40) } \\
\text { welding heads, (1) part staging table, (22) support } \\
\text { stands, (2) cutoff saws. }\end{array}$ \\
\hline Area 2 & 1 & $\begin{array}{l}\text { Transmission line subassembly: Install copper braid over inner tube, } \\
\text { install SC braid over copper braid, install outer tube segments over } \\
\text { cable braid }\end{array}$ & $\begin{array}{l}\text { (1) storage rack, (44) support stands, (1) part } \\
\text { staging table, (1) conductor spooler, (1) outer } \\
\text { swage }\end{array}$ \\
\hline Area 3 & 1 & $\begin{array}{l}\text { Transmission line subassembly: Expand inner tube using ball } \\
\text { swaging, }\end{array}$ & (1) storage rack, (44) support stands, (1) ball swage \\
\hline Area 4 & 1 & $\begin{array}{l}\text { Transmission line drive conductor cryostat subassembly: using } \\
\text { transmission line produced at station 3, install suspension spiders, } \\
\text { install shield segments, install shield cooling tubes, install } \\
\text { superinsulation, install vacuum tube segments. }\end{array}$ & $\begin{array}{l}\text { (1) storage rack, (44) support stands, (1) 70K tube } \\
\text { straightened, (1) MLI applicator, (1) part staging } \\
\text { table, (1) tube installer }\end{array}$ \\
\hline Area 5 & 3 & Leak check of welded tubes produced at station 1 & $\begin{array}{l}\text { (6) leak check carts, (1) storage rack, (1) manifold } \\
\text { and adapters. }\end{array}$ \\
\hline Area 6 & 1 & Drive conductor bender: bend end of conductor into dogleg shape & $\begin{array}{l}\text { (1) storage rack, (1) support stand, (2) bending } \\
\text { frames }\end{array}$ \\
\hline Area 7 & 1 & $\begin{array}{l}\text { Cryopipe subassembly: merge cryo pipes, return transmission line } \\
\text { subassembly, support spiders, shield segments, MLI blankets }\end{array}$ & $\begin{array}{l}\text { (1) storage rack, (44) support stands, (1) blanket } \\
\text { handler }\end{array}$ \\
\hline Area 8 & 1 & $\begin{array}{l}\text { Vacuum tube subassembly: weld } 12 \mathrm{~m} \text { extrusion pieces together to } \\
\text { make } 68 \mathrm{~m} \text { lengths }\end{array}$ & $\begin{array}{l}\text { (1) storage rack, (1) staging table, (5) welding } \\
\text { machines, (44) support/alignment stands. }\end{array}$ \\
\hline Area 9 & 1 & $\begin{array}{l}\text { Vacuum tube seam welding: install getter pumps, seam weld } \\
\text { together half tubes produced at station } 12 \text {, weld end adapters }\end{array}$ & $\begin{array}{l}\text { (1) storage rack, (1) assembly table, ( 1) seam } \\
\text { welder, (2) end welder, (2) cut off saws }\end{array}$ \\
\hline Area 10 & 1 & $\begin{array}{l}\text { Leak check station: bake out and leak check completed vacuum } \\
\text { pipe }\end{array}$ & $\begin{array}{l}\text { (1) storage rack, (2) leak check carts, (1) bake out } \\
\text { insulator, (44) support stands }\end{array}$ \\
\hline Area 11 & 5 & $\begin{array}{l}\text { Main assembly station: a) merge drive and return transmission line } \\
\text { subassemblies, iron subassemblies, cryo subassembly; align and } \\
\text { weld vacuum vessel. b) end assembly, connect promary } \\
\text { transmission line shield cooling line to 70K header, assemble end } \\
\text { componenets, fit and weld end vacuum vessel. c) leak check of } \\
\text { completed vacuum vessel. }\end{array}$ & $\begin{array}{l}\text { a): (1) iron staging and transport, (44) support } \\
\text { stands, (5) welding machines, (15) temporary end } \\
\text { supports b): (1) support stand, (1) welding } \\
\text { machine, (1) alignment jig c): (1) leak check cart, } \\
\text { (1) adapter fitting }\end{array}$ \\
\hline Area 12 & 2 & $\begin{array}{l}\text { Warm measurement stand: install probe assembly and power } \\
\text { magnet, measure and record field }\end{array}$ & $\begin{array}{l}\text { (2) probe insertion alignment fixtures, (1) power } \\
\text { supply, (1) DAC system }\end{array}$ \\
\hline Area 13 & 1 & $\begin{array}{l}\text { Cold measurement stand: install cryo lines to feed and return boxes, } \\
\text { install probe assemble, power magnet, measure and record field }\end{array}$ & $\begin{array}{l}\text { (1) feed box, (1) return box, (2) probe insertion } \\
\text { alignment fixtures, (1) power supply, (1) DAC } \\
\text { system, (1) refrigeration system }\end{array}$ \\
\hline Area 14 & 1 & $\begin{array}{l}\text { install beam tube assemblies: comprees beam tube and insert in pole } \\
\text { gap, secure in place }\end{array}$ & (1) storage rack, (2) compression insertion fixtures \\
\hline
\end{tabular}

The factory will have special handling requirements. Since the magnets and their subassemblies are $65 \mathrm{~m}$ long, and will need to be transported laterally between areas, no columns can be located within the primary assembly area. A custom hoist system is required to lift and transport the long subassemblies between the various assembly area stations. Storage areas, associated with each assembly area, will be needed to house finished subassemblies before 
moving to the next higher-level assembly area. Most stations will require a staging area where the various component parts for the day's production are marshaled. Figure 5.28 shows, schematically, an elevation of a section of the plant, showing the workstations in nine areas.

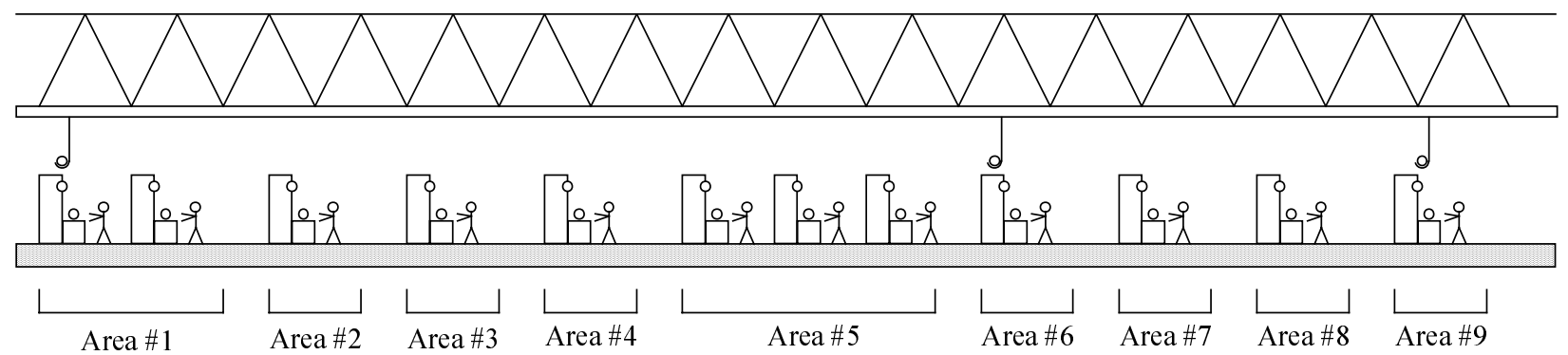

Figure 5.28. Transmission line magnet assembly factory elevation.

Storing and staging into tunnel. The completed and tested magnets will be stored until they need to be transported into the tunnel. Installation is not expected to begin until some time after magnet production is underway. Consequently, space has been allocated to allow a sufficient number of completed magnets to be stored in preparation for installation. Support stands will allow the magnets to be stacked vertically. A custom hoist system (described previously under assembly and test area) will move the magnets from the test stand to the storage space. An area is provided to allow transfer from the storage area to a specially built transporter rig (see Section 5.1.8). Magnet installation in the tunnel is expected to take place at a peak rate of 4 magnets per day, significantly faster rate than the production rate.

\subsubsection{Factory Throughput}

Each magnet construction factory is designed to produce one magnet per day, accomplished in approximately 2 shifts. As with any normal production scenario, initial throughput would be much lower than one magnet per day, with the schedule eventually increasing to reach the peak production levels.

Table 5.16 lists, for each area, the number of technicians and times necessary to accomplish each operation, the number of technician-hours required per day to manufacture one magnet, and the number of stations needed in each area to complete the required tasks. All activities shown in Table 5.16 are accomplished in two, 8 hour shifts per day.

Reference [40] shows detailed schedules for each sub-assembly manufactured for the transmission line magnet, with a breakdown of elapsed time for each individual operation described in Section 5.1.6.3. "Throughput" schedules for each work area are also shown.

At the end of each day, enough components or subassemblies are completed in each area to produce one magnet. The subassemblies are stored on a rack near each station after they are completed. A third shift is used to move the components to the appropriate area of operation for the next day's work. Maintenance of tooling is also done on the third shift or weekends. Table 5.16 does not include these third shift activities. 
Table 5.16. Technician resources required to produce one magnet per day.

\begin{tabular}{|c|c|c|c|c|c|c|c|}
\hline Area & Operation & $\begin{array}{l}\text { Techs per } \\
\text { operation }\end{array}$ & $\begin{array}{l}\text { Tech time } \\
\text { used per } \\
\text { operation } \\
\text { (hrs) }\end{array}$ & $\begin{array}{l}\text { Number of } \\
\text { operations } \\
\text { per day }\end{array}$ & $\begin{array}{l}\text { Total } \\
\text { Tech } \\
\text { hours per } \\
\text { day }\end{array}$ & $\begin{array}{l}\text { Techs } \\
\text { assigned } \\
\text { to area }\end{array}$ & $\begin{array}{l}\text { Stations } \\
\text { in area }\end{array}$ \\
\hline 1 & Tube Welding & 2 & 3 & 8 & 48 & 4 & 2 \\
\hline 2 & Transmission Line Subassembly & 2 & 4 & 2 & 16 & 2 & 1 \\
\hline 3 & Ball Swaging of Lines & 2 & 4 & 2 & 16 & 2 & 1 \\
\hline 4 & Cryostat Assembly & 2 & 16 & 1 & 32 & 2 & 1 \\
\hline 5 & Leak Checking of Components & 2 & 2.7 & 6 & 32 & 2 & 3 \\
\hline 6 & Drive Line Bending & 2 & 8 & 1 & 16 & 2 & 1 \\
\hline 7 & Cryopipe Subassembly & 3 & 16 & 1 & 48 & 3 & 1 \\
\hline 8 & BT/Vac Cham Azimuthal Weld & 2 & 4 & 4 & 32 & 2 & 1 \\
\hline 9 & BT/Vac Cham Seam Welding & 2 & 4 & 2 & 16 & 2 & 1 \\
\hline 10 & BT/Vac Cham Bakeout \& Lk Chk & 2 & 4 & 2 & 16 & 2 & 1 \\
\hline 11 & $\begin{array}{l}\text { Iron \& Struct Support Installation, } \\
\text { End Assembly, Leak Check Vac } \\
\text { Vessel }\end{array}$ & 3 & 56 & 1 & 168 & 12 & 5 \\
\hline 12 & Warm Magnet Measurements & 2 & 32 & 1 & 64 & 4 & 2 \\
\hline 13 & Cold Magnet Measurements & 2 & 80 & 0.1 & 16 & 2 & 1 \\
\hline 14 & Beam Tube/Vac Cham Install & 2 & 4 & 2 & 16 & 2 & 1 \\
\hline Total & & & & & 536 & 43 & 22 \\
\hline
\end{tabular}

\subsubsection{Magnet Assembly}

Assembly of the arc dipole magnet for the VLHC Stage-1 accelerator is summarized in this section. It is described in more detail in reference [39].

\section{A. Assembly of drive transmission line for Stage-1 VLHC}

The transmission line consists of four components made by outside vendors: inner tube sections, a superconducting braid, a copper braid, and outer tube sections.

Mount six 12-meter long Invar pipes together into one continuous section at Area \#1. Weld using automatic rotary welding heads, and inspect mechanically. Tooling pulls the copper and superconducting braids over the Invar tube at Area \#2. The outer tube is fed, in 12-meter sections, over the assembly, then returned to Area \#1 to be welded, then leak checked at Area \#5. The welded transmission line is moved to Area \#3 for ball swaging. 3 carbide balls, each of increasing diameter, are pulled through the inner tube, swaging out the diameter and compressing the braid between the two tubes. The line is moved to Area \#4 for the cryostat assembly. Support rings and aluminum thermal shield sections are automatically fed onto the transmission line from the end. Trace tubes for $60 \mathrm{~K}$ helium are installed on the sides. 30 layers of superinsulation are individually spiral wrapped onto the shield, while the cryostat rotates on the assembly table. Tooling punches holes in the insulation as it is being wrapped, to accept the penetrations from the support rings. The extruded aluminum vacuum jacket is then fed over the insulated assembly in 12-meter sections, returned to Area \#1 and welded, then leak checked at Area \#5. The end of the drive conductor is bent into a dogleg shape at Area \#6. It is then moved to storage to wait for installation into the iron at Area \#11. 
Assembly of the transmission line will require somewhat complex tooling, which must perform seven types of automatic processes: automatic rotary welding, feeding parts longitudinally onto pipes, pulling copper and superconducting braid onto pipes, installing trace tubes, wrapping superinsulation, pulling carbide balls through the assembly to swage the inside diameter, and bending the line into the dogleg shape. Pictorial representations of each of these processes are shown in reference [39].

\section{B. $\quad$ Assembly of the return transmission line}

The return transmission line design is similar to the drive transmission line, except a slightly larger size. The return transmission line is not surrounded by an individual cryostat, as was the drive line. Instead, it is included in the cryopipe assembly. The fabrication scenario is identical to that given for the drive assembly, excluding the cryostat.

\section{C. $\quad$ Fabrication of cryopipe assembly}

Weld the $6.5 \mathrm{~K}$ flow return pipes, the $40 \mathrm{~K}$ shield supply header, and the $70 \mathrm{~K}$ shield return header pipes together longitudinally in Area \#1, and leak check in Area \#5. Transport the three pipes, together with the Return Transmission line, to the assembly station Area \#7 [39]. Mount and align the four pipes. Feed 34 spiders over the pipes, spaced every two meters. Attach shield sections. They are each two meters long, and will clip on between each spider. Attach braids to intercepts from $4.5 \mathrm{~K}$ with connection to $6.5 \mathrm{~K}$. Apply superinsulation. Inspect, and transport to storage area.

\section{Installation of iron and structural support over the cryopipe assembly and transmission lines.}

At Area \#11 the Transport Drive Transmission Line and Cryopipe Assembly are positioned in space using special tooling. The 12-m long Iron and Structural support subassemblies slide over the prepositioned drive line and cryopipe assemblies, are positioned and attached.

When installed, the transmission line, complete with cryostat, needs to be positioned with an accuracy of $\pm 0.5 \mathrm{~mm}$ with respect to the iron laminations. This will be accomplished by using a series of steel balls, which will contact the outside surface of the transmission line cryostat and fit into pockets stamped into the iron laminations. The balls, cryostat, and iron laminations can easily be manufactured to a tolerance acceptable to accomplish the accuracy necessary between the transmission line and the iron. The specific tooling and procedure still need to be developed, since this process was not used on the initial prototype.

Welds are then made at each junction between the magnet support tubes (vacuum vessel). The vacuum vessel is leak checked, and the entire assembly is inspected.

It is presumed that the iron and structural support subassembly, including vacuum vessel, will be prefabricated in 12-m sections in industry and supplied to the final assembly site for assembly in the 65-m long magnet. As the accuracy of lamination profile and stackup are paramount in achieving the design parameters some discussion is warranted.

The pole tip profile is required to be reproducible to within 20 microns. While this tolerance is not achievable globally for all features of the stamped laminations, manufacturing experience indicates that it is possible for the limited range over which the pole surface is defined. The 
laminations are to be stacked directly on the base datum feature for the profile. This minimizes the tolerance buildup due to the stacking operation. The design of the core assembly tie bars and welding of the spacer bars to the laminations draw the laminations toward the reference datum from which the profile is defined, assuring that the basic dimension is maintained accurately.

The internal welding of the spacer bars to the laminations poses some unique problems associated with physically fitting the required automated welding equipment within the small space. Equipment will need to be designed that fits within the limited space. A properly designed apparatus will produce very repeatable results.

\section{E. End assembly}

Still at Area \#11, connect drive transmission line shield cooling line to $70 \mathrm{~K}$ header. Assemble end components. Fit up and weld end vacuum vessel. Leak check completed vacuum vessel.

\section{F. $\quad$ Assembly of the beam tube and vacuum pumping chamber}

Start at Area \#8. Beam Tube sections are received in 12-m long extrusions. Two pieces must be seam welded together to make the final assembly [39]. Inner and outer pieces are butt welded together into 68-meter long sections. Welds and interior surface quality is inspected, while the assembly is still in two pieces. Getter pumps are installed into outer piece. Then beam tube halves are connected and seam welded longitudinally at Area \#9. Leak check and beam tube bake out takes place at Area \#10.

\section{G. Measurement of the magnetic field of the assembly}

All magnets are measured warm, either magnetically or with mechanical measurements of the pole tip profile and alignment. Cold testing will be performed on a limited fraction of the total production. Measurements must be done before the beam tube and vacuum pumping chamber is installed, because the test probe must be placed in the area occupied by the beam tube. Separate stations are used for warm and cold testing and appropriate space has been allocated in the facilities layout. A key concern is isolation of the measurement area from sources of electrical noise, which are typically from welding, electric motors, etc. The testing operation is estimated to take 30 hours for a warm test and 70-80 hours for a cold test, although this might be speeded up. More details on measurements and testing follow in Section 5.1.7.

\section{H. Installation of the beam tube and vacuum pumping chamber}

Beam Tube Assemblies are mounted onto the Structural Support and installed from the sides at Area \#14. On half of the assemblies, the beam tube must be "sprung" into position, but then will stay in position after being installed. On the other half, they will slide into position easily, but will need to be secured afterward. See Figure 5.1 and Section 5.2.4.1. The completed magnet is then transported to the storage area to await installation into the tunnel.

\subsubsection{Magnetic Measurements and Testing}

We focus discussion on testing of the combined function magnets during production since these magnets are the dominant element of the Stage-1 machine. Because of their length and small bore, these combined function C-magnets are also the most difficult to measure. Measurement 
and testing of other magnetic elements in the ring are can be accommodated by existing equipment and approaches.

Unlike conventional superconducting magnets, the conductor of the combined function magnets (transmission line) is not in a high field region and quench training is not a significant issue for magnet performance. Thus magnetic field measurements dominate the magnet testing process, both as a quality control measure and as characterization necessary for machine operation.

Warm measurements will be performed on all magnets. This must be done before the beam tube and vacuum pumping chamber is installed, because the test probe must be placed in the area occupied by the beam tube. Roughly $10 \%$ of magnets will be measured with the transmission line cooled to cryogenic temperatures allowing characterization of the field to full current. The remainder will be measured with the transmission line at room temperature. During initial stages of production when the fabrication rate is lower, we will cold test all magnets. As rates approach one magnet per day, the fraction of total magnets cold tested will be determined by test facility capacity and test needs as indicated by initial results.

We first discuss a conceptual design for a magnetic measurement system to be used for the combined function magnets. We then follow with a brief overview of the approach to cold testing and conclude with a few remarks on other test and measurement considerations.

\subsubsection{Measurement System}

As described above, magnetic measurements are the primary concern of the test program.

While one would expect to perform an extensive series of measurements, possibly using several measurement techniques, during the R\&D phase of magnet development, we have chosen to present here one concept for measurements of production magnets. This concept utilizes a rotating coil. We define the most important issues related to this concept and examine the consequences. A brief discussion of other approaches follows.

The main parameters of the arc dipole magnets are $\sim 2 \mathrm{~T}$ field, $\leq 12 \%$ horizontal gradient, $65 \mathrm{~m}$ length, and $20 \mathrm{~mm}$ gap. Measurement of the integrated dipole field is needed with an accuracy of a few parts in $10^{4}$. Desired measurement accuracy for the higher order harmonics is 0.1 units. ${ }^{1}$ We begin by examining the implications of requirements for field strength measurements. Based on this discussion we present a concept for a measurement system. Given this system we then address the issue of measurement of field harmonics.

With respect to measurement of the integrated field strength, there are two issues. The fractional accuracy of the measured field goes as $\Delta R / R$ where $R$ is the radius of the rotating coil [39]. This requires that the radius of the probe be known with high accuracy. Typical manufacturing and measurement uncertainty for coil placement in magnetic measurement apparatus is $\sim 0.001$ inch $(25 \mu \mathrm{m})$ which translates into a field error of $0.3 \%$ for a probe of $10 \mathrm{~mm}$ maximum radius. At least an order of magnitude greater precision $(0.03 \%$ strength error $)$ is required. This level of precision can only be achieved using a calibration magnet in which the strength is known at least as well as one need know it in the subject magnets. ${ }^{2}$ The second issue is unique

\footnotetext{
${ }^{1}$ One unit of harmonic corresponds to $10^{-4}$ of the main field, measured at a reference radius (in our case) of $1 \mathrm{~cm}$.

${ }^{2}$ Calibration in both a dipole and quadrupole magnet are required due to the large gradient which we want to measure with high accuracy.
} 
to measurement of combined function magnets. Due to the horizontal field gradient, an error in the horizontal position of the probe translates into an error in measured field strength. In a magnet with $10 \% / \mathrm{cm}$ gradient, measurement of the strength to 5 parts in $10^{4}$ requires positioning the probe with $0.05 \mathrm{~mm}$ accuracy after averaging over the length of the magnet. Although difficult, this can be achieved using mechanical fixtures referenced to the magnet laminations. Vertical placement of the probe is not as critical.

The main conclusions from the preceding discussion are that (1) a rotating coil of the size we are considering can be calibrated with sufficient precision, and (2) that the horizontal position of the probe can be set to the required accuracy with mechanical fixtures. For reasons we list below, we choose to insert the rotating probe into the aperture from the side rather than from the end. The probe is supported on a frame attached to the magnet as shown in Figure 5.29 .
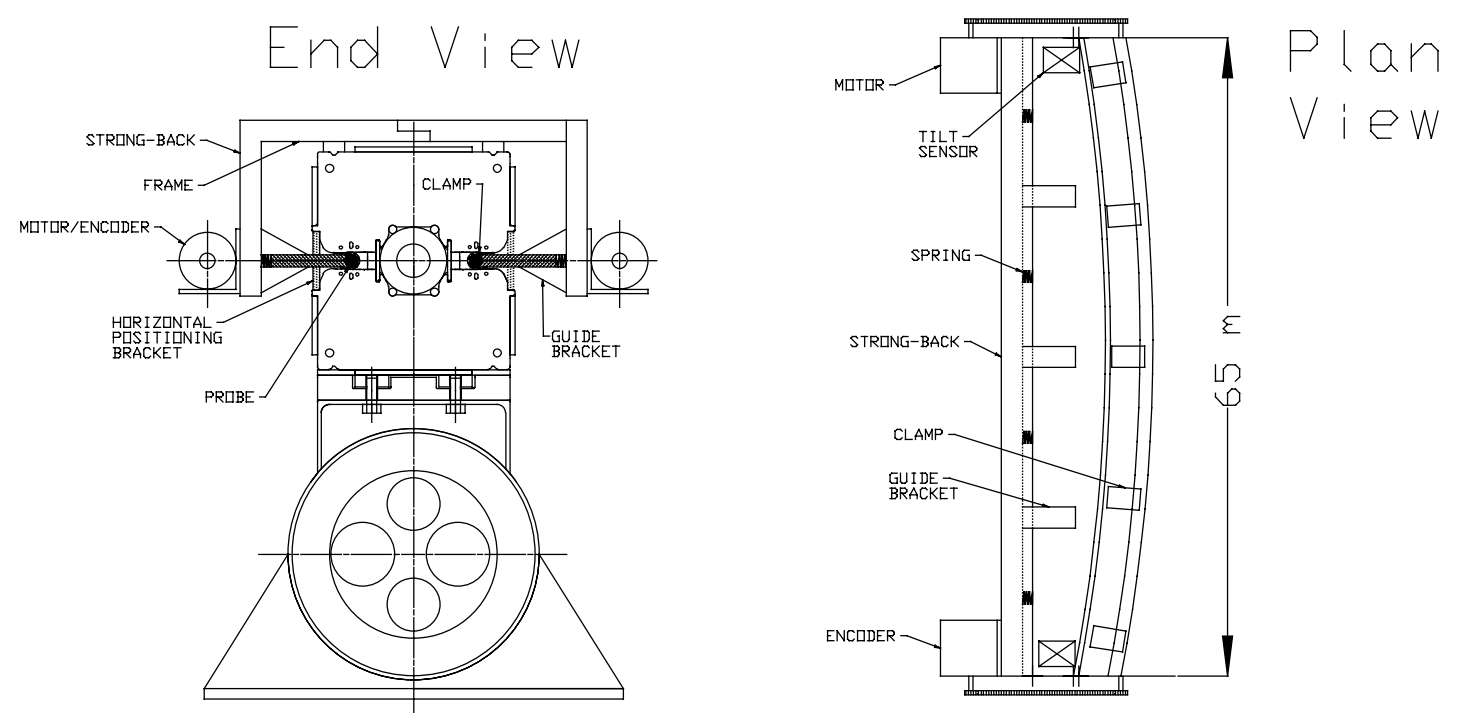

Figure 5.29. Measurement fixture. Note that the magnet sagitta is highly exaggerated.

Principle elements of the design are the following:

- The probe body is clamped to a precisely machined fixture ('strong-back') on an edge which follows the magnet sagitta.

- The strong-back slides horizontally in a series of guide brackets ('jaws') attached to the vertical member of the frame. The strong-back is spring loaded so that it and the probe are forced against the magnet laminations at the edge of the magnet gap, referencing the horizontal probe position with respect to the laminations.

- The vertical position of the probe is defined by the precision with which the frame is attached to the magnet and the precision of the jaws relative to the vertical member of the frame and the horizontal surfaces of the strong-back. A tilt sensor is mounted on the strongback to monitor its alignment.

- The motor for rotation and encoder for probe angle readout are mounted on the frame and connected to the probe by a mechanism such as a toothed belt or right angle drive. Other drive components could also be mounted outside the magnet bore (e.g. gearboxes). 
- Development of slip rings small enough to fit the probe bore is assumed although one might choose to digitize and transmit the signals from inside the bore. A pre-amplifier circuit sized to fit the bore is also assumed allowing a boost in signal size.

A consequence of insertion from the side is that one loses a few extra mm of probe radius due to the reduction in gap at the magnet edge of the focusing magnet. One also needs to allow space for the clamps holding the probe to the strong-back and for clearance during insertion.

Given a probe radius, we need to examine the accuracy of the measurement of field harmonics. From [41] we see that probe calibration matters little in measuring error harmonics. Accuracy of $10 \%$ or so is adequate. This requires knowing the probe radius to $0.1 \%$. What is of greater interest is the evaluation of the interplay of signal size with harmonic measurement. This has implications for the required level of excitation current during test. For a reasonable set of probe parameters, pre-amplifier gain of 100, measurement accuracy of 0.1 unit through $\mathrm{n}=9$ requires at least $200 \mathrm{~A}$ excitation current [41]. This should be possible for room temperature measurements. We would no doubt increase the gain and run at the highest tolerable current for increased accuracy. Hysteretic effects in the steel may be the limiting factor in the measurement accuracy at this low field ( 70 Gauss, or 1/15 of the injection field).

A probe length of $6.5 \mathrm{~m}$ was assumed above. Ten measurements are then required to construct the integral field. The time required for measurement is estimated as 2.5 hours with 2 crews of 3 technicians using 4 sets of measurement apparatus [41]. This certainly allows for the required production throughput. Automation and optimization of procedures will reduce the time required. We also note that the apparatus described could be used for either warm or cold measurements. The only special consideration for cold measurements would be to construct the strong-back of a nonmetallic material to prevent the formation of eddy currents during ramping.

We have chosen a probe inserted into the magnet bore from the side and supported by an external frame. An alternative approach would be a "mole." This probe type, having drive and angular alignment systems built in, was developed for measurements of SSC [42] and LHC magnets [43]. The obvious advantage of this approach is that the probe can be larger by as much as $25 \%$ as insertion is from the magnet end rather than the side. A larger probe produces larger signals. However, development of drive apparatus operating in a strong magnetic field is neither trivial nor cheap. We have no doubt that this could be done; however, we have chosen a simpler approach that removes elements of the measurement system other than slip rings and pre-amplifiers from the bore accepting as a consequence somewhat smaller signals.

\subsubsection{Cold Testing}

As a quality control measure and to characterize the magnetic field of a sample of the magnets to full current, we plan to cold test at least $10 \%$ of produced magnets. The peak rate of 2 magnets built per day requires 1 magnet cold test per week. Cooldown and warmup times are short (about an hour) due to the low cold mass, so testing time will be dominated by setup and measurement times. If an accurate measurement of the heat leak is required, it may take a couple of days for the multilayer insulation and vacuum to stabilize.

As discussed in the introduction, quench training is not a significant issue for the superferric design employed. However, it is important to have a full set of field measurements of magnets produced early in the program to check the overall design, iron saturation, yoke 
fabrication, and related effects on the field quality and transfer function. This increased testing also produces a statistically significant warm-cold correlation for the field harmonics. As the production rate rises, measurements check mechanical properties of the magnets as well as properties of the materials used to build them; and the set of measurements can be reduced to those necessary for quality assurance.

The cold parts of the magnet are the transmission line and return bus and their relatively small cryostat. Refrigeration requirements are modest; cool down and warm up times, short. The associated test equipment - cryogenic end boxes, transfer lines, pumps, and refrigerators is simple and inexpensive when compared to facilities developed for testing 'conventional' superconducting magnets. Cold testing can be done in a small area in the production facility in conjunction with warm measurements rather than in a large, separate cold test laboratory. The actual time spent in hooking up, cooling down, testing, warming up, and disconnecting the magnet is small when compared with conventional superconducting magnets. A peak production cold test cycle occupying the test stand for one five day week would allocate a two shift day to each of these tasks: installation and connection of the magnet on the stand, cool down, testing, warm up, removal.

\subsubsection{Other Testing Considerations}

One of the difficult issues in design and construction of a magnet system of the scale proposed is the understanding of its overall reliability. Failure rates for the magnets must be very small if collider availability is to be kept at the level necessary to carry out the physics program.

Determining failure rate is itself a difficult task as it is unrealistic to plan for the number of tests necessary to 'prove' that the magnets as constructed will meet the lifetime reliability requirements for the collider (even assuming that we could agree on a 'proof'.) However, since the iron yoke design and peak fields are similar to conventional magnet designs in use for decades, we do not envision reliability problems here. Key components will be subjected to 'lifetime' testing - equivalent magnetic and thermal cycles (if appropriate) -to verify that they meet requirements. While it is impossible to argue the statistical validity of a small sample of test magnets, accelerated life testing of a few early magnets will be used to validate design and provide feedback early in production should any degradation in performance be detected.

\subsubsection{Magnet Installation}

More than three thousand $67.75 \mathrm{~m}$ superferric magnets and their associated subsystems must be installed in the arcs of the $233-\mathrm{km}$ accelerator ring. This installation must be tightly coordinated with tunnel excavation, outfitting, and magnet manufacturing. The scale of this work and the difficulty of subsequent maintenance require techniques that ensure high quality installation resulting in high reliability of the accelerator.

In addition, about 1000 conventional magnets (Section 5.1.5) are to be installed in the local straight sections and two transfer lines. This installation is comparable to existing accelerators, nearby to surface access, and less constrained by schedule. 
The baseline assumptions adopted for the arc magnet installations are:

- Beneficial occupancy of the tunnel takes place after the tunnel is dry and safe. Specifically, the excavation, muck removal, roof support, grouting, ventilation, sump installation, emergency egress systems, etc. should be complete.

- The first installation activities complete the safety and transportation infrastructure. These include emergency and work lighting, communication, environmental safety monitoring, and trolley power lines for transport vehicles. At this point the tunnel can be used by workers without extraordinary training.

- The second phase of installation provides infrastructure in advance of actual magnet installation. This includes magnet stands, power cables, local electronics modules, helium gas return header, and a sufficiently accurate survey and alignment network.

- Magnets are transported into the accelerator tunnel one-by-one and installed onto prealigned adjustable support stands. The magnets arrive with the majority of subassembly installation and test work completed at the factory. Specifically this includes transmission line and cryogenic pipe assembly and test, hi-pot and vacuum tests, vacuum system bake out, and cable installation and instrumentation checkout on the magnet (Section 5.2).

- Final installation of each magnet into the string involves: 1) rough alignment; 2) making the necessary interconnections for the transmission line, beam pipes, cryo-pipes and vacuum jackets; 3) installation of the skid containing the corrector magnets; 4) connection of the magnet cables to the electronics modules, 6) testing of the corrector supplies, instrumentation, and network readout, and 5) final alignment.

- System checkout proceeds in phases. 1) a re-verification of the electrical network, highpot test, and beam vacuum tightness is performed after each half-cell is assembled; 2) the insulating vacuum is tested after installation of each vacuum break (every 8 magnets or $540 \mathrm{~m}$ ), a cryogenic performance and current load test is performed after each 10-km magnet string is completed

A basic constraint on the installation is that it be consistent with the addition of the Stage- 2 magnets at a later time. Since the second stage High Field Ring will modify tunnel layout, we must consider this upgrade while planning the Stage-1 work so that installed equipment will not make this upgrade too complicated. A tunnel cross section with both Low Field and High Field accelerators installed is shown in Figure 5.30.

\subsubsection{Tunnel Infrastructure Installation}

Before magnet installation a number of systems must be completed. Hangers must be mounted for the cable trays, transport trolley rail, and the helium gas return header. Alignment requirements for these hangers are in the $2-5 \mathrm{~cm}$ range and can be provided with the construction survey monuments at quad locations, and stretched strings or lasers for the alignment of intermediate brackets.

The baseline scenario uses manual labor and generic unit costs for bracket and piping installation. However, opportunity exists for optimizing the process. Repetitive bracket installation could take place with automatic drilling, installation, and bolting equipment. Long sections of pipes, trolley rail, and cable trays could be preassembled in the magnet factory and placed on 
these brackets with a long vehicle and mechanical assists, thereby minimizing the number of in-tunnel pipe welds, trolley rail joints, etc.

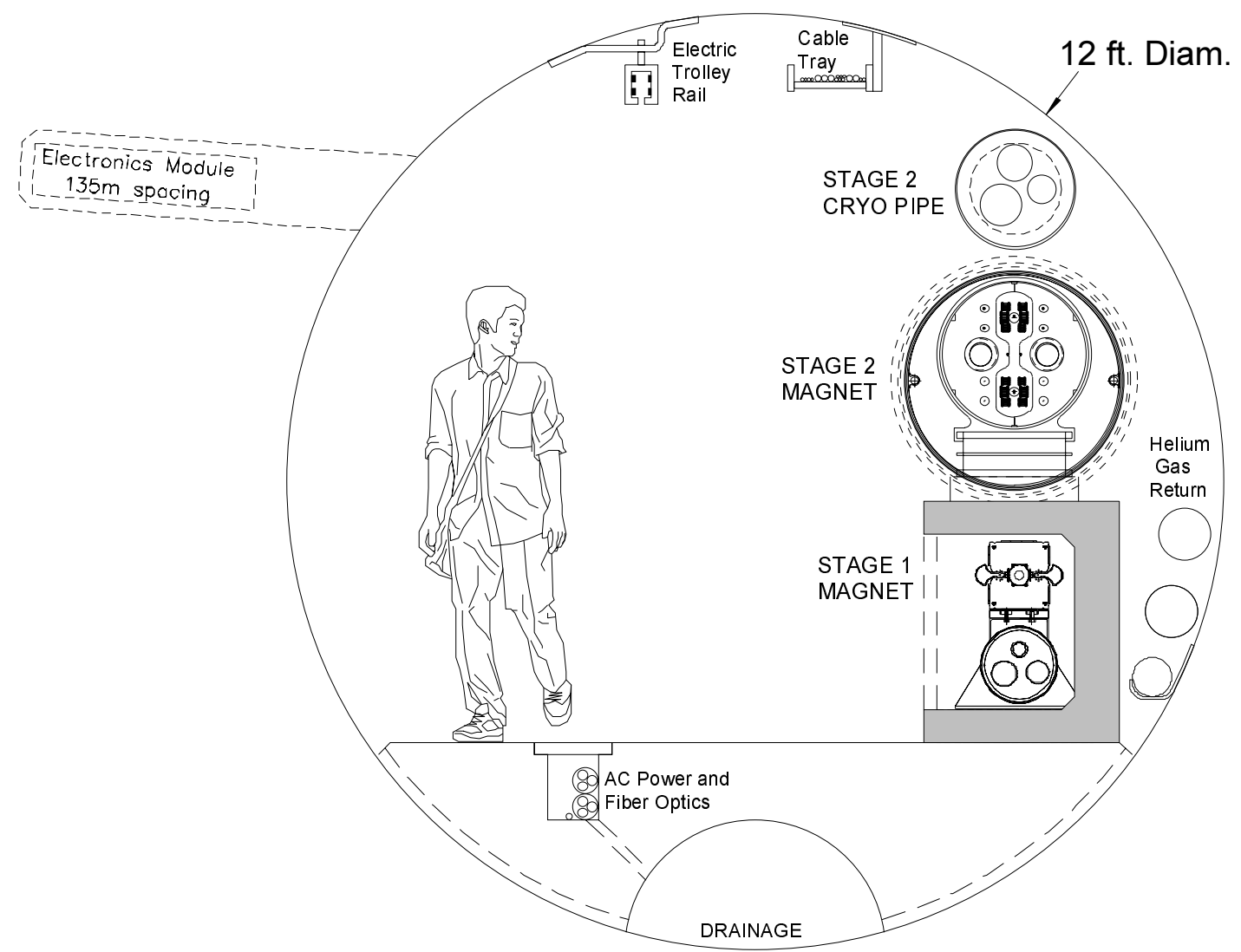

Figure 5.30. Tunnel cross-section after the Stage-2 upgrade. The evolution of the tunnel from Stage-1 to Stage-2 is shown in Figures 2.4 and 2.5.

Cable laying required in the tunnel is minimized as described in Section 5.2.3.2. Longdistance $(10 \mathrm{~km})$ power cable and optical fiber runs are laid in a trench in the floor by pulling a trailer with a large spool. Power and fiber connections to the electronics modules every are made by unspooling long vendor-supplied harnesses with prefabricated breakouts every $135 \mathrm{~m}$. Waxed-wire fire safety cables are suspended from the cable tray near the top of the tunnel.

The time at which electronics modules are installed and commissioned is somewhat flexible. The modules should be functional by the time of final magnet checkout so that they can be used to monitor insulating and beam vacuum, temperature, etc. If they play an important role in tunnel communications and environmental monitoring they should be installed earlier. An early installation date encourages earlier system debugging, but an electronics design frozen several years before project completion will be painfully obsolete by the time of commissioning.

Prior to magnet installation, survey monuments must be embedded into the tunnel wall with positions known to sub-mm accuracy, as described in Section 7.7. Magnet support stands are pre-installed and aligned to the accuracy of $\pm 1 \mathrm{~mm}$ in both transverse planes. 


\subsubsection{Tunnel Transportation System}

An electric trolley system with rubber-tired vehicles is chosen for transport of magnets and personnel. The system is similar to that recommended for the SSC [44] and also chosen for the LHC [45]. An overhead enclosed safety rail [46] operating at 400-750 VDC [47] provides power for magnet tractor/trailers, personnel transport vehicles, and temporary power for installation work lights, welding, etc.

The 30 ton magnet weight is less that the 35 ton LHC magnet so vehicle power requirements are similar. Drive requirements are $30 \mathrm{~kW}$ assuming a total vehicle weight of 50 tons, a rolling coefficient of friction of 0.02 [44], a drive train efficiency of $85 \%$ and a peak speed of $2.5 \mathrm{~m} / \mathrm{sec}(9 \mathrm{~km} / \mathrm{h})$. Trolley power up to $100 \mathrm{~kW}$ is supplied at the $10 \mathrm{~km}$ alcoves, allowing up to three vehicles to operate simultaneously in each $10 \mathrm{~km}$ sector. Copper conductors $\sim 1 \mathrm{~cm}^{2}$ are required in the rail to limit line losses to $<10 \%$. Details of the magnet transportation vehicles are discussed in the next section.

In addition to the overhead rail, limited battery power allows the vehicle to traverse unpowered regions such as splices and equipment bypasses. The battery is continuously recharged during normal operations. In emergency situations battery power is sufficient for the transport tractor to drop the magnet and travel $5 \mathrm{~km}$ to the personnel egress points.

Personnel transport vehicles similar to [44] will provide emergency egress, fire and ODH refuge, work break and sanitary facilities for installation crews.

\subsubsection{Magnet Handling and Transportation Considerations}

Because of the unprecedented magnet length and flexibility, careful attention must be devoted to their transportation from the factory to the place of installation in the tunnel. Magnets will be handled and transported using specially designed fixtures to prevent excessive stress concentrations and damage.

Allowable limits on local static deformation and torsion of the magnet are shown in Table 5.17. In the first three cases in the table, a force (or force couple) was applied it to a single set of magnet support/adjusters while the magnet cross section was held fixed $\pm 6 \mathrm{~m}$ away at the position of the adjacent magnet supports.

Table 5.17. Limits of relative displacement of magnet parts over \pm 6 -m length.

\begin{tabular}{|l|l|l|}
\hline Mode & $\begin{array}{l}\text { Allowable } \\
\text { range }\end{array}$ & Limitation type \\
\hline Horizontal & $\pm 3 \mathrm{~mm}$ & $\begin{array}{l}\text { Relative displacement of upper and lower } \\
\text { magnet halves is more than } 100 \mu \mathrm{m}\end{array}$ \\
\hline Vertical & $\pm 4 \mathrm{~mm}$ & $\begin{array}{l}\text { Stress in magnet structural elements } \\
\text { exceeds safe fraction of yield limit }\end{array}$ \\
\hline Twist & $\pm 16 \mathrm{mrad}$ & $\begin{array}{l}\text { Stress in magnet structural elements } \\
\text { exceeds safe fraction of yield limit }\end{array}$ \\
\hline $\begin{array}{l}\text { Smooth } \\
\text { Bend }\end{array}$ & $\begin{array}{l}\sim 1 \mathrm{~km} \\
\text { Radius }\end{array}$ & $\begin{array}{l}\text { Stress in magnet structural elements } \\
\text { exceeds safe fraction of yield limit }\end{array}$ \\
\hline
\end{tabular}


Taking into account its length, the magnet structure is quite flexible. The vertical sag can exceed the safe limits from the above table, just due to its own weight, if one of the supports is removed. On the other hand, a smooth continuous bend with $1 \mathrm{~km}$ radius of curvature produces a $50 \mathrm{~cm}$ sagitta in the magnet with acceptable internal stresses. To significantly reduce the risk of magnet damage during transportation, a $60 \mathrm{~m}$ long aluminum "lifting fixture" frame is used. The frame increases overall rigidity and protects the magnet from vertical bending damage. Figure 5.31 shows the frame moving inside the tunnel. Figure 5.32 shows the "A-Frame" structure of the beam that permits it to be either lifted or driven off of the magnet.

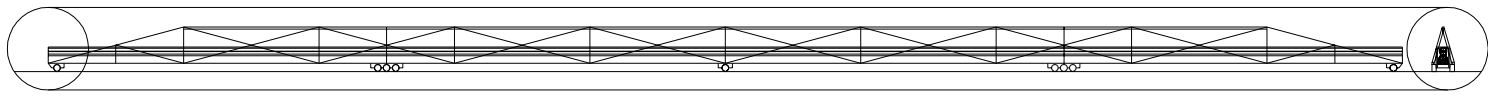

Figure 5.31. Magnet transportation frame, which acts as a "lifting fixture" to uniformly support the magnet and prevent stress concentrations during transport and installation. The frame is stiff enough to allow the magnet to be picked up and supported at two points without damage. The major load is taken by the two sets of wheels at the $25 \%$ and $75 \%$ points, with other smaller wheels to damp oscillations.

During transportation, dynamic loading effects have the potential to increase stress levels in the magnet. During transportation and installation, it is necessary to monitor and correct relative displacement of magnet parts to the limits well below those of Table 5.17. This is accomplished with an active shock absorber system. Several non-powered wheels installed along the frame use position controlled hydraulic cylinders to support the frame with the magnet inside during transportation. Inclinometers installed on the carts provide needed information to a feedback system about relative position of the neighboring carts.

Accelerometers installed near critical points on the magnet detect any mechanical oscillations and slow down or stop transportation if any danger to magnet is suspected. Through the coordinated action of the cylinders it is possible to lower the magnet to its final destination in a controlled manner. This system is also effective at limiting vertical deflections when the transportation vehicle enters and exits the $4 \%$ slope of the installation ramp.

Two motorized trucks on both sides of the frame, each with engine power of $60 \mathrm{~kW}$ and a typical operating power of $15 \mathrm{~kW}$, pull the frame during transportation from production facility to the designated location in the tunnel. An alternative (chosen by the SSC and LHC) is to use a general-purpose towing tractor pulling a passive magnet trailer. This allows the towing vehicle to be used as an egress vehicle if the personnel transporter fails.

An automatic guidance system is desirable to reduce the chances of pilot error on monotonous drives through the tunnel. The simplest system can use the overhead electric rail as a position reference. Another option, chosen by the LHC, is to use the magnetic field of a guide wire buried in a groove in the floor. Ultrasonic position detectors that sense nearby walls are commonly used on mobile vehicles in factory automation. These require no tunnel infrastructure and provide automatic shutdown if unexpected obstructions are encountered. These have been successfully tested in robotic tunnel guidance experiments undertaken as part of VLHC R\&D at Fermilab [48].

\subsubsection{Initial Magnet Installation}

The installation scheme shown in Figure 5.32 corresponds to the simplest case when magnet installation occurs with a clear space upstream or downstream of the installation point. 


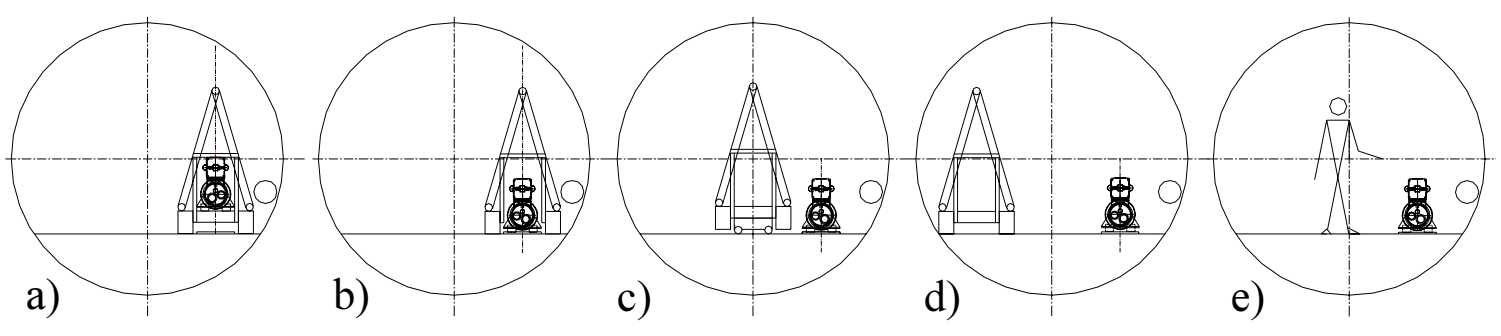

Figure 5.32. Initial installation scheme that is possible when the upstream or downstream magnet slot is empty. Installation vehicle a) drives up to the location along the beam line, b) deposits the magnet on the pre-aligned stands, and c-d) departs by initially moving along the beam line, then moving if needed to the center lane to pass any pre-installed magnets. Final connections e) may be made by separate work crews working off-shift or in different sections of the tunnel.

Magnets are driven to their location along the beam line and put on the support blocks without any transverse movement (Figure 5.32b). Unloaded frames are then backed out along the installation pathway (c). In this case, each next magnet can start its movement to the assembly place without waiting for the unloaded frame and the transport vehicles. Two magnet transport vehicles and an escape or a crew delivery vehicle can move in the tunnel simultaneously and independently. This scenario provides maximum flexibility for organizing optimal delivery traffic.

\subsubsection{Magnet Interconnections}

Three different types of interconnections are to be made after magnets are installed. First, the two 67-m magnets in each half-cell must be connected to form a common electrical, cryogenic and vacuum system. See the right-hand side of Figure 5.11. This is a "hard-welded" joint with no bellows in the vacuum shell, cryogenic piping, or beam pipes. There is a 1-m space available for this interconnection. Second, at the half-cell points (Figure 5.11) the superconducting transmission line dog-legs downward into the cryo service pipe enclosure to clear a $3.5-\mathrm{m}$ space for conventional correction magnets. Finally, in the straight sections where special magnets are installed there is need for several modes of transmission line positioning. The interconnection scheme does not differ greatly among these cases (see Section 5.1.2.3).

The basic steps of this procedure are listed below:

- connection of the transmission line inner Invar pipes for both drive and return lines

- splicing of the superconducting cables

- installation of electrical insulation and performing a high-pot test

- connection of cryogenic piping

- installation of the $50 \mathrm{~K}$ screen and superinsulation

- sealing and leak checking the insulating vacuum. 

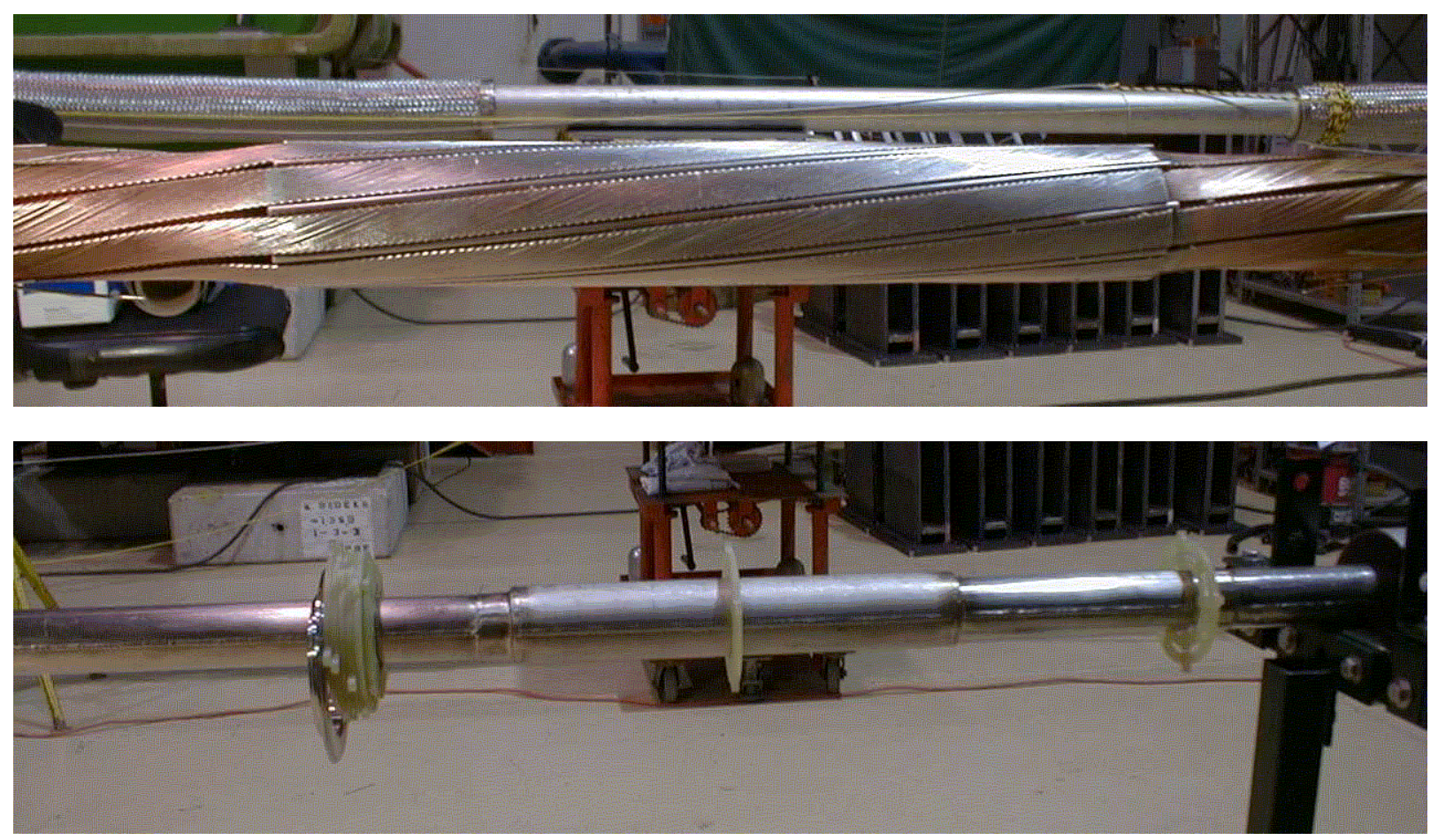

Figure 5.33. Splice of 100-kA conductor on 17-m test loop. Top: soldered spiral-wrapped cables. Bottom: completed interconnection after weld and leak check complete.

Automatic welding machines will produce high quality vacuum-tight connections of all helium pipes. An appropriate fixture for the 100-kA conductor splicing between the magnets must be designed and thoroughly tested to guarantee $100 \%$ reliability for these connections. The magnet interconnection appears to be the most labor-intensive procedure in the process of magnet installation, so R\&D could profitably be devoted to making this procedure simple. A high voltage test ( $5 \mathrm{kV}$ to ground) is to be made for each magnet before final closing of the external vacuum jackets of the interconnecting boxes.

\subsubsection{Corrector Magnet Skids}

In the baseline scenario, a skid containing six corrector magnets is installed at the $3.5 \mathrm{~m}$ gap between half-cells (every other magnet or $135 \mathrm{~m}$ ). These magnets are factory assembled and pre-aligned on a common support structure, with beam pipes, BPMs and bellows installed in the magnet gaps. All correctors and beam instrumentation will be electrically tested and cabled at the factory as described in Section 5.2.3.2. A description of the correctors can be found in Section 5.1.3. A total of 1568 corrector blocks will be transported and installed. A single magnet transport vehicle can carry 16 skids, so 100 trips are required. A two-ton capacity fork lift or equivalent hydraulic fixture will be used to install the corrector magnet blocks on their support stands. The skids will be aligned via locating pins in the previously aligned laminations of the arc magnets. Then beam pipe interconnections can be made following by a vacuum leak test.

Depending on the number of cryogenic problems encountered it may be desirable to defer corrector and beam vacuum installation until cryogenic system testing is completed. 


\subsubsection{Magnet Replacement}

A more complicated mechanical situation arises in the (hopefully rare) situation that a magnet needs to be replaced after installation, or is installed as the last magnet in an otherwise complete arc. In this case the magnet will be transported parallel to the magnets that have already been installed (Figure $5.34 \mathrm{a}$; installed magnets are not shown). Using hydraulic and control systems on the transportation trailers, the magnet will be lowered down to temporary support stands installed beneath the carts (b). After the frame is removed (c), the magnet is to be shifted horizontally and placed on the permanent stands with the use of a specially designed hydraulic pulling system connected to the permanent support blocks (d, e). Control equipment similar to that used during transportation will synchronize the system during this operation. This procedure will also be used to replace a Stage-1 magnet after Stage-2 installation is complete.

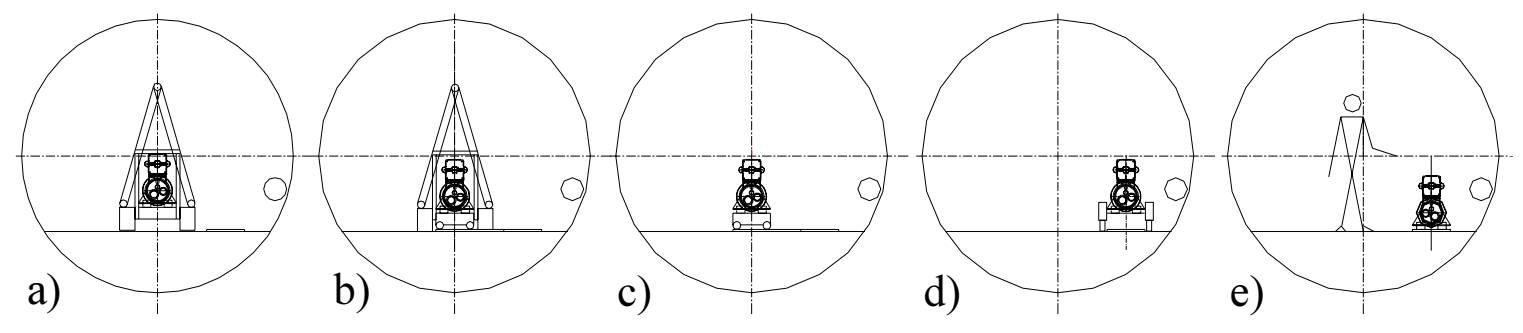

Figure 5.34. Magnet replacement scheme.

Magnet replacement will require the following work at the magnet interconnects:

- backfill the beam and insulating vacuum sectors with dry nitrogen and cut off beam pipes of the replaced magnet

- open the connection space

- remove super-insulation

- cut all the cryo-pipes and transmission lines of a the magnet to replace

- remove replaced magnet moving it horizontally out of the magnet string

- install new magnet following the repair scenario of magnet installation

- make interconnections as described above.

A reasonable goal for the magnet replacement procedure is to complete it during one 8-hour shift of multi-crew work. Pump-down and leak checking operations may extend this time. The overall magnet replacement time will be dominated by cryogenic warm-up and cool-down.

\subsubsection{Arc Magnet Alignment}

During installation, the $65-\mathrm{m}$ magnet is bent in the horizontal plane to the radial position predefined by the pre-aligned support stands. It is assumed that the support stand rough alignment is done with the help of a semi-automated surveying system using available survey monuments in the tunnel.

Arc magnets are ready for final alignment after correction magnets are installed and vacuum pipe interconnections are made. Each magnet must be installed so that the magnet reference center is within $\pm 0.25 \mathrm{~mm}$ from the nominal orbit position. For each magnet, an 
acceptable roll angle is less than $1 \mathrm{mrad}$ rms or less per adjuster. Conventional optical techniques (theodolites and laser trackers) for alignment have angular errors in the range of 10 microns per meter, corresponding to \pm 300 microns at the magnet ends from a setup at the center of the magnet. This would be marginally adequate to meet the magnet alignment specification of 250 microns rms over the length of the magnet. However this accuracy can be exceeded using the hydrostatic leveling and stretched wire techniques described below.

For each magnet there will be a support every 6 meters with horizontal, vertical and roll adjustment capability. The magnets will be built "laser straight" in the factory. The magnets will be installed in the tunnel with a $15.57-\mathrm{mm}$ sagitta over the 65 -meter length. The stiffness of the magnet will allow an independent range of motion of approximately $\pm 3 \mathrm{~mm}$ horizontally or vertically at each adjuster. Motions outside this range will require coordinated movements of nearby adjusters. The magnet position will be roughed in using optical techniques along the beam line relative to the internal tunnel network. At each of the support stands there will be a hydrostatic leveling pot for vertical alignment, a capacitive stretched wire pick up for horizontal alignment, and an electronic gravity sensor for roll. As the adjustment screws are turned there will be real time readout of both systems to allow for the positioning of each segment of the magnet. $A \pm 3 \mathrm{~cm}$ range of the adjusting screws will be sufficient to put in the sagitta and to compensate for installation errors in the magnet stands as well as drift and settling of the tunnel ( $\pm 1 \mathrm{~cm}$ maximum) over a 20-year lifetime.

Vertical alignment can be made using a hydrostatic leveling technique, which makes use of fluid-filled containers with capacitive readouts. This technique allows vertical alignment within $\pm 100 \mu \mathrm{m}$ for each magnet. For larger scale, corrections taking into the account nonuniformity in the Earth's gravitational field must be made. This will require careful study of the Geoid and the change in the density of the earth in the region of the machine. Corrections to the vertical can then be imposed on each magnet.

Magnet roll can be adjusted using a technique similar to the one used during leveling of the magnets. It can help to establish a real time display of the roll of the magnet at each adjustment station. Given a base support of $500 \mathrm{~mm}$ and a resolution of 2 microns for the hydrostatic levels a roll angle of \pm 4 micro-radians is achievable. This exceeds the $1 \mathrm{mrad}$ requirement for acceptable vertical closed-orbit distortion.

Horizontal alignment is done using the stretched wire technique. A small diameter (less than $1 \mathrm{~mm}$ ) copper beryllium wire mechanically attached to both ends of the magnet forms the reference base. Two wires separated vertically can be used to increase the signal to noise ratio. Using capacitive pickup electrodes at each support stand, it will be possible to adjust the horizontal position of each magnet to the desired accuracy. Alignment of the entire 65-meter magnet to within \pm 100 microns should be possible. To set the sagitta of $15.6 \mathrm{~mm}$ for the magnet all the adjustments must be made at the same time. Starting with optical tooling the rough sagitta can be set to an accuracy of \pm 300 microns. Once this is done the stretched wire system can be installed. Each capacitive pick up will have a different offset from the magnet to account for the sagitta. The final adjustment will involve moving all adjusters at the same time.

A degree of automation is possible. The simplest approach is for the person at the central display of magnet alignment data to give directions to one or more workers with wrenches. A more sophisticated alternative is to manually place motorized wrenches at each of the adjusters, then run automated software procedures to bring the entire magnet into alignment. If these 
systems were permanently installed and coupled with motorized adjustment stands they could be used as an active alignment that continually aligns the magnets as the machine runs. Beam position monitor information could also be used.

\subsubsection{Straight Sections and Transfer Line Installation}

Special magnets of several types are to be installed in the ten straight sections and two beam injection lines (see Section 5.1.5). Straight section focusing quadrupoles and injection line dipole and Lambertson magnets are conventional magnets. The maximum weight of these magnets does not exceed 5 tons and their length is less than 6 meters, so they are installed on their support stands using conventional techniques.

Separation/recombination dipoles and the abort Lambertson use the transmission line for excitation. To minimize labor related to cryo interconnections, these magnets are assembled in the tunnel by installing steel magnet cores around preinstalled transmission line and vacuum pipes. Individual pieces have lengths up to 6 meters and weigh less than 5 tons. Dipole alignment is non-critical but the Lambertson septa must be accurately aligned.

Finally there are eight 10-meter, superconducting, high gradient, low-beta IR quadrupoles described in 5.1.4. Each quadrupole will weigh about 25 tons and is rigid enough to be transported to the place of installation using two high load trailers and two transport vehicles of the type used for the arc magnet transportation. The alignment requirement for these quadrupoles is $\pm 100 \mu \mathrm{m}$ so two adjustable support stands will be used. The alignment procedures are complicated by the presence of the detectors and could be similar to those used at the LHC.

Because sections that use special magnets are located closer to the Fermilab and Far Side sites than accelerator arcs, installation of these magnet can be done independently of the installation of arc magnets.

\subsubsection{Installation Schedule}

The number and size of working crews needed to install the magnet in a 3-4 year term has been estimated. Several crews must work simultaneously to make the total installation time acceptable.

At least two access points at the opposite sides of the ring are required. The simplest magnet delivery scenario can be realized when assembly starts from the middle of the half-arcs connecting Fermilab site and Far End site and goes in four directions simultaneously. If magnet replacement is required after the magnet string is installed, a more complicated scheme must be used. In this case magnet traffic will go down the tunnel with already installed magnets, and additional measures must be taken to allow parallel work of an assembly crew. The magnet installation procedure in this case also is more complicated because it requires magnet movement across the tunnel.

Because of a high average magnet transportation distance inside the tunnel (about $30 \mathrm{~km}$ ), only one magnet per day can be installed by each installation vehicle. Connection of the installed magnets in a string can be made simultaneously for several magnets. 


\subsubsection{System Commissioning}

The described procedure will ensure interconnections of a high quality; nevertheless, after each $20-\mathrm{km}$ magnet string assembly is completed, a cryo-test must be performed. This cryo-test will involve a pressure test, relief valve setting tests, magnet string cooling down, heat leak measurement, and transmission line current test. Cold vacuum leaks could be detected at this point. Cryogenic plant performance and magnet string quench tests will be also conducted.

A temporary installation of the 100-kA holding supply and current leads (Figure 5.42) easily fits in the underground caverns eventually intended for the Stage-2 dump resistors. The 2.5V holding supply will ramp a $20-\mathrm{km}$ magnet string to $100 \mathrm{kA}$ in 40 minutes. This allows fullcurrent testing of a complete quench protection cell of the accelerator.

The beam pipes are interconnected after the cryogenic test passes and the correction magnet block is installed. Because the system is not baked in situ, the static vacuum will improve steadily over time and it is desirable to have most of the system under vacuum for as long as possible before collider ring commissioning.

\subsection{Accelerator Systems}

The systems distributed around the ring circumference include cryogenics, arc instrumentation, corrector power supplies, and beam vacuum systems. The "once per turn" systems are located primarily in the straight sections on the Fermilab site. These include RF, injection, extraction, and once-per-turn instrumentation.

A completely conventional approach is taken to the standard utilities in the straight sections. Warm iron/copper magnets use LCW cooling and standard power supplies. Electronics and major power supplies are located in underground shielded instrumentation rooms that are accessible with beam on. A larger tunnel, conventional power systems, and generous cable trays provide the infrastructure for RF systems, injection and extraction kickers and Lambertsons, beam crossovers, beam halo scraping, beam current and beam profile monitoring. Concentration of these major subsystems at the Fermilab site makes maintenance and accessibility requirements similar to other Fermilab accelerators. These systems are modeled on existing designs from recent projects for which the specifications and costs are well known.

Four distributed systems contain notable new features which will be described in some detail: the cryogenic system which is smaller and simpler than comparable systems, the beam stop which must deal with the large kinetic energy in the beam, the arc instrumentation which is distributed as electronics modules in "holes in the wall" at each quad location, and the beam damper system which is distributed around the ring circumference.

\subsubsection{Cryogenic System}

\subsubsection{Cryogenic System Description}

The magnet's superconducting transmission line is cooled by pressurized supercritical helium at 4.5-6.0 K. The heat load of the magnet system is removed by the sensible heat of the 
supercritical helium stream. The fluid operates just outside the critical region expanding the helium stream as it passes through the transmission line, producing a large effective heat capacity. The tunnel cryogenics are an all-piping system with very simple topology. Figure 5.36 shows the temperature profile in the transmission line.

The Stage- 1 cryogenic system is significantly simpler and lower-powered than the LHC or SSC systems. A rough comparison of the cryogenics of various machines, normalized to beam energy, is given in Table 5.18. For this comparison, the refrigeration for the SSC operating at $\mathrm{L}=10^{34}$ was crudely estimated by adding the synchrotron load scaled from the VLHC.

Table 5.18. Comparison of cryogenics per TeV beam energy for various machines.

\begin{tabular}{|c|c|c|c|c|c|c|c|}
\hline & & Tevatron & HERA & LHC & $\operatorname{SSC}\left(10^{33}\right)$ & $\operatorname{SSC}\left(10^{34)}\right.$ & VLHC-1 \\
\hline Refrigeration (4.5 $\mathrm{K}$ eqv.) $/ \mathrm{TeV}$ & $\mathrm{kW} / \mathrm{TeV}$ & 27 & 38 & 21 & 10 & 12 & 4 \\
\hline Helium Inventory/TeV & tons/TeV & 4 & 21 & 14 & 16 & 16 & 4 \\
\hline Cold Mass/TeV & tons/TeV & 600 & 5,000 & 5,143 & 5,000 & 5,000 & 286 \\
\hline Magnetic Stored Energy/TeV & $\mathrm{MJ} / \mathrm{TeV}$ & 400 & 1,000 & 1,629 & 600 & 600 & 171 \\
\hline
\end{tabular}

As shown in Figure 5.35, cryogens are fed from six refrigerator plants spaced at 38-km intervals around the ring. For efficiency reasons, refrigeration is provided at two temperature levels. Refrigeration at a higher temperature range $(40-70 \mathrm{~K})$ provides cooling for a heat shield that is used to intercept much of the heat that would otherwise be absorbed by the lower temperature superconductor. Each plant provides refrigeration for an upstream and downstream magnet string 19-km long. Each string is sub-divided into two loops, referred to as "near" and "far" loops.

\subsection{Helium Distribution System}

The helium distribution system is designed to distribute refrigeration to the magnet system with a minimal temperature variation. The distribution system consists of cryogenic valve boxes, transfer lines, and warm helium header.

\section{Cryogenic Valve Boxes}

Cryogenic valve boxes are used to redistribute the helium between the conductor loops for steady-state operation and perform various transient state operations. These boxes are located every $9.5 \mathrm{~km}$ and house the control valves and instrumentation required for each circuit. Boxes vary in configuration dependent upon location in the magnet string. These boxes are relatively simple devices mainly providing a transition for flow redistribution. 


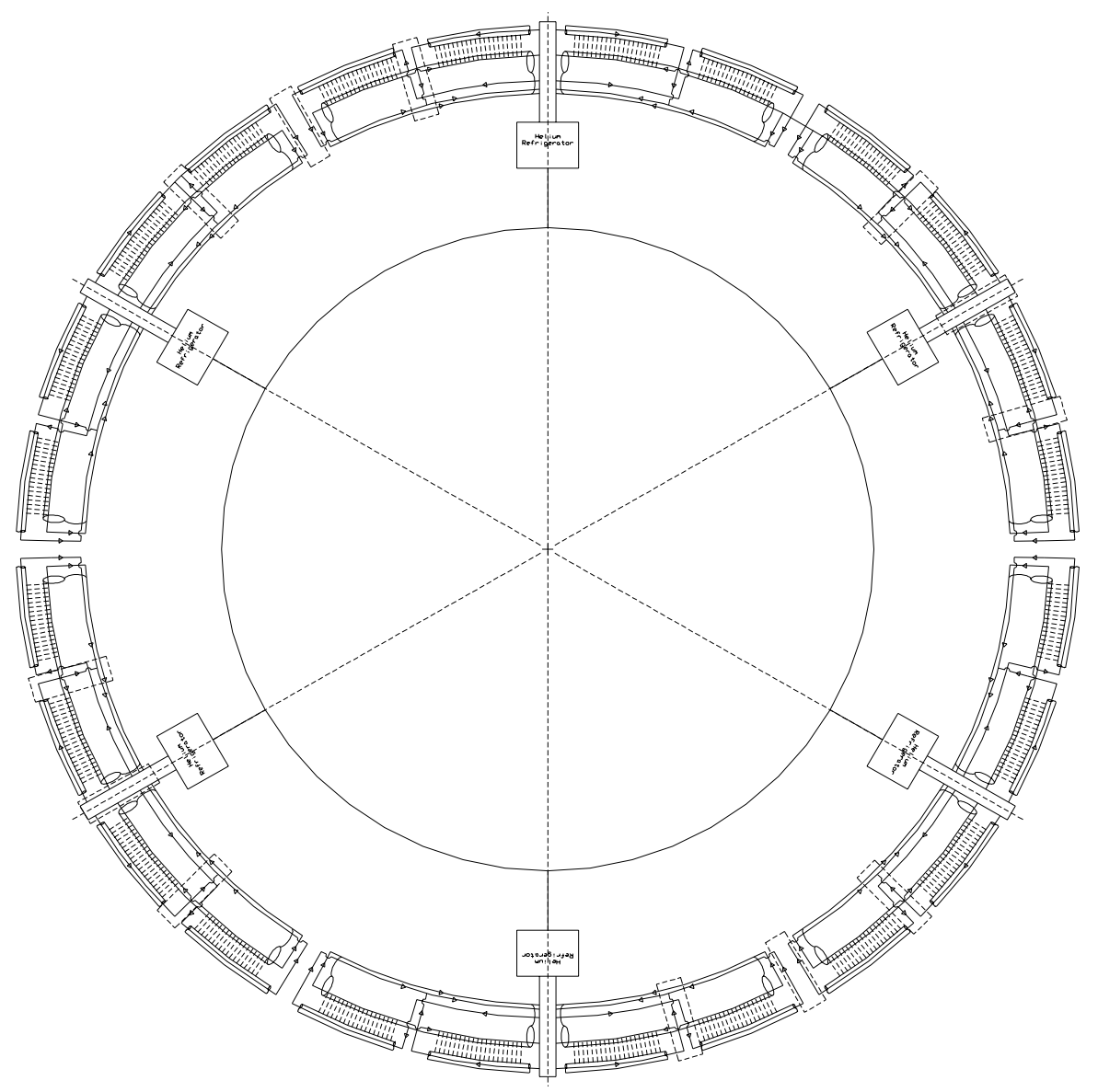

Figure 5.35. Cryogenic layout of the Stage-1 VLHC. Six plants are spaced at 38-km intervals around the ring. Each plant provides refrigeration equivalent to $9.6 \mathrm{~kW}$ at $4.5 \mathrm{~K}$. Nominal operating wall power is $2 M W$ at each plant. One plant is on-site at Fermilab and has additional capacity for detectors, IRs, $R F$, and superconducting current leads.

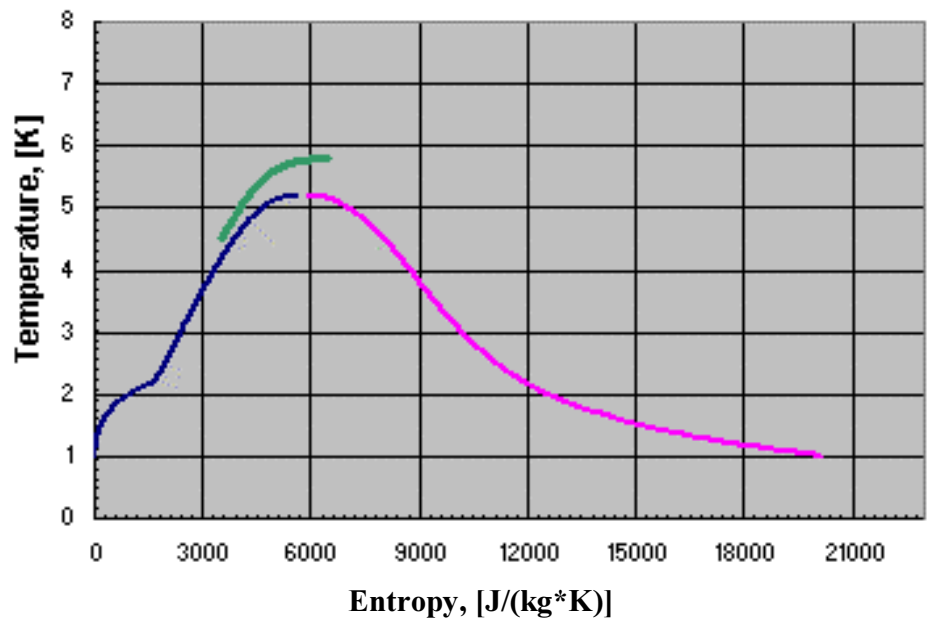

Figure 5.36. Temperature profile of the supercritical flow in the VLHC transmission line. 


\section{Cryogenic Transfer Lines Inside the Magnet}

Transfer lines are a part of the magnet assembly. A line contains five circuits: $4.5 \mathrm{~K}$ superconductor supply line (near loop only), $6.0 \mathrm{~K}$ superconductor return line, $40 \mathrm{~K}$ shield supply line, $70 \mathrm{~K}$ shield return line, and a vacuum circuit. Each section of transfer line is equal in length to a magnet (67.5 meters) and is attached to the magnet assembly as described in Section 5.1.2.3.

- The $70 \mathrm{~K}$ return line is a 0.102-m OD stainless steel pipe connected to an extruded aluminum shield that surrounds all the inner lines. The shield is wrapped with double-sided aluminized Mylar (dimpled and perforated). The longitudinal contraction of the line is compensated with guided formed bellows in the interconnects. During this shrinkage, the G-10 support structure is allowed to move within the vacuum space.

- The $40 \mathrm{~K}$ shield supply line is a 0.089-m OD stainless steel pipe supported on the G-10 spiders from the aluminum shield. Bellows are used at the end of each 67.5-m module to compensate for thermal contraction and expansion.

- $\quad$ The $4.5 \mathrm{~K}$ supply (near loop only) and 6.0 K superconductor return line are Invar tubes that are also anchored at the midpoint of the module. These lines are of concentric design. The $6.0 \mathrm{~K}$ helium flows between two concentric Invar tubes

- $\quad$ The $4.5 \mathrm{~K}$ supply line is suspended via G-10 spiders from a $6.0 \mathrm{~K}$ inner line.

\subsection{Helium Pressure Relief and Venting System}

Relief valves for each of the five circuits are located every 540 meters, as seen in Figure 5.37. These valves will vent into the warm helium header that is itself protected every $1 \mathrm{~km}$ at $3 \mathrm{MPa}$ (30 bar) from over pressurizing. Each relief is conventional in nature and set to the MAWP of its given circuit. The primary need for these reliefs are for trapped volume and loss of insulating vacuum scenarios. The warm header is further protected at the refrigerator (above ground) at a set pressure of $0.2 \mathrm{MPa}$ (2 bar), venting to the atmosphere.

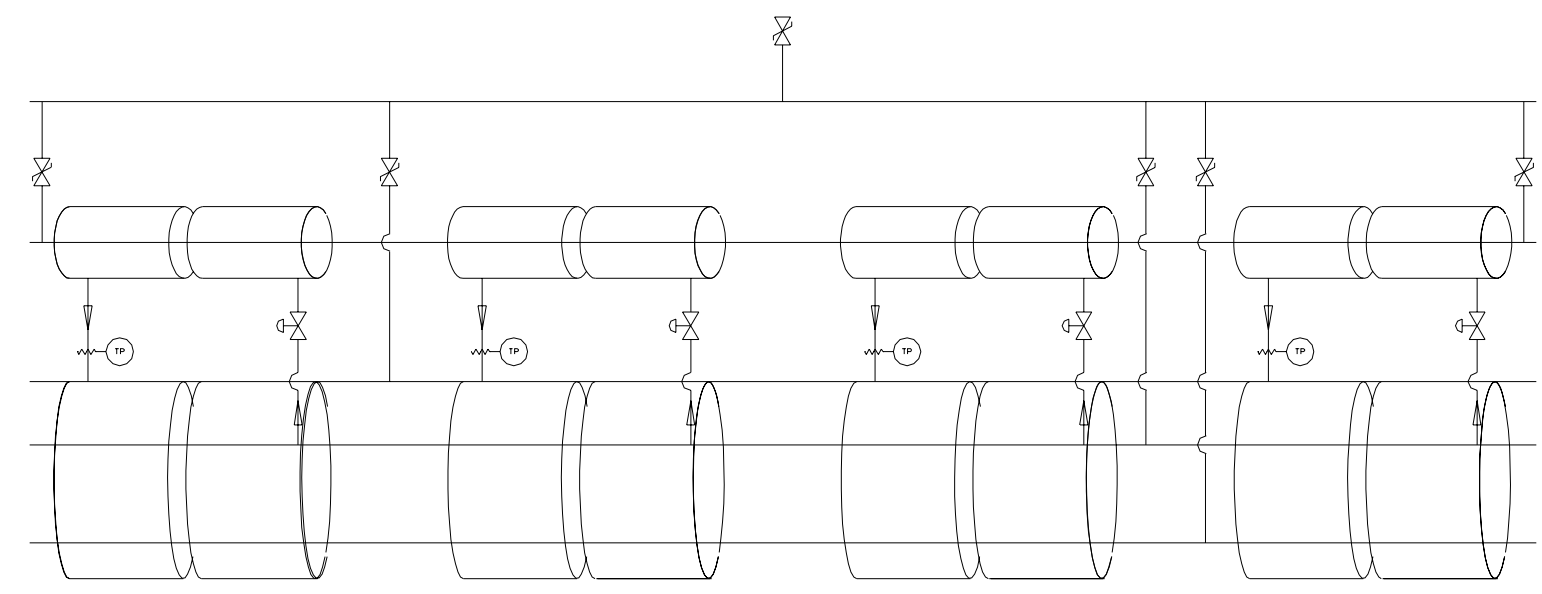

Figure 5.37. Helium shield flow and pressure relief valves.

Quench protection of the transmission line is discussed in Sections 5.1.2.2 and 5.2.2.3. The $100 \mathrm{kA}$ transmission line conductor contains enough copper that it can be safely shut down with a 1 second time constant following a quench. A number of detailed quench simulations 
have been performed. The peak temperature is $\sim 250 \mathrm{~K}$. The worst-case peak pressure comes from a situation in which a mis-steered beam (kicker misfire) causes the beam to oscillate sideto-side in the beam pipe and scrape heavily in a series of locations separated by a half-betatron wavelength. In this case the peak temperature is unaffected, but a worst-case pressure occurs when the hydrodynamic shock waves from two adjacent quenches collide at the midpoint between quenches. The peak pressure is below the $4 \mathrm{MPa}$ (40 Bar) pressure rating of the transmission line. Thus no helium needs to be vented anywhere, even for a long period following the quench. The pressure burst from the hydrodynamic shock wave, as registered on pressure sensors along the transmission line, should provide a convenient method of identifying where the quench occurred.

\subsection{Heat Shield Cooling System}

Pressurized $40 \mathrm{~K}$ helium is used to intercept the radiation and conduction heat leak to the transmission line. Helium is supplied from the refrigerator at $1.7 \mathrm{MPa}(17 \mathrm{bar})$ and returns at less then $70 \mathrm{~K}$ and $1.3 \mathrm{MPa}$ (13 bar). Flow is supplied via a $0.089 \mathrm{~m}$ OD tube and distributed via a pair of $6 \mathrm{~mm}$ OD lines every 270 meters carrying $2.0 \mathrm{~g} / \mathrm{sec}$. This scheme provides shield cooling for every two magnets. Control valves and temperature sensors are included every 270 meters for heat shield flow control based on temperature requirements.

\subsection{Transmission Line Heat Load Estimates}

Transmission line heat load estimates are divided into two components: the primary heat load on the $4.5 \mathrm{~K}$ system and the heat load to the $40 \mathrm{~K}$ shield system. The calculated heat loads are presented in Table 5.19.

Table 5.19. VLHC design study calculated transmission line heat loads.

\begin{tabular}{|c|c|c|c|}
\hline & & Primary 4.5K & Secondary $40 \mathrm{~K}$ \\
\hline \multicolumn{4}{|l|}{ STATIC } \\
\hline & \multicolumn{3}{|l|}{ Near Loop } \\
\hline & \begin{tabular}{l|l} 
& Mechanical Supports, $\quad[\mathrm{mW} / \mathrm{m}]$ \\
\end{tabular} & 53 & 670 \\
\hline & Superinsulation, $\quad[\mathrm{mW} / \mathrm{m}]$ & 15 & 864 \\
\hline & \multirow[b]{2}{*}{ Far Loop } & & \\
\hline & & & \\
\hline & \begin{tabular}{l|l} 
& Mechanical Supports, $\quad[\mathrm{mW} / \mathrm{m}]$ \\
\end{tabular} & 53 & 670 \\
\hline & Superinsulation, $\quad[\mathrm{mW} / \mathrm{m}]$ & 13 & 864 \\
\hline \multicolumn{4}{|c|}{ DYNAMIC } \\
\hline & Beam Loss, $\quad[\mathrm{mW} / \mathrm{m}]$ & 2 & 1 \\
\hline & Superconductor Splice, [mW/m] & 7 & - \\
\hline
\end{tabular}

\subsection{Cryogenic Loads for On-Site Refrigeration Plant}

The extra loads for the on-site refrigeration plant are summarized in Table 5.20. The total refrigeration power ( $4.5 \mathrm{~K}$ equivalent) is comparable to a single additional cryo plant. 
Table 5.20. Extra loads for the on-site refrigeration plant.

\begin{tabular}{|l|c|c|c|c|}
\cline { 2 - 5 } \multicolumn{1}{c|}{} & $\begin{array}{c}\mathbf{4 . 5 ~ K} \\
\text { (static) }\end{array}$ & $\begin{array}{c}\mathbf{4 . 5} \mathbf{~ K} \\
\text { (dynamic) }\end{array}$ & $\begin{array}{c}\mathbf{4 0 ~ K} \\
\text { (shield) }\end{array}$ & $\begin{array}{c}\text { Liquefaction } \\
\text { (g/s) }\end{array}$ \\
\hline Current Leads (Sec. 5.2.2.2) & - & - & - & $15 \mathrm{~g} / \mathrm{s}$ \\
\hline IR Beam Debris (Sec. 5.1.4.2) & - & $2.4 \mathrm{~kW}$ & $0.5 \mathrm{~kW}$ & \\
\hline IR Static Heat Load (rough est.) & $0.5 \mathrm{~kW}$ & - & $10 \mathrm{~kW}$ & \\
\hline RF Cavities (Sec. 5.2.6) & $0.48 \mathrm{~kW}$ & $1.76 \mathrm{~kW}$ & - & \\
\hline On-site Transfer Lines (6 km) & $0.2 \mathrm{~kW}$ & - & $12 \mathrm{~kW}$ & \\
\hline \hline TOTAL & $1.2 \mathrm{~kW}$ & $4.2 \mathrm{~kW}$ & $25 \mathrm{~kW}$ & $15 \mathrm{~g} / \mathrm{s}$ \\
\hline
\end{tabular}

It is notable that most of the on-site cryogenic loads are dynamic and are not present during the injection cycle when the Tevatron is ramping. It is therefore plausible that most or all of the on-site cryogenic load could be serviced by load shifting from the existing Fermilab Central Helium Liquefier.

\subsection{Refrigeration System}

The refrigeration system is composed of six $4.5 \mathrm{~kW}$ at $4.5 \mathrm{~K}$ capacity facilities equally spaced around the ring. Each plant will also provide $100 \mathrm{~kW}$ of $40 \mathrm{~K}$ refrigeration used in the shielding of the magnet system.

This provides approximately a 50\% margin above predicted steady state requirements. This margin is required to ensure continued operation when the heat load increases either due to events such as a magnet quench or vacuum leak, or because refrigeration capacity falls below its intended design value (i.e. decreasing machine efficiency or inefficient heat transfer due to contamination). With this scheme, all six refrigerators must be operational for the accelerator to function. No plant redundancy is built into this design.

Figure 5.38 shows the refrigeration process schematic. The process helium is compressed from suction pressure of $0.101 \mathrm{MPa}(1.01 \mathrm{bar})$ up to an intermediate pressure of $0.4 \mathrm{MPa}(4.0$ bar) by means of a first stage compressor discharging into a second stage, which compresses the helium to $1.85 \mathrm{MPa}$ (18.5 bar). Turbo machinery is used for the refrigeration plants while positive displacement machines comprise the compressor system. There are two cold boxes: the upper and lower cold boxes. Each cold box contains five plate-and-fin type heat exchangers and three expanders. To avoid return helium gas instabilities due to the elevation change from the tunnel to the surface, the lower cold box will be located at the tunnel level. This requires a dedicated space of $432 \mathrm{~m}^{3}$ ( $6 \mathrm{~m}$ along the beam by $6 \mathrm{~m}$ transverse by $12 \mathrm{~m}$ high) located near the accelerator tunnel.

These plants are well within existing commercial vendor capabilities. They are simpler but technologically similar to those of LEP, LHC, SNS, and HERA, and contain no nitrogen precooling. The cold box configuration is a horizontal carbon steel shell enclosure housing all heat exchangers, piping, valves, cold ends of turbo expanders and vessels operating at cryogenic temperatures. The equipment within the cold box and the inner face of the vacuum jacket are 
covered by multi layer aluminized Mylar. The cold boxes are designed to withstand the outside atmospheric pressure while they are evacuated and are also protected against overpressure by safety valves. All internal valves, filters, turbo expanders, bayonets and major pipe connections are fixed at the top plate and are accessible from a structural steel platform along the length of the cold box.

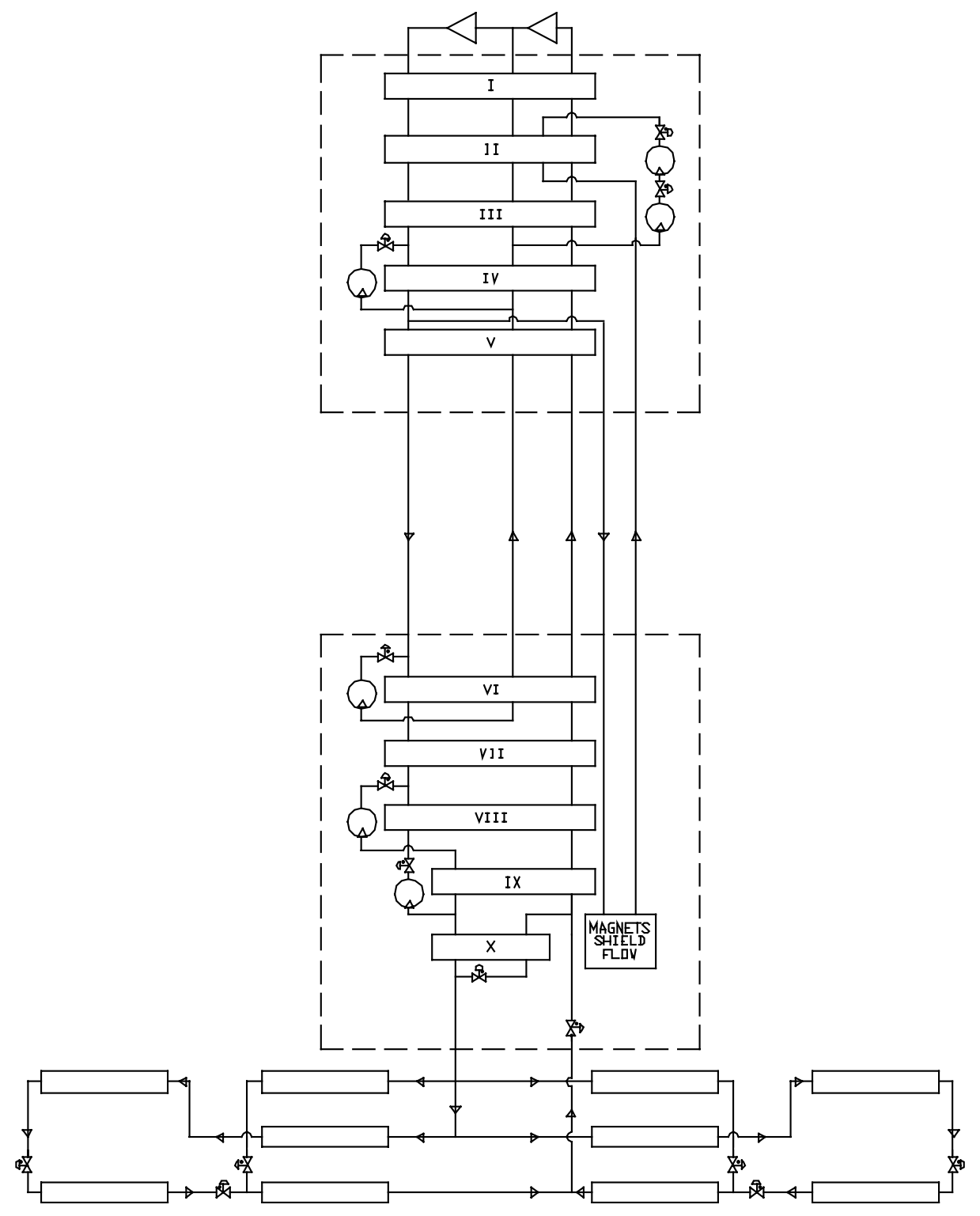

Figure 5.38. Refrigeration process flow and transmission line flow schematic. A split cold box similar to LEP is used to make the process insensitive to altitude changes between the tunnel and surface. The transmission line flows extend $\pm 19 \mathrm{~km}$ from the cryo plants. The flow is split into two parallel loops: the "near" loops that extend $\pm 9.5 \mathrm{~km}$ from the cryo plant, and the "far" loops that service magnets between $9.5 \mathrm{~km}$ and $19 \mathrm{~km}$ from the cryo plants.

The turbo expanders are self activated gas bearing type, superior in their performance to oil bearing turbines. Each turbo expander is a small single-stage centrifugal turbine braked by a direct-coupled, single stage centrifugal compressor. The turbine housing is vertically mounted 
to a plate at the cold box top, projecting into its vacuum space. Within the cold box are brazed aluminum heat exchangers, pressure vessels containing an adsorbent to remove impurities and the cold end of gas-bearing turbo expanders. Part of the outer shell cover is flanged and has an $O$ ring seal, and is removable for complete access to all cold box internal components and piping. Two panels will be mounted on the structural steel platform next to the cold box, one containing all instrument terminations and transmitters, and the other an assembly of purge and defrost valves. Provisions for purification of the process helium is provided at each plant as well as oil removal systems. A commercial control system package will permit the unattended operation of these plants.

Table 5.21. Predicted power requirements for ring cryogenics.

\begin{tabular}{|c|c|c|c|c|c|c|}
\hline & \multicolumn{4}{|c|}{ Magnets } & \multirow[t]{3}{*}{ Shield } \\
\hline & & \multicolumn{2}{|c|}{ Transmission Line } & \multicolumn{2}{|c|}{ Current Return Bus } & \\
\hline & & Near Loop & Far Loop & Near Loop & Far Loop & \\
\hline Temp in & {$[\mathrm{K}]$} & 4.50 & 4.52 & 5.44 & 5.40 & 37.00 \\
\hline Press in & [bar] & 4.00 & 3.80 & 2.00 & 2.50 & 17.00 \\
\hline Enthalpy in & {$[\mathrm{J} / \mathrm{g}]$} & 12.09 & 12.09 & 33.92 & 27.18 & 207.28 \\
\hline Entropy in & {$\left[\mathrm{J} /\left(\mathrm{g}^{*} \mathrm{~K}\right)\right]$} & 3.52 & 3.56 & 8.19 & 6.69 & 14.76 \\
\hline Temp out & {$[\mathrm{K}]$} & 5.57 & 5.39 & 5.80 & 5.54 & 70.00 \\
\hline Press out & [bar] & 2.80 & 2.50 & 1.90 & 2.10 & 13.00 \\
\hline Enthalpy out & {$[\mathrm{J} / \mathrm{g}]$} & 26.40 & 26.66 & 37.79 & 34.24 & 381.66 \\
\hline Entropy out & {$\left[\mathrm{J} /\left(\mathrm{g}^{*} \mathrm{~K}\right)\right]$} & 6.44 & 6.59 & 8.96 & 8.18 & 18.71 \\
\hline Predicted heat load & {$[\mathrm{W} / \mathrm{m}]$} & 0.044 & 0.044 & 0.024 & 0.022 & 1.534 \\
\hline Distance & {$[\mathrm{m}]$} & 19400 & 19400 & 19400 & 19400 & 38800 \\
\hline Design total heat & {$[\mathrm{kW}]$} & 0.85 & 0.85 & 0.47 & 0.43 & 60 \\
\hline Mass flow & [g/sec] & 60 & 59 & 120 & 60 & 341 \\
\hline \multirow[t]{2}{*}{ Design ideal power } & {$[\mathrm{kW}]$} & 103 & 107 & 27 & 53 & 345 \\
\hline & & Magnets & Shield & & & \\
\hline Predicted heat load & {$[\mathrm{kW}]$} & 2.6 & 60 & & & \\
\hline Heat uncertainty factor & {$[-]$} & 1.25 & 1.25 & & & \\
\hline Design Heat Load & {$[\mathrm{kW}]$} & 3.2 & 74 & & & \\
\hline Design mass flow & [g/sec] & 120 & 348 & & & \\
\hline Design ideal power & {$[\mathrm{kW}]$} & 291 & 345 & & & \\
\hline $4.5 \mathrm{~K}$ equiv design power & [kW] & 4.43 & 5.26 & & & \\
\hline Efficiency (fraction Carnot) & {$[-]$} & 0.28 & 0.28 & & & \\
\hline Nominal operating power & {$[\mathrm{kW}]$} & 1039 & 1233 & & & \\
\hline Overcapacity factor & {$[-]$} & 1.3 & 1.3 & & & \\
\hline Installed operating power & $(\mathrm{kW})$ & 1351 & 1603 & & & \\
\hline \multicolumn{4}{|c|}{ Operating wall plug power for one sector (MW) } & \multicolumn{3}{|c|}{2.0} \\
\hline \multicolumn{4}{|c|}{ Installed wall plug power for cryogenics for one sector (MW) } & \multicolumn{3}{|c|}{3.0} \\
\hline \multicolumn{5}{|c|}{ Operating wall plug power for cryogenics for entire accelerator (MW) } & \multicolumn{2}{|l|}{11.8} \\
\hline \multicolumn{5}{|c|}{ Installed wall plug power for cryogenics for entire accelerator (MW) } & 17.7 & \\
\hline
\end{tabular}

\subsection{Helium Compressor System}

The compression package will consist of three oil flooded rotating screw compressors. One compressor will compress from $0.11 \mathrm{MPa}(1.1 \mathrm{bar})$ to $0.4 \mathrm{MPa}(4.0 \mathrm{bar})$, and the two other will compress in parallel from $0.4 \mathrm{MPa}(4.0 \mathrm{bar})$ to $1.85 \mathrm{MPa}(18.5 \mathrm{bar})$. The first stage compressor 
is smaller than the two second stage machines. Each compressor skid contains the compressor, main drive motor, oil reservoir/separator vessel, and lubrication system mounted on a common rigid skid base. The base plate provided is suitable for installation on a flat pad without grouting. The helium enters the compressor and is compressed through the rotating screw compressor, also flooded with oil. The oil seals the rotor and absorbs some heat of compression. The compressed warm helium passes through a water-cooled heat exchanger and then into a bulk oil separator. The oil is also passed through a separate water-cooled heat exchanger. Each compressor is direct driven by an electric induction electric motor. The compressor assemblies are equipped with automatic inlet and outlet block valves and automatic recycle valves.

The high-pressure helium from the compressors passes through a skid mounted oil removal system. The oil removal system consists of three stages of coalescing filter housings followed by a vessel containing activated carbon. All entrained oil is filtered out of the gas stream by the three stages of filters. The small amount of oil in the vapor phase is removed by the activated carbon. The total amount of oil contained in the helium leaving the oil removal assembly is less than $10 \mathrm{ppb}$.

\subsection{Helium Liquid and Gas Storage and Inventory Management}

The inventory for the entire cryogenic system is estimated to be 75 tons of liquid helium, which is twenty times the Tevatron inventory. Each cryogenic plant will have three $37.8 \mathrm{~m}^{3}$ liquid helium storage dewars, and fifteen gas tanks with a capacity of $113.6 \mathrm{~m}^{3}$. This will provide storage for $143 \%$ of the total helium inventory. The gas storage serves as make-up/kick back during normal operations, and as a buffer/storage during quenches and warm up of a single loop. The liquid storage station will have pumps and heat exchangers necessary to liquefy/warm up cold gas during upset conditions. During the planned warm-up of the entire ring, the inventory will be liquefied into the dewars. In case of a sector warmup, the inventory can be distributed between five sector gas/liquid storage vessels via the cooldown header. The tunnel transfer line and the cooldown header can be used to hold up to $40 \%$ of inventory at pressure below the MAWP of any component in the system.

\subsection{Cryogenic Control System}

A well-proven process control system will be used. The cryogenic system in each sector will be controlled by a commercially available distributed control system (DCS). Commercial program logic controllers will be used for local control of the cryogenic hardware in the event that a high-speed network connection is unavailable. The cryogenic control system will provide full automation of any operation mode, including transient modes. The accelerator high-speed network will be used for communication and data exchange between sectors.

\subsubsection{Design Operating Conditions}

\subsection{Steady State}

Helium is supplied from a refrigerator at $4.5 \mathrm{~K}, 0.4 \mathrm{MPa}$ (4 bar) and returned at $5.8 \mathrm{~K}, 0.19$ $\mathrm{MPa}$ (1.9 bar). Supercritical supply helium is fed from the helium plant to four parallel loops. Each loop is comprised of $9.5 \mathrm{~km}$ of magnet and transmission line. There are cryogenic connecting boxes at the beginning and end of each $9.5 \mathrm{~km}$ section. At the exit of the refrigerator 
the helium process is split into four paths. There are upstream and downstream magnet strings that are mirror images of each other. The typical flow schematic of a magnet string is shown in Figure 5.39.

As helium enters the upstream or downstream magnet strings the flow is split into two parts: half enters the $4.5 \mathrm{~K}$ transmission line circuit (for the near loop) and half enters the $4.5 \mathrm{~K}$ current return circuit contained in the magnet (for the far loop). There is expected to be an approximately $1 \mathrm{~K}$ gradient across each loop. These loop flows are then re-distributed at valve boxes and become the return $6.0 \mathrm{~K}$ flow.

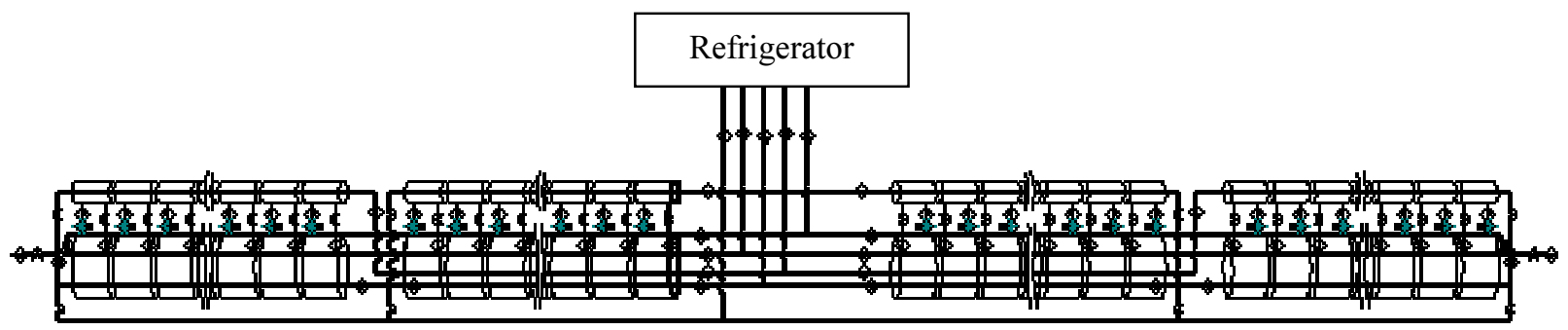

Figure 5.39. Flow schematic of the 38-km sector serviced by one cryogenic plant.

The cooling scheme relies on a balance between heat leak and pressure drop. Any scenario causing an upset in the heat load (i.e. vacuum degradation) can be compensated by increasing the helium flow rate and supply pressure for the proper loop. The refrigerator has been oversized by $50 \%$ to compensate for these types of upset conditions.

\subsection{System-Wide Purification}

Purification of this machine will be done in two steps. First, each sector will be pumped and backfilled five times to 25 inches of mercury via pumpouts on every helium circuit at each valve box located every $10 \mathrm{~km}$. This method has been proven to work in the Tevatron. Levels of contamination less than 100 ppmv are achieved with this first step by using roughing pumps. The second step is dilution purification. A purifier with a nitrogen cooled charcoal bed will be located at each refrigeration plant. The discharge gas of the compressor system will be circulated through this purifier and then will be distributed to the magnet/transmission line system. Contamination instrumentation, such as arc cells used on the Tevatron, will be used to monitor the ppmv levels in each sector. The system will be ready for cool down when contamination levels are $<5 \mathrm{ppm}$. It is estimated that the purification for a sector in the VLHC will be 72 hours.

\subsection{System-Wide Cool down}

Cool down of the VLHC will be done in three stages. The first stage involves the cool down of the helium plants. The second stage is the cool down of the transmission line to $40 \mathrm{~K}$. Finally the transmission line will be cooled to operating temperatures.

In the first stage, cooling is provided by turbines and should not take more than 24 hours per plant. In the second stage, the plant is set up in the liquefaction mode and $40 \mathrm{~K}$ gas at 0.4 $\mathrm{MPa}$ (4 bar) is sent via cool down valves located at each valve box back to the compressor suction warm gas header located in the tunnel. Gas flows out from the refrigerator through the 
near loop transmission line, the $6.5 \mathrm{~K}$ return, the current return bus, and the shield circuits, and then returns via the warm header. When the temperature at the end of the "near" loop (measured at the valve box) is $70 \mathrm{~K}$ the flow is then re-directed to the "far" loop and into the return header. As the "far" loop temperature reaches $70 \mathrm{~K}$ the second cooldown stage can be started.

During the third cool down stage, the plant is re-tuned for an outlet temperature of $4.5 \mathrm{~K}$. The flow pattern for the second stage is the same as the first stage. As the temperature for each loop is at $7 \mathrm{~K}$ the cool down valves can be closed and the flow will be looped back in refrigerator mode. Total cool down time is expected to be on the order of 10 days.

\subsubsection{Magnet Power Supplies, Current Leads, and Quench Protection}

Stage-1 power supplies are summarized in Table 5.22 below. The major item is the single $100 \mathrm{kA}$ supply for the transmission line magnets. The Interaction Region power supplies, current leads and quench protection are discussed in Section 5.1.4. Total Stage-1 power supply requirements (both ramping and steady state) are about a third of the Fermilab Main Injector.

Table 5.22. Power supply, current leads and quench protection summary for the Stage-1 machine.

\begin{tabular}{|c|c|c|c|c|}
\hline & $\begin{array}{l}\text { Transmission Line } \\
\text { Power Supply }\end{array}$ & $\begin{array}{l}\text { IR Quadrupole } \\
\text { Power Supplies } \\
\text { (Sec. 5.1.4) }\end{array}$ & $\begin{array}{l}\text { Straight section } \\
\text { Warm Magnet } \\
\text { Power Supplies }\end{array}$ & $\begin{array}{c}\text { Corrector } \\
\text { Magnets } \\
\text { (warm copper) }\end{array}$ \\
\hline Number & 1 & 2 & 16 approx. & 12,000 \\
\hline Location & FNAL & at Experiments & Straights & $\begin{array}{c}\text { Quad } \\
\text { locations }\end{array}$ \\
\hline Voltage per supply & 62 & $10 \mathrm{~V}$ typ. & $1000 \mathrm{~V}$ typ. & 100 Typ \\
\hline Current per supply & $100 \mathrm{kA}$ & $25 \mathrm{kA}$ & 200 A typ & 2 A typ \\
\hline Ramping MVA (tot) & $6.2 \mathrm{MVA}$ & $1 \mathrm{MVA}$ & - & - \\
\hline DC Power (total) & $0.4 \mathrm{MW}$ & $0.6 \mathrm{MW}$ & $7.4 \mathrm{MW}$ & $2.1 \mathrm{MW}$ \\
\hline LCW Consumption & - & - & 150 liters/sec & (air cooled) \\
\hline Quench Detection & 1 Circuit at PS & 2 circuits/quad & - & - \\
\hline Quench Protection & Dumps@20km & 4 heaters/quad & - & - \\
\hline SC Current leads & $100 \mathrm{kA}$-pair & 60 kA-pair & - & - \\
\hline $\begin{array}{l}\text { Peak Ramping } \\
\text { Supply Power }\end{array}$ & \multicolumn{4}{|c|}{$17.7 \mathrm{MW}$} \\
\hline $\begin{array}{l}\text { Total Power Supply } \\
\text { Power in Collision }\end{array}$ & \multicolumn{4}{|c|}{$10.5 \mathrm{MW}$} \\
\hline
\end{tabular}

\subsubsection{Main Transmission Line Power Supply}

The low inductance $(3 \mu \mathrm{H} / \mathrm{m}, 0.6 \mathrm{H}$ ring total) and low stored energy $(10 \mathrm{~kJ} / \mathrm{m}, 3 \mathrm{GJ}$ total $)$ of the single-turn transmission line magnet allow the entire ring to be powered from a single supply [49]. See Figure 5.40. A voltage of $\pm 62 \mathrm{~V}$ ramps the machine up or down in 1000 seconds. The design current of $100 \mathrm{kA}$ at flattop exceeds the $87.5 \mathrm{kA}$ required for the transmission line magnet to reach its design field. The supply will be located on site at Fermilab in a shielded underground room approximately $25^{\prime}$ x $50 \mathrm{ft}$ in size. The ramping power and footprint of the power supply is comparable to one of the six Main Injector power supply buildings. Superconducting current leads will be located immediately adjacent to the supply to minimize 
power loss in warm buswork. Superconducting buswork will carry the current through a short connecting conduit to the transmission line magnets in a manner identical to the MS6 test string $[50]$.

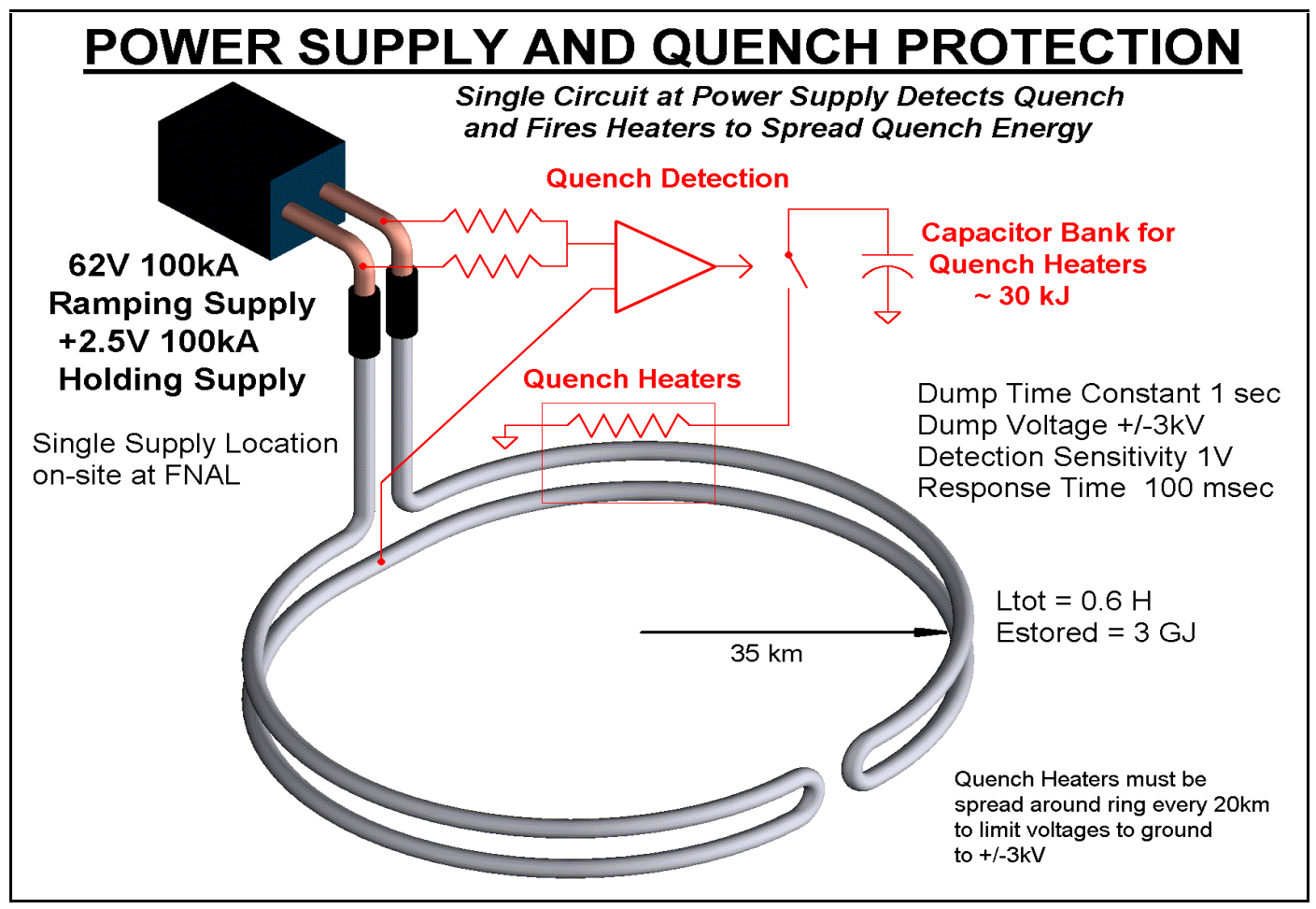

Figure 5.40. 100-kA power supply and quench protection for transmission line magnets.

The power supply is actually two supplies in series. The main ramping supply generates the positive or negative voltage to transfer the inductive energy into and out of the magnets. It is not used when the magnet is at constant current. The second "holding" supply maintains the current during long periods at flat top and injection, and provides only the voltage necessary to overcome resistive losses in the current leads, warm buswork, superconducting splices, and the shorting SCR switch across the ramping supply. This approach minimizes the cost of the ramping supply, simplifies the power distribution system, and moves the tightest regulation and ripple requirements to the precision holding supply.

The Main Ramping Supply design (Figure 5.41) is based on paralleling 8500 A modules of design similar to Main Injector supplies. This approach permits spare modules to be taken off line for repair, and allows the use of commercially available $10 \mathrm{kA} \mathrm{DCCTs} \mathrm{for} \mathrm{current} \mathrm{regula-}$ tion. A 2-quadrant 12-phase SCR bridge returns inductive power to FNAL as the magnets ramp down. Ramping systems are designed to have rms power sized to be halfway between the peak (6.2 MVA) and the rms (3.1 MVA). This has proven to be a reliable design methodology for similar supplies at Fermilab. The rms feeder load of 4,800 KVA is less than one feeder cable for the source. 


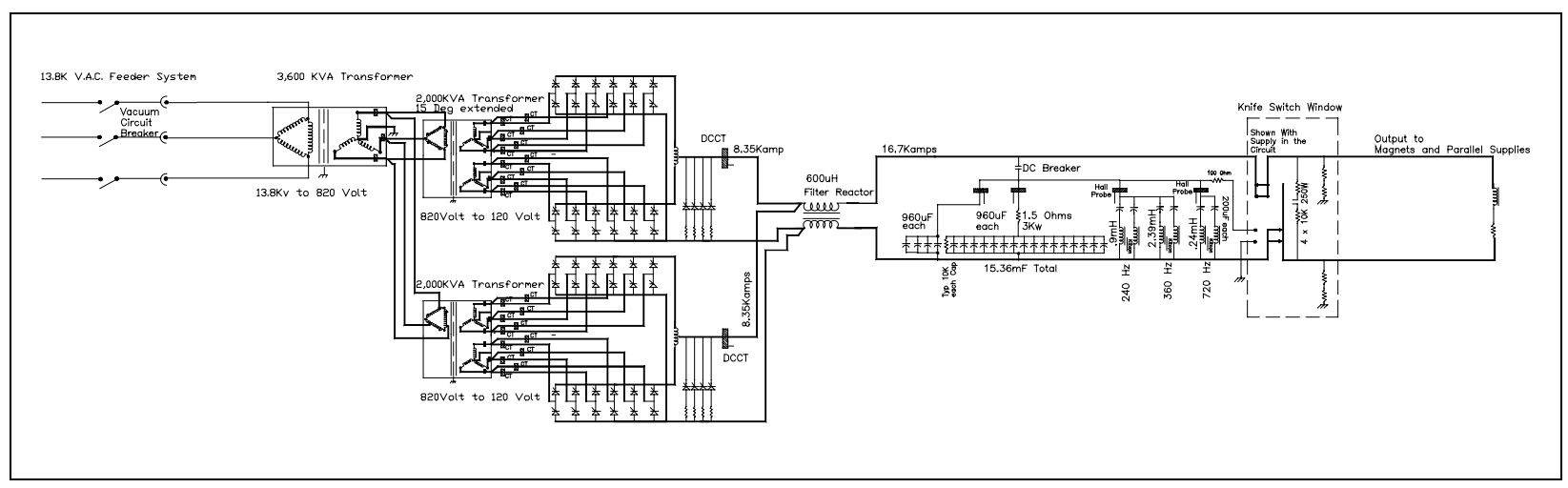

Figure 5.41. 100-kA ramping supply schematic (one 2 x 8.5-kA module shown).

The $+2.5 \mathrm{~V}$ holding supply is essentially a copy of the $100 \mathrm{kA}$ switching converter [51] constructed for the magnet string test. The device (Figure 5.42) consists of a $450 \mathrm{VDC}, 500 \mathrm{~kW}$ bulk supply followed by ten $10 \mathrm{kA}$ DC-DC converter modules in parallel. This two-stage regulation scheme provides immunity to variations in input line voltage. The modules use IGBT chopper bridges, tape-wound cores and Schottky power diodes.

The switching frequency is $\sim 2 \mathrm{kHz}$. To minimize output ripple the 10 modules are phased to switch sequentially. This pushes the fundamental ripple frequency up to 10x the switching frequency or $\sim 20 \mathrm{kHz}$. The switching will be phase locked to the revolution frequency so that beam emittance growth due to power supply ripple will be negligible. The control system for this switcher will be integrated into that of the main ramping supply to simplify regulation and load hand-off.

\subsubsection{Current Leads}

Superconducting current leads are required for both the main transmission line and for the IR quadrupoles. A single pair of $100 \mathrm{kA}$ current leads is required for powering the transmission line. These could either be conventional copper leads identical to the leads developed for the MS6 transmission line test facility [50], or could be a specially built High-Temperature Superconductor (HTS) version using the $50 \mathrm{~K}$ transmission line shield flow as the inter-stage temperature. The cryogenic wall power associated with the conventional $100 \mathrm{kA}$ leads is approximately $300 \mathrm{~kW}$. This could be reduced by a factor of 4 with HTS leads [52]. Payback time for the electrical cost savings from HTS leads is approximately 3 years.

IR quadrupoles can use commercially available HTS leads similar to the LHC. The total requirement for current leads in the Stage- 1 machine is about $150 \mathrm{kA}$-pair, or about $1 / 12^{\text {th }}$ of the current leads required by the LHC. 

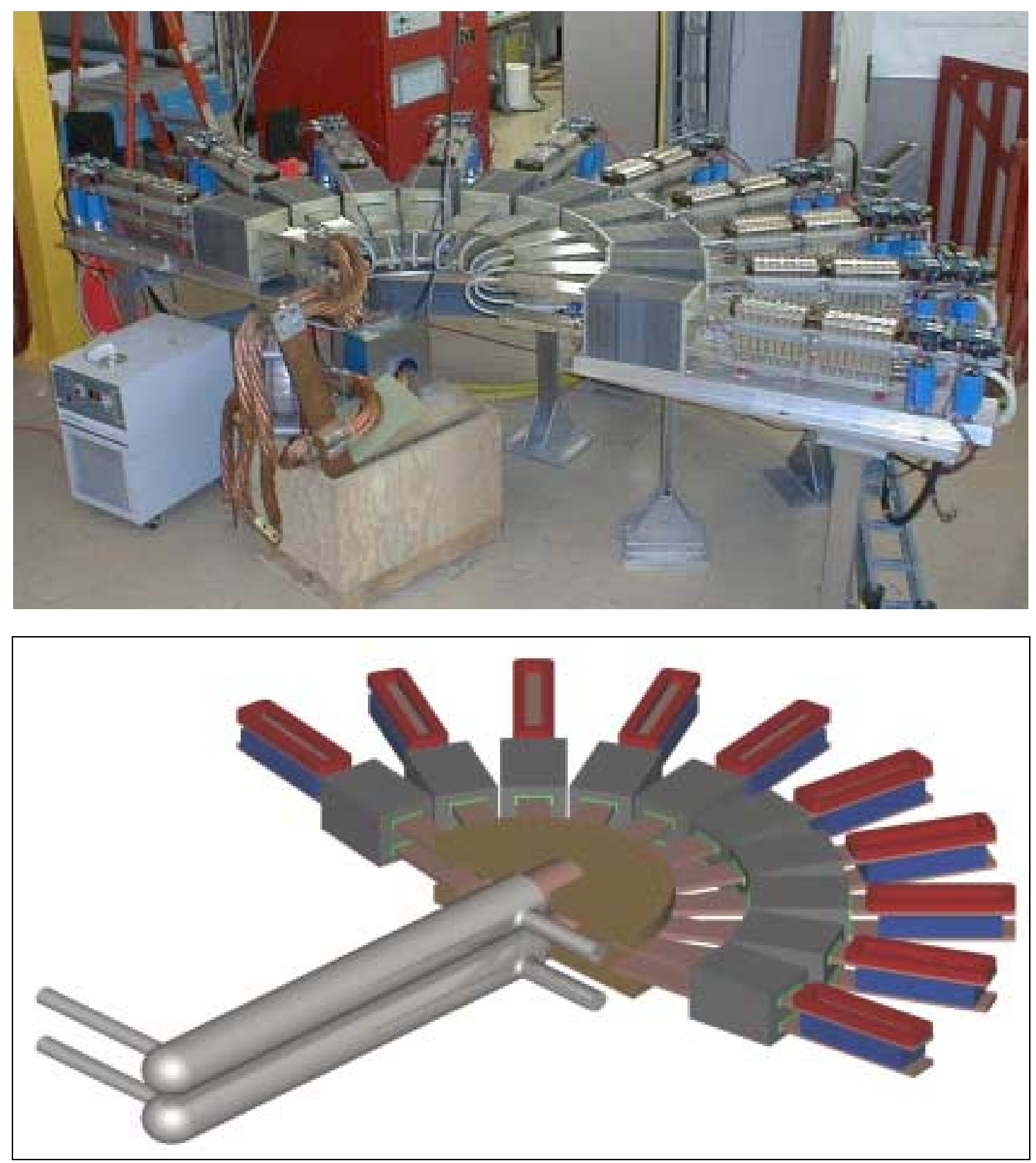

Figure 5.42. 100-kA superconducting current leads and switching converters developed for the MS6 test string. A more compact arrangement of the leads and power converter will be small enough to be placed in the tunnel and used for partial-ring commissioning.

\subsubsection{Quench Protection}

The only superconducting magnets of the Stage-1 collider are the transmission line magnets and the IR Quadrupoles. Protection of the IR quads with heaters is described in Section 5.1.4.

The low inductance and low stored energy of the transmission line magnet allows a simple and inexpensive quench protection system. The magnets themselves require no internal quench detection or protection circuitry. Ring-wide quench detection is performed by a single circuit 
located at the power supply terminals. Quenches are detected by a voltage imbalance at the power supply (Figure 5.40). When a quench is detected, magnetic energy is extracted with a 1second L/R time constant by cryogenic "dump resistors" spaced at $20 \mathrm{~km}$ intervals around the ring. This keeps the peak temperature and pressure in the quenched section of the conductor at acceptable levels as described in Section 5.1.2.2. Quench protection system parameters are given in Table 5.23. The theory of operation is explained in [53] and a detailed write-up is given in [54].

Table 5.23. Stage-1 quench detection and protection parameters.

\begin{tabular}{|l|l|}
\hline Transmission Line Current & $100 \mathrm{kA}$ \\
\hline Magnetic Stored Energy @ $100 \mathrm{kA}$ & $10 \mathrm{~kJ} / \mathrm{meter}, 2100 \mathrm{MJ} / \mathrm{ring}$ \\
\hline Magnet Inductance at low field & $3 \mu \mathrm{H} / \mathrm{m} 600 \mathrm{mH} / \mathrm{ring}$ \\
\hline Energy Dump Time Constant & $1 \mathrm{~second}$ \\
\hline Peak Voltage To Ground during Dump & $\pm 3 \mathrm{kV}$ \\
\hline $\mathrm{I}^{2} \mathrm{t}$ during dump & $5 \mathrm{x} 10^{9} \mathrm{~A}^{2} \mathrm{~s}$ \\
\hline Peak Temperature of Conductor During Quench & $250 \mathrm{~K}$ \\
\hline Peak Pressure of Helium During Quench & $35 \mathrm{Bar}$ \\
\hline Pressure relief during quench & Every 2 cells $(500 \mathrm{~m})$ \\
\hline Effective Copper Cross Section of Conductor & $3 \mathrm{~cm}^{2}$ \\
\hline Quench Detection Threshold & 1 Volt \\
\hline Quench Detection Method (primary) & Analog bucking with midpoint of current return bus \\
\hline Quench Detection Method (backup) & Deviation from V=LdI/dt at power supply terminals \\
\hline Energy Dump Resistance & $60 \mathrm{~m} \Omega$ per location, \\
\hline Dump Switch Locations & at cryoplants and midpoint of arc (20 km spacing) \\
\hline Dump Switches & Quenched superconducting cables 65 m long \\
\hline Superconducting Dump Switch Conductor & $1: 1$ CuNi:NbTi “Switch Wire" \\
\hline Cryogenic Dump Resistor Thermal Mass & $65-\mathrm{m}$ long, 3-cm ID, 5.5-cm OD Invar pipe \\
\hline Final Dump Temperature after Dump from 100 kA & $325 \mathrm{~K}$ \\
\hline LHe Required for recovery after Dump from 100 kA & 1600 liters (less if shield flow used for pre-cooling) \\
\hline
\end{tabular}

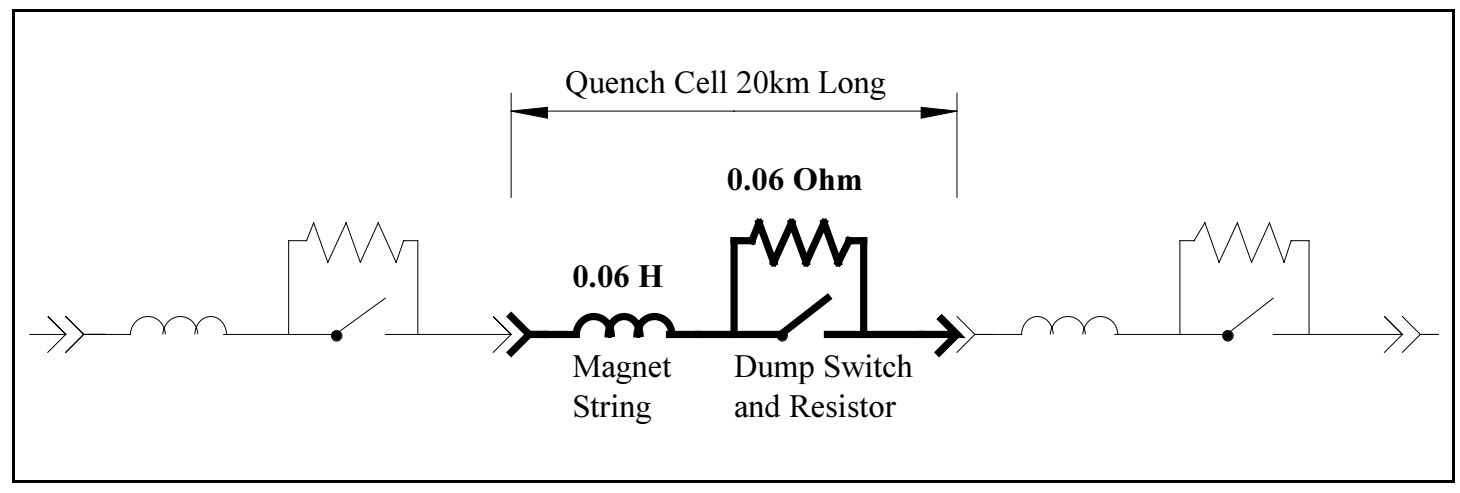

Figure 5.43. Quench protection cell of the transmission line magnet.

The "dump switches" are deliberately quenched sections of transmission line conductor built with $\mathrm{Cu}-\mathrm{Ni}$ stabilized $\mathrm{NbTi}$ "switch wire"[55] which has a high normal state resistance. Current is transferred to a thick-walled Invar pipe that provides thermal mass. These dumps are efficiently recooled using helium from the cryogenic shield flows and transmission line flows, with the warm gas vented into the return header. 
The protection circuit for the $100 \mathrm{kA}$ current leads and power supply is integrated into the quench protection system. When an overtemperature or overvoltage is detected in the current leads, nearby copper buswork, or power supply components, the power supplies are shut off and a ring wide dump is forced. The required response time is equal to the thermal time constant of the leads or approximately 100 seconds.

It is instructive to compare this quench protection system to those of other accelerators. The LHC for example [56] requires a crate of quench detection electronics in the tunnel underneath each dipole in the tunnel. Each LHC magnet has more than 10 voltage taps and instrumentation feed-throughs. The LHC also requires a much larger array of energy dump resistors, dump switches, and current leads around the circumference of the ring. Despite the larger machine circumference, the quench protection system of the Stage-1 magnet is far simpler and should be more reliable.

\subsubsection{Straight Section (Warm Magnet) Power Supplies}

The straight sections use warm iron/copper quadrupoles and a small number of other magnets. In each straight section all $\mathrm{F}$ and $\mathrm{D}$ quadrupoles are powered in series by a single supply. The IR straight sections have a larger number of independently programmable "tuning quads" but the total power and LCW usage is approximately the same as a normal straight section. The power supplies are located in shielded rooms (one per straight section) which contain $\sim 500 \mathrm{~kW}$ of power supplies and are accessible with beam on. These are single quadrant supplies. Given the limited scope of these supplies it is desirable to make them fully redundant, especially those at the far side of the ring from FNAL. A detailed breakdown is given in [57].

Dipoles and quad correctors magnets identical to the arcs are provided at each quad location. Dipole magnets used to separate and cross over the beams are powered by the $100 \mathrm{kA}$ transmission line and require no separate supplies.

\subsubsection{Water Cooling (LCW) System}

The "on-site" and "far side" straight sections require LCW systems. No LCW system exists in the arcs. Major heat loads are the resistive magnets, power supplies, and the RF klystrons. Loads are detailed in [57] and summarized in Table 5.24. The overall scope of the onsite LCW system is comparable to the MI-60 LCW system of the Fermilab Main Injector. The far site $\mathrm{LCW}$ system is about half that size.

The layout of the LCW system is sketched in Figure 5.44. The on-site straight section is connected to the Main Ring LCW system and can use the FNAL infrastructure for filling and makeup. Existing (unused) Main Ring ponds exceed requirements for power dissipation. New ponds and purification systems are required at the far side sites. The vertical drop generates a 150 psi pressure head, which is overcome by locating the circulation pumps at tunnel elevation and pumping the return flow upwards. The beam stop and beam collimation cooling systems require separate pumped loops for radioactive water (RAW) which will be heat exchanged with the main cooling water flow to maintain isolation. 
Table 5.24. LCW and power dissipation summary for Stage-1.

\begin{tabular}{|l|l|c|c|c|}
\hline & Heat Load & Total Loads & Total kW & LCW Flow (I/min) \\
\hline \multirow{4}{*}{$\begin{array}{l}\text { On-Site } \\
\text { Straight } \\
\text { Sections }\end{array}$} & Resistive Magnets & 188 & 4480 & 3200 \\
\cline { 2 - 5 } & Power Supply Cooling & 22 & 500 & 600 \\
\cline { 2 - 5 } & RF Klystrons & 16 & 3760 & 6400 \\
\cline { 2 - 5 } & Ream Stop & 16 & 5600 & 4500 \\
\cline { 2 - 5 } & Total (on-site) & 1 & 500 & 600 \\
\hline \multirow{3}{*}{$\begin{array}{l}\text { Far Side } \\
\text { Straight }\end{array}$} & Resistive Magnets & 227 & 14840 & 15300 \\
\cline { 2 - 5 } Sections & Power Supply Cooling & 176 & 3320 & 3500 \\
\cline { 2 - 5 } & Beam Collimation & 9 & 550 & 600 \\
\cline { 2 - 5 } & Total (far side) & - & 40 & 50 \\
\hline
\end{tabular}

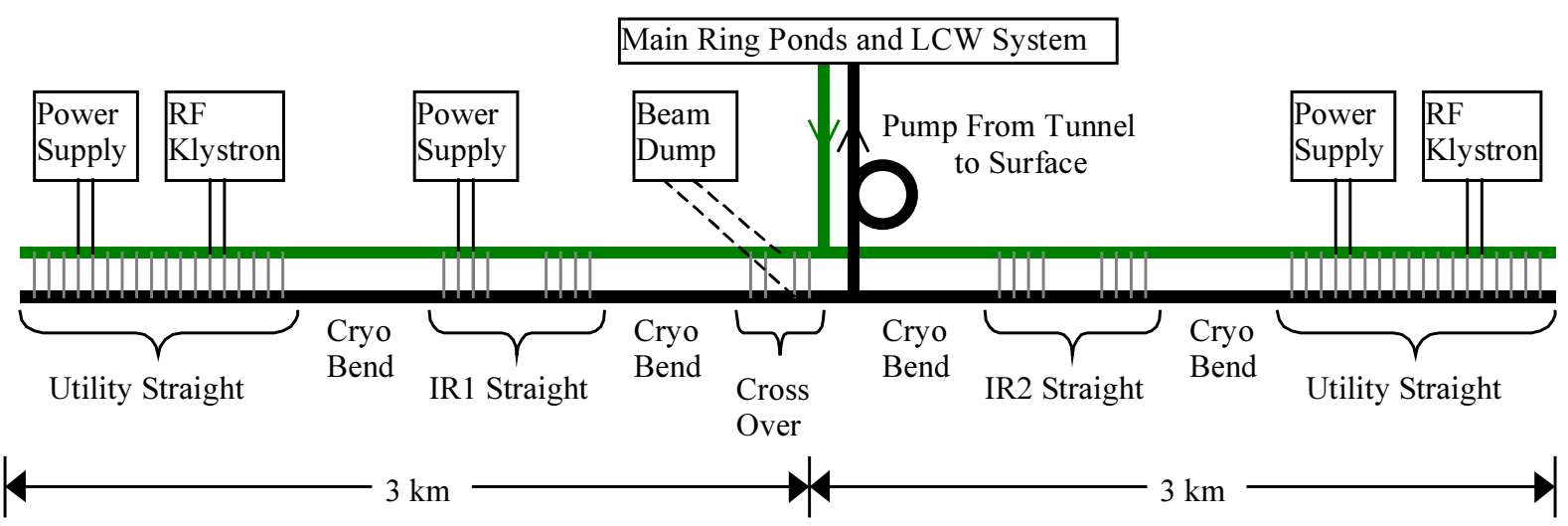

Figure 5.44. LCW system layout for on-site straight section. The far side LCW system is similar except that the RF loads are absent, the beam dump load is replaced by the smaller load from the beam collimation system, and an independent system of ponds and makeup water is needed.

\subsubsection{Arc Instrumentation and Power Distribution}

\subsubsection{Reliability and Maintenance Considerations in the Arcs}

The long arcs of bending magnets have specialized and limited requirements. The once-perturn subsystems and their cabling, power and cooling requirements are absent. No LCW system is required. Only the repetitive instrumentation and corrector power supplies at each of the 1760 "half-cell locations" (every $135 \mathrm{~m}$ around the ring at the location of beta-max in each half-cell) are required. Infrastructure for power and communications occurs at alcoves at $10 \mathrm{~km}$ intervals. 
Reliability is at a premium for the arc instrumentation due to the large size of the machine. Repair technicians at a centrally located facility require a 20 mile drive to reach the surface facility and begin access into the tunnel. Travel time will not be small compared to the 1.5 hour time needed to refill the machine and reaccelerate the beams. For this reason fully redundant capability is provided for all mission-critical subsystems in the arcs. Power distribution is provided to each module by two independent loop feeders with auto-resetting circuit breakers and remote disconnects. Two independent power supplies are provided for each corrector magnet and control function. Redundant network connections to each module are provided. The critical function of beam loss monitoring is provided by redundant sensors read out from alternate half-cell modules. The system design is such that even the failure of a handful of local electronics modules will not require immediate maintenance.
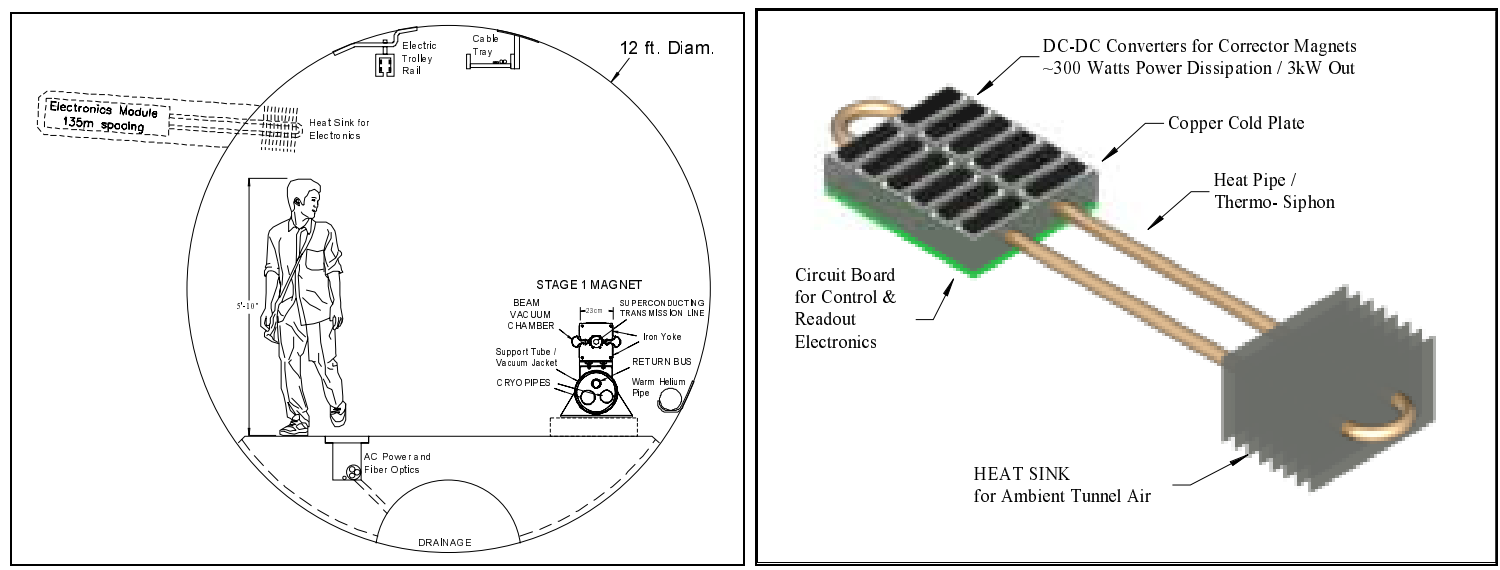

Figure 5.45. Electronics module at each half-cell location (every $135 \mathrm{~m}$ ) contains beam instrumentation and DC-DC power converters for corrector supplies. Modules are in a radiation-shielded "hole in the wall" and dissipate their power in the ambient tunnel air via a heat pipe/thermosiphon and heat sink.

These electronics are located in a radiation-shielded modules buried in "holes-in-the-wall" at each half-cell location. All cables for instrumentation and corrector magnets are preassembled on the magnet and factory tested before installation. Cryogenic thermometry and a valve controller are used at every other half-cell location $(270 \mathrm{~m})$ to regulate the $50 \mathrm{~K}$ shield flow. Every $10 \mathrm{~km}$ there is a walk-in alcove associated with a tunnel egress point that contains conventional electronics racks and provides network connection, tunnel safety systems, bulk DC power for the instrumentation modules, and cryogenic instrumentation for the cooldown valve box. Average power dissipation is approximately $15 \mathrm{~W}$ per meter of tunnel, which can be air cooled.

\subsubsection{Cabling in the Arcs}

At the Fermilab Main Injector, the cable tray to each service building contains 316 cables with a total cable cross sectional area of $180 \mathrm{~cm}^{2}$ [58]. Over $95 \%$ of these cables service Beam Position Monitors (BPMs), Beam Loss Monitors (BLMs), ion pumps, corrector magnets, and temperature and vacuum monitoring. The superconducting Tevatron also has a large cable plant associated with quench protection and cryogenic monitoring and controls. The installation, termination, and testing of these cables are a significant cost and schedule item. 
The Stage-1 design eliminates most cabling costs by multiplexing the signals to a local electronics module (Figure 5.45) at each half-cell boundary. Signals are digitized locally and sent to the control room via network fiberoptics. Instrumentation and corrector magnets are clustered at half-cell locations and cable runs are typically 5 meters. Cable harnesses are prefabricated by outside vendors and mounted on the magnets and tested in the factory.

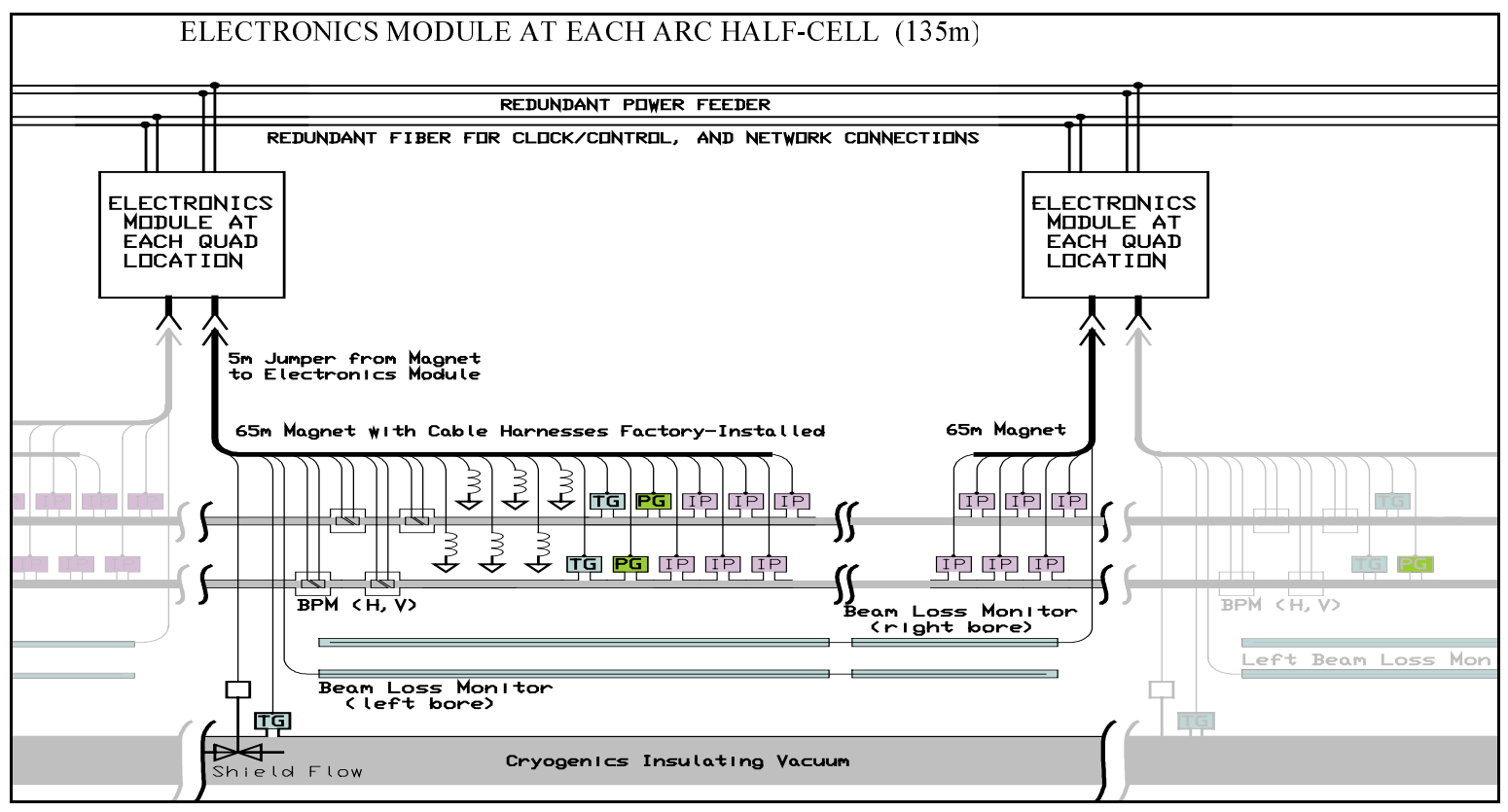

Figure 5.46. Cabling layout on the transmission line magnet. Cables and instrumentation are preinstalled and factory-tested on the 65-m magnets. Each magnet has a 5-m "extension cord" which jumpers to an electronics module located in a hole in the wall at each half-cell location (135-m spacing).

\subsubsection{Corrector Power Supplies}

The warm (air-cooled copper) correction magnets (Sec. 5.1.3) dominate the power consumption in the arcs. The electronics module at each half-cell location has 6-8 corrector magnet loads with a maximum installed power of approximately $1400 \mathrm{~W}$. Average corrector power is $<1000 \mathrm{~W}$ in a realistic operating scenario. At maximum current approximately $90 \%$ of the power is dissipated in the magnets themselves with the remaining $10 \%(\sim 150 \mathrm{~W})$ in the power supply converters. Instrumentation and control power at each half-cell location is estimated to be $\sim 100 \mathrm{~W}$ for a total of $250 \mathrm{~W}$ dissipated in the module. Power at this level can be passively carried away from the electronics (Figure 5.45) via a heat pipe [59] and removed by the tunnel airflow of $\sim 1 \mathrm{~m} / \mathrm{sec}$.

\subsubsection{Power Distribution in the Arcs}

Redundant DC-DC power converters are provided both for module control power and for each trim magnet. Power can be drawn from either of two redundant $1 \mathrm{kV}$ DC feeders that loop the ring (see Figure 5.47). Power supplies are connected in parallel at each load. Under normal 
circumstances the load is shared and each power supply operates at $<50 \%$ of rated load, encouraging long lifetimes. Under failure conditions the load is taken over by a single supply which operates near its design rating (or less, if the corrector magnet setting is below 100\%).

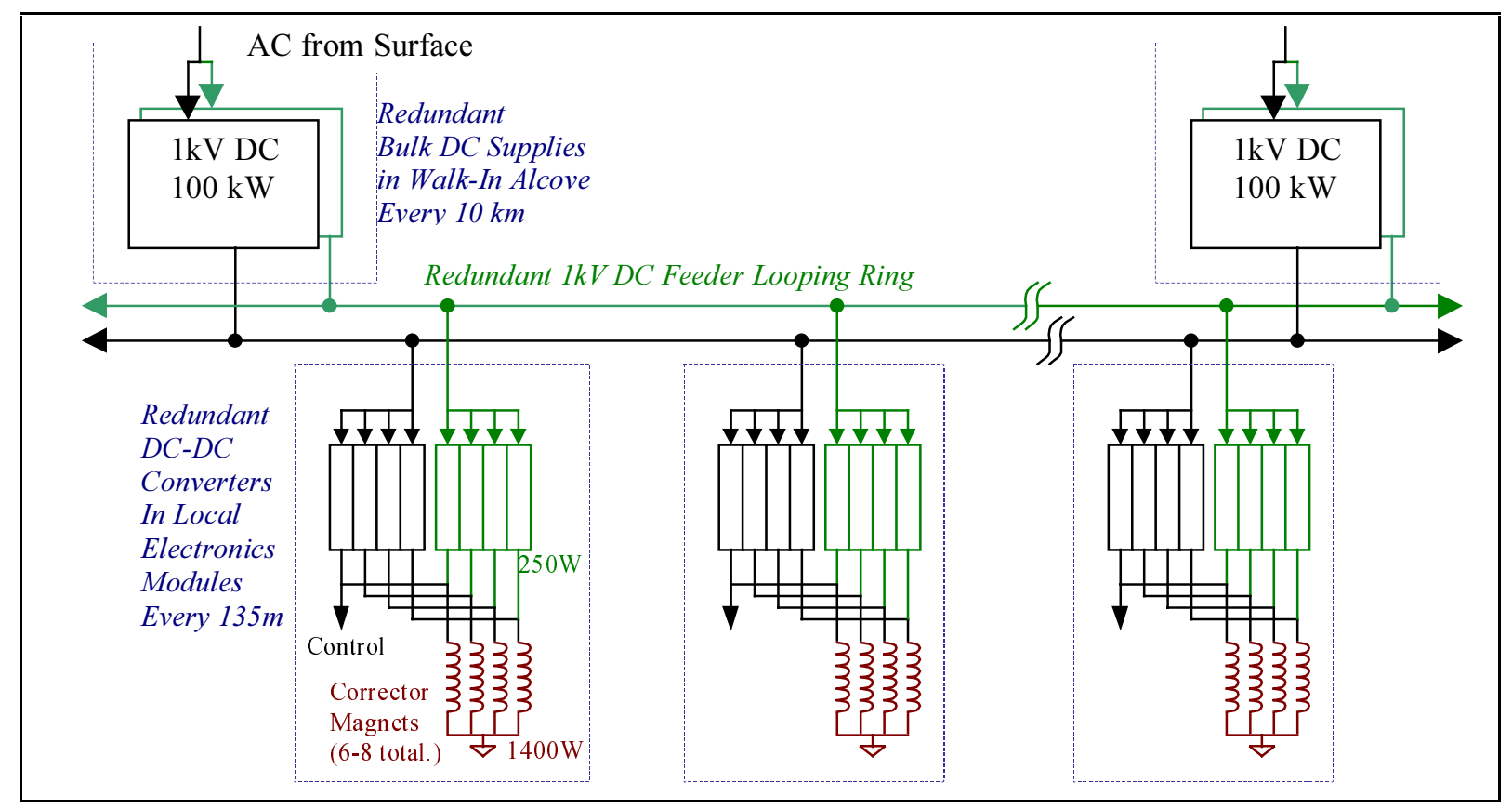

Figure 5.47. DC power distribution scheme in arcs. Redundant bulk DC supplies in walk-in alcoves every $10 \mathrm{~km}$ supply a redundant loop feeder. Each local electronics module contains two DC-DC converters for each load. Under normal conditions all supplies share loads and run at less than 50\% of rated capacity. By means of auto-resetting circuit breakers (not shown), the system can tolerate short or open circuits on the loop feeders, a failure of one or both bulk supplies at any alcove, a failure of the $A C$ surface feed to any single alcove, and individual failures on many corrector supplies.

Medium voltage $1 \mathrm{kV} \mathrm{DC}$ is chosen for power distribution to the electronics modules at each half-cell location. The DC is provided by $100 \mathrm{~A}$ bulk supplies every $10 \mathrm{~km}$ located in racks of electronics in walk-in alcoves. Two redundant bulk supplies are provided at each location, and two independent distribution loops are provided to route the power to the modules. Under normal conditions $25 \mathrm{~A}$ is sourced in either direction onto each of the 500 MCM loop feeders, and less than 3\% of the power is lost in the distribution cabling. Under fault conditions one or both of the bulk supplies at one of the $10 \mathrm{~km}$ locations can fail, with the slack being taken up by adjacent supplies in the loop feeders. A short-circuit fault in one segment of the loop feeders can be tolerated by letting its circuit breaker trip and relying on the remaining loop feeder. Each module is protected with fault current limiting via automatically resetting "solid-state fuses" [60].

The advantages of DC power in this application are as follows. The main power consumption is due to corrector power supplies that naturally operate as DC-DC converters. These are more compact than line-transformer based supplies, an important consideration for the local electronics modules. Modern DC-DC converters [61] have the following parameters: power density $200 \mathrm{~W} / \mathrm{in}^{3}$, efficiency $\sim 90 \%$, cost $\sim \$ 0.35 /$ watt. In fact, essentially all modern AC devices (e.g., fluorescent light ballasts, computers, high current welders, motor controllers) 
rectify the AC line power and then chop the signal using DC-DC techniques. Rectification at $60 \mathrm{~Hz}$ requires bulky capacitors and produces current spikes with power factor and noise problems that complicate AC distribution. DC power distribution makes the most efficient use of the copper in distribution cables, which is a major cost component in power distribution. DC power also simplifies the design of loop feeders, which may be powered from separate segments of the power grid without regard to the relative phase of the incoming AC power. Finally, by phase-locking the switching frequencies of the DC-DC converters to a frequency near the revolution frequency of the machine, the effects of power supply noise on emittance growth can be essentially eliminated as discussed in Section 5.2.2.1.

\subsubsection{Beam Position Monitors (BPMs)}

Both X and Y BPMs (Figure 5.48) will be provided every $135 \mathrm{~m}$ at the corrector region at each half-cell boundary (Figure 5.2). Although measurement of the second coordinate only improves the closed-orbit distortion by $10 \%$ [62], the added redundancy obtained by an independent readout of both coordinates will be beneficial given the small aperture and the travel distance to repair failed BPMs.

Button style beam pickups similar to those used on electron machines and the LHC are adequate. These devices are nonlinear outside the central $30 \%$ of their aperture. Given anticipated closed orbit distortions $<1 \mathrm{~mm}$ this is not a problem. In a few special locations near beam transfers where off-axis orbits must be measured, split-plate BPMs with better linearity will be used.

The BPM calibration offset can be verified in situ using the independently powered quadrupole correctors at each half-cell. The BPM can be accurately fiducialized to the warm iron of the corrector. At injection energy, the quad steering associated with a $1 \mathrm{~mm}$ orbit offset through the corrector generates an easily measured $3 \mathrm{~mm}$ orbit distortion as the corrector is powered.

$\mathrm{BPM}$ readouts using either $\mathrm{AM} / \mathrm{PM}$ or Log Amp electronics are acceptable. In the case of Log-Amp readout both $\log (\mathrm{A} / \mathrm{B})$ and $\log (\mathrm{A})+\log (\mathrm{B})$ would be digitized to provide both position and intensity readout. In the case of AM/PM conversion a separate channel (possibly a log amp) is required to provide intensity information. In either case an in-situ calibration is desirable. The calibration circuit injects equal signals of adjustable amplitude into both channels to accurately determine the zero-offset response at different input strengths. A recent example of log-amp readout made for the SLAC B-factory fit on a 2.7" $\mathrm{x} 4.7$ " board that also included calibration circuitry [63].

Digitization bandwidth is determined by the first turn and transient response desired. A digitization rate $>25 \mathrm{MHz}$ can be supported by the DMA channels of today's Digital Signal Processors (DSPs), with no external components other than the flash ADC. A 10-bit digitization of $\log (\mathrm{A} / \mathrm{B})$ would provide $20 \mu \mathrm{m}$ least count resolution. Power dissipation for four channels of 10-bit $40 \mathrm{MHz}$ digitization would be $300 \mathrm{~mW}$ total [64].

The cable run from the BPM to the local electronics module is about $5 \mathrm{~m}$. This is long enough that the signal must be terminated but short enough that normal RG-58 can be used. The cable runs are short enough that no cable trimming for phase matching is required if $\mathrm{AM} / \mathrm{PM}$ electronics are selected. 

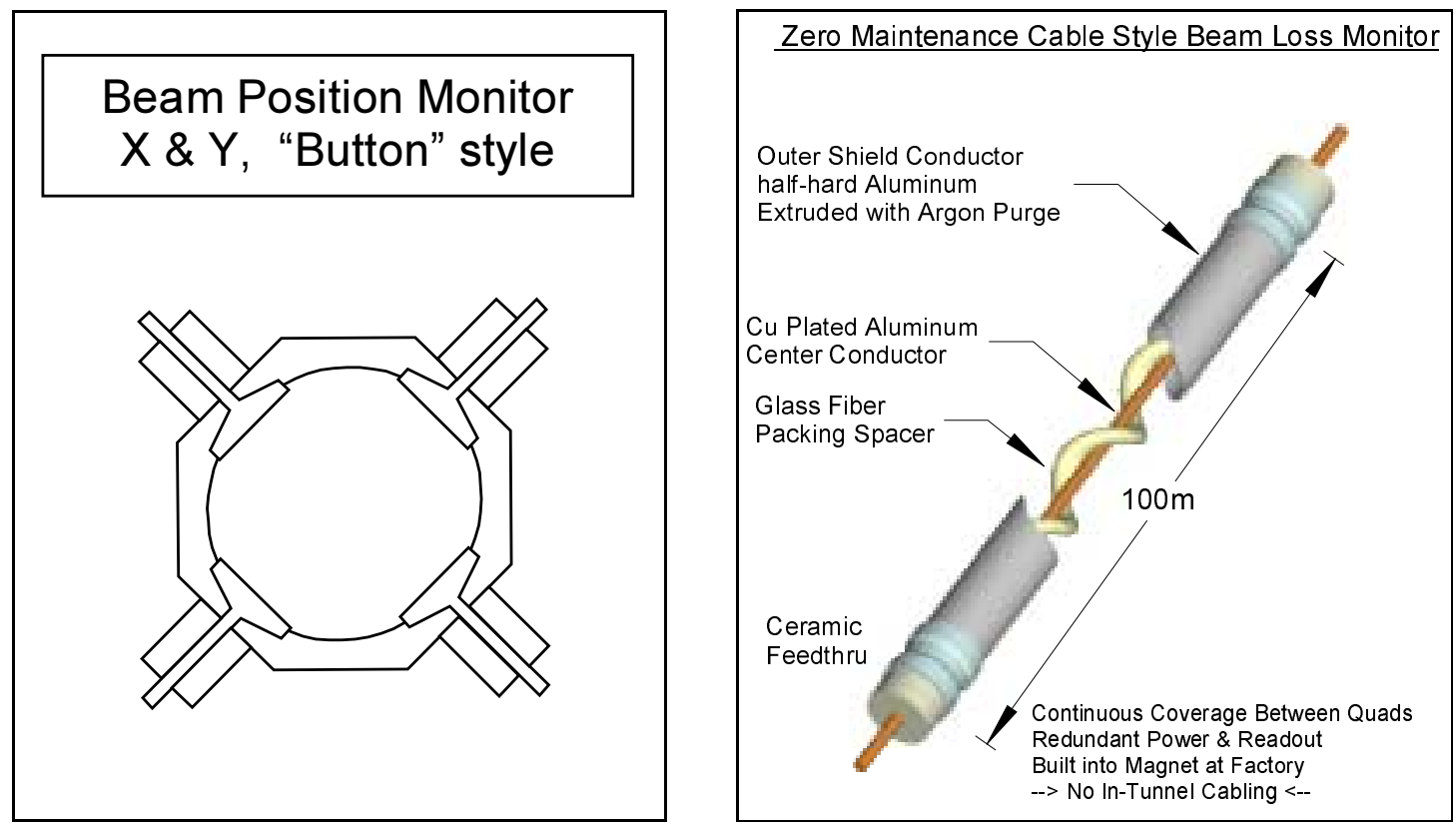

Figure 5.48. Beam Position Monitor (BPM) and Beam Loss Monitor (BLM) for Stage-1 magnets. The BPM is a capacitive "button" style typically found on small aperture beam pipes. The X and Y pickups are rotated by 45-degrees to avoid the synchrotron radiation (this is common at light sources). The $B L M$ is a permanently sealed Argon filled cable-style ionization chamber. Two redundant BLMs run the full length of the magnet. It features all metal and ceramic construction similar to the Tevatron BLMs to allow the device to operate unattended for years (no gas bottle to change).

\subsubsection{Beam Loss Monitors (BLMs)}

The BLM function in the arcs is mission-critical in that no blind spots may exist which might allow chronic beam loss to go undetected. We therefore choose a cable style BLM. This is essentially a long ionization chamber that can provide complete coverage along the length of the magnet. The cable will be all metal/ceramic construction (Figure 5.48) so that no gas-bottle changing will be required. For redundancy, two independent cable BLMs are provided. Each runs the full length of the magnet but they are read out at alternate quad locations. This will provide complete BLM coverage even when the electronics module at a single location goes incommunicado. Separate BLMs on each side of the magnet provide some differential sensitivity to beam losses in each bore. Electronics required include a $2 \mathrm{kV} / 100 \mu \mathrm{A}$ Cockroft-Walton DC-DC converter module, an op-amp integrator, and a medium bandwidth $\sim 100 \mathrm{kHz} 14$-bit ADC. Power dissipation should be $<2 \mathrm{~W}$.

\subsubsection{Ion Pump Power Supplies \& Vacuum Gauges}

Each $135 \mathrm{~m}$ half-cell contains 12 ion pumps (22.5 m pump spacing in each bore). These are powered and read out from the local electronics module. Each pump requires a $5 \mathrm{kV}$ supply with a starting current of up to $30 \mathrm{~mA}$ but an operating current less than $0.1 \mathrm{~mA}$. The power for all 12 pumps is provided by a single transformerless bulk supply (a 5-stage Cockroft-Walton converter operating from the $1 \mathrm{kV}$ DC feeder). High voltage DC relays (Cotto 5501) isolate each pump and give the capability of providing the full starting current sequentially to each supply. Individual current readback on each pump is provided via an array of $\mathrm{V} / \mathrm{F}$ converters 
operating at HV with an optically isolated data link to the DSP. Vacuum gauge readout for the beam and insulating vacuum will also be incorporated into the local electronics module.

Failure of the bulk supply shuts down all ion pumps in a 135-m half-cell. Pumping from the NEG strip remains intact. This results in a 50x rise in the pressure of a single gas species $\left(\mathrm{CH}_{4}\right)$ over the length of one half cell, equivalent to a $3 \%$ rise in the average pressure of $\mathrm{CH}_{4}$ in the ring and $<1 \%$ drop in the overall beam lifetime. Redundancy in the ion pump power supplies is therefore unnecessary.

\subsubsection{Network \& Clock Connections}

The electronics module at each quad location contains a DSP computer with an optical fiber connection running a network protocol stack. This CPU can store, average, spectrum analyze, and data-compress all digitized information before transmitting over a conventional fiber network. A separate dedicated fiber handles the FNAL-specific real time signals such as clock, event triggers, and beam abort heartbeat signals. These will be handled in hardware logic by a Programmable Logic Device (PLD) in the electronics module.

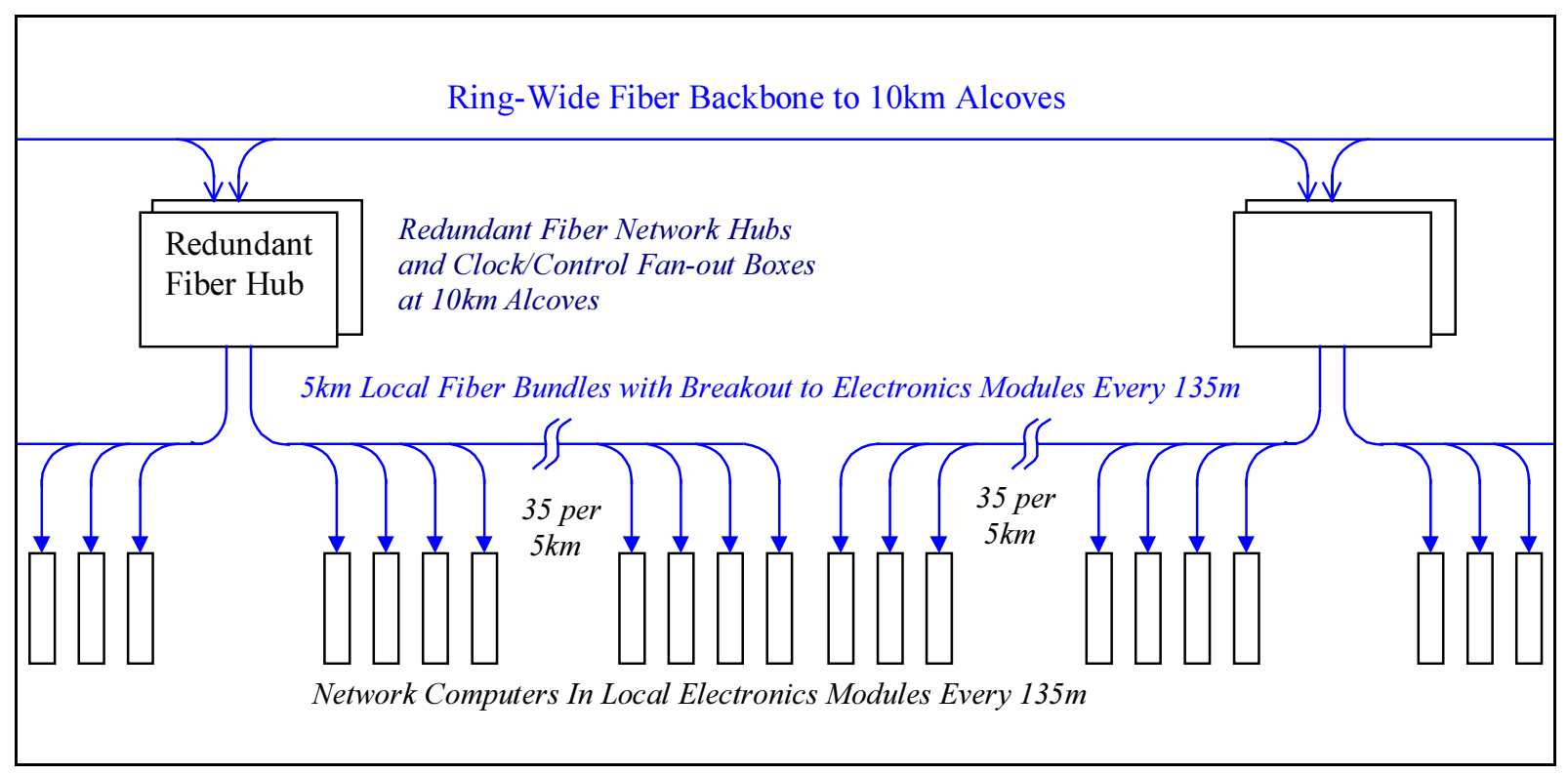

Figure 5.49. Fiberoptic clock and network connections in the tunnel, including redundant hubs and clock fanouts at 10-km alcoves.

A minimal set of fiber connection to each module consists of one network connection plus one dedicated fiber for delivering Fermilab-specific signals. For cost estimation purpose we have assumed four direct point-to-point fiber connections between each quad location and redundant network hubs at located in the $10-\mathrm{km}$ alcoves. The fiber cable will be a pre-assembled harness 5-km long with 4-fiber breakouts at quad locations every $135 \mathrm{~m}$. Installation will consist of laying the fiber harness in the cable tray immediately below the electronics modules (Figure 5.49) and plugging the breakout into each electronics module. 
The long-haul fiber backbone interconnecting the $10-\mathrm{km}$ alcoves around the ring will be buried with the power duct in the invert. This provides additional radiation shielding to compensate for the longer distance of the backbone fiber runs (see Section 5.3.3.2).

\subsubsection{Local Electronics Module Miscellaneous Functions}

The networked CPU in each electronics module allows additional features to be added at relatively low cost. In many cases this requires adding only an interface IC, transducer and software to the local electronics module. The engineering effort for this is not unreasonable given the large quantity of local electronics modules. Some possibilities are:

- Tunnel temperature, humidity, and airflow transducers

- Integrated radiation dose monitor for the electronics module (FET + resistor)

- Microphones for detecting quenches and water problems

- Speakers to communicate with tunnel crews

- RF modules to communicate with the cell phones and PDAs of tunnel crews

- Cryogenic sensors and valve controls (presently estimated as a separate system)

- Vacuum valve controls (presently estimated as a separate system).

\subsubsection{Beam Vacuum System}

The beam vacuum system is divided into two sections. In the $225 \mathrm{~km}$ of transmission line magnets an extruded-aluminum warm vacuum system similar to LEP and electron storage rings is used. In the $8 \mathrm{~km}$ of straight sections synchrotron radiation is absent and the vacuum system will be a conventional stainless steel system similar to those commonly used at Fermilab.

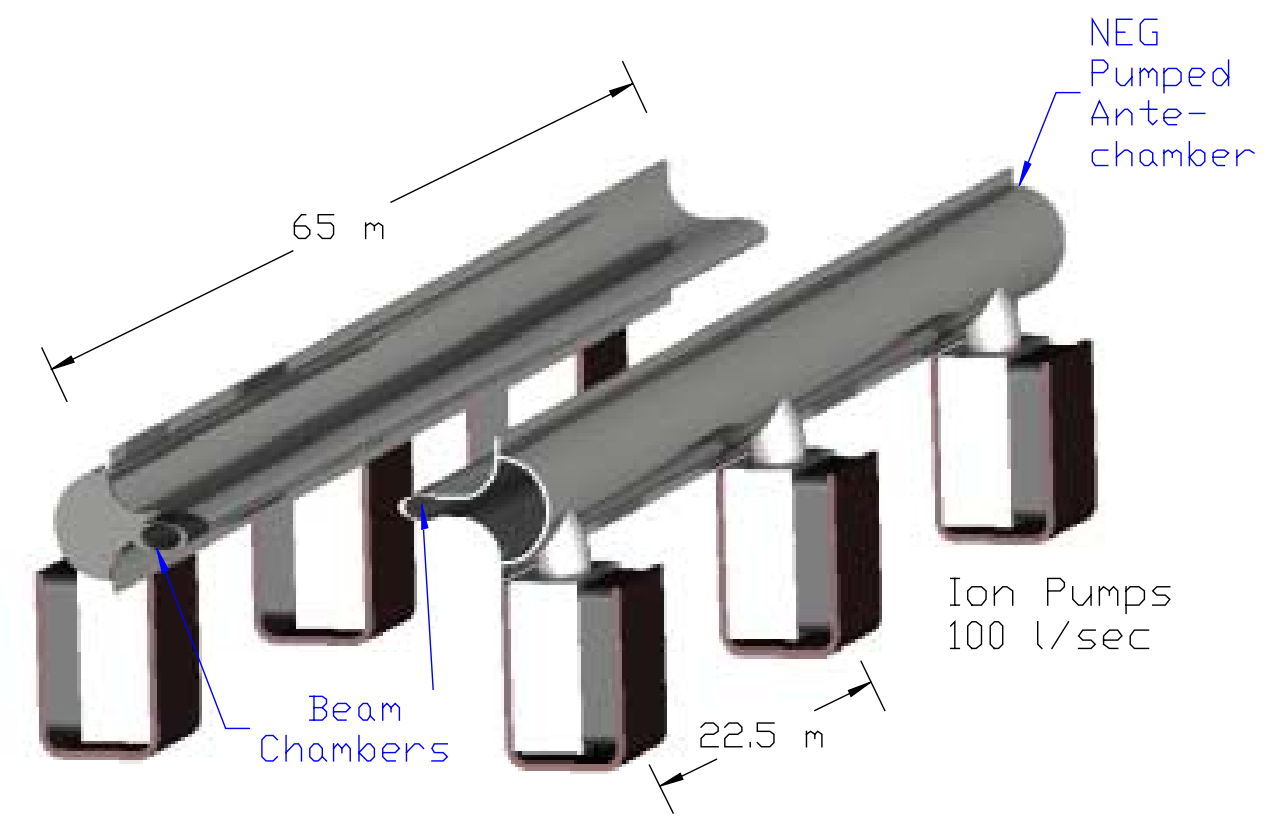

Figure 5.50. Extruded aluminum beam vacuum system in the transmission line magnets. 


\subsubsection{Arc Magnet Vacuum System}

An extruded aluminum vacuum system (Figure 5.50) is used for the $225 \mathrm{~km}$ of arc magnets (450 km of beam pipe). The design of this system is driven by vacuum desorption from synchrotron radiation, the small aperture of the magnet gap, beam impedance and cost. Aluminum is chosen for reasons of economy and beam impedance. The system is pumped by a nonevaporable getter (NEG) strip located in an antechamber of the extrusion. Methane and noble gasses are pumped by $100 \mathrm{l} / \mathrm{s}$ sputter-ion pumps spaced every $22.5 \mathrm{~m}$. The antechamber dimensions are chosen to provide adequate vacuum conductance to transport synchrotron-desorbed methane to the ion pumps. Pump power and vacuum gauges are discussed in 5.2.3.7.

The parameters and performance of this system have been analyzed in $[65,66]$. Inputs to the calculation included: 1) the measured synchrotron-induced desorption of major gas species from aluminum [67] as a function of the integrated dose of $E_{\text {CRIT }}=86 \mathrm{eV}$ photons, 2) the measured pumping speeds for NEG strip as a function of absorbed gas [68], 3) calculated conductances of the extrusion antechamber, 4) ion pump speeds for methane, and 5) scattering cross sections for the relevant gas species. This approach has given good agreement with the measured performance of LEP [69]. The result of the calculation (Figure 5.51) is that a beamgas scattering lifetime greater than 200 hours will be achieved after approximately one week of operation. During the "beam cleaning" period the beam current will be increased from an initial $50 \mathrm{~mA}$ to the design value of $190 \mathrm{~mA}$ in five successive fills. The beam power scattered into the cryogenic system (Section 5.3.3) will not be a limiting factor during this time period.

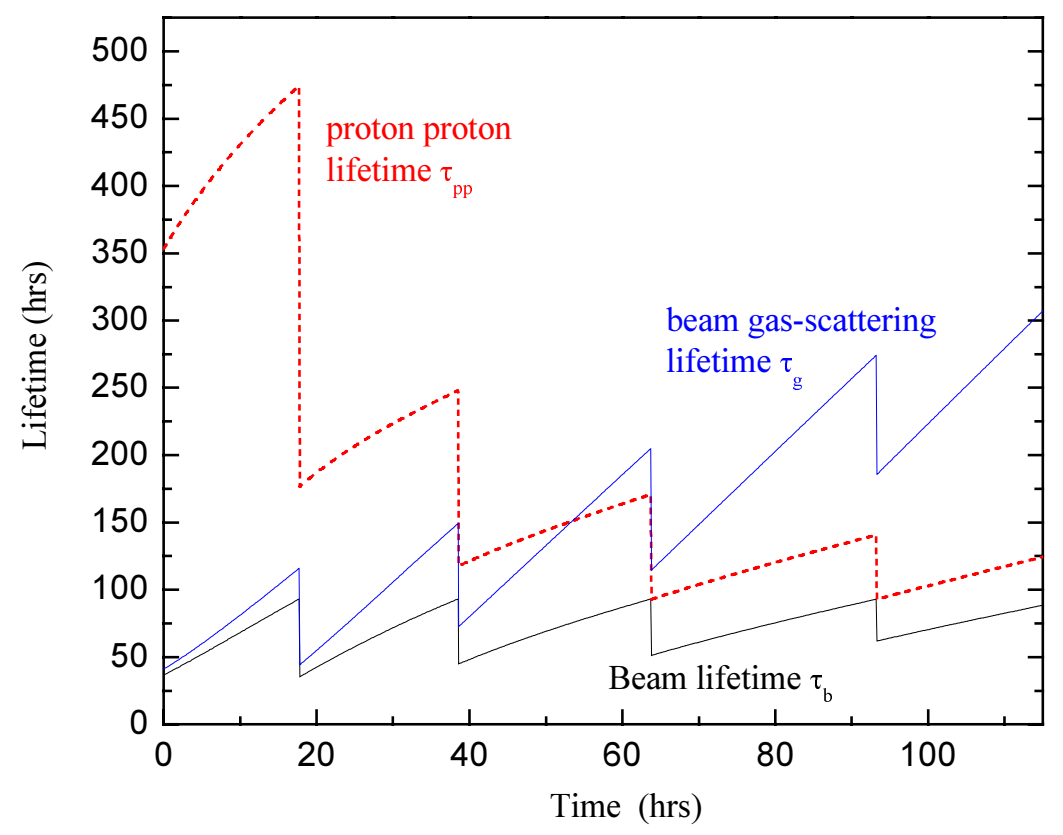

Figure 5.51. Beam scrubbing scenario for Stage-1 aluminum vacuum chamber. During the first 5 fills the beam current is increased from $50 \mathrm{~mA}$ to the design value of $190 \mathrm{~mA}$. As synchrotron radiation desorption cleans the walls of the beam chamber, the vacuum lifetime increases to $>200$ hours.

Production of the aluminum vacuum system will be integrated into the magnet factory. Tunnel labor is minimized by pre-assembling $65 \mathrm{~m}$ lengths of extruded pipe which are installed in the magnets, cabled, and tested at the factory. Beam pipe extrusions $12 \mathrm{~m}$ in length will be 
received, inspected, and cleaned using an environmentally safe alkaline cleaning procedure developed at the APS at Argonne and used on the Fermilab Recycler [70]. The sections will be welded together to form $65 \mathrm{~m}$ lengths that include the Ion Pumps, NEG strips, feed-throughs and end transition sections. The assembly will be sealed, pumped and baked at $200^{\circ} \mathrm{C}$ for 12 hours in a $65 \mathrm{~m}$ long vacuum oven similar to the one built for the Recycler beam pipes.

The vacuum pipe assembly is then snapped into the C-magnet gap (Figure 5.52) in such a way that the beam chamber is pre-loaded against the magnet laminations. The preload prevents any possible loss of vertical aperture inside the magnet. The beam pipe has to be deformed slightly to get it inside of the horizontally focussing gradient magnet. This has been analyzed [71] in ANSYS and stresses are acceptable even for annealed aluminum.

The design does not provide for an in-situ bakeout. After factory bakeout the magnets will be stored and transported with the beam pipes capped off and under vacuum, and the beam pipes will be let up to dry nitrogen and the caps cut off to make the field joints in the tunnel. Under these circumstances an in-situ bakeout gains about a factor of 2 in initial dynamic outgassing [72], and gains very little after the beam scrubbing has completed [73]. A similar conclusion has been reached at the ELETTRA light source [74]. Our R\&D program should verify this.

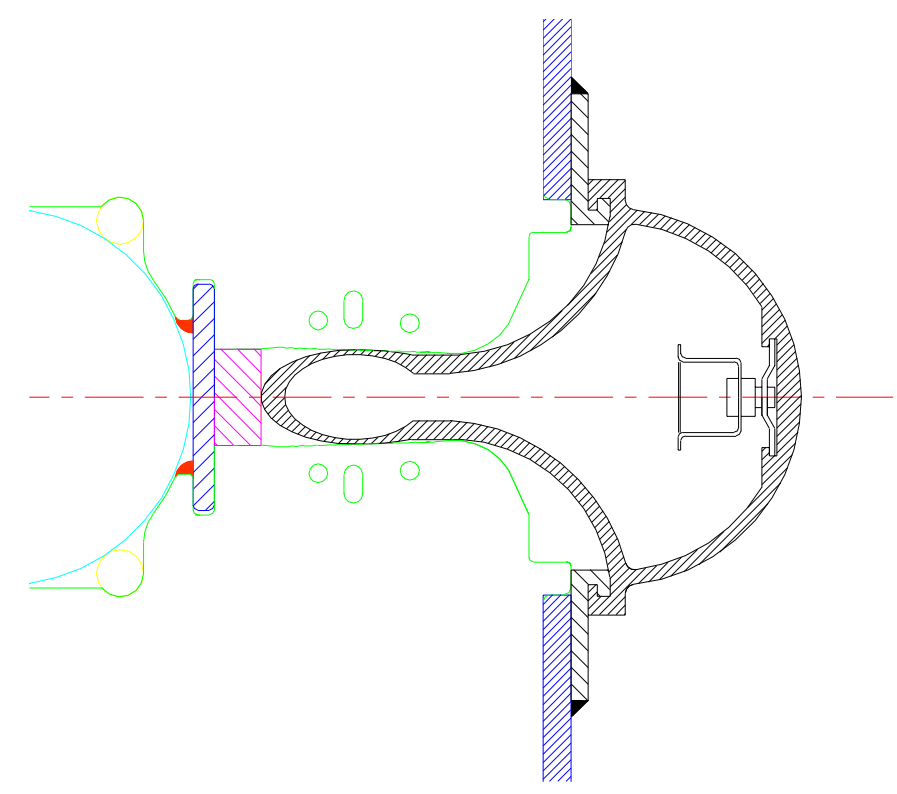

Figure 5.52. Cross section of beam pipe extrusion inserted into the magnet. During insertion the beam pipe is elastically deformed to fit it into the gradient magnet.

\subsubsection{Straight Section Vacuum System}

Synchrotron radiation is absent or reduced in most of the $8 \mathrm{~km}$ of straight sections and the vacuum system will be a conventional baked stainless steel / ion pump system similar to those commonly used at Fermilab. The $10 \mathrm{~cm}$ aperture of the $135 \mathrm{~m}$ stainless steel pipes between quadrupole locations is large enough that the beam impedance, alignment, and vacuum conductance are not a problem. The reduced apertures at the quadrupoles and corrector locations will be handled with $30 \mathrm{~cm}$ funnel transitions, which may be copper-plated stainless, or aluminum. 


\subsubsection{Once-per-Turn Instrumentation}

A number of standard instrumentation systems occur only once per ring. In general these can be copied from existing systems in the Tevatron and/or Main Injector. The $53 \mathrm{MHz}$ bunch structure and modest beam currents $(\sim 1 / 3$ of Tevatron fixed target) provide no special challenges. Readout electronics for these systems is located in shielded rooms at the Utility Straight Sections. A warm beam pipe is used everywhere except at the IR triplet and superconducting RF cavities, so the instrumentation interface is simple. Physical layout of these systems is not heavily constrained since quadrupole spacing is typically $150 \mathrm{~m}$, the beam pipes are separated by $\sim 45 \mathrm{~cm}$ for much of the utility straight sections, and the transmission line and current return have been moved away from the beam to reduce fringe field. These are discussed more fully in [75].

Table 5.25. Once-per-turn instrumentation parameters.

\begin{tabular}{|l|l|l|l|}
\hline FUNCTION & Occurrences & Readout Frequency & Comments \\
\hline Tune Measurement & a few/ring & $10 \mathrm{~Hz}$ & Use Arc Module DSPs for FFT? \\
\hline Beam Current Toroids & 1/ring & & Also on injection lines \\
\hline Sampled Bunch Display & $1 /$ ring & $1 \mathrm{~Hz}$ & \\
\hline Fast Bunch Integrator & $1 /$ ring & $1 \mathrm{~Hz}$ & \\
\hline Synchrotron Light Monitor & 1/ring & $1 \mathrm{~Hz}$ & \\
\hline Ion Profile Monitor & 2/ring & $.1 \mathrm{~Hz}$ & Small beam size may be challenging \\
\hline Flying Wires & 1/ring & $\sim$ few per store & \\
\hline
\end{tabular}

\subsubsection{Radio Frequency Systems}

Either superconducting or warm copper cavities produce workable RF acceleration systems for the Stage-1 machine [76]. During the design study acceptable parameters and costs based on the B-factory RF systems were developed [77]. The system described here is based on superconducting RF (SCRF). The advantages of SCRF are a higher energy efficiency during the long periods at storage flattop, and more flexibility to produce RF overvoltage to control beam instabilities if this proves to be an advantage.

The RF system parameters and some RF relevant machine parameters are summarized in Table 5.26. The RF frequency is $371.7 \mathrm{MHz}$, the seventh harmonic of $53.1 \mathrm{MHz}$. Overall the VLHC RF system is similar to the LHC RF [78,79], but with somewhat relaxed requirements for higher-order mode damping due to lower total beam current. The superconducting cavities are manufactured using niobium on copper technology developed at CERN. As in the case of LHC, there will be separate RF systems for counter-rotating beams. The beam separation in LHC is $420 \mathrm{~mm}$. If we just scale cryomodule dimensions with RF frequency, we get beam separation of $453 \mathrm{~mm}$.

To minimize the effect of the transient beam loading, cavities with high stored energy are preferred. The LEPII RF system experience [80] and tests of LHC [79] and SOLEIL [81] cavities show that it is reasonable to expect operating gradients as high as $8 \mathrm{MV} / \mathrm{m}$. For VLHC we chose a gradient of $7.75 \mathrm{MV} / \mathrm{m}$ (3.125 MV per cell). Sixteen single-cell superconducting cavities are needed for each beam in the Stage-1 machine and 64 cavities are needed for each beam in the Stage-2 machine. The low $R / Q$ of the LHC cavity helps to increase its stored energy. Still, the large $(10 \%)$ beam gap will produce a very strong periodic modulation of $\mathrm{RF}$ 
phase of $27^{\circ}$ for Stage- 1 and $10^{\circ}$ for Stage-2. This modulation is larger than at the LHC. A special RF feedback loop around the amplifier-cavity system assisted by the programmed phase modulation a la LHC [82] will be necessary to suppress this effect.

Table 5.26. Summary of RF system parameters.

\begin{tabular}{|c|c|c|c|c|}
\hline & \multicolumn{2}{|c|}{ Stage-1 } & \multicolumn{2}{|c|}{$\begin{array}{r}\text { Stage-2 } \\
\end{array}$} \\
\hline & Acceleration & Storage & Acceleration & Storage \\
\hline Beam current & \multicolumn{2}{|c|}{$190 \mathrm{~mA}$} & \multicolumn{2}{|c|}{$68.9 \mathrm{~mA}$} \\
\hline Beam energy & $0.9-20 \mathrm{TeV}$ & $20 \mathrm{TeV}$ & $10-87.5 \mathrm{TeV}$ & $87.5 \mathrm{TeV}$ \\
\hline Acceleration time & $1000 \mathrm{sec}$ & & $2000 \mathrm{sec}$ & \\
\hline Acceleration per turn & $14.8 \mathrm{MV}$ & & $39.4-18.15 \mathrm{MV}$ & \\
\hline Acceleration power (both beams) & $5.62 \mathrm{MW}$ & & $4.134 \mathrm{MW}$ & \\
\hline Synch. rad. loss per turn & & $0.03 \mathrm{MeV}$ & & $12.37 \mathrm{MeV}$ \\
\hline Total s.r. power (both beams) & & $13 \mathrm{~kW}$ & & $2.1 \mathrm{MW}$ \\
\hline Revolution frequency & \multicolumn{4}{|c|}{$1286.5 \mathrm{~Hz}$} \\
\hline Bunch length, rms & $142 \mathrm{~mm}$ & $66 \mathrm{~mm}$ & $81.9 \mathrm{~mm}$ & $33.7 \mathrm{~mm}$ \\
\hline Synchrotron tune & 0.00845 & 0.00179 & .00280 & .00189 \\
\hline Synchrotron frequency & $10.87 \mathrm{~Hz}$ & $2.30 \mathrm{~Hz}$ & $3.60 \mathrm{~Hz}$ & $2.43 \mathrm{~Hz}$ \\
\hline Bunch frequency & \multicolumn{4}{|c|}{$53.1 \mathrm{MHz}$} \\
\hline Number of buckets & \multicolumn{4}{|c|}{41280} \\
\hline Bunch spacing & \multicolumn{4}{|c|}{$5.646 \mathrm{~m}, 18.8 \mathrm{~ns}$} \\
\hline RF harmonic number & \multicolumn{4}{|c|}{288960} \\
\hline RF frequency $(7 \times 53.1)$ & \multicolumn{4}{|c|}{$371.7 \mathrm{MHz}$} \\
\hline RF wavelength & \multicolumn{4}{|c|}{$80.65 \mathrm{~cm}$} \\
\hline RF voltage & $50 \mathrm{MV}$ & $50 \mathrm{MV}$ & $50 \mathrm{MV}$ & $200 \mathrm{MV}$ \\
\hline Accelerating gradient & \multicolumn{4}{|c|}{$7.75 \mathrm{MV} / \mathrm{m}$} \\
\hline Voltage per cavity & \multicolumn{4}{|c|}{$3.125 \mathrm{MV}$} \\
\hline$R / Q$ & \multicolumn{4}{|c|}{$89 \mathrm{Ohm}$} \\
\hline$Q$ factor at $8 \mathrm{MV} / \mathrm{m}$ & \multicolumn{4}{|c|}{$2 \times 10^{9}$} \\
\hline Number of cavities & \multicolumn{2}{|c|}{$32(16+16)$} & \multicolumn{2}{|c|}{$128(64+64)$} \\
\hline Cavities per cryostat & \multicolumn{4}{|c|}{4} \\
\hline RF cavity wall losses & \multicolumn{4}{|c|}{$55 \mathrm{~W}$} \\
\hline Cryostat static heat leak & \multicolumn{4}{|c|}{$60 \mathrm{~W}$} \\
\hline Total cryogenic heat load & \multicolumn{2}{|c|}{$2.24 \mathrm{~kW}$} & \multicolumn{2}{|c|}{$8.96 \mathrm{~kW}$} \\
\hline Beam power per cavity & $176 \mathrm{~kW}$ & $0.406 \mathrm{~kW}$ & $42.4 \mathrm{~kW}$ peak & $16.4 \mathrm{~kW}$ \\
\hline Number of $500 \mathrm{~kW}$ klystrons & \multicolumn{2}{|c|}{$16(8+8)$} & \multicolumn{2}{|c|}{$16(8+8)$} \\
\hline Number of cavities per klystron & \multicolumn{2}{|c|}{2} & \multicolumn{2}{|c|}{8} \\
\hline
\end{tabular}

Each cryomodule will accommodate four single-cell cavities with cell-to-cell distance of $3 \lambda / 2$. The overall length of the cryomodule is approximately $5.6 \mathrm{~m}$. The static heat leak of the cryomodule is $60 \mathrm{~W}$ and the RF cavity wall losses are $55 \mathrm{~W} /$ cavity, which yields a total cryogenic load of $280 \mathrm{~W}$ per cryomodule. The overall cryogenic heat load (without losses in cryogen distribution system) is $2.24 \mathrm{~kW}$ for Stage-1 and $8.96 \mathrm{~kW}$ for Stage-2.

Due to the rather small RF power per cavity, we propose to use $500 \mathrm{~kW}$ klystrons with two cavities per klystron in Stage-1 and eight cavities per klystron in Stage-2. The number of klystrons required is 16 for each stage. A $500 \mathrm{~kW} \mathrm{CW}$ klystron was jointly developed and constructed recently by CERN and SLAC for testing LHC cavities [83]. The klystron has a high 
efficiency of $68 \%$ and thus the required $\mathrm{AC}$ power is $735 \mathrm{~kW}$ to generate RF output power of $500 \mathrm{~kW}$. Then the maximum total wall-plug AC power for klystrons is $11.76 \mathrm{MW}$ total for both beams.

The klystron collector cooling water flow of $\geq 4001 / \mathrm{min}$ yields a total cooling water requirement of $6400 \mathrm{l} / \mathrm{min}$. RF water loads will need an additional $4000 \mathrm{l} / \mathrm{min}$ (during beam deceleration only) and ferrite circulators will consume $480 \mathrm{l} / \mathrm{min}$. Thus the total amount of deionized water required for the RF system is $10,880 \mathrm{l} / \mathrm{min}$ per ring.

To estimate the cost of Stage-1 cryomodules we will use the formula [84]

$$
C=k \cdot(0.8)^{\log (L)} \sqrt{\frac{1300 M H z}{f[M H z]}} \cdot L
$$

Here $L$ is the active length of accelerating structure; $k=200 \mathrm{k} \$ / \mathrm{m}$ is the cost factor. As one can see, the cryomodule cost scales approximately linearly with total length of the RF structure. The total cost of cryomodules for Stage-1 is then equal to $4.2 \mathrm{M} \$$. The RF chain cost (including klystrons, high voltage power supplies, low level electronics, and RF distribution) is $\$ 1 / \mathrm{W}$ and totals to $8 \mathrm{M} \$$.

The VLHC RF system would greatly benefit from R\&D on improving the technology of sputtering niobium on copper with the goal to decrease if not completely eliminate the phenomenon of the $Q$ slope. Special efforts should be devoted to understanding the nature of this effect. Success of this R\&D would allow an increase of the accelerating gradient without seriously increasing the cryogenic heat load. Also, an R\&D program is required to develop RF controls for heavy beam loading. Careful analysis of transients during injection, acceleration and beam storing is necessary and may necessitate having an individual klystron for each cavity [79].

\subsubsection{Beam Dampers}

In the last 30 years high intensity storage rings have become absolutely dependent on beam damper circuits. The Tevatron in fixed-target mode, the Booster, and the LHC will not operate anywhere near design intensity without them. At synchrotron light sources and B-factories the beams are lost within milliseconds without dampers. It is thus not surprising that the VLHC requires damper circuits to reach design luminosity for both Stage-1 and Stage-2 operation. Fortunately this technology is well developed. One new element which does occur in the Stage1 VLHC is the necessity to distribute the dampers for one type of instability (the coupled-bunch resistive-wall instability) around the large circumference of the ring. While this provides some level of redundancy (since the beam is stable even if one of the distributed circuits fails) it is a new element which will be described below. Other dampers are conventional.

\subsubsection{Distributed Transverse Dampers for Resistive-Wall Stability}

Growth of transverse motions of bunches in the LF ring will be suppressed with active feedback systems [85]. The strongest beam impedance arises from resistive-wall. At the frequency of the first lower-sideband coupled-bunch mode, which is (1-0.235) $f_{0}=0.99 \mathrm{kHz}$, the transverse beam impedance is $(7.84+1.27 \mathrm{j}) 10^{10} \mathrm{ohm} / \mathrm{meter}$. This causes an exponential growth rate 
of $2.53 \times 10^{3}$ per second for a fully filled machine. Prompt cancellation of this growth is provided by six localized feedback stations spaced around the ring circumference. At each station, radial and axial damping are the same, and for each there are two feedback systems displaced azimuthally by $270^{\circ}$ in betatron phase to address both 'sine' and 'cosine' phases of the oscillation; this makes the damping tolerant of tune changes that might be desired.

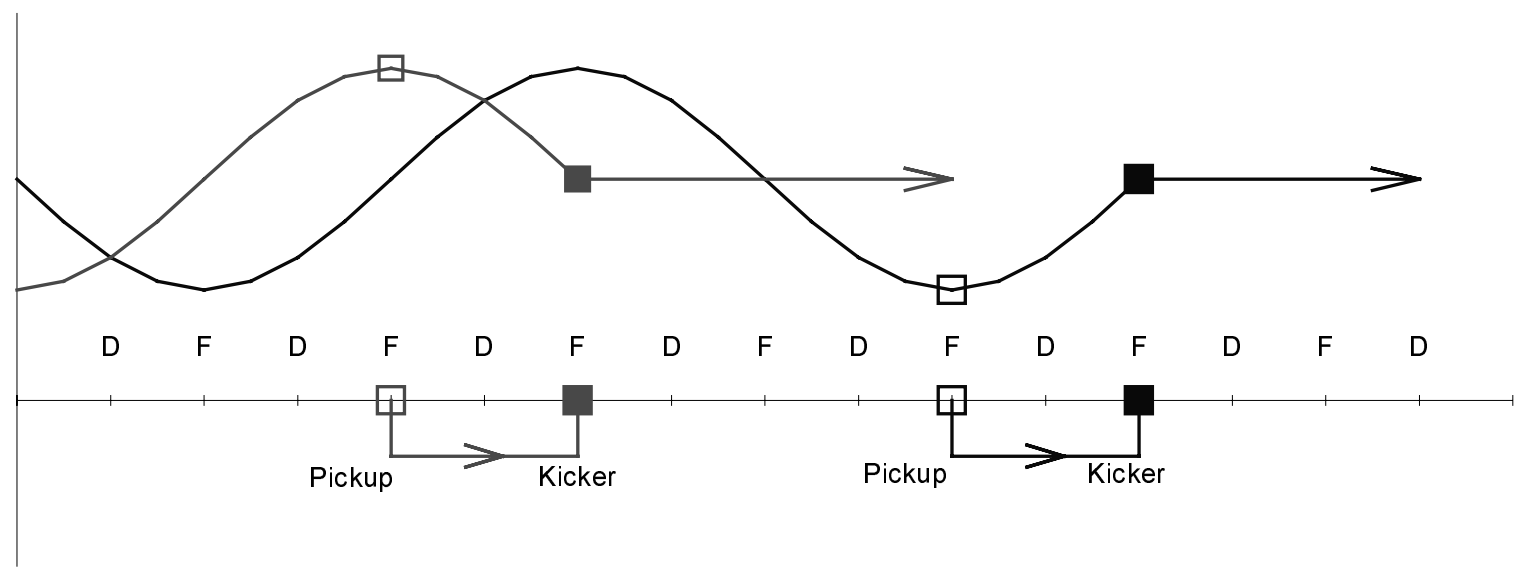

Figure 5.53. Layout of pickup and kicker positions for the horizontal coordinate at one station. Incoming beams with sine-like or cosine-like oscillations have their positions sensed by pickups at cell boundaries (F-locations) and are kicked back towards the design orbit after one-quarter betatron oscillation. An identical set of vertical pickups and kickers operates at D-locations. Six identical stations are distributed around the ring, one at each Stage-1 cryoplant location.

In localized feedback, a kicker is located downstream from the pickup by $90^{\circ}$ in betatron phase (one lattice cell) and the signal is transmitted promptly from pickup to kicker through foam-insulated coax to minimize delay. Delay in the 271 meters of cable is $180 \mathrm{~ns}$ to which one adds about $50 \mathrm{~ns}$ for electronic processing and amplification. Although with this delay the signal from one bunch is delivered to the $12^{\text {th }}$ following bunch, the phase delay at $1 \mathrm{kHz}$ is a negligible 1.5 milliradian. For higher-frequency sidebands the phase delay increases, becoming a significant $1 / 8$-oscillation at $550 \mathrm{kHz}$. The localized feedback systems will be used up to 500 $\mathrm{kHz}$; at that frequency the impedance has become small enough to be controlled by a conventional one-turn bunch-by-bunch system that extends up to the needed $26.5 \mathrm{MHz}$ bandwidth of coupled-bunch modes.

The hardware in a local feedback system consists of (1) a pair of capacitive pickup plates, (2) electronics and cable to the kicker, and (3) a 4-meter long stripline-pair kicker. The pickup strips are $25 \mathrm{~cm}$ long and connect to high-impedance amplifiers. The signals are then differenced and the unipolar pulses are stretched to half the interval between bunches to give a baseband signal for the kicker. At the lowest, strongest mode, the required feedback loop-gain is $G=0.287$ and to damp injection errors of $0.1 \mathrm{~mm}$ calls for a transverse kick of $83.3 \mathrm{kV}$. The kicker delivers this with a power input of $1.10 \mathrm{~kW}$. Injection in bunch trains separated by 2 seconds produces unwanted transients, especially if there is closed-orbit offset. Bunch signals will be recorded and analyzed to generate a correction for the local closed-orbit corrector; this will zero the closed-orbit well before the second train of bunches is injected. After the ring is filled, additional rejection of very low frequency excursions will be made by the low-frequency 
cutoff of the feedback electronics; this is possible with baseband operation as we have used here. For damping both radial and axial motions at 6 stations, 24 systems are required for each circulating beam.

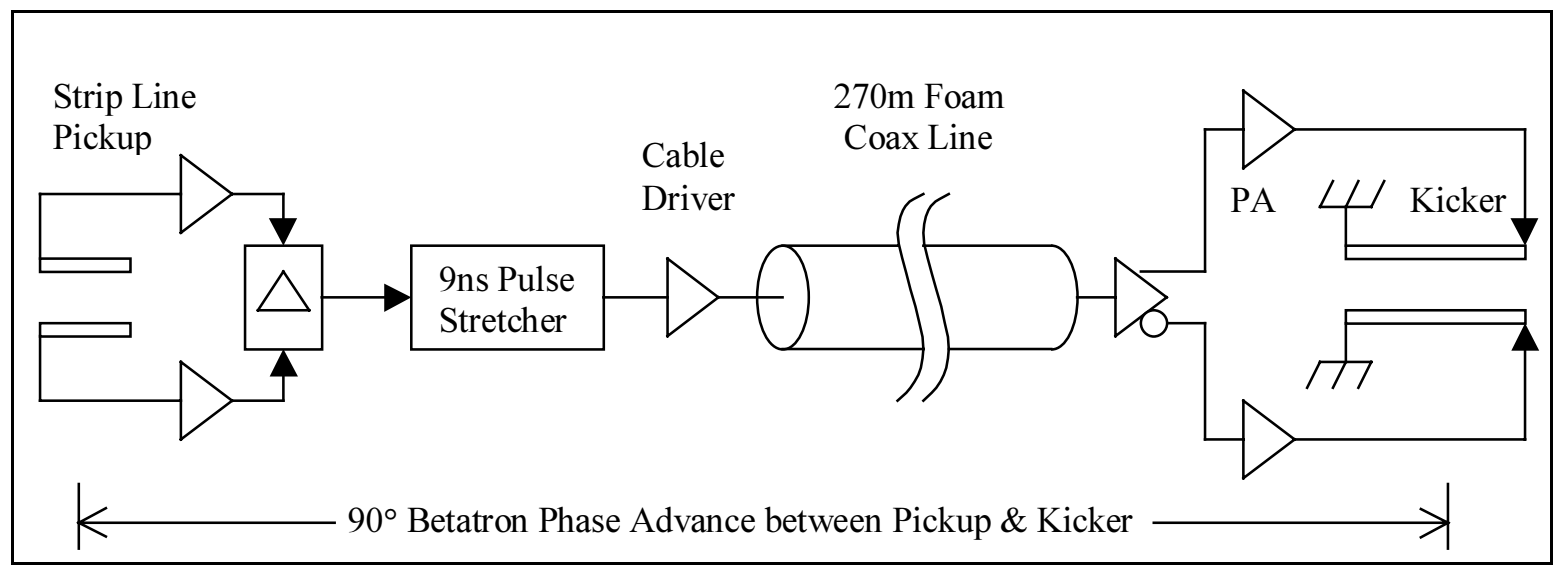

Figure 5.54. Electronic elements of the coupled-bunch feedback system.

Pickup and kicker are located at points of maximum betatron amplitude in slightly modified standard arc cells. The modifications (Section 3.2.1.4) involve removing $5 \mathrm{~m}$ of magnet iron at the boundaries of 8 successive half-cells. An additional quadrupole corrector is used at each location to repair damage to the $\beta$-functions caused by the missing focusing. The $\sim 10 \%$ dispersion wave generated by the missing bend automatically cancels outside the insertion by virtue of the $360^{\circ}$ phase advance across the 8 half-cells. The damper system uses at most 6 of the 8 available free spaces. Placing the damper pickups and kickers where the dispersion is nonzero is not believed to cause difficulties although this is will be the subject of continuing study.

At the coupled-bunch mode frequency of $500 \mathrm{kHz}$, the growth rate has been reduced to 57 per second or 0.044 per turn. This and all higher coupled-bunch modes are suppressed by the one-turn bunch-by-bunch feedback system that operates over the range $53.1 \pm 26.5 \mathrm{MHz}$. The kicker is a quarter-wavelength long at this frequency, 1.41 meter. The required kick for $0.1 \mathrm{~mm}$ excursion is $26.4 \mathrm{kV}$ and power is 513 watt. Power amplifiers suitable for this application are commercially available. The pickup is the same length as the kicker and feedback gain is 0.108 . Radial and axial systems are similar.

One can ask if electronic noise in these dampers will add emittance during a long collision time. Fortunately, although the loop gains are high, the small aperture and peak bunch current of 8.4 ampere make the pickup signal strong and large electronic gain is not needed. The peak current gives a pickup sensitivity $I Z_{L} g / b \sqrt{2}=2.6 \times 10^{4}$ volt/meter. Input thermal power with an amplifier noise figure of $1 \mathrm{~dB}$ is $1.2 \mathrm{kTB}=5.2 \times 10^{-21} \mathrm{~B}$, where $B$ is the bandwidth. Equating this to the pickup signal power with equivalent rms noise amplitude $X_{N}$ gives $<X_{N}^{2}>=3.7 \times 10^{-28} B$. The higher energy at collision reduces the gain to $\mathrm{G}=0.013$. The 12 feedback systems would then cause an emittance growth rate, without allowing for damping, of

$$
\frac{d \varepsilon}{d t}=12 \frac{f_{0} \gamma}{2 \beta}<G^{2} X_{N}^{2}>=2.1 \times 10^{-17} \mathrm{~m} / \mathrm{sec}
$$


Since the nominal emittance is $1.7 \times 10^{-6}$, this dilution rate is of no concern. It would actually be smaller because of damping by the feedback. The broadband system produces a similarly negligible emittance growth.

\subsection{Radiation, Machine Protection, and Beam Abort}

\subsubsection{Overview}

The 2.8 GJ of kinetic beam energy in the Stage- 1 machine is approximately eight times that of the LHC. Under normal circumstances roughly $50 \%$ of this energy is gradually dissipated in beam-beam collisions at the IRs. A few percent of the energy is lost diffusely due to beam-gas collisions around the ring, intercepted by beam collimation inserts which scrape away beam halo particles, and dissipated in the RF loads as the beam is decelerated. Somewhere between $40 \%$ (intentional beam abort at the end of the store at normal operation) and $100 \%$ (unintentional beam abort at certain circumstances) of the beam energy can be deposited in the external beam absorbers.

A very small fraction of the machine circumference exceeds the limit $(\sim 1 \mathrm{~W} / \mathrm{m}$ of beam losses) for normal "hands-on" maintenance [86]. These regions include the small-angle regions of the IRs, the beam collimation system, and a small region of arc magnets downstream of the beam collimation system. The average radiation load in these components is a few times higher than the LHC, and the shielding and handling requirements comparable. LHC experience will be useful in optimizing these designs.

Under accidental conditions there is enough energy to cause severe damage to the magnet and surrounding environment. This is not a new situation for high energy colliders. The ISR beams had enough energy to repeatedly burn holes in the machine [87]. The magnitude of the problem has increased however. The beam of the Stage-1 machine carries enough energy that in principle it could liquefy 400 liters of stainless steel (whereas the LHC beam can only liquefy 50 liters).

Under worst-case accident assumptions the beam will leave the machine and strike the tunnel wall. We have begun to study the mechanical and radiological consequences. See Section 5.3.3. A single shot beam accident releases beam energy equivalent to losing the Main Injector beam at one place for about 4 hours. This is not outside the scope of existing shielding requirements at Fermilab, and should not be major problem for a tunnel in deep bedrock.

Tunnel groundwater activation from single-pulse accidents appears not to be a concern. A preliminary calculation indicates that it would be possible to lose the beam in a single spot, immediately transfer the activation into the tunnel water sump pit, and pump out the activated water without violating applicable Federal guidelines. Another way of saying this is that you could dump the beam into the tunnel sump cistern and the water would still be safe to drink. (This is of course a very pessimistic scenario, since in reality it will take days or weeks for the groundwater to collect in the sump, by which time most of the radioactivity will have vanished). 
Effective strategies have evolved to deal with machine protection issues culminating with the Tevatron, SSC, and LHC. These issues have been revisited for VLHC energies $[88,89]$ and the results are summarized here.

A robust and redundant extraction system removes the beam whenever the position becomes unstable or anomalous losses are detected. This protects the machine from anything that happens "slowly" (in milliseconds to seconds) such as magnet quenches, RF or power supply failures, vacuum failures or corrector magnet trips. Such a system has prevented the Tevatron (so far) from having a hole burned through it. This is a standard feature, which continues to be essential at VLHC energies.

The one credible accident that occurs on a rapid time scale involves an abort kicker module misfire. The incompletely extracted beam will rattle around the machine and hit various welldefined limiting apertures such as extraction septa, IR quadrupoles, and beam collimators. The probability of this will be reduced in the LHC by replacing Thyratrons with solid-state kicker power supplies, and appropriate fail-safe logic which in principle prevents a single module misfiring. Since the probability of this cannot be reduced to zero, a set of "sacrificial collimators" are placed upstream of the vulnerable components. These are replaceable graphite structures that absorb and diffuse the beam energy. This strategy is essential at the SSC/LHC and will be necessary and sufficient for the VLHC.

Three qualitatively new features emerged while reviewing the machine protection situation at VLHC energies. First, the graphite core of the beam absorber may be damaged by a single pulse in the case of a sweeper magnet failure. The core must be protected (Section 5.3.2.2) with a replaceable sacrificial collimator immediately upstream. Secondly, in the case of a sweeper magnet failure the beam window in the extraction channel will have a hole melted in it. This is not a safety issue, but in this accident scenario the beam vacuum must protected with an automatically closing gate valve and the window(s) must be replaced. Finally, the beam collimation system (Section 5.2.3.4) will require water cooling for the jaws of the secondary collimators.

\subsubsection{Beam Abort System}

\subsubsection{Extraction}

It turns out to be quite straight forward to kick the beams out of the machine towards absorbers. The magnet parameters and layout are discussed in Section 5.1.5.4.2. Like the Tevatron, SSC and LHC abort systems, we use fast kicker magnets to switch the circulating beam into the other aperture of Lambertson magnets. The circulating beam goes through the field-free hole in the Lambertson magnets, and the extracted beam is bent upward in the Lambertson magnets so as to clear the first quadrupole in the downstream half of the straight section. A full aperture extraction kick is used and the separation of the circulating and extracted beams is $25 \mathrm{~mm}$ at the entrance to the Lambertson magnets. Thus any beam which is circulating anywhere in the physical aperture will be extracted. Special large-aperture warm-iron-and-copper quadrupoles are used near the Lambertsons so that no aperture restriction is presented to the circulating beam. 
Normally the beam extraction is synchronized with an "abort gap" in the beam that has a length equal to the rise time of the extraction kicker. During certain control system failures it may be necessary to unconditionally extract the beam. This sweeps the beam across the extraction septum, which must survive without damage. For the VLHC the stress on the extraction septum is roughly equal to the LHC (since the beam energy per unit time is similar) and does no damage.

The requirements for the reliability of a one-turn extraction mechanism are comparable to the SSC [90] and LHC [91]. The extraction kicker is broken into 10 independent modules, with any 7 out of 10 sufficient for a safe abort. Solid-state pulsers (as opposed to Thyratrons) will be used to minimize accidental prefires. Three Musketeer logic ("All-for-one, and one-for-all!") guarantees that any single module firing will automatically trigger the rest of the modules.

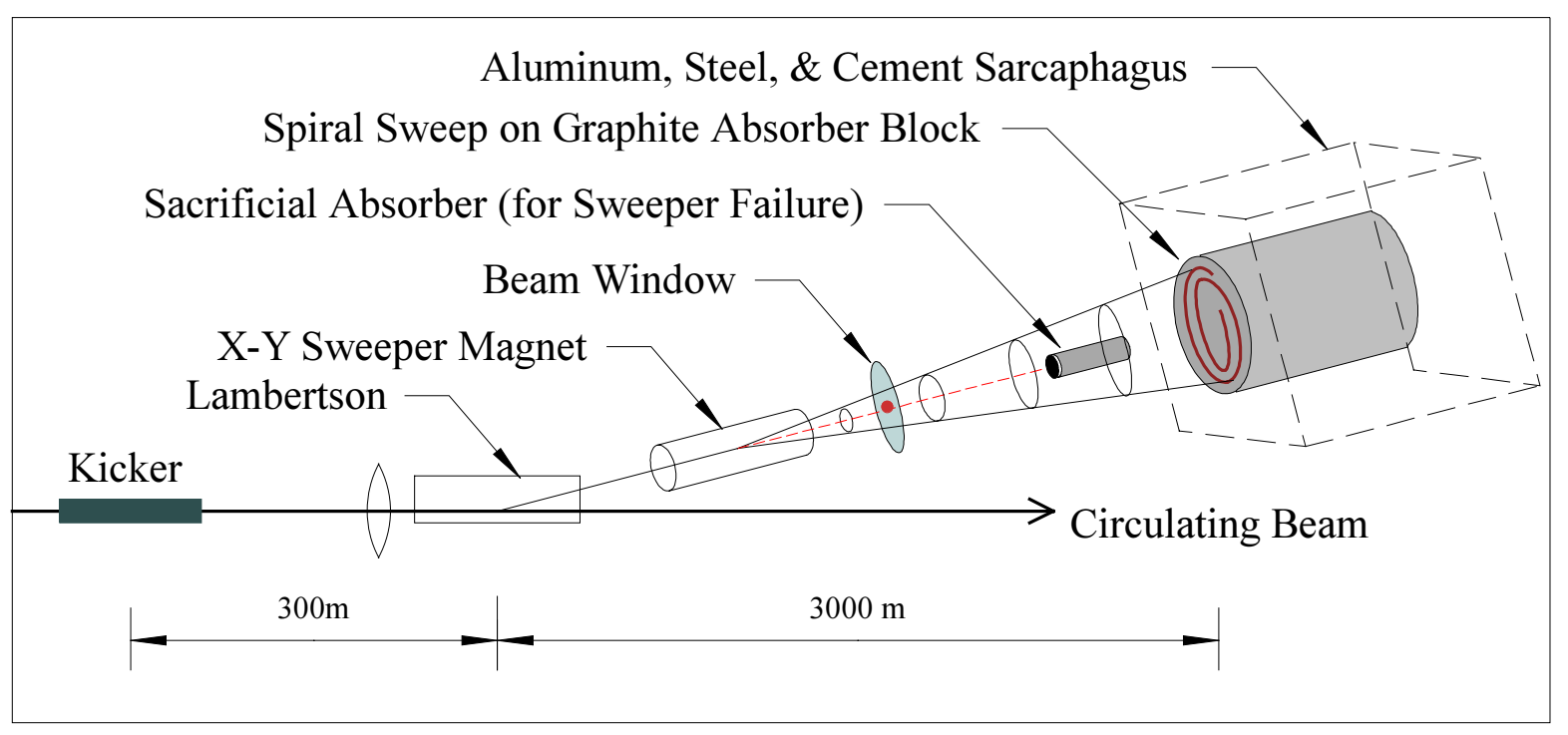

Figure 5.55. Schematic layout of beam abort channel including kickers, Lambertson septa, extraction beam sweeping, beam window, sacrificial rod, and graphite beam absorber. Under normal circumstances the extracted beam is swept in a spiral pattern to spread the energy across the graphite dump. If the sweeper magnet fails, the beam travels straight ahead into a sacrificial graphite rod, which takes the damage and must be replaced.

\subsubsection{Beam Absorber}

For $\mathrm{TeV}$ beams, the natural choice for the absorber is graphite, as at the Tevatron, SSC and LHC. The major difficulty lies in making the beams big enough that they will not crack a graphite absorber. A standard absorber consists of a graphite core, contained in an aluminum jacket with water cooling, followed by adequate steel and cement to protect ground water. The graphite core is rectangular, with dimensions $10 \times 1.2 \times 1.2 \mathrm{~m}$. The thickness of steel shielding around the aluminum container is about $1.3 \mathrm{~m}$ on each side and extends about $3 \mathrm{~m}$ downstream. At present we consider a "4-in-1" design where extracted beams from both directions hit absorber cores in a common sarcophagus. The Stage- 1 and Stage- 2 machines share a common absorber for each direction (see Figure 2.2).

Activation and cooling water considerations are driven by the average beam power on the target. The $300 \mathrm{~kW}$ average power (Section 7.4.1) corresponds to one full-energy abort of both 
beams every day and is equal to the $300 \mathrm{~kW}$ design value for the Main Injector beam absorber. The overall design is patterned on the Main Injector absorber that has proven satisfactory and meets ES\&H requirements for this power level.

The graphite dimensions and beam size necessary to contain the showers without cracking the graphite were found by detailed simulations with the MARS14 code. The design goal was to keep the maximum temperature rise at the axis of the graphite core per spill below 1300$1500^{\circ} \mathrm{C}$ (from successful Tevatron dump experience and theoretical shock wave considerations). For a purely Gaussian beam with a spill that is stationary in transverse position, the beam sigma would need to be $19 \mathrm{~cm}$ in both $\mathrm{x}$ and $\mathrm{y}$ for the maximum temperature rise in the core of $1330^{\circ} \mathrm{C}$. Expanding the beams to this size with defocusing quadrupoles is out of the question.

\subsubsection{Beam Sweeping System}

A spiral beam sweeping scheme similar to the SSC and LHC is adopted to spread the beam energy across the absorber core. The magnets are described in Section 5.1.5.4.2. A horizontal and a vertical sweeper, $90^{\circ}$ out of phase, both oscillate with decaying amplitudes. Ideally the frequency should increase as the radius of the spiral decreases in order to keep the temperature rise constant. Since this is electrically difficult, a suitable compromise is to limit the inner radius of the spiral to half that of the outer radius and accept a factor of two higher temperature rise at the inner radius. An estimate indicates that an outer radius of $30 \mathrm{~cm}$ would be adequate to keep the temperature below $1500^{\circ} \mathrm{C}$. If the beam sigma was $0.5 \mathrm{~cm}$ in both planes, the frequency of these sweepers would be $9.7 \mathrm{kHz}$.
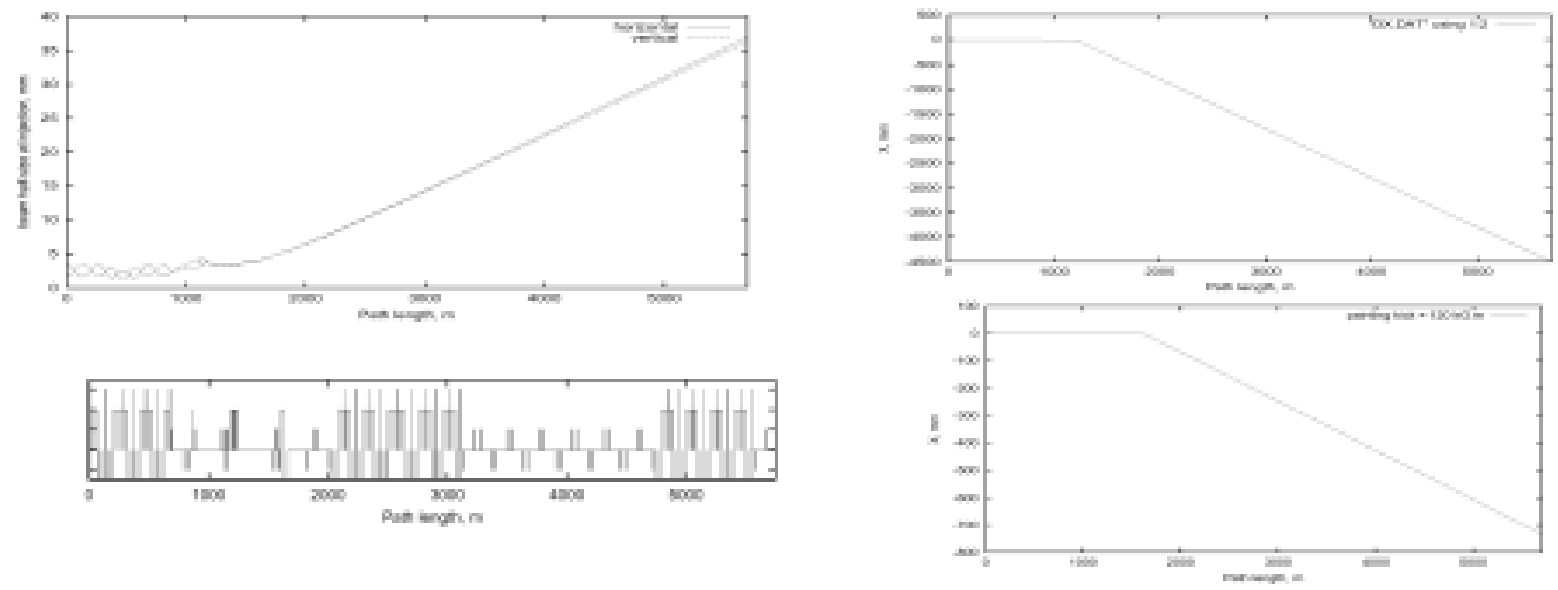

Figure 5.56. Beam sweeping system optics. Left: 3-sigma beam half-size at 1 TeV injection (beam size at $20 \mathrm{TeV}$ is 4.5x smaller), and beam line layout. Right: beam displacement with respect to the direction of extraction straight section and maximum sweeping kick in the horizontal or vertical plane.

In the case of a failure of both coordinates of the sweeper system the beam would damage the graphite absorber core [92]. To prevent this, a sacrificial graphite rod $5 \mathrm{~cm}$ in diameter and $4 \mathrm{~m}$ long is positioned on the beam axis immediately upstream of the main absorber. See Figure 5.55. Normally the extracted beams will spiral around this rod without hitting it. If the beam is 
extracted with all sweeping magnets off, the beam damage will be confined to the sacrificial rod. The rod will be housed in a metal box to prevent the spread of radioactive debris.

A beam window will not be protected in case of sweeping system (or defocusing quadrupole) failure, if the effective beam sigma on the window is less than $0.5 \mathrm{~cm}$. The ring vacuum can be preserved by rapid-acting gate valves and/or multiple windows in series. Each window gets a small hole drilled in it when the sweepers fail, and pumping between the windows isolates ring vacuum until a slow gate valve closes. Alternatively, differential pumping with several wire meshes would do the same job. No personnel safety issues are involved.

\subsubsection{Radiation and Beam Loss}

\subsubsection{Quenches from Beam Loss in Transmission Line Magnets}

The warm-iron design of the Transmission line magnet is less sensitive to radiation-induced quenches than ordinary superconducting magnets. To determine tolerable beam loss in the arcs, MARS14 simulations are done in the lattice both at injection $\left(1 \mathrm{TeV}, \sigma \mathrm{x}, \mathrm{y}=1.4 \mathrm{~mm}, \alpha_{\text {inc }}=0.7\right.$ $\mathrm{mrad})$ and top energy $\left(20 \mathrm{TeV}, \sigma \mathrm{x}, \mathrm{y}=0.3 \mathrm{~mm}, \alpha_{i n c}=0.15 \mathrm{mrad}\right)$. Corresponding materials and magnetic field distributions have been implemented into a 3-D model. Inward and outward beam losses were considered both for inner and outer beam pipes (see Figure 5.57).
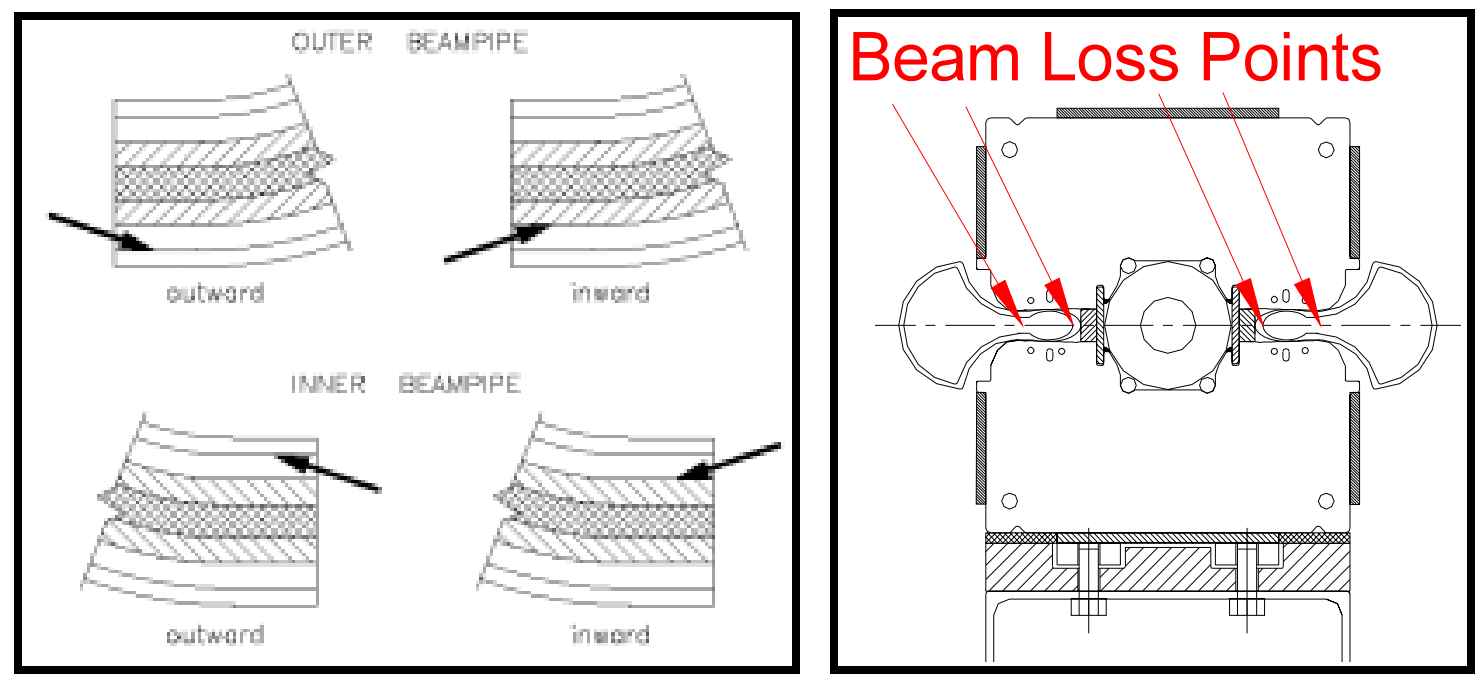

Figure 5.57. Beam loss geometries simulated in the Stage-1 VLHC magnets.

Simulations show that the superconductor in the transmission line magnets is rather well protected radially by warm iron. The energy deposition is diffuse and the peak temperature is relatively low at the hottest spot in the showers downstream of where the proton hits the beam pipe. Therefore, the tolerable beam loss is significantly higher than in a conventional cosinetheta type magnets. Table 5.27 shows fast $(1 \mathrm{msec})$ and slow $(0.1 \mathrm{sec})$ beam loss rates needed to initiate a SC magnet quench at injection and top energy in the Stage-1 ring. For comparison, the values for the Tevatron at $0.9 \mathrm{TeV}$ are shown. This comparison assumes that the quench limits are the same in the VLHC conductor and the Tevatron dipoles. This assumption is 
probably pessimistic since the braided cable of the VLHC can re-route the current around a quenched region on the magnet midplane, whereas a cosine-theta magnet cannot.

Table 5.27. Quench-inducing loss thresholds (protons per pulse) for the Stage-1 VLHC and Tevatron.

\begin{tabular}{|l|c|c|}
\hline & Fast Loss (ppp) & Slow Loss (ppp) \\
\hline $0.9 \mathrm{TeV}$ & $9 \times 10^{8}$ & $3 \times 10^{9}$ \\
\hline $20 \mathrm{TeV}$ & $2.5 \times 10^{7}$ & $7.5 \times 10^{7}$ \\
\hline Tevatron & $4 \times 10^{7}$ & $3 \times 10^{8}$ \\
\hline
\end{tabular}

\subsubsection{Cryogenic Heat Load from Beam Losses}

Heat load from beam-beam interaction debris in the IR quads is included explicitly in the discussion in Section 5.2.1. The distributed heat load from beam losses in the arcs is also a potential concern. At $20 \mathrm{TeV}$ roughly $5 \%$ of the energy from beam losses is estimated to be absorbed in the cryogenic transmission line. If $1 \%$ per hour of the $(3+3)$ GJ of beam energy were lost uniformly in the arcs, this amounts to a total heat load of $800 \mathrm{~W}(3.5 \mathrm{~mW} / \mathrm{m}$ average). This is a 5\% perturbation on the $5 \mathrm{~K}$ heat load. The collimation system (Section 5.3.4) is expected to localize essentially all beam halo losses into collimators in warm sections, thereby eliminating that heat load from the cryo system.

The collimation system will be ineffective against losses from inelastic beam-gas scattering. With the expected vacuum lifetime (Section 5.2.4.1) of $>200$ hours, the cryogenic heat load will be $<2 \mathrm{~mW} / \mathrm{m}$, less than $3 \%$ of the $4.5 \mathrm{~K}$ heat load. If continued $\mathrm{R} \& \mathrm{D}$ reduces the $4.5 \mathrm{~K}$ heat leak this will become an increasingly important part of the total.

\subsubsection{Radiation Damage to Tunnel Electronics}

Section 5.2.3 describes electronics housed in small enclosures ("holes in the wall") at each halfcell location. This approach was studied at UNK [93] and the electronics was found to survive under rather conservative assumptions about their radiation hardness. A recent interesting development [94] is that modern CMOS is found to be intrinsically rad-hard due to the absence of long-term charge trapping at the gate oxide layer in sub-micron devices. The LHC is apparently contemplating placing unshielded electronics under each dipole, and is initiating a study of the radiation hardness of commercially available electronics [95]. Depending on LHC operational experience, the rather modest costs of the module enclosures might be saved by eliminating the enclosures.

Radiation damage to fiber optics placed in the tunnel was studied at the SSC [96]. It was found that placing the fibers into the trough in the invert guaranteed their survival even for upgrades to 10x the SSC luminosity. We have followed this approach (Section 5.2.3.8). 


\subsubsection{Beam Collimation System}

Even in good operational conditions, a finite fraction of the beam will leave the stable central area of the accelerator aperture because of intra-beam scattering, small-angle beam-gas interactions along the circumference, collisions in the IPs, RF noise, ground motion and resonances excited by the accelerator imperfections. These continuously generate a beam halo. As a result of beam halo interactions with limiting apertures, hadronic and electromagnetic showers initiated in accelerator and detector components will cause accelerator related background in the detectors, magnet heating and accelerator and environmental irradiation. The design strategy of the VLHC is that the beam losses are controlled as much as possible by localizing them in a dedicated beam collimation system. This minimizes losses in cryogenic parts of the accelerator, and drastically reduces the source term for radiation hazard analysis in the rest of the lattice. The technology for these systems has been well developed for the Tevatron, SSC, and LHC.

For the VLHC a complete beam cleaning system which provides for both betatron and momentum scraping has been designed and simulated [97,98]. The three-stage beam collimation system consists of $5 \mathrm{~mm}$ thick primary tungsten collimators placed at $7 \sigma_{\mathrm{x}, \mathrm{y}}$ and $3 \mathrm{~m}$ long copper secondary collimators located in an optimal phase advance at $9.2 \sigma_{\mathrm{x}, \mathrm{y}}$ and aligned parallel to the circulating beam envelope. Two more supplementary collimators are placed in the next long straight section to decrease particle losses in the low- $\beta$ quadrupoles and in the accelerator arc. They are located at $14 \sigma_{x, y}$ to intercept only particles scattered out from the secondary collimators.
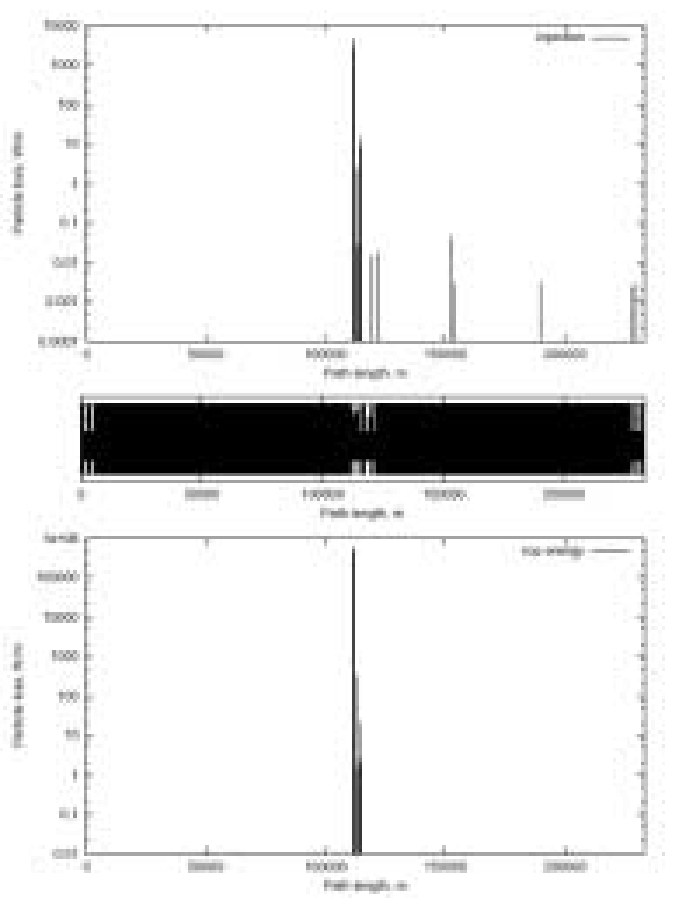

Figure 5.58. Beam loss distribution along the accelerator at injection (top) and at collisions (bottom).
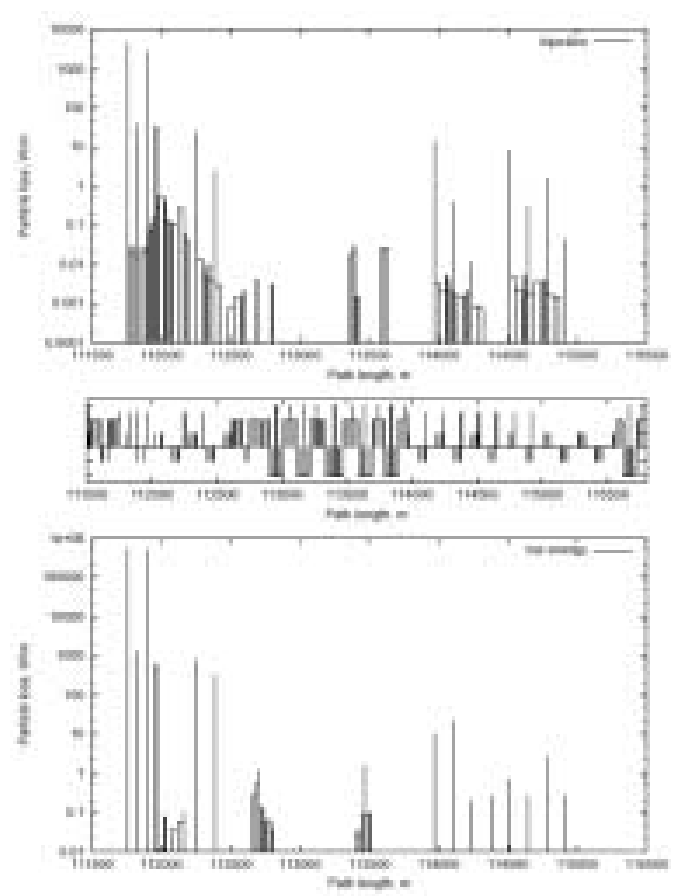

Figure 5.59. Beam loss distributions in the collimation section at injection (top) and at collisions (bottom). 
Figure 5.58 shows that the simulated performance of the system meets the goal of essentially eliminating losses away from the cleaning insert. The main reason for this excellent performance is that the system uses a lattice insert specifically designed for this purpose, rather than something that has been retrofit into an existing machine such as the Tevatron. The maximum beam loss in the arc section behind the collimation region (Figure 5.59) is $1 \mathrm{~W} / \mathrm{m}$ in two 21.5-m long dipoles and one 6-m long quadrupole, and $0.2 \mathrm{~W} / \mathrm{m}$ in two $21.9-\mathrm{m}$ long gradient magnets.

The beam cleaning system was designed with the goal of reducing losses and detector backgrounds near the high luminosity insertions. Without the supplementary collimators, about $61 \mathrm{~W}$ of beam power $\left(2 \times 10^{7}\right.$ protons/sec) are lost upstream of the detectors. Including the supplementary collimators in our calculations, however, $6 \times 10^{4}$ particles are lost in the collimators, and we did not see any loses in the vicinity of interaction point. Further investigations should be done to determine ultimate particle backgrounds in the IP with the collimation system.

The primary collimation insert will be located in a far side straight section conjugate to the one of the on-site Utility Straight Sections. Secondary collimators will be located in this section as well as in special locations near the IRs. The secondary collimator will absorb approximately $20 \mathrm{~kW}$ of beam power and will require water cooling, probably thorough an isolated RAW (radioactive water) loop heat exchanged with the LCW flow. Shielding of the collimators will require approximately $1 \mathrm{~m}$ of radial iron followed by $50 \mathrm{~cm}$ of radial concrete over a length of $\sim 15 \mathrm{~m}$ along the beam line to allow free passage of rad-trained personnel. This will require locally enlarging the tunnel cross section but should not require a bypass tunnel.

\subsubsection{IR Protection}

A sophisticated system must be designed to protect the IR SC magnets, particularly from kicker misfires. As in the LHC [99], it will consist of a front collimator, inner and intermediate absorbers in the inner triplet, and of a neutral beam dump and several collimators in the outer triplet. The similarity of the Stage-1 IR layout to the LHC makes it likely that a similar protection layout would be workable. The length of these sacrificial collimators will need to be increased due to the increased energy and decreased beam spot size of the VLHC beams.

\subsubsection{Worst-Case Beam Accidents}

Work has begun on understanding the implications of a "worst-case" beam accident in the VLHC. The assumption is that some unspecified agent causes the beam to be kicked out of the machine with a rise time fast compared to the revolution frequency, so that the normal beam abort does not have time to act. This agent is also assumed to possess a "kicker flat top" accurate enough $(<1 \%)$ that the beam is effectively held in one place for the duration of the accident. Under these assumptions the beam will rapidly melt a hole in the magnet and impact the tunnel wall at near grazing incidence $(3-5 \mathrm{mrad})$.

A preliminary observation is that the nearby cryogenic piping would rupture due to the rapid temperature rise. This would also be true of the Tevatron, HERA, and the LHC so it is important to keep this mysterious agent out of these machines as well. The cryo piping is contained inside carbon-steel structures (vacuum jackets and magnet iron) which would limit 
collateral damage. It would probably not even be noticed from the outside of the magnet. This is not our biggest problem.

A second observation is that the "beam drilling" scenario (in which a stable beam vaporizes a small channel deep into the target) is impossible at grazing incidence, even if the beam were perfectly extracted on a fixed trajectory. This beam drilling scenario is expected when the beam is normally incident onto a semi-infinite slab of material such as a beam absorber block. However at grazing incidence, the imbalance of the mechanical forces near the beam impact point (due to local heating of the rock) will cause a rock chip to "spall" out from the tunnel wall. This spalling behavior has been observed during rock excavation tests with electron beams [100] and follows this simple mechanical model. This spalling effectively sweeps fresh material across the beam and guarantees that non-vaporized material will be available to initiate the shower, even if the beam is perfectly extracted. Thus the pattern of energy deposition can be calculated accurately enough by assuming that the shower initiates at a more-or-less fixed position near the point of grazing incidence.

A MARS calculation has been performed (Figure 5.60) to evaluate the energy deposition under the assumption that both the rock and beam position remain fixed. The simulation indicates that a region 8 meters long and about $15 \mathrm{~cm}$ in radius are heated to the melting point of dolomite. Obviously it will splatter to the floor. The next step in the calculation (in progress) is to use ANSYS to evaluate the thermal stresses in the surrounding rock and estimate the amount of rock that breaks off from thermal stress. The rise time of the heat pulse (1 machine revolution or about $0.8 \mathrm{msec}$ ) allows the mechanical stresses to relieve themselves on the scale of a couple of meters, so a static mechanical analysis is approximately valid.
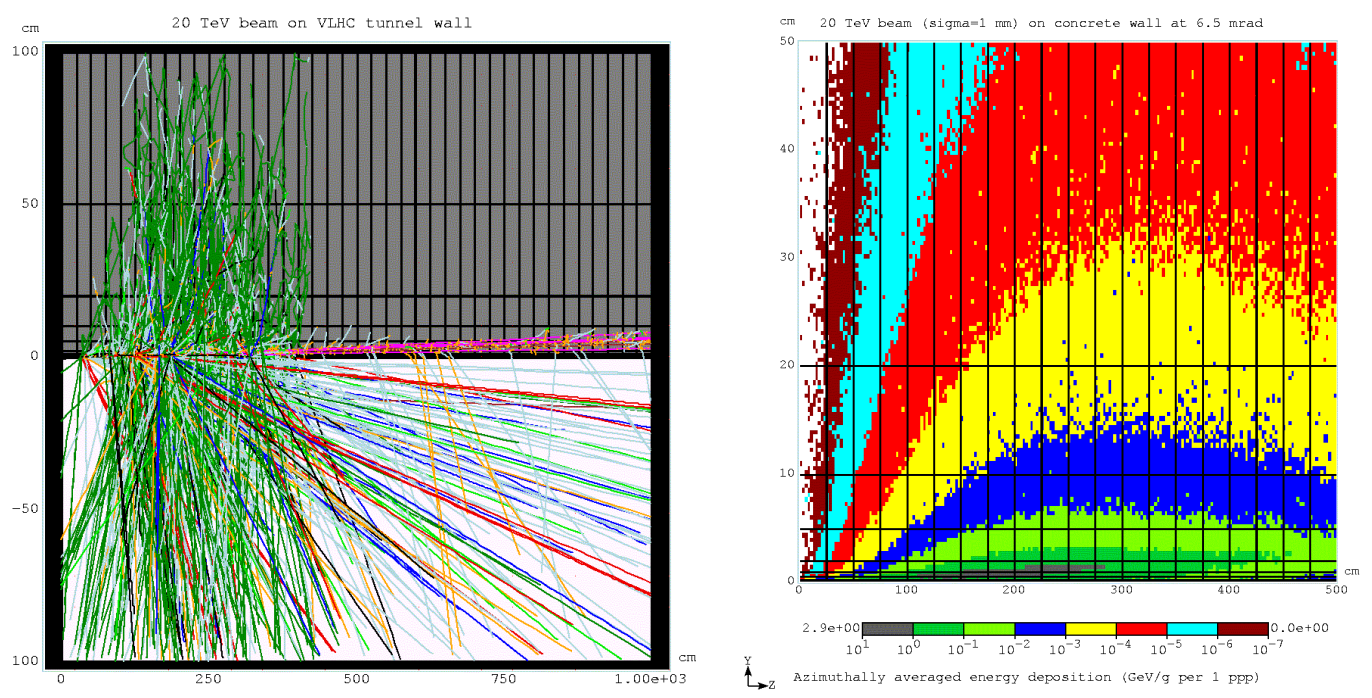

Figure 5.60. Stage-1 VLHC beam at 6.5 mrad grazing incidence on tunnel wall. The left picture shows particle tracks; the right picture is a map of energy deposition.

A more realistic situation in which the beam angle sweeps by even a few milliradians during extraction changes the situation significantly. In this case the heating is distributed into a large enough rock mass that the only a very small region (of order a centimeter wide) approaches the melting point. The picture becomes that of a destroyed magnet, a centimeter-wide 
scar on the tunnel wall, and an amount or residual radiation comparable to that of a 4-hour continuous beam loss at the Fermilab Main Injector.

\subsubsection{General ES\&H Considerations}

\subsubsection{Introduction}

The VLHC with its two stages present a number of important considerations in the general area of environment, safety, and health. It is the intent of this section to identify the character of these challenges in a general way. Some of the considerations which must be taken into account are very similar to those that have been encountered and solved during the construction and operation of other facilities at Fermilab and at similar laboratories elsewhere in the United States and worldwide. Other novel issues have not been encountered on the same scale and require particular attention to assure their timely resolution in a manner that is cost-effective and that meets the approval of the public. In this section, both the conventional and the novel issues are discussed. With adequate planning in the conceptual design stages, these problems can be adequately addressed in a manner that merits the support of the Laboratory, the Department of Energy, and the public.

\subsubsection{Procedural/Regulatory Matters}

The actual design, construction, and operation of the VLHC will have to meet a number of procedural/regulatory milestones to assure timely and continued support by the public and by the Department of Energy. ES\&H requirements are currently set forth as a part of Fermilab's Work Smart Standards, incorporated in the contract between the Universities Research Association and DOE. Currently, the Work Smart Standards are reviewed annually to assure that they adequately address the hazards of the laboratory, including those of any new facility. If changes are necessary they are negotiated and the URA-DOE contract is revised accordingly. These standards include listings of applicable Federal and State Regulations as well as internally developed policies and national standards [101]. Of course, the contract under which Fermilab might operate in the future is likely to change in ways that could modify the applicable requirements.

$A$. Environmental Protection Procedural/Regulatory Matters

All DOE activities are subject to the requirements of DOE's regulations for implementing the National Environmental Policy Act (NEPA) [102]. A new project of this magnitude will be the subject of an Environmental Assessment (EA). A review will be done of all possible impacts of this project on the environment and the public. The required analysis is broad in scope and includes societal impacts along with those topics that are more generally associated with environmental protection such as the discharges of pollutants, effects upon wetlands and floodplains, and exposures of people to chemicals and radioactive materials. It will include a review of the alternatives of carrying out the project elsewhere or not at all. This process is centered on the production of a comprehensive document but also includes the participation of the public by methods that will be chosen by DOE. A likely result will conclude that that the preparation of an Environmental Impact Statement (EIS) is necessary since the VLHC extends 
far beyond the present boundary of the Fermilab site. The EIS process is generally considered to be an arduous one, but one that can be followed to a successful conclusion. The preparation of an EIS is certain to be a large task having significant cost, customarily accomplished using external resources. Regardless of the eventual path of the NEPA process, project funds cannot be issued to support a Congressional "line item" project beyond the early conceptual stage prior to the successful completion of the NEPA process. It is thus crucial that this process be conducted in a manner that is honest and which comprehensively addresses the potential concerns of members of the public. A good working relationship with the Department of Energy is also a necessity to a successful result.

Other procedural requirements apply in the area of environmental protection. These will be more certainly identified as a part of the NEPA process but early planning may well serve to avert problems later. DOE facilities are generally subject to Federal and State environmental protection regulations promulgated chiefly by the U. S. Environmental Protection Agency (USEPA) and the Illinois Environmental Protection Agency (IEPA). Some of the Federal and State permits apply during the construction stages, others apply during operations, and some apply during both stages. Permits are likely to be required to cover such topics as storm water discharges, discharges of cooling water, wetlands mitigation, releases of air pollutants for both non-radioactive pollutants and for radionuclides, and construction in any floodplains. Archaeological sites might need further investigation and study prior to the commencement of construction. The preparation of the applications for these permits and approvals is generally straightforward. Early coordination with the project design team should greatly facilitate completion of the associated milestones. The VLHC is likely to be initially viewed by the public and by Federal and State regulators as a poorly understood, esoteric technology. In recognition of this and also because it will have an impact beyond the geographical boundaries of the present Fermilab site, early coordination with the Federal and State regulatory agencies is recommended as a vehicle to promote enhanced understanding of the nature and impact of the facility.

$B$.

\section{Safety and Health Procedural/Regulatory Matters}

In accordance with Fermilab's Work Smart Standards, the Laboratory will be required to prepare an assessment of the ES\&H issues associated with this project in the form of a Safety Assessment Document (SAD). Given the size, scope and cost of the VLHC, the preparation of a Preliminary Safety Assessment Document (PSAD) is needed. The purpose of the PSAD is to identify the relevant ES\&H issues at an early stage and propose how they might be mitigated. A final Safety Assessment Document (SAD), then, documents the resolution of all the pertinent issues raised by the PSAD. Environmental issues are customarily integrated into the $\mathrm{PSAD} / \mathrm{SAD}$ process to promote program cohesiveness. It is nearly certain that DOE will choose to review these safety documents by utilizing an external review team. Just prior to facility operation, a readiness review will be conducted in similar fashion using an external review team. Unlike NEPA assessment activities, PSAD/SAD activities generally begin after funds are issued. Nevertheless, careful consideration of PSAD/SAD in the design can only result in beneficial results. Efforts should be taken at early stages to promote consistency between the conclusions of the NEPA assessment and the safety and health documentation.

DOE is presently "self-regulating" in the areas of industrial safety and occupational radiation protection. There is a possibility that during the development of the next accelerator 
facility, DOE activities might become subject to "external" regulation in these areas, as well as in occupational radiation protection. It is difficult at this time to anticipate the form such external regulation might take or which agencies might be involved.

\subsubsection{Occupational Safety During Construction}

The VLHC requires a large amount of tunneling in bedrock units, most of it horizontal or nearly so, and other tunnels with slopes. In addition to the safety requirements pertaining to construction activities, Federal regulations pertaining to underground operations (e.g., "mining" activities) come into play. Solutions to these issues are being developed to address challenges of this type encountered in the excavation for the NuMI project. These include the standard concerns about tunneling safety and material movement as the tunneling proceeds. Tunnel Boring Machines (TBMs) will be used for most of the tunnel. For some portions of the underground facility the drill and blast method will most economical. Both methods have associated occupational safety considerations. Provisions for emergency response including underground rescues will be needed. Egress issues relevant to protection of the construction workers will be of paramount importance.

It is clear that stringent measures must be taken to prevent flooding both during the construction period and thereafter. This problem must be addressed in harmony with the related environmental protection concerns (see Section 5.3.7.4). Downward slopes in the bedrock units will require attention to the prevention of uncontrolled movements of heavy objects downhill. While such control measures are well within those encountered in mining operations elsewhere, they are novel at accelerator laboratories.

During project construction, industrial radiography, a tool commonly used in general industry, is likely to be employed to assure the quality of pipe welds. Such radiographic operations, which typically use radioactive sources of high activity and relatively hazardous compared with the sources commonly used in particle physics experiments, would need to be conducted in compliance with the pertinent requirements of the State of Illinois in order to control the hazard to personnel. In the course of construction, other radiation-generating devices such as soil density gauges and media water-content probes might also need to be used. Standard procedures pertaining to such activities should be applied.

\subsubsection{Environmental Protection During the Construction}

A small portion of the VLHC will be located near the surface, in the glacial till, while most of it will be located deep underground in various rock strata. For the portions near the surface, construction may proceed by cut and fill techniques similar to those employed to build most of the present facilities at Fermilab. Erosion control measures similar to those in practice for a number of years will be employed in accordance with good engineering practice and Federal and State regulations. Dust from any spoil piles must be kept under control. Likewise, a storm water management plan will need to be developed. Noise from construction activities is not expected to be significantly larger than that associated with normal civil construction activities in the vicinity of Fermilab. The NEPA process will result in a determination of the impact of the project on wetlands and or floodplains. It may be necessary for compensatory man-made wetlands to be created. 
Tunneling in the bedrock units will result in the removal of a considerable volume of rock. The management of the spoil is a major issue that must be addressed and provisions for its proper stockpiling provided. In particular, concerns about dust may be more severe for this material, largely pulverized rock. The duration of this storage may be temporary for the spoil that is of marketable quality and longer for that reused at Fermilab or disposed. This should be carefully planned in accordance with Fermilab's longstanding tradition of placing high importance on aesthetic issues. The management of spoil materials will need especially vigorous attention off site. The placement of such a project in any aquifers results in the need to protect drinking water resources from contamination during construction. Also, the "de-watering" of tunnels, as the construction proceeds will require measures to prevent the depletion of wells and also to manage effectively any additional water discharges from this source.

The storm water management plan will need to take into account any releases of groundwater generated in the course of "de-watering" the tunnel. Careful hydrogeologic studies need to be performed to understand the interplay of the construction of the project with the various aquifers. This must be done to establish with certainty that the construction activities will not cause significant perturbations of the local individual and municipal water supplies. The exact depth of the various aquifers is not known in detail at all locations and accurate measurements will be needed. The results can be used to plan a strategy for preventing the tunnel from serving as a possible path of cross-contamination from one aquifer to another. During construction activities, precautions are needed to guarantee that spills of chemicals, including lubricants and fuels from the construction equipment, are captured before they enter the groundwater.

Tunneling activities can generate considerable noise and vibration. For blasting techniques, quantitative standards apply to the amplitude of the vibrations that are allowable at the surface. Noise exposure, both occupational and to the public at the site boundary and at off site locations will be an issue that needs to be addressed.

\subsubsection{Occupational Safety During the Operations}

$A$.

\section{"Ordinary" Occupational Safety Hazards}

In this section, the focus is on the issues that have been successfully addressed before, at Fermilab and elsewhere by well-known techniques.

- The facility will use high current electrical circuits on a large scale. Present techniques in managing power distribution and providing means to effectively lock out supplies should be adequate to address the electrical hazard.

- Radio-Frequency (RF) generation and distribution equipment will be used extensively. Present techniques for controlling possible exposures to non-ionizing radiation should be sufficient.

- Large amounts of cables, transformers and electrical switchgear will be installed underground. Current methods for addressing fire protection concerns should be adequate.

- Long tunnels will be present. There is a need to adequately address Life Safety Code/fire protection issues to assure adequate provisions for egress and adequate means of prevention of and response to fires. 
- There will be movements and alignment of large, heavy components. There is a need to include considerations in the design related to ease of movement of equipment to facilitate the prevention of injuries.

$B$. Novel Occupational Safety Hazards

This section is directed to those occupational safety hazards that are not generally encountered at accelerator facilities. These will require consideration in the early planning stages in order to be addressed in an efficient manner.

- The VLHC requires the extensive use of superconducting materials and cryogens. While these technologies are relatively new, a number of accelerators worldwide have developed techniques adequate for addressing them. Provisions will need to be made for the safe release of cryogens to the surface both during normal operations and in the event of quenches. Current accelerator facilities have developed mechanisms for using skilled engineers to independently review such systems for safety during the design and commissioning stages. The result has been the development of a number of standard engineering practices to mitigate both direct cryogenic hazards and the accompanying oxygen deficiency hazards (ODH).

- As during construction, the strong desire to minimize the number of exit points will render adequate design and engineering with respect to the Life Safety Code imperative. It is likely that special means of communication underground will need to be provided, "refuge" locations incorporated into the design, and adequate means of transport of personnel, both healthy and injured, to the surface established. These provisions will need to incorporate recommendations of a qualified fire protection professional at the time of the development of the conceptual design in order to assure adequate allowances for their costs. Later involvement of fire protection engineering will also be needed as the detailed design proceeds.

\subsubsection{6. $\quad$ Ionizing Radiation Safety During Operations}

$A$.

\section{Prompt Radiation Shielding}

The siting of the VLHC deep underground will provide adequate passive shielding to attenuate prompt radiation to levels acceptable to the members of the public. The shielding of proton accelerators needs to attenuate the neutrons produced at large angles. At the forward angles, given the copious production of muons, and the increased importance of range-energy straggling at high energies [103], the shielding requirements must be well-understand at the earliest possible stage in the design and in the NEPA process.

Current Department of Energy requirements are not well matched to discussions of radiation fields that exist beyond the boundaries of DOE sites. DOE has specified the annual limits on the radiation dose equivalent that can be received by occupational workers and members of the public (see Regulation 10 CFR 835) [101,104]. These limits, in all situations expressed to date, pertain to the dose equivalent delivered to people or to locations where people could reasonably be. For individual members of the public, the primary limit is 100 mrem $(1 \mathrm{mSv})$ in a year, not including man-made, medical, or enhanced natural radioactivity. Special reporting requirements apply when the annual dose equivalent received by an individual exceeds 10 mrem $(0.1 \mathrm{mSv})$ in a year. DOE has expressed the view that non-occupational annual doses to 
members of the public are not expected to exceed a few mrem in a year. In light of public concerns about radiation exposures, any new facility should be designed to keep the dose that could be reasonably received by actual members of the public to as small a value as possible.

\section{B. Residual Radioactivity of Components}

In the high-energy region, most, but not all, of the radiation effects scale roughly with the beam power. In particular the effects of high residual activity levels should be carefully taken into account at early stages of design of beam cleaning and abort systems and other locations of possible high beam loss. Doses that might be received by workers are an important subject that must be assessed as a part of the NEPA process. The need to minimize the generation of radioactive wastes and to eliminate the creation of wastes that contain materials that are toxic or hazardous, and which contain radioactive waste, is an important concern.

$C$. Airborne Radioactivity

Federal Regulations promulgated by the USEPA have established an annual limit on dose equivalent of $10 \mathrm{mrem}(100 \mu \mathrm{Sv})$ to any member of the public that can be received as a result of operations of DOE facilities such as accelerators [105]. Further, the same regulations impose stringent continuous monitoring requirements if the annual dose equivalent to any member of the public is to exceed $0.1 \mathrm{mrem}(1 \mu \mathrm{Sv})$ in one year. In addition, if the level of $0.1 \mathrm{mrem}$ in one year is to be exceeded, then an application for approval to construct and a notification of startup must both, in proper sequence, be submitted to the USEPA. Given the extent of the VLHC beyond the present boundaries of the Fermilab site, careful attention must be paid to the production of airborne radioactivity.

\section{Radioactivity in Soil and Groundwater}

The VLHC siting requires that the production of radioactivity in hydrogeologic units be given careful attention. Before the exact footprint of any chosen facility is irrevocably determined, detailed hydrogeologic studies should be conducted to determine the relevant parameters precisely, as they are known to vary significant in the vicinity of the Fermilab site. It is clear that protection of groundwater against contamination with radioactivity merits early, detailed attention in project design to assure satisfaction of likely public concerns. It may well be that the most prudent choice of design objectives might be far below present regulatory standards for drinking water.

\subsubsection{7. $\quad$ Non-Radiological Environmental Protection Issues During Operations}

Operations of the facility should be planned in a way that incorporates proper measures to control the generation of non-radioactive wastes. Further, there is a need to address potential spills of hazardous or toxic materials in a way that fully protects members of the public and of environmental resources. The detailed attention to these issues will be required as part of the NEPA process and the designs should provide information of the quality needed to support all required permit applications to State and Federal environmental regulatory agencies. In any enclosures located deep underground the cross-contamination of the various aquifers must be 
prevented and any de-watering operations must assure that local community or individual drinking water supplies are not perturbed.

\subsubsection{Summary}

The construction and operation of the VLHC present a number of ES\&H challenges. Many of these have been encountered, and effectively addressed, at other accelerator facilities. Some of the problems are common to other recent projects undertaken at Fermilab and elsewhere that have resulted in the need to develop new methods to address them. Given the scale of the VLHC some of these issues may have a greater importance than found in the present experience. With adequate planning in the design stages, these problems can be adequately addressed in a manner that merits the support of the Laboratory, the Department of Energy, and the public.

\section{References}

[1] R.R. Wilson, Proceedings of Snowmass 1982.

[2] E. Malamud, ed., "The Pipetron: a Low-Field Approach to a Very Large Hadron Collider," Snowmass '96 (a.k.a. pink book).

[3] G.W. Foster, "Transmission Line Magnet Status Report," VLHC Information Packet for HEPAP Subpanel, Fermilab 1998.

[4] G.W. Foster, V.S. Kashikhin, E. Malamud, P. Mazur, H. Piekarz, J. Fuerst, R. Rabehl, P. Schlabach, J. Volk, "The 100 kA VLHC Transmission Line Magnet Superconducting Cable Test Facility," MT-16, IEEE Trans. of Applied Superconductivity, Vol.10, No.1, March 2000, pp.318-321.

[5] R. Walker, "50 m Invar Thermal Cycling Report," TD-01-037, 2001.

[6] N. Shatil, "Quench Simulation of VLHC Transmission Line Magnet (Final Report)," TD-01-025, 2001.

[7] J. DiMarco, G.W. Foster, V. Kashikhin, A. Makarov, P. Schlabach, "Measurements of a Crenellated Iron Pole Tip for the VLHC Transmission Line Magnet," PAC 99, pp. 3327 - 3329.

[8] G.W. Foster, V.S. Kashikhin, I. Novitski, "Design of a 2 Tesla Transmission Line Magnet for the VLHC," MT-16, IEEE Trans. of Applied Superconductivity, Vol.10, No.1,March 2000, pp.202-205.

[9] G.W. Foster, V.S. Kashikhin, V.V. Kashikhin, "Pole Profile Optimization of VLHC Transmission Line Magnet," EPAC 98.

[10] G.W. Foster, "Experience with Permanent Magnets in the 8-GeV Line and the Recycler Ring," EPAC '98.

[11] V.S. Kashikhin, "Corrector wires in holes-in-the-poles for transmission line magnets," TD-01-038, 2001.

[12] V. Tsvetkov, "Transmission Line Magnet Mechanics," TD-01-039, 2001.

[13] V. Tsvetkov, "Alignment of the Transmission Line Magnet in the tunnel," TD-01-040, 2001.

[14] V.S. Kashikhin, "Mechanical model 2 test results," TD-01-041, 2001.

[15] V.S. Kashikhin, "Transmission Line Magnet for VLHC. Magnetic Forces and Magnet Deflection," TD-01$042,2001$.

[16] M. McAshan, "Cryogenic System for the 3 TeV Injector Study," Fermilab VLHCPUB-87,1997.

[17] ULTEM 2300 is a $40 \%$ glass-filled polyetherimide marketed by GE plastics. It was developed for use in SSC quadrupole support posts at BNL. See J. Sondericker et al., Advances in Cryogenic Engineering, Vol. 40, pp. 1099-1105, Plenum Press, New York (1994).

[18] G.W. Foster, "Mechanical Load Cycling Tests of the Transmission Line Spiders," TD-01-043, 2001.

[19] I. Novitski, "Analysis of the spider support," TD-01-044, 2001.

[20] E.C. Quimby et al., "VJR/VJRR Design, Construction, Installation, and Performance," ACE, vol. 43, New York, 1998. 
[21] G. Grygiel et al., "Status of the TTF Cryogenic System," ACE vol.41, New York, 1996.

[22] K. Hosoyama et al., "Development of a High Performance Transfer Line System," ACE vol. 45, 2000.

[23] M. Chorowski, "Risk analysis of the LHC cryogenic system," ACE 2000.

[24] V. Parma et al., "The Insulation Vacuum Barrier for the Large Hadron Collider Magnet Cryostats," ICEC 18.

[25] B. Jenny et al., "A Composite Vacuum Barrier for the LHC Short Straight Section," $A C E$ vol.41, New York 1996.

[26] "Technical Specification for a Compound Cryogenic Helium Distribution Line for the Large Hadron Collider (LHC)," IT-2399/lhc/lhc, Geneva, Switzerland, December 1997.

[27] R.C. Niemann, "Model SSC Dipole Magnet Cryostat Assembly at Fermilab," Supercollider 1, New York 1989.

[28] N. Andreev, "Compact superconducting correctors with cold ferromagnetic screens," TD-01-046, 2001.

[29] G. Ambrosio et al., "Preliminary Proposal of a $\mathrm{Nb}_{3} \mathrm{Sn}$ Quadruple Model for the Low-Beta Insertions of the LHC," INFN Report, INFN/TC-95/25, Sept. 13, 1995.

[30] R. Scanlan, "Conductor Development Program," Low Temperature Superconducting Workshop, Santa Rosa, CA, Nov. 8-10, 2000.

[31] M. Canali, L. Rossi, "DYNQUE: a computer code for quench simulation in adiabatic multicoil superconducting solenoids," INFN Report, INFN/TC-93/06, 1993.

[32] L. Imbasciati, G. Ambrosio, P. Bauer, V. Kashikhin, A. Zlobin, "Quench Protection of the Common Coil Racetrack (CCRT-1): Results of Adiabatic Model Calculations," TD-00-056, FNAL (Sept. 2000).

[33] G. Ambrosio, G. Bellomo and L. Rossi, "A $300 \mathrm{~T} / \mathrm{m} \mathrm{Nb}_{3}$ Sn Quadrupole for the Low-beta Insertions of the LHC," Proc. of EPAC96, Sitges (Barcelona), p. 2290.

[34] Nikolai Mokhov, private communication.

[35] Bill Turner, private communication.

[36] V.V. Kashikhin, I. Terechkine, "Superconducting straight section quadrupole magnet for low-field VLHC," FNAL, TD-01-008, Feb. 2001.

[37] Conceptual Design of the Superconducting Super Collider. SSC-SR-2020, DE93 007234.

[38] J. Volk, "Injection transfer line magnets," TD Note TD-01-027, FNAL, 2001.

[39] J. Carson, R. Bossert, “A Proposed Production Procedure for Stage 1 Transmission Line Magnets," TD-01$028,2001$.

[40] J. Carson, R. Bossert, "Throughput for Stage 1 Transmission Line Factory," TD-01-029, 2001.

[41] P. Schlabach, "Magnetic Measurements During Production of VLHC Low Field Combined Function Magnets," TD-01-007, 2001.

[42] G. Ganetis et al., "Field Measuring Probe for SSC Magnets," Particle Accelerator Conference, 1987.

[43] L. Walkiers, "Measurements for the Acceptance Tests of the LHC Superconducting Magnets," Eleventh International Magnet Measurement Workshop, 1999.

[44] Brown \& Root, Installation \& Transport Proposal for the SSC.

[45] Supply of Cryo-Magnet Transport Vehicles and Unloading Equipment for the LHC Tunnel, LHC-HMU-CI0001 .

[46] An example of a commercial safety rail can be found at http://www.vahleinc.com/pdf/Catalog4C.pdf.

[47] Examples of tram systems and drive components operating in this voltage range can be found at http://w4.siemens.de/ts/products_solutions/light_rail tram.

[48] G.W. Foster, PAC 2001 invited talk.

[49] S. Hays, "100 kA Power Supply Options for the VLHC," TD-01-047, 2001.

[50] H. Piekarz et al., "Summary of Transmission Line Magnet R\&D Activities at MS-6," TD-01-048, 2001.

[51] G.W. Foster, S. Hays, H. Pfeffer, "Design of a Compact 100 kA Switching Power Supply for Superconducting Magnet Tests," TD-01-045, 2001. 
[52] J. G. Weisand (ed.), Handbook of Cryogenic Engineering, p. 411.

[53] K. Koepke, P. Mazur, A. Zlobin, Snowmass '96.

[54] G.W. Foster, H. Piekarz, "Quench Protection of the Transmission Line Magnet," TD-01-049, 2001.

[55] Switch wire is used on persistent-current MRI magnets. Commercial sources include SW-18 and SW-1 from Supercon Inc, Shrewsbury, MA, and Oxford Instruments, www.oxinst.com.

[56] See the LHC PAC '99 papers. See also the proceeding of the workshop on powering the LHC, http://hcp.web.cern.ch/lhcp/TCC/PLANNING/TCC/Power2000/welcome.htm.

[57] I. Terechkine, "LCW and power summary for the VLHC-1 Straight Sections," TD-01-050, 2001.

[58] Fermilab Main Injector Technical Design Report, Table 3.9.1.

[59] Typical laptop computers have heat pipes in them. See http://www.thermacore.com/papers.htm.

[60] Auto-resetting fuses are sold (for example) by: http://www.circuitprotection.com.

[61] Specifications quoted are single-quadrant DC-DC converters from Vicor, Inc. www.vicor.com.

[62] Fermilab Main Injector Note MI-75.

[63] Medvenko \& Smith, Beam Instrumentation Workshop 2000, p.503.

[64] Specifications quoted are from http://products.analog.com/products/info.asp?product=AD9203.

[65] W. Turner, "Beam Tube Vacuum in Low Field and High Field Very Large Hadron Collider," LBNL-39482, UC414, presented at the Snowmass Conference, CO, October 1996.

[66] M. Pivi and W.C. Turner, "Beam Tube Vacuum in a Very Large Hadron Collider Stage 1," Version 2.1, 12 April 2001.

[67] J. Gomez-Goni, O. Gröbner and A.G. Mathewson, "Exposure of a baked Al lined stainless steel chamber to photons," CERN, Vacuum Technical Note 94-10 pg. 9, AT-VA/JGG.

[68] C. Benvenuti, "Molecular Surface pumping: the getter pumps," CAS CERN Accelerator School - Vacuum Technology, CERN Yellow Book, pg. 48.

[69] J.C. Billy, J.P. Bojon, O. Gröbner, N. Hilleret, M. Jimenez, I. Laugier, P. Strubin, "The LEP Vacuum System: a Summary of 10 Years of Successful Operation," Proc. EPAC 2000.

[70] G.P. Jackson, ed., "The Fermilab Recycler Technical Design Report”, FNAL-TM-1991.

[71] I. Novitski, "Vacuum pipe stresses in the low field VLHC", TD-01-051, 2001.

[72] A. Chao and M. Tigner, Handbook of Accelerator Physics and Engineering, pg.225.

[73] Oswald Gröbner pvt. comm.

[74] A. Gambitta et al., "Experience with Aluminum Vacuum Chambers at ELETTRA," EPAC 2000, Vienna, Austria; and J. Miertusova et al., "How to Save Money Designing a Vacuum System for a Third Generation Synchrotron Light Source," EPAC 1996.

[75] Bob Goodwin, Peter Lucas, Elliott McCrory and Mike Shea, "Thoughts on Controls/Instrumentation for 3 TeV booster/VLHC," http://fnalpubs.fnal.gov/archive/vlhc/VLHCPub-14.html, July 1997.

[76] V. Shiltsev, "Summary of the Working Group II: RF, Instabilities and Feedback Systems," VLHC Accelerator Technology Workshop, TJNAF, Feb.1999. http://www.vlhc.org/tjef/wg2_summary.pdf.

[77] A. Jackson, contribution to the VLHC Design Study, unpublished.

[78] The LHC Study Group, "The Large Hadron Collider - Conceptual Design Report,” CERN/AC/95-05 (LHC), 1995.

[79] D. Boussard and T. Linnecar, "The LHC Superconducting RF System," Proceedings of the 1999 Cryogenic Engineering and International Cryogenic Material Conference (CEC-ICM'99), 12-16 July 1999, Montreal, Canada.

[80] P. Brown et al., "Performance of the LEP200 Superconducting RF System," Proceedings of the 9th Workshop on RF Superconductivity, 1-5 November 1999, Santa Fe, NM, USA.

[81] P. Bosland et al., "Completion of the SOLEIL Cryomodule," Proceedings of the 9th Workshop on RF Superconductivity, 1-5 November 1999, Santa Fe, NM, USA. 
[82] D. Boussard, "RF Power Requirements for a High Intensity Proton Collider," Proceedings of the 1991 Particle Accelerator Conference, pp. 2447-2449.

[83] H. Frischholz, W.R. Fowkes and C. Pearson, "Design and Construction of a $500 \mathrm{~kW} \mathrm{CW,} 400 \mathrm{MHz}$ Klystron to be Used as RF Power Source for LHC/RF Component Tests," Proceedings of the 1997 Particle Accelerator Conference, pp. 2911-2913.

[84] H. Padamsee, "SRF for Muon Colliders," Proceedings of the 9th Workshop on RF Superconductivity, 1-5 November 1999, Santa Fe, NM, USA.

[85] G. Lambertson, "Active feedback systems for Stage-1VLHC," TD-01-052, 2001.

[86] N.V. Mokhov and W. Chou, ed., "Beam Halo and Scraping," Proc. of ICFA Workshop, Lake Como, Wisconsin (1999).

[87] Bryant \& Johnsen, Circular Accelerators and Storage Rings, p. 308.

[88] A.I. Drozhdin and N.V. Mokhov, "Energy Deposition Issues in the Very Large Hadron Collider," Workshop on VLHC, Fontana, Wisconsin, February 22-25, 1999.

[89] A.I. Drozhdin, N.V. Mokhov, C.T. Murphy, S. Pruss, "Beam Abort For a 50 X50 TeV Hadron Collider", http://fnalpubs.fnal.gov/archive/vlhc/VLHCPub-51.ps.

[90] I.S. Baishev, A.I. Drozhdin, N.V. Mokhov, B. Parker, R.D. Richardson, J. Zhou, "Dealing with Abort Kicker Prefire in the Superconducting Super Collider," PAC 93, Washington, DC, May 1993.

[91] The Large Hadron Collider, Conceptual Design, CERN/AC/95-05(LHC) October 20, 1995.

[92] D.C. Wilson, C.A. Wingate, J.C. Goldstein, R.P. Godwin, N.V. Mokhov, "Hydrodynamic Calculations of 20-TeV Beam Interactions with the SSC BEAM Dump," Proc. of IEEE Particle Accelerator Conference, $\mathrm{p}$. 3090. (1993). A legendary SSC video contains a visualization of the extracted SSC beam drilling a hole in the absorber block when the sweeping magnet system fails.

[93] N. Mokhov, pvt. comm.

[94] R. Yarema, pvt. comm.

[95] LHC Talk at Beam Instrumentation Workshop '00.

[96] S. Hunt, S. Crivello, N. Mokhov, "Radiation Effects on Optical Fiber Links at the SSC," Nucl. Instr. Meth. in Phys. Research, A352 (1994) 329-243.

[97] A.I. Drozhdin and N.V. Mokhov, "The VLHC Beam Collimation System," TD-01-053, 2001.

[98] A.I. Drozhdin, N.V. Mokhov and A.A. Sery, "The Very Large Hadron Collider Beam Collimation System," Workshop on VLHC, Fontana, Wisconsin, February 22-25, 1999.

[99] N.V. Mokhov and J.B. Strait, "Towards the Optimal LHC Interaction Region: Beam-Induced Energy Deposition," Proc. of IEEE Particle Accelerator Conference, p. 124 (1997).

[100] Electron Beam Tunnel Boring papers from LBL, circa 1975.

[101] "Fermilab Work Smart Standards Set," Fermi National Accelerator Laboratory, http://wwwlib.fnal.gov/library/protect/worksmart.html, November 15, 1999.

[102] United States Code of Federal Regulations, Title 10, Part 1021, "Department of Energy National Environmental Policy Act Implementation Procedures," 1997.

[103] A. Van Ginneken, P. Yurista, and C. Yamaguchi, "Shielding calculations for multi-TeV hadron colliders," Fermilab Report FN-447 (1987).

[104] U. S. Department of Energy, "Radiation Protection of the Public and the Environment," DOE Order 5400.5, January 7, 1993.

[105] United States Code of Federal Regulations, Title 40, Part 61, Subpart H, "National Emissions Standard for Hazardous Air Pollutants (NESHAP) for the Emission of Radionuclides other than Radon from Department of Energy Facilities," 1989. 


\section{Chapter 6. Stage-2 Components}

\subsection{Superconducting Magnet Systems}

The application of superconductivity for high-energy accelerators started more than 20 years ago. At the present time four machines, the Tevatron (FNAL), HERA (DESY), NUKLOTRON (JINR) and RHIC (BNL) are in operation, and LHC (CERN) is under construction. All these machines utilize NbTi superconducting (SC) magnets cooled by LHe at a nominal temperature of 1.9 or $4.5 \mathrm{~K}$. NbTi has an upper critical magnetic field of $14-15 \mathrm{~T}$ at $0 \mathrm{~K}(10 \mathrm{~T}$ at $4.2 \mathrm{~K})$ and a critical temperature of $9.5 \mathrm{~K}$. Thanks to the excellent mechanical properties of $\mathrm{NbTi}$, traditional electromagnet technologies could be used in the construction of these magnets. Accelerator dipole magnets based on this technology provide 7.5-8 T maximum field (6-7 T operating field) at $4.5 \mathrm{~K}$ and the 10 $10.5 \mathrm{~T}$ maximum field (8-9 $\mathrm{T}$ operating field) at $1.9 \mathrm{~K}$ of the required field quality. These magnets behave reliably and reproducibly in thermal and current cycles over long periods of time in hard radiation environments. Thousands of short and full-scale (6-15 $\mathrm{m}$ long) NbTi magnets have been fabricated, tested and used in large accelerator/collider projects, beginning with the Tevatron, and continuing through RHIC and LHC. The technology of NbTi magnets has been successfully demonstrated in mass production in industry (HERA, RHIC) at affordable costs. Although NbTi can be used in Stage-2 for relatively low-field magnets such as multipole correctors, NbTi magnets do not reach the nominal field of $10 \mathrm{~T}$ chosen for the main arc dipoles.

Alternative superconductors for high field accelerator magnets are the A15 materials, $\mathrm{Nb}_{3} \mathrm{Sn}$ in particular. $\mathrm{Nb}_{3} \mathrm{Sn}$ has a high upper critical magnetic field of $23-24 \mathrm{~T}$ at $4.2 \mathrm{~K}$ and a critical temperature of $18 \mathrm{~K}$. It is produced commercially on a fairly large scale. Formation of the $\mathrm{Nb}_{3} \mathrm{Sn}$ phase and its properties (brittleness) determine two possible approaches to magnet fabrication: wind-and-react or react-and-wind techniques. $\mathrm{Nb}_{3} \mathrm{Sn}$ accelerator magnets employing different coil designs and utilizing both wind-and-react and react-and-wind fabrication techniques are being investigated at various U.S. and European HEP laboratories and universities [1-7]. In the past 5-8 years, magnetic fields above $10 \mathrm{~T}$ have been achieved. The magnet group at Twente University (Netherlands) achieved $11 \mathrm{~T}$ at $4.3 \mathrm{~K}$ with a two-layer cos-theta dipole model [8]. The LBNL group built a four-layer cos-theta dipole model that reached fields above $12.4 \mathrm{~T}$ at $4.3 \mathrm{~K}$ and above $13 \mathrm{~T}$ at $1.9 \mathrm{~K}$ [9]. A common coil $\mathrm{Nb}_{3} \mathrm{Sn}$ racetrack structure at LBNL recently reached $12 \mathrm{~T}$ field at $4.2 \mathrm{~K}$ [10]. Progress in raising the critical current of $\mathrm{Nb}_{3} \mathrm{Sn}$ strands [11] and in developing magnet technologies for brittle superconductors make it possible to design cost-effective $\mathrm{Nb}_{3} \mathrm{Sn}$ accelerator magnets with a nominal field of 10-12 $\mathrm{T}$. Although only a few short models have been fabricated and tested up to now, the positive results of this work make $\mathrm{Nb}_{3} \mathrm{Sn}$ conductor very attractive for Stage- 2 magnets.

Since the construction of Stage 2 is many years from now, new emerging SC materials and technologies could be considered in the future. The most promising of the new materials include $\mathrm{Bi}_{2} \mathrm{Sr}_{2} \mathrm{CaCu}_{2} \mathrm{O}_{\mathrm{x}}$ (BSCCO-2212), with $\mathrm{T}_{\mathrm{c}} \sim 85 \mathrm{~K}$, and $\mathrm{Bi}_{2} \mathrm{Sr}_{2} \mathrm{Ca}_{2} \mathrm{Cu}_{3} \mathrm{O}_{\mathrm{x}}$ (BSCCO-2223), having $\mathrm{T}_{\mathrm{c}} \sim 110 \mathrm{~K}$. Kilometer-length quantities of these materials have been made in the form of multifilamentary tapes or round strands, suitable for a magnet 
using the flat racetrack coil design [11]. Another material, YBCO, shows even more promising properties. Very high critical current densities have been achieved at high fields in YBCO short samples. However, commercial production of this material lags well behind BSCCO. An appropriate magnet technology for this material has yet to be invented. Cost of all HTS materials is very high and their production rate is too low at the present time to be considered for Stage- 2 magnets. In small quantities these materials could be used for special Stage-2 magnets required in both Stage 1 and Stage 2, such as the interaction region (IR) magnets.

\subsubsection{Stage-2 Magnet System}

The Stage-2 magnet system consists of SC arc dipoles, quadrupoles and corrector magnets as well as special SC magnets such as dispersion suppression magnets, IR magnets, and special correctors. A discussion of magnet field quality and tolerances is presented in Section 3.3.

Based on the chosen lattice and machine operation parameters, Stage- 2 arc magnets should meet the following general requirements:

- operation field range 1-10 $\mathrm{T}$ for arc and DS dipole magnets

- field gradient range 45-400 T/m for arc and DS quadrupole magnets

- good field quality in the operational range

- coil aperture $\geq 40 \mathrm{~mm}$, sufficient for installation of the vacuum chamber and beam screen

- vertical bore arrangement

- operation temperature range 4.5-5.5 K

- sufficient critical current and critical temperature margin for reliable operation under high heat load from synchrotron radiation

- restricted temperature and voltage change during a quench

- robust mechanical design at affordable magnet cost

Proton beams in Stage 2 circulate in opposite directions in two crossing storage rings. These storage rings can be designed based on single-aperture magnets placed in separate cryostats (SSC) [12] or based on double-aperture magnets with common iron yoke and cryostat (LHC) [13]. The second approach allows reducing the size, weight and cost of magnet and cryostat. Since all these parameters are important for the VLHC, this approach was chosen for the Stage-2 high field magnets.

\subsubsection{Arc Dipole Magnets}

The arc dipoles use a cold yoke design based on the common coil approach. The design meets the Stage-2 VLHC requirements.

Flat racetrack coils are shared between two vertically positioned apertures. The common coil dipole design described here is a simple single-layer coil divided into three blocks by wide spacers. This simplifies design and fabrication technology, and allows the possibility of using reacted brittle $\mathrm{Nb}_{3} \mathrm{Sn}$ cable, solving many mechanical problems, and at the same time achieving excellent field quality [14]. 
The coils are made of rectangular Rutherford-type cable with $60 \mathrm{Nb}_{3} \mathrm{Sn}$ strands, each $0.7 \mathrm{~mm}$ in diameter. The bare cable width is $22.22 \mathrm{~mm}$ and the thickness is $1.250 \mathrm{~mm}$. The insulation is $0.10 \mathrm{~mm}$ thick and made of fiberglass tape.

Each coil consists of 58 turns grouped into three blocks and separated by $6 \mathrm{~mm}$ thick spacers. There are also two $3 \mathrm{~mm}$ thick spacers in each middle block. The pole blocks are shifted horizontally inwards by $5 \mathrm{~mm}$ with respect to the middle blocks. The size and position of blocks and spacers were optimized to provide the maximum transfer function, minimum coil volume and small low-order geometrical harmonics within the largest possible gap. The gap between pole blocks is $40 \mathrm{~mm}$, determining the magnet aperture. The use of the reacted $\mathrm{Nb}_{3} \mathrm{Sn}$ cable dictates a minimum bending radius of $90 \mathrm{~mm}$ for the chosen strand size and thus sets the aperture separation to $290 \mathrm{~mm}$.

The iron yoke is split vertically into two pieces. To provide contact between the collared coil and the yoke at all temperatures, the gap between yoke pieces is always open. Special holes are used for correction of iron saturation. The yoke inner surface and outer radius, geometry and position of the correction holes were optimized to provide good field quality, low fringe fields, maximum transfer function and smallest yoke size. Figure 6.1 shows the flux distribution in a 2D cross-section of the arc dipole. A top/bottom yoke asymmetry relative to the magnet aperture will cause a skew quadrupole component in the case of symmetric coils. This component was reduced using a small top/bottom block asymmetry with respect to the mid-plane of each aperture.

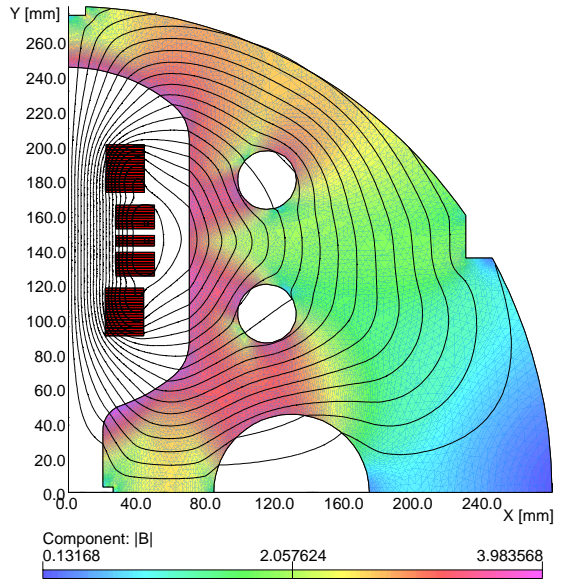

Figure 6.1. Magnetic design of the arc dipole.

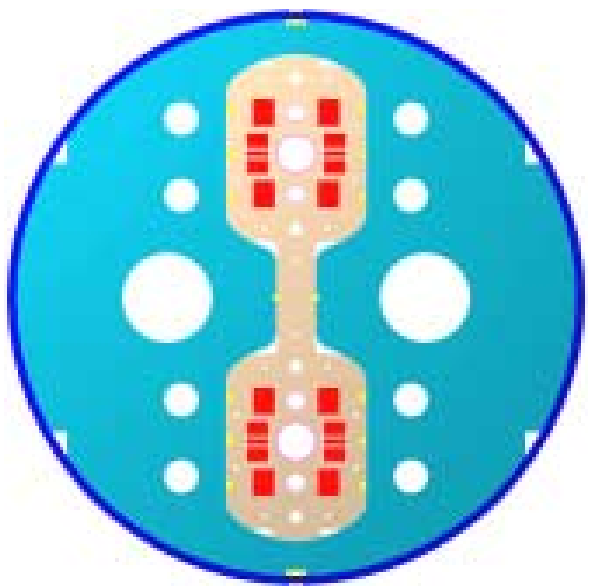

Figure 6.2. Cross-section of the arc dipole.

This design allows the use of a stress management strategy. Coil blocks, surrounded by the $0.5 \mathrm{~mm}$ thick electrical insulation, are placed inside a strong collar structure. The collar structure has round holes for the beam pipe and the cooling channels. During assembly the collar structure provides the vertical prestress and protects the coil from the horizontal and vertical over-compression. During operation it prevents an accumulation of the vertical Lorentz forces and a force transfer from the pole blocks to the mid-plane blocks. It also intercepts a significant part of the horizontal Lorentz forces reducing stresses in the yoke and skin. At all conditions, stress in the coil is less than $150 \mathrm{MPa}$, which is safe for the $\mathrm{Nb}_{3} \mathrm{Sn}$ cable [15]. 
Both coils are wound directly into the support structure and impregnated with epoxy. The collared coil assembly is placed inside the iron yoke surrounded by a $10 \mathrm{~mm}$ thick stainless steel skin. Thick end plates welded to the skin restrict the longitudinal motion of the coils. The horizontal pre-compression of the collared coil is provided by the stainless steel skin via the iron yoke. The main calculated parameters are summarized in Table 6.1, and the 2D cross-section of the magnet cold mass is shown in Figure 6.2.

Table 6.1. Arc dipole parameters.

\begin{tabular}{|l|c|}
\hline $\mathrm{B}_{\text {nom }}, \mathrm{T}$ & 10 \\
\hline $\mathrm{I}_{\text {nom, }}, \mathrm{kA}$ & 23.5 \\
\hline Aperture, mm & 40 \\
\hline Aperture separation, mm & 290 \\
\hline Magnetic length, $\mathrm{m}$ & 16.15 \\
\hline Iron yoke OD, mm & 560 \\
\hline Stored energy @10 $10 \mathrm{~T}, \mathrm{~kJ} / \mathrm{m}$ & $2 \times 414$ \\
\hline Inductance @ $10 \mathrm{~T}, \mathrm{mH} / \mathrm{m}$ & $2 \times 1.5$ \\
\hline
\end{tabular}

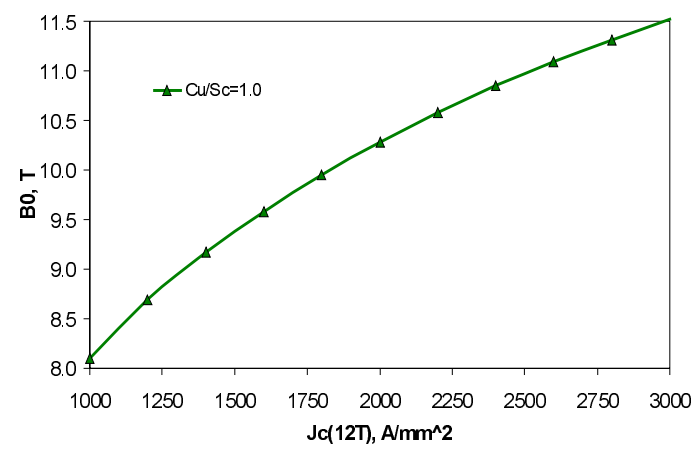

Figure 6.3. Arc dipole short sample limit.

Maximum (quench) field in the magnet aperture at $4.2 \mathrm{~K}$ vs. the critical current density of SC strand in the coil is shown in Figure 6.3. At 15\% $\mathrm{I}_{\mathrm{c}}$ degradation [16,17], a nominal field of $10 \mathrm{~T}$ with $10 \%$ margin will be achieved using the new $\mathrm{Nb}_{3} \mathrm{Sn}$ strands with $\mathrm{J}_{\mathrm{c}}(12 \mathrm{~T}, 4.2 \mathrm{~K})=3 \mathrm{kA} / \mathrm{mm}^{2}[18]$.

The magnetic field in the dipole bore is described according to the expression

$$
B_{y}+i B_{x}=B_{0} \sum_{n=1}^{\infty}\left[b_{n}+i a_{n}\right] \cdot\left(\frac{z}{R_{r e f}}\right)^{n} .
$$

Calculated geometrical harmonics and their RMS spread at $1 \mathrm{~cm}$ radius for $\pm 50 \mu \mathrm{m}$ random coil block displacements for the arc dipole are shown in Table 6.2.

Table 6.2. Relative dipole systematic and random geometrical harmonics at $1 \mathrm{~cm}$ radius, $10^{-4}$.

\begin{tabular}{|c|c|c|c|}
\hline Harmonic number, $\mathrm{n}$ & Systematic, $\mathrm{b}_{\mathrm{n}}$ & Systematic, $\mathrm{a}_{\mathrm{n}}$ & RMS $\left(\sigma_{\mathrm{a}, \mathrm{b}}\right)$ \\
\hline 1 & - & 0.003 & 1.040 \\
\hline 2 & 0.000 & - & 0.360 \\
\hline 3 & - & -0.005 & 0.141 \\
\hline 4 & 0.001 & - & 0.046 \\
\hline 5 & - & +0.002 & 0.017 \\
\hline 6 & 0.001 & - & 0.006 \\
\hline 7 & - & 0.010 & 0.003 \\
\hline 8 & -0.026 & - & 0.002 \\
\hline 9 & - & 0.002 & 0.000 \\
\hline
\end{tabular}


The effect of iron saturation on the magnet transfer function and low order field harmonics is shown in Figure 6.4. The coil magnetization effect is shown in Figure 6.5. The reduction of the dipole transfer function at the nominal field of $10 \mathrm{~T}$ is about $9.2 \%$. The iron saturation effect on the low order harmonics in the operating field range 1-10 $\mathrm{T}$ is effectively suppressed by optimizing the yoke size and correction hole geometry. The coil magnetization effect at $\mathrm{B}>1 \mathrm{~T}$ is small even with the currently availble $\mathrm{Nb}_{3} \mathrm{Sn}$ strands having a large effective filament diameter of $\sim 100 \mu \mathrm{m}[19]$.

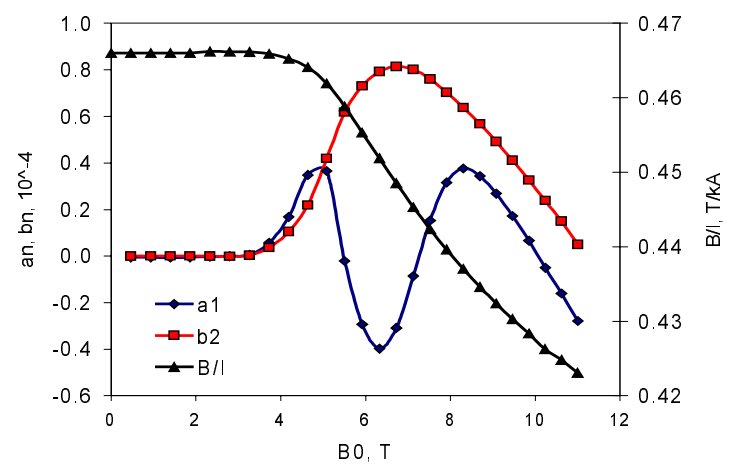

Figure 6.4. Iron saturation effect.

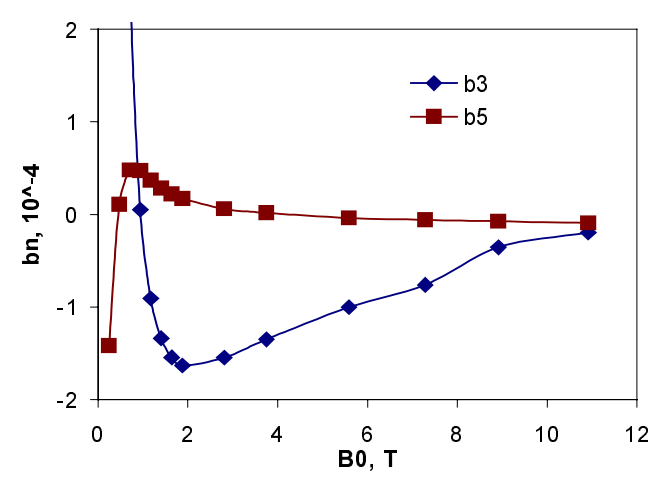

Figure 6.5. Coil magnetization effect.

\subsubsection{Arc Quadrupole Magnets}

The 2-in-1 arc quadrupole magnet design with vertical bore arrangement and FF or DD functions [20] was developed to use together with the arc dipole magnet described above. The magnet design is based on a two-layer shell-type coil and cold iron yoke, and meets the Stage- 2 requirements.

The coil bore diameter is $43.5 \mathrm{~mm}$. Each coil quadrant consists of 13 turns. The interlayer spacer is $0.28 \mathrm{~mm}$ thick and the additional mid-plane insulation layers are $2 \times 0.125$ $\mathrm{mm}$ thick. Each coil quadrant has a floating pole and two wedges in the inner layer in order to minimize low order field harmonics. Coil ends have a block-wise layout of turns optimized to reduce the maximum field in the ends and to improve end field quality.

The coil utilizes a keystone Rutherford-type cable made of $28 \mathrm{Nb}_{3} \mathrm{Sn}$ strands, each 1 $\mathrm{mm}$ in diameter. The bare cable width is $14.24 \mathrm{~mm}$, the thickness is $1.800 \mathrm{~mm}$ and the keystone angle is 0.91 degree. The cable is wrapped with special insulation suitable for the long high-temperature heat treatment. The nominal thickness of the cable insulation is $0.25 \mathrm{~mm}$. Both layers of half-coil are made from one cable piece without an inter-layer splice.

Two collared coils are placed in $144 \mathrm{~mm}$ diameter circular holes separated by $290 \mathrm{~mm}$ inside the common iron yoke. Special holes are used for correction of iron saturation. The iron yoke was split vertically into two pieces to allow assembly of two coils in one yoke. In order to reduce the effect of gap variation on the field quality, the vertical gap between the two iron pieces is always closed. Figure 6.6 shows the flux distribution in the optimized 2D cross-section of the arc quadrupole magnet. 


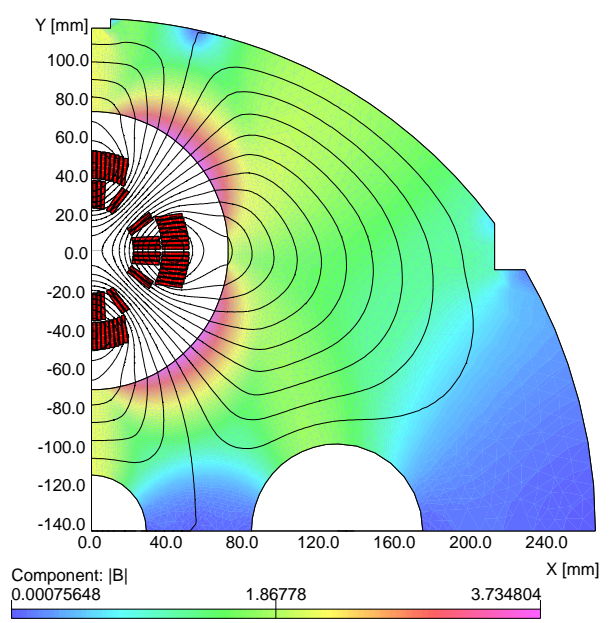

Figure 6.6. Arc quadrupole magnetic design.

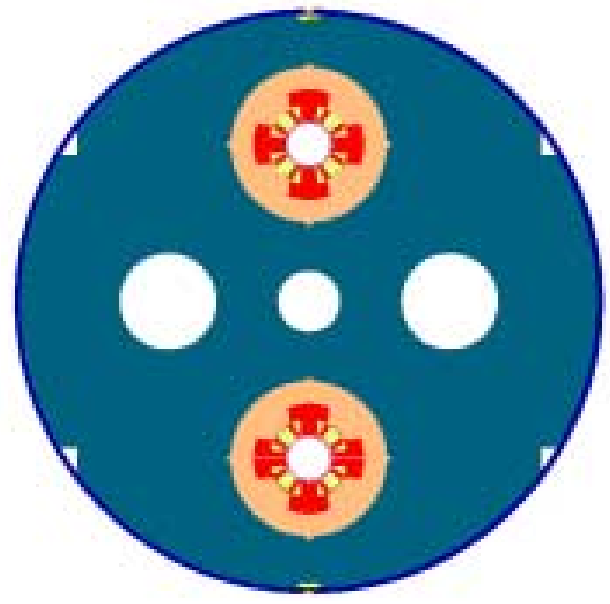

Figure 6.7. Arc quadrupole cross-section.

The magnet body is prestressed and mechanically supported by means of stainless steel collar laminations. Tapered keys lock collar laminations in two perpendicular directions. Thick aluminum end cans provide prestress and mechanical support of the coil ends. Coil prestress is less than $150 \mathrm{MPa}$ in all conditions [21].

The collared coil is centered inside the yoke with the help of alignment keys. No additional radial support from the iron yoke is required. The yoke outer diameter is $530 \mathrm{~mm}$. Yoke laminations feature large round holes for LHe flow and special cutouts required for precise alignment. The two-piece iron yoke is enclosed by a $6 \mathrm{~mm}$ thick stainless steel skin. To restrict coil longitudinal motion, two thick stainless steel end plates are welded onto the skin. The cross-section of the arc quadrupole is shown in Figure 6.7. The main calculated parameters are summarized in Table 6.3.

Table 6.3. Arc quadrupole parameters.

\begin{tabular}{|l|c|}
\hline $\mathrm{G}_{\text {nom }}, \mathrm{T} / \mathrm{m}$ & 400 \\
\hline $\mathrm{I}_{\text {nom }}, \mathrm{kA}$ & 27.2 \\
\hline Aperture, mm & 43.5 \\
\hline Aperture separation, mm & 290 \\
\hline Magnetic length, $\mathrm{m}$ & 9.16 \\
\hline Iron yoke OD, mm & 530 \\
\hline Stored energy @4400T/m, kJ/m & $2 \times 209$ \\
\hline Inductance @4400T/m, mH/m & $2 \times 0.57$ \\
\hline
\end{tabular}

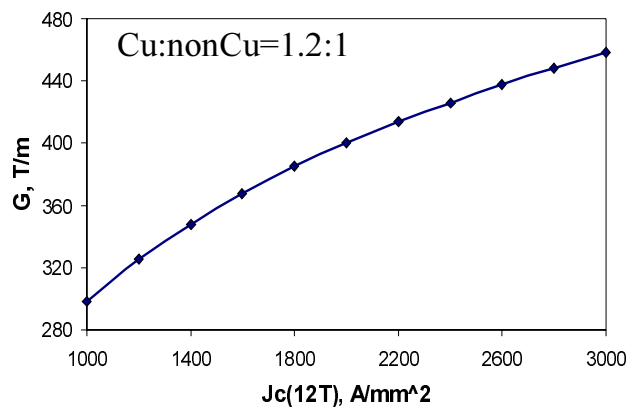

Figure 6.8. Arc quad short sample limit.

Maximum (quench) field gradient in the magnet aperture at $4.2 \mathrm{~K}$ vs. the critical current density of $\mathrm{Nb}_{3} \mathrm{Sn}$ strand in the coil is shown in Figure 6.8. Assuming 10\% $\mathrm{I}_{\mathrm{c}}$ degradation [16], a nominal gradient of $400 \mathrm{~T} / \mathrm{m}$ with $10 \%$ margin will be achieved using R\&D $\mathrm{Nb}_{3} \mathrm{Sn}$ strands with $\mathrm{J}_{\mathrm{c}}(12 \mathrm{~T}, 4.2 \mathrm{~K})=3 \mathrm{kA} / \mathrm{mm}^{2}$ [18]. Calculated geometrical harmonics and 
their rms spread at $1-\mathrm{cm}$ radius for $\pm 50 \mu \mathrm{m}$ random coil block displacements for the arc quadrupole are summarized in Table 6.4.

Table 6.4. Quadrupole systematic and random geometrical harmonics at 1-cm radius, $10^{-4}$.

\begin{tabular}{|c|c|c|c|}
\hline Harmonic number, $\mathrm{n}$ & Systematic, $\mathrm{b}_{\mathrm{n}}$ & Systematic, $\mathrm{a}_{\mathrm{n}}$ & $\mathrm{rms}, \sigma_{\mathrm{a}, \mathrm{b}}$ \\
\hline 2 & - & - & 1.82 \\
\hline 3 & - & - & 0.82 \\
\hline 4 & - & - & 0.38 \\
\hline 5 & -0.0003 & - & 0.19 \\
\hline 6 & - & - & 0.07 \\
\hline 7 & - & - & 0.026 \\
\hline 8 & - & - & 0.013 \\
\hline 9 & -0.0039 & - & 0.001 \\
\hline
\end{tabular}

The effect of iron saturation at high fields on the magnet transfer function and low order field harmonics is shown in Figure 6.9. The coil magnetization effect is shown in Figure 6.10.

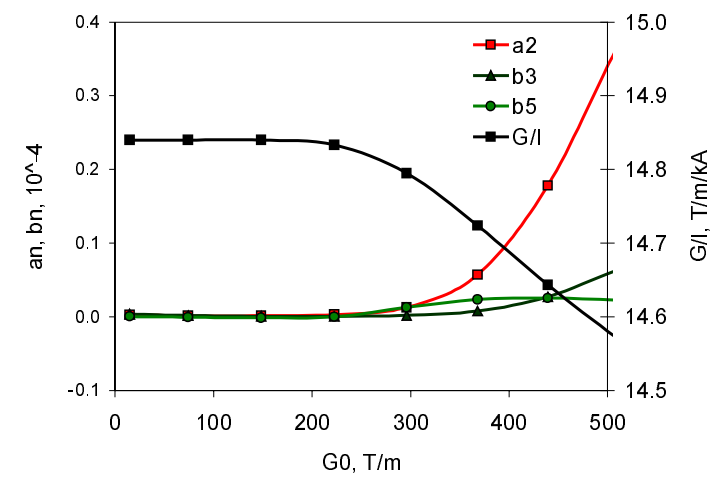

Figure 6.9. Iron saturation effect.

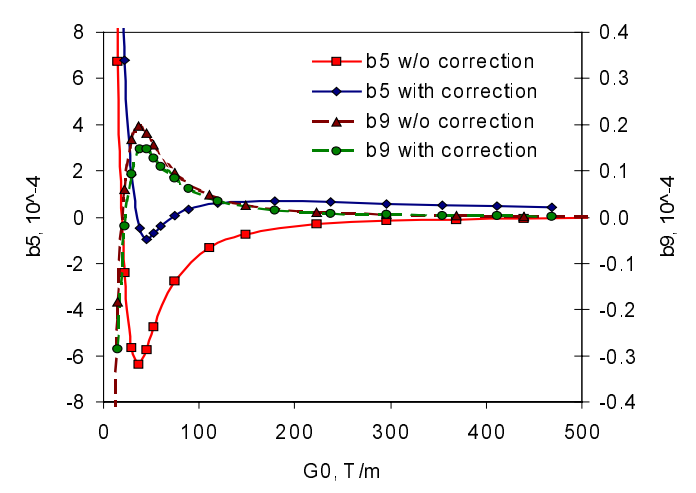

Figure 6.10. Coil magnetization effect.

Reduction of the quadrupole transfer function at the nominal field gradient of $400 \mathrm{~T} / \mathrm{m}$ is about $1 \%$, smaller than the reduction of the dipole transfer function. To provide a constant $\mathrm{G} / \mathrm{B}$ ratio in the operation field range, the arc quadrupole magnets will be powered independently from the arc dipole magnets. The iron saturation effect on the normal and skew low order harmonics at field gradient $\mathrm{G}<400 \mathrm{~T} / \mathrm{m}$ is effectively suppressed by optimizing the yoke size and correction hole geometry. The coil magnetization effect at $\mathrm{G}>45 \mathrm{~T} / \mathrm{m}$ is minimized using a simple passive correction system based on iron strips [22]. Additional reduction will be achieved by reducing the effective filament diameter in $\mathrm{Nb}_{3} \mathrm{Sn}$ strands.

\subsubsection{Arc Corrector Magnets}

The correction system required for Stage-2 consists of two subsystems: an arc correction system and an IR correction system. This section describes the correction magnets to be used in the arc, including the dispersion suppressor regions. The arc correction system includes dipole, quadrupole and sextupole correctors combined in different packages: a) 
dipole-sextupole package for the arc cells and b) dipole / skew-quadrupole package for DS cells. Two possible design approaches, nested vs. lumped coil arrangement, and two different superconductors have been studied [23]. The lumped approach was chosen as the most simple and effective for magnet fabrication and correction system operation. It is expected that all correctors included in spool pieces will be powered from individual power supplies. To simplify the power supply and current lead designs, as well as corrector quench protection, the operational current of correctors was limited to $100 \mathrm{~A}$. Since the operational temperature varies from 4.5 to $5.5 \mathrm{~K}$, corrector temperature margin is a critical issue. $\mathrm{NbTi}$ and $\mathrm{Nb}_{3} \mathrm{Sn}$ were compared in providing maximum corrector strength with sufficient critical current margin and sufficient temperature margin. It was found that $\mathrm{NbTi}$ can provide sufficient operational margin. Based on this and taking into account the availability of well-developed technologies for NbTi multipole correctors, they were chosen as a baseline approach for Stage-2 corrector magnets.

The cross-sections of the dipole, quadrupole and sextupole correctors are shown in Figure 6.11. Corrector operational parameters are summarized in Table 6.5.
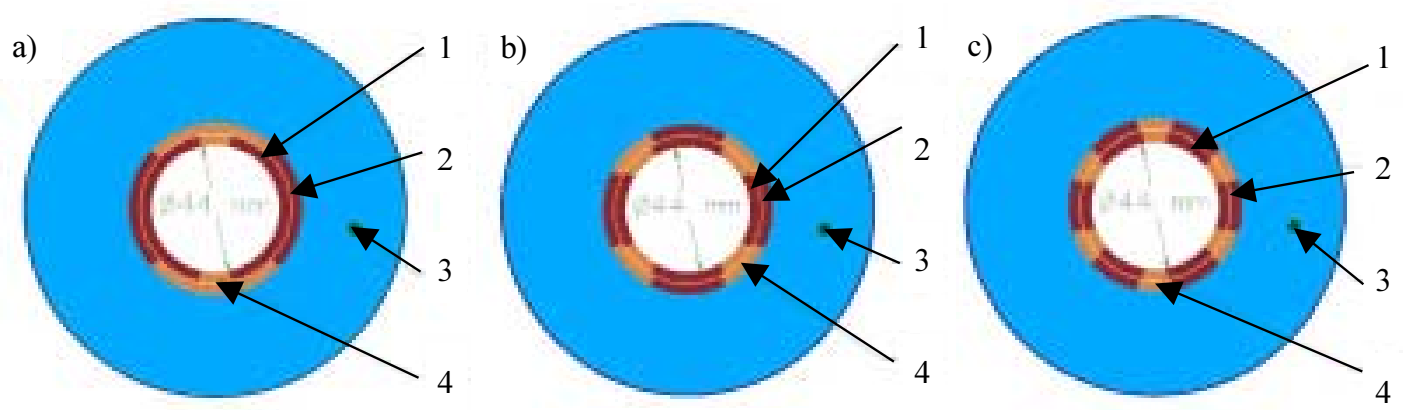

Figure 6.11. Dipole (a), quadrupole (b), and sextupole (c) corrector cross-section. The bore diameter for all 3 magnets is $44 \mathrm{~m} .1-$ coil $\left(\Delta r_{\text {coil }}=2 \times 3.6 \mathrm{~mm}\right) ; 2-$ insulation $\left(\Delta r_{\text {ins }}=0.5 \mathrm{~mm}\right)$; 3 - iron yoke $\left(R_{\text {in }}=30 \mathrm{~mm}, R_{\text {out }}=65 \mathrm{~mm}\right) ; 4$ - pole piece.

All coils have a two-layer design. In order to simplify the coil winding and optimize the coil packing factor, a flat single-layer ribbon cable is used having the parameters listed below. After coil fabrication, strands are connected in series to provide the required low nominal operation current. The strand and cable parameters are:

- Bare strand diameter $-0.3 \mathrm{~mm}$

- Insulated strand diameter $-0.36 \mathrm{~mm}$

- $\mathrm{Cu} / \mathrm{nonCu}$ ratio - 2:1

- $\mathrm{Jc}(12 \mathrm{~T}, 4.2 \mathrm{~K})-2.75 \mathrm{kA} / \mathrm{mm}^{2}$ (SSC)

- Number of strands - 10

- Width $-3.6 \mathrm{~mm}$

- Mid thickness $-0.36 \mathrm{~mm}$

- Insulation $-0.025 \mathrm{~mm}$ 
Table 6.5. Stage-2 corrector parameters.

\begin{tabular}{|c|c|c|c|}
\hline Parameter & $\begin{array}{c}\text { Sextupole } \\
(\mathrm{n}=2)\end{array}$ & $\begin{array}{l}\text { Quadrupole } \\
(\mathrm{n}=1)\end{array}$ & $\begin{array}{c}\text { Dipole } \\
(n=0)\end{array}$ \\
\hline Required integrated strength, $\mathrm{T}-\mathrm{m} / \mathrm{cm}^{\mathrm{n}}$ & 0.74 & 0.95 & 2.3 \\
\hline Critical current $\mathrm{I}_{\mathrm{c}}(4.2 \mathrm{~K}), \mathrm{A}$ & 150 & 136 & 155 \\
\hline Nominal operation current $\mathrm{I}_{\text {nom }}, \mathrm{A}$ & 55 & 50 & 40 \\
\hline Critical current margin $\mathrm{I}_{\mathrm{c}} / \mathrm{I}_{\mathrm{n}}$ & 2.7 & 2.7 & 4.3 \\
\hline Critical temperature margin $\Delta \mathrm{T}_{\mathrm{c}}=\mathrm{T}_{\mathrm{c}}-5.5, \mathrm{~K}$ & 1.5 & 1.5 & 2.5 \\
\hline Nominal strength, $\mathrm{T} / \mathrm{cm}^{\mathrm{n}}$ & 0.8 & 1.0 & 2.2 \\
\hline Magnetic length, $\mathrm{m}$ & 0.93 & 0.95 & 1.1 \\
\hline Inductance $\mathrm{L}, \mathrm{H} / \mathrm{m}$ & 1.4 & 2.5 & 5.4 \\
\hline Stored energy @10A, J/m & 70 & 126 & 271 \\
\hline Current density in $\mathrm{Cu}$ matrix, $\mathrm{A} / \mathrm{mm}^{2}$ & 1167 & 1061 & 845 \\
\hline
\end{tabular}

The main correction elements (dipole and sextupole coils or dipole and quadrupole coils) are combined in packages with a total length of $\sim 2 \mathrm{~m}$ and placed in spool pieces near each quadrupole in the arc and DS cells. Adjacent to each arc focusing quadrupole spool piece are horizontal dipole and focusing sextupole windings. Adjacent to each arc defocusing quadrupole there is a spool piece with vertical dipole and defocusing sextupole windings. Spool pieces in DS cells consist of dipole and normal or skew quadrupole windings. Other correctors required in special regions of the Stage- 2 arc could be combined with the main correctors and installed in the same corrector assembly.

\subsubsection{IR Magnets}

There are two possible approaches to the collision optics in Stage 2: one is symmetric doublet optics suited for the flat beams and the other is traditional anti-symmetric optics suited for round beams. The layouts of IR doublets and triplets, as well as the discussion of these two approaches from the accelerator physics viewpoint and machine luminosity considerations, are presented in Chapter 3.

The parameters of various IR magnets required for flat beam optics are summarized in Table 6.6. As can be seen from this table, the magnet parameters are very challenging. Achieving them will require development of innovative magnet designs and new superconducting and structural materials with unique properties. Some possible approaches to this problem are discussed in Section 10.2. The parameters of various IR magnets required for the case of round beam optics are summarized in Table 6.7.

The single bore separation dipole $\mathrm{D} 1$ is similar to the $\mathrm{Nb}_{3} \mathrm{Sn}$ separation dipole magnet with 80-mm bore being developed for the second generation of the LHC IR [7]. In the vertical bore arrangement chosen for Stage- 2 in this study, this magnet has to provide a horizontal field of $10 \mathrm{~T}$. The other separation dipole D2 is a double bore magnet with a horizontal field in each aperture of $10 \mathrm{~T}$. This magnet could be a modification of the arc dipole magnet based on the costheta design shown in Figure 10.4. Quadrupoles Q4, Q5, Q6 and Q7 are double bore magnets with the same cross-section and nominal field gradient as the arc quads but different magnetic length. 
Table 6.6. Design parameters of Stage-2 interaction region magnets (flat beam optics).

\begin{tabular}{|c|c|c|c|c|c|}
\hline Magnet & Field, T & Gradient, T/m & Aperture, $\mathrm{mm}$ & Length, $\mathrm{m}$ & Type \\
\hline D1A & 16 & - & 25 & 12.1 & Single bore \\
\hline D1B & 12 & - & 50 & 6.0 & Single bore \\
\hline D2 & 12 & - & 50 & 11.1 & 2-in-1 \\
\hline Q1A & - & 400 & 30 & 12.4 & 2 -in-1 \\
\hline Q1B & - & 600 & 30 & 12.4 & 2 -in-1 \\
\hline Q2A & - & 600 & 30 & 7.9 & 2 -in-1 \\
\hline Q2B & - & 600 & 30 & 7.9 & 2-in-1 \\
\hline
\end{tabular}

Table 6.7. Design parameters of Stage-2 interaction region magnets (round beam optics).

\begin{tabular}{|c|c|c|c|c|c|}
\hline Magnet & Field, T & Gradient, T/m & Aperture, mm & Length, $\mathrm{m}$ & Type \\
\hline Q1 & - & 400 & $60 ?$ & 23.58 & Single bore \\
\hline Q2a, Q2b & - & 400 & $60 ?$ & 18.98 & Single bore \\
\hline Q3 & - & 400 & $60 ?$ & 23.58 & Single bore \\
\hline D1 & 10 & - & $80 ?$ & $?$ & Single bore \\
\hline D2 & 10 & - & $40 ?$ & $?$ & 2 -in-1 \\
\hline Q4 & - & 400 & 40 & 10.47 & 2-in-1 \\
\hline Q5a, Q5b & - & 400 & 40 & 15.09 & 2-in-1 \\
\hline Q6a, Q6b & - & 330 & 40 & 15.09 & 2-in-1 \\
\hline Q7 & - & 375 & 40 & 10.47 & 2-in-1 \\
\hline
\end{tabular}

The most challenging magnets are the inner triplet quadrupoles. These magnets should provide the same nominal field gradient of $400 \mathrm{~T} / \mathrm{m}$ as the arc quads but in a significantly larger aperture. Scaling the parameters of the large aperture quadrupole magnet described in Section 5.1.4.1, one can see that the required field gradient of $400 \mathrm{~T} / \mathrm{m}$ could be achieved in the twolayer quadrupole design with an aperture of $60 \mathrm{~mm}$ or larger using cable with similar or better parameters. Good field quality in these magnets is realizable. The $60-\mathrm{mm}$ aperture should be sufficient for the required beam separation determined by the beam sizes and crossing angle as well as for the insertion inside the magnet an absorber. The absorber should be able to intercept a sufficient amount of the radiation-induced heat load at temperature higher than nominal in order to reduce the heat load on the IR cryogenic system.

The IRs will also contain a number of corrector magnets. The higher order harmonic correctors will be based on multi-layer coils within the same cold mass. These correctors will use $\mathrm{Nb}_{3} \mathrm{Sn}$ superconductor to provide large operational margins (critical temperature and critical current) required in the IR with the high level of radiation heat deposition.

\subsubsection{Cryostat and Spool Pieces}

The cryostat serves to support the magnet cold mass, thermal shield(s) and cryogenic piping within the vacuum vessel, and to insulate the cold mass from heat radiated and conducted from the environment. It must function reliably during storage, shipping and handling, normal operation, quenches, and seismic excitations, and must have affordable cost. The major components of 
the cryostat are the vacuum vessel, thermal shields, multi-layer insulation, cryogenic piping, interconnections, and suspensions.

Figure 6.12 shows conceptual layouts of the arc dipole and quadrupole cryostats [24]. Both cryostats are $958 \mathrm{~mm}$ wide and $1012 \mathrm{~mm}$ high. Their lengths are approximately $17 \mathrm{~m}$ and $9 \mathrm{~m}$ and total estimated weights are 42 tons and 22 tons respectively. The DS magnets have similar cross-sections, but are obviously shorter and lighter.
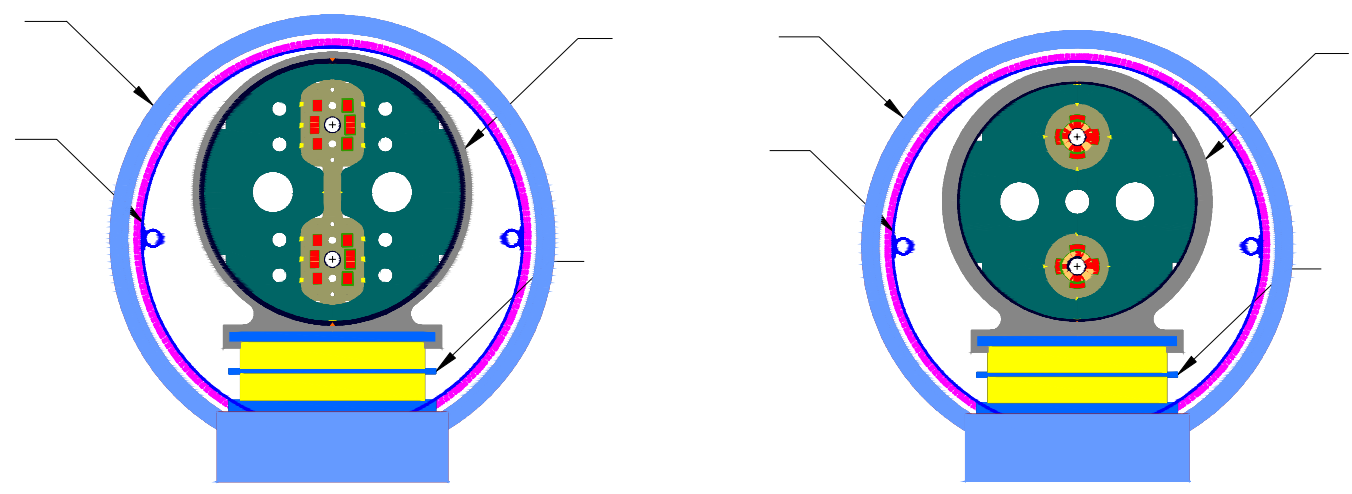

Figure 6.12. Stage-2 arc dipole (left) and arc quad (right) cryostat cross section: 1 -vacuum vessel; 2 - $80 \mathrm{~K}$ shield; 3 -cold mass; 4 -support post.

Table 6.8. Estimated static heat loads $(\mathrm{W} / \mathrm{m})$.

\begin{tabular}{|l|c|c|}
\hline Temperature & $80 \mathrm{~K}$ & $4.5 \mathrm{~K}$ \\
\hline Support conduction & 1.5 & 0.3 \\
\hline Radiation and gas conduction & 2.7 & 0.2 \\
\hline Total & 4.2 & 0.5 \\
\hline
\end{tabular}

Table 6.8 summarizes thermal design criteria for the Stage-2 arc magnet cryostats. The design shipping and handling load is $1 \mathrm{~g}, 2 \mathrm{~g}$ and $0.1 \mathrm{~g}$ in lateral, vertical and axial directions respectively. Magnet alignment criteria are similar to HERA, RHIC and LHC magnets.

The vacuum vessel contains the insulating vacuum, supports all other systems and serves also as a pressure containment vessel in the event of a failure in an internal cryogen line. The vessel is $914 \mathrm{~mm}$ OD with $12 \mathrm{~mm}$ wall. The length of the vacuum vessels will be determined as the lattice and interconnect requirements are finalized.

The cryostats have a single thermal shield cooled by He gas nominally at $80 \mathrm{~K}$. The shields intercept heat radiated from the $300 \mathrm{~K}$ surface of the vacuum vessel and conducted through the support system. Aluminum extrusions serve as the supply and return for the shield flow. The shield itself is attached to the cryostat at the support structure. To minimize thermal gradients around the circumference the shell must have high thermal conductivity. Thermal analyses were performed using $\mathrm{Cu}$ and $\mathrm{Al}$. Based on thermal analysis results and cost considerations the selected material is 6061-T6 Al, $3.175 \mathrm{~mm}$ thick. The shields are segmented along the length to minimize the effect of distortions during cooldown caused by the asymmetric cooling of the supply tubes. The shield extrusions are anchored at one of the magnet supports and are free to slide axially at the other to allow for thermal contraction.

The cold mass is covered by a single blanket of 10 layers of double aluminized Mylar separated by Nylon spacer material. The blankets are secured using Velcro strips. Similarly, two 
blankets of 15 layers each will cover the $80 \mathrm{~K}$ shield. Two blankets are used to stagger the longitudinal seams.

Table 6.9 lists the sizes for each of the cryostat pipes. The pressure and flow parameters are reported in Section 6.2.1.

Table 6.9. Cryostat pipe sizes.

\begin{tabular}{|l|c|c|c|l|}
\hline Description & OD $(\mathrm{mm})$ & ID $(\mathrm{mm})$ & Thickness $(\mathrm{mm})$ & Notes \\
\hline Vacuum vessel & 914.0 & 890.0 & 12.0 & Carbon steel \\
\hline Dipole cold mass & 580.0 & 560.0 & 10.0 & Including outer shell \\
\hline Quadrupole cold mass & 542.0 & 530.0 & 6.0 & Including outer shell \\
\hline 4.5 K supply & 88.9 & 85.6 & 1.651 & 2 at each end dome \\
\hline 80 K shield supply & 38.1 & 31.8 & 3.175 & Aluminum extrusion \\
\hline 80 K shield shell & 830.0 & 823.7 & 3.175 & Aluminum shell \\
\hline
\end{tabular}

The suspension system serves as the structural attachment for all cryostat systems to the vacuum vessel, which in turn anchors them to the support platform. The design emphasis was on meeting the allowed suspension system conduction heat load, satisfying the structural requirements, and maximizing the suspension stiffness. The conceptual designs for the Stage- 2 arc dipoles and quadrupoles uses support posts similar to those developed for SSC dipole cryostats and to those employed in LHC arc dipoles and quadrupoles. These supports are easy to manufacture, very strong and provide good thermal resistance to minimize heat load to the cold mass. Figure 6.12 conceptually illustrates cross-sections that utilize this support.

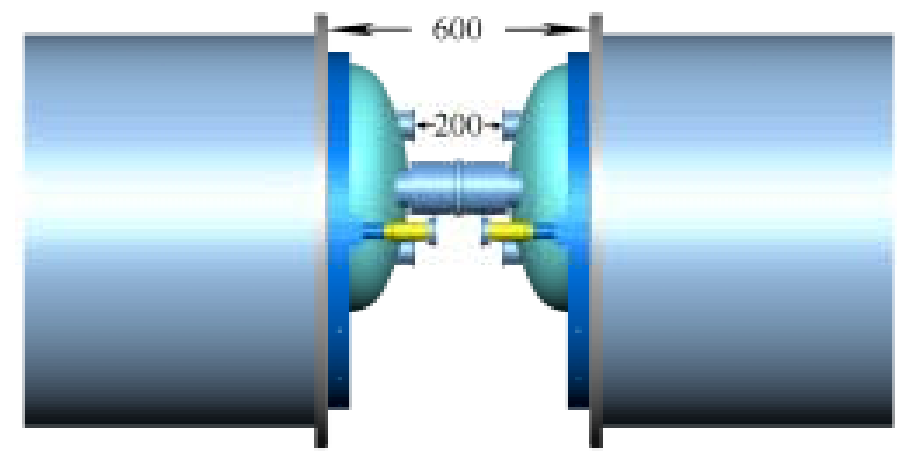

Figure 6.13. Dipole cryostat interconnect.
Figure 6.13 illustrates the interconnect of two arc or DS dipoles. The beam screen and electrical bus connections are not shown. One of the challenges of the interconnect is the beam screen connections between magnets. The details of these connections are unclear at this time, but necessitate connection of large number of small tubes carrying cold He gas at high pressure. Each pipe is anchored axially near the center of the magnet assembly.

In the case of a 17-m long dipole this means the interconnect bellows need to accommodate approximately $50 \mathrm{~mm}$ of axial cooldown contraction for stainless steel assemblies like the cold mass and $70 \mathrm{~mm}$ for aluminum assemblies like the thermal shield. Bellows are required on each cold mass, shield, and cryogenic pipe to allow for this expansion. Bellows are either hydroformed or welded stainless steel. Generally hydroformed bellows are used except where electrical connections requiring greater access are made up between magnets. In these locations welded bellows offer greater flexibility. For the aluminum extrusions on the shield, aluminum to stainless steel transition joints are required for the bellows connection. Lateral instability is a concern for interconnect bellows and requires the use of internal squirm protectors on each bellows assembly. 
Shield bridges that span the gap between adjacent magnets minimize the radiative heat transfer in the interconnect area. These bridges are extensions of the magnet shields, modified to contain their respective bellow ODs if necessary. A sliding joint between bridge sections on adjacent magnets accommodates contraction during cooldown. Each is covered with the same multi-layer insulation (MLI) used throughout the body of the magnet. Each shield bridge also has a pressure relief to prevent pressure buildup in the event of an internal piping failure. They are located in the upper half of the shield sections in order to prevent liquid spills from impinging directly onto the vacuum vessel wall.

A spool piece is required at the end of each arc quadrupole to house the corrector packages, provide access to the cryogenic transfer lines, and a variety of other service-related functions. A preliminary list of spool requirements is shown below:

- Corrector package - 2 in each spool piece

- Corrector leads - 2 pairs of 100 A HTS leads per corrector

- Beam separation same as dipole and quadrupole after cooldown

- Vacuum break - every other spool

- Cross connection to transfer lines and warm pipes

- Vacuum pumpout ports - insulating and beam vacuums

- Vacuum gauge connections

- Instrumentation - thermometers for LHe and shield, pressure taps

- One $90 \mathrm{~mm}$ LHe pressure relief

- Vacuum relief

- HTS dipole $(25 \mathrm{kA})$ and quadrupole $(30 \mathrm{kA})$ power leads.

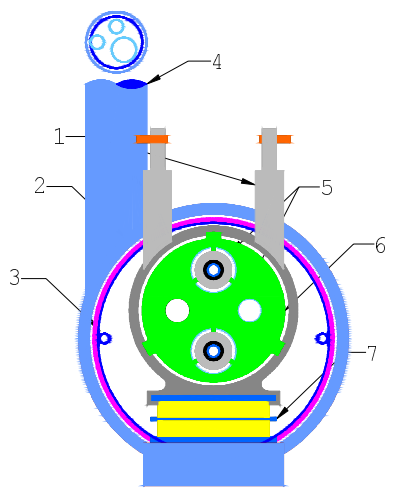

Figure 6.14. Spool piece cross-section. It consists of 4 pairs of HTS current leads (1), vacuum vessel (2), $80 \mathrm{~K}$ shield(3), connection to transfer line (4), corrector packages (5), cold mass support spider (6), and support post (7).
The spool piece cross-section is shown in Figure 6.14. The nominal length of a normal spool is $2.5-3 \mathrm{~m}$. Spools will exist in several varieties, e.g. with and without vacuum breaks, with and without high-current dipole and quadrupole leads, etc. However, they will share many common design features with other spools and with the arc magnets. A preliminary design goal is to utilize as many features of the dipoles and quadrupoles as possible. The preliminary design shown in Figure 6.14 utilizes the same vacuum vessel and shield cross sections and the same support geometry as the arc magnets. 


\subsubsection{Magnet Quench Protection}

The large energy stored in SC magnets is dissipated after quench in the normal zones, heating the coils and producing a voltage drop between the turns and between the coil and ground. Quench heaters installed in the magnet help to distribute this energy deposition throughout the coil, protecting it from overheating and preventing insulation damage from the high voltage and thermo-mechanical stress.

The maximum temperature and voltage were arbitrarily set to $300-400 \mathrm{~K}$ (similar to the LHC): $1000 \mathrm{~V}$ to ground and $100 \mathrm{~V}$ turn to turn. The high current density in the Cu-matrix of the conductors, requires effective quench detection, quick heater operation and a fast current decay in order to keep peak temperatures below the limit. The quench protection analysis is based on a quench detection time of $5 \mathrm{~ms}$ and a heater delay time of $35 \mathrm{~ms}$. To provide such a short delay time the heaters are placed in the high field regions and cover almost $100 \%$ of the coil. The results of quench protection calculations for the arc magnets are summarized in Table 6.10; the details are reported in $[25,26]$.

Table 6.10. Calculated quench characteristics.

\begin{tabular}{|l|c|c|}
\hline \multicolumn{1}{|c|}{ Parameter } & Arc Dipole & Arc Quad \\
\hline $\mathrm{QI}(300 \mathrm{~K}), \mathrm{MA}^{2} \mathrm{~s}$ & 42 & 40 \\
\hline $\mathrm{QI}(40 \mathrm{~ms}), \mathrm{MA}^{2} \mathrm{~s}$ & 22 & 30 \\
\hline $\mathrm{T}_{\text {peak }}, \mathrm{K}$ & 350 & 400 \\
\hline $\mathrm{T}_{\text {bulk }}, \mathrm{K}$ & 125 & 100 \\
\hline $\mathrm{V}_{\mathrm{cg}}, \mathrm{V}$ & 240 & 125 \\
\hline $\mathrm{V}_{\mathrm{tt}}, \mathrm{V}$ & 40 & 63 \\
\hline Decay time $\tau, \mathrm{ms}$ & 106 & 80 \\
\hline
\end{tabular}

The heaters used in Stage-2 magnets are similar to the LHC dipole heaters [27]. One heater for the arc dipole consists of two stainless steel strips $15 \mathrm{~mm}$ wide, $25 \mu \mathrm{m}$ thick, and $17 \mathrm{~m}$ long connected in parallel. One heater for the quadrupole consists of two 15-mm wide, 25- $\mu \mathrm{m}$ thick, and 10-m long stainless steel strips connected in series. To reduce the maximum voltage applied to quench heaters, they are periodically covered along their length with copper layers. The stainless to copper ratio is 1:3 (1 regular heater segment of the twist pitch length followed by a copper coated section of 3 twist pitch lengths). In the dipole case, 8 heaters are placed on the inner edge of the coil. In the quadrupole case, 4 heaters are placed between the two layers.

Table 6.11. Heater power supply parameters.

\begin{tabular}{|l|c|c|}
\hline \multicolumn{1}{|c|}{ Parameter } & Arc Dipole & Arc Quad \\
\hline $\mathrm{C}_{\text {tot }}, \mathrm{mF}$ & 19 & 9.4 \\
\hline $\mathrm{U}_{\text {tot }}, \mathrm{V}$ & 1000 & 1000 \\
\hline $\mathrm{U}_{\mathrm{op}}, \mathrm{V}$ & 700 & 700 \\
\hline $\mathrm{E}_{\mathrm{op}}, \mathrm{kJ}$ & 4.6 & 2.3 \\
\hline Decay time, $\mathrm{s}$ & 0.052 & 0.058 \\
\hline Peak current, $\mathrm{A}$ & 252 & 113 \\
\hline Peak power, $\mathrm{W} / \mathrm{cm}^{2}$ & 138 & 111 \\
\hline
\end{tabular}

Heater power supply parameters are given in Table 6.11. Each power supply for the dipole heaters consists of 16 capacitors (4.7 mF, $500 \mathrm{~V}$ each); 8 sets of 2 series capacitors are connected in parallel. Each power supply for the quad heaters consists of 8 capacitors $(4.7 \mathrm{mF}, 500 \mathrm{~V}$ each); 4 sets of 2 series capacitors are connected in parallel. 
For a total insulation thickness of $0.5 \mathrm{~mm}$ between the heater and the cable, the heater heats the cable edge to $11.5 \mathrm{~K}$ after $35 \mathrm{~ms}$ [27]. At currents below the nominal, the heater delay time for a given peak temperature in the magnet increases such that the same heater will be able to induce a quench. The total number of capacitors for the arc dipole protection hardware is 128 or 2.5 times the amount of capacitors in the LHC. In the case of the arc quadrupole 64 capacitors are required.

Dipoles and quadrupoles in dispersion suppressor cells have the same cross section as arc dipoles and quadrupoles but slightly different length. Analysis shows [25] that the arc magnets can operate within the above limitations for a length up to $20 \mathrm{~m}$. Based on that, quench protection of dipoles and quads in dispersion suppressor cells will be provided by the same method as for arc magnets.

Since corrector magnets will be powered from individual power supplies their protection will be provided by using low voltage power supplies with internal dump resistors. The quench protection scheme for the IR magnets will be developed later when IR magnet design and parameters are determined.

\subsubsection{Magnet Production and Testing}

Stage 2 will require $\sim 11,400$ (+ spare) double aperture dipoles, $\sim 1700$ (+ spare) double aperture quadrupoles, and about 1700 spool pieces with $\sim 11,000-13,000(+$ spare) correctors of different types. To be able to fabricate all these magnets in 5 years the average production rate must be at least 3000 magnets per year or 8-12 magnets per day. This is an order of magnitude higher than the planned production rate for the LHC (and also for HERA and RHIC). The high production rate will require participation in the Stage-2 magnet production of many large, medium and small industrial companies.

The large number of superconducting magnets will require procurement of large amounts of superconductor and structural materials. The dipole magnets will require $\sim 50,000 \mathrm{~km}$ of cable or $\sim 3,300,000 \mathrm{~km}(9,500$ metric tons $)$ of $0.7-\mathrm{mm} \mathrm{Nb}_{3} \mathrm{Sn}$ strand. The quadrupole magnets will require $\sim 10,000 \mathrm{~km}$ of cable or $\sim 300,000 \mathrm{~km}\left(1,300\right.$ metric tons) of $1-\mathrm{mm} \mathrm{Nb}_{3} \mathrm{Sn}$ strand. Different magnet designs utilize $\sim 45,000$ tons of stainless steel (w/o cryogenics) and $\sim 350,000$ tons of iron (including cryostat). The superconductor volume (or cost) is thus the major parameter that will affect the cost and schedule of magnet and accelerator construction.

Before installation, all magnets go through quality control procedures, production tests and pre-installation tests. Production tests in industry include:

- magnetic and electrical measurements of all collared coils at room temperature

- magnetic and electrical measurements of all cold masses at room temperature

- final warm magnetic, electrical measurements, vacuum and pressure tests, geometry control of all fully assembled magnets in cryostats at room temperature

Pre-installation tests at Fermilab include control geometry, magnetic and electrical parameters after transportation, and magnet cold tests.

Standard measurement techniques and equipment will be used for the production and pre-installation tests. To provide these tests at an acceptable rate, both the magnet production factories 
and the VLHC Laboratory have to be equipped with precise measurement apparatus and skilled personnel consistent with the machine construction schedule. To test $\sim 13,000$ dipoles and quadrupoles during 5 years will require an average test rate of 2600 magnets per year. Assuming a turn-around time per test bench and per double aperture magnet of $200 \mathrm{~h}$ (as for LHC) and 7200 working hours (300 days) per year, 73 cold test stands will be required. This number is a factor of 4.5 larger than expected for LHC magnet tests. The number of cold test stands could be reduced if a high reproducibility of main magnet parameters is achieved. Then only $(\sim 10-20 \%)$ of the magnets will have to be cold tested.

\subsubsection{Magnet Installation, Including Survey and Alignment}

The magnet installation procedure includes magnet transportation from storage to final position in tunnel, magnet placement and alignment, interconnection of individual magnets, vacuum and electrical tests, and survey. Installation of the large number of magnets requires systems and techniques that minimize cost and labor while preserving high accuracy and reliability. Taking into account that sizes and weights of the Stage- 2 magnets are close to those of LHC magnets, and that the VLHC tunnel cross section is similar to LHC, the installation procedures, machines and tools similar to LHC will be used for Stage 2.

In order to reduce the time and cost of magnet installation, removal procedures and installation tests, significant attention at this stage was concentrated on the simplification of the magnet interconnect design, especially the optimization of the number of splices as well as the number and parameters of interconnecting pipes.

\subsection{Accelerator Systems}

There are many systems issues which a complete Stage- 2 design needs to consider, including RF, cryogenics, vacuum, power supplies, instrumentation, feedback and controls, operational scenarios and safety systems. For this report, we report on studies performed on three key systems: cryogenics, discussed in Section 6.2.1; vacuum, including details of the beam-screen and photonstops, described in Section 6.2.2; and the final section discusses magnet power supply and quench protection issues.

\subsubsection{Stage-2 Cryogenic System Concept}

\subsubsection{Introduction}

This study of possible high field VLHC cryogenic systems relies heavily on previous reports about VLHC cryogenics by M. McAshan and P. O. Mazur [28,29,30]. Comparisons are frequently made with LHC parameters not only because it is the most recent large accelerator cryogenic system design, but because the thorough analyses done in support of the LHC cryogenic system provide an excellent baseline for comparison. One of the most extensively used LHC documents was Ref. [31]. 


\subsubsection{System Layout}

The high field VLHC design effort will use 12 cryogenic plant locations as the working assumption for the cryogenic system layout. Such an arrangement creates 24 cryogenic strings of approximately $9.7 \mathrm{~km}$ length each.

Tom Peterson

19 March 2001

High Field VLHC cell flow concept with S. Zlobin electrical scheme
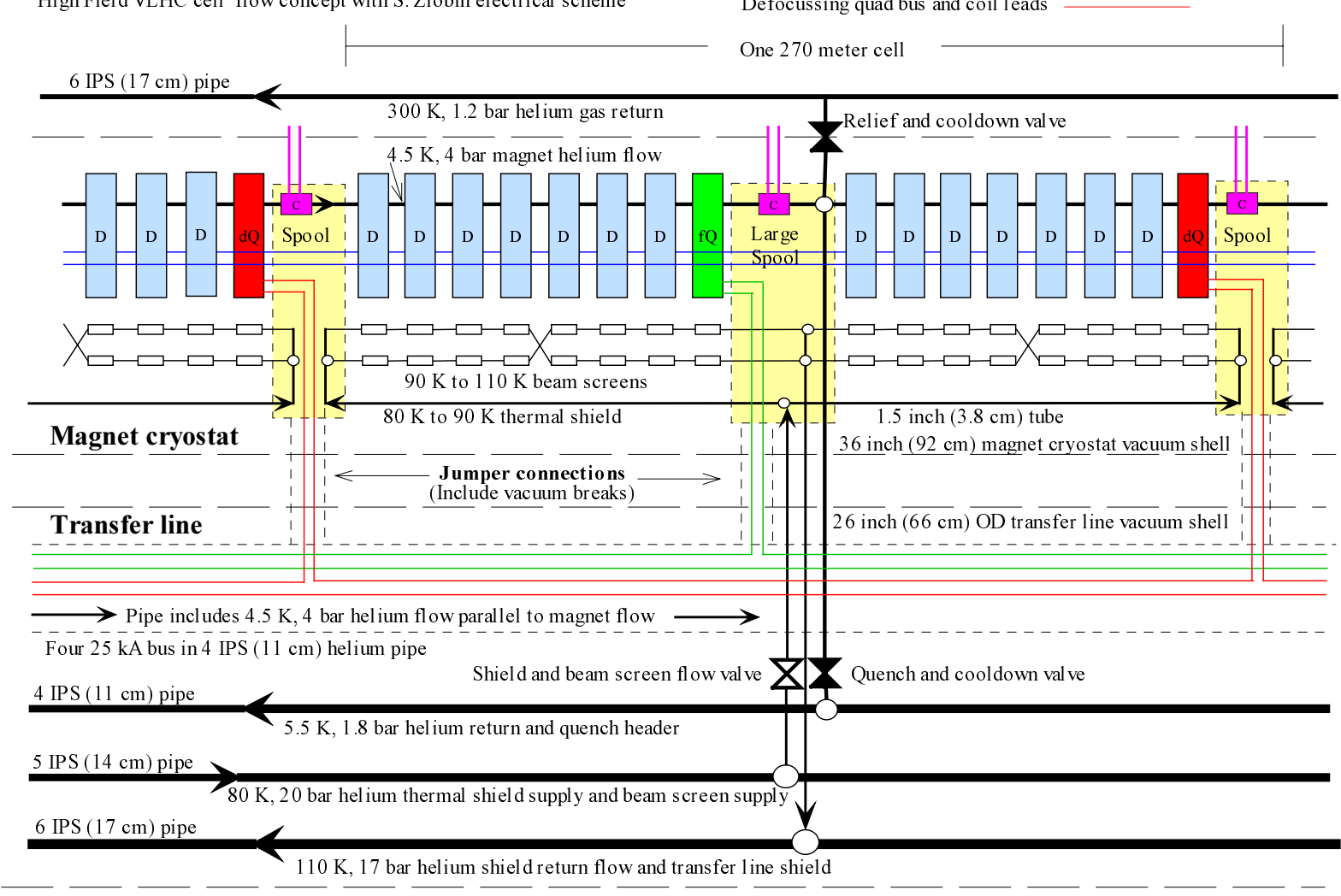

Figure 6.15. VLHC high field cryogenic schematic for one cell.

Shown in Figure 6.15 is a concept for a flow and electrical bus schematic for one cell of the high field VLHC. We avoid the use of recoolers or two-phase helium by allowing a $1 \mathrm{~K}$ rise in the helium flow through the magnet cold mass. Magnets are cooled by a large flow rate of supercritical, $4.5 \mathrm{~K}$ helium.

A nominally $80 \mathrm{~K}$ to $110 \mathrm{~K}$ helium stream cools the thermal shields and beam screens. High pressure (20 bar) helium is selected for the shield and beam screen since the higher pressure and density permit more mass flow in a given pipe size. Nevertheless, pressure drops for the $80 \mathrm{~K}$ to $110 \mathrm{~K}$ flow should be small so that most of the pressure remains available for expander work upon returning to the cold box.

This flow scheme provides four parallel beam screen cooling flow paths in each cell, two in each 135 meter long half-cell. A crossover is shown in each half-cell. The alternative to the crossover is balancing valves controlled by temperature sensors in the spool piece. Depending on how the beam screen passes through the interconnect, valves might be less costly than the crossover, but for now we will show the crossover. 
This scheme puts all the large cold pipes and most of the electrical bus in a separate transfer line, so one does not have to deal with them at each magnet interconnect. It requires one jumper from the transfer line to the magnet string every $270 \mathrm{~m}$ for helium pipe connections, and one jumper every half-cell, or every $135 \mathrm{~m}$, for electrical bus connections. Jumper connections containing electrical bus will require flow plugs to prevent helium flow short-circuits. The helium pipe containing four $25 \mathrm{kA}$ bus may be cooled with a fraction of the $5.5 \mathrm{~K}, 1.8$ bar return flow or with a parallel supply flow of $4.5 \mathrm{~K}, 4$ bar helium. A $300 \mathrm{~K}$ helium gas return pipe is shown for lead flows and relief valve flow collection. A $300 \mathrm{~K}, 20 \mathrm{bar}$, helium gas line (not shown in Figure 6.15) might be required for inventory management.

The quench valve spacing of one every 270 meters appears to be adequate based on an analysis of quenching in $25 \mathrm{kA} \mathrm{Nb}_{3} \mathrm{Sn}$ magnets [32]. Large quench valves would be required, with a port size of at least $60 \mathrm{~cm}$, but quench pressures would remain under 20 bar.

All of the lines utilize helium as the coolant. Our initial decision is not to use nitrogen in the tunnel, but one might make good use of it above ground for cool-down and load leveling. Although it presents safety problems for the tunnel, one might reconsider the use of nitrogen in the tunnel in the thermal shields and/or for recooling helium from the beam screens, in order to reduce the huge helium inventory of this system.

\subsubsection{Estimated Heat Loads}

The heat load numbers in Table 6.12 are scaled from LHC heat loads as reported in Reference [31]. A thermal model $[33,34,35]$ scales static heat loads to the thermal shields and cold mass for VLHC based on LHC experience. The synchrotron radiation heat load estimate comes from beam dynamics studies (Section 6.2.2 and Reference [38]). An analysis finding optimum beam screen temperature is also reported in [38].

The heat load to the thermal shield of the arc magnets in LHC at nominally $60 \mathrm{~K}$ is $3.7 \mathrm{~W} / \mathrm{m}$. Scaling to our high-field $\mathrm{Nb}_{3} \mathrm{Sn}$ magnet concept, we find $4.2 \mathrm{~W} / \mathrm{m}$ as the predicted static heat load to the VLHC magnet thermal shield. To the LHC transfer line at $60 \mathrm{~K}$, the heat load is 2.7 $\mathrm{W} / \mathrm{m}$. Our VLHC transfer line is smaller in diameter than the LHC transfer line by 0.80 , so we use $0.80 \times 2.7=2.2 \mathrm{~W} / \mathrm{m}$ to the transfer line thermal shield.

For the magnet cold mass heat load estimate, static and dynamic heat loads for LHC scale up with our higher current and higher luminosity in VLHC, adding $0.15 \mathrm{~W} / \mathrm{m}$ to the $0.35 \mathrm{~W} / \mathrm{m} \mathrm{LHC}$ number, yielding $0.50 \mathrm{~W} / \mathrm{m}$ for VLHC. In addition, we have a warmer beam screen than LHC, adding another $0.33 \mathrm{~W} / \mathrm{m}$ (total for two beams), for a total of $0.83 \mathrm{~W} / \mathrm{m}$.

To the beam screen we estimate $10 \mathrm{~W} / \mathrm{m}$ total for both beams due primarily to synchrotron radiation. Image current heating and multipacting are negligible compared to the $5 \mathrm{~W} / \mathrm{m} / \mathrm{beam}$ synchrotron radiation. As indicated in Figure 6.15 and in Table 6.12, the beam screen operational temperature in this flow scheme is between $87-107 \mathrm{~K}$, at the predicted optimum range. Studies [35] have shown the optimal magnet thermal shield temperature (in terms of minimizing overall refrigeration power) to be about $63 \mathrm{~K}$, consistent with the LHC selection of thermal shield temperature. In order to reduce the number of pipes through this VLHC system, the thermal shield and beam screen are placed in series with the thermal shield first. The high heat load on the beam screen results in its optimum temperature dominating that for the screen and thermal 
shield combined. Thus, the optimum thermal shield average temperature when in series with the beam screen is $83 \mathrm{~K}$.

Table 6.12. Predicted heat loads and power requirements.

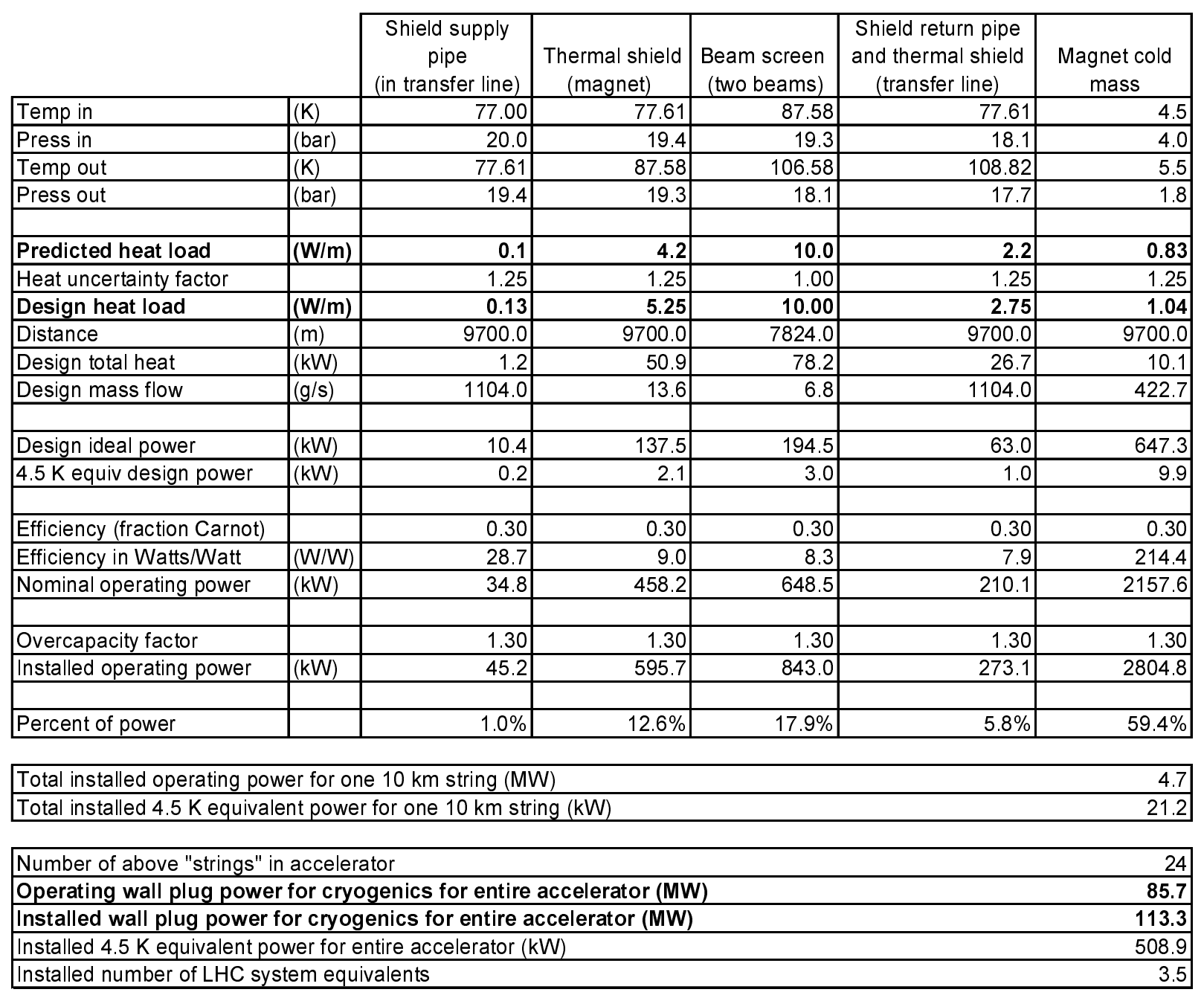

\subsubsection{Current Leads}

There is one $25-\mathrm{kA}$ dipole circuit per $10-\mathrm{km}$ string and two $25-\mathrm{kA}$ quadrupole circuits per $20 \mathrm{~km}$. The 25-kA dipole circuit requires a pair of current leads at each end of the $10-\mathrm{km}$ string. Thus, each cryo plant location has eight $25-\mathrm{kA}$ leads and each $10-\mathrm{km}$ turnaround point has 4 leads. We assume six 100 A corrector leads per beam per spool piece, with about 75 spools in one $10-\mathrm{km}$ sector, for a total of 900 corrector leads per sector. LHC current lead test data [36] and Fermilab HTS lead test data [37] were used to scale cooling requirements for Stage-2 current leads. The relatively low number of high-current leads and the use of HTS leads make the total cooling power for the current leads only about $4 \%$ of the cryogenic system power. Lead details are not shown in Table 6.12, but lead cooling power is included in the totals in the table. 


\subsubsection{Cooling Power Requirements and Plant Sizing}

Table 6.12 shows that the heat load estimates and the $233-\mathrm{km}$ circumference of the VLHC give rise to a system requiring about $113 \mathrm{MW}$ of installed cooling power, equivalent to about two large cryoplants at each of the 12 locations. Such a system would total about 3.5 times the cooling capacity of the LHC cryogenic system. Note the applied factors: the uncertainty factor of 1.25 on heat loads other than the beam screen, the Carnot efficiency of 0.30 which is high but achievable with today's technology (LHC reports 0.3 for their helium systems), and the overcapacity factor of 1.3. Overcapacity is the cooling capacity reserve required for availability, maintenance, control, and non-optimal operation. LHC uses a factor of 1.5. We have reduced that to 1.3 in light of the large proportion of heat load due to synchrotron radiation, over which we have control.

\subsubsection{Cool-Down and Warm-Up}

Cool-down times are estimated to be on the order of two months using liquefier capacity. Each $20 \mathrm{~km}$ of high field VLHC has roughly 35,000 tons of cold mass (about equal to LHC), requiring the removal of about $3 \times 10^{12}$ Joules to cool to $80 \mathrm{~K}$. Some reduction in time can be gained with the use of above-ground $\mathrm{LN}_{2}$ precooling, but extremely large amounts of $\mathrm{LN}_{2}$ storage would be required. The extremely large cold mass will force long cool-down and warm-up times. "Warm iron" magnets could offer significant advantages in reducing cool-down and warm-up times and hence system repair times and system availability.

\subsubsection{Helium Inventory}

The inventory for the entire machine is estimated to be 0.65 million $\mathrm{kg}$ or 5 million liquid liters equivalent. This is about 85 times the Tevatron inventory, 7 times the LHC inventory, and about 0.056 of the annual U.S. production of helium, costing about $\$ 12$ million. We found in this study that one of the problems with reducing the number of cryogenic plant sites from 12 to 6 is that doubling the lengths of cryogenic loops, along with doubling the mass flow to maintain the same exit temperature, requires significantly larger diameter pipes. As a result, the helium inventory for such a system is approximately twice this one, about 10 million liquid liters instead of 5 million liquid liters. Twenty-four $10 \mathrm{~km}$ magnet strings might not be precisely the optimum division of the Stage-2 cryogenic system, but we believe it is closer to the cost optimum than twelve $20-\mathrm{km}$ strings.

\subsubsection{Conclusions and Recommendations}

The large total system power, the magnet string lengths, total cold mass, and total helium inventory for a Stage- 2 cryogenic system go well beyond that of previous systems. This cryogenic system is technically feasible in that the required machinery assembled into smaller, similar systems exists. But system design and optimization on this scale will require significant effort and R\&D.

Recommendations for cryogenic system R\&D may be found in Section 10.2.4. One particular area of $\mathrm{R} \& \mathrm{D}$, removal of synchrotron radiation at room temperature, deserves special mention. At $\$ 0.114 / \mathrm{kWh}$, power would cost $\$ 1.00$ per Watt-year, so the "installed power" shown in MW could be approximately the annual power bill in M\$. Thus, one can see that it would be 
very desirable to reduce the installed power to significantly less than the presently estimated 113 MW. The heat load with the biggest potential for reduction by some innovative method is probably the synchrotron radiation. Developing methods for removing some significant fraction of the synchrotron radiation at room temperature could permit a dramatic reduction in cryogenic system size (both power and inventory) for a high field VLHC.

\subsubsection{Vacuum System}

\subsubsection{Synchrotron Radiation}

With a proton energy of $87.5 \mathrm{TeV}$ and a bending radius of $29.9 \mathrm{~km}$, the critical energy of the synchrotron radiation emitted by the protons is $8 \mathrm{keV}$, which corresponds to a wave-length of $\sim 1.6 \mathrm{C}$. At a peak current of $57.4 \mathrm{~mA}$ each beam releases $4.7 \mathrm{~W} / \mathrm{m}$ and $1.2 \times 10^{16}$ photons $/ \mathrm{sec} / \mathrm{m}$ in the bending magnets. The total synchrotron radiation power emitted by the two beams is $9.4 \mathrm{~W} / \mathrm{m}$. This, combined with additional heat load contributions such as resistive wall power loss and emittance growth-related loss due to multipactoring electrons and ion-induced desorption - estimated to total $<1 \mathrm{~W} / \mathrm{m} /$ beam, would be an excessive heat load for the cold mass. Thus, a beam-screen, maintained at a higher temperature by a flow of gaseous helium, is inserted inside the magnet cold bore to intercept this power.

The synchrotron radiation photons impinging on the beam-screen desorb gas molecules from the near surface layer and convert them into a steadily increasing surface density of loosely bound (physisorbed) states. These physisorbed surface molecules are easily redesorbed by the photons, deteriorating the quality of the vacuum in the beam chamber. Therefore, the beamscreen will be perforated over a few percent of its surface, to allow cryopumping to the $5 \mathrm{~K}$ cold bore surface, where the vapor pressure of all gases (except $\mathrm{H}_{2}$ and $\mathrm{He}$ ) is negligible and the condensed gas is shielded from synchrotron radiation and ion bombardment.

\subsubsection{Beam-Screen Cooling}

\subsection{Optimum Beam-Screen Temperature}

The cooling of the beam-screen is a key issue in the VLHC. The beam-screen refrigeration power requirement is the leading contributor to the total cryo-budget. The beam-screen refrigeration power requirement is a strongly varying function of the beam-screen operational temperature. The optimum beam-screen temperature balances between the heat load absorbed by the beam-screen refrigeration system and the heat load absorbed by the cold mass. At low beamscreen temperature, the heat load is absorbed mainly by the beam-screen, at low thermodynamic efficiency and thus at high cost. For a high beam-screen temperature, the cost of beam-screen refrigeration is reduced, but a significant part of the heat load is transferred from the beam-screen by conduction and radiation to the cold mass, where it is extracted with low thermodynamic efficiency. The thermodynamic optimum beam-screen temperature in Stage- 2 was calculated to be $86 \mathrm{~K}[38]$ for the case of $5 \mathrm{~W} / \mathrm{m} /$ beam.

The baseline Stage-2 cryo-concept (Section 6.2.1) foresees that the beam-screens are cooled in series with the cold-mass thermal shield by the same high pressure helium gas. Although both systems have different optimum operational temperatures when separated, the optimum (aver- 
age) operating temperature of the cold mass thermal shield in the combined system is $83 \mathrm{~K}$, and the ensuing average beam-screen temperature is $98 \mathrm{~K}$. At this temperature the beam-screen refrigeration system requires a total wall plug power of $15 \mathrm{MW}$ (both beams). In these conditions the heat load transferred from two beam-screens to the cold mass is $0.26 \mathrm{~W} / \mathrm{m}$ (conduction) and $0.07 \mathrm{~W} / \mathrm{m}$ (radiation). At a cold mass temperature of $5 \mathrm{~K}$, the evacuation of the heat load transferred from both beam-screens to the cold mass represents a wall plug power requirement of 14.4 MW for the complete ring.

\subsection{Concept for a Beam-Screen Cooling System}

Following a calculation procedure outlined in [38], the beam-screen cooling system requires $\sim 12 \%$ of the area enclosed by the cold bore (ID=34 mm), or $110 \mathrm{~mm}^{2}$ in cross-sectional area, to be taken up by the coolant at an average temperature of $100 \mathrm{~K}$. The coolant is high pressure gaseous helium, entering the beam-screen cooling system at $87 \mathrm{~K}, 19$ bar and exiting at $106 \mathrm{~K}$, 18 bar. The beam-screen cooling system length is assumed to be the length of a half-cell $(135 \mathrm{~m})$. The total flow rate per beam-screen is $6.6 \mathrm{~g} / \mathrm{s}$. In the calculations the stainless steel cooling duct wall thickness was assumed to be $0.5 \mathrm{~mm}$.

\subsubsection{Vacuum}

\subsection{Synchrotron Radiation}

The high photon flux is the dominant source of gas-desorption. On the other hand, it is very effective in cleaning the exposed surfaces via photo induced desorption. The use of superconducting magnet technology results in the amenity of a low cold bore temperature of $\sim 5 \mathrm{~K}$, at which most gases have a very low saturation vapor pressure. Therefore, most gases get trapped at the cold bore walls and remain there until the magnets are warmed up again to room temperature. The pumping capacity of such a cryo-pump is very large and compatible with the accelerator lifetime. In addition, the high beam-screen temperature decreases the rate of re-adsorption of gas on the beam-screen and thus facilitates the cleaning process. The higher saturation vapor pressure of $\mathrm{H}_{2}$ at $5 \mathrm{~K}$ on the other hand results in the limitation that $\mathrm{H}_{2}$ cannot be cryo-pumped beyond one monolayer. Therefore the cold bore will have to be warmed up to room temperature at regular intervals (some years) to allow regeneration of the cryo-pumping surfaces.

\subsection{Residual Gas Pressure}

The residual beam-tube pressure must be low enough to limit beam-gas scattering to a level at which the lifetime of the beam is not significantly affected, and at which the radiation into the magnet cold mass remains below a stipulated limit. Estimations of the bounds on the beam tube gas pressure for the most common UHV gases are listed in Table 6.13. The beam lifetime limiting pressure is calculated, stipulating that the beam-gas scattering lifetime be five times larger than the luminosity lifetime ( 7.5 hours). The beam lifetime reduction stemming from beam-gas scattering related emittance growth is neglected because of strong radiation damping. The limiting pressure to restrict the power radiated into the magnet cold mass due to beam-gas scattering is calculated, stipulating that the beam-gas scattering power be less than $0.1 \mathrm{~W} / \mathrm{m} /$ beam. According to the table, the limiting pressure in the VLHC has to be in the nTorr range for the most common UHV gases. 
Table 6.13. Numerical bounds on the beam tube gas pressure for the most common UHV gases in Stage-2, calculated for each gas "alone" at ambient room temperature equivalent pressure.

\begin{tabular}{|lcc|}
\hline Gas & $\begin{array}{c}\text { Max pressure for a beam gas scattering } \\
\text { lifetime of } 5 \times 7.5 \text { hours (nTorr) }\end{array}$ & $\begin{array}{c}\text { Max pressure for a beam gas scattering } \\
\text { power of } 0.1 \mathrm{~W} / \mathrm{m} / \text { beam (nTorr) }\end{array}$ \\
\hline $\mathrm{H}_{2}$ & 31.8 & 50.3 \\
$\mathrm{CH}_{4}$ & 5.9 & 9.3 \\
$\mathrm{H}_{2} \mathrm{O}$ & 5.5 & 8.7 \\
$\mathrm{CO}$ & 3.8 & 6 \\
$\mathrm{CO}_{2}$ & 2.4 & 3.8 \\
\hline
\end{tabular}

\subsection{Photo Desorption and Pumping Speed}

Photo-induced desorption due to synchrotron radiation with a critical energy of $8 \mathrm{keV}$ is the most important source of vacuum contamination in the Stage-2 VLHC. Calculations based on procedures formerly developed in [39], have recently [40] been performed to estimate the quantity of gas desorbed by synchrotron radiation from a copper coated beam-screen. Copper can be prepared (baked) to have a small initial photo-desorption coefficient $\eta_{0}$ (e.g. $\sim 0.0035 \mathrm{H}_{2}$ molecules per photon) which offers the advantage of a low secondary electron emission coefficient (reducing the electron cloud effect). It is believed that the initial photo-desorption coefficient does not increase further with the critical energy of the photons, above a few $\mathrm{keV}$, a range for which reliable measurements exist [41].

The photo-desorbed gas load has to be matched by a pumping speed to remain within the specified pressure bounds. The $\mathrm{CO}$ equivalent pumping speed required to keep the heat dissipation in the magnet below $0.1 \mathrm{~W} / \mathrm{m}$ after one third of an operational year ( $\mathrm{I} \cdot \mathrm{t}=48$ A-hours) is 5.6 lit/sec-m. For $\mathrm{H}_{2}$ this pumping speed is $21 \mathrm{lit} / \mathrm{sec}-\mathrm{m}$. During the commissioning phase of the machine the photo-desorption rate is larger, requiring a larger pumping speed and/or a smaller beam current. A possible commissioning beam- current profile (for a $\mathrm{CO}$ equivalent pumping speed of $61 \mathrm{l} / \mathrm{sec}-\mathrm{m}$ ) was proposed [40]. The current in the profile is calculated for the condition that the beam-gas scattering power always remains below $0.1 \mathrm{~W} / \mathrm{m}$. It consists of three 15 hour cycles with initial currents of $30 \mathrm{~mA}, 38 \mathrm{~mA}$ and $54 \mathrm{~mA}$. The total conditioning time is thus 45 hours.

\subsection{Cryo-Pumping}

Due to its low saturation vapor pressure, $\mathrm{H}_{2}$ cannot be cryo-sorbed on a $5 \mathrm{~K}$ surface beyond one monolayer $\left(\sim 3 \times 10^{15} \mathrm{H}_{2} / \mathrm{cm}^{2}\right)$. Pumping speeds of $61 \mathrm{lit} / \mathrm{sec}-\mathrm{m}$, as required for conditioning (see above), can be achieved through cryo-pumping the gas onto the cold $(5 \mathrm{~K})$ magnet bore. The beam-screen surface fraction occupied by pumping slots to obtain $60 \mathrm{lit} / \mathrm{sec}-\mathrm{m}$ is $\sim 1.5 \%$. The integrated gas-load set free by the synchrotron photons during a 20 year accelerator operation is $\sim 1.1 \times 10^{19} \mathrm{H}_{2}$ molecules/m. This represents 3.3 mono-layers of $\mathrm{H}_{2}$. This gas-load can be managed by periodic warm-up of the machine to allow regeneration of the cryo-pumping surfaces. 


\subsubsection{Conceptual Beam-Screen Design}

We propose a vacuum system design (Figure 6.16), similar to the LHC design [42], using a perforated liner operating at $\sim 100 \mathrm{~K}$. Beam-stability issues related to the "higher" temperature of the beam-screen are being investigated. The beam-screen is a 1-mm thick non-magnetic stainless steel $\left(\mu_{\mathrm{r}}<1.005\right)$ tube with a round cross-section, flattened top and bottom. The space between it and the surrounding cold bore contains the cooling pipes carrying the forced flow supercritical helium. The pipes, e.g., made of stainless steel, are laser welded to the beam-screen. The beamscreen is centered within the cold bore by steel/bronze rings, every $0.5 \mathrm{~m}$. The assumed magnet bore ID is $40 \mathrm{~mm}$. A 1.5-mm gap is needed to allow the insertion of the cold bore into the magnet. Then, to withstand the quench-pressure, the cold bore tube needs a wall thickness of $1.5 \mathrm{~mm}$, hence the cold bore inner diameter becomes $34 \mathrm{~mm}$. The supports require an additional $0.75-\mathrm{mm}$ gap between the cold bore and the beam-screen. The remaining aperture for the beamscreen becomes $32.5 \mathrm{~mm}$. The inner dimensions of the beam-screen are $30.5 \mathrm{~mm}$ in width and $20 \mathrm{~mm}$ in height.

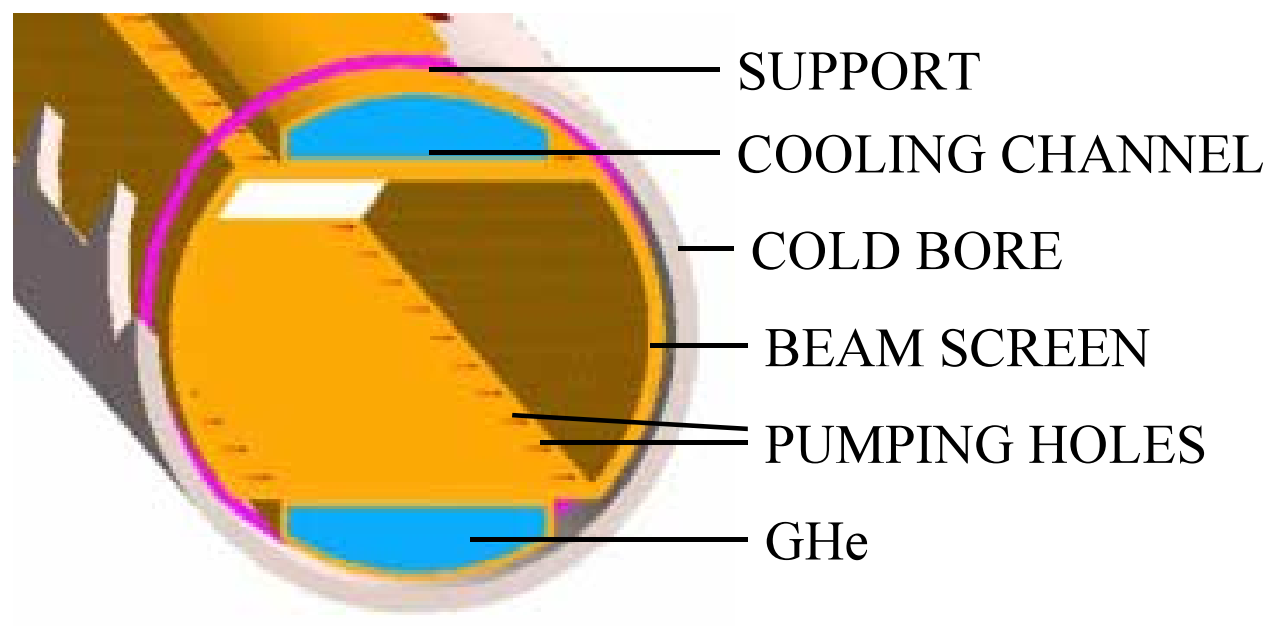

Figure 6.16. A schematic view of the beam-screen showing supports, cooling pipes and pumping slots.

To minimize resistive wall heating and longitudinal beam impedance the interior surface of the screen is covered by a thin co-laminated layer of high purity copper. The pumping slots are rounded (1.5 $\mathrm{mm}$ wide, $8 \mathrm{~mm}$ long)) and randomly distributed to reduce beam-coupling impedance and resonance effects. To obtain a pumping speed of $60 \mathrm{lit} / \mathrm{sec}-\mathrm{m}, 120$ holes are required per meter of beam-screen (e.g. 4 rows of 30 holes, one hole every $5 \mathrm{~cm}$ ). As in the case of LHC [42] the beam-screen is fabricated from sheet metal with stamped cooling slots. It is cold-rolled with the copper foil and cold-pressed into the shape of a half tube, which is then welded together. To eliminate the effects of heat load variations between the apertures, the cooling tubes should cross-over to the other aperture at every half cell (as proposed in Section 6.2.1). 


\subsubsection{Photon-Stops}

\subsection{Justification}

The strong synchrotron radiation in Stage-2 results in a refrigeration power requirement of $\sim 25 \mathrm{MW}$. Intercepted at room temperature, the synchrotron radiation heat load would represent a reduced power at the plug of $\sim 1.9 \mathrm{MW}$. Motivated by the considerable cost saving potential, the use of room temperature operated photon-stops, which are commonly used in synchrotron light sources [43], is under evaluation. Photon-stops are devices that protrude into the beam tube at the end of each bending magnet and scrape off the synchrotron light beam emitted in the second magnet up-stream from their location. A preliminary feasibility study was recently performed [44], showing that, given the arc bending radius in the VLHC of $29.9 \mathrm{~km}$, it is possible to place photon-stops between the magnets with a length and aperture compatible with the current VLHC design. We believe that synchrotron radiation in the VLHC is a sufficiently serious issue to justify the use of both a traditional beam-screen as well as photon-stops. In the case that the photon-stops are retractable, the synchrotron heat load can be shared in a controlled way between both systems which would allow operation of the accelerator at energies and luminosities above nominal.

\subsection{Geometrical Issues}

A geometrical model was based on the assumptions that 1) the bending magnets are straight and positioned such that the average distance between the ideal particle orbit and the magnet center is always minimal, and 2) all particles are moving very close to the ideal orbit. The model estimates the maximum permissible magnet length and the minimum distance between the photon-stop and the beam. Given that the photon-stops are placed at the beginning of the 3-m long straight section between the magnets and a $30-\mathrm{mm}$ horizontal beam-screen aperture, the maximum permissible bending magnet length is $14 \mathrm{~m}$, which is compatible with the maximum magnet length imposed by quench protection and transportation constraints. In this condition, the synchrotron radiation emitted from the beginning of the second up-stream magnet will heat the photon-stop at its base (where it enters the beam tube) and the radiation emitted from the end of the same magnet will hit its tip. The SR emitted by the magnet up-stream from the photon-stop passes through the gap between the photon-stop tip and the beam. The emission angle of the SR is approximately $1 / \gamma \sim 10 \mu \mathrm{rad}$. The total radial extension of the photon-stop is $1 \mathrm{~cm}$, such that its (minimum) distance from the particle orbit becomes $\sim 5 \mathrm{~mm}$.

\subsection{Engineering Design}

Assuming that a single photon-stop intercepts the synchrotron radiation heat load emitted by one beam over the length of one magnet, the thermal load is given by $\sim 5 \mathrm{~W} / \mathrm{m}$ times the magnetlength of $70 \mathrm{~W}$. The required heat exchange surface is $\sim 1 \mathrm{~cm}^{2}$ at a coolant flux of $0.2 \mathrm{liter} / \mathrm{sec}$, given a cooling tube wall thickness of $1 \mathrm{~mm}$ and a cooling tube diameter of $10 \mathrm{~mm}$ [45]. The coolant assumed in this calculation is water at room temperature; the coolant temperature rise is $5 \mathrm{~K}$. The peak heat flux of $0.6 \mathrm{MW} / \mathrm{m}^{2}$ remains safely below the critical heat flux of forced flow water $\left(\sim 5 \mathrm{MW} / \mathrm{m}^{2}\right)$. The largest $\mathrm{SR}$ power fraction is transported by photons with a wavelength of $0.5 \mathrm{C}$. The radiation length of steel, used as a reference material in the thermal calculation, is $\sim 0.04 \mathrm{~g} / \mathrm{cm}^{2}$ or $0.05 \mathrm{~mm}$. The synchrotron radiation hits the absorber in a $\sim 0.5-\mathrm{mm}(\mathrm{rms})$ thin strip. The absorber surface material needs sufficient thermal conductivity and thickness to 
distribute the heat load over a larger area to dilute the surface heat flux density to the coolant. Heaters, wrapped around the cooling tubes, are required to prevent the cooling liquid from freezing when no synchrotron radiation heat load is present. A preliminary engineering design that satisfies these thermal considerations was presented [45].

According to this design, the photon-stop is a T-shaped piece, consisting of a $\sim 6 \mathrm{~cm}$ short copper tube shaped like the beam-screen (but without pumping slots) with flanges at the ends, to which a $\sim 0.5 \mathrm{~m}$ long warm finger, the photon-stop, is attached perpendicularly. The photon-stop enters the beam-screen from the side, where it is hit by the synchrotron radiation (Figure 6.17).

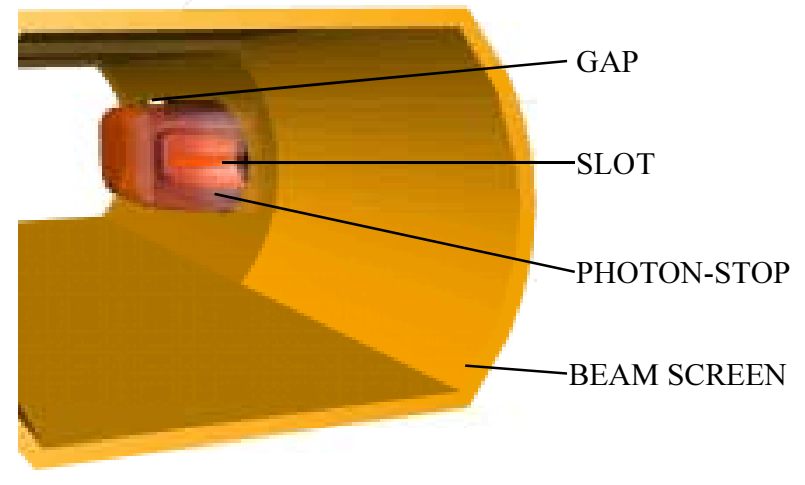

\section{Figure 6.17. Photon-stop sketch according to the engineering design proposal.}

The photon-stop assembly consists of an outer hull with flanges on top and bottom to fix it to the cryostat hull and the short beamscreen piece with vacuum tight seals. Into this hull the core of the photon-stop is inserted. The core piece consists of the cooling tubes with the radiation absorber at the end. The short beam-screen piece is inserted into a specially prepared gap in the beam-screen / cold-bore assembly and welded at each end. The cold-bore tube is discontinued over the $\sim 10 \mathrm{~cm}$ of the photon-stop to reduce the heat influx from the $100 \mathrm{~K}$ beam-screen / photonstop system.

The bellows, required to allow the beam tubes to contract during magnet cool-down, are placed in sections further away from the photon-stop. The beam-screen cooling tubes have to be guided out of the beam-screen to bypass the bellows, usually in the form of flexible tubes. For the photon-stop we propose that the cooling tubes also bypass the photon-stop system. Furthermore, it is assumed that the photon-stop will be placed close to the magnet anchoring point. This offers the advantage of not having to cope with longitudinal thermal contraction effects.

At the heart of the photon-stop is the radiation absorber, a hollow cylinder machined at one end into a special shape. The inside of the end piece is hollow to allow the cooling liquid to extract the heat deposited by the synchrotron radiation on its outer surface. The wall thickness of the absorber is $\sim 2 \mathrm{~mm}$. It is made from copper to obtain a low initial photo-desorption coefficient and high thermal conductivity. To reduce the thermal emissivity and the photo-desorption coefficient, the outer surface of the end piece requires a high grade polishing. The shape of the absorber was optimized to yield low impedance. It has the shape of a prism (Figure 6.17), with axial / radial / azimuthal extensions of $35 \times 10 \times 10 \mathrm{~mm}$ with respect to the beam tube, and rounded edges.

A major issue regarding the absorber is scattering of the low energy (long wavelength) spectrum of the SR. Measures aimed at the reduction of the scattered photon-fraction have to be pursued. One possibility, for example, is to make the absorber piece hollow, with an entry slot for the photons. For such a photon-catcher to be efficient, a more detailed knowledge of the geometrical characteristics of the photon beam is required. 


\subsection{Photon-Stop Impedance}

The longitudinal impedance of the photon-stop has to be small to 1) reduce the impedance related power loss per turn, and 2) avoid beam instabilities, such as the microwave instability. The transverse impedance has to remain below beam instability thresholds, such as for the transverse mode coupling instability (TMCI). A major issue is whether the photon-stops can be treated as independent. In the coupled case the sum of the impedances of all coupled devices would be the relevant parameter, and thus the impedance limitations would be more stringent. A first estimation of the coupling strength can be obtained from the damping length of the first order mode. The damping length was calculated [46] to be $4.2 \mathrm{~cm}$, which is sufficiently smaller than the projected distance between the photon-stops of $\sim 14 \mathrm{~m}$, such that coupling is suppressed.

The beam tube impedance of a copper coated, round beam tube (ID=30 mm) is of the order of a few ohms. The cut-off frequency of such a pipe is $2 \pi \cdot 7.6 \mathrm{GHz}$, which is larger than the largest characteristic beam frequency in the VLHC, the bunch-length frequency at collision of $2 \pi \cdot 5.8 \mathrm{GHz}$. A simple, analytical model to calculate the impedance of a "half ellipsoidal" protrusion in the beam pipe [47] predicts, for a total number of 14500 photon-stops and a longitudinal extension of the protrusion of $3.5 \mathrm{~cm}$ and a radial/azimuthal extension of $1 \mathrm{~cm}$, the longitudinal impedance $Z_{\mathrm{II}} / \mathrm{n}$ becomes $25 \mathrm{~m} \Omega$. The transverse impedance for all photon-stops together becomes $Z_{\perp}=8 \mathrm{M} \Omega / \mathrm{m}$.

An estimate [46] of the microwave instability threshold bunch population is $3 \times 10^{12}$, two orders of magnitude larger than the Stage-2 bunch population. Numerical wake calculations, using MAFIA $^{\odot}$ [46], indicate a TMCI threshold bunch population of $4.5 \times 10^{11}$, still larger than the design peak number of protons per bunch. We believe that the photon-stops will not cause TMCI.

Trapped modes are of concern in cavity-like structures, e.g., holes in the beam pipe and sharp edges. We believe that photon-stops, with the appropriate design, will not be a major cause of trapped modes.

The impedance calculations were performed assuming that the photon-stops are made of copper and have indicated the viability of this concept. Another possible approach to the design of this device would be the use of dielectric materials with low electrical permittivity and high thermal conductivity, such as sapphire or diamond. Although this approach would be advantageous from an impedance standpoint, complications are expected to arise in conjunction with the removal of surface charge and the lack of knowledge of the photo-desorption coefficients. In addition these solutions could be more expensive.

\subsection{Photon-Stop Vacuum Issues}

The strong synchrotron radiation flux impinging on the photon-stop results in a fast clean-up of the photon-stop surface. However, it is necessary to pump the desorbed gas load. According to the engineering design proposed, the beam-screen in the photon-stop system has no pumping holes. The desorbed gas load has to diffuse $\sim 5 \mathrm{~cm}$ to each side into the beam tube to reach sections where active pumping to the cold-bore removes the gas from the beam area. The high temperature of the photon-stop $(\sim 350 \mathrm{~K})$ and the beam-screen $(\sim 100 \mathrm{~K})$ certainly prevents readsorption of the desorbed gases and thus the pumping task is simplified. The high surface temperatures result as well in a high molecular speed, accelerating the gas diffusion into the adjacent beam-screen regions, where the pumping occurs. On the other hand the high 
temperature of the photon-stop raises the thermal desorption rate. Vacuum simulations are required to determine if the longitudinal diffusion is sufficient to ensure a proper vacuum in the photon-stop sections. It will certainly be necessary to perform a high temperature heat treatment (bake-out) of the photon-stop insert before installation.

\subsection{Photon-Stop R\&D Issues}

An R\&D plan has been presented, that lists the most important tasks on the path toward the realization of a photon-stop for Stage-2 VLHC [48]. These tasks include 1) a detailed investigation of the characteristics of Stage-2 SR to allow for an optimization of the absorber shape, 2) continued impedance calculations to improve the understanding of the effects of the photon-stop on beam stability, especially with the use of improved absorber shapes aimed at a reduction of the photon scattering rate, 3 ) vacuum simulations to investigate the magnitude of the pressure bump in the photon-stop sections and to calculate a suitable conditioning profile, 4) (warm) testing of prototypes in a synchrotron source photon beam, 5) cryo-testing of the prototypes, and finally 5) full blown vacuum tests in a Stage-2 vacuum system mock-up exposed to SR, as have been performed for the SSC and LHC projects.

\subsubsection{Magnet Power Supply and Quench Protection}

\subsubsection{Stage-2 Electrical schemes}

Arc dipoles and short straight section quadrupoles will be grouped into electrical circuits ordered for efficient electrical distribution, space utilization, and minimization of cryogenics and cooling water requirements. Power supplies for these circuits will be located in ground level service buildings at 12 feeder locations corresponding to the cryogenic plants (Section 6.2.1). Circuit boundaries will match cryogenic boundaries; this will enable 1/12 sections of the ring to be commissioned independently, and will also sectorize the maximum quench load on the system. Additional low current supplies will be required for corrector magnets. Finally, power supplies are needed to energize quench protection strip heaters for the superconducting dipole and quadrupole circuits.

\subsubsection{Arc Dipole and Quadrupole Power Schemes}

The arc dipoles (Section 6.1.1) will be powered in 24 circuits, 2 circuits per feeder location as shown in Figure 6.18. Each circuit will consist of approximately 450 dipoles in series with a 25 $\mathrm{kA}$ power supply, a reference magnet for monitoring the field harmonics, and an energy extraction system. The characteristics of each circuit are shown in Table 6.14.

The twin dipole apertures will be internally bused in series, with a cold bypass diode across the magnet terminals to electrically isolate the magnet in the event of a quench. Each circuit will have two room temperature extraction resistor banks, located symmetrically in the circuit. One bank will be located near the power supply, while the other bank will be located in an alcove halfway between the feeder access ports. Each bank is capable of dissipating $~ 3$ GJ of stored energy. The resistance and location of the extraction circuits determine the peak voltage to ground as well as the discharge time of the circuit. Limiting the peak voltage to $\pm 1 \mathrm{kV}$ requires 
two $85 \mathrm{~m} \Omega$ dump resistors. Using the LHC dipoles circuits as a scale, each circuit will require 9 times the LHC energy extraction.

Power supply requirements depend on the total stored energy, and the current vs. time profile used to reach the peak operating field. With a constant ramp rate, $150 \mathrm{MW}$ of power will be required for the 2000 second ramp to the operating field of the dipole circuits. Using a "constant power" $(\mathrm{I}=\mathrm{K} \sqrt{t})$ time vs. current profile reduces the peak power consumption by a factor of two, but puts an unacceptably large voltage requirement on the dipole power supplies and increases the accelerator RF power requirements at lower currents. A linear ramp (constant RF accelerating voltage) followed by a "constant power" ramp looks like an attractive compromise. The break between the linear and constant power portion of the ramps can be optimized based on the relative cost of supplying RF and dipole current power.

The quadrupoles will be divided into 24 circuits. As shown in Figure 6.19, focussing and defocusing quadrupole circuits will be separated to facilitate tuning. The focussing- defocusing circuit pair covers $1 / 12$ of the accelerator and is matched to two dipole circuits and a corresponding cryogenic supply. A single extraction circuit for each quadrupole circuit will be located in the power supply service building. As shown in Table 6.15, the power supply and extraction requirements are modest compared to the dipole circuitry.

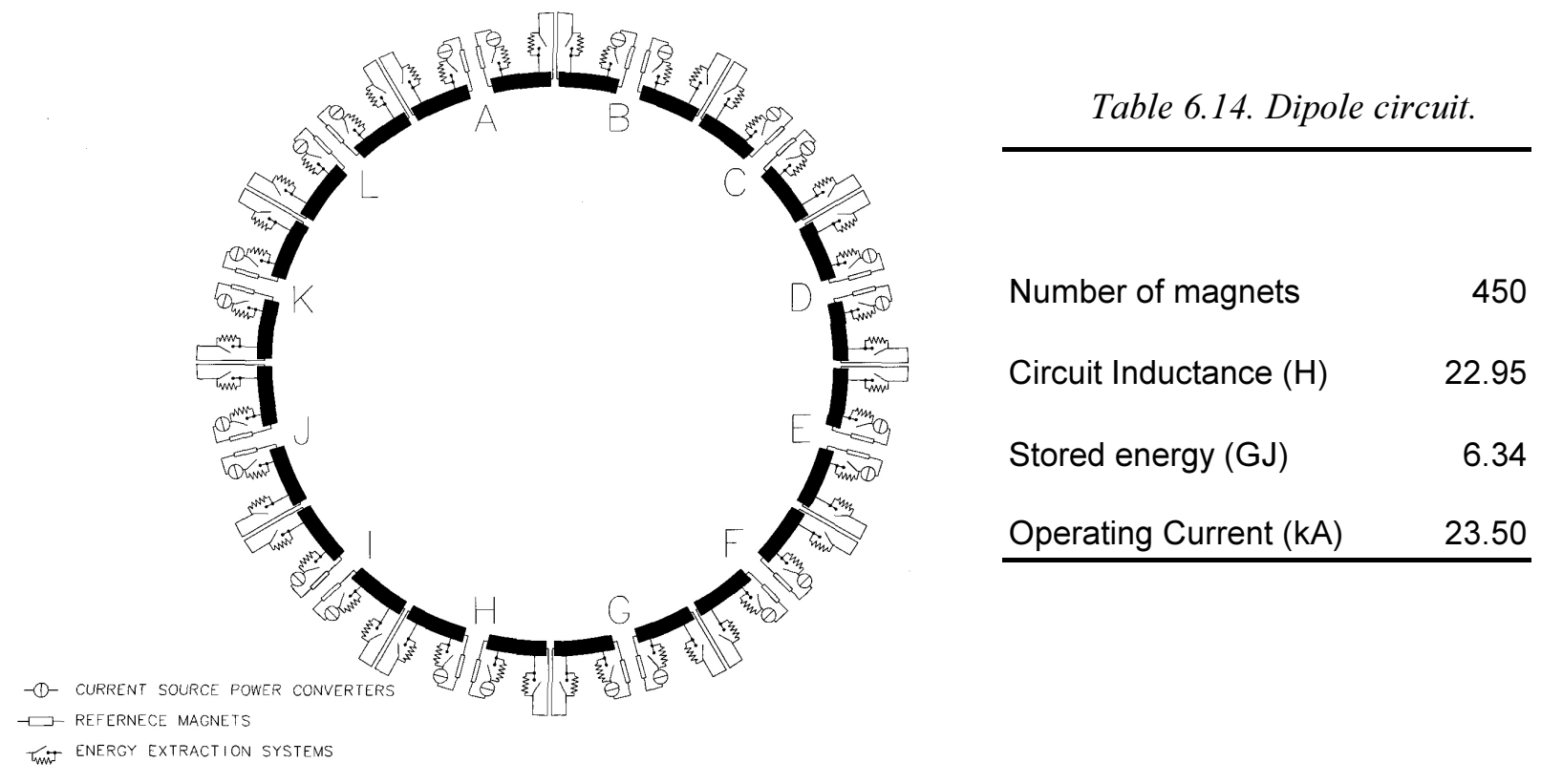

Figure 6.18. Electrical distribution for dipole circuits. Power supplies are located in ground level service buildings $A-L$. 


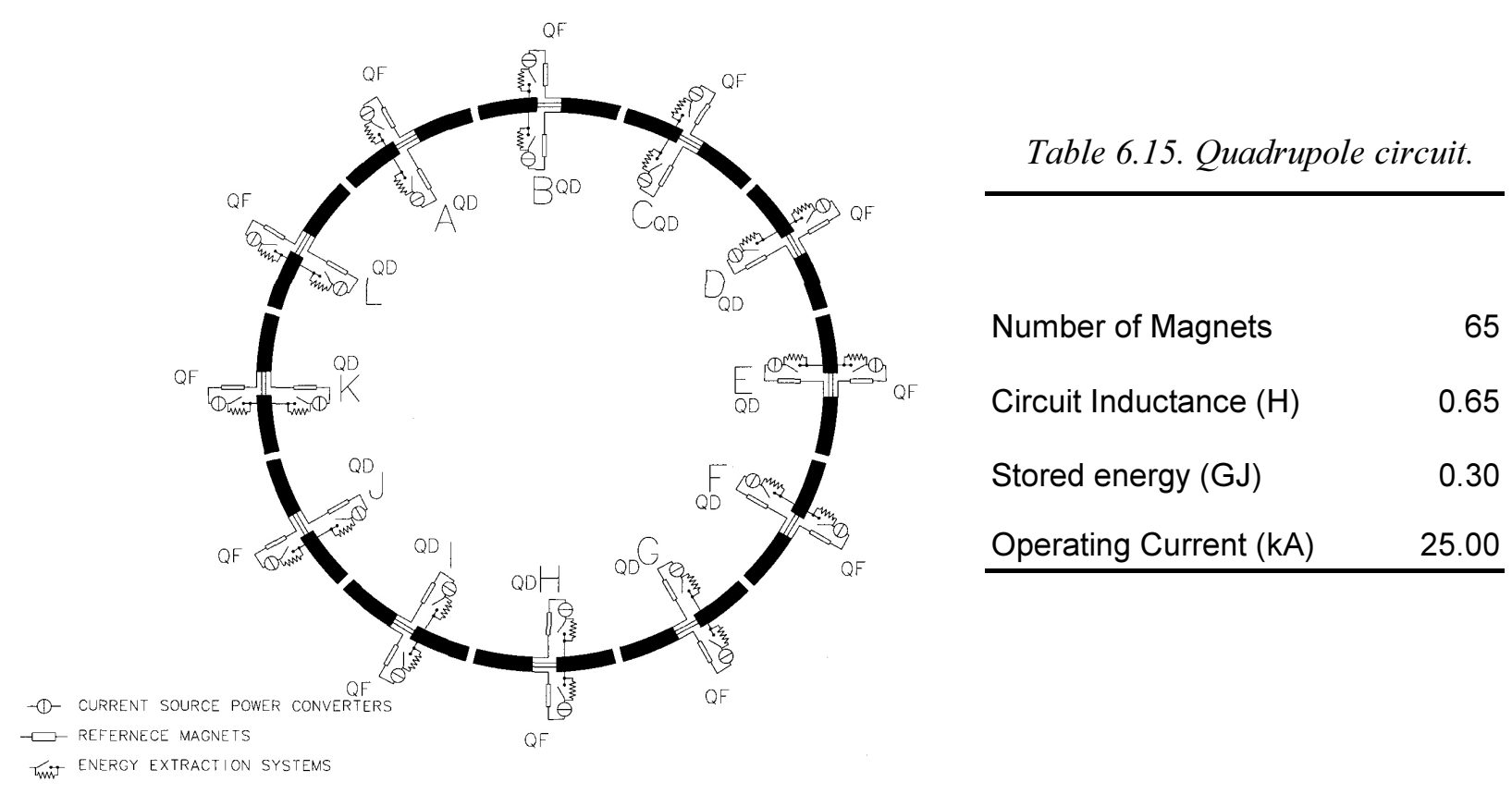

Figure 6.19. Electrical distribution for arc quadrupoles.

\subsubsection{Corrector Powering}

The correctors required for Stage-2 are described in Section 3.5 and in Section 6.1.4. Dipole, quadrupole and sextupole correctors will be packaged into spool pieces and will be locally powered. Currents will be less than 100 amps. Power supplies should be kept as close as to the spool pieces as possible, space and radiation permitting to reduce the power loss in the leads.

\subsubsection{Bus work}

The dipole and quadrupole bus work will consist of superconductor stabilized with copper. The amount of copper is sized to protect the bus for excessive temperature in the event of a bus quench. Based on the LHC dipole experience, the required copper cross section for the dipole and quadruple bus would be $\sim 900 \mathrm{~mm}^{2}$ and $200 \mathrm{~mm}^{2}$ respectively. Dipole bus will likely be placed inside the magnet coldmass. Unlike the dipole bus, the two quadrupole buses could be routed through an auxiliary bus port parallel to the dipole/quadrupole cold mass but inside the cryostat. It is then possible to have these buses as a continuous piece spanning many contiguous cryogenic elements, simplifying the bus manufacturing and eliminating several splices. The bus configuration is shown in Figure 6.15.

\subsubsection{Quench Protection Powering}

Quench protection strategy centers around the use of strip heaters. Strip heaters are thin copper clad stainless steel strips that are in good thermal contact with the coils. Banks of capacitors are discharged into the heaters in order to quench the coils and thus distribute the quench energy more uniformly throughout the dipoles and quadrupoles. Studies have been performed in order to determine the required heater coverage and power supplies. The power supplies for the dipoles quadrupoles are comparable in voltage and capacitance (within a factor of 2.5) of those require 
for the LHC magnets [49]. The power supplies and quench detection electronics should be located as close to the magnets as possible, space and radiation limits permitting, to reduce quench detection signal noise as well as power loss in the heater leads.

\section{References}

[1] G. Ambrosio et al., "Development of the $11 \mathrm{~T} \mathrm{Nb}_{3}$ Sn Dipole Model at Fermilab," MT-16, IEEE Transactions on Applied Superconductivity, v. 10, No. 1, March 2000, p.298.

[2] G. Ambrosio et al., "Development of React \& Wind Common Coil Dipoles for VLHC," ASC'2000, IEEE Transactions on Applied Superconductivity, v. 11, No. 1, March 2001, p. 2172.

[3] S.A. Gourlay et al., "Design and Fabrication of a $14 \mathrm{~T}, \mathrm{Nb}_{3} \mathrm{Sn}$ Superconducting Racetrack Dipole Magnet," MT16, IEEE Transactions on Applied Superconductivity, v. 10, No. 1, March 2000, p.294.

[4] S. Caspi et al., "Design of a $\mathrm{Nb}_{3}$ Sn High Gradient Low-beta Quadrupole Magnet," MT-15, Proceedings, Oct 2024, 1997, Beijing, China, p.175.

[5] R. Gupta et al., "Common coil magnet Program at BNL," ASC'2000, IEEE Transactions on Applied Superconductivity, v. 11, No. 1, March 2001, p. 2168.

[6] T. Elliott et al., "16 Tesla $\mathrm{Nb}_{3}$ Sn Dipole Development at Texas A\&M University," IEEE Transactions on Applied Superconductivity, June 1997, p. 555.

[7] A. den Ouden et al., "A 10 T Model Separator Magnet for the LHC," MT-15, Proceedings, Oct 20-24, 1997, Beijing, China, p.137.

[8] A. den Ouden \& H. ten Kate, "Application of $\mathrm{Nb}_{3} \mathrm{Sn}$ Superconductors in High-Field Accelerator Magnets," IEEE Transactions on Applied Superconductivity, June 1997, p. 733.

[9] A. McInturff et al., "Test Results for a High Field (13T) $\mathrm{Nb}_{3}$ Sn Dipole," Particle Accelerator Conference (PAC97), Vancouver, Canada, 1997, p. 3212.

[10]A. Lietzke et al., "Racetrack Coil Technology Validation (RT-1) Test Results," Proc. of $2^{\text {nd }}$ VLHC Magnet Workshop, Fermilab, May 2000 (http://vlhc.org).

[11]R.M. Scanlan et al., "Conductor Development for High Field Dipole Magnets," MT-16, IEEE Transactions on Applied Superconductivity, v. 10, No. 1, March 2000, p.288.

[12] Superconducting Super Collider, Conceptual Design, SSC Central Design Group, SSC-SR-2020, March 1986.

[13] The Large Hadron Collider, Conceptual Design, The LHC Study Group, CERN/AC/95-05 (LHC), 20 October 1995.

[14] V.V. Kashikhin and A.V. Zlobin, "Magnetic Designs of 2-in-1 $\mathrm{Nb}_{3}$ Sn Dipole Magnets for VLHC," ASC'2000, IEEE Transactions on Applied Superconductivity, v. 11, No. 1, March 2001, p. 2176.

[15]I. Novitski et al., "Design and Mechanical Analysis of a Single-Layer Common Coil Dipole for VLHC," ASC'2000, IEEE Transactions on Applied Superconductivity, v. 11, No. 1, March 2001, p. 2276.

[16]E. Barzi et al., "Strand Critical Current Degradation in $\mathrm{Nb}_{3}$ Sn Rutherford Cables," ASC'2000, IEEE Transactions on Applied Superconductivity, v. 11, No. 1, March 2001, p. 2134.

[17]P. Bauer et al., "Fabrication and Testing of Rutherford-Type Cables for React and Wind Accelerator Magnets," ASC'2000, IEEE Transactions on Applied Superconductivity, v. 11, No. 1, March 2001, p. 2457.

[18] G. Ambrosio et al., "Superconductor Requirements for the HFM Program at Fermilab," Fermilab, TD-99-073, December 151999.

[19]E. Barzi et al., "Study of $\mathrm{Nb}_{3}$ Sn Strands for Fermilab's High Field Dipole Models," ASC'2000, IEEE Transactions on Applied Superconductivity, v. 11, No. 1, March 2001, p. 3595.

[20] V. Kashikhin and A.V. Zlobin, "Conceptual Designs of 2-in-1 $\mathrm{Nb}_{3}$ Sn Arc Quadrupole Magnets for VLHC," Fermilab, TD-01-019, March 2001.

[21]D. Chichili, "Mechanical Analysis of FF Arc-Quadrupole for VLHC Stage-2," Fermilab, TD-01-012, March 5, 2001. 
[22] V.V. Kashikhin and A.V. Zlobin, "Correction of the Persistent Current Effect in $\mathrm{Nb}_{3}$ Sn Dipole Magnets," ASC'2000, IEEE Transactions on Applied Superconductivity, v. 11, No. 1, March 2001, p. 2058.

[23] A.V. Zlobin, TD note on VLHC-2 correctors.

[24] T. Nicol, "VLHC Arc Dipole and Quadrupole Cryostats," Fermilab, TD-01-016, March 2001.

[25]P. Bauer et al., "Quench Protection Calculations for Fermilab's $\mathrm{Nb}_{3}$ Sn High Field Magnets for VLHC - Part 1," Fermilab, TD-01-003, February 2001.

[26] P. Bauer et al., "Quench Protection Calculations for Fermilab's $\mathrm{Nb}_{3}$ Sn High Field Magnets for VLHC - Part 2," Fermilab, TD-01-004, February 2001.

[27]L. Imbasciati, G. Ambrosio, and P. Bauer, "Quench heater design considerations for VLHC magnets," Fermilab, TD-01-022, April 2001.

[28] M. McAshan and P. O. Mazur, "Cryogenic Systems for the High Field RLHC Study Cases," 1996 Snowmass Study.

[29] M. McAshan and P. O. Mazur, "Cryogenic Systems for the Low Field RLHC Study Cases," 1996 Snowmass Study.

[30] M. McAshan, "Cryogenic Systems for the 3 TeV Injector Study," VLHCPUB-87, Dec 1997.

[31] L. Tavian and U. Wagner, "LHC sector heat loads and their conversion to LHC refrigerator capacities," LHC Project Note 140 (1998).

[32] Roger Rabehl, VLHC quench study and "Cooldown of a VLHC-2 Magnet String," personal communications.

[33] C. Darve, A. Poncet, D. Willems, "CTM3, the latest evolution of the cryostat thermal model: thermal performance measurements of the first run from March to May 1998," Technical Note 98-19, 03/1999.

[34]C. Darve, G. Ferlin, M. Gautier, L.R. Williams, "Thermal performance measurements for a 10 meter LHC dipole prototype (Cryostat Thermal Model 2)," LHC-Project-Note-112, 11/1997.

[35] C. Darve, P. Bauer, T. Nicol, T. Peterson, "VLHC Thermal Shield Cooling," Fermilab Tech. Div. note TD-01035, 2001.

[36] Amalia Ballarino and Albert Ijspeert, "Design and Tests on the 30 to 600 A HTS Current Leads for the Large Hadron Collider," CERN-LHC-Project-Report-78, 24 Oct 1996.

[37] G. Citver et al., "Thermal tests of 6-kA HTS current leads for the Tevatron," Advances in Cryogenic Engineering, Vol. 45B, (2000), pp. 1549 - 1556. (Also FERMILAB-Conf-99-284)

[38] C. Darve et al., "VLHC Beam-screen Cooling," Fermilab Technical Division Note TD-01-005, 02/01.

[39] W.C. Turner, "Beam Tube Vacuum in Low Field and High Field Very Large Hadron Colliders," LBNL-39482, UC 414, presented at the Snowmass Conference, Oct. 1996.

[40] P. Bauer et al., "Vacuum Calculations for the VLHC - stage 2," Fermilab, Technical Division Note, TD-01-011, $04 / 01$.

[41] A. Mathewson, O. Groebner, P. Strubin, R, Marin, R. Souchet, AIP Conf. Proc., 236, 313, 1990.

[42] O. Groebner, "The LHC Vacuum System," Proceedings of the Particle Accelerator Conference 1997.

[43] S. Sharma et al., "High Heat-Load Absorbers for the APS Storage Ring," Proceedings of the $1^{\text {st }}$ International Workshop on Mechanical Engineering Design of Synchrotron Radiation Equipment and Instrumentation, PSIWurenlingen, Switzerland, July 2000.

[44]I. Terechkine, "Synchrotron Radiation in the VLHC," Fermilab, Technical Division, unpublished note, 01/01.

[45] P. Bauer et al., "A Photon-stop for VLHC-2 - Engineering Design - Part 1," Fermilab, Technical Division Note, TD-01-023, 04/01.

[46]P. Bauer, N. Solyak, "A Photon-stop for the VLHC-2 - Impedance Calculations," Fermilab Technical Division Note TD-01-014, 04/01.

[47] S. Kurennoy, in M. Tigner, A. Chao, eds., "Handbook of Accelerator Physics and Engineering," World Scientific, 1998, p. 207.

[48] P. Bauer et al., "Photon-Stop R\&D Plan," Fermilab Technical Division Note TD-01-030, 05/01.

[49] P. Bauer, L. Imbasciati, G. Ambrosio, V. Kashikhin, A. Zlobin, M. Lamm, "Quench Protection Calculations for Fermilab's $\mathrm{Nb}_{3}$ Sn High Field Magnets for VLHC, Part II," TD-01-004 FNAL (March 2001). 


\section{Chapter 7. Conventional Construction and Facilities}

\subsection{Geology of the Fermilab Region}

The geologic studies for the Illinois proposal for the SSC [1,2] concluded that the main tunnel ( $87 \mathrm{~km}$ circumference) and experimental facilities should be sited in the Galena-Platteville dolomite which is a uniform, competent tunneling unit. A subsequent geotechnical study for the Next Linear Collider Project [3] and generic site studies for future accelerator facilities at Fermilab [4] also reached the same conclusion. The only less-than-ideal feature was the depth of construction (approximately 400 feet below the surface) to reach this dolomite layer. However, due to its longer circumference $(233 \mathrm{~km})$, a VLHC might extend beyond the region where it is practical, or possible, to site the tunnel entirely in the Galena-Platteville. An earlier geological study for various VLHC configurations was reported by Conroy [5]. There has been substantial experience in the Chicago area $[6,7]$ in tunneling and underground caverns in the Silurian dolomite, Maquoketa shale, and Galena-Platteville dolomite. There is a lack of corresponding local similar experience, or even knowledge of the mechanical/structural engineering properties of the underlying units consisting of the Ancell, Middle Confining unit, and the Franconia formation.

There is a general sloping of the till, Silurian, Maquoketa, Galena-Platteville, Ancell sequence of layers from the West (higher elevation above mean sea level) to the East (lower elevation) to Lake Michigan. However, to the Northwest and West of Fermilab are located the Troy and Rock Bedrock Valleys, where the Silurian, Maquoketa, and Galena-Platteville layers have been completely cut away by glaciers and filled to the underlying sandstones with Glacial drift. The Sandwich Fault Zone lies to the Southwest of Fermilab, passing through Sandwich, Illinois, and running roughly NW to SE. This fault zone does end approximately SSE of Fermilab. To the Southwest of the Sandwich Fault Zone, the underlying Ancell and Middle Confining layers are upthrust to meet the Glacial drift at the bedrock surface, and even the Franconia layer can impact tunnels traversing the fault zone.

The Des Plaines disturbance, as shown in Figure 7.1, is an unusual structure, possibly an ancient meteor impact. The rocks in the disturbance, in an area about $51 / 2$ miles in diameter, are reported to be intensely faulted. This disturbance was encountered during construction of the TARP tunnels, but did not cause any significant problems.

The study region is in an area of the central mid-continent that is tectonically stable and seismically relatively quiescent. The seismic hazard posed to structures in this region is very low. Active faults are not known in the study area, and the last movement on the Sandwich Fault Zone has been demonstrated to be more than 200,000 years ago. The closest known earthquake source zones capable of producing ground motions of any significance to engineering design or operational requirements are located several hundred miles to the south. 


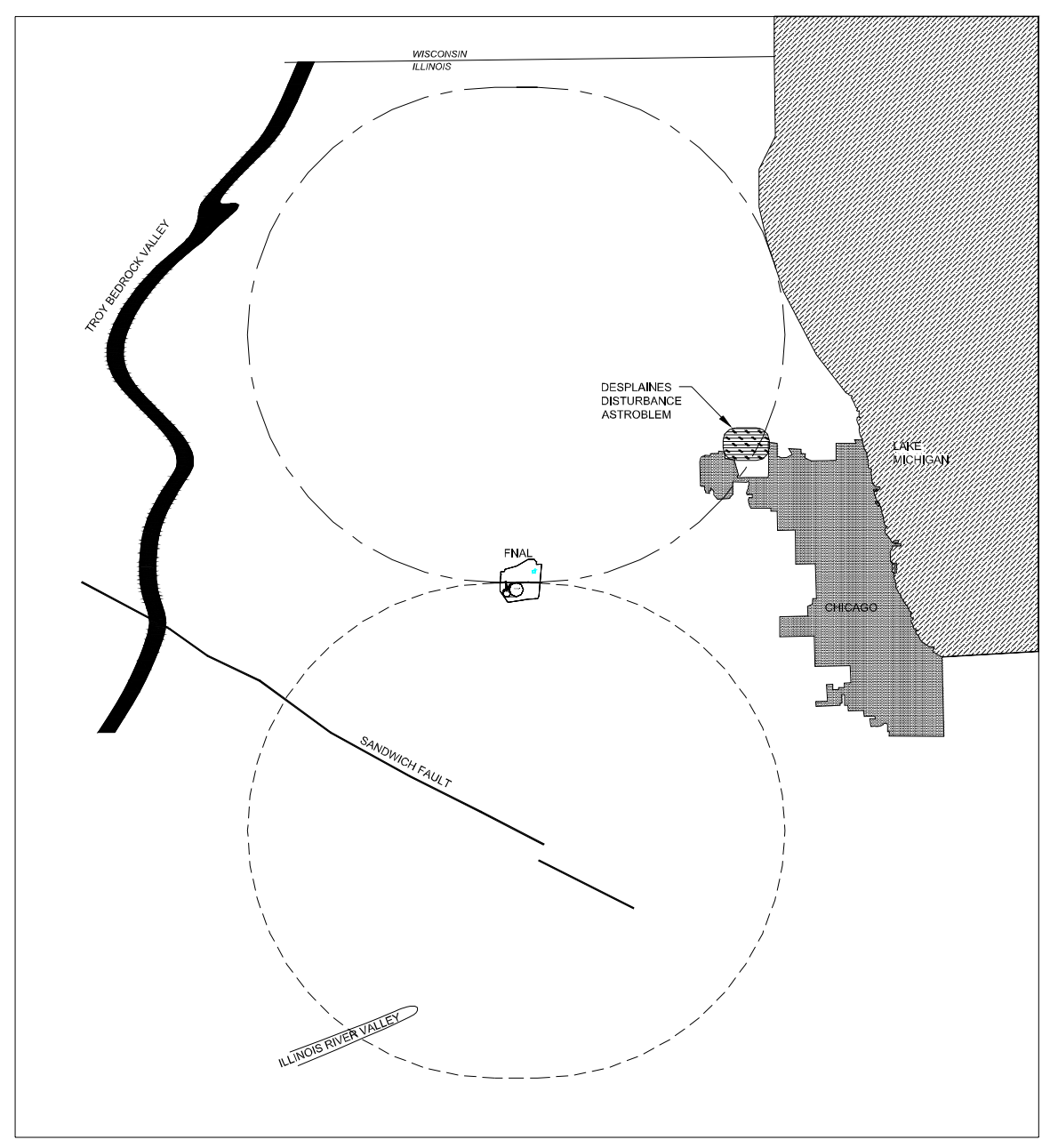

Figure 7.1. General geologic features of Northeastern Illinois with two alternative ring orientations under study.

If we restrict consideration to rings that would be contiguous to the existing Tevatron, only a VLHC ring with its center oriented to the North of Fermilab (North Ring) could be completely contained within the Galena-Platteville dolomite. This ring would have a roughly North-South strike axis passing through Fermilab with a tilt of approximately $0.2 \%$ grade to stay within the dolomite. Lake Michigan and the Wisconsin border would bound this ring. Any ring with its center oriented farther to the West (even NNW) would be impacted by the bedrock valleys. Possible rings oriented to the South or West would traverse the Sandwich fault into the strata underlying the Galena-Platteville. 


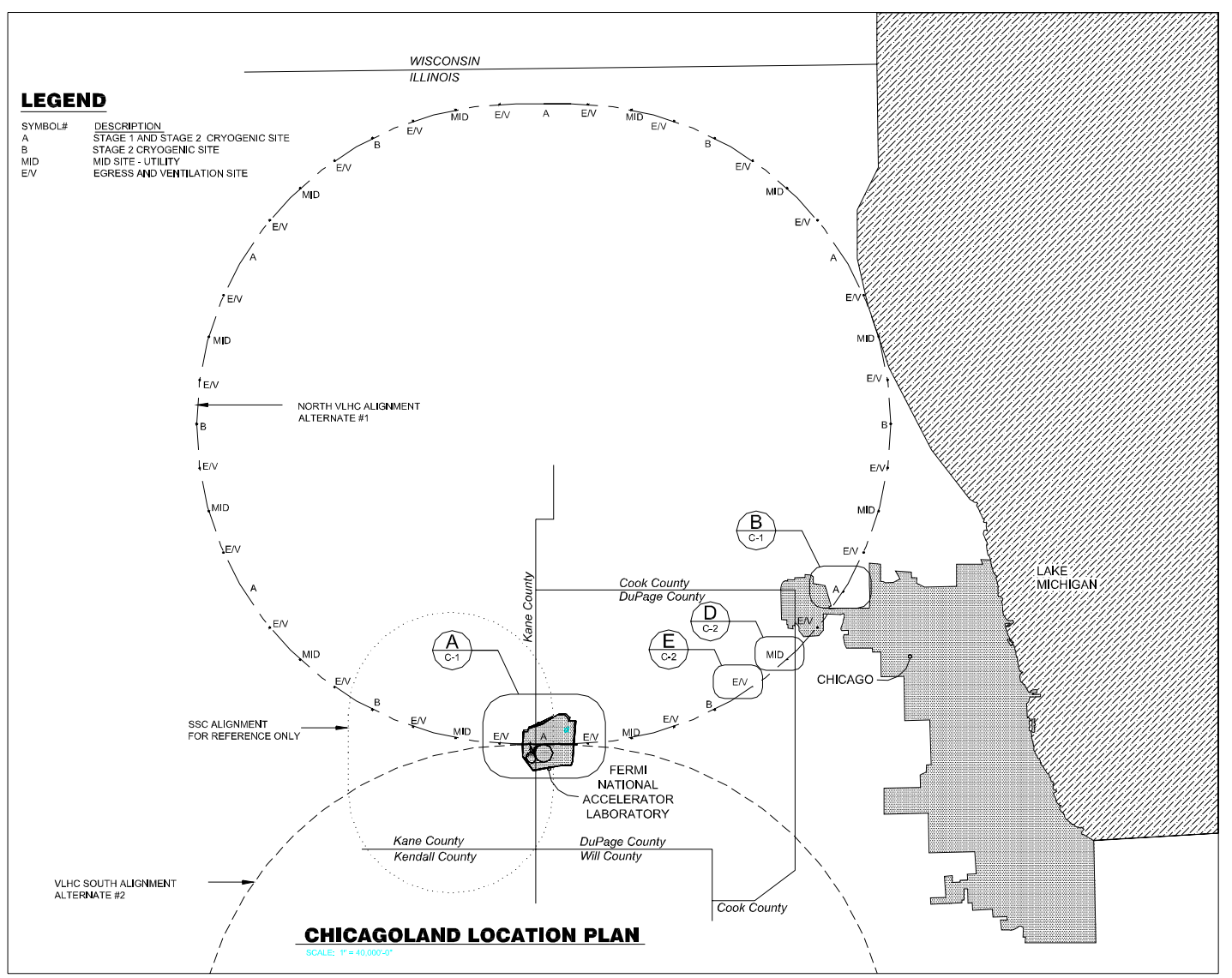

Figure 7.2. Schematic of the VLHC 233- $\mathrm{km}$ racetrack $(R=35 \mathrm{~km}$, two 6-km straights) depicting off-site service areas for North ring configuration.

Figure 7.2 shows the proposed configuration for the off-site service areas for the North ring. An approximate ring description is two half circles of radius $35 \mathrm{~km}$ joined by two long straight sections, each $6 \mathrm{~km}$ long. A more precise description of the proposed footprint is the major arcs bend the beams 174 degrees and the remaining 6 degrees of bending occurs in the $6 \mathrm{~km}$, which is a cluster of 5 straight sections separated by four bends. These clustered straight sections are for injection, abort, RF cavities, interaction regions for two experiments, and for the transfers between Stage 1 and Stage 2. Approximately equally spaced around the circumference are 12 cryo sites, labeled A-sites and B-sites. The six A-sites are needed in Stage 1; the full 12 sites are required in Stage 2. Spaced between the 12-cryo sites are 12 addition utility mid-sites.

In order to undertake a tunnel costing and feasibility study for various orientations of a VLHC ring, two layouts and vertical strata lampshades were prepared [8]. The simplest configuration is a tilted $(0.2 \%$ incline $)$ North Ring, which stays completely in Galena-Platteville dolomite. The second is a horizontal North Ring which transitions between the following media: Maquoketa shale, Silurian dolomite, Maquoketa shale, Galena-Platteville dolomite, and back into Maquoketa shale. The third is a tilted South Ring, which crosses the Sandwich Fault (only once) into the sandstone Ancell aquifer (wet) and the dolomitic sandstone Middle Confining unit and possibly the Franconia formation. These rings are shown in plan in Figure 7.1 
and in section in Figures 7.3 and 7.4. Elevation parameters and the percentage of tunneling medium for each of these rings are listed in Table 7.1.

Another design constraint of this costing exercise deals with the positioning and depth of the caverns for the two experimental halls. It is desirable to have these located at the Fermilab site to be able to cluster the long straight sections (for injection/abort and interaction regions) and to utilize the Fermilab campus for the related experiment fabrication and staging buildings, support utilities, and equipment shaft facilities. This also helps to minimize off-site surface land requirements.

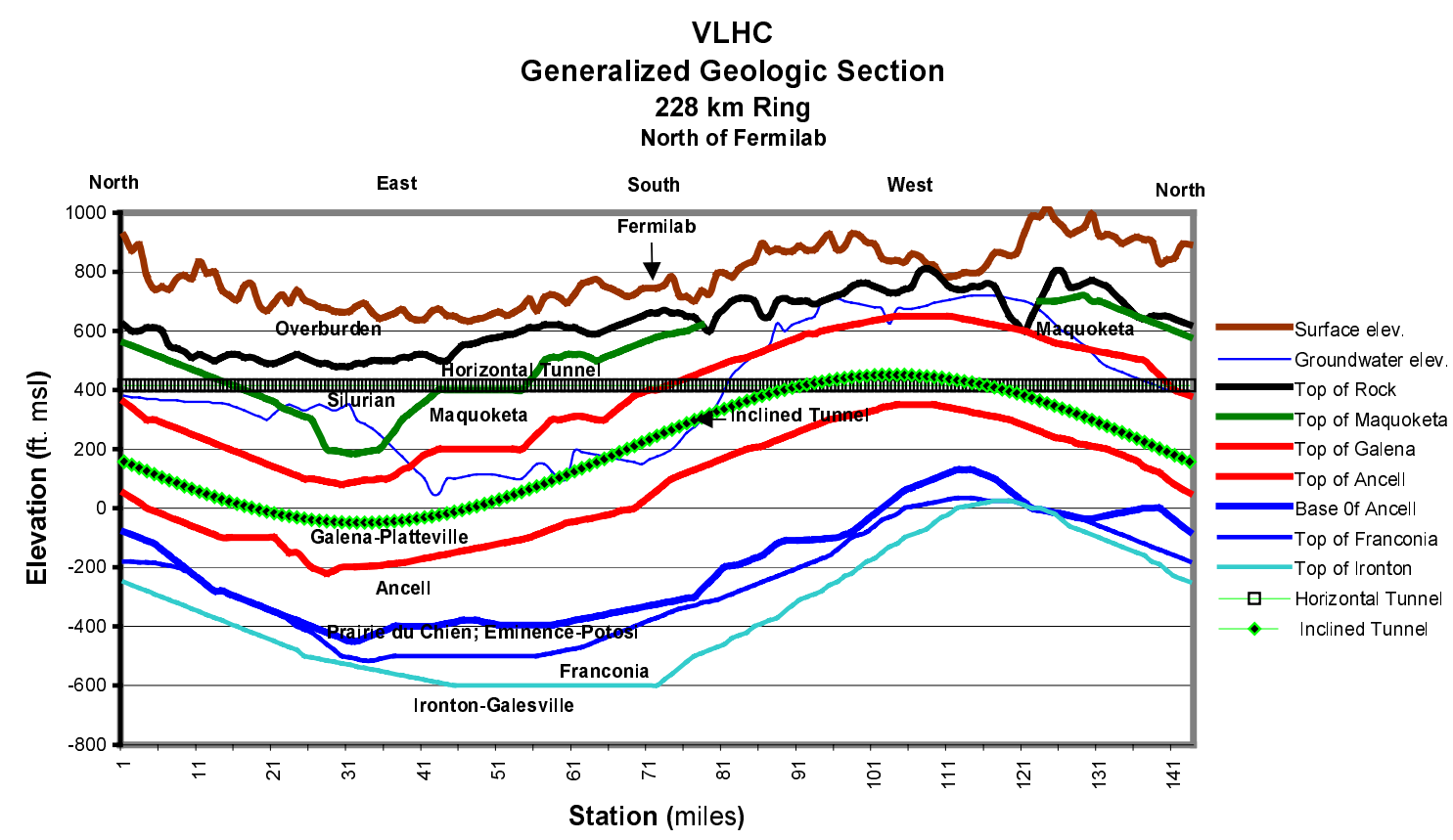

Figure 7.3. Lampshade diagram for North Ring orientation for horizontal and inclined tunnels.

Structural features and thickness of the strata supporting the spans of the experimental halls or caverns [2] will determine the elevations at which the caverns can feasibly be sited. This, of course, impacts the elevation of the accelerator tunnel at the site of the cavern. A general rule of thumb is that there should be a depth of rock (dolomite) strata above the cavern at least equal to the span of the cavern. Previous studies [1,2] have concluded that for 75 foot high chambers, roof spans up to 125 feet are feasible using standard methods of roof arching and rock supports. This assumed that the orientation axis of the cavern bisects the major joint sets. The joints run approximately NE-SW $\times$ NW-SE so these criteria would be satisfied by VLHC ring orientations to the North, South, or West. Possible options in elevation for the experiment caverns are depicted in Figure 7.5. 
Table 7.1. Elevation parameters and strata fraction for three tunnel models.

\begin{tabular}{|c|c|c|c|}
\hline & $\begin{array}{l}\text { South Ring } \\
0.08 \% \text { incline }\end{array}$ & $\begin{array}{l}\text { North Ring } \\
\text { horizontal }\end{array}$ & $\begin{array}{c}\text { North Ring } \\
0.2 \% \text { incline }\end{array}$ \\
\hline elevation at Fermilab $(\mathrm{ft} \mathrm{msl})$ & 510 & 415 & 244 \\
\hline depth at Fermilab (feet) & 235 & 330 & 501 \\
\hline average depth (feet) & 277 & 371 & 585 \\
\hline r.m.s. depth (feet) & 61 & 99 & 121 \\
\hline minimum depth (feet) & 118 & 219 & 342 \\
\hline maximum depth (feet) & 406 & 603 & 779 \\
\hline \multicolumn{4}{|l|}{ Strata (approximate \%) } \\
\hline Silurian dolomite & $29 \%$ & $27 \%$ & \\
\hline Maquoketa shale & $34 \%$ & $27 \%$ & \\
\hline Galena-Platteville dolomite & $21 \%$ & $46 \%$ & $100 \%$ \\
\hline Ancell - St. Peter sandstone & $6 \%$ & & \\
\hline $\begin{array}{l}\text { Middle Confining layer: Prairie du Chien, } \\
\text { Eminence-Postosi dolomitic sandstones }\end{array}$ & $10 \%$ & & \\
\hline
\end{tabular}

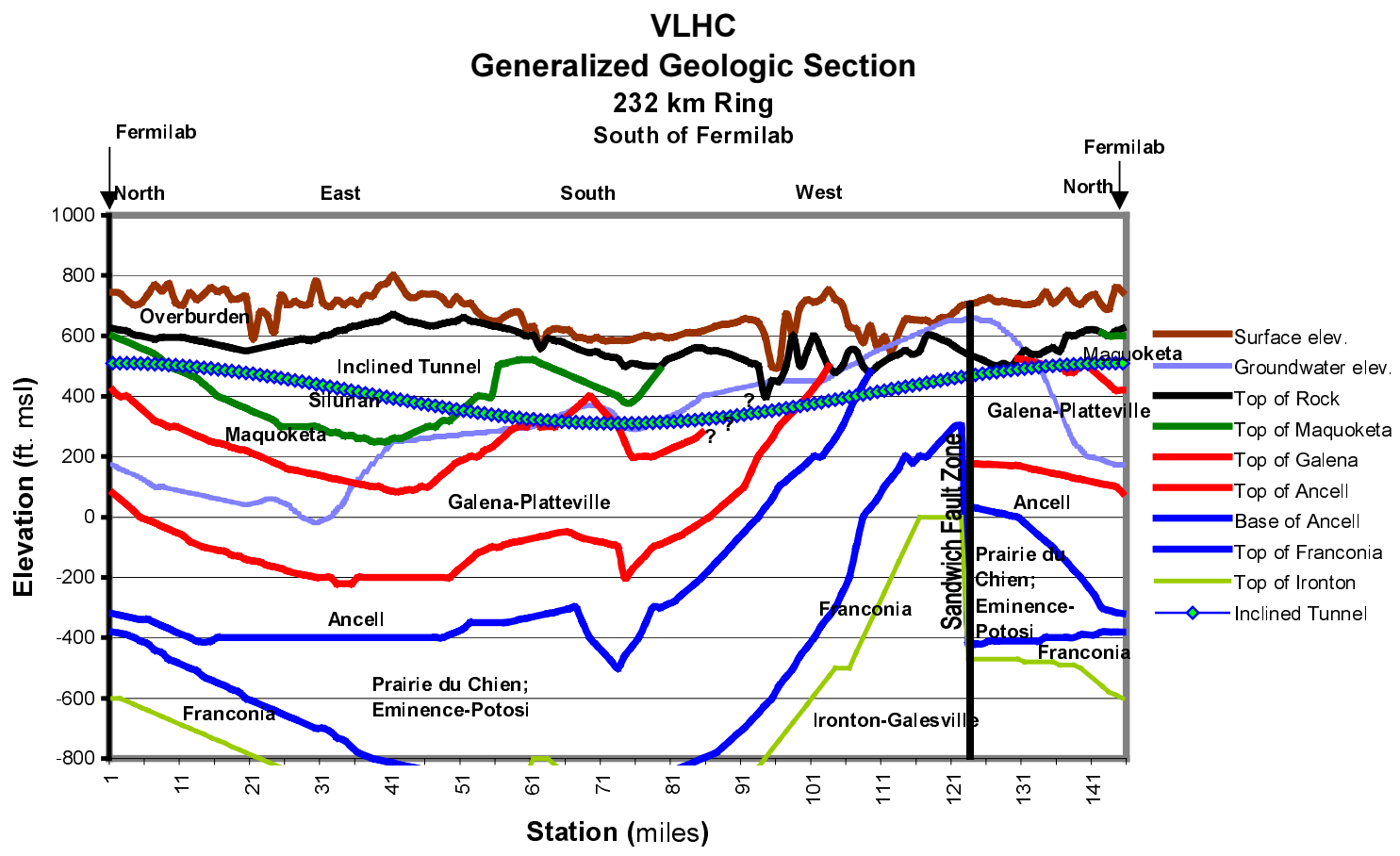

Figure 7.4. Lampshade diagram for South Ring orientation with inclined tunnel.

As a working hypothesis for this study, a time scale of 5 years is assumed for completion of the underground construction, including main tunnel, special tunnels, and all caverns and enclosures. It is anticipated that component installation and commissioning in finished sections of the tunnel could be accomplished in parallel with continued construction in other sections. At this stage of the study, engineering design has not begun for any of these underground elements.

Analogously to the 1997 study of a $34 \mathrm{~km}$ VLHC booster tunnel by the Kenny Construction Company [9], we have entered into a tunnel costing exercise with CNA Consulting Engineers 
[10] to study the expected cost ranges and cost drivers of the three tunnel configurations listed above. By studying the three different tunnels, we hope to learn the unit costs (per mile, per shaft, lined/unlined, etc.) of a tunnel in an excellent media, that of a tunnel transitioning between three different rock media, and that of a tunnel which combines rock media, traversing the Sandwich Fault, and the sandstone and dolomitic sandstones, both within the aquifer, and beyond. These data will allow optimizations and tradeoffs with respect to siting costs. The tunnel cost estimating report will be published elsewhere.

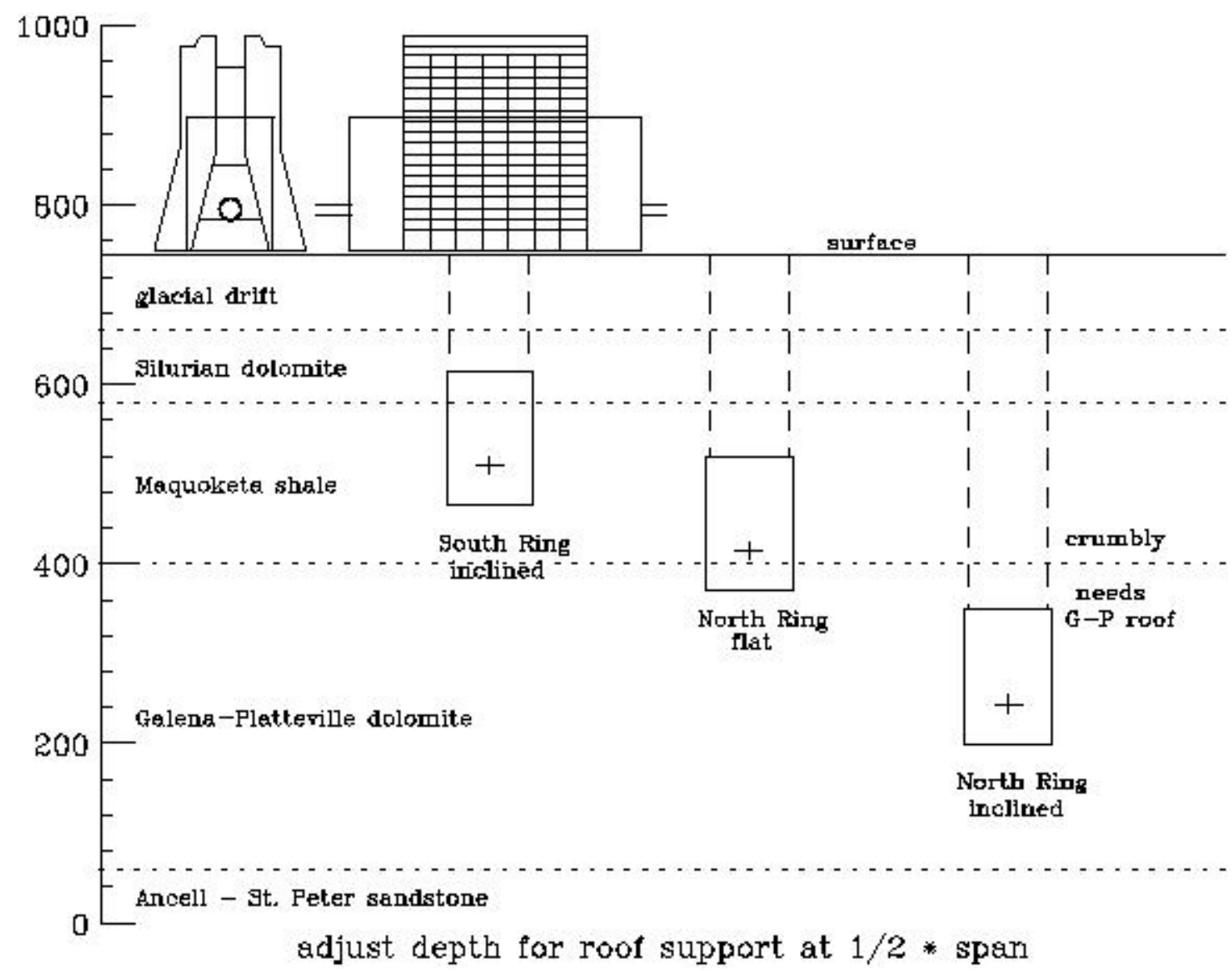

Figure 7.5. Vertical siting of the experimental halls/caverns (and therefore VLHC).

\subsection{Collider Tunnel and Enclosures}

Since the largest volume of the underground construction is the main collider tunnel, we will first concentrate on that component. A preliminary 12 foot clear diameter tunnel cross section is shown in Figure 7.6. However, a major purpose of this cost estimating study is to determine the sensitivity of cost to tunnel diameter. As shown in Figure 7.7 the ring will be adjacent to the Fermilab Tevatron. It will serve as the injector to the Stage-1 (low field) ring. The major straight sections will be clustered (5 each cluster) at Fermilab and at the opposite side. Keeping many of these special tunnels, the abort/beam stops, and the experimental areas at Fermilab will 
optimize utilization of the existing infrastructure and minimize offsite underground construction and shafts. The cluster opposite will contain only the Stage-1 to Stage-2 beam transfer and possibly beam scrapers. Much of these opposite side straight sections can be reserved for future needs. The cluster of straight sections near Fermilab is illustrated in Figures 7.8. and 7.9. Although one configuration for Tevatron injection is shown in these figures and used for the cost estimating exercise, other possible geometry options were discussed in Chapter 4.

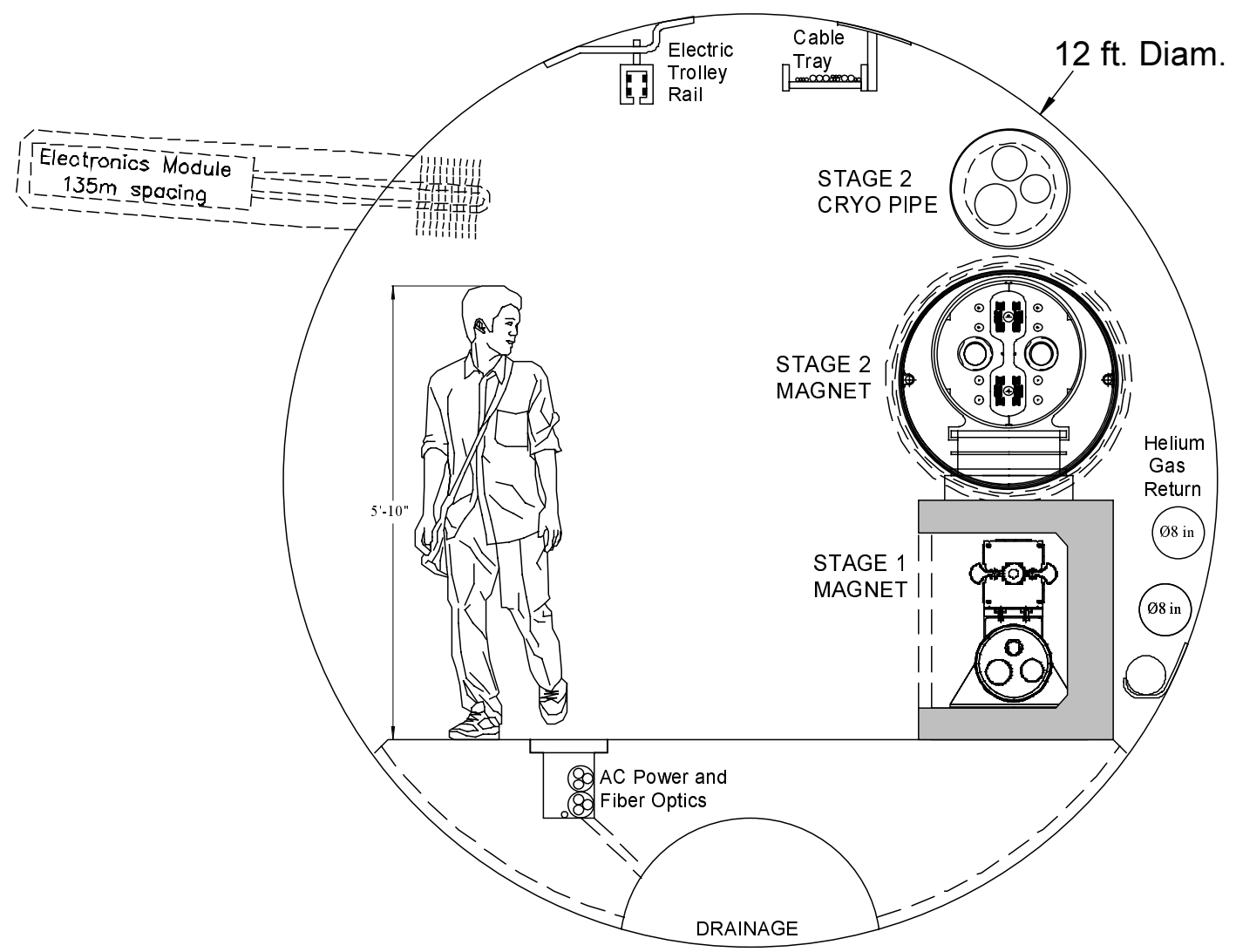

Figure 7.6. VLHC tunnel cross section showing LF (Stage-1) and HF (Stage-2) colliders. The electronics modules with heat sinks projecting into the tunnel occur only every $135 \mathrm{~m}$.

The main tunnel will have a minimum finished inner clear diameter of 12 feet with 10 -foot wide invert floor (Figure 7.6). Tunnel center alignment will be required to \pm 4 inches. The engineering study [10] will estimate the cost differential between 12 and 16 foot diameter tunnels. The tunnel is specified to have an average groundwater influx of not more than 50 gallons per minute per mile. This may require grouting or even lining of some tunnel sections to reduce the groundwater influx to this specification. Dehumidification $(<50 \%$ relative humidity) of the tunnel air will likely be required. The four utility straight sections (injection, abort, RF, and Stage-1 to Stage-2 beam transfers) will likely require additional transverse dimensions, up to 25 feet $\times 25$ feet square cross section. (The SSC design for RF caverns had a 25 foot horseshoe shape). These can be finished by drill and blast after initial tunnel construction by tunnel boring machine. Similarly, at the 12 cryo sites and at the 12 mid-sites, additional underground caverns will be required for tunnel power distribution, cryogenics components, and quench protection systems. 


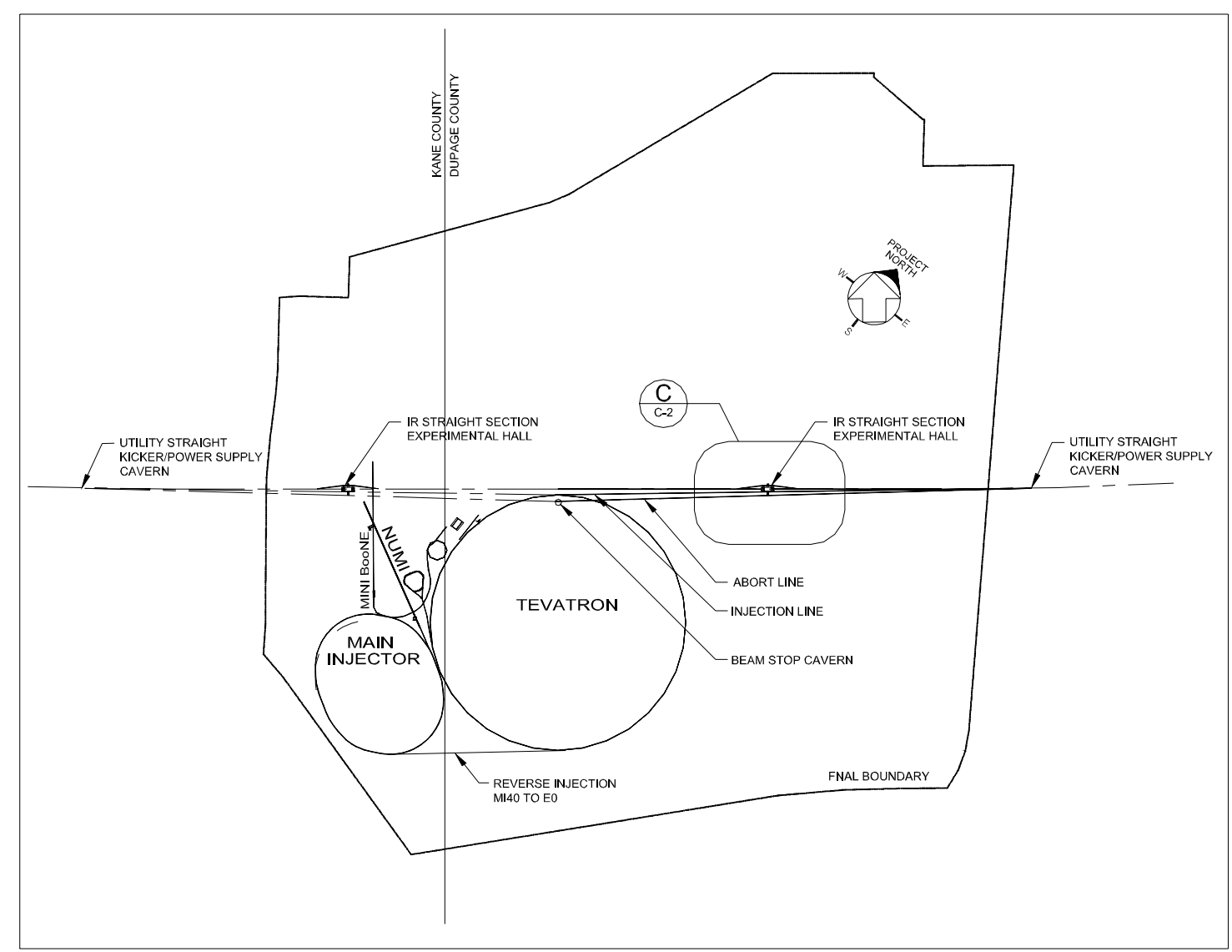

Figure 7.7. Extraction from Tevatron to North Ring and location of experimental halls/caverns.

It is anticipated that there will be twelve 30 -foot diameter major equipment access shafts, with a clear $6 \mathrm{ft} \times 30 \mathrm{ft}$ aperture, distributed around the ring at the sites of the 12 cryogenics plants, as shown in Figure 7.2. The rest of the aperture of these shafts will be filled with equipment elevator, cryogenics, power, communications, and sump discharge utilities, plus an isolated emergency egress staircase. There may also be up to 4 magnet delivery shafts for the Stage-2 dipoles (16 meters long) distributed around the ring. Dipoles for both Stage 1 and Stage 2 will access the tunnel through the injection ramps to the surface. There may be a similar additional installation ramp at the cluster of straight sections opposite Fermilab. For tunnel ventilation and emergency egress, there will be approximately $48 \mathrm{E} / \mathrm{V}$ shafts (at A-sites, Bsites, mid-sites, plus an additional 24 between) of 15-foot diameter separated by 3 miles maximum. At the Superconducting Super Collider Laboratory (SSCL) the standard was 2.7 miles (see Section 7.8). These egress shafts will be provided with elevators, but, due to the depths, not all will have parallel staircases. The tunnel ventilation system will also provide heat removal cooling for the conventional corrector magnet elements for Stage 1 and their power supplies distributed around the ring.

In addition, at each of the 1710 half-cells, separated by 135.5 meters, there will be a $1-\mathrm{ft}$ diameter $\times 5$-ft deep electronics drawer for instrumentation and power supplies for the correction elements and other local machine components. See Section 7.4.9 and Figure 5.45. 


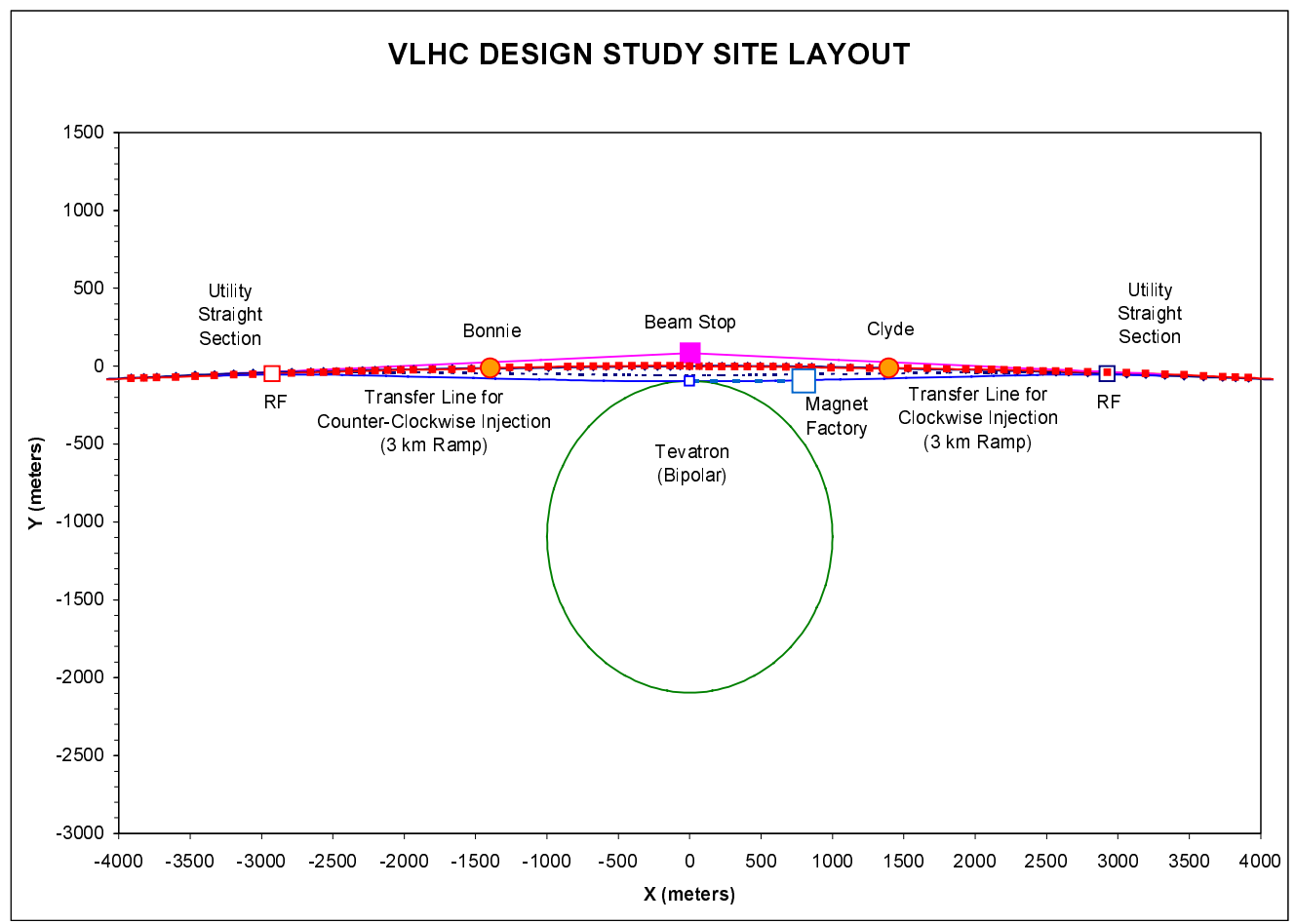

Figure 7.8. Straight section geometry adjacent to Tevatron. See Figure 7.9 for (distorted scale) details.

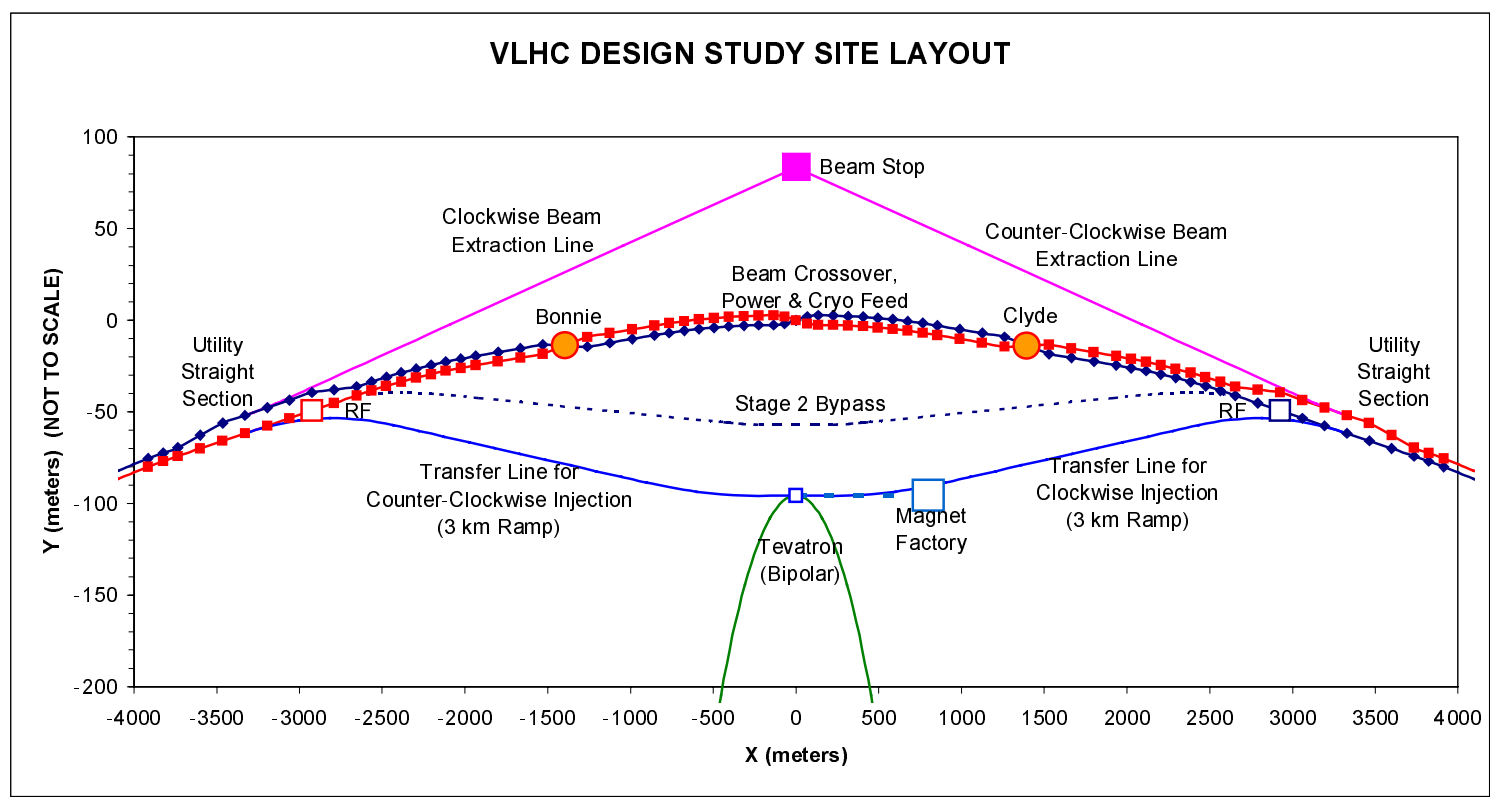

Figure 7.9. Schematic (distorted scale) geometry of straight sections and Stage-2 bypass line. 
Table 7.2. Five straight sections and intervening arcs at Fermilab.

\begin{tabular}{|c|c|}
\hline Major Arc & 174.375 degrees bend - to/from far side cluster of straight sections \\
\hline $\begin{array}{l}\text { Utility Straight } \\
1.38 \mathrm{~km}\end{array}$ & $\begin{array}{l}\text { CW Stage-1 Injection, CW-1 beam scraping, CCW-1 abort, } \\
\text { CW-2 abort, RF cavities with parallel PS and RF Klystron cavern } \\
\text { larger tunnel cross section }-25 \mathrm{ft} \times 25 \mathrm{ft}\end{array}$ \\
\hline $\begin{array}{l}\text { Bend Section } \\
1.00 \mathrm{~km}\end{array}$ & $\begin{array}{l}28.419 \mathrm{mrad} \text { bend at } 35 \mathrm{~km} \text { radius } \\
\text { stub to Stage-2 Low-field ring Bypass tunnel }\end{array}$ \\
\hline $\begin{array}{l}\text { IR Straight } \\
1.64 \mathrm{~km}\end{array}$ & $\begin{array}{l}\text { experimental hall/cavern - } 100 \text { meters long } \\
\text { with equipment bypass tunnel }-0.55 \mathrm{~km} \text { at } 25 \mathrm{~m} \text { bypass }\end{array}$ \\
\hline $\begin{array}{l}\text { Bend Section } \\
0.72 \mathrm{~km}\end{array}$ & $20.668 \mathrm{mrad}$ bend at $35 \mathrm{~km}$ radius \\
\hline $\begin{array}{l}\text { Short Straight } \\
0.27 \mathrm{~km}\end{array}$ & $\begin{array}{l}\mathrm{CW} \text { and } \mathrm{CCW} \text { beams cross-over, changing magnet apertures } \\
\text { may require slightly enlarged tunnel }\end{array}$ \\
\hline $\begin{array}{l}\text { Bend Section } \\
0.72 \mathrm{~km}\end{array}$ & $20.668 \mathrm{mrad}$ bend at $35 \mathrm{~km}$ radius \\
\hline $\begin{array}{l}\text { IR Straight } \\
1.64 \mathrm{~km}\end{array}$ & $\begin{array}{l}\text { experimental hall/cavern - } 100 \text { meters long } \\
\text { with equipment bypass tunnel }-0.55 \mathrm{~km} \text { at } 25 \mathrm{~m} \text { bypass }\end{array}$ \\
\hline $\begin{array}{l}\text { Bend Section } \\
1.00 \mathrm{~km}\end{array}$ & $\begin{array}{l}28.419 \mathrm{mrad} \text { bend at } 35 \mathrm{~km} \text { radius } \\
\text { stub to Stage- } 2 \text { Low-field ring Bypass tunnel }\end{array}$ \\
\hline $\begin{array}{l}\text { Utility Straight } \\
1.38 \mathrm{~km}\end{array}$ & $\begin{array}{l}\mathrm{CCW}-1 \text { Injection, CCW-1 beam scraping, CW-1 abort, } \\
\mathrm{CW}-2 \text { abort, } \mathrm{RF} \text { cavities with parallel PS \& RF Klystron cavern } \\
\text { larger tunnel cross section }-25 \mathrm{ft} \times 25 \mathrm{ft}\end{array}$ \\
\hline Major Arc & 174.375 degrees bend - to/from far side cluster of straight sections \\
\hline
\end{tabular}




\subsection{Injection Line Tunnels (Various Scenarios), Beam Abort Lines, Equipment Access Ramps, and Stage-2 Low-Field Ring Bypass}

Options for injecting from the Tevatron to the VLHC are discussed in Section 4.3. For whatever option is chosen, the Tevatron tunnel will have a new line(s) tangential to it. These lines will pitch downward to the VLHC elevation as shown in Figure 7.10. The utility straight section enclosure and interfaces are detailed in Figure 7.11. The major slope of this injection tunnel could be up to $4.2 \%$ grade, sufficiently flat to serve as part of the equipment access $\operatorname{ramp}(\mathrm{s})$. There will be an incentive to cross the boundary between glacial drift and bedrock surface as steeply as possible to reduce both complexity of construction and ground water influx at the interface. The beam injection tunnels will also serve as the equipment access ramps which would branch off from the injection tunnels and continue to the surface. It will be important to design and schedule the construction of this geometry to allow VLHC component installation during beam operations of the Tevatron.

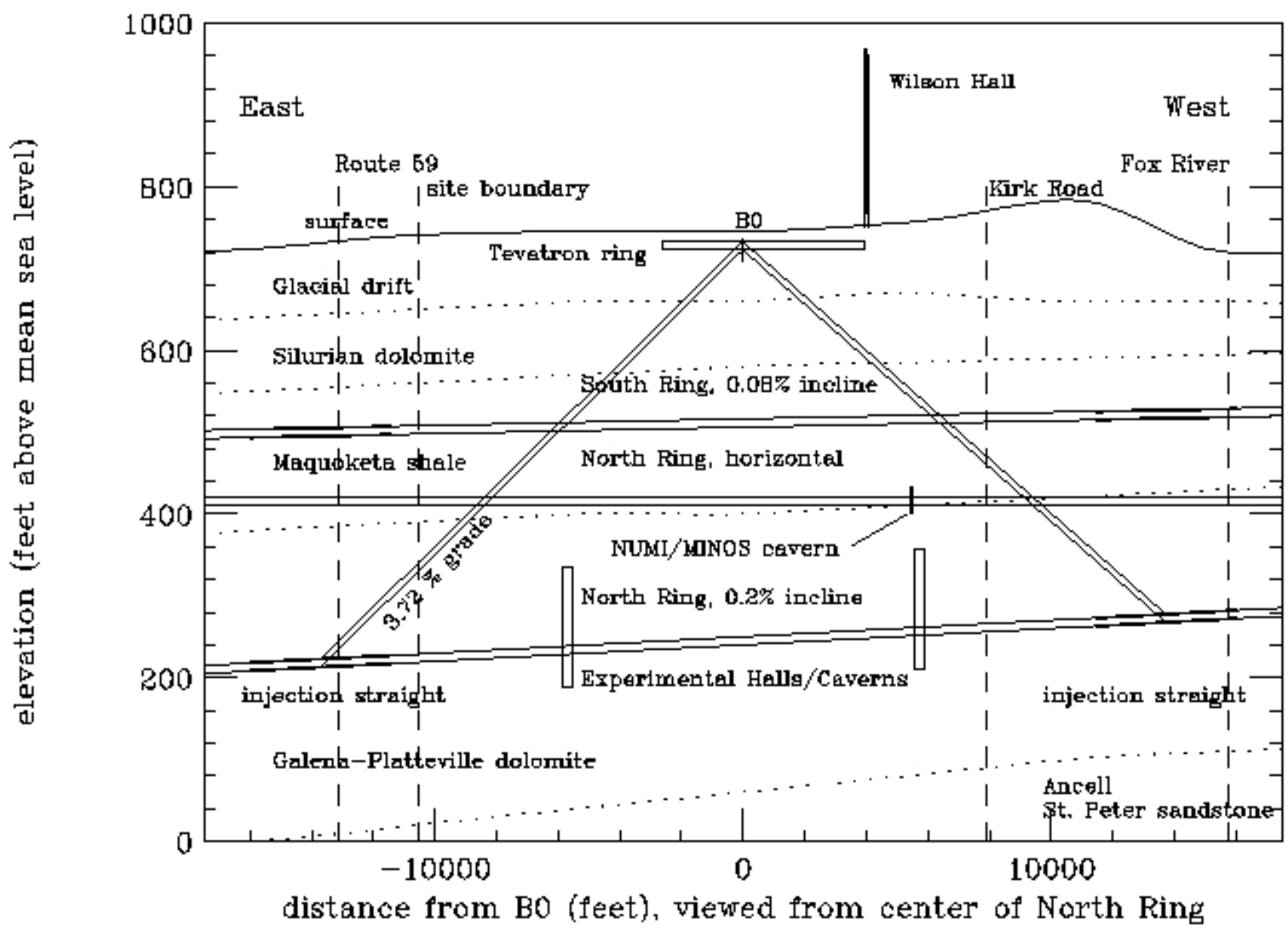

Figure 7.10. Schematic of injection ramps for inclined North Ring configuration.

There also will be two sets of straight tunnels connected to the two near-Fermilab Utility Straight Sections. One set will be for the injection lines from the Tevatron to the Stage-1 lowfield ring and equipment access ramps to the surface. The other set will be for the abort beam lines of both the Stage- 1 and Stage- 2 high-field ring. Figure 7.10 illustrates the bipolar operation and extraction from the Tevatron. Table 7.2 also includes the length of arcs for unipolar 
single beam and unipolar two extracted beams configurations. Some of the lines for injection, abort, and equipment ramps could likely share some common tunnel sections. However, the lines are now listed independently. The interfaces between these tunnels have not yet been designed. To facilitate construction and installation, it will be important to configure the equipment ramps to allow access to the VLHC tunnel while the Tevatron program is in operation.

It is planned to operate the Stage-1 VLHC with the low-field, superferric magnet ring. For Stage 2, a high-field superconducting ring will be added. The low-field ring will serve as injector to the high-field ring. The beam transfer from low-field ring to high-field ring is anticipated to occur in the straight sections opposite Fermilab. In order to remove the second, non-colliding beam and reduce crowding in the experimental areas and also allow possible access to the experimental halls/caverns while the low-field ring is performing accelerator studies, a small section (approximately $7 \mathrm{~km}$ ) of the low-field ring will move to a bypass tunnel. In Stage 2 additional and stronger bends will be added to the low-field ring in this region to match its beam arc length to that of the high-field ring through the main tunnel. In the current model, stubs will be provided at the 28.4-mrad bend enclosures to allow later construction of this bypass tunnel for the low-field ring in Stage 2. No such bypass is planned for the far-side cluster of straight sections. All of these special tunnel sections are anticipated to be of the same inner finished diameter as that of the main tunnel.

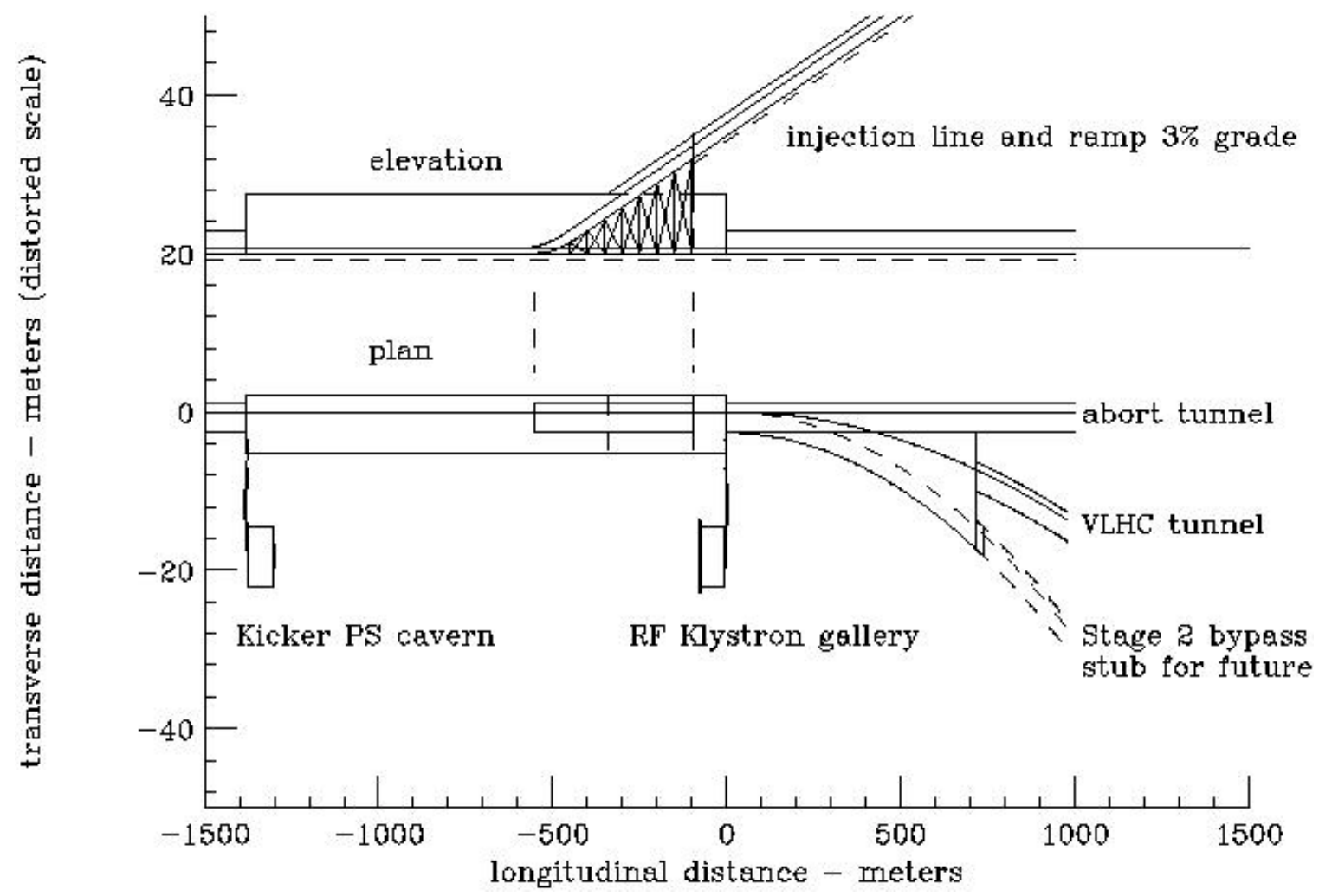

Figure 7.11. Utility straight section cavern, interfaces, and injection/equipment ramp. 


\subsection{Accelerator Utility Caverns (Includes Special Requirements For Abort System)}

Where detailed specifications were lacking at this time, many of the design features for the SSCL [11] were used as a temporary model.

Table 7.3. Special tunnel sections (same finished diameter as main tunnel).

\begin{tabular}{|l|c|l|l|}
\hline Function & $\#$ & Length ea. & Comments - all located at Fermilab site \\
\hline Access Ramps & 2 & $<1 \mathrm{~km}$ & $\begin{array}{l}\text { Branch from Injection Lines near surface, } \\
\text { up to 4\% grade to surface (745 ft msl) } \\
\text { (possibly 1 access on side opposite Fermilab) }\end{array}$ \\
\hline Abort Lines & 2 & $3.7 \mathrm{~km}$ & at tunnel level to common abort/beam stop cavern \\
\hline Injection Lines & 2 & $\begin{array}{c}\text { Varies: } \\
3.5 \mathrm{~km} \\
\text { or } 5.7 \mathrm{~km} \\
\text { or } 3.7 \mathrm{~km}\end{array}$ & $\begin{array}{l}\text { Tevatron }(722 \mathrm{ft} \text { msl) to VLHC Utility Straight Section } \\
\text { (bipolar configuration) } \\
\text { (unipolar with } 3.5 \mathrm{~km} \text { radius of curvature) } \\
\text { (unipolar with two extracted beams configuration) }\end{array}$ \\
\hline Exp. Hall Bypass & 2 & $0.55 \mathrm{~km}$ & radius = 2.0 km, offset =25 meters \\
\hline LF Ring Bypass & 1 & $7.0 \mathrm{~km}$ & Stage-2 construction only, radius = 2.0 km \\
\hline
\end{tabular}

\subsubsection{Beam Stop Enclosure (1)}

A single beam stop enclosure will be located on the Fermilab site at the elevation of the main tunnel. It will house the water cooled beam stop, which will simultaneously service the four rings: low-field $\mathrm{CW}$, low-field $\mathrm{CCW}$, high-field $\mathrm{CW}$, and high-field $\mathrm{CCW}$. The maximum time-averaged power anticipated for the beam stop is estimated to be $100 \mathrm{~kW}$ for the Low Field Ring and an additional $200 \mathrm{~kW}$ for the High Field Ring. This is based on one full intensity, full energy, two-beam extraction per day. The beam stop enclosure is fed by standard diameter tunnels from the inboard ends of the two Utility Straight Sections at the Fermilab site. By putting all four beam stops at the same location, this plan minimizes the number of places where high energy densities and high radiation fields are encountered, and keeps this under the Fermilab site. The size of the beam stop enclosure will be $16 \mathrm{~m} \times 16 \mathrm{~m} \times 40 \mathrm{~m}(\mathrm{~W} \times \mathrm{H} \times \mathrm{L})$. It will have the necessary shielding and water-impervious retention linings to prevent radioactive contamination of the bedrock or groundwater, either by prompt or residual radiation or by coolant leakage.

A reservoir cavern below the cavern elevation will prevent contamination of the tunnels due to leakage of beam stop coolant. This reservoir cavern will allow monitoring of the radioactivity and other contamination levels before proper discharge. There will be a 30 foot diameter equipment access shaft and a 15 foot diameter personnel access shaft at this enclosure site. 


\subsubsection{RF Klystron Tube Enclosure (2)}

SSCL specifications are used in this section. Adjacent to the utility straight sections, the Klystron Tube Galleries house the RF driver tubes and power supplies for the RF cavities. The Klystron galleries are intended to be accessible by personnel for servicing systems during operation. The position of the gallery is illustrated in Figure 7.11. The Klystron Galleries are 25 feet $\times 25$ feet $\times 240$ feet long, serviced by a 30 -foot diameter equipment and personnel shaft. This length will accommodate RF-systems for both Stage-1 and Stage-2 machines. They are located a minimum of 30 feet from the accelerator tunnel and are each joined to the RF Cavern by four 30-inch diameter feed throughs, and a 12-foot diameter labyrinth corridor. The total electrical power levels and LCW cooling capacities needed for the RF systems are $4 \mathrm{MW}$ for Stage 1 and an additional 40 MW for Stage 2, distributed over the two enclosures.

\subsubsection{Kicker Magnet Power Supply Enclosures (4)}

SSCL specifications are used in this section. The Kicker Magnet Power Supply Enclosures house the power supplies for injection and abort (at the Fermilab site) and for beam transfer from Low-Field to High-Field Rings (opposite side). These enclosures are adjacent to the four Utility Straight Sections. The position of the enclosure is illustrated in Figure 7.11. The Kicker Power Supply Enclosure is 25 feet $\times 25$ feet $\times 200$ feet long, serviced by a 15 -foot diameter equipment and personnel shaft. This length will accommodate both Stage- 1 and Stage- 2 machines. It is a minimum of 30 feet from the accelerator tunnel and is joined to the Utility Straight Section by four 12-inch diameter feed throughs, and a 12-foot diameter labyrinth corridor. Since the kickers are energized by capacitor discharge at a low duty factor, power requirements are minimum and air cooling seems to be sufficient.

The pairs of Kicker Magnet Power Supply Enclosures and RF Klystron Tube Enclosures at the Fermilab site may be combined into single caverns.

\subsubsection{A-site and B-site Cryo Systems Caverns (12)}

Although most of the cryogenic refrigeration equipment will be located on the surface, caverns will be required at the elevation of the VLHC rings for additional cryogenics refrigeration equipment to compensate for the pressure drop due to gravity. For Stage 1, it is anticipated that there be 6 such caverns of dimensions $40 \mathrm{ft}$ transverse $\times 20 \mathrm{ft}$ long $\times 40 \mathrm{ft}$ high located at the A-sites. For Stage 2, it is anticipated that there be 12 such caverns of dimensions $40 \mathrm{ft}$ transverse $\times 20 \mathrm{ft}$ long $\times 40 \mathrm{ft}$ high located at the A-sites and the B-sites. Since these caverns will house vital rotating machinery requiring frequent maintenance, they will be located at 30 feet from the tunnel. This will provide adequate radiation shielding to allow occupancy by personnel during operations of the VLHC. At each of these cryo system caverns, there will be approximately 4 penetrations of 30 inch diameter for transfer of cryogens to the VLHC tunnel. There will be a major $30-\mathrm{ft}$ diameter equipment, utilities, and access shaft, along with a Yshaped switchback for access to the tunnel and radiation shielding. This is depicted in Figure 7.12. There will likely be at least 4 magnet delivery shafts for the Stage-2 dipole magnets (16 meters long) distributed among the A-sites and/or B-sites. 


\subsubsection{Groundwater Collection Caverns and Pumping Stations (6)}

The handling of groundwater seepage during the lifetime of the VLHC project will be a major concern. The tunnel design specifies a maximum average of 50 gallons per minute per mile, or a total of 7,200 gallons per minute. This flow will be directed through conduits through the invert floor to six collection and pumping stations at the A-sites of the cryogenics plants. A gentle slope of the tunnel floor is required to drive this water to the collection/pumping sites. It is necessary to be able to collect at least 1 day's flow of groundwater seepage (1.728 million gallons $=277,400 \mathrm{cu}$. ft. per day for each of 6 pumping sites), in the event of failure of power or water systems. At each of the 6 pumping sites, there will be groundwater collection reservoir caverns. These will have dimensions with a volume equivalent to $65 \mathrm{ft} \times 65 \mathrm{ft} \times 65 \mathrm{ft}$. Emergency back-up generator power will be dedicated for pumping, possibly even a mobile generator. Given this average water influx rate, the power, summed over the 6 sites, for pumping to the surface for the entire tunnel is estimated to be $1.6 \mathrm{MW}$. If required, any monitoring of groundwater influx for contamination, either conventional or radioactive, can be sampled at the collection caverns before discharge. If advantageous, pumping facilities could be distributed over both the $6 \mathrm{~A}$-sites and the $6 \mathrm{~B}$-sites. To facilitate maintenance, the groundwater pumps should be accessible during accelerator operations. See Figure 7.12.

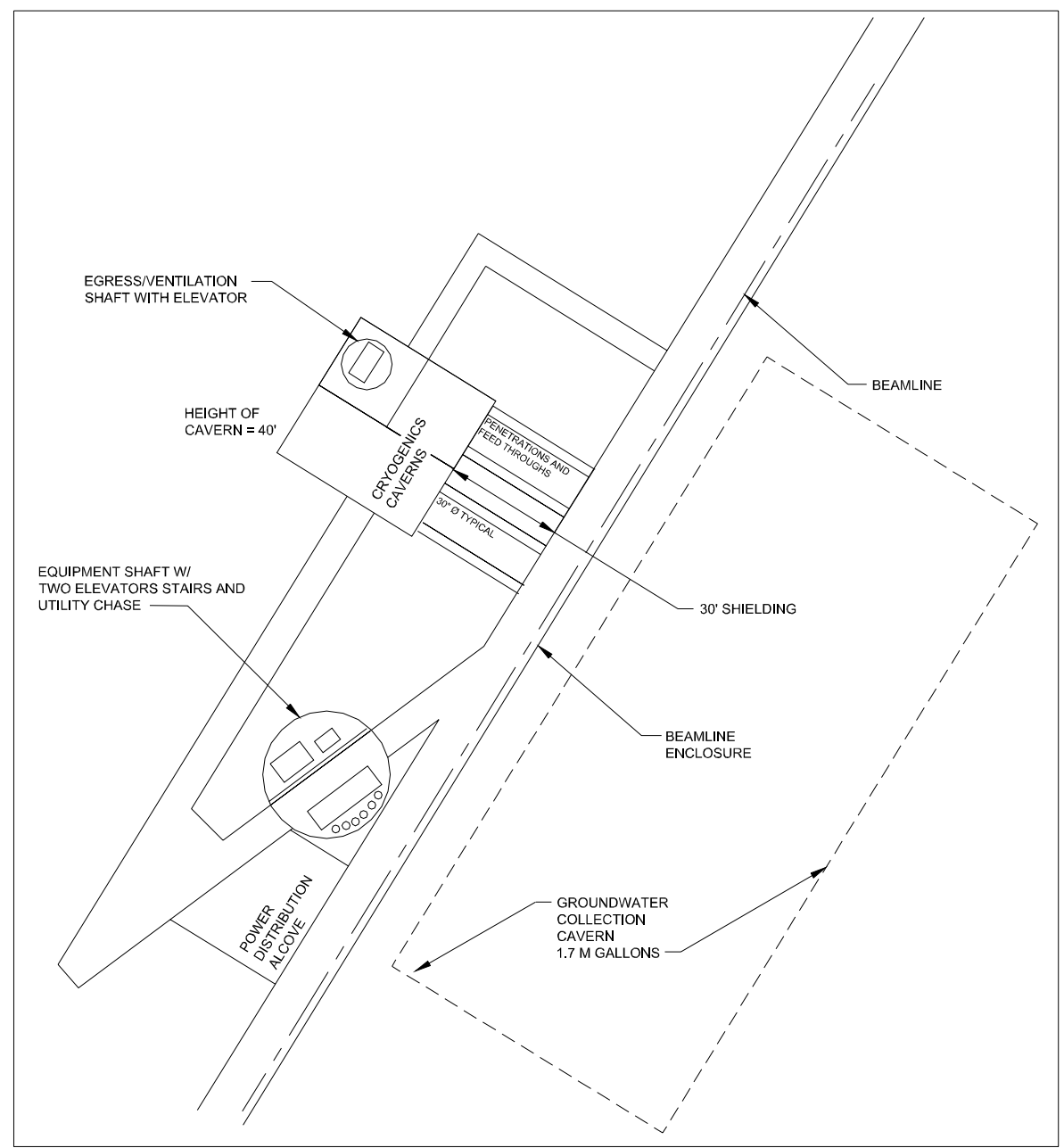

Figure 7.12. A-site and B-site underground enclosures/caverns. 


\subsubsection{AC Power Distribution Alcoves (24)}

These are alcoves cut into the aisle side of the tunnel wall every $10 \mathrm{~km}$, each $27 \mathrm{ft}$ transverse $\times$ $25 \mathrm{ft}$ long $\times 10 \mathrm{ft}$ high, which house transformers, switch gear, and breakers to step down the $13.8 \mathrm{kV}$ feeders fed through the tunnel from the cryo sites to 480/277 and 120/208 VAC for further distribution around the ring. For redundancy, there will be four $13.8 \mathrm{kV}$ transformers per alcove. All components will be dry, without oil, to minimize fire hazards and environmental concerns below ground. Although the caverns are sized for the full Stage-1 plus Stage-2 complement, half of these components would initially be installed, with the second half installed for Stage 2. This cavern is shown as part of Figure 7.12 and Figure 7.13. These AC power distribution alcoves will be located at the A-sites, B-sites, and mid-sites. There will also be a trolley-type electrical bus on the ceiling, running the length of the tunnel, to provide power for the vehicles for personnel, component transport and installation, and logistical support. This could also provide distribution for welding, temporary lighting, and other applications not requiring high quality power.

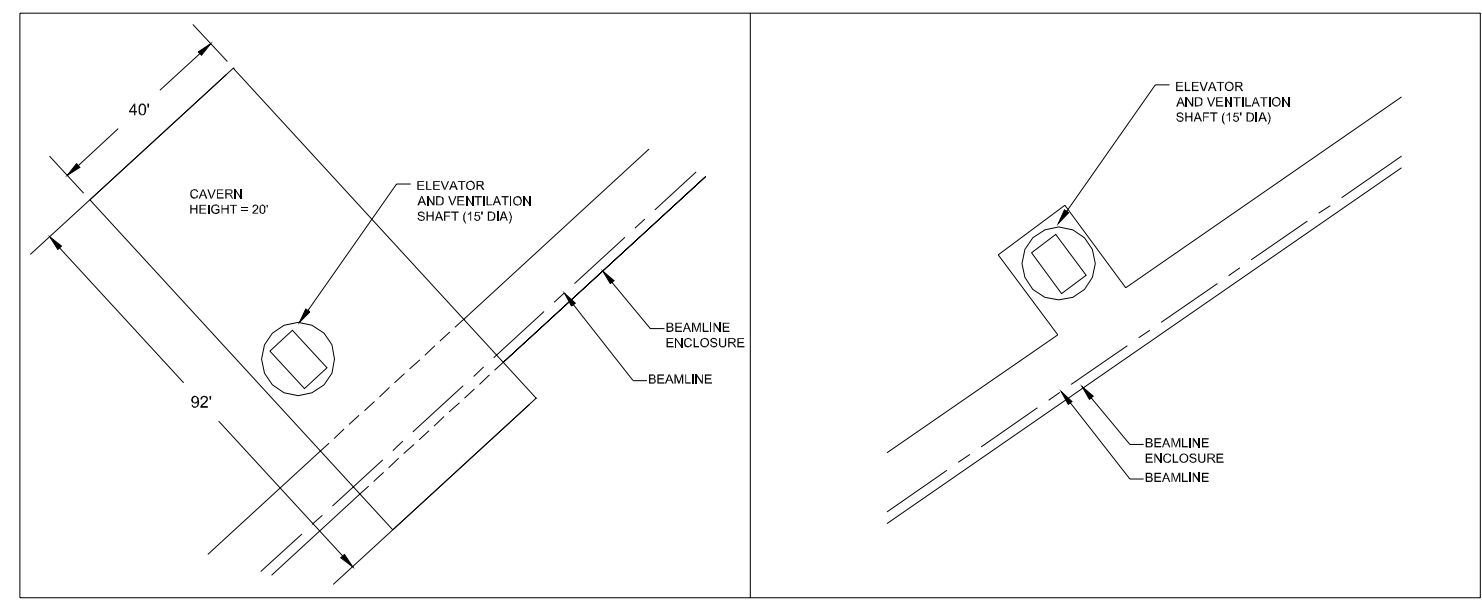

Figure 7.13. Mid-site and E/V underground enclosures/caverns.

\subsubsection{Cryo Valve Alcoves (12)}

For each of the 12 mid-sites, between the A-sites and B-sites, there will be small alcoves, $10 \mathrm{ft}$ transverse $\times 20 \mathrm{ft}$ long $\times 15 \mathrm{ft}$ high, cut into the tunnel wall behind the accelerator rings with access stairs and catwalks. These allow more room for the installation of the cryogenic turnaround boxes and valves for magnet cool down and flow control and manipulation of bayonet cryogenic couplers. There will be an additional cavern $30 \mathrm{ft}$ transverse $\times 20 \mathrm{ft}$ long $\times 20 \mathrm{ft}$ high to house the personnel staircase/beam overpass and a small chain fall crane for equipment. This cavern is shown as part of Figure 7.13. These cryo valve alcoves will be located only at the mid-sites.

\subsubsection{Quench Resistor Caverns (12) - Stage 2}

For Stage 2, quench protection resistors will be placed at the mid-sites. This will require caverns of size $40 \mathrm{ft} \times 30 \mathrm{ft} \times 15 \mathrm{ft}(\mathrm{W} \times \mathrm{L} \times \mathrm{H})$ to house the resistors and cooling systems. 
This cavern is shown as part of Figure 7.13. These quench resistor caverns will be located only at the mid-sites.

\subsubsection{Electronics Drawers (1710)}

Small cylindrical enclosures, $5 \mathrm{ft}$ deep $\times 1 \mathrm{ft}$ diameter, are bored into the aisle side of the tunnel wall. These will house low power supplies for the trim and corrector magnets for both rings, along with instrumentation and controls. The electronics components will be isolated from the tunnel by a fire/access door, and the small heat load will be cooled by convective cooling of a heat exchanger plate by the tunnel air flow. The electronics drawers will be humidity controlled. The equipment contained in this electronics drawer will be on rolling racks allowing access when extracted into the tunnel aisle. This is illustrated in Figure 7.6.

\subsection{Experiment Caverns and Bypasses}

SSCL specifications are used in this section. There is provision for two experiment caverns and installations in the IR straight sections at the Fermilab site. These caverns, major access shafts, and associated surface structures for experimental apparatus fabrication, staging, and operations, will be located on the Fermilab site to minimize land procurement. The model chosen is that of the designs of the caverns for the GEM and SDC experiments at the SSC [11,12]. It is understood that these caverns were optimized for a $20 \mathrm{TeV} \times 20 \mathrm{TeV}$ collider, analogous to the Stage-1 VLHC Collider, and specifically not for the $87.5 \mathrm{TeV} \times 87.5 \mathrm{TeV}$ Stage-2 VLHC Collider. At this stage of this exercise, it is not fully appreciated how the cavern needs scale with collider energy. The current model assumes that there is only one pair of experiment caverns, placed along the tunnel of the Stage-2 ring. For Stage 2, the low-field ring will be displaced through its low-field ring bypass tunnel, away from the experiment caverns. There will not be interaction regions or experiment caverns in the Stage-2 low field bypass. For Stage 2 , the experiments will have to upgrade to the four times higher energy. The upgrade will include a vertical change in the position of the interaction point, due to the difference in elevations of the low-field and high-field rings.

Structural features and thickness of the strata supporting the spans of the experimental halls or caverns [2] will determine the elevations at which the caverns can feasibly be sited. This, of course, determines the elevation of the collider tunnel at the site of the cavern. A general rule of thumb is that there should be a depth of rock (dolomite) strata above the cavern at least equal to the span of the cavern. The previous study has concluded that for 75-foot high chambers, roof spans up to 125 feet are feasible using standard methods of roof arching and rock supports. This assumed that the orientation axis of the cavern bisects the major joint sets. The joints run approximately NE-SW $\times$ NW-SE so this criterion would be satisfied by VLHC ring orientations to the North, South, or West. Possible options in elevation for the experiment caverns are depicted in Figure 7.5.

Cost optimization of the combination of geology, structural strength of strata, height of dolomite in spans, strength of walls, requirements of radiation shielding, and equipment access all determine the elevation of the experiment caverns and the collider tunnel elevation at the Fermilab site. 
The experiment caverns are envisioned to be $30 \mathrm{~m} \times 45 \mathrm{~m} \times 100 \mathrm{~m}(\mathrm{~W} \times \mathrm{H} \times \mathrm{L})$ similar to the SSC model depicted in Figure 7.14.

The current model does not have special underground cryogenic plants to service superconducting experiment magnet systems. Special cryo plant alcoves would be added parallel to, and isolated from the experimental halls. Similarly, the experimental halls would be isolated from the accelerator tunnel to minimize the Oxygen Deficiency Hazards associated with the superconducting accelerators.

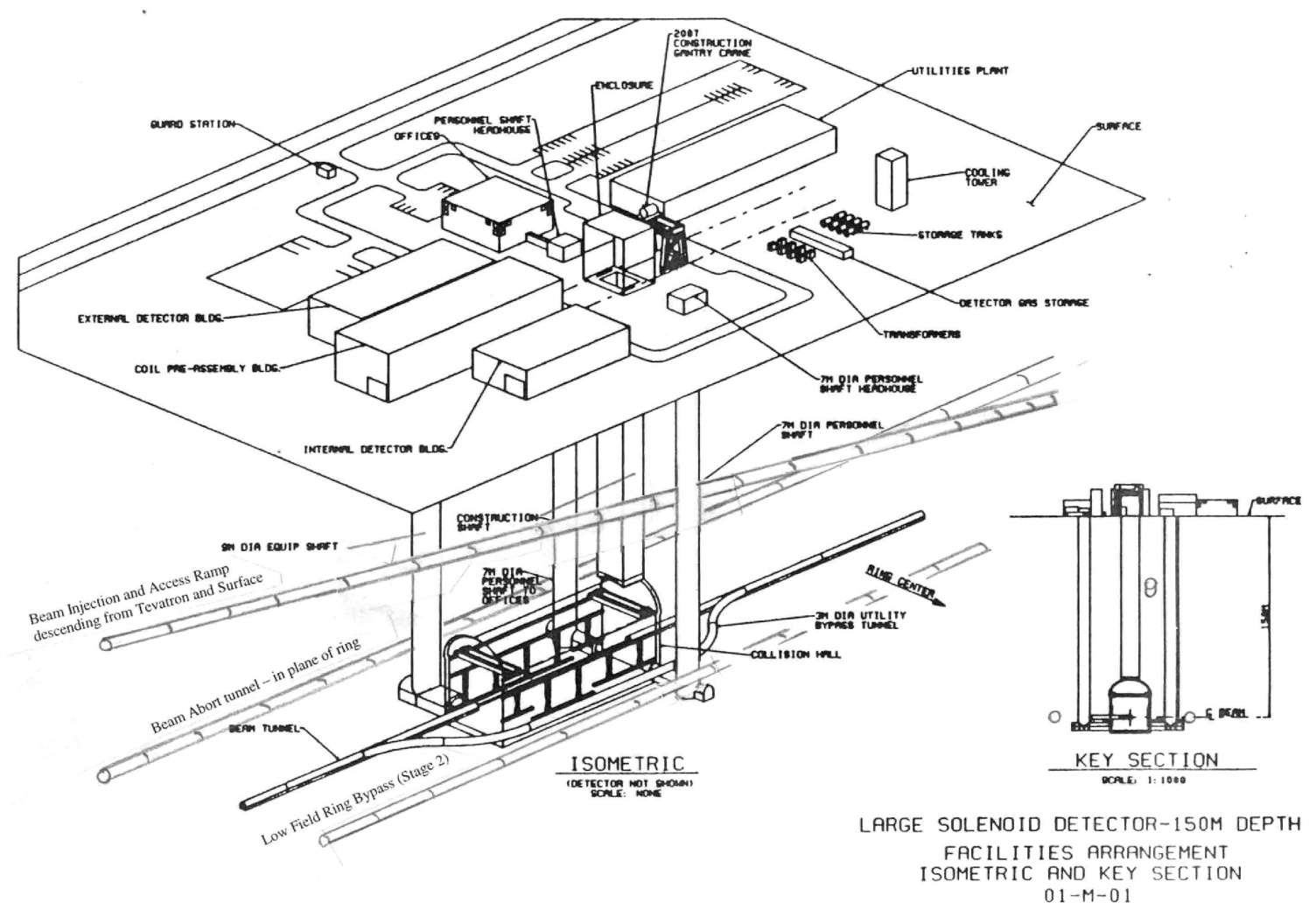

Figure 7.14. Experimental area, isometric view, modeled on SSC generic Large Solenoid Detector.

A tunnel bypass, to allow free access and transport of accelerator personnel, utilities, and equipment without entering the experiment caverns will be included. A 25 -meter maximum offset can be accommodated with 2-km radius bends over a total bypass length of 550 meters (including the 100-meter length of the experimental halls). This would be of the same diameter as the main tunnel. Likewise, the tunnels for the abort line and the sloped tunnels for the injection lines/access ramps will pass nearby the experimental halls. 
Table 7.4. Experimental facilities (SSC specs).

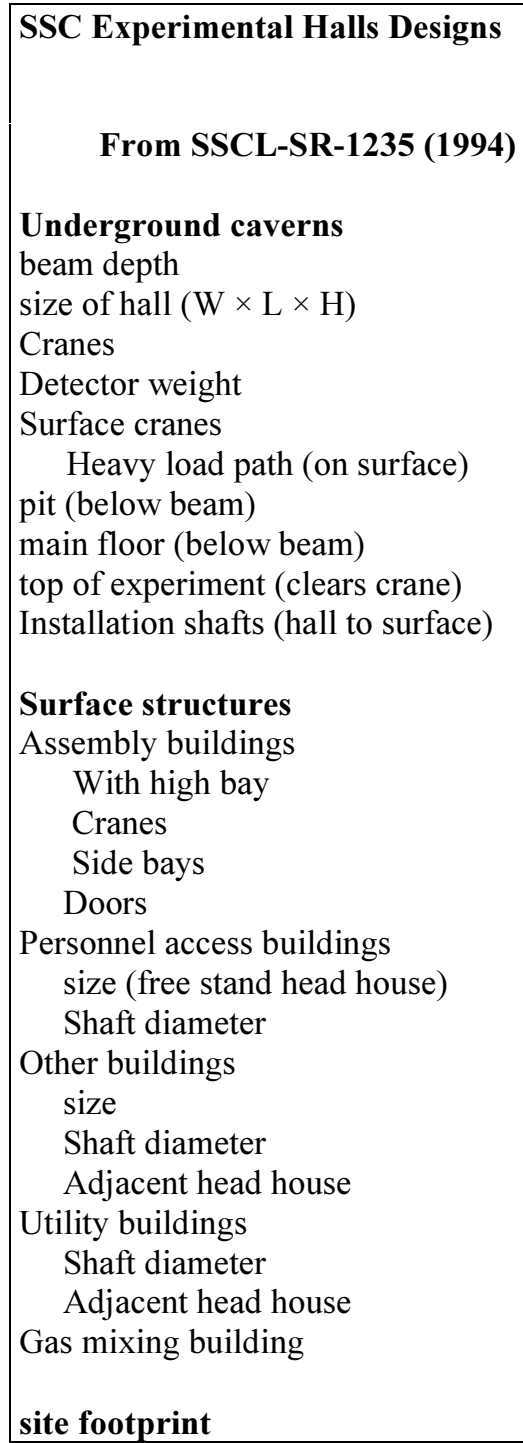

GEM IR-5
50
$30 \times 100 \times 45$
$2 * 100 / 20$
11,000
gantry ??
1600
NA
-13
+10.5
$2 * 28 \times 18$

GEM North

$64 \times 110$

$30 \times 110$

$2 * 45 / 10$

1 story

??

SDC IR-8
50
$30 \times 105 \times 45$
$2 * 100 / 20$
40,000
gantry $100 / 20$
$? ?$
-13.9
-6.8
+10
$2 * 11 \times 18$

SDC AB

$60 \times 134$

$30 \times 134$

$50 / 100+220 / 5$

1 story

$14 \times 12+18 \times 12$

$10 \times 11$

$13 \times 5$
$13 \times 18$

EQ access

$15 \times 20$

utility $13 \times 17.5$

NA

$24 \times 80$

NA

$\mathrm{NA}$

$16 \times 13$

\section{9 meters dia.}

Detector Ops

$24 \times 442$ story

cable $9 \mathrm{~m}$ dia

$12 \times 11$

$24 \times 80$

$10 \mathrm{~m} \mathrm{dia}$

$14 \times 14$

$17 \times 10$

26

\section{units \\ meters \\ $\mathrm{m} \times \mathrm{m} \times \mathrm{m}$ \\ Tons \\ Tons \\ Tons \\ Tonnes \\ meters \\ meters \\ meters \\ $\mathrm{m} \times \mathrm{m}$}

units

$\mathrm{m} \times \mathrm{m}$

$\mathrm{m} \times \mathrm{m}$

tons

$\mathrm{m} \times \mathrm{m}$

$\mathrm{m} \times \mathrm{m}$

$\mathrm{m} \times \mathrm{m}$

$\mathrm{m} \times \mathrm{m}$

meters dia

$\mathrm{m} \times \mathrm{m}$

$\mathrm{m} \times \mathrm{m}$

meters dia

$\mathrm{m} \times \mathrm{m}$

$\mathrm{m} \times \mathrm{m}$

\subsection{Surface Buildings, Utilities, Factories, and Footprints}

\subsubsection{Stage 1}

The required surface buildings include the 12 cryogenics service areas, small service areas for the beam stops, RF Klystron tube and the Kicker Power Supply caverns. One of the A-site cryogenics plants and the beam stop and RF Klystron/Kicker PS service areas are anticipated to be on the Fermilab site. In addition, the emergency egress/ventilation shafts will require minimum head houses and footprints, both on- and off- the Fermilab site. The cryogenic service areas will provide equipment and personnel access, collider power supplies, and groundwater pumping stations. For Stage 1, there will be only one plant at each cryogenics Asite. This will minimize the square-feet of building space and power and cooling requirements, and even the land footprint requirements. However, since it seems more appropriate to consider 
land acquisition as a single activity, the total land needs for the Stage 2 are indicated. The major power and heat load will be for the helium compressors. These requirements, also, are greatly reduced for the Stage-1 VLHC. The choice of cooling methodology, either cooling ponds or air cooling towers, will likely be dictated as much by the local aesthetics of a possibly suburban environment as by cost considerations. There will be one power supply station for the $100 \mathrm{kA}$ magnet bus. This will be located at the cryogenics A-site at Fermilab. It will consist of a building, $75 \mathrm{ft} \times 40 \mathrm{ft}$, and an outdoor pad, $20 \mathrm{ft} \times 25 \mathrm{ft}$ for transformer and oil containment. A superconducting transmission line will carry this current to the tunnel via a vertical shaft.

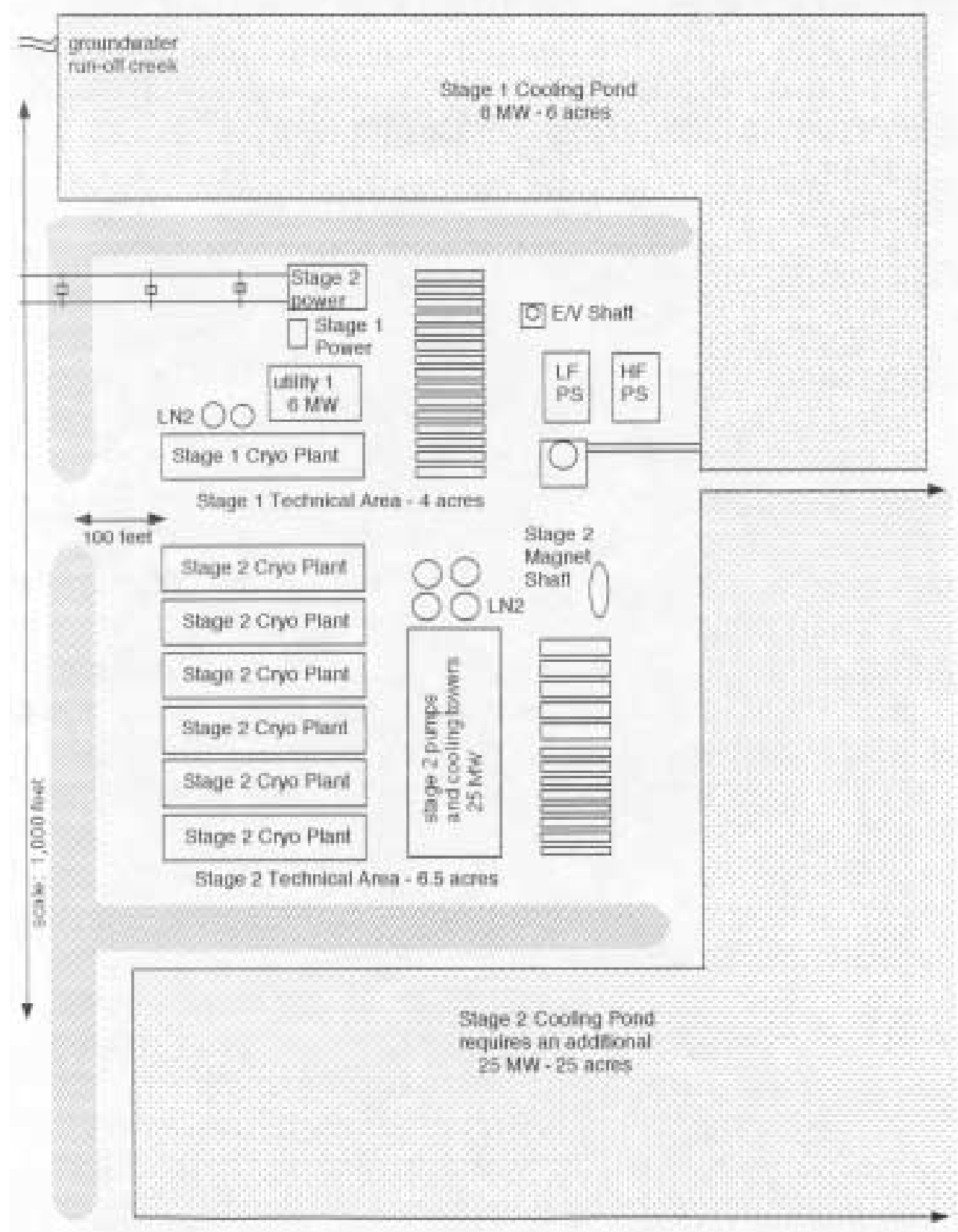

Figure 7.15. Cryogenics and utility plants at A-sites for Stage 1 and Stage 2. 
Adjacent to the two access ramps at the Fermilab site (and possibly at an additional access ramp opposite), there will be the need for a warehouse, assembly, cryo testing, and storage building for final fabrication of the long magnet and cryogenics systems to be installed in the tunnel. See Section 5.1.6. This building will measure $690 \mathrm{ft}$. x $460 \mathrm{ft}$. with a variety of cranes and lifting devices with capacities up to 50 tons. The size, capacity, and complexity of the cryogenics test station/facility associated with these factories for Stage-1 components will be comparable to that of the Fermilab Magnet Test Facility (MTF).

Table 7.5. Sites, functions, and features.

\begin{tabular}{|c|c|c|c|}
\hline Site & Reference & \# & Function and Features \\
\hline \multirow[t]{10}{*}{ A-site } & Sec. 7.4 .4 & 6 & (4) - $30 \mathrm{ft}$ EQ shaft, E/V shaft, AC power distribution cavern, \\
\hline & Sec. 7.4.5 & & - cryogenics cavern, Y-labyrinth, cryogenics penetrations, \\
\hline & Fig. 7.12 & & - beam-on access to cryogenics caverns, \\
\hline & & & - groundwater collection and pumping station, \\
\hline & & & - surface: Stage-1 Cryo Plant, Stage-2 Cryo Plant, \\
\hline & & & Stage-2 Magnet Power Supplies. \\
\hline & & & (1 - at Fermilab, crossover straight) - same as (4) above plus \\
\hline & & & - Stage-1 Magnet Power Supplies \& LCW system. \\
\hline & & & (1-opposite Fermilab, crossover) - same as (4) above plus \\
\hline & & & - LCW system for straight section components. \\
\hline \multirow[t]{5}{*}{ B-site } & Sec. 7.4 .4 & 6 & $30 \mathrm{ft}$. EQ shaft, E/V shaft, AC power distribution cavern, \\
\hline & Fig. 7.12 & & Cryogenics cavern, Y-labyrinth, cryogenics penetrations, \\
\hline & & & beam-on access to cryogenics caverns, \\
\hline & & & surface: Stage-2 Cryogenics Plant \& Stage-2 PS only \\
\hline & & & (undeveloped for Stage 1). \\
\hline \multirow[t]{2}{*}{ mid-site } & $7.4 .6-8$ & 12 & E/V shaft, AC power distribution cavern, cryo valve alcove, \\
\hline & Fig. 7.13 & & quench resistor cavern and cooling system \\
\hline $\mathbf{E} / \mathbf{V}$ & 7.2 & 48 & $15 \mathrm{ft}$ diameter shaft, elevator w/auxiliary power system, \\
\hline (Egress \& & Fig. 7.13 & & HVAC fans, dehumidification, air cooling tunnel heat loads \\
\hline Ventilation) & & & (total of 48 includes those at A-sites, B-sites, and mid-sites). \\
\hline Utility Straight & 7.4 .2 & 4 & Kicker PS Cavern (2 each - at and opposite Fermilab): \\
\hline \multirow[t]{5}{*}{ Section } & 7.4 .3 & & $-15 \mathrm{ft}$ shaft, penetrations to tunnel, Y-labyrinth, \\
\hline & Fig. 7.10 & & - beam-on accessible. \\
\hline & Fig. 7.11 & & RF Klystron Cavern (2 each - only at Fermilab): \\
\hline & & & $-30 \mathrm{ft}$ EQ shaft, penetrations to tunnel, Y-labyrinth, \\
\hline & & & - beam-on accessible, LCW cooling for RF systems. \\
\hline Beam Stop & 7.4 .1 & 1 & $30 \mathrm{ft}$ EQ shaft \& E/V shaft, radioactive water retention, \\
\hline Cavern & & & beam stop cooling: $100 \mathrm{~kW}$ Stage 1 (plus $200 \mathrm{~kW}$ Stage 2) \\
\hline Electronics & 7.4 .9 & 1710 & $1 \mathrm{ft}$ diameter $+5 \mathrm{ft}$ deep - air cooled by tunnel HVAC \\
\hline Drawers & Fig. 7.6 & & \\
\hline Experimental & 7.5 & 2 & see Table 7.4 \\
\hline Caverns & Fig. 7.14 & & \\
\hline Magnet Factory & 7.6 & $1(2)$ & one factory at Fermilab, possibly a second at opposite side. \\
\hline
\end{tabular}

\subsubsection{Stage 2}

The additional facilities for Stage 2 will include greatly increasing the capacity of the six cryogenics A-sites, adding similar enlarged cryo plants at the six B-sites and increasing the 
utilities for the RF Klystron/Kicker PS caverns. There will also be Stage-2 magnet power supply buildings, of similar size and utilities to those of Stage 1, located at each of the twelve cryogenics service sites.

A summary of the various sites, their functions, and underground and surface construction features is presented in Table 7.5.

\subsection{Alignment Issues}

\subsubsection{Tunnel Alignment During Construction}

The technology exists today to attain the specified \pm 4 inches global positional accuracy (bullseye) and relative alignment of the tunnel sections around the ring. This is commonly attained in rapid-transit subway systems and even for elevation of sewer tunnels [10]. This is accomplished with GPS surface measurements translated through normal construction shafts (including horizontal offsets) into a laser reference line and positioning the tunnel boring machine (TBM) relative to that line. By the time of VLHC tunnel construction, it is anticipated that this laser reference and TBM tracking/positioning will be fully computer automated to follow preprogrammed trajectories including curves and slopes within line of sight. Nevertheless, it still will be necessary for the VLHC staff to constantly monitor the construction contractors' tunneling progress, especially with regard to relative alignment of separated tunnel segments being dug simultaneously.

\subsubsection{Reference Network and Component Alignment}

\subsubsection{General Considerations}

This section is a discussion of the errors encountered in machine installation and techniques of building networks for machine alignment. Estimation of possible errors is based on experience from the Main Injector project. Mechanical aspects and specialized alignment tooling are discussed in Section 5.1.8.8.

The ability to transport beam around any synchrotron is directly dependent on how well each component of the machine is installed. The total installation error can be expressed as

$$
\sigma^{2}=\sigma_{\mathrm{n}}^{2}+\sigma_{\mathrm{m}}^{2}+\sigma_{\mathrm{f}}^{2}+\sigma_{\mathrm{s}}^{2}
$$

where:

- $\sigma_{\mathrm{n}}$ are errors in the network

- $\sigma_{\mathrm{m}}$ are errors due to measurement between control points and magnet fiducials

- $\sigma_{\mathrm{f}}$ are errors due deviation between the magnet fiducials and the magnetic center

- $\sigma_{\mathrm{s}}$ are errors due to stand adjustment.

The errors in the stand adjustment $\left(\sigma_{\mathrm{s}}\right)$ are a mechanical design issue. There is a trade-off between cost of the stand and the ability to precisely position the magnet. The ability to move a 
65-meter long magnet without causing undue stress is discussed in Section 5.1.8.3. Also, the lattice designers should determine how precisely to align the dipoles relative to the quadrupoles for a separated function machine. It may be easier to have a lamination pack that can be added to or subtracted from the magnet once it is installed to make up for errors. Once these values are determined a detailed design for a stand can evolve.

The errors between the magnet fiducial and the magnetic center $\left(\sigma_{f}\right)$ are dominated by the quality of the lamination stamping. Experience from the Fermilab Main Injector (FMI) indicates that this can be controlled to $\pm 0.025 \mathrm{~mm}$. This should be an acceptable error for the VLHC.

The errors between the control points and the fiducials $\left(\sigma_{\mathrm{m}}\right)$ are determined by the instruments used and the skill of the people doing the measurements. For the Main Injector, a laser tracker was used for the final installation of all magnet elements. This device is a laser interferometer using a set of motor driven mirrors and a feedback system to follow a corner reflector from a base location to a desired spot. The ultimate accuracy of such a device is \pm 10 microns with an angular resolution of 0.2 milliradians. This is for short distances (less than 50 meters) and in controlled atmospheric conditions. To obtain the best results with such a device a dense network of monuments must be available near the magnets such that multiple and redundant measurements can be made. One of the major advantages of a laser tracker is that a computer logs the data directly thereby avoiding transcription errors. Errors are virtually eliminated if, in addition, a bar code system is in place for the magnets. A database will need to be developed to track the offsets from the magnet to the network. This database will also need to have the values determined from the construction of each magnet and magnetic field measurement.

The dominant error is the network itself $\left(\sigma_{n}\right)$. Since ancient Egypt, all survey networks have the same structure. They consist of a set of inter-linking triangles where the distance from the start to any point in the network is determined by either triangulation or trilateration. Triangulation consists of measuring the length of a base line and all the angles of all the triangles. The law of sines is then used to calculate the distances between each vertex (monument) of the triangles. Since the sine of angles near 0 degrees varies rapidly, angles less than 30 degrees or greater than 150 degrees should be avoided. It is obvious that this is impossible inside the VLHC tunnel.

Trilateration consists of measuring the distances between the monuments in a network and at least one angle. These distances are then reduced to a plane coordinate system for error checking. By using a laser interferometer such as a Kern Mekometer errors are $(0.2 \mathrm{~mm}+0.2 \mathrm{x}$ $10^{-6} \mathrm{x}$ distance measured in $\mathrm{mm}$ ). For the long, narrow geometry of the VLHC tunnel, this is the preferred method.

Using an arc radius of 36,924 meters the longest line of sight (LOS) in the tunnel will be 941.4 meters. The LOS will set the base of the primary network in the tunnel. Given that the magnet end points will be occupied with instruments the exact length of the base line will be slightly shorter. It will be possible to measure from the base line center point to either end point with the Mekometer with an error of $\pm 0.39 \mathrm{~mm}$. These primary base lines will serve a similar purpose to the Murphy line [13] in the Tevatron. They should be located parallel to the quadrupoles in each section. This will keep the necessary offsets constant for each quadrupole.

In the region of dipoles and quadrupoles the primary network will be densified, that is more monuments will be installed near the magnets to give redundant measurements. The accuracy 
of these monuments will depend directly on the primary base line. Error analysis of the Main Injector network [14] indicated a tunnel monument position should be better than $\pm 0.3 \mathrm{~mm}$.

\section{Quad center}

\section{End point}

\section{End point}

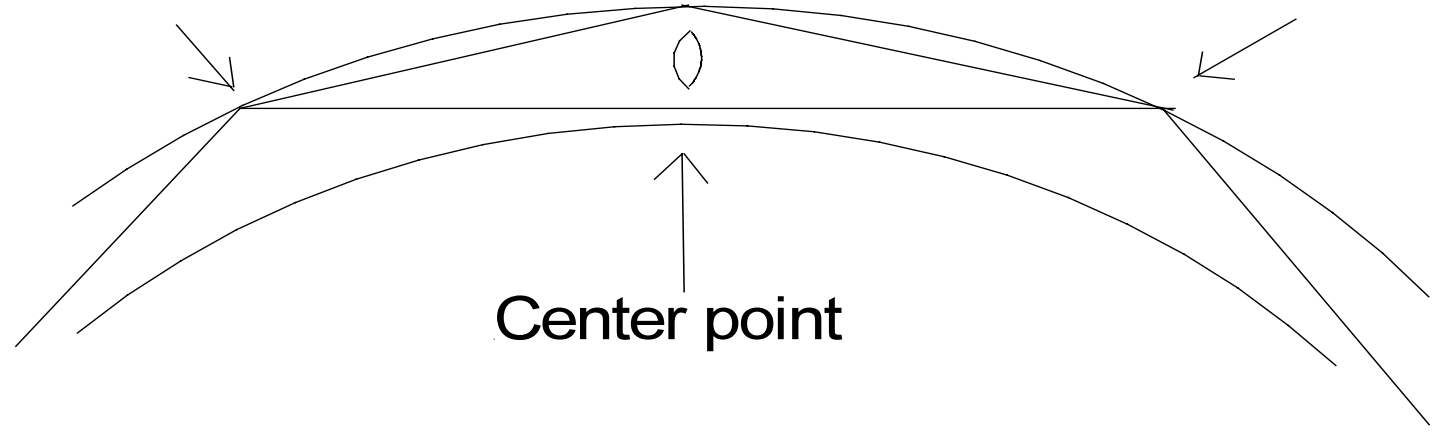

Figure 7.16. Alignment schematic for technical components.

In an ideal world, each of these baseline points would have an alignment hole located directly over it so that solid connection to the outside network can be made. This could require, in principle, up to 250 alignment holes. Given the need to minimize surface disruption, fewer alignment holes will be specified. It is important that connection to the surface be made. A strong surface network using GPS and standard survey techniques will strengthen the underground network [13]. At the other extreme, alignment holes directly over the tunnel reference system were not used at CERN's 27-km long LEP/LHC. Instead, the surface control was transferred through vertical shafts at 8 service and experimental areas and then horizontally transferred to the tunnel control network.

Consideration should be given to the fact that IDOT District 2 has been installing a Class I GPS network consisting of 250 monuments [15]. This is in the area north of Interstate 80 and south of the Wisconsin border, west of the Kane-DeKalb county line to the Mississippi River. The errors between the monuments in this area are less than 1 part per 100,000. This can be used as part of the above ground network for the VLHC and save time and effort in the construction phase.

Given the size of the VLHC ring, geodetic techniques will be required to ensure that the above ground network and the tunnel network are designed and installed properly. Care must be taken to account for variation in the geoid and gravity over the entire site. Both will be significant for this machine. It is very possible that there will be significant variations in the local vertical line due to change in the rock density. This needs to be monitored during construction, and measured after the tunnel is complete.

The exact errors in the above ground and primary underground network can be modeled once a detailed lattice and tunnel layout are known.

The requirements for alignment of magnets in the VLHC is approximately $250 \mu \mathrm{m}$ transverse to the beam with a rotation along the beam axis of under $1 \mathrm{mrad}$. Achieving the latter is a standard exercise and requires no particular effort. Therefore we concentrate on the first criterion. 
Magnets at Fermilab are routinely surveyed to $500 \mu \mathrm{m}$. Achieving $250 \mu \mathrm{m}$ would require careful construction of reference points on the magnets and relating the physical references to the pre-determined magnetic center but neither of these problems involves surveying or alignment. The relevant difficulty arises from aligning magnets over the ring circumference. No matter what magnet one starts from a "random walk" error of $250 \mu \mathrm{m}$ per magnet adds to large errors over the ring. A standard geodetic check is "closure", that is traversing the ring once and checking the height of the first magnet measured against its height measured at the end of the surveying chain. Surveys in the Tevatron and Main Injector typically achieve a few $\mathrm{mm}$ of closure; in the VLHC we might therefore naively expect a few $\mathrm{cm}$, which would be unacceptable.

It is important to note that there are two sorts of error:

- Slow walks over a long distance. These will be controlled by the methods discussed below but can be handled through correction magnets.

- Individually mis-aligned magnets will be caught locally and present no special challenges.

\subsubsection{External Global Network}

Judging from our experience at the Tevatron, we would design exterior monuments to anchor our internal measurements located roughly every few km along the circumference. These exterior monuments could be referenced by a variety of techniques both to each other and to other parts of the accelerator complex. Both horizontal and vertical control can then be transferred into the tunnel from the outside control network. For comparison, Figure 7.17 shows the outside control network for the FMI. One may expect additional control points within the area of the ring for the larger VLHC. Experience with the existing laboratory network shows that we can maintain control to about a millimeter over several miles across the site using techniques developed at Fermilab and SLAC. These points serve to anchor the internal measurements and prevent the random walk from growing out of bounds.

\subsubsection{Internal Control Network}

The FNAL tunnels use a system of tie rods and bolts. The tie rods are placed in the walls of the tunnel and the bolts are placed in the floor. The FMI required 463 wall and floor monuments so we would expect an order of magnitude more in the VLHC. The only significant problem with the system arises from the assumption that the walls and floor do not move relative to each other or to the magnets and that there is no overall twist of the tunnel. The external control network will constrain the size of such effects but it will be necessary to monitor the overall motion of the tunnel. We have seen the FMI tunnel sink by a few millimeters on average since construction with local perturbations of three times this much arising from construction on the site and the loading and unloading of dirt for construction. Again long-wavelength motion is not a significant problem but local twists and shifts are problematic for machine operations. 


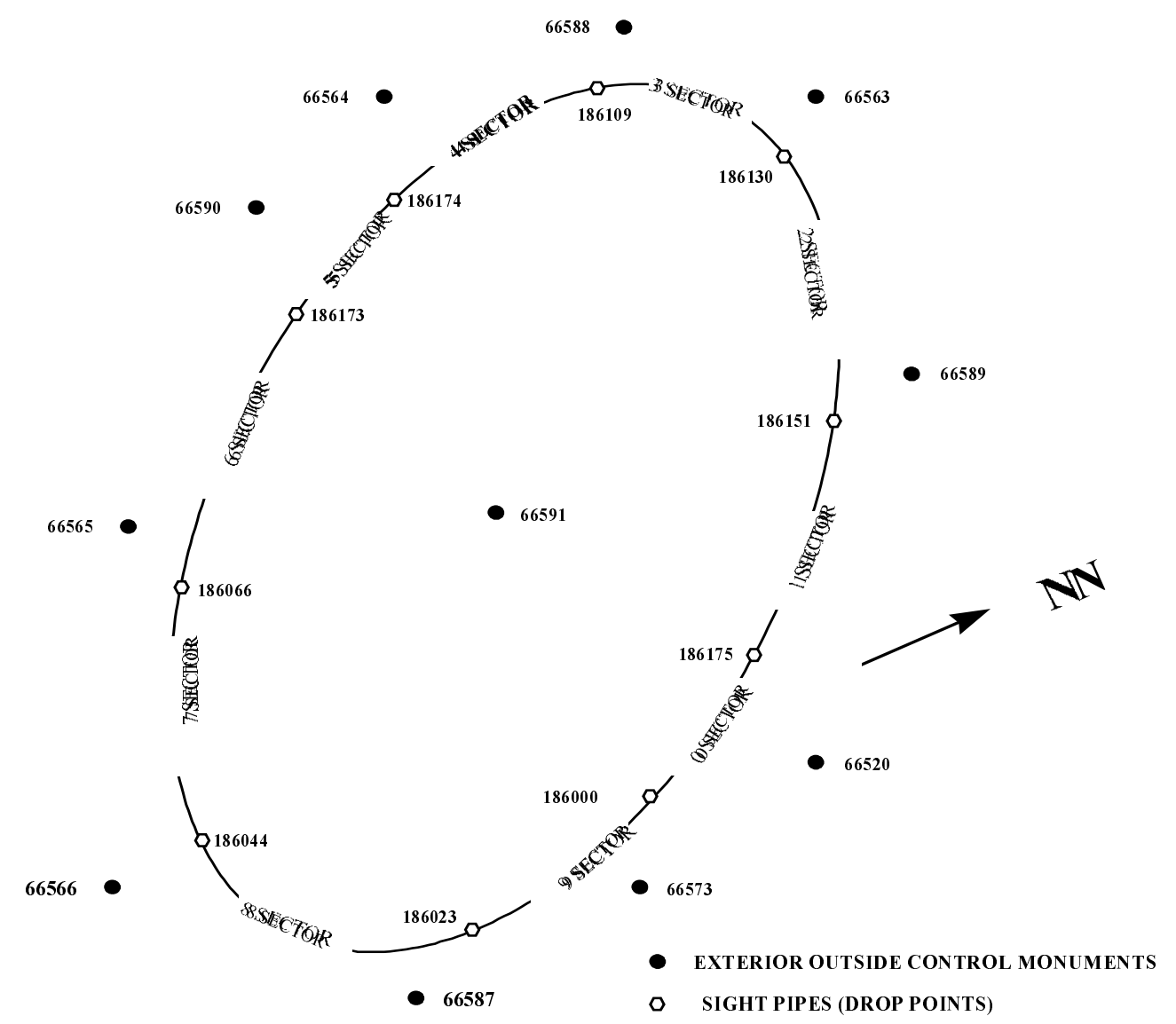

Figure 7.17. FMI external control, drop points (sight pipes, alignment holes), and sector designations.

The actual surveying and alignment of the magnets relies on a laser tracker. A laser tracker uses interferometry to locate a magnet in three dimensions. Combined with optical tooling and the tie rod and bolt system we would construct a network as shown below. Three laser trackers reference the tie rods and bolts relative to each other along the tunnel length as in Figure 7.18. In normal operations we do not measure the locations of the magnets relative to the wall control system but this could be added at the cost of increased time. A survey in the FMI or Tevatron takes approximately a week, with an additional week for analysis. We estimate 40 man-weeks for a full alignment of the VLHC. Such work could go on in parallel using multiple crews.

Using this system we believe a survey to the requisite $250 \mu \mathrm{m}$ locally, with closure of order $1 \mathrm{~cm}$ could be achieved by building on the experience in the Tevatron/FMI complex. The most challenging features would be the scale of the effort combined with careful design of the aboveground network and its referencing to the tunnel coordinates. 


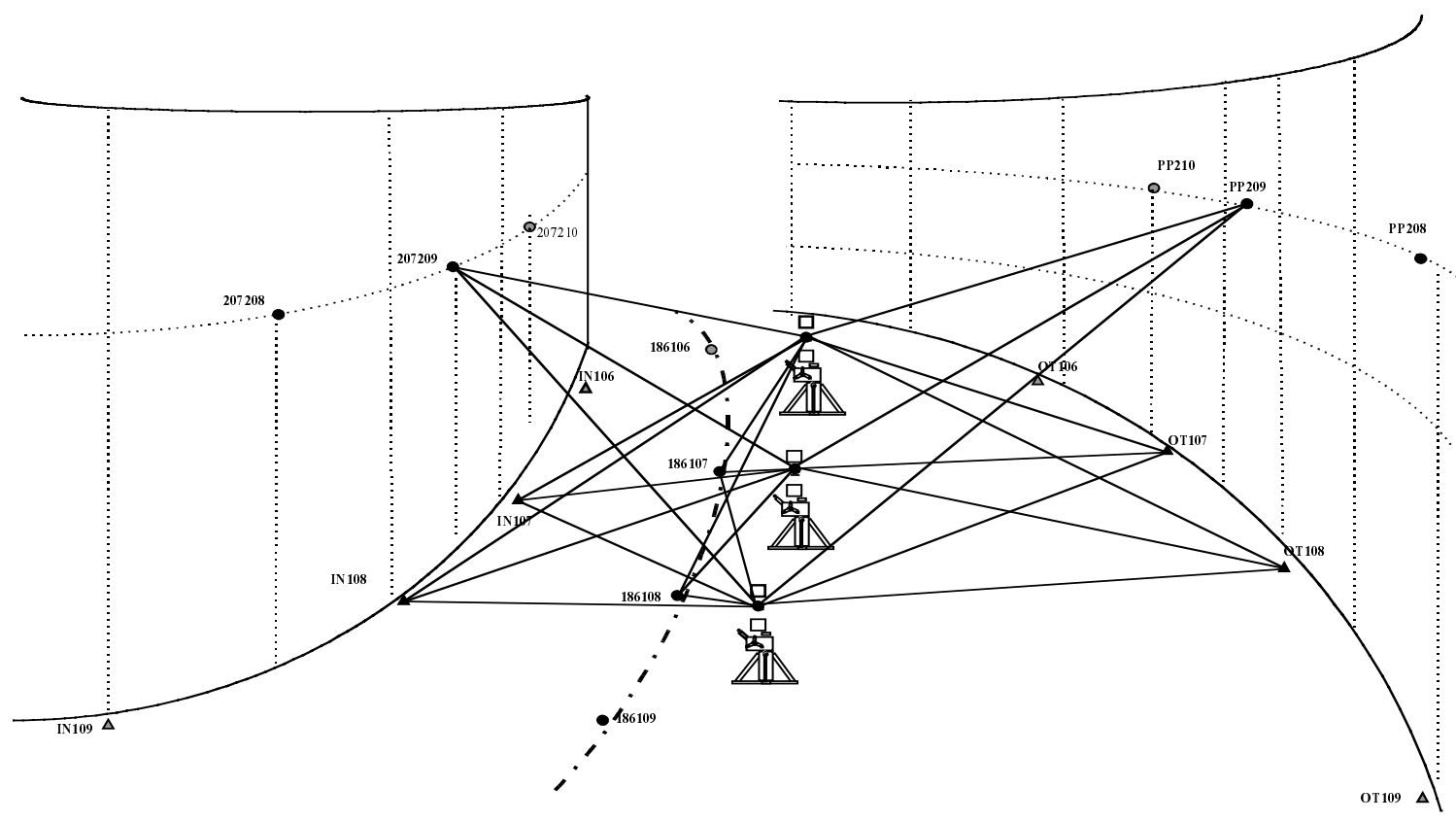

Fig.7.18. Control stations common to three laser tracker setups in the FMI tunnel.

We have worked out a preliminary network for the VLHC which allows us to quantitatively estimate the error on an individual magnet relative to the global network as a function of the number of external alignment holes. We can control the global walk to $2.1 \mathrm{~mm}$ over $8.5 \mathrm{~km}$ of tunnel.

Table 7.6: Global walk vs. distance between alignment holes.

\begin{tabular}{|c|c|c|}
\hline \multicolumn{2}{|c|}{ Distance Between Alignment Holes } & Position Error (mm) \\
\hline $135 \mathrm{~km}$ & $\Rightarrow 2$ alignment holes & 7.0 \\
\hline $68 \mathrm{~km}$ & 4 alignment holes & 4.3 \\
\hline $34 \mathrm{~km}$ & $\Rightarrow 7$ alignment holes & 4 \\
\hline $17 \mathrm{~km}$ & $\Rightarrow 14$ alignment holes & 3.2 \\
\hline $8.5 \mathrm{~km}$ & $\Rightarrow 28$ alignment holes & 2.1 \\
\hline
\end{tabular}

\subsection{ES\&H Issues During Construction, Installation, and Opera- tions (Worker Health \& Safety, Wetlands Restoration, Muck Disposal, Egress, etc.)}

For this exercise, ES\&H issues have been merely identified without detailed study or specification of proposed solutions. Most of the ES\&H issues both during construction and during operations of a VLHC are discussed for generic new accelerator facilities by the Fermilab Committee on Site Studies [16]. This section is intended to focus on issues peculiar to the VLHC and its scale that are in addition to the issues discussed by that Committee. Although it is anticipated that much further ES\&H work will be needed to optimize the costs and operations of a VLHC and satisfy standards to be applicable at the relevant time scales, for the 
purposes of this study, many of the ES\&H requirements of the SSCL [17,18] were assumed for the specifications and costing exercises.

VLHC design/specification issues with ES\&H impact start with the finished diameter of the main tunnel. A larger diameter will optimize installation, operations, and maintenance of the accelerator systems, and improve emergency egress and response. However, increasing the tunnel diameter may increase the overall project cost. Personnel access and egress are issues of the number and placement of minor shafts. After much negotiation, the Department of Energy accepted a minimum egress/ventilation shaft spacing of 2.7 miles for the final operating configuration for the SSC. The Chicago area deep reservoir project (TARP) construction required a temporary personnel access/egress shaft serviced by a crane with man-cage at a maximum of 5000 feet from the deadheaded boring face. Such temporary egress shafts are filled-in upon completion and final finishing of that nearby tunnel section.

In this study, it is anticipated that there will be minimum fire and smoke hazards in the tunnel. The main accelerator bus power supplies will be located on the surface. Power distribution transformers, switch gear, and breakers located locally in the tunnel will be oil-free. Smaller power supplies and electronics will be in special fire and smoke resistive electronics drawers, and interconnecting cables of low toxicity and of low smoke generation. The special underground enclosures for RF klystrons and kicker power supplies, cryogenic systems, and the experiment caverns could be equipped with local automatic fire suppression systems and local ventilation systems. The envisioned VLHC cryogenics systems will not use liquid nitrogen. The cryogenic magnet systems will only be energized while the tunnel is unoccupied. The Stage-1 quench reliefs of the liquid helium systems will be through high pressure piping within the cryogenic transfer lines. With reduced fire, smoke, and oxygen deficiency hazards, it is anticipated that during and after installation of the accelerator components, personnel entering the tunnel will carry sufficient oxygen supplies nearby on their motorized access vehicles, removing the need for safety refuges [18]. The SSCL had refuges, each with two hour fire door and two hour emergency air supply for up to 7 people located not greater than 2500 feet apart, between the emergency egress shafts.

After completion of construction, tunnel ventilation will be provided at the $1 / 3$ volume change per hour level during collider operations (unoccupied), and at the 1 full volume change per hour level during personnel accesses for installation and maintenance. This ventilation is needed to purge $\mathrm{C}^{11}$ resulting from collider operations and possibly $\mathrm{CO}_{2}$ and Radon naturally released from the tunnel rocks. Flow design, controls, and barriers will have to be designed to isolate ventilation problems.

The handling of groundwater will be a major environmental concern throughout the VLHC project. Much of the tunnel, although below the piezometric surface (water table), will be in the Galena-Platteville dolomite, which is classified as an aquitard. Still, grouting of cracks will be required to reduce the post-construction groundwater influx to workable pumping limits. Other strata such as the sandstone and dolomitic sandstone, below the water table, of the South Ring configuration will be problematic from structural and water influx rate viewpoints and likely will require fully lined tunnel sections.

During tunnel construction, there will be additional water influx rates before tunnel grouting and lining, especially at the injection/equipment access ramps where the long and shallowangle traversal of the glacial till-bedrock surface interface may be problematic before tunnel 
lining. There will be the issues of dewatering the aquifer both during construction and operations, prevention of aquifer contamination during construction, and radiological contamination of the soil and aquifer during collider operation. Not only will the volume of water discharged during construction require serious attention, the quality of this discharge, in terms of $\mathrm{pH}$ and suspended solids and other environmental limits, will likely be an issue. Engineered solutions will be required for channeling, storage, pumping, and disposal of groundwater influx into existing creeks nearby capable of accepting this water discharge. The VLHC operational specification for groundwater seepage is an average discharge of 50 gallons per minute per mile of tunnel, corresponding to a total of 7,200 gpm total, distributed over 6 pumping sites, each at 1,200 gpm. Optimization studies will have to be undertaken to balance additional construction costs for water exclusion versus operating costs for dewatering costs over the lifetime of the VLHC, including environmental impacts.

The question of possible radioactive contamination of groundwater systems must be addressed. Preliminary estimates of radionuclide concentrations for a single maximum accidental loss at a point of full energy, full intensity beams indicate that for aquitards (such as Silurian, Maquoketa, and Galena-Platteville strata), an exclusion zone for wells of about 100 feet from the VLHC ring will ensure that federal limits for radionuclide concentration in drinking water will not be exceeded. Similar calculations for contamination due to losses in aquifers will need to be undertaken, including specific local water flow migration and dilution models. Preliminary calculations also indicate that even if all radionuclides produced in a single worst case beam loss accident were to immediately be deposited in one of the full groundwater seepage collection caverns, the concentrations of tritium and $\mathrm{Na}^{22}$ would be comparable to federal limits for surface discharge. Levels and procedures regarding residual radiation of components and walls, and contamination due to beam losses are part of normal radiological operations of this accelerator.

For the Stage-2 high-field VLHC, each of the 12 remote cryogenics sites will be consuming approximately $25 \mathrm{MW}$ electrical power, mainly to run the helium compressors. This power will have to be dissipated to the environment, either by air-cooling towers, cooling ponds (approximately 25 acres each), or combinations of towers and ponds, depending on the particular environmental needs at each cryogenics site.

The disposal of spoils or muck from the underground construction will be an issue. The spoils will have various fractions of clay, dolomite, shale, sandstone, etc. The Illinois SSC Proposal [19] evaluated four scenarios, three of which were to refill nearby previous mining operations and the fourth was in landscaping around the shafts and remote sites. Sale of the dolomite for use as construction material may, or may not, be feasible, depending on market conditions.

Constructing a tunnel will have major surface impact for personnel and equipment access and muck removal. Some of these surface sites will not be needed, or with reduced footprint, after construction. It will be important to have a plan to return these lands back to the citizens of Illinois in a useable and environmentally acceptable condition. 


\subsection{Model of Construction Schedule}

A preliminary impression of a possible construction schedule is based largely on the Kenny study for the $3 \mathrm{TeV}$ VLHC Booster Tunnel [9]. The schedule presented as part of that study included a 34-km circumference tunnel in Galena-Platteville dolomite, four major shafts, and two injection ramps. Other portions of the VLHC civil construction, such as experiment caverns and adits, cannot be extrapolated from the Kenny study, and at this time are only estimates. CNA Engineers [10] are providing costing and scheduling estimates for the entire scope of the VLHC project, including three geological sitings for the tunnel.

This section is intended to provide a straw-man model to begin to understand how the VLHC construction could be accomplished in a finite time scale.

For this schedule, it is assumed that day-one, year $=0$, occurs after an Architectural, Engineering, \& Construction Management contractor has been hired, the final construction designs have been prepared, land procurements and easements have been obtained, and the bidding process for the construction contracts has begun.

It is envisioned that the VLHC construction will be broken up into some number of underground construction contracts. Geologic factors will be considered to attempt to have each contract utilize only one method of tunnel excavation. As a preliminary model, the $233-\mathrm{km}$ ring tunnel could be broken into 6 contracts of approximately $39 \mathrm{~km}$ each, 4 contracts of $58 \mathrm{~km}$ each, or 8 contracts of $29 \mathrm{~km}$ each. Using the Kenny model [9], the tunnel boring phase (TBM) of these contracts would be 5 years, 7 years, and 4 years, respectively, including one year for contractor mobilization and preliminary shafts before beginning the boring operation. These individual TBM crews could start and end at the A-sites or B-sites. There may be advantages to reconfigure to larger number of smaller, or shorter length/duration, tunneling contracts. In the remainder of this section, 6 TBM crews are assumed for the main-ring tunnel.

In addition, there would be another contractor to handle the more complicated Utility Straight through Utility Straight sections (U2U) plus two more contractors for the experimental halls. It is not apparent whether it is optimal to have the TBM contractors also have responsibility for the adjacent side caverns, or whether that is better handled as separate contracts. Managing all these separate, but somewhat interacting contracts will be a challenging coordination and management task.

Although the VLHC ring has natural 6-fold, 12-fold, 48-fold symmetries, the choice of two magnet fabrication plants and associated ramps for transport of the 67 meter long Stage- $1 \mathrm{LF}$ magnets implies that with more than 4 tunneling contractors, two or more sections of the tunnel will be isolated, in that magnets cannot be delivered to these isolated sections until tunnel sections between the sections in question and the magnet ramps are completed. This complication in sequencing tunnel construction and magnet installation will imply a undesired lengthening of the time scale for overall VLHC project completion. The alternative is to add a third magnet factory, with 6 tunneling and 6 installation crews all working in parallel.

In Figure 7.19, a tunnel construction and magnet placement schedule is presented for $1 / 6$ th $(38.75 \mathrm{~km})$ of the VLHC ring. This assumes that four positions for vertical removal of muck/spoils will be used for this section of construction. This would correspond to a maximum of about $10 \mathrm{~km}$ of horizontal muck conveyor to reach the vertical take-out shaft. Although contractors could utilize longer horizontal runs, moving the vertical take-out after every $10 \mathrm{~km}$ 
will allow the tunnel finishing tasks, grouting and laying of the invert floor, and the installation of utilities, AC power, lighting, HVAC, etc., and even the beginning of magnet installation to proceed in parallel with tunnel boring.

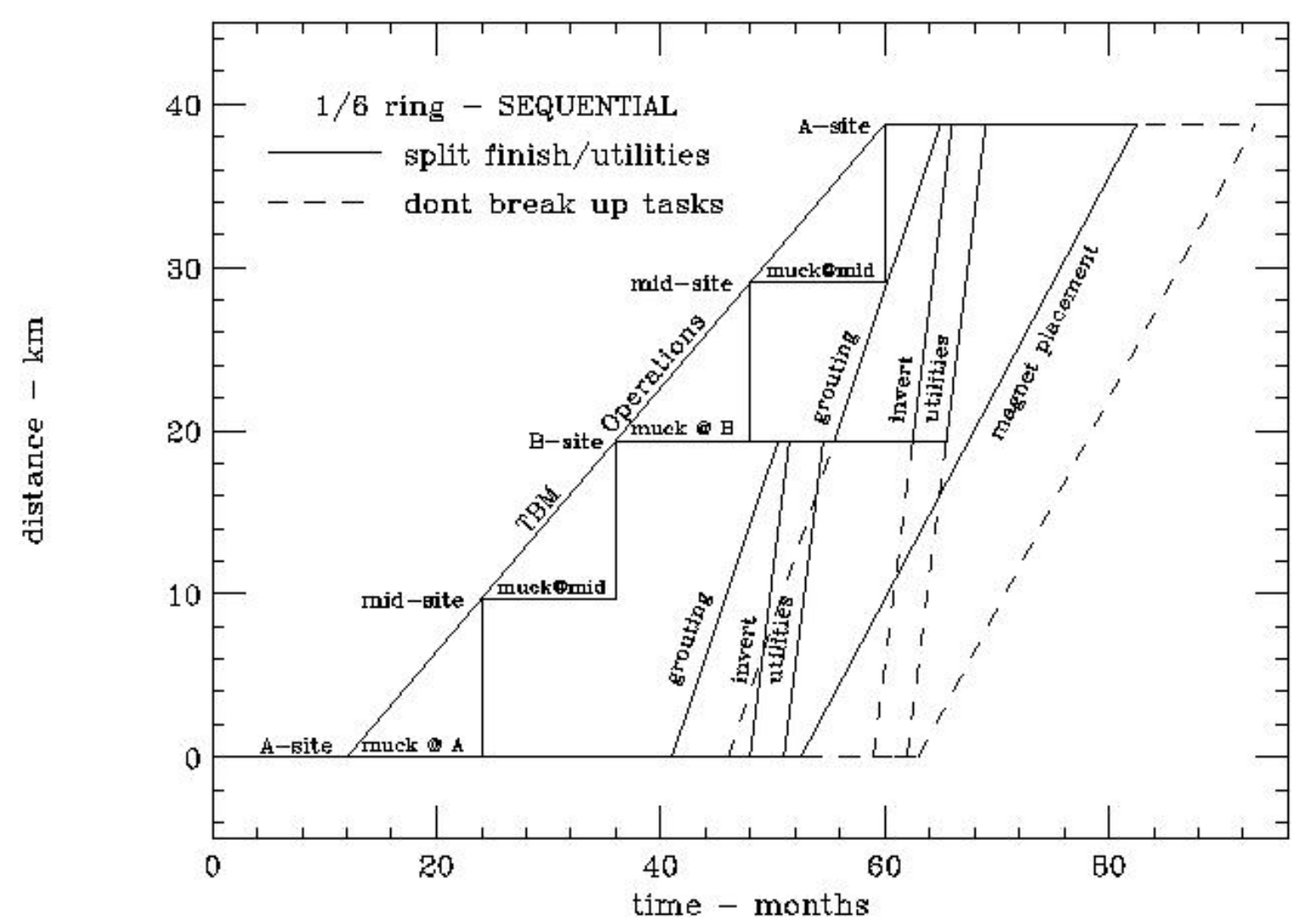

Figure 7.19. Tunneling schedule for $1 / 6^{\text {th }}$ of VLHC ring based on Kenny study of a 34-km ring [9].

This model assumes that the construction of the side caverns can proceed in parallel with minimal mutual interference with nearby TBM operations. This also assumes that the tunnel finishing and utilities operations will begin as soon as boring of the first half of this section is complete. The downside of this early finishing is that these operations will outpace the progress of the tunnel boring and there will be a delay before starting the finishing of the second half of this section. This might incur extra expenses, but it would escalate beginning of magnet installation by almost one year compared to waiting to perform the finishing/utilities operations as one continuous task. (This later start for finishing/utilities, still in parallel with TBM operations, is depicted as the dashed lines in the above figure.)

A more complicated construction area is at the Fermilab site between the utility straight sections (inclusive). A possible scheduling sequence for this $\mathrm{U} 2 \mathrm{U}$ section is depicted in Figure 7.20. This would be done by the 7-th TBM contractor in this model. The most time-critical feature is completion of the injection/installation ramps to the surface and the outboard sections of the widened utility straight tunnels in order to be ready for magnet installation. The completion of the RF klystron tube caverns, the kicker magnet power supply caverns, the equipment and utility bypasses around the experimental halls, the abort lines and beam stop cavern, experimental halls, and the stub-outs for the Stage-2 LF ring bypass (to be installed later) will 
occur after that milestone. Since there are no injection lines at the opposite side magnet factory, the equipment ramp is envisioned to have a much simpler design which could represent a small additional contract or be added to one of the TBM contracts starting from that position. The eighth and ninth underground contractors would have responsibility for each of the two experiment caverns.

A model construction schedule is presented in Figure 7.21. The 3.5 year time scale for construction of the experiment caverns is a pure guess at this time. There is a concern that construction and technical outfitting of the magnet factories will be completed only $3 / 4$ year before tunnel installation of magnets would begin. Finally, the isolated B and E sectors are noted, illustrating how magnets would be filled from the adjacent sectors sequentially. In this report, it has been assumed that there would be 4 crews for magnet installation (one magnet per crew per one shift per day). Cryogenic lines would also need to be installed during this same time period.

\subsection{Construction Engineering \& Design Challenges}

Little real engineering study or design has been devoted so far toward the conventional construction aspects of the VLHC. There are several outstanding issues that must be addressed to go beyond the simplified models presented. Of paramount importance is to reduce the overall cost. This can be done by simplifying the underground construction, performing R\&D on tunneling methodology [20]. This includes optimizing TBM utilization, especially in the region between utility straights.

There is no design yet for the Stage-2 abort. Its design will impact on the design of the utility straight section cavern or widened tunnel. Other issues to be addressed are the stub for the LF bypass for Stage-2. The LF bypass ring with stronger magnets will cross the enclosure at floor level and will need ramps across the aisle.

There are installation issues to resolve. How many magnet delivery shafts will be needed to install the 16-m long Stage-2 HF dipoles? What are the details of the injection/equipment ramp interfaces to the Tevatron and the surface? The experiment caverns will house enormous detectors deep underground. What are the optimal shaft dimensions for installation and assembly of the detector components?

The optimal size for the Stage- 2 experiment caverns where $175 \mathrm{TeV}$ collisions take place needs engineering and physics design optimization of the roof span. The beam heights in the Stage- 1 and Stage- 2 colliders are different but could be made the same where they pass through the detectors. This would have the consequence of introducing a small amount of vertical dispersion, and its effect would require further study. A better option may be to design the detectors so that they could be adjusted in the Stage-2 upgrade to match the new beam height. 

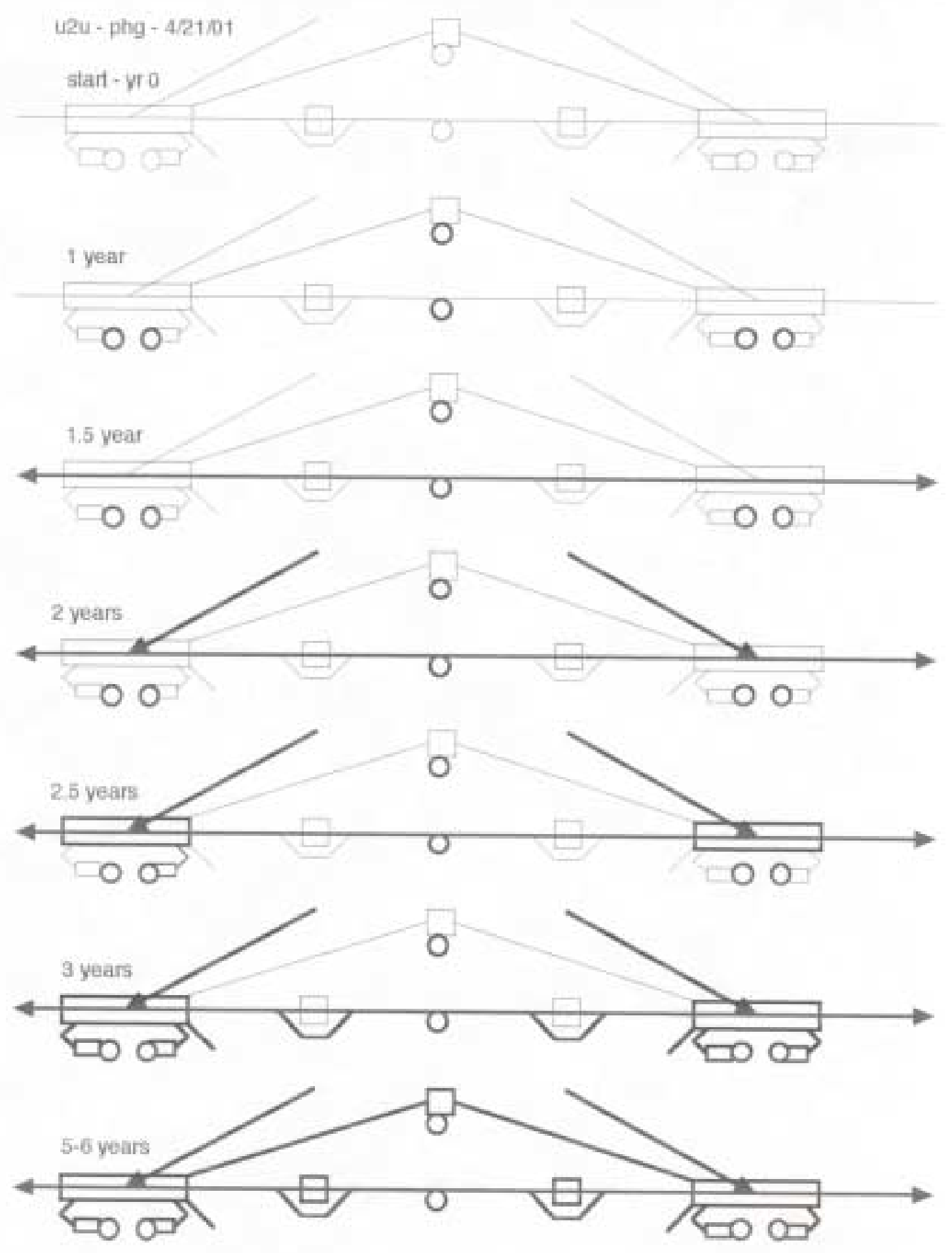

Figure 7.20. A time-line cartoon depicting progress in the utility straight to utility straight section at Fermilab. Bold indicates completion of civil construction. 


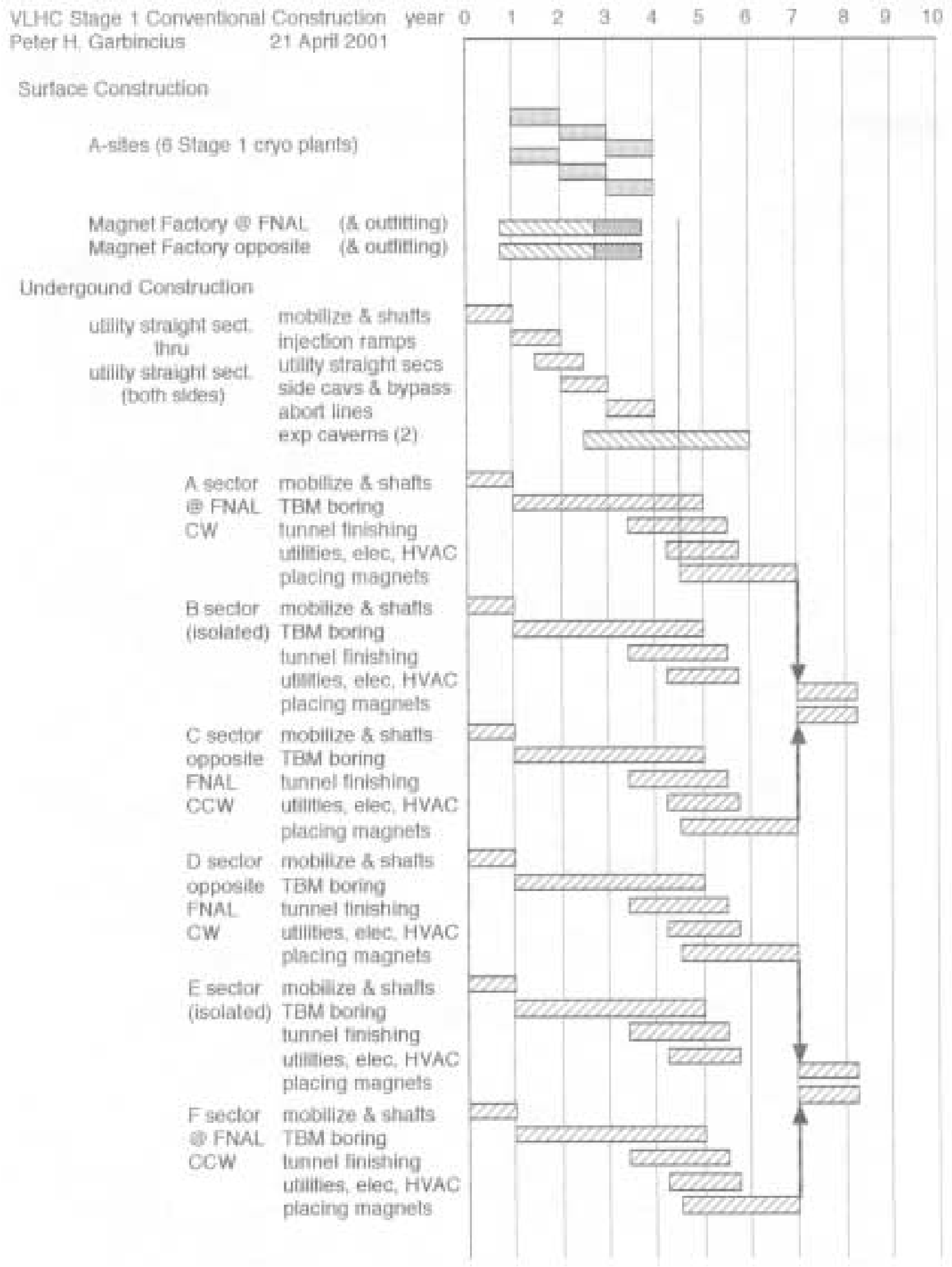

Figure 7.21. Preliminary schedule for VLHC conventional construction. 


\subsection{Cost and Risk Reduction}

A major cost driver for the overall VLHC construction is the underground tunneling. Any improvement in the technology or methodology of constructing the tunnel is likely to have an impact on reducing the overall cost of the project. A preliminary study has already been completed by the Robbins Company, a major international supplier of tunnel boring machinery [20].

Uncertainties in the cost estimate for the construction reflect the state of knowledge of the underground conditions and quality of the rock, soil, and especially water content. "Average" conditions were assumed in this study. However, before a more realistic cost estimate can be prepared, the particular conditions for a specific proposed site must be quantitatively measured. An optimization between the extent (up-front cost) of such studies and acceptable risk will then be required.

\section{References}

[1] J.P. Kempton et al., "Geological-Geotechnical Studies for Siting the Superconducting Super Collider in Illinois: Preliminary Geological Feasibility Report," ISGS Environmental Geology Notes, EGN-111 (1985).

We also thankfully acknowledge the contributions of Joe Lach (Fermilab) to this study, including sharing his experiences with the Illinois SSC proposal, providing reference materials, and arranging personal contacts and site visits.

[2] Geotechnical Summary to the Proposal to Site the Superconducting Super Collider in Illinois, Harza Engineering Company and ISGS (1987).

[3] Next Linear Collider (NLC) Project, North-South Alignment, Geotechnical Study, Harza Engineering Company (2000).

[4] Siting Considerations for Future Accelerator Facilities at Fermilab, FCSS, DRAFT 2 (01/17/01), dated XXXX-XX, Chapter 2. Geology and Underground Construction Methodologies, FERMILAB-TM-2142.

[5] Peter J. Conroy, "Characterization of Fermi Region Geology," Second Annual VLHC Meeting, Port Jefferson, New York, October, 2000.

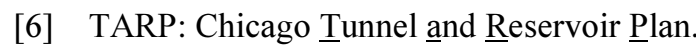

[7] Summary of the NuMI Project, the Fermilab NuMI Project Staff and the MINOS Collaboration, NuMI-678, Version 5.1 (2000).

[8] Peter J. Conroy, geo-technical consultant, Elmhurst, Illinois.

[9] J. Lach et al., "Cost Model for a 3 TeV VLHC Booster Tunnel," FERMILAB-TM-2048 (1998), based on a study by the Kenny Construction Company.

[10] CNA Consulting Engineers, Charles R. Nelson and Associates, 2800 University Avenue S.E., Minneapolis, Minnesota 55414.

[11] Superconducting Super Collider Laboratory, SSCL-SR-1056, Site Specific Conceptual Design, July 1990.

[12] Superconducting Super Collider, A Retrospective Summary, 1989-1993, SSCL-SR-1235, April 1994, Chapter 14. Experimental Areas.

[13] C.T. Murphy, "How the Tevatron was Aligned," MI-note 0089. Also, private communication.

[14] Virgil Bocean, "Geodesy and Alignment Concepts for the Fermi Main Injector," MI-note 0093.

[15] www.smithengineering.com

[16] Siting Considerations for Future Accelerator Facilities at Fermilab, FCSS, DRAFT 2 (01/17/01), dated XXXX-XX, FERMILAB-TM-2142, Chapter 4, Potential Environmental, Safety, and Health Issues.

J.D. Cossairt et al., "Environmental, Safety, \& Health Considerations for a New Accelerator Facility," FERMILAB-Conf-01/051-E (April, 2001). 
[17] Superconducting Super Collider, A Retrospective Summary, 1989-1993, SSCL-SR-1235, April 1994, Chapter 28, Environment, Safety, and Health, and Quality Assurance.

[18] L. Coulson and T. Toohig, "SSC Egress Provisions and Features for Evaluation of Equivalency as per Chapter 7 of NFPA $101 \mathrm{M}, "$ 5/29/90.

[19] L.M. Curran and S.B. Bhadwat, "Disposal of Material to be Excavated from the Illinois SSC," ISGS (1987).

[20] Tunneling Cost Reduction Study, The Robbins Company, prepared for Fermi National Accelerator Laboratory, July, 1999. 


\section{Chapter 8. VLHC Experiments and Detector Issues}

The Stage-1 VLHC with $40 \mathrm{TeV}$ and Stage 2 with $175 \mathrm{TeV}$ center of mass energy will, as each stage comes on line, provide the world's highest energy proton proton collisions. Experience with large-scale collider detectors built over the last two decades demonstrates that at these frontier exploration energies general-purpose detectors are best. The major reasons for this choice is space in the collider ring for experiments, their high cost, and the long time scale for construction and operation. As discoveries are made physics priorities will change over the detector lifetime. The number of general-purpose experiments for the VLHC is a compromise between available resources and the need for confirmation of new results and discoveries. We assume that the VLHC will have two general-purpose detectors. These will be designed for Stage 1 and then upgraded or replaced for Stage 2. In this chapter we describe the major experimental parameters for the Stage- 1 detectors, then outline the experimental challenges for Stage 2 and conclude with the accelerator detector interface requirements for Stage 1.

\subsection{Description of Experiment Parameters for Stage 1}

The goal of a general-purpose collider detector is to measure the largest possible number of independent quantities for each trigger event. These include e/ $\mu$ identification, sign of charge and momentum, detection and measurement of isolated photons, measurement of jet energies and directions, identification of jets with b-hadrons, determination of charged particle multiplicities, and detection of non-interacting neutrals. The major parameters for VLHC detectors have been discussed at Snowmass 1996 [1] and at the 1997 VLHC Physics and Detector Workshop held at Fermilab [2]. The SSC detectors, SDC [3] and GEM [4], were designed to achieve the above stated goals, while operating at a luminosity of $10^{33} \mathrm{~cm}^{-2} \mathrm{~s}^{-1}$. Detectors currently under construction at LHC, CMS [5] and ATLAS [6], are designed for a center of mass energy of $14 \mathrm{TeV}$ and luminosity $10^{34} \mathrm{~cm}^{-2} \mathrm{~s}^{-1}$ equal to the Stage-1 VLHC design. Therefore, the major parameters for the VLHC detectors can be based on the large amount of R\&D and design work done for the above experiments.

Experimentally detectable objects are charged tracks and clusters of electromagnetic and/or hadronic energy. Detector parameters can be optimized based on a set of benchmark physics processes, e.g. Higgs particle production or the search for supersymmetry. We are looking for the heaviest particles at an energy frontier machine. These are produced almost at rest in the center of mass system and therefore the acceptance for the decay products is basically proportional to the detector solid angle coverage. Based on this assumption rapidity coverage up to $\sim 3$ is sufficient for $\sim 90 \%$ detection efficiency. An exception to this rule is neutrino detection. In order to reach good missing energy resolution, calorimetry acceptance up to a rapidity of $\sim 5$ is needed. For detection of charged tracks we are aiming at good momentum resolution up to the maximum energy of the decay objects. Typically this energy is $5-10 \%$ of the center of mass energy; so for a $40 \mathrm{TeV}$ machine we are interested in reconstruction of particles with momentum up to $\sim 3 \mathrm{TeV} / \mathrm{c}$. 
A hypothetical generic VLHC detector is described beginning from the central region of the detector: the central tracking system. Typically this region consists of two sub-detectors: a precision vertex detector and a tracking system for momentum measurement. Vertex detection is very important. Decay of many heavy objects occurs via decay into massive b quarks. In order to reduce backgrounds from production of light objects (copious at such energies) detection of $\mathrm{b}$ quark decay a few $\mathrm{mm}$ to $\mathrm{cm}$ away from the original vertex is necessary. For precision vertex detectors, silicon detectors are mainly used. Some of them [6] have areas of hundreds of square meters. As the radiation dose in the central rapidity region depends weakly on the colliding beam energy and linearly on luminosity LHC type detectors (with some modifications) could be used for the Stage-1 VLHC. In order to increase radiation hardness and improve coordinate resolution, silicon pixel detectors are under extensive R\&D study [7].

Reconstruction of charged particle tracks and their momentum measurement is performed in the tracking system. There are two major goals: pattern recognition in a high multiplicity environment and precision momentum measurement in the TeV region. Different types of gaseous detectors, silicon or scintillation fiber detectors could be used in this region. Momentum resolution about $5-10 \%$ could be reached with such technologies for momenta up to $\sim 3 \mathrm{TeV} / \mathrm{c}$ and three sigma charge identification up to $\sim 15 \mathrm{TeV} / \mathrm{c}$. For momentum measurements there is a solenoidal magnetic field in the central area of the detector. Typical values for the field are in the range $2-4 \mathrm{~T}$. Mechanical forces in the magnet limit this field. The presence of the superconducting solenoidal magnet requires a liquid He supply to the detector area and appropriate cryogenic plant, so special cavern(s) are required close to the experimental halls (see Section 7.5).

Calorimetry is used to measure the energy of electrons, gammas and jets as well as missing energy in the event. As the depth of the hadronic shower (which defines calorimeter thickness) depends logarithmically upon particle energy, the overall size of the calorimeters is similar to SSC and LHC experiments. Calorimeter energy resolution is proportional to $1 / \sqrt{ }(E)$ and improves with energy increase up to a constant limit, typically a few percent or less. Therefore calorimetry becomes a precision tool for measurement of event parameters at very high energy. Currently most of the large collider detector calorimeters are liquid Ar or scintillation/Cerenkov light type. As the collision energy increases, heavy gas calorimeters could become efficient and be an inexpensive solution for ionization calorimetry. The radiation dose for the Stage-1 VLHC in the central calorimeter region is manageable, although in the very forward region special radiation hard techniques, like tungsten with quartz fibers, will have to be used.

The muon detection system is located outside the calorimeter. Muon tracks are bent in the muon magnet/absorber, enabling the trigger to select high momentum muons. The muon magnets also improve muon momentum resolution at very high energy and provide track points at large distances from the interaction region. One of the most challenging problems in muon detection in the $\mathrm{TeV}$ energy range is that at these energies, muons start to behave like electrons, irradiating electromagnetic debris along their tracks in the absorbers. This debris complicates reconstruction of muon hits, especially at the trigger level and requires multiple hit measurement along the muon track as well as special shielding materials.

An important part of the detector design is the shielding necessary to reduce background. A major source of background is interaction of proton fragments with the beam pipe and other interaction region components. Special shielding design can reduce background flux by orders 
of magnitude [8], reducing radiation dose to the detector components and improving triggering and track reconstruction capabilities.

One of the major challenges for detector operation at such high luminosity is the large number of interactions per crossing. At a luminosity of $10^{34} \mathrm{~cm}^{-2} \mathrm{~s}^{-1}$ and beam crossing time of $18.8 \mathrm{~ns}$, the average number of interactions per crossing will be 19 (Section 1.3). While most of these events are soft minimum bias events, they complicate central tracker triggering and reconstruction. Calorimetry and muon systems are less affected by high multiplicity events. It is basically impossible to separate events from the same crossing based only on timing information. On the other hand, in order to reduce overlap of events from different crossings, achieving detector and electronics resolution time less than the beam crossing time is at premium. This is especially true at the triggering level where only limited information about the event is available. Ideally charge collection time from the detector should also be less than the beam crossing time.

Radiation flux is another major challenge for the VLHC detectors. Table 8.1 compares the estimated dose at different distances from the beam pipe for one year of running at design luminosity for the Stage-1 VLHC and LHC detectors. In the central rapidity region the dose values are comparable, so detector technologies developed for the LHC detectors could be adopted for the VLHC as well.

Table 8.1. Radiation dose in Mrad per year for LHC and Stage-1 VLHC at $90^{\circ}$.

\begin{tabular}{|l|c|c|}
\hline & LHC & VLHC \\
\hline Vertex detector $(\sim 10 \mathrm{~cm})$ & 3 & 5 \\
\hline Central tracking $(\sim 50 \mathrm{~cm})$ & 0.25 & 0.3 \\
\hline EM calorimeter $(\sim 150 \mathrm{~cm})$ & 0.06 & 0.1 \\
\hline Hadron calorimeter $(\sim 250 \mathrm{~cm})$ & $2 \times 10^{-3}$ & $\sim 3 \times 10^{-3}$ \\
\hline
\end{tabular}

An important part of any experiment is the trigger system. It should reduce the initial interaction rate of about $50 \mathrm{MHz}$ to a manageable rate of about $50 \mathrm{~Hz}$ for writing events to tape. With a high occupancy of detector elements and a large number of readout channels a typical event size is in multi Mbyte range. This translates to a Gbyte per second tape rate. It is important to select only interesting classes of events at the trigger level and reduce the huge data sample manipulation after events are written to tape. So, triggering capabilities are an important consideration in detector design and special fast trigger electronics need to be developed.

A general detector design based on the above considerations is shown in Figure 8.1. This concept is well established and proven based on the Fermilab and CERN collider experiments.

One of VLHC design parameters is the size of the detector cavern. This will be mainly determined by the muon system. For high precision muon momentum resolution, the required size of the hall is large. Table 8.2 shows hall sizes together with muon momentum resolution. Based on these numbers and taking into account the evolution of the detectors from Stage-1 to Stage-2, the experimental caverns should have a length of about $60 \mathrm{~m}$ and diameter of about $30 \mathrm{~m}$ plus additional space for personnel and equipment access (Section 7.5). 


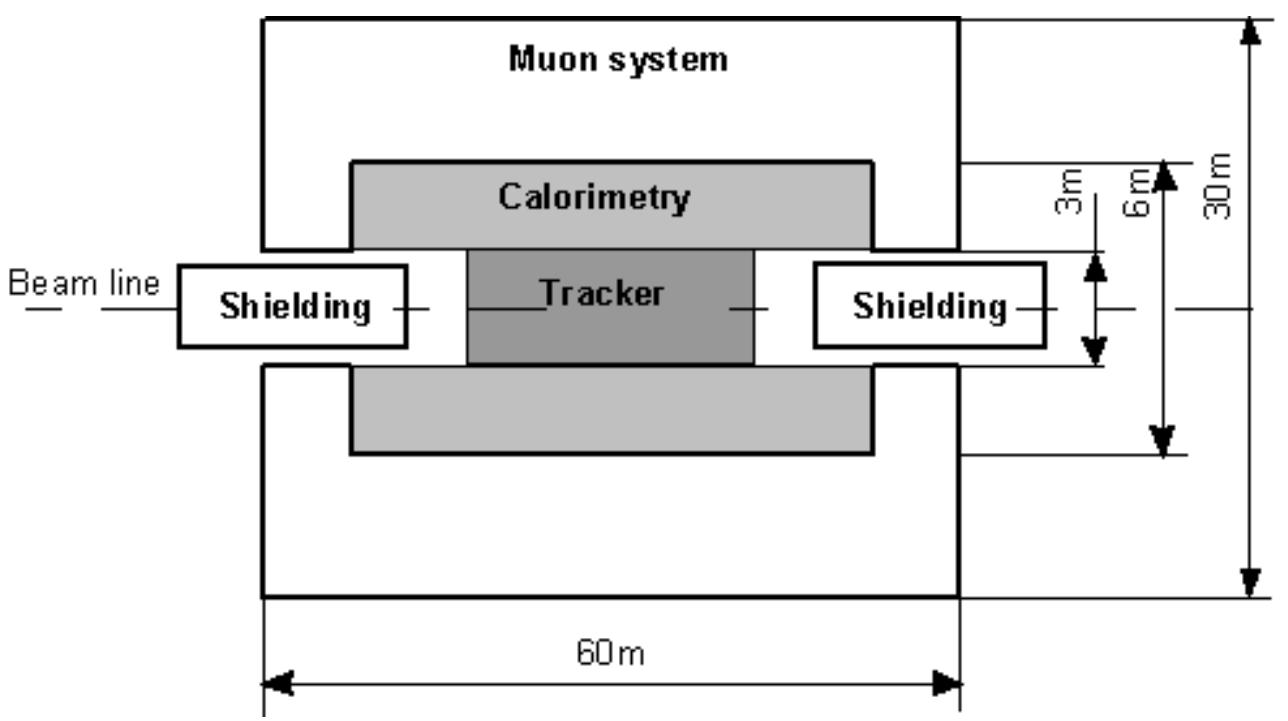

Figure 8.1. Layout of a general purpose VLHC detector.

Table 8.2. Hall sizes and momentum resolution at momentum of $1 \mathrm{TeV} / \mathrm{c}$.

\begin{tabular}{|l|c|c|}
\hline Experiment & Hall size, $m^{3}$ & $d p / p, \%$ \\
\hline SDC (SSC) & $29 \times 29 \times 50$ & 11 \\
\hline GEM (SSC) & $24 \times 24 \times 76$ & 10 \\
\hline CMS (CERN) & $26 \times 26 \times 60$ & 5 \\
\hline ATLAS (CERN) & $25 \times 25 \times 53$ & 8 \\
\hline
\end{tabular}

Today's detector technology provides a wide selection of choices for the Stage-1 VLHC detectors. Experience obtained in designing SSC and LHC detectors is very important. ATLAS and CMS designs could be used for Stage-1 VLHC with minor modifications. In order to reduce detector cost and improve operational parameters, detector R\&D is important. For each of the detector subsystems R\&D studies should concentrate on providing high radiation tolerance, resolution time below $19 \mathrm{~ns}$ beam crossing time, high segmentation as well as reliable long term operation and low construction and operational costs. Development of very fast electronics to digitize signals from detectors, select events with interesting topology with an input rate of $50 \mathrm{MHz}$ should be part of the R\&D program as well.

\subsection{Description of Major Experimental Challenges for Stage 2}

The upgrade from Stage-1 to Stage-2 will increase the luminosity a factor of two and the center of mass energy nearly a factor of four. These increases in energy and luminosity provide a significant increase in the collider physics reach [2], but bring major challenges to detect and reconstruct events. In this section we will discuss how the increase in energy and luminosity will affect detectors.

From 40 to $175 \mathrm{TeV}$, both the charged track multiplicity and the inelastic cross section increase [9], and the luminosity is twice as large. As a result, the radiation dose in the central region is a factor of $\sim 3$ higher than in Stage-1. Figure 8.2 shows the energy flux per unit of 
rapidity for $100 \mathrm{TeV}$ center of mass. Most of the energy is deposited in the forward region. Dose in the calorimeter located at a rapidity of $\sim 5$ will be about 1 Trad per year, a serious experimental challenge.

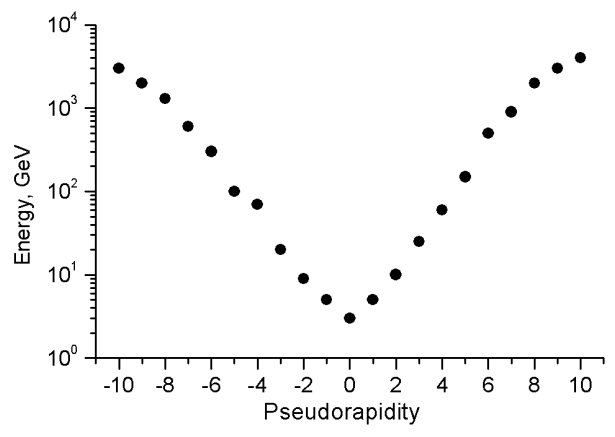

Figure 8.2. Calculated energy flux per unit of rapidity for pp collisions at energy $100 \mathrm{TeV}$.

The average number of minimum bias interactions per crossing for Stage 2 will be about 49 , a factor of three increase from Stage 1. This large number of interactions per crossing creates pile up of events in the tracking detectors as well as in the calorimeter. Figure 8.3 shows the energy deposition in a calorimeter cone, $\left(\Delta \phi^{2}+\Delta \eta^{2}\right)^{1 / 2}$ of 0.4 in the central rapidity region for 1000 overlapped events at an energy of $100 \mathrm{TeV}$. At a luminosity of $2 \times 10^{34} \mathrm{~cm}^{-2} \mathrm{~s}^{-1}$ the average energy deposition in a 0.4 cone will be about $350 \mathrm{GeV}$. This "pedestal" level will fluctuate due to statistical fluctuation in the number of events per crossing as well as with changes in luminosity. While challenging this task is similar to operation of calorimeters at LHC where the energy flux will be about factor of $\sim 5$ lower. Muon system pile up of events is less important, as the probability to a have high-energy muon in a minimum bias event is $\sim 10^{-5}$.

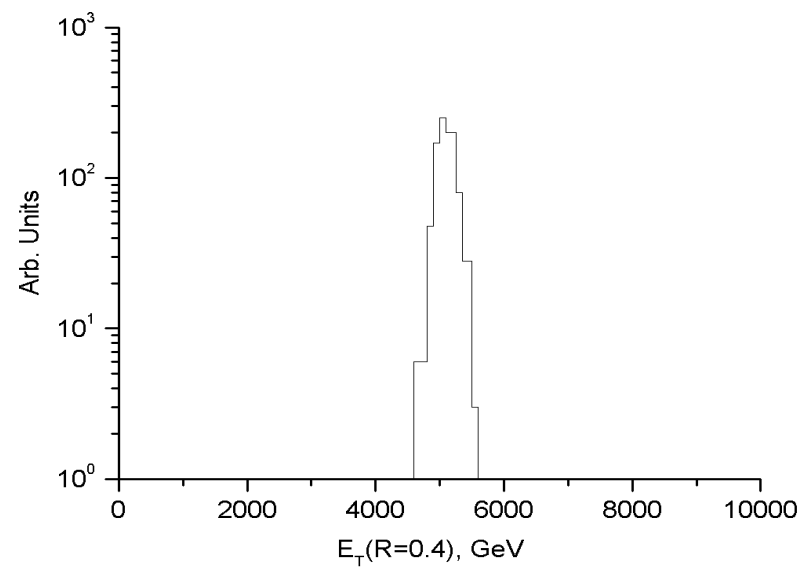

Figure 8.3. Energy deposition in a cone 0.4 for $10^{3}$ overlapped events at 100 TeV center of mass energy.

With increase in energy the detectable decay products will have higher energy as well. Calorimetry energy resolution is improving as $1 / \sqrt{ }($ E $)$ and will be limited by the constant term and pile up fluctuations. The real challenge will be momentum measurement of the charged particles and muons. If the energy of the detectable objects we are looking for increases proportional to the center of mass energy we will need momentum resolution of about $10 \%$ for $5-10 \mathrm{TeV}$ particles. Presently designed detectors at $10 \mathrm{TeV}$ (see Table 8.2) will have 50\% resolution at 
best. So, improvements in charged particle detection (precision of tracking detectors, value of magnetic field, and/or size of the lever arm) are needed. Another approach is to think about detectors based mainly on calorimeter information.

In summary, Stage 2 of the VLHC will be similar to Stage 1 in terms of occupancies and radiation dose in the central region as luminosity, inelastic cross section, and charged particle multiplicities each increase by a small factor. Calorimetry will become very precise with resolution limited by event pile up and the energy resolution constant term, while charged particle momentum measurements in the region $1-10 \mathrm{TeV}$ will require serious design and R\&D efforts.

\subsection{Machine-Detector Interface Requirements for Stage 1}

There is a set of accelerator and detector interface parameters, important for efficient detector operation. The most important of these are: the dimensions of the beam interaction region, beam abort due to abnormal losses, location of the low beta quads, shielding from background from collisions in accelerator components and background from the accelerator tunnel.

The beam interaction region is described in two planes: perpendicular to the beam pipe and along the beam direction. For triggering on displaced vertices and missing energy it is preferable to keep the beam diameter below $50 \mu \mathrm{m}$. It is also important to maintain beam center location stable to within $1 \mathrm{~mm}$ during the store and locate the beam to within a few $\mathrm{mm}$ with respect to the beam pipe center. Usually these requirements are easily met. The size of the interaction region along the beam pipe is not as well defined. A smaller interaction region has advantages and disadvantages. Advantages are better missing $\mathrm{E}_{\mathrm{t}}$ resolution and smaller detector size. Disadvantages are overlap of events in the tracking detector, and more difficult pattern recognition in the muon and calorimeter systems. Usually the compromise length is $\sigma \sim 30 \mathrm{~cm}$, which means that $99 \%$ of events occur in a region $\pm 1 \mathrm{~m}$ around the detector center.

Some of the detector elements are sensitive to radiation, especially those elements of the tracking system located close to the beam pipe. In order to prevent excess irradiation of these components during unexpected high loss a feedback loop to the accelerator control room will be provided. If the integrated dose or the irradiation rate increase above specific levels the beam will be aborted to prevent damage to the detector. Such systems exist at all hadron colliders and are well developed.

The requirement of hermetic calorimetry to measure missing energy sets the calorimeter pseudorapidity coverage at about $\pm 5[5,6]$. In order to locate calorimeters close enough to the beam pipe as well as provide space for access to the detector elements the low beta quads should be located at a distance of about $20 \mathrm{~m}$ from the center of the interaction. A more specific number for this parameter will be determined from specific detector design.

There are two major sources of background hits in the muon detectors which are difficult to reduce: (1) sprays from hadronic and electromagnetic showers developed along detector cracks and along the beam pipe from accelerator components and (2) backgrounds coming from the tunnel produced by beam losses along accelerator. Cracks between sub-detectors will be minimized. Nevertheless there will be background from these cracks and originating from the collision point. This background will be reduced with a tapered beam pipe as well as shielding 
along the beam pipe. There is considerable experience in designing such shielding $[3,4,5,6,8]$. Reduction in charged particle flux up to two orders of magnitude, especially in the muon detectors, can be achieved.

In order to define an acceptable background flux of particles from the tunnel, this flux has to be compared with backgrounds coming from the interaction region, taking into account the effect of shielding. Typical measured/estimated background flux on the muon detectors from the interaction region is $(0.1-1) \times 10^{6}$ particles $\mathrm{m}^{-2} \mathrm{~s}^{-1}[5,10]$. The flux of particles from the tunnel should be below this number. In the case of the CDF and D0 experiments, $2 \mathrm{~m}$ of thick concrete shielding in the tunnel on both sides of the detector was needed in order to reduce background to an acceptable level. Results of MARS [11] calculations of the background flux in the Tevatron agree well with observations, so as soon as the interaction region lattice and beam parameters are known, backgrounds can be calculated and ways explored for reducing them.

\section{References}

[1] D. Denisov and S. Keller, "Summary of the Very Large Hadron Collider Physics and Detector Subgroup," Proceedings, Snowmass 1996.

[2] G. Anderson et al., "Summary of the Very Large Hadron Collider Physics and Detector Workshop," Preprint Fermilab 1997, FERMILAB-CONF-97/318-T.

[3] E. Berger et al., SDC Technical Design Report, SSCL-SR-1215, 1992.

[4] W. Lefmann et al., GEM Technical Design Report, SSCL-SR-1219, 1993.

[5] G. Bayatian et al., The Compact Muon Solenoid Technical Proposal, CERN/LHCC 94-38, 1994.

[6] W. Armstrong et al., Atlas Technical Proposal, CERN/LHCC 94-43, 1994.

[7] http://wcc.oeaw.ac.at/, $9^{\text {th }}$ Vienna Conference on Instrumentation, to be published, 2001.

[8] V. Sirotenko et al., "D0 Shielding Optimization: Simulations and Measurements," D0 Note 3417, 1998.

[9] W.A. Barletta and H. Leutz, eds., Supercolliders and Superdetectors, World Scientific, 1993.

[10] D. Denisov, private communication about D0 detector tunnel shielding.

[11] N. Mokhov, “The MARS Code System User's Guide, Version 13(95)", Fermilab-FN-628, 1995. 


\section{Chapter 9. Cost Analysis of the Stage-1 VLHC}

\subsection{Uses and Limitations of the Cost Analysis for This Study}

Within the scope of this design report, the development of a preliminary cost estimate of the major cost elements is useful and reasonable. A cost estimate at this early stage of the design and development is inherently limited, but can nevertheless give a "ballpark" figure for the total cost of the collider. Furthermore, reasonable crosschecks with known costs and historical data for similar systems can serve to give one confidence in the result. Hence, the costs associated with individual systems can and should be accurate even at this early stage. The major weaknesses of making estimates without complete and proven designs, and without extensive fabrication experience, is the high probability of leaving out many of the minor subsystems and necessary infrastructure and activities that go to make the whole out of the sum of the parts. Even in that case, comparisons with complete accelerators and cost estimates of mature designs, viewed from a high level, can be used to verify costs, and, in particular, to set upper bounds, provided that the historical data are accurate. The results can then be used, along with physics analyses and technical evaluations, as input into the important matter of deciding which facilities the high-energy physics community should build. Finally, and perhaps most importantly, analysis of the major cost drivers is useful as a means to identify systems where further R\&D and engineering can net substantial reductions in cost.

\subsection{Identification of the Cost Drivers}

The nine major cost drivers, listed in Table 9.1, were chosen as a result of the past experience of the participants in the design study. Most of them are obvious, such as the underground construction, surface construction, and the main magnet systems and associated cryogenics. The unusually long magnets and large tunnel circumference led us to believe that there might be important issues to discover in the installation activity, such as the need for sophisticated tooling or very long travel times. The vacuum system is conventional and room temperature, but its extensive size requires many components, and hence, the possibility of high cost. The interaction regions were chosen because they are likely to be complex-and therefore interesting - and considerably more costly than the total cost of their components.

Once the high-level cost drivers were identified, engineers and scientists used the technical descriptions to make cost estimates. If possible, we obtained quotes from industry as aids in the estimates. This was particularly true for very large subsystems, such as steel and superconductor for the magnets, and the refrigerators for the cryogenic system. In many cases, recent purchases and contracts were used as guides to the costs of components, such as the cost of assembling steel laminations into yoke subassemblies, which was estimated from the known cost of the same activity for the Fermilab Main Injector. Labor, overhead and profit was, of course, included in the cost of delivered subsystems estimated by industry. Other labor, e.g., for the final assembly, test and installation of the magnets was estimated by engineers using models created by them for the activity being estimated. Standard Fermilab labor rates were used in those cases, including complete fringe benefits but not overhead or other indirect costs. 
Table 9.1. The major cost drivers and the associated technical description section.

\begin{tabular}{|l|c|}
\hline \multicolumn{1}{|c|}{ Cost Driver } & Report Section \\
\hline Main Magnets & $5.1 .1,5.1 .6,5.1 .7$ \\
Corrector Magnets \& Special Magnets (Injection, etc.) & $5.1 .3,5.1 .5$ \\
Interaction Regions & 5.1 .4 \\
Refrigerators & 5.2 .1 \\
Cryogenic Systems & 5.2 .1 \\
Vacuum System & 5.2 .4 \\
Installation & 5.1 .8 \\
Civil Construction - Above Ground & $7.5,7.6$ \\
Civil Construction - Below Ground & $7.2-7.5$ \\
\hline
\end{tabular}

The cost drivers were developed by estimating the cost to build the current design, as presently known, in FY2001 dollars, at FY2001 prices. There is no assumption of future piece cost savings due to the successful completion of current or future R\&D programs, or manufacturing scale-up possibilities. This estimate assumes that a complete design exists, and all major R\&D programs necessary to prove and complete the design have been finished before the start of construction. The estimate is for production and installation only. In places where the bottoms up estimates as developed do not appear consistent with experience, modifications were made to bring them more in line with past experience. Only the direct costs for the major cost drivers are estimated. Engineering, design, inspection and administration (EDI\&A) and indirect costs such as G\&A are not included, but are estimated in the analysis section as a complement of professional and support personnel over the duration of the project. Finally, the estimates do not include commissioning, pre-operations, $R \& D$, detectors or land acquisition; nor does it include escalation and contingency.

These rules make this cost analysis consistent with a so-called "European" or raw cost model, which excludes all of the factors we have excluded, including EDI\&A and contingency. This is an appropriate way to get at an estimate useful for a comparisons of costs of different facilities, since almost all of the adjustments necessary to get a "U.S." cost estimate are multiplicative and apply to all raw estimates equally. The one exception is the assignment of appropriate contingency. Contingency is specific to each project and depends on a risk analysis that takes into account the state of the design, engineering, prototype fabrication and certain economic factors at the time the risk analysis is done.

\subsection{Models for Estimating the Cost Drivers}

The cost estimate for the largest cost element, the underground construction, was done by a collaboration of CNA Consulting Engineers, Minneapolis, MN, and Hatch-Mott-McDonald, Toronto, Canada, under contract to Fermilab. They estimated three ring orientations, as described in Chapter 7, not because those are the preferred sites - there are no preferred sites as yet - but in order to get a range of costs due to different physical and geological features in the Fermilab area. The result is not only an estimate that defines a range of costs for the underground construction, but also a set of building blocks that can be used to estimate the cost of underground construction with different ring orientations, different ring designs and different included features. The cost number for underground construction in Table 9.2 is the 
approximate average of the costs for the three ring orientations, which did not differ from each other by more than $\$ 100$ million. The underground construction includes all necessary shafts and ramps, and the underground adits and two large collision halls, in addition to conventional outfitting costs such as HVAC, AC power distribution, groundwater pumping, staircases, elevators and so forth. The underground construction also includes the cost of an AE/CM firm, based on 17.5 percent of the underground construction cost. This firm manages the underground construction. The Laboratory EDI\&A needed to oversee the AE/CM firm is not included. The surface buildings, including the six cryogenic service buildings and the surface features above the collision halls were estimated based on footprint area by Fermilab's Facilities Engineering Section, using standard rates similar to those used for recent accelerator estimates. Those estimates include utility installation, such as power and water.

The main magnet estimate includes costs for fabrication and procurement of all main and dispersion suppressor dipoles in the Stage 1 ring, including both the magnet and the directly associated cryogenic piping and return conductor. The model used is similar to the one used to build the Main Injector at Fermilab. Large subassemblies appropriate for shipping over roads, such as 11-m yoke sections or beam tubes, are built in industry and delivered to a final assembly building near an installation ramp at the VLHC site. The final assembly and testing is done at that factory site and either stored temporarily or immediately installed in the tunnel. Costs for the correctors and straight section magnets are separately included. We assume that most of them will be built in industry. The interaction-region magnets are also separately estimated. Since they are technically challenging, they will probably be built either at Fermilab or another laboratory with extensive superconducting magnet experience.

The estimate for the cryogenic system was split into two pieces - the refrigerator package and the pipes and valves in the tunnel not included in the magnet system. This was done because the refrigerator package can be estimated by industry and fits a well-known cost curve as a function of power, while the delivery system is very design specific. The refrigerator package includes the fabrication, installation, and commissioning of the six refrigerators required for the ring, and the additional refrigerators required for the interaction regions. The tunnel cryogenic system estimate includes the fabrication, installation and checkout of components necessary to deliver cryogenics from the refrigerators up to and including the distribution box at the end of each magnet string.

Frequently, we had to make somewhat arbitrary decisions as to which system a particular component fell. For example, all the pipes for the cryogenic system that are in the magnet are included in the magnet system. The vacuum system estimate includes all vacuum components, including the beam tube extrusion. Hence, the magnet system does not include the beam tube as part of its cost. The installation estimate covers magnet installation, including magnet stands, alignment, and special tooling for handling the 65-m magnets; and the installation of tunnel infrastructure, such as lighting, trolley rails, cable trays and pipe hangers. In order to get a total cost estimate, we had to include an estimate for the total of the "non-drivers," that is, the minor subsystems. We did this by taking the ratio of those minor accelerator systems-power supplies, RF, instrumentation, controls, safety systems, beam abort line and dump, and accelerator utilities - that were part of the SSC 1990 Site Specific Cost Estimate [1] to the accelerator systems we did estimate. Those minor systems amounted to less than 10 percent of the SSC Collider cost. 


\subsection{Results and Analysis}

Table 9.2 lists the results of the cost driver exercise. The most important thing to notice is that more than half the total cost is in the civil construction, and almost all of that is in the underground part. Traditionally, 40 percent or more of underground construction is in labor costs. R\&D to reduce the number of workers by using straightforward automation techniques common in other industries and even in the construction industry could significantly reduce costs, while at the same time improving safety because fewer workers will be underground. Also, the details show that half the underground cost is in features other than the arcs, such as the necessary collision halls, adits and breakouts added to the tunnel. This indicates that discipline and coordination between accelerator designers and tunnel builders to eliminate unnecessary special features could significantly reduce costs.

Table 9.2. The estimated costs of the major cost drivers for Stage-1 VLHC.

\begin{tabular}{|l|c|c|}
\hline \multicolumn{1}{|c|}{$\begin{array}{c}\text { Stage-1 VLHC } \\
\text { Cost Driver }\end{array}$} & $\begin{array}{c}\text { Cost Estimate } \\
\text { (in FY2001 MS) }\end{array}$ & $\begin{array}{c}\text { Fraction of Total } \\
\text { Stage-1 Cost }\end{array}$ \\
\hline Total Cost & 4,138 & $100 \%$ \\
Construction - Below Ground & 2,125 & $51.4 \%$ \\
Construction - Above Ground & 310 & $7.5 \%$ \\
Main Arc Magnets & 792 & $19.1 \%$ \\
Correctors \& Special Magnets & 112 & $2.7 \%$ \\
Refrigerators & 95 & $2.3 \%$ \\
Other Cryogenic Systems & 22 & $0.5 \%$ \\
Installation & 232 & $5.6 \%$ \\
Vacuum System & 154 & $3.7 \%$ \\
Interaction Regions & 26 & $0.6 \%$ \\
Other Accelerator Systems & 270 & $6.5 \%$ \\
\hline
\end{tabular}

"The below-ground construction cost is the approximate average for the total underground construction in the three different ring orientations, based on $12 \mathrm{ft}$. diameter tunnels. The maximum cost difference is less than $\$ 100$ million. The cost includes 17.5 percent for an AE/CM firm.

The second cost driver is the main arc magnet system. The cost of these magnets is about $\$ 900$ per Tesla-meter, much less than the present-day cost of a cos-theta NbTi dipole, which is around $\$ 2500 / \mathrm{T}^{\circ} \mathrm{m}$. The cost of the transmission-line magnet is dominated by steel laminations, not by superconductor as in other styles. Research into the use of commodity steel for these magnets, or improvements in stamping and stacking that small-scale magnet builders have not used in the past are likely places to look for cost reductions.

Continuing the work on the engineering study will sharpen the cost estimate and further focus the R\&D on the most important parts of the VLHC. This is extremely important. 
Additionally, increasing the R\&D to a point where industry can reasonably be involved will also sharpen the cost estimate and reduce the needed contingency. Both of these efforts must continue at a more vigorous pace.

\subsection{The Reality Checks}

The relatively low cost of the Stage 1 VLHC is a bit surprising when compared to that presented recently by the TESLA Collaboration for a superconducting linear collider at much lower energy. This is largely so because magnets are much less costly than RF cavities and RF power systems, and tunnels are less costly than magnets. It pays to make some comparisons with other machines or cost estimates. We have attempted to put our cost analysis side-by-side with the baseline cost estimate of the collider ring of the SSC. That cost estimate was mature by 1991, and in spite of many rumors to the contrary did not significantly change between 1991 and the end of the project.

In order to make the comparison, we used the estimates in reference [1], adjusted to contain only the collider-ring costs, and to include the fractions of the accelerator-wide costs that apply to the collider ring [2]. The results were then deconstructed and reconstructed into categories that are parallel to the VLHC system categories. For example, corrector magnets in the SSC baseline cost estimate were not part of the magnet system, but were included in the accelerator system estimate. One can only imagine why. After the reconstruction, the SSC baseline was inflated from 1990 to 2001 dollars by the consumer price index (CPI), a 35 percent increase. The actual inflation may be somewhat less. The producer price index between 1990 and 1998, the last year with complete data, show significantly lower inflation, more like 15 percent. Since large projects like the SSC and the VLHC would procure much of its material directly from manufacturers, that lower rate would apply in some cases. The system-by-system cost comparisons are shown by percent of the total cost in Table 9.3. The SSC total cost inflated to 2001 dollars is $\$ 3.79$ Billion, very close to the same as the VLHC at the same center-of-mass energy, even though the distribution of costs in civil construction and magnets is almost exactly reversed.

Table 9.3. A comparison by major system of the Stage-1 VLHC costs and the SSC baseline cost escalated to FY2001 dollars.

\begin{tabular}{|c|c|c|}
\hline \multicolumn{1}{|c|}{ Collider System } & $\begin{array}{c}\text { Fraction of total } \\
\text { Stage-1 VLHC Cost }\end{array}$ & $\begin{array}{c}\text { Fraction of Total } \\
\text { SSC Collider Ring Cost }\end{array}$ \\
\hline Total Cost & $100 \%$ & $100 \%$ \\
Construction - Below Ground & $51 \%$ & $15 \%$ \\
Construction - Above Ground & $8 \%$ & $5 \%$ \\
All Magnets (except IR) & $22 \%$ & $61 \%$ \\
All Other Collider Systems & $19 \%$ & $19 \%$ \\
Total Cost in FY2001 MS & $\$ 4,138$ & $\$ 3,790$ \\
\hline
\end{tabular}


The appearance that the inflation-adjusted SSC cost and the Stage-1 VLHC cost is a bit artificial because of the uncertainty in the cost estimates and in the cumulative inflation rate.

Nevertheless, there is no denying that they are close. Some conclusions can be drawn from this. First, it implies that the Stage-1 VLHC cost is not wildly wrong. Second, it says that less costly low-field magnets and simple magnet-related systems compensate for the higher cost of the big tunnel required for the Stage-1 VLHC. This is very good news, because the large-circumference tunnel will be a major cost driver for the $200 \mathrm{TeV}$ Stage-2 VLHC. As we hoped when the concept of a staged VLHC was born, paying for the large-circumference tunnel during Stage-1 is not a significant cost penalty, and it will save over $\$ 2$ Billion during construction of the 200 $\mathrm{TeV}$ collider.

\section{References}

[1] Report on the Superconducting Super Collider Cost and Schedule Baseline, DOE/ER-0468P, January, 1991. [2] Dr. John Marriner performed this exercise and kindly shared his spreadsheets with the VLHC Study. 


\section{Chapter 10. R\&D Programs and Related Studies}

The purposes of Research and Development programs and engineering studies are to reduce technical risk and cost, and to improve performance. The following sections briefly discuss what we see now as the most important R\&D that should be vigorously pursued for both Stage- 1 and Stage- 2 . There is a distinct difference in the nature of the R\&D between Stage-1 and Stage-2; the work on the low-energy collider emphasizes systems issues, engineering and cost reduction, while the R\&D for the high-energy machine is strongly slanted toward the development of components. This difference is appropriate, because the components of the Stage- 1 collider are relatively straightforward, whereas the Stage- 2 machine requires cuttingedge components, particularly magnets.

\subsection{R\&D for the Stage-1 VLHC}

Because of the simplicity of the magnet and accelerator systems of the Stage- 1 collider, there are only a few risk-associated R\&D studies needed. Most of the R\&D effort is devoted to reducing costs.

\subsubsection{Tunneling R\&D and Engineering}

The major cost driver in the Stage-1 collider is tunneling and other underground construction. Large investments in underground construction R\&D and engineering studies are easily justified because even small improvements in efficiency will save considerable cost and avoid significant financial risks. Furthermore, the R\&D results would be useful to a broad range of business and government far beyond high-energy physics. It might be possible to find funding from sources outside of HEP to support this R\&D. The R\&D is of two types: improvements in the current tunnel boring machine and conveyor belt muck removal systems, and trying new and innovative methods that might, if successful, reduce cost and improve safety.

Typically, forty percent or more of tunneling costs are in labor. Hence, development of automated or semi-automated procedures looks very promising. Such procedures could also result in improved safety, since fewer workers would be underground and at risk of injury. This is not fancy technology that will be difficult to develop and sell to the conservative tunneling industry, but relatively straightforward application of existing techniques that are widely used in other industries.

From a study carried out for Fermilab [1] cost drivers in conventional tunneling in Fermilab region geology are well understood. Improvement in tunnel boring machine (TBM) utilization can be achieved by fully instrumenting the TBM and trailing gear with pressure sensors, flow meters in the hydraulic and water systems, vibration sensors placed on all critical devices, and TV cameras positioned to watch all moving components. The TBM can be remotely operated from any place on the construction site. This dramatically reduces labor costs and improves job safety. Maintenance can be done as scheduled. Breakdowns aren't as costly, because you don't have large crews standing around waiting for a repair to be completed. The cost per meter of tunnel can be significantly reduced [2]. A Fermilab group has formed an informal collaboration with the Kenny Construction Company to instrument a TBM and gather its data in a TARP Tunnel under construction [3]. 
There are also many possibilities for the development of better tools and machinery to make tunneling faster, safer and cheaper. In a systematic R\&D program aimed at reducing tunnel costs, new approaches to tunneling can be explored. A visionary goal is to have no people underground except during maintenance. The mining industry is moving toward totally robotic systems but this technological evolution has not yet reached the construction industry. A modified TBM with corner cutters $[4,5]$ will produce a flat floor and allow use of a diesel-fume free battery operated, rubber tired autonomous muck removal and transportation system.

Close collaboration between accelerator designers and tunnel engineers can also cut costs. The many additions, side caverns and adits that an accelerator requires drive up the cost of underground construction. Careful engineering of the accelerator in conjunction with the tunnel may be able to eliminate some of these and significantly reduce costs. This study will involve a multi-disciplinary team of accelerator scientists, engineers and underground construction experts.

\subsubsection{Vacuum System}

The vacuum system for the Stage-1 collider is a surprising cost driver. Development and studies of different methods to reduce its cost are worthwhile. These could include simpler and less costly ion pumps and improved beam-tube materials and fabrication techniques. There are a number of possibilities. There may be practical non-evaporable getter material (NEG) that will pump semi-inert gasses such as methane. It would also be worthwhile to investigate the possibility that NEG strips exposed to synchrotron radiation and/or secondary electrons can pump methane. If so, this would eliminate the need for ion pumps. Another fruitful area of investigation is thin film NEG coatings which are under development at CERN. At a minimum these could be useful in special regions of the machine where conventional pumping solutions are difficult or impossible. The possibility that these coatings could replace the NEG strips, and possibly eliminate the need for ion pumps when exposed to synchrotron radiation, should also be investigated. Photodesorption coefficients of thin film NEG coated beam tubes and the pumping speed of these coatings in the presence of synchrotron radiation need to be documented.

The possibility of using the already present cryogenics of the magnet transmission line for a beam tube cryopump was recognized at the beginning of the study but it was felt that the cost of the cold to warm transitions and thermal shields would exceed the cost of the system that was analyzed. Perhaps this question should be reexamined. Unless the cryopump temperature is equal to or less than $\sim 3 \mathrm{~K}$, cryosorber will need to be added to pump hydrogen beyond one monolayer.

\subsubsection{Beam Stability}

There are a number of potential beam stability issues in the Stage- 1 collider. As discussed in Chapter 3, these have been solved in the design study by rebunching the beam in the Tevatron before injection into the collider, using a uniform filling sequence, and by using an audio frequency tune-equalization quadrupole in the Stage- 1 collider. None of these steps seem difficult or expensive, but they do need to be studied, both on paper and by experiments on existing machines. In addition, there are some other promising possibilities, particularly TMCI feedback systems, that are even less costly and also deserve study. 
Studies at Fermilab that might lead to reduced transverse beam emittance would allow lower current in the Stage-1 collider and have many good effects, including reducing the possibility of beam instabilities.

\subsubsection{Magnetic Field Quality}

Superferric magnets are unlikely to have many field-quality problems except near saturation. Nevertheless, we have not yet made a magnet with good field quality using the correct cross section for the design-study lattice. There is a contract with the D.V. Efremov Scientific Research Institute (St. Petersburg, Russia) to make two 6-meter long magnets with accurate laminations. It might be useful at the same time to build and test cross sections with some fastturnaround fabrication method, such as wire EDM machining. A study of the sensitivity of the magnetic field to steel chemistry and production tolerances would also be informative and might lead to cost-savings. Many of these measurements have been carried out on the magnet test bench shown in Figure 10.1. In this apparatus, 10 turns of water-cooled conductor are used to emulate the $100 \mathrm{kA}$ single-turn conductor in the Transmission Line magnet. It is a simple matter to make yokes of any desired pole shape and size, clamp them around the conductor, and make accurate measurements.

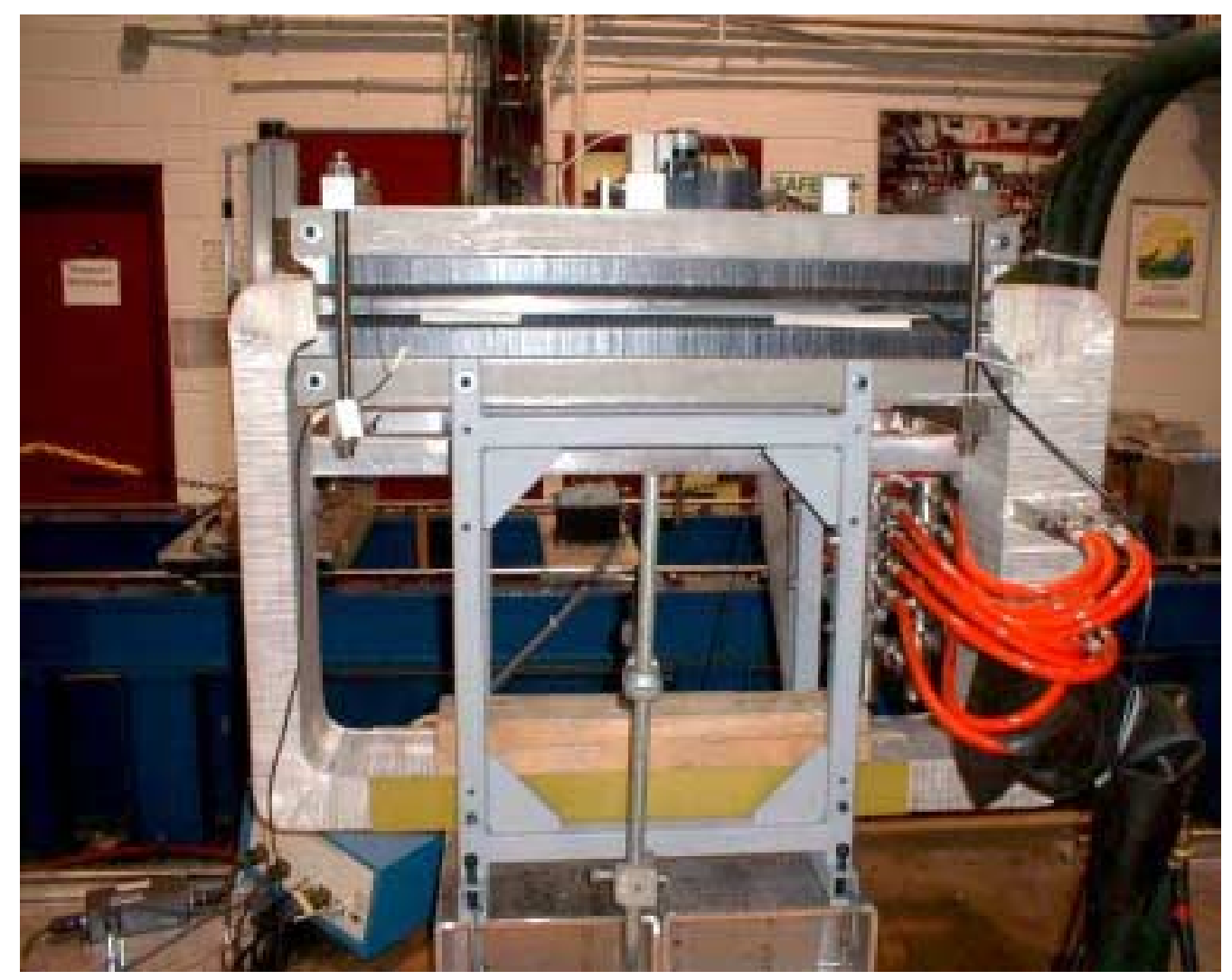

Figure 10.1. Apparatus for magnetic field quality measurements. 


\subsubsection{High-Gradient IR Quadrupoles}

The interaction regions of the VLHC require high-gradient quadrupoles that seem feasible but are beyond the present state-of-the-art. These magnets need to be developed, and since such developments have traditionally taken many years, the R\&D should start now. The target gradient for these magnets, $300 \mathrm{~T} / \mathrm{m}$, requires $\mathrm{Nb}_{3} \mathrm{Sn}$ of improved performance, and hence, a superconducting materials R\&D program is also required in parallel. The U.S. LHC collaboration has proposed developing quadrupoles of similar performance for a luminosity upgrade of the LHC. If that effort is funded, it may accomplish the necessary R\&D for the VLHC as well.

Fermilab is particularly well-positioned to lead the high-gradient quadrupole R\&D effort. In recent years, we have built the infrastructure necessary to do this work-reaction ovens, a strand-testing facility, a compact cable-making machine, winding and curing tooling for model and full-sized magnets, and a vertical dewar test facility (Figure 10.2) that will soon have 30 kA capability. In addition, if wide cables are required, LBNL is the world leader in cablemaking $\mathrm{R} \& \mathrm{D}$, and has a facility to make cables containing up to 60 strands. This infrastructure at the two labs allows the flexibility of design and fast turn-around necessary for a successful R\&D program involving such challenging magnets.

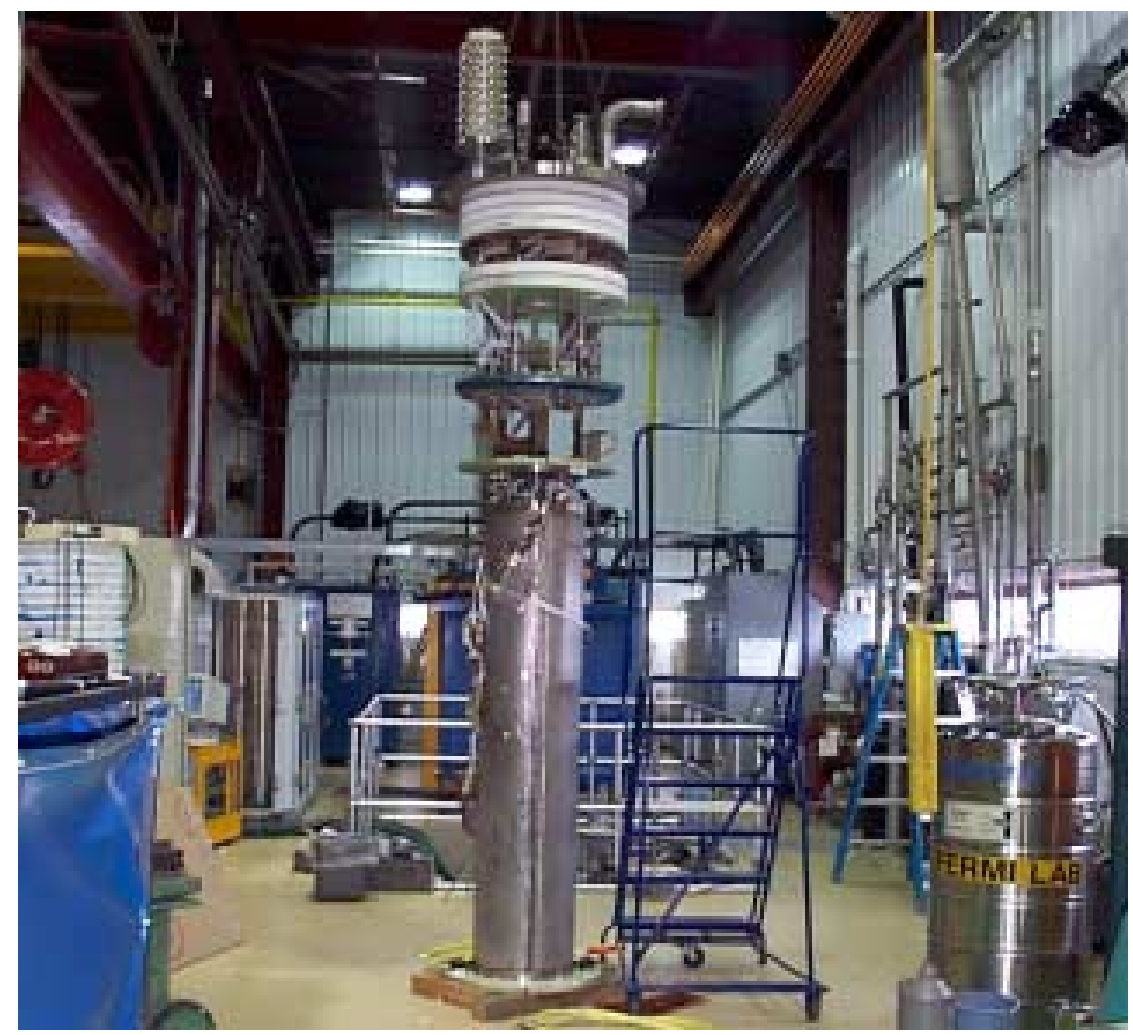

Figure 10.2. The Vertical Magnet Test Facility with a model LHC IR quadrupole ready to be inserted for testing. 


\subsubsection{Magnet Production, Handling and Operation}

The very long magnets discussed in the design study raise a number of issues that are certainly soluble but need both detailed engineering and some R\&D. These include the welding of the half-cores from the inside to permit an open-gap magnet; design of the cryogenic pipes and support system for large thermal contractions; beam pipes and transmission lines with short or non-existent bellows; the use of thicker laminations, extruded steel yokes, and less costly lowcarbon steel to reduce the cost of the steel yokes; and engineering and prototyping of handling, transportation and installation needs. The R\&D plan must contain the fabrication and operation of long magnets in a "string test" that will prototype all of the issues involving the production, handling, installation and operation of the transmission-line magnets.

\subsubsection{Cryogenic System}

Figure 10.3 shows the apparatus used for developing the transmission line. The large magnet is used as the primary of a transformer to induce $100 \mathrm{kA}$ in the single-turn superconducting transmission line. During the past 12 months several different transmission-line configurations were successfully tested in the current loop to $100 \mathrm{kA}$ and beyond.

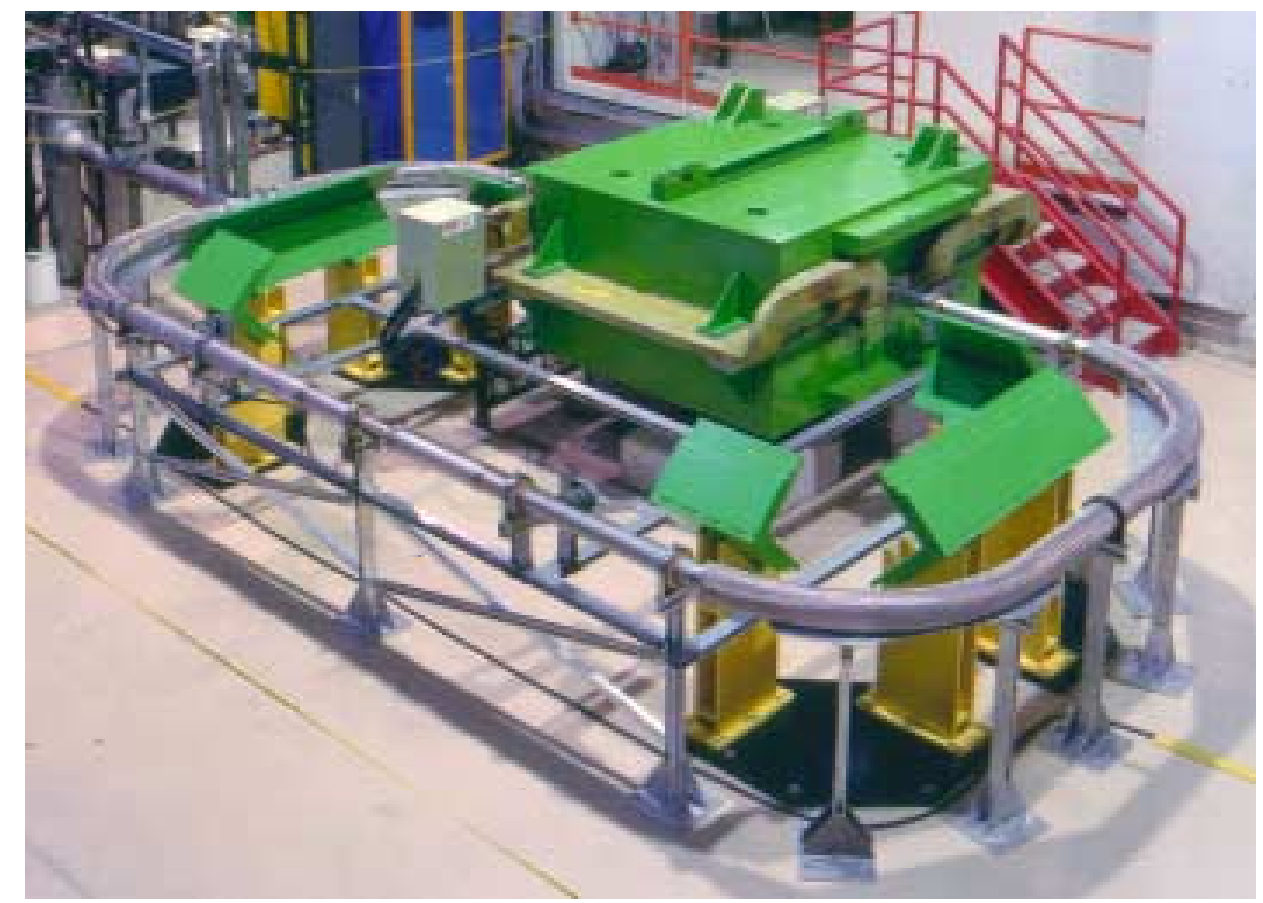

Figure 10.3. The test apparatus used at MW-9 for developing the transmission line.

The transmission-line magnet can, in principle, have a very small heat load, which leads to small cryogenic plants spaced far apart, and low operating costs. Several concepts exist for further optimization of this heat leak. With the plants spaced $40 \mathrm{~km}$ apart, there is the possibility of flow instabilities if there are regions of high relative heat load due to assembly errors or bad vacuum. These issues can only be understood and resolved by the combined use of computer simulation, bench-top laboratory experiments and large-scale demonstrations, using components fabricated in a realistic production environment. The results of these efforts 
will be fed back into the production and quality assurance methods, and will influence the designs of the instrumentation and controls systems, including software for stable control and recovery during upset conditions. The test string planned for operation at the MS-6 location at Fermilab will answer many of these questions. It will have magnets and transmission line mock-ups connected to a well-instrumented refrigerator close by. It is expected to be fully operational in mid-2002, although earlier tests will be done using helium from dewars.

Using turbo compressors could increase the efficiency of the cryogenic plant. In order to use turbo compressors, helium must be mixed with high molecular-weight gasses at the inlet of the machine and effectively separated at the outlet. It is necessary to develop a reliable separation/purification system to keep contamination of the helium below the required level.

As mentioned above, one of the issues of operating with supercritical helium is control of instabilities. One way to avoid these problems is to operate at higher temperature, in gaseous helium. It seems possible to add superconductor to the transmission line, which will have a small cost impact, permitting the transmission line to operate at a higher temperature. This would make control easier and the refrigerator more efficient, and may lead to a net cost reduction.

\subsubsection{Public Acceptance and Outreach}

One of the major hurdles that must be overcome before any big machine can be built is convincing the public that building and operating the collider under their homes and neighborhoods is both safe and environmentally sound. As a community, we have not been very adept at this activity in the past, but we will need to be in the future. A program of understanding the attitudes and real concerns of our neighbors and interacting with them in constructive ways must be started. In addition, understanding what other labs, such as CERN and DESY have done to permit them to construct off their sites would be useful.

Even though we believe the VLHC to be the least expensive way to advance the energy frontier, it is not cheap. Getting the public and political support necessary to be funded will be a very challenging task. It must begin by educating and informing American citizens and their leaders of the importance of this research to their culture, civilization and well-being. As a community, we need to lead in this effort and not leave it up to the funding agencies. Again, understanding what our citizens and leaders view as important and how we might fit in among those perceptions is the first step.

\subsubsection{Other Engineering Studies}

There are numerous issues for the construction and operation of the VLHC that can be solved by engineering studies that will not involve much R\&D. In many cases, these are as important and challenging as the R\&D itself. Among them are studies of the integration of construction and installation to reduce the total construction period. This is a complex organizational problem that could be studied through computer simulation. Other computer simulation studies might include construction and operational safety, which could inform us about the number of necessary egress points, the design of tunnel vehicles, and even safety and administrative practices. 
Studies and simulations of beam accidents and their effect on the environment are absolutely necessary (see Section 5.3.6), as are studies of the environmental impacts of the actual construction of the underground and surface facilities. These studies must include the issue of groundwater handling and radiological contamination; for a detailed discussion, see Section 7.8.

The cost analysis presented in this study is very preliminary. It must be improved by continued iteration and deeper detail. In addition, there are other staging options and designs beside the one chosen for this study. It is important that some effort be invested early in the program to understand what the VLHC might look like and cost if those other designs were used. Exercises of this type must go hand-in-hand with discussions of high-energy physics goals and the politics of international collaboration and planning.

\subsection{R\&D for the Stage-2 VLHC}

\subsubsection{High Field Magnet R\&D}

The Stage- 2 magnet system configuration with the vertical bore arrangement adopted in this study dictated the configuration of the superconducting magnets described in Section 6.1. Arc dipole magnets are based on the common coil design and react-and-wind fabrication technique. This is regarded at this time as the most innovative and cost effective approach, although it requires significant efforts to prove it experimentally. Extensive R\&D efforts in this direction are in progress at Fermilab, LBNL and BNL.

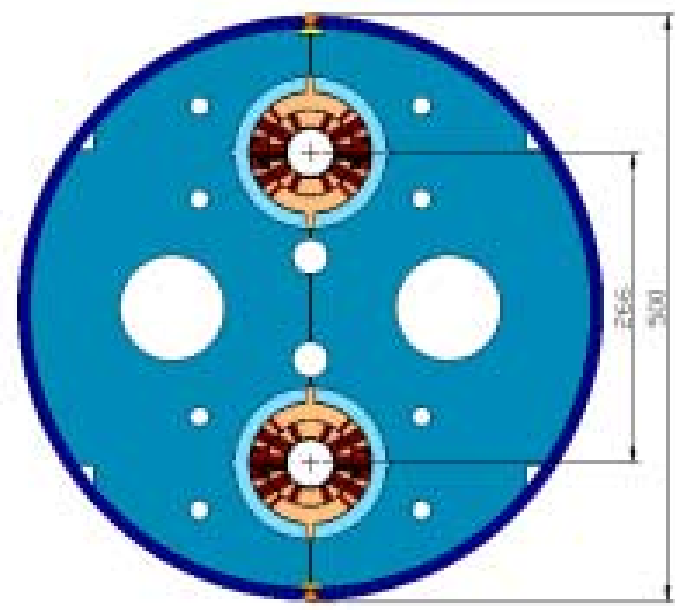

Figure 10.4. Cos-theta cold-warm yoke dipole with vertical bore arrangement. The yoke cold part is shown in the picture, the $15 \mathrm{~mm}$ thick yoke warm part with $I D=680 \mathrm{~mm}$ is not shown.

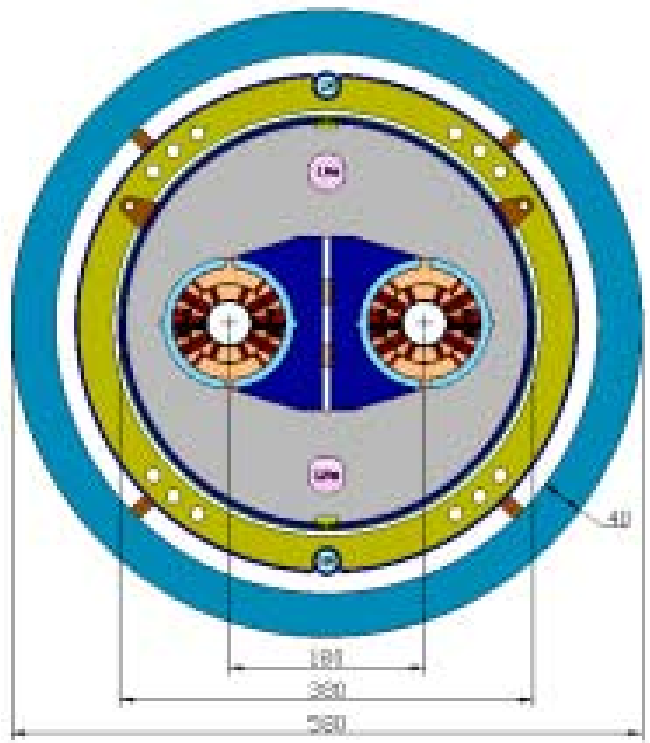

Figure 10.5. Cos-theta warm yoke dipole with minimized size and weight.

There are also other magnet design approaches based on the traditional cos-theta (shelltype) coil geometry which allows both horizontal and vertical bore arrangements as shown in Figure 10.4 and Figure 10.5. These magnets are being developed at Fermilab for the VLHC and meet the Stage-2 VLHC requirements, including operating field range, field quality, critical 
current and critical temperature margin, quench protection, etc. [6]. The designs and parameters of arc quadrupole magnets that match these dipole magnets are described in $[7,8]$.

Due to the small bending radii in the cos-theta type coils, we are forced to use the windand-react technique in order to avoid a large degradation of the cable critical current during coil winding. For the same coil bore diameter, the cos-theta coil configurations allow a reduction of the coil cross section area by about 20 percent with respect to the block-type coil. These coils can be placed much closer to each other, especially in the horizontal bore arrangement, reducing the iron yoke size and the total magnet size, weight and cost. The most straightforward design for the warm yoke [9] is shown in Figure 10.5.

The model magnet R\&D programs carried out in several U.S. national labs and universities have to address the main question: which design approach and fabrication techniques are the best for Stage-2 VLHC? The Stage-2 magnet R\&D program will consist of three traditional phases: Phase 1 - short model R\&D; Phase 2 - full-scale prototype fabrication and tests; and Phase 3 - magnet string (half-cell) test. It will require funding at the level of $\$ 5$ million- $\$ 10$ million per year for five to 10 years to study different magnet designs and technologies, to select the best arc-magnet design, to fabricate and test a series of full-scale prototypes, to build and test a magnet system half-cell to demonstrate the feasibility of the Stage-2 magnet system. Results of this program will provide the basis for the cost estimate and schedule of the Stage-2 VLHC.

\subsubsection{Strand and Cable R\&D}

To build the high field SC magnets with the required Stage- 2 nominal field and gradient and reliable operational margins, the superconducting strands must provide certain parameters [10] discussed in Section 6.1 and summarized in Table 10.1.

Table 10.1. Strand target parameters for the Stage-2 high field magnets.

\begin{tabular}{|c|c|}
\hline Parameter & Value \\
\hline Strand diameter & $0.300-1.000 \mathrm{~mm}$ \\
\hline Critical current density $\mathrm{J}_{\mathrm{c}}(4.2 \mathrm{~K}, 12 \mathrm{~T})$ & $>3000 \mathrm{~A} / \mathrm{mm}^{2}$ \\
\hline Effective filament diameter $\mathrm{d}_{\mathrm{eff}}$ & $<40 \mu \mathrm{m}$ \\
\hline $\mathrm{Cu}$ stabilizer & $>50 \%$ \\
\hline Residual resistivity ratio (RRR) & $>100$ \\
\hline
\end{tabular}

$\mathrm{Nb}_{3} \mathrm{Sn}$ is currently the most likely superconducting material for the Stage- 2 high-field Superconducting magnets, because of its properties and commercial availability. At this time there are three technologies that may reach the desired technical goals: Internal Tin (IT), Modified Jelly Roll (MJR), and Powder-in-Tube (PIT). In the second half of 2000, a Superconductor R\&D National Program for HEP applications was started by the DOE [11]. This program is focussed on reaching the conductor target parameters presented in Table 10.1 and on reducing $\mathrm{Nb}_{3} \mathrm{Sn}$ strand cost. This program has proved remarkably successful. In only a few months of R\&D, U.S. companies IGC and OST improved the critical current density of $\mathrm{Nb}_{3} \mathrm{Sn}$ strands by $30 \%$. Figure 10.6 shows the progress in time of $\mathrm{Nb}_{3} \mathrm{Sn}$ strand critical current 
density since 1984. A trendline extrapolation suggests that reaching the goal of $3000 \mathrm{~A} / \mathrm{mm}^{2}$ is possible by the 2005 .

The critical current density of $\mathrm{Nb}_{3} \mathrm{Sn}$ strands is controlled by a few parameters, such as the volumetric fraction of the $\mathrm{Nb}_{3} \mathrm{Sn}$ phase that can be packed in the non-copper part of a strand, the strand heat treatment procedure, and the flux-pinning mechanisms. All of these parameters are now under investigation and optimization [12-14] in order to reach the required $\mathrm{J}_{\mathrm{c}}$.

A critical parameter responsible for the persistent current effect in magnets and strand stability is the strand magnetization determined by $\mathrm{J}_{\mathrm{c}} \cdot \mathrm{d}_{\mathrm{eff}}$, where $\mathrm{d}_{\mathrm{eff}}$ is an effective filament diameter. For the $\mathrm{Nb}_{3} \mathrm{Sn}$ strands produced using the most cost effective technologies such as IT or MJR, $\mathrm{d}_{\text {eff }}$ is quite large about $100-120 \mu \mathrm{m}$, and only PIT provides a small $\mathrm{d}_{\text {eff, }}$ on the level of $50 \mu \mathrm{m}$, which significantly decreases the magnetization effects, but results in increased strand cost [15]. An optimization of the strand design and technology aimed at reducing $\mathrm{d}_{\mathrm{eff}}$ in the high $\mathrm{J}_{\mathrm{c}} \mathrm{Nb}_{3} \mathrm{Sn}$ strands is an important next step of the conductor R\&D program.

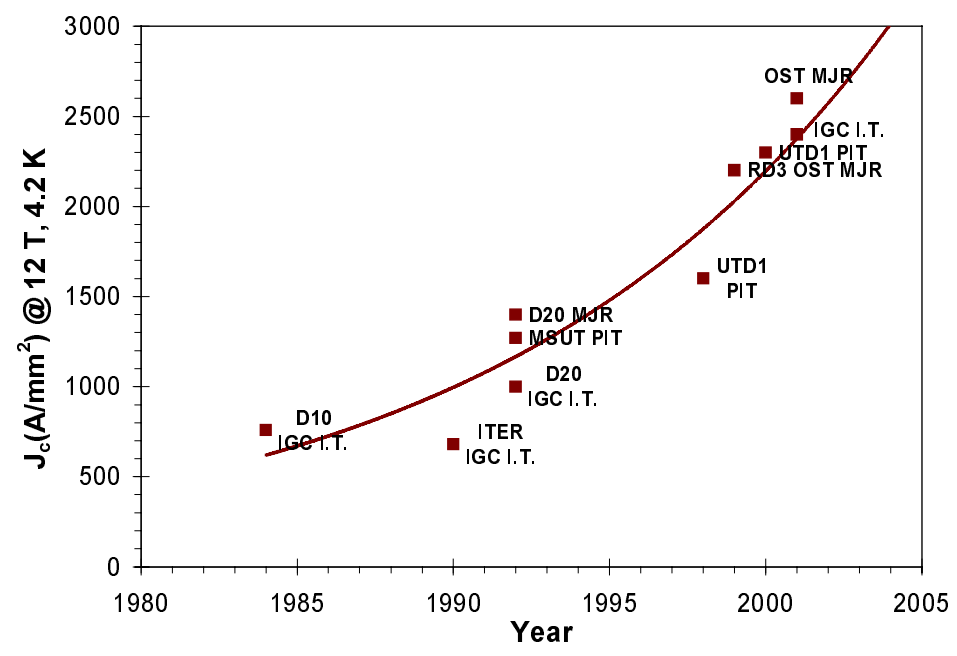

Figure 10.6. Improvement in $J_{c}(4.2 \mathrm{~K}, 12 \mathrm{~T})$ of $\mathrm{Nb}_{3} \mathrm{Sn}$ since 1984.

It is necessary during magnet and cable fabrication and operation to reduce the critical current degradation relative to that of the original virgin strands due to strain as well as to control the interstrand resistance responsible for the thermal and electromagnetic coupling between the strands. In the wind-and-react technique, both strand plastic deformation during cabling (before reaction), and cable compression in the coil during magnet fabrication and operation (after reaction, due to coil pre-compression and Lorenz force) contribute to $I_{c}$ degradation and interstrand resistive coupling. In the react-and-wind method, there is also $\mathrm{I}_{\mathrm{c}}$ degradation due to the bending strain introduced during winding. The cable R\&D program goals are summarized in Table 10.2. Cable studies performed at Fermilab and LBNL show significant progress in developing SC cable for accelerator magnets during last 1-2 years. The results obtained prove that cable $R \& D$ goals will be certainly achieved [16-19].

As was shown in section 6.1.8, the cost of $\mathrm{Nb}_{3} \mathrm{Sn}$ strands is one of the major cost drivers of the Stage-2 magnet system. NbTi strands for accelerator magnets, thanks to previous HEP projects and the MRI magnet business, can be purchased in large quantities for about $\$ 100 / \mathrm{kg}$. The small quantity price (e.g., 2 tons purchased for the U.S. LHC program) is as much as 50\% 
higher - about $\$ 150 / \mathrm{kg}$. It is apparent that lower prices in the future for this conductor is unlikely to be realized, since most of the cost savings and property improvements available for $\mathrm{NbTi}$ have already been realized. The status of $\mathrm{Nb}_{3} \mathrm{Sn}$ is in stark contrast to $\mathrm{NbTi}$. The quantities being purchased annually are small at present (about 0.5 ton for accelerator magnets, two tons for MRI magnets, and two tons for fusion energy magnets). This factor of 100 lower volume means that the economies of scale available for NbTi do not exist. Consequently, at this time, $\mathrm{Nb}_{3} \mathrm{Sn}$ conductor costs about $\$ 750 / \mathrm{kg}$.

Table 10.2. Cable R\&D goals.

\begin{tabular}{|c|c|}
\hline Parameter & Value \\
\hline Number of strands & $28-60$ \\
\hline Packing factor & $87-90 \%$ \\
\hline Cabling degradation & $<10 \%$ \\
\hline Bending degradation & $<10 \%$ \\
\hline Compression degradation & $<10 \%$ \\
\hline Strand crossover resistance & $>10 \mu \mathrm{Ohm}$ \\
\hline
\end{tabular}

The goal of the HEP Conductor Development Program is to demonstrate that $\mathrm{Nb}_{3} \mathrm{Sn}$ costs can be reduced to the $\$ 150 / \mathrm{kg}$ range when the production processes are scaled-up to those used for $\mathrm{NbTi}$. In order to demonstrate this, the key is to scale up the unit process quantities, i.e. billet sizes, to the $\mathrm{NbTi}$ equivalent, and also to procure the raw materials in quantities that will show economies of scale. The necessary technological steps and the costs to do this, as well as the overall process costs are discussed in reference [20]. These scale-up efforts will require about $\$ 1$ million per year for three years for each process that is selected for scale-up. However, the cost savings projected for a VLHC-size project will return this investment many times over, and may be the critical factor determining the feasibility of such a project.

\subsubsection{IR Magnets for Flat Beam Optics}

The parameters of various IR magnets required for the flat beam optics are given in Table 6-6. The layout is shown in Figure 3.10. The following are the major design considerations of these IR magnets:

- Small aperture (especially in quadrupoles for generating high gradients)

- Brittle superconductors that must be used for generating high field/gradient

- Large Lorentz forces (associated with high fields)

- Small separation between the two apertures (associated with the doublet optics)

Given the importance of these few magnets, state-of-the-art superconductors are used in the design. The ends of conventional cos-theta designs put a practical limit on the minimum aperture, particularly in quadrupole magnets made with brittle material. To overcome this and other limitations, Stage-2 VLHC IR doublets are based on non-traditional magnet designs with racetrack coils. These are conductor friendly designs with large bend radii and are suitable for containing large Lorentz forces. 
The minimum separation between the two apertures in first quadrupole from the interaction point determines the layout of the entire interaction region and the maximum $\beta$-function for the given optics, since it sets the distance of that quadrupole from the IP. In addition, it also establishes the maximum pole-tip field of this and other magnets. In conventional 2-in-1 designs, the minimum separation is determined by the conductor width required for generating field gradient and the support structure required for containing large Lorentz forces. In the proposed design the amount of conductor between the two apertures is much smaller than on any other side and no support structure is required between the two apertures. This brings a large reduction in spacing (by about a factor of five) between the two apertures. The cross section of the proposed design is shown in Figure 10.7. In order to facilitate large bend radii, the return path of all turns is further away from the aperture. Field contours and field lines in the aperture of this magnet are shown on the right in Figure 10.7. The design is based on reactand-wind $\mathrm{Nb}_{3} \mathrm{Sn}$ superconductor with a current density in the coil of $2500 \mathrm{~A} / \mathrm{mm}^{2}$ at $12 \mathrm{~T}$. The magnets based on these design principles use a much larger amount of conductor than in a conventional design. However, the cost of conductor is not a major issue in designing a few critical high performance magnets.
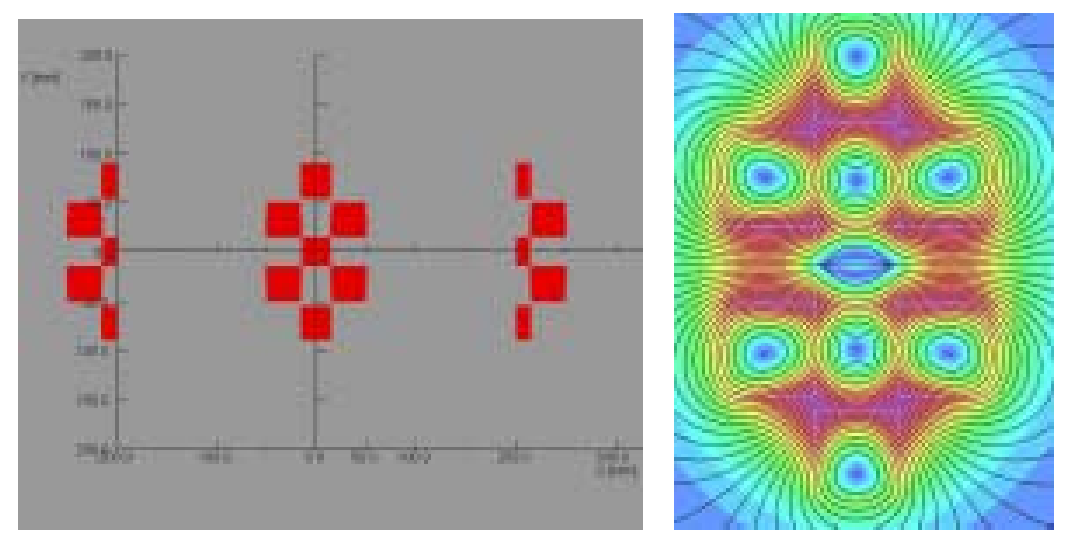

Figure 10.7. A conceptual design of 2-in-1 Q1A (left) and flux distribution in the coil region of the magnet (right). The design minimizes the spacing between two apertures.

This design also introduces a strong coupling and cross talk between the two apertures. The superimposition of a dipole field on the quadrupole coils increases the peak field in the conductor and reduces the maximum achievable gradient. The maximum gradient in $\mathrm{Q} 1 \mathrm{~A}$ (minimum separation) is, therefore, $400 \mathrm{~T} / \mathrm{m}$ as compared to $600 \mathrm{~T} / \mathrm{m}$ in Q1B, Q2A and Q2B where this effect is much smaller. The goal is to minimize the cross talk induced harmonics with the exception of the dipole field. By symmetry, the normal even harmonics and skew odd harmonics are theoretically zero. The target field harmonics will be obtained either by design or by correction. As the design evolves, the harmonics are expected to be reduced. All four quadrupoles will have a different cross section.

The VLHC IR region uses three types of dipole magnets. The design of the insertion region dipoles is also based on racetrack coils. D1A, D1B (one each on each side) are single aperture dipoles and D2 (two on each side) is a 2-in-1 dipole. The operating field in the smaller aperture $(25 \mathrm{~mm}$ ) dipole D1A is $16 \mathrm{~T}$ (quench field $\sim 18 \mathrm{~T}$ ) and it uses High Temperature Superconductor (BSCCO-2212) in a hybrid design. The operating field in the larger aperture 
(50 $\mathrm{mm}$ ) dipole D1B is reduced to $12 \mathrm{~T}$ to reduce the Lorentz forces in larger aperture. This field can be entirely obtained by $\mathrm{Nb}_{3} \mathrm{Sn}$ superconductor.

\subsubsection{Cryogenic-Related R\&D for a High Field VLHC}

The large total cryogenic system power, the magnet string lengths, total cold mass, and total helium inventory for a Stage-2 cryogenic system go well beyond that of previous systems. Scaling up standard cryogenic methods and systems to the size of a VLHC results in some new problems which call for R\&D. The following is a list of possible areas for R\&D in cryogenics for a high field VLHC.

1. It would be very desirable to reduce the installed cryogenic system power to significantly less than the presently estimated $105 \mathrm{MW}$. The heat load with the biggest potential for reduction by some innovative method is the synchrotron radiation load at $100 \mathrm{~K}$. Developing methods for removing a fraction of the synchrotron radiation at room temperature could permit a dramatic reduction in cryogenic system size (both power and inventory) for a high-field VLHC.

2. Static heat load (thermal radiation and conduction) reduction might be possible. One should investigate the possible utilization of techniques developed for MRI or by NASA (e.g., trace cooling of supports and/or trace cooling of an inner shield). It could also be beneficial to fund a development effort toward better multilayer insulation (MLI) and MLI installation.

3. Improve cryogenic plant efficiency. Although there is no thermodynamic advantage in a heavier gas as a working fluid in a cycle, there might be some advantage of a higher molecular weight refrigerant (nitrogen or neon) in permitting the use of very large-scale air separation compressors and expanders. Hans Quack (cryogenics professor at University of Dresden, Germany) states [21]: "At a paper, which I am going to present at the CEC in Madison, I am going to propose a new system with a mixture of helium and neon as refrigerant. Such a refrigerant is nearly as efficient as helium, it allows power recovery of the turbines and a turbo compressor as main compressor and one can go down to $27 \mathrm{~K} . "$

4. Improve helium screw compressor efficiency. This R\&D item was listed at the Bloomington workshop in 1994 and the Jefferson Lab workshop in 1999. Quoting from the 1999 report [22]: "Screw compressor efficiency (FY94 recommendation): This one component accounts for more than half of total inefficiency of a refrigeration system. We use screw compressors rather than the more efficient reciprocating compressors because of their very high reliability. The root problem is that the He market is so small compared to Freon that it is difficult to get the vendor's R\&D attention."

5. Another R\&D item from the 1999 Jefferson Lab workshop which is also worth listing is a study of flow instabilities in long systems. Density wave instabilities could cause serious trouble for a VLHC, and a thorough understanding of this phenomenon would be required to ensure good design. 


\subsubsection{Synchrotron Radiation and Vacuum}

Almost twenty years ago, when people started studying very high energy colliders like the SSC, the LHC and the Eloisotron, it was immediately recognized that the effect of synchrotron radiation on the beam tube vacuum and the cryogenic system would be a major issue and $R \& D$ challenge [23-26]. Numerous experiments and calculations have been done since that time, and they still remain major issues for the design and operation of high energy hadron colliders.

The cryogenic issues fall into two categories: removal of heat from the beam-tube liner, and the total heat load. These are complex but well understood cryogenic issues involving cryogen flow, thermal conductivity, pressure drop, and other parameters. So many parameters, in fact, that the solutions must be experimentally verified in addition to being simulated on computers. We anticipate, however, that these simulations of this complex system will be very useful in converging on a proper design.

The vacuum system is more difficult to understand, and must be experimentally verified. The usual solution proposed, but which will be tested first in the LHC approximately six years from now, is to have a beam-tube liner, shown in Figure 10.8, which intercepts the synchrotron radiation at elevated temperature (to take advantage of improved Carnot efficiency), and which has small perforations that permit gas to escape and be trapped, cryopumped and shadowed between the liner and the beam tube. This system must be tested at synchrotron light sources, although a lot will be learned during the early operation of the LHC. Among the issues are pumping speed, desorption coefficients and clean-up time.

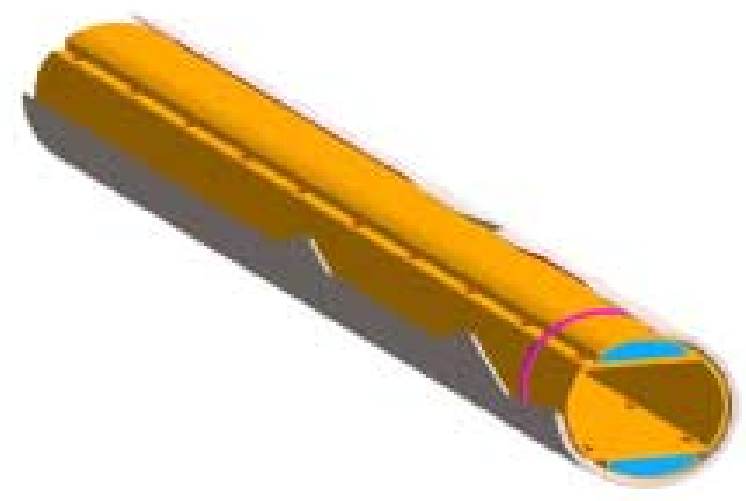

Figure 10.8. A model of the VLHC beam-tube liner.

A photon stop has been proposed as a possibility to intercept the intense synchrotron radiation in the Stage-2 VLHC [27]. The photon stop, shown in Figure 10.9, is a piece of metal or semiconductor that protrudes into the beam pipe between magnets, and intercepts all or most of the synchrotron radiation before it hits the beam-tube liner. The advantage of the photon stop is that it may be able to be operated at temperatures close to $300 \mathrm{~K}$, thus saving significant amounts of cryogenic power, possibly more than 30 percent. A first pass engineering design of such a device has been completed [28]. The results of numerical impedance calculations [29] give additional support to the viability of the approach. 


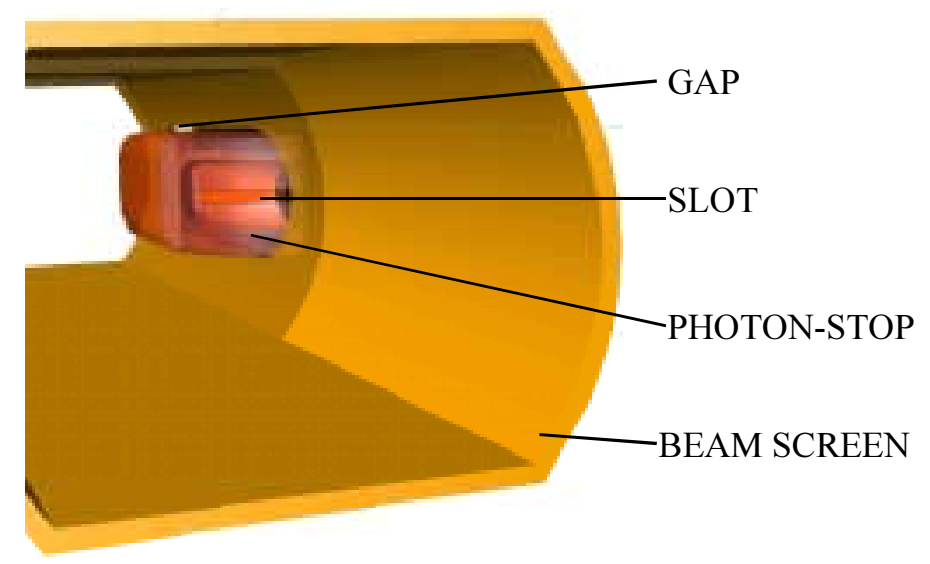

Figure 10.9. A model of a proposed VLHC photon stop, to be placed between magnets to absorb synchrotron radiation at $300 \mathrm{~K}$.

An Argonne National Laboratory-Fermilab collaboration proposes a multi-step R\&D plan towards the realization of such a device [30]. In the first step, a photon stop prototype would undergo tests in the Advanced Photon Source (APS) photon beam in a device that could be operated wholly at room temperature. Simultaneously, cryogenic tests of prototypes could take place at Fermilab. A photon beam is not required for such tests. Full-blown cryogenic and vacuum test series of the photon-stop in a VLHC-like setting is proposed as the final stage. An important part of the R\&D is to understand the effect of the photon stops on the beam stability. This can be accomplished with a combination of sophisticated calculations and bench or beam measurements.

\section{References}

[1] J. Lach, R. Bauer, P. Conroy, C. Laughton, E. Malamud, "Cost Model for a 3 TeV VLHC Booster Tunnel," Fermilab TM-2048, April 11, 1998.

[2] The Robbins Company, "Tunnel Cost Reduction Study," July 16, 1999.

[3] Joseph Lach, "Instrumentation of a 17ft Diameter Tunnel Boring Machine in the Chicago Dolomite."

[4] G.W. Foster, "An Unmanned, Battery-Operated TBM Tunneling Scenario," October 6, 1998. (VLHCPub-85).

[5] G.W. Foster, M.P. May, "Generic Accelerator tunneling R\&D at Fermilab," August 1999.

[6] V.V. Kashikhin and A.V. Zlobin, "Magnetic Designs of 2-in-1 $\mathrm{Nb}_{3}$ Sn Dipole Magnets for VLHC," IEEE Transactions on Applied Superconductivity, v. 11, No. 1, March 2001, p. 2176.

[7] D.R. Chichili et al., "Mechanical Design and Analysis of Fermilab 2-in-1 Shell-Type $\mathrm{Nb}_{3}$ Sn Dipole Models," IEEE Transactions on Applied Superconductivity, v. 11, No. 1, March 2001, p. 2288.

[8] V. Kashikhin and A.V. Zlobin, "Conceptual Designs of 2-in-1 $\mathrm{Nb}_{3} \mathrm{Sn}$ Arc Quadrupole Magnets for VLHC," Fermilab, TD-01-019, March 2001.

[9] V. Kashikhin and A.V. Zlobin, "2-in-1 Arc Dipole and Quadrupole Magnets for VLHC based on the shelltype coils and vertical bore arrangement," Fermilab, TD-01-034, May 2001.

[10] G. Ambrosio et al., "Superconductor Requirements for the HFM Program at Fermilab," Fermilab, TD-99-073, December 15, 1999.

[11] R.M. Scanlan et al, "Conductor Development for High Field Dipole Magnets," IEEE Transaction on Applied Superconductivity, vol. 10, p.288, March 2000. 
[12]M. T. Naus, P. J. Lee and D. Larbalestier, "The Influence of the Starting Cu-Sn Phase on the Superconducting Properties of Subsequently Reacted Internal-Sn $\mathrm{Nb}_{3} \mathrm{Sn}$ Conductors," IEEE Transactions on Applied Superconductivity, v. 11, No. 1, March 2001, p. 3569.

[13]E. Barzi et al., "Heat Treatment Optimization of Internal $\mathrm{Tin}^{\mathrm{Nb}_{3}} \mathrm{Sn}$ Strands," IEEE Transactions on Applied Superconductivity, v. 11, No. 1, March 2001, p. 3573.

[14] J. McDonald and E. Barzi, "A Model for Jc in Granular A-15 Superconductors," IEEE Transactions on Applied Superconductivity, v. 11, No. 1, March 2001, p. 3884.

[15]E. Barzi et al., "Study of $\mathrm{Nb}_{3}$ Sn Strands for Fermilab's High Field Dipole Models," IEEE Transactions on Applied Superconductivity, v. 11, No. 1, March 2001, p. 3595.

[16]E. Barzi et al., "Progress report on superconducting strand and cable R\&D," Fermilab, TD-01-013, February $14,2001$.

[17] D. R. Dietderich et al., "Critical Current of Superconducting Rutherford Cable in High Magnetic Fields with Transverse Pressure," IEEE Trans. on Appl. Superconductivity, v. 9, pp. 122-125, 1999.

[18] P. Bauer et al., "Fabrication and Testing of Rutherford-Type Cables for React and Wind Accelerator Magnets," IEEE Transactions on Applied Superconductivity, v. 11, No. 1, March 2001, p. 2457.

[19]E. Barzi et al., "Strand Critical Current Degradation in $\mathrm{Nb}_{3}$ Sn Rutherford Cables," IEEE Transactions on Applied Superconductivity, v. 11, No. 1, March 2001, p. 2134.

[20] R.M. Scanlan, "Conductor cost/performance status report for Snowmass 2001," LBNL SCMAG 737, February 2001.

[21]H. Quack, private communication.

[22]D. Arenius et al., "VLHC Accelerator Technologies Workshop, Jefferson Lab, February 8-11, 1999, Cryogenics and Beam Screens Working Group," VLHCPUB-214.

[23] V. Anashin, O. Groebner et al., "Synchrotron Radiation Induced Gas Desorption from a Prototype LHC Beam Screen at Cryogenic Temperature", CERN, LHC Project Note 7, Sept. 1995.

[24] W.C. Turner, "Beam Tube Vacuum in Future Superconducting Proton Colliders," SSCL Preprint 564, Oct. 1994.

[25]D. Bintinger, H. Jostlein, P. Limon, D. Trbojevic, "Status of the SSC Photodesorption Experiment," SSC-102mc, Dec. 1986.

[26]D. Bintinger, P. Limon, R.A. Rosenberg, "Photodesorption from copper-plated stainless steel at liquid-helium temperature and at room temperature," J. Vac. Sci. Technol. A, 7, 1, 59-63 (Jan. 1989).

[27]I. Terechkine, "Synchrotron Radiation in the VLHC," Fermilab, Technical Division Note, unpublished, 01/01.

[28] P. Bauer et al., "A Photon-Stop for Stage-2 - Engineering Design - Part 1," Fermilab, Technical Division Note TD-01-023, 04/01.

[29] N. Solyak et al., "A Photon-Stop for Stage-2 - Impedance Calculations," Fermilab, Technical Division Note TD-01-014, 04/01.

[30]P. Bauer, et al., "Photon Stop R\&D Proposal," Fermilab Technical Division Note TD-01-30. 